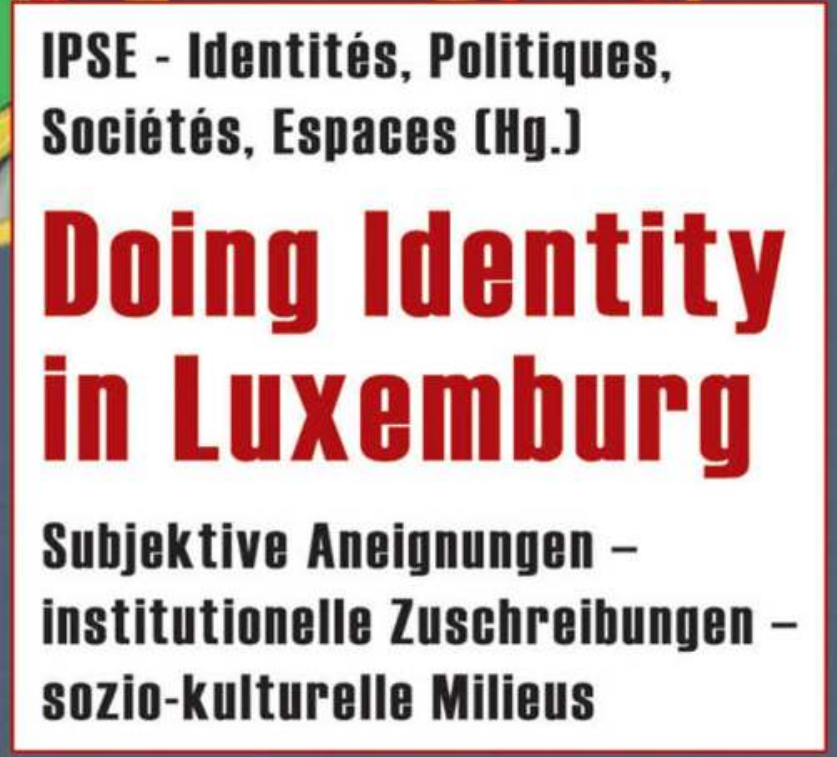

[transcript $]$ Kultur und soziale Praxis 
IPSE - Identités Politiques Sociétés Espaces (Hg.)

Doing Identity in Luxemburg 


\section{Wissenschaftlicher Beirat:}

Wilhelm Amann, Sonja Kmec, Sophie Neuenkirch, Agnès Prüm, Rachel Reckinger, Christian Schulz, Christian Wille

\section{Autorinnen und Autoren:}

Wilhelm Amann, Christel Baltes-Löhr, Viviane Bourg, Marion Colas-Blaise, Paul Dell, Fernand Fehlen, Paul Di Felice, Sylvie Freyermuth, Peter Gilles, Georg Mein, Sonja Kmec, Fabienne Lentz, Agnès Prüm, Gian Maria Tore, Sebastian Reddeker, Rachel Reckinger, Christian Schulz, Sebastian Seela, Heinz Sieburg, Melanie Wagner, Christian Wille

\section{Übersetzerinnen und Übersetzer:}

Marion Gretscher, Eva Nossem, Dietmar Zehl 
IPSE - Identités Politiques Sociétés Espaces (Hg.)

\section{Doing Identity in Luxemburg}

Subjektive Aneignungen - institutionelle Zuschreibungen sozio-kulturelle Milieus 


\section{Bibliografische Information der Deutschen Nationalbibliothek}

Die Deutsche Nationalbibliothek verzeichnet diese Publikation in der Deutschen Nationalbibliografie; detaillierte bibliografische Daten sind im Internet über http://dnb.d-nb.de abrufbar.

\section{(c) (1) $\Theta \Theta$}

Dieses Werk ist lizenziert unter der Creative Commons Attribution-NonCommercial-NoDerivs 4.0 Lizenz (BY-NC-ND). Diese Lizenz erlaubt die private Nutzung, gestattet aber keine Bearbeitung und keine kommerzielle Nutzung. Weitere Informationen finden Sie unter https://creativecommons.org/licenses/by-nc-nd/4.o/deed.de Um Genehmigungen für Adaptionen, Übersetzungen, Derivate oder Wiederverwendung zu kommerziellen Zwecken einzuholen, wenden Sie sich bitte an rights@transcript-publishing.com

Die Bedingungen der Creative-Commons-Lizenz gelten nur für Originalmaterial. Die Wiederverwendung von Material aus anderen Quellen (gekennzeichnet mit Quellenangabe) wie z.B. Schaubilder, Abbildungen, Fotos und Textauszüge erfordert ggf. weitere Nutzungsgenehmigungen durch den jeweiligen Rechteinhaber.

\section{(C) 2010 transcript Verlag, Bielefeld}

Umschlaggestaltung: Kordula Röckenhaus, Bielefeld Korrektorat: Kirsten Hellmich, Bielefeld

Satz: Alexander Masch, Bielefeld

Druck: Majuskel Medienproduktion $\mathrm{GmbH}$, Wetzlar

Print-ISBN 978-3-8376-1448-0

PDF-ISBN 978-3-8394-1448-4

https://doi.org/10.14361/9783839414484

Gedruckt auf alterungsbeständigem Papier mit chlorfrei gebleichtem Zellstoff. Besuchen Sie uns im Internet: https://www.transcript-verlag.de Unsere aktuelle Vorschau finden Sie unter www.transcript-verlag.de/vorschau-download 


\section{Inhalt}

\section{Vorwort}

Rachel Reckinger, Christian Schulz, Christian Wille | 7

\section{Identitätskonstruktionen erforschen}

Rachel Reckinger und Christian Wille | 11

\section{Sozio-kulturelle Milieus in Luxemburg}

Wilhelm Amann, Fernand Fehlen, Georg Mein 37

\section{Sprachen und Identitäten}

Peter Gilles, Sebastian Seela, Heinz Sieburg, Melanie Wagner | 63

\section{Räume und Identitäten}

Marion Colas-Blaise, Sylvie Freyermuth, Sonja Kmec, Gian Maria Tore,

Christian Schulz | 105

\section{Bilder und Identitäten}

Wilhelm Amann, Viviane Bourg, Paul Dell, Fabienne Lentz, Paul Di Felice, Sebastian Reddeker $\mid 165$

\section{Alltagskulturen und Identitäten}

Christel Baltes-Löhr, Agnès Prüm, Rachel Reckinger, Christian Wille | 235

\section{Identitätskonstruktionen in Luxemburg}

Rachel Reckinger, Christian Schulz, Christian Wille | 295

Autoren/-innen | 299 



\section{Vorwort}

\section{Rachel Reckinger, Christian Schulz, Christian Wille}

Was charakterisiert denn nun die Luxemburger und Luxemburgerinnen? Mit dieser Frage wurden wir in den letzten Jahren immer wieder konfrontiert, wenn von den Arbeiten zu diesem Buch berichtet wurde. Offenbar weckt das Vorhaben, sozio-kulturelle Identitäten zu erforschen, Erwartungen, die wir enttäuschen werden. Denn das Anliegen dieses Buches besteht nicht in der Festschreibung der vermeintlich stypischen Luxemburger/-innen<, sondern in der Untersuchung von Identitätskonstruktionen auf unterschiedlichen Ebenen des sozialen Miteinanders. Dabei geht es um Prozesse der Aneignung oder Zuschreibung, die von den Subjekten selbst, aber auch von öffentlichen Institutionen vollzogen werden. Solche Prozesse, die den Forschenden oft nur als Momentaufnahme zugänglich werden, wurden in unterschiedlichen Gesellschaftsbereichen aufgedeckt, und dies stets im Kontext der Luxemburger Gesellschaft. Damit bildete nicht Luxemburg in seiner wie auch immer gearteten Verfasstheit den Ausgangspunkt der folgenden Kapitel, sondern die Frage nach sozio-kulturellen Identitäten und ihren Artikulationsformen. Diese Perspektive wird umso einsichtiger, wenn die durch Arbeitsmigration bedingte Vielfältigkeit, der multilinguale Kontext oder die internationalen Verflechtungen und somit die Pluralisierung der Identitätsangebote in der Luxemburger Gesellschaft berücksichtigt werden.

Dieses Buch geht aus einem dreijährigen Forschungsprojekt mit dem Titel »IDENT - Sozio-kulturelle Identitäten und Identitätspolitiken in Luxemburg« hervor. Die Idee zum IDENT-Projekt entstand im Jahr 2006 im Zuge der Konstituierung der Forschungseinheit (Unité de Recherche) »IPSE - Identités, Politiques, Sociétés, Espaces« (UR IPSE) innerhalb der Fakultät für Sprachwissenschaften und Literatur, Geisteswissenschaften, Kunst und Erziehungswissenschaften der Universität Luxemburg. Die Kurzbezeichnung »IPSE« verweist bereits auf ihre Ausrichtungen: Sie wendet sich besonders dem Themenfeld der Identitätskonstruktionen $\mathrm{zu}$, wobei diese aus verschiedenen thematischen Blickwinkeln und unter interdisziplinärem Zugriff erforscht werden. Das IDENT-Projekt bildete neben anderen Aktivitäten der damals jungen Forschungseinheit einen hervorragenden Rahmen, um die Arbeitsfähigkeit der UR IPSE unter Beweis zu stellen. So waren 
über 20 Forscher/-innen aus zwölf Disziplinen ${ }^{1}$ an dem Großprojekt beteiligt, dessen Mehrwert - wie noch zu sehen sein wird - in der thematischen und methodischen Vielfalt der Bearbeitung des Forschungsgegenstands liegt. Während der dreijährigen Projektarbeit wurden - wenn auch nicht immer einfache - disziplinenübergreifende Lernprozesse angestoßen, die sich in den Beiträgen dieses Buches widerspiegeln. Dafür maßgeblich war die Neugierde, Offenheit und vor allem die Courage der beteiligten Forscher/-innenpersönlichkeiten, auch fachfremdes Terrain zu betreten und sich hier zu behaupten. Die interdisziplinäre Zusammenarbeit hat jedoch nicht nur die Perspektiven der Forscher/-innen erweitert, ebenso ist der föderative Charakter des IDENT-Projekts hervorzuheben. So bildeten die zahlreichen Arbeitsgruppen und Workshops, die regelmäßig und stets ergebnisorientiert stattfanden, einen Rahmen, in dem sich die Kollegen/-innen von fachlicher und persönlicher Seite zunehmend besser kennenlernten. Dies förderte auch die Zusammenarbeit in anderen Zusammenhängen des Forschungsalltags und festigte das Selbstverständnis der Forschungseinheit IPSE. Dieser kurze Einblick in die Genese und Funktionsweise des IDENT-Projekts hilft den Lesern/-innen, die Beiträge in diesem Buch einzuordnen, die aus einer rein disziplinären Sicht z.T. (noch) ungewöhnlich erscheinende methodisch-konzeptionelle Wege gehen. Darin sehen die über 20 Autoren/-innen, die aus mindestens vier unterschiedlichen nationalen Universitätssystemen stammen, jedoch die Besonderheit ihrer Zusammenarbeit, die in weiteren Projekten dieses Zuschnitts verfeinert und weiterentwickelt werden soll.

Die hier präsentierten Ergebnisse der Identitätsforschung sind in den Themenfeldern Sprachen, Räume, Selbst- und Fremdbilder sowie Alltagskulturen anzusiedeln. Ihnen vorgeschaltet ist eine ausführliche Erläuterung der IDENTProjektarchitektur in konzeptioneller, methodischer und forschungspraktischer Hinsicht, die den roten Faden für die nachfolgenden Kapitel bildet (Kap. 2). Dazu gehört die für die Luxemburgische Gesellschaft erstmalige Auseinandersetzung mit sozio-kulturellen Milieus (Kap. 3), die anhand anschaulicher Profile dargelegt und auf die sich die Autoren/-innen im Weiteren beziehen werden. So werden sprachenbezogene Identitäten in verschiedenen sozio-kulturellen Milieus und ihre Ausdrucksformen im öffentlichen Raum beleuchet, ebenso wie die gesellschaftliche Sprachen-Debatte in Printmedien (Kap. 4). Auf einem konstruktivistischen Verständnis aufbauend wird außerdem der >Raum Luxemburg< nachgezeichnet, so wie ihn der touristische Diskurs auf verschiedenen Ebenen konturiert, und den raumbezogenen Identitätskonstruktionen der Luxemburger Wohnbevölkerung gegenübergestellt (Kap. 5). Die sich anschließende Untersuchung von visuellen und sprachlichen Bildern wird interdiskurstheoretisch gerahmt und deckt Mechanismen der Identitätskonstruktion in den Bereichen Wirtschaft, Kultur und Mi-

1 Dabei handelt es sich im Wesentlichen um Anglistik, Gender Studies, Geografie und Raumplanung, Germanistik, Geschichte, Interkulturelle Kommunikation, Luxemburgistik, Medienwissenschaft, Romanistik, Soziologie und Visuelle Kunst. 
gration auf (Kap. 6). In alltagskultureller Hinsicht schließlich werden auf einem praxeologischen Kulturbegriff aufbauend Konsum- und Genderidentitäten sowie Alteritätsverhältnisse untersucht (Kap. 7). In einer abschließenden Zusammenschau der Forschungsergebnisse werden Rückschlüsse auf Prozesse der Identitätsbildung in Luxemburg und im Spannungsfeld von zugeschriebenen und angeeigneten Identitäten gezogen (Kap. 8).

Besonderer Dank gilt den Personen, ohne die das IDENT-Projekt nicht möglich gewesen wäre bzw. die es mitgetragen und unterstützt haben. Dazu zählen diejenigen Einwohner/-innen Luxemburgs, die an unseren quantitativen und qualitativen Befragungen teilgenommen haben, und alle, die uns mit Rat und Tat über die Projektphasen hinweg begleitet haben, insbesondere Ralf Bläser, Pascale Fack, Daniel Gardemin, Jean-Marie Klinkenberg, Charles Margue, Antonella Di Pasquale, Jürgen Pohl, Michael Vester und viele andere, die nicht namentlich genannt werden. Der Université du Luxembourg, stellvertretend ihrer Vizerektorin für Forschung, Luciënne Blessing, sowie dem Dekan der Fakultät für Sprachwissenschaften und Literatur, Geisteswissenschaften, Kunst und Erziehungswissenschaften, Michel Margue, danken wir für die großzügige Unterstützung des Vorhabens. Nicht zuletzt möchten wir uns für die professionelle Zusammenarbeit mit dem Verlag und für die Leistungen der Übersetzer/-innen bedanken, die aus einem mehrsprachigen $^{2}$ und von verschiedenen disziplinären sowie universitären Traditionen gefärbten Manuskript eine deutsche, französische und englische Fassung angefertigt haben.

2 | Die Wahl der Sprachen, in denen die Beiträge originär verfasst worden sind, stand den beteiligten Forschern/-innen frei. 



\title{
2. Identitätskonstruktionen erforschen
}

\author{
Rachel Reckinger und Christian Wille
}

\subsection{Theoretisch-Konzeptionelle Annäherung AN IDENTITÄTEN}

\section{Identitäten in Luxemburg}

Im Mittelpunkt dieses Buches stehen Identitäten in Luxemburg. Der hier gewählte Plural deutet bereits an, dass es weder um die Festschreibung einer >nationalen Identität $<$ noch um die Bestimmung >des Luxemburgers/der Luxemburgerin « gehen kann. Vielmehr stehen Prozesse der Identitätskonstruktion im Zentrum bzw. ihre für die Forschenden empirisch zugänglichen Ausdrucksformen auf unterschiedlichen Ebenen. Hier schließt sich die Frage an, weshalb der Untersuchungskontext >Luxemburg< zur Betrachtung von Identitäten gewählt wurde. Oder in anderen Worten: Was macht Luxemburg unter dem Aspekt der Identitätskonstruktion interessant? Zur Annäherung an diese Frage gilt es zunächst, den Prozess der Enttraditionalisierung und seine Auswirkungen auf Identitäten zu klären, und schließlich auf einige Besonderheiten der Luxemburger Gesellschaft einzugehen.

Hinsichtlich des Identitätsbegriffs kursieren in der Forschungslandschaft unterschiedliche Deutungen. Diese lassen sich - wie später zu sehen sein wird nach verschiedenen Gesichtspunkten ordnen, womit bereits ein Charakteristikum der >Identitätsfrage< angesprochen wird: der Versuch, Ordnung(en) herzustellen. Solche Ordnungsversuche bedienen sich selbst oder durch andere zugewiesener bzw. abgelehnter Kategorien, die wie auch immer geartete (Gruppen-)Zugehörigkeiten markieren. Diese können sich auf Regionen, Nationen, Berufe, Familie, Geschlechter, ästhetische Stile u.v.m. beziehen, womit die auf verschiedenen Ebenen kursierenden Identitätsangebote >verarbeitet< werden. Solche Verarbeitungsprozesse sind aber komplexer geworden aufgrund zunehmender Differenzierung, Individualisierung und Pluralisierung der Gesellschaften. Mit diesen Stichworten wird der Prozess der Enttraditionalisierung gefasst, der die scheinbar traditionellen Vorgaben im Hinblick auf Identitäten infrage stellt. Angesprochen wird damit 
das Relativieren von nicht hinterfragten Normalitäten durch die Potenzierung von Identitätsangeboten, die aus der globalen Zirkulation von Waren, Bildern, Symbolen, Ideen und Lebensstilen resultiert. Mit der Fragmentierung und Durchmischung von sinnstiftenden >Heimaten <, Traditionen und Sinnwelten, die vielerorts unter dem Begriff der Postmoderne verhandelt werden, kann die Vorstellung stabiler und in sich ruhender Identitäten bzw. Ordnungen nicht mehr aufrecht erhalten werden. Vielmehr werden Identitäten als subjektive Eigenleistungen bzw. als Konstruktionsaufgaben - im Sinne von >Doing Identity< - aufgefasst, die Eickelpasch und Rademacher (2004) sowie Keupp et al. (1997; ${ }^{2006)}$ mit dem Begriff der »Identitätsarbeit« in Verbindung bringen.

Identitäten gleichen in einer zerrissenen Welt der Spätmoderne nicht fertigen Behausungen mit einem dauerhaften Fundament und einem schützenden Sinn-Dach, sondern permanenten, lebenslangen Baustellen, auf denen die [...] Individuen ohne festgelegten Bauplan und unter Verwendung vorhandener Bausätze und Sinnangebote sich (bis auf weiteres) eine Unterkunft schaffen. Je nach situativem und biografischem Erfordernis sind An- und Umbauarbeiten fällig. (Eickelpasch/Rademacher 2004: 14)

Die Beiträge in diesem Buch knüpfen hier an und basieren auf der These, dass Luxemburg geradezu eine Laborsituation zur Untersuchung von Prozessen der Identitätskonstruktionen unter enttraditionalisierten Bedingungen bietet. Damit angesprochen wird die Pluralisierung der Identitätsangebote innerhalb der Luxemburger Gesellschaft durch ihre vielfältigen Verflechtungen auf sozialem, kulturellem, politischem und wirtschaftlichem Gebiet, die spätestens seit 1900 augenfällig werden. Denn Ende des 19. Jahrhunderts wanderten die ersten Italiener/-innen und Deutschen im Zuge der fortschreitenden Industrialisierung in das zuvor ausschließlich landwirtschaftlich geprägte Luxemburg ein, so dass damals bereits knapp 6o \% der Stahlarbeiter im Süden des Landes von Ausländern gestellt wurden. Weitere Einwanderungswellen - insbesondere aus Italien - folgten in den Wachstumsperioden der 1920er Jahre und nach dem Zweiten Weltkrieg. Aufgrund der hohen Arbeitskräftenachfrage im industriellen Sektor holten in den 1950er Jahren viele Italiener ihre Familien nach. Im Zuge der in den 1970er Jahren einsetzende Zuwanderung von Portugiesen/-innen, die bis heute die größte Gruppe der ansässigen ausländischen Mitbürger/-innen stellen, konnten die Italiener/-innen zusehends gesellschaftlich aufsteigen. (Willems/Milmeister 2008; Zahlen 2008)

Während bereits im Jahr 1970 die Bevölkerung Luxemburgs ein Fünftel ansässige Ausländer/-innen zählte und mit der Krise (1973) die mit der Zuwanderung verbundenen Probleme zunehmend in den Blick rückten, gerieten zusätzlich die Grenzgänger/-innen aus den benachbarten Regionen Saarland und RheinlandPfalz, Lothringen und Wallonien ins Blickfeld. Ihr Anteil an den Erwerbstätigen lag bis Anfang der 1980 er Jahre noch unter $10 \%$, im Zuge der rasanten Entwicklung des Dienstleistungssektors nach der Blüte der Eisen- und Stahlindustrie wuchs ihre Zahl in den 1980er Jahren explosionsartig an: Im Jahr 1990 stellten Grenzgän- 
ger/-innen bereits $20 \%$ der Erwerbstätigen, 2009 machen die 147.000 Deutschen, Franzosen und Belgier fast die Hälfte der Erwerbstätigen im Großherzogtum aus.

Neben der klassischen Zuwanderung und der Grenzgängerbeschäftigung verzeichnet das Großherzogtum seit einigen Jahren auch einen starken Zuwachs an hochqualifizierten Arbeitskräften. Sie arbeiten weitgehend in Führungspositionen und werden von ihren Unternehmen ins Ausland entsendet oder kommen als sinternationale Arbeitsnomaden< ins Großherzogtum. Diese hochmobile Gruppe, zu der Manager/-innen internationaler Unternehmen ebenso zählen wie EU-Beamte, rekrutiert sich weitgehend aus europäischen Ländern. Am wichtigsten Standort der EU-Institutionen, dem in den $1960 e r$ Jahren erschlossenen Kirchberg-Plateau in Luxemburg-Stadt, und an weiteren Standorten im Süden und Zentrum der Stadt arbeiten bspw. 11.000 Personen in der EU-Verwaltung. (Chilla 2009) Ebenso sind zahlreiche Hochqualifizierte im privatwirtschaftlichen Sektor und hier insbesondere in der Finanzbranche beschäftigt: Die Banque Centrale de Luxembourg zählte bspw. im Jahr 2008 unter ihren Führungskräften vier Fünftel ausländische Mitbürger/-innen. (Fehlen/Pigeron-Piroth 2009: 6) Der Finanzsektor löste bereits in den $1970 e r$ Jahren die bis dahin dominierende Eisen- und Stahlindustrie ab und strahlte spätestens seit den 1980er Jahren auch auf andere Branchen ab. So erfuhr der Dienstleistungssektor im Großherzogtum einen beeindruckenden Aufschwung und die ehemals landwirtschaftlich und industriell geprägte Gesellschaft durchlief in kurzer Zeit einen nahezu beispiellosen und tiefgreifenden Wandlungsprozess unter tertiärem Vorzeichen.

Die erläuterte Präsenz von Hochqualifizierten aus aller Welt, einschließlich der EU-Beamten, hat heute in Luxemburg spürbare Auswirkungen. Sie verleihen dem Land einen internationalen Charakter, insbesondere der Hauptstadt, die im Vergleich zu anderen europäischen Städten dieser Größe weitaus kosmopolitischer ist. Des Weiteren gewinnt das Englische neben den üblichen Sprachen Französisch, Deutsch und Luxemburgisch zunehmend an Bedeutung, besonders im internationalen Finanzsektor und in sich entwickelnden »Expatriates-Communities« (Fehlen/Pigeron-Piroth 2009: 6). Das kulturelle Angebot ist demnach sehr vielfältig und multikulturell geprägt, auch wenn es dem Großereignis »Luxemburg und die Großregion - Kulturhauptstadt Europas 2007 « nur bedingt gelang, das Großherzogtum aus seiner >avantgardistischen Provinzialität< zu heben. Des Weiteren ist das City-Management der Stadt Luxemburg bestrebt, das bekannte UNESCO-Weltkulturerbe in den Lebensalltag der Einwohner/-innen zu integrieren und es für Touristen/-innen stärker aufzuwerten; in den städtischen Museen des Landes und in neuen dezentral organisierten Kultureinrichtungen entsteht eine kritische intellektuelle Masse. Die Minette-Region im Süden des Landes vollzieht einen Wandel durch die kulturellen Aufwertungen, durch die Ansiedlung von Dienstleistungsunternehmen und nicht zuletzt durch den anstehenden Umzug der Universität Luxemburg auf das Gelände der ehemaligen Industriebrache Esch-Belval, auf dem sich Tradition und Moderne architektonisch miteinander vereinen. Ebenso setzen die ländlichen Regionen im Norden, Westen und Osten des Landes zukunftsorien- 
tierte Entwicklungsstrategien über selbstbewusste Trägerinstitutionen und Bürgerbeteiligungen um. Kurz: Luxemburg durchläuft vielschichtige Umbrüche, bei wenig variierender politischer Besetzung der Regierungsstühle, was eine weitere Besonderheit des Großherzogtums widerspiegelt.

Dieser Tour d'horizon als ein Ausblick auf die folgenden Kapitel deutet die vielfältigen und sich permanent wandelnden Identitätsangebote in Luxemburg bereits an. Besonders hervorzuheben sind - neben anderen Besonderheiten der Luxemburger Gesellschaft - die durch Arbeitsmigration bedingte Pluralität, der multilinguale Kontext sowie die internationalen Verflechtungen auf nahezu allen gesellschaftlichen Gebieten. Diesen ist - bedingt durch die Geschichte, geografische Lage und Kleinheit des Landes - jeweils eine transnationale Dimension inhärent. So kann die Luxemburgische Gesellschaft geradezu exemplarisch als eine >Gegend $<$ in >transnationalen Landschaften $<\left(>\right.$ scapes $\left.<^{1}\right)$ aufgefasst werden, in der traditionelle Ordnungskategorien zunehmend ihre Gültigkeit verlieren. Vor diesem Hintergrund wird das oben erläuterte Bild der Identitätsarbeit bzw. wird >Doing < im Hinblick auf Identitäten im Folgenden aufgegriffen, um den hier zugrunde gelegten Flüchtigkeits- und Konstruktionscharakter von Identitäten herauszustellen.

\section{Theoretische Verortung von Identitäten}

Das Thema »Identität« hat seit geraumer Zeit Hochkonjunktur - sowohl als alltagsweltlich gelebte Frage als auch als Forschungsgegenstand. Wenngleich die untrennbaren Fragen »Wer bin ich (geworden)?/Wer bin ich nicht (geworden)?« und »Wer sind wir (geworden)?/Wer sind wir nicht (geworden)? «(vgl. Abels 2006; Straub/Renn 2002) eine ontologische Konstante der menschlichen Reflexivität darstellen und spätestens seit der Antike schriftlich dokumentiert sind (Keupp et al. 32006 ), so haben sie in der Spätmoderne einen neuen Stellenwert erlangt. In der Tat »stellt Identität eine anthropologische Notwendigkeit dar und keine Erscheinung der Moderne [...]. Subjekte beziehen ihre Handlungsfähigkeit zu allen Zeiten und in allen Kulturen aus ihrem Sinn dafür, wer sie sind. Allerdings wird dieser Sinn in der Neuzeit in besonderem Maße fraglich [...].« (Rosa 2007: 50) Die Folge dieser hier angedeuteten fortgeschrittenen Enttraditionalisierung, Differenzierung und Individualisierung von Sozialstrukturen im westlichen Kapitalismus (Straub/Renn 2002) ist eine erhöhte Selbstgestaltung in allen Lebensbereichen, deren ambivalente Verantwortung zunehmend bei der Einzelperson liegt (vgl. Ahbe 1997; Bauman 1999). Diese Eigenverantwortung bedeutet gleichzeitig ein Mehr an Wahlfreiheit und ein Mehr an Infragestellung, da sowohl ein Gelingen als auch ein Misslingen der so geformten Lebensführung möglich ist. Oder in ande-

1 | Unter dem Begriff 'scapesı fasst Appadurai miteinander vernetzte und veränderbare 'Landschaftenı, um die sich im Zuge der Globalisierung herausbildenden sozialen, kulturellen oder wirtschaftlichen Figurationen zu beschreiben. Er unterscheidet zwischen rethnoscapesı, ,financescapes`, 'mediascapes` und ideoscapes`. (Vgl. Appadurai 1998) 
ren Worten: Einerseits zieht dieser Umstand eine konstante und nie abgeschlossene »alltägliche Identitätsarbeit« (Keupp et al. ${ }^{32006}$; Straus/Höfer 1997) nach sich, andererseits ist diese immer nur als »riskante Chance« (Keupp et al. ${ }^{32006}$; Eickelpasch/Rademacher 2004) zu betrachten.

Trotz dieser Pluralisierung der Möglichkeiten von Identitätskonstruktion bleibt deren Reichweite an die Quantität und Qualität der sozialen Interaktionen sowie wirtschaftlichen und alltagskulturellen Ressourcen gebunden - und somit an strukturelle Kapitalien der sozialen Ungleichheit (Bourdieu 1992; 1972) -, anhand denen sich Identität in Mechanismen des »Sich Erkennen, Erkannt- und Anerkanntwerden « (Greverus 1995: 219) konstituiert ${ }^{2}$. Somit beinhalten Identitätskonstruktionen eine doppelte Ambivalenz: Wegen erodierender vorgefertigter Pfadabhängigkeiten gibt es einerseits einen Zwang zur Wahl, die dennoch Gelingen oder Scheitern birgt, und andererseits die Freiheit der Wahl, die dennoch sozio-kulturell überformt ist. Richard Sennett bringt dieses ambivalente Verhältnis humorvoll auf den Punkt: »In der Moderne übernehmen die Menschen die Verantwortung für ihr Leben, weil sie den Eindruck haben, es hänge von ihnen ab.« (1996:48)

Somit avancierte >Identität<, als »das je spezifische Selbst- und Weltverhältnis sozialer Subjekte« (Rosa 2007: 47), ab den 1950er Jahren und insbesondere ab den $1980 e r$ Jahren zu einem zentralen Forschungsgegenstand in den Sozial- und Kulturwissenschaften. Kanonisch wird konzeptionell zwischen personaler und kollektiver Identität unterschieden (vgl. Luckmann 2007); also zum einen »die Art und Weise, wie sich der Einzelne als Individuum verstehen soll« - entwicklungspsychologisch ${ }^{3}$ und historisch-soziokulturell - und zum anderen »die Form des Selbstverstehens, in dem sich der Einzelne als Teil eines Kollektivs definiert« (Reckwitz 2001: 21). Dieses Kollektiv kann strukturell (z.B. Gender, Alter, Gesundheitszustand etc.), sozial (z.B. Familie, Netzwerke, Milieuzugehörigkeit, Erwerbstätigkeit, Bildungsstand etc.), alltagskulturell (z.B. Intimität, Normvorstellungen, Werte und Vorlieben, Konsum- und Lebensstil, Wertegemeinschaft wie religiöse Gemeinden oder zivilbürgerliche, politische Vereinigungen, Freizeitvereine etc.), national, ethnisch etc. sein. Alltagsweltlich jedoch sind diese - lediglich heuristisch getrennten - Erfahrungsebenen eng miteinander verflochten:

2 | So verstandene Identitätskonstruktionen stehen in Zusammenhang zur "postmodernen Folie und damit zu Prozessen der Performanz, der Wiederholung, der Subversion, der Anerkennung, des Verhaftet-Seins, als Pendeln zwischen Potenzialitäten und Faktizitäten, zwischen Differenzen und Ähnlichkeiten, zwischen Varianzen und Kontinua, eingebettet in Macht- und Wissensstrukturen“ (Baltes-Löhr 2006: 64). Um diese bewegliche Vielschichtigkeit zu verdeutlichen, schreibt sie metaphorisch: "Das Muster entsteht beim Weben." (Ebd.: 37)

3 | Wir blenden hier die entwicklungspsychologische Perspektive aus, da sie den Rahmen der von uns praktizierten Interdisziplinarität überschreitet. 
L'un des paradoxes de l'identité personnelle est précisément de s'exprimer par l'appartenance à des groupes et donc par le croisement d'identités collectives (je suis un homme, libraire, père de famille, militant politique, amateur d'opéra, d'origine italienne etc.). ${ }^{4}$ (Halpern 2009: 13)

Wir begreifen jedoch das Kollektive im Identitätsdiskurs weniger als durch die Art der Gruppenzugehörigkeit gegeben - in einer Schritt-für-Schritt-Metapher, die, linear und getrennt, vom Individuum über Interaktions- und Affinitätsgruppen bis hin zu gesellschaftlich-kulturellen Großgruppierungen reicht ${ }^{5}$ - sondern eher durch den permanenten Bezug auf gesellschaftlich geformte Wert- und Normvorstellungen, Ressourcen und Wissensbestände in jeder Einzelhandlung. Je nach Analysegegenstand kann diese Einzelhandlung von Individuen oder von Gruppen ausgehen. Entscheidend erscheint uns hier das konsequente empirische Registrieren der »Wir-Schicht« (Elias 1986) in jeder Einzelhandlung, die als Identitätsprojekt angesehen werden kann, ohne dass dieser Aspekt den handelnden Subjekten zwingend bewusst sein muss. Demnach schreiben wir uns in Forschungstraditionen ein, die auf theoretischer Ebene vorrangig die »Balance zwischen individuellen Ansprüchen und sozialen Erwartungen« (Abels 2006: 254; vgl. Krappmann ${ }^{10} 2005$ ) thematisieren und die sich nicht auf die funktionalen (Mehrfach-)Zugehörigkeiten (Goffman 2003; Lahire 1998), die sich in der Spätmoderne vervielfältigt haben, beschränken.

Die von uns gewählte Sichtweise auf kollektive Identität unterscheidet sich in genau diesem Punkt von alltagsweltlich und oftmals ideologisch-politisch genutzten Diskursen, in denen zumeist vereinheitlichend und reifizierend argumentiert wird. Der Begriff >Identität< - vor allem im Singular - ist dabei emotional aufgeladen und benennt zu kurz greifende Aussagen wie etwa: »Dörfliche Identität« soll gewahrt werden, »nationale Identität« scheint bedroht, »eine europäische Identität« wird beschworen. >Identität< bezieht sich nach diesem Verständnis auf ein vorausgesetztes Gemeinschaftsgefühl einer Gruppe, deren Mitglieder homogenisiert werden; individuelle Entwicklungsmöglichkeiten werden ihnen dabei abgesprochen und alternative Loyalitäten ausgeblendet. Sie fungieren weniger als vollwertige und reflexive Mitträger/-innen einer bestimmten Gemeinsamkeit denn vielmehr als quantitatives Alibi für Vereinnahmungen im Namen der Gruppe. Vor allem jedoch wird auf eine - gar naturalisierte - Essenz verwiesen (vgl. Weinreich/ Saunderson 2003; Reckwitz 2001), die empirisch nicht überprüft worden ist und von der aus abstrahierend und universalisierend weiterdiskutiert wird.

4 | Deutsch: "Eins der Paradoxa von personaler Identität ist gerade, dass sie sich über Gruppenzugehörigkeiten ausdrückt und somit über die Kreuzung kollektiver Identitäten (ich bin ein Mann, Buchhändler, Vater, politisch engagiert, Opernliebhaber, von italienischer Herkunft etc.)."

5 | Halpern 2009 oder Ruano-Borbolan 1998 stehen exemplarisch für diese Herangehensweise. 
Dieser soziale Tatbestand hat ein gewisses wissenschaftliches Misstrauen gegenüber dem Begriff der kollektiven Identität nach sich gezogen (vgl. Kmec 2007; Kaufmann 2004; Brubaker 2001; Giesen 1999; Bayart 1996), dem eine inhärente »Tendenz zum Fundamentalismus und zur Gewalt (Niethammer 2000: 625) anhafte. Wenngleich man die Position vertreten könnte, dass kollektive Identitäten per se nicht existieren (da sie keine leiblichen, sprach- und handlungsfähigen Entitäten bilden), so ist ihre soziale und kulturelle Performativität doch real (vgl. Meyran 2008), da sie gerade wegen ihrer »Nebulosität bestens für eine ideologische Diktion geeignet« (Straub 2004: 293) und deshalb doch erforschenswert sind. Niethammer empfiehlt daher, »dass wir, anstatt irgendeine kollektive Identität zu beschwören, >wir« sagen«, da »Wir-Aussagen [...] vor allem in ihrer Subjektivität leichter erkennbar und dadurch diskutabel« sind, d.h., sie eignen sich weniger zur »Totalisierung einer speziellen Gruppe«. (2000: 628-629) Bayart schlägt vor, von identitären Strategien (vgl. 1996) zu sprechen anstelle von einer >selbstständigen< Identität, MacClancy von »modes of identification« (2004: 64), die den subjektzentrierten, relationalen, aktiven und motivierten Aspekt von Identitäten betonen, sowie ihre fragmentierte und plurale Prozesshaftigkeit. Straub präzisiert:

Unter einer kollektiven oder Wir-Identität verstehen wir das Bild, das eine Gruppe von sich aufbaut und mit dem sich deren Mitglieder identifizieren. Kollektive Identität ist eine Frage der Identifikation seitens der beteiligten Individuen. Es gibt sie nicht ran sich،, sondern immer nur in dem Maße, wie sich bestimmte Individuen zu ihr bekennen. [...] Nach der nahe gelegten Auffassung sind kollektive Identitäten Konstrukte, die nichts anderes bezeichnen, als eine näher zu spezifizierende Gemeinsamkeit im praktischen Selbst- und Weltverhältnis sowie im Selbst- und Weltverständnis Einzelner. (2004: 299)

Rosa fasst schließlich zusammen: Obwohl Bausteine personaler Identität sich auf Kollektive beziehen, trägt »jede Einheitsunterstellung [...] einen potentiell ideologischen und normierenden Charakter. [...] Individuen und Gruppen sind daher stets zu einer dialogischen (und konflikthaften) Klärung kollektiver Identität gezwungen.« (2007: 51-52)

Aus den o.g. Gründen haben wir kollektive Identität nicht als direkten Forschungsgegenstand gewählt, sondern uns - bescheidener, aber empirisch fundiert - auf sogenannte >Identitäten ${ }^{6}$ konzentriert. Dabei haben wir die Wechselwirkungen und Diskrepanzen zwischen institutionell >zugeschriebenen $<$ und alltagsweltlich >angeeigneten $<$ Identitäten untersucht - ob diese nun kollektiv (in welcher Konstellation auch immer) oder individuell sind. Diese neutrale und plurale Formulierung soll verdeutlichen, dass in unseren Augen die konstruktivistische und

6 | Es geht hierbei nicht um 'nationale Identitätı, sondern um 'Identitäten in Luxemburg`, nicht um 'kulturelle Identitätı, sondern um das Zusammenleben (im Sinne von Interaktionen und Repräsentationen) alltagsweltlicher Identitätsmuster von Menschen unterschiedlicher Herkunft und unterschiedlichen Ausrichtungen in Luxemburg. 
nicht-essentialistische >konzeptionelle Annäherung an die Identitätsthematik entscheidender ist als der Fokus auf die Bezugseinheiten innerhalb dieser Thematik. Außerdem ist die von uns gewählte Perspektive fruchtbar für die Analyse sowohl von personalen als auch kollektiven >Identitätsmustern $<$ die als Eckwerte auf einem dynamischen Spektrum - mit seinen Übergängen und Transgressionen - begriffen werden. Der Begriff der >Identitätsmuster< legt nahe, dass empirisch gewisse Strukturen von Identitäten aufzufinden sind; es sind deren jedoch mehrere und sie sind mit einer gewissen Handlungsfreiheit in individueller Eigenleistung, jedoch in der Interaktion mit >meaningful others $<$, ausformbar - eben das Gegenteil von >einer Identität<, die angeboren wäre und die man für immer >in sich trüge< (vgl. Avanza/Laferté 2005; Brubaker 2001). Eine ähnlich gelagerte Metapher wie die der >Identitätsmuster< ist die der >Patchwork-Identität<. Sie soll ausdrücken, dass von den

einzelnen Personen eine hohe Eigenleistung bei diesem Prozess der konstruktiven Selbstverortung zu erbringen ist. Sie müssen Erfahrungsfragmente in einen für sie sinnhaften Zusammenhang bringen. Diese individuelle Verknüpfungsarbeit nennen wir Identitätsarbeit‘, und wir haben ihre Typik mit der Metapher vom 'Patchworkı auszudrücken versucht. (Keupp et al. ${ }^{3} 2006:$ 9-10)

Empirisch gehen wir von - persönlich interviewten bzw. mittels Fragebogen befragten - Individuen aus und interessieren uns für deren Entwürfe, Aushandlungen und Repräsentationen von Identitäten, verstanden als »subjektiver Konstruktionsprozess [...], in dem Individuen eine Passung von innerer und äußerer Welt suchen« (Keupp et al. ${ }^{32006: ~ 7) . ~ D i e ~ a k t i v e ~ I n a n s p r u c h n a h m e ~ s o l c h e r ~ I d e n t i t a ̈ t s m u s t e r ~ g i b t ~}$ den von Selbstsorge geprägten Individuen Orientierung in einem komplexen Rollensystem. (Vgl. Abels 2006) Da jedoch sowohl die gesellschaftlichen Gegebenheiten wie die individuellen Denk-, Handlungs- und Wahrnehmungsschemata (vgl. Bourdieu 1980) synchron und diachron - wenn auch nicht beliebig7 - wandelbar sind, können die resultierenden Identitäten immer nur »temporär« (Keupp et al. 32006: 276; vgl. Rosa 2007), »transitorisch«, »prozesshaft« (Straub 2004; Straub/ Renn 2002), »prekär« oder »prinzipiell unvollständig« (Straub 2004: 280) sein. Auch ihre Selbst- und Fremdbewertung als mehr oder weniger gelungen sind flexibel, historisch, sozial und kulturell wandelbar, d.h. kontingent. (Vgl. Straus/Höfer

7 | Straub nennt dieses Phänomen sehr treffend "strukturell verankerte Beweglichkeit" (2004: 281). Gesellschaftliche Bedingungen und individuelle Aspirationen und Ressourcen sind wohl im Wandel; dieser Wandel ist relativ, da er soziale Ungerechtigkeiten dennoch perpetuiert. Indem die gesamte Sozialstruktur sich in Richtung Liberalisierung und Kulturalisierung des Alltäglichen verschiebt, haben die Individuen zwar das subjektive Empfinden einer Veränderung, die sozio-kulturellen Milieu-Zugehörigkeiten bleiben jedoch weitgehend ähnlich gelagert (vgl. Vester et al. 2001) und "setzen ein bestimmtes Möglichkeitsfeld der Identitätsentwicklung" (Straus/Höfer 1997: 218). 
1997) Sie können als lebenslang anhaltende und in Interaktionen immer wieder neu entworfene »Projekte« oder »Aspirationen « gelten - als eine Momentaufnahme eines andauernden Proze $\beta$ von »Selbsttechnologien « (Foucault 1994; 1984). Wenngleich der so verstandenen Identitätsarbeit eine »Selbstdistanzierung durch Selbstreflexion und Selbstkritik« (Straub 2004: 282; vgl. Giddens 1991; Bauman 1999) zugrunde liegt, so bedarf sie nicht einer konstanten Aufmerksamkeit der Subjekte. Denn

Subjekte arbeiten (indem sie handeln) permanent an ihrer Identität. Deren Basis(akte) bestehen aus situativen Selbstthematisierungen, die unser Denken und Handeln kontinuierlich begleiten. (Straus/Höfer 1997: 273)

Demnach müssen die individuellen »Antworten auf die (praktische) Identitätsfrage [...] nicht unbedingt explizit artikuliert werden « (obwohl dies oft in narrativem und diskursivem Modus der Fall ist); sie können »dem Handeln auch implizit sein bzw. handelnd zum Ausdruck gebracht werden«. (Straub 2004: 280)

Doch gleich, wie wenig oder wie stark dieser Selbstbezug reflektiert ist, er ist eine »Sinnfrage« (Reckwitz 2001: 22), die alltagsweltlich die soziale und kulturelle Form des Bemühens um Kontinuität und Kohärenz annimmt, »angesichts der Vielfalt lebensweltlicher Selbsterfahrungen und der Abnahme gesellschaftlich verfasster Kohärenzmodelle« (Straus/Höfer 1997: 270).

Dieser Anspruch muss nicht unbedingt widerspruchsfrei sein; vielmehr geht es darum, eine sinnhafte Balance zwischen synchroner Kohärenz und Flexibilität einerseits und diachroner Kontinuität und Wandel zu finden. (Rosa 2007: 48) Die Dynamik dieses »stimmige[n] aber kontingente[n]« (Straub 2004: 287) Gefüges entspricht eher der >Selbstheit< (oder Ipseität, vom lateinischen >ipse<, selbst), denn der >Gleichheit< (oder Identität, vom lateinischen >idem<, gleich, identisch). Ricoeur (1990) verweist auf diese doppelte Bedeutungsebene des Identitätsbegriffs, >idem < und >ipse<, um herauszustellen, dass letztere Dimension den Akzent eher auf Wandelbarkeit und Subjektivität setzt und einen Bezug zur Alterität mitdenkt.

Wir schreiben uns in dieses Ipseitäts-Verständnis von Identität(en) ein, weil es unseres Erachtens signalisiert, dass wir die von Reckwitz thematisierte latente Gefahr zeitgenössischer Identitätstheorien vermeiden möchten. Er unterscheidet zwischen >klassischen< Konzeptionen (1940er bis 1970er Jahre), die »universalistisch und kompetenztheoretisch orientiert und auf das Problem des Verhältnisses zwischen Individuum und sozialen Zwängen sowie das Problem der temporalen Konstanz zentriert « waren, und Modellen ab den 1970er Jahren, die eher »hermeneutisch und historisch orientiert sowie auf das Problem des kontingenten Selbstverstehens bezogen « sind. (2001: 25) Diese aktuellen Theorien seien einem doppelten Überinterpretationsrisiko ausgesetzt: einerseits durch die »Dramatisierung der Stabilität von Differenzen«, wobei ein kulturalistischer Essentialismus nahegelegt wird; andererseits durch die »Dramatisierung der permanenten Veränderbarkeit von Identitäten«, d.h. durch das »Bild eines hyperflexiblen, seine Identitäten 
austauschenden Subjekts [...], das den Boden der Alltagspraktiken zu verlassen scheint«. (2001: 34-35) Der Verweis auf Ipseität soll hier daran erinnern, dass die von uns erforschten Identitäten die jeweiligen Differenzen inhärent als Komplement mittragen (vgl. Rosa 2007) und dass sie in ihrer sozialen Eingebundenheit und Milieuzugehörigkeit empirisch ermittelt werden.

Zusammenfassend ist festzuhalten, dass wir, um den relationalen Charakter von Identitätsmustern zu betonen, unser Augenmerk auf das verwobene Wechselspiel von internen Selbstverständnissen und -verhältnissen sowie externen Einwirkungen gerichtet haben - oder, anders ausgedrückt: auf das Wechselspiel von »Bottom-up-Identifizierungen « (oder »identification with«) und »Top-down-Identifikationen« (oder »identification of«). (Hark 1999) Die zirkulierenden Identitätsentwürfe und -angebote - hier in Form von Repräsentationen und Aushandlungen analysiert - sind intrinsisch dialogisch und politisch. Es werden »Machtkämpfe um die Bedeutung, Stellung und den Wert von Lebensformen, Eigenschaften, Tätigkeiten und Verhaltensweisen« (Rosa 2007: 52) verhandelt. Identitäten werden insbesondere in sprachlichen und alltagskulturellen Aushandlungen sowie in räumlichen Repräsentationen und in der Konfrontation zwischen Selbst- und Fremdwahrnehmung gestaltet, weshalb diese Themenfelder in diesem Buch empirisch vertieft und mit dem Querschnittsthema der sozio-kulturellen Milieus (Vester et al. 2001) verzahnt werden. Die aktuell stattfindenden, aktiven Prozesse der jeweiligen Identitätsarbeiten des bewussten Seins stehen dabei im Zentrum - eben das >Doing Identity<.

\section{Analytische Kategorien und Forschungsdesign}

Vor dem Hintergrund der generellen Einordnung des hier zugrunde gelegten Identitätsbegriffs wurde dieser für die interdisziplinäre Projektarbeit operationalisiert. Dabei wurden zwei Perspektiven eingenommen, die nur analytisch voneinander zu trennen sind und aus der sich eine dritte Perspektive ergibt, die für Aussagen über aktuelle und ggf. zukünftige Gesellschaftsentwicklungen zentral erscheint. Dabei handelt es sich einerseits um das Konzept der zugeschriebenen und angeeigneten Identitäten und andererseits um ihre Wechselwirkungen. Mithilfe des Konzepts der zugeschriebenen Identitäten werden Zuschreibungen und die Konstruktionsmechanismen sogenannter >wünschenswerter Identitäten< untersucht, wie sie sich in politisch-medialen Diskursen manifestieren. Das bedeutet, das Forschungsinteresse richtet sich hier auf identitätsbezogene Zuschreibungsprozesse durch Vektoren in unterschiedlichen gesellschaftlichen Feldern. Das Konzept der angeeigneten Identitäten bezieht sich andererseits auf die in der sozialen Praxis artikulierten Identitätsmuster. Von besonderem Interesse sind hierbei die sogenannten >gelebten Identitäten < der Luxemburger Wohnbevölkerung in verschiedenen sozio-kulturellen Milieus, die - so die Ausgangsthese - quer zu milieuspezifischen Gliederungen verlaufen. Die kontrastive Gegenüberstellung von zugeschriebenen und angeeigneten Identitäten nach den jeweils betrachteten thematischen Feldern 
vermag schließlich die Wechselwirkungen und ggf. die Divergenzen zwischen beiden analytischen Kategorien aufzuzeigen, welche als Annäherung an die gesellschaftliche Praxis im Sinne eines >structure-agency link < (vgl. Giddens 1997) auf dem Gebiet der Identitätskonstruktion zu verstehen ist.

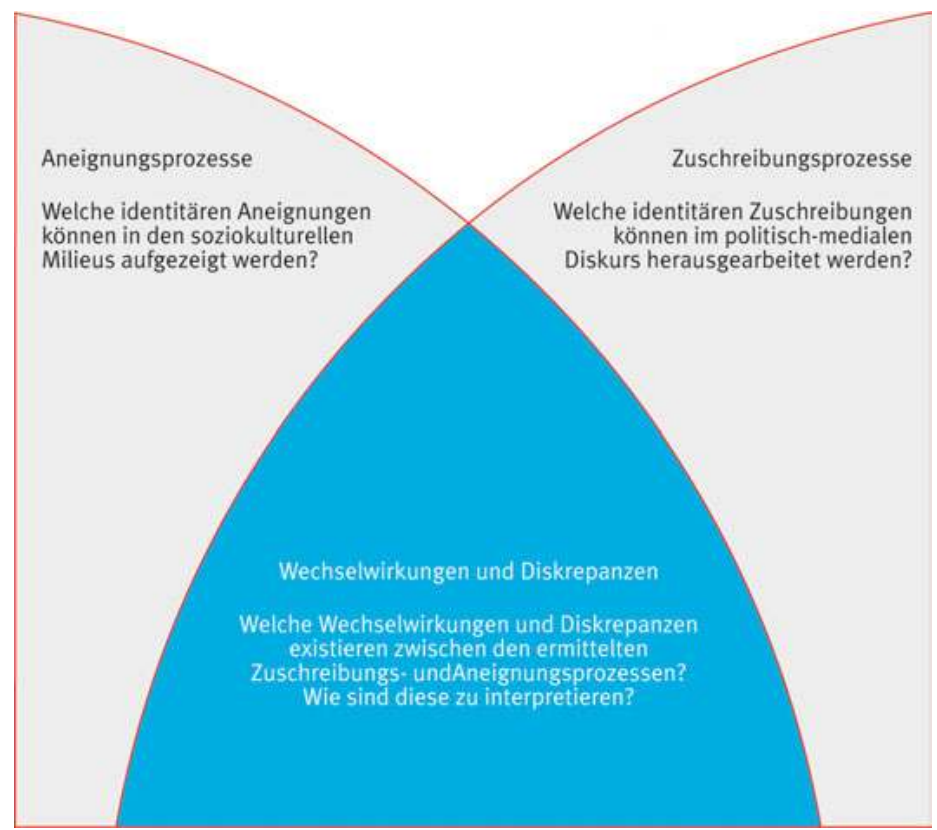

Abbildung 1: Heuristik von Prozessen der Identitätskonstruktion

Aufbauend auf die analytische Grundlegung des oftmals diffus erscheinenden Identitätsbegriffs haben sich vor dem Hintergrund der in der Forschungseinheit IPSE vertretenen Disziplinen fünf thematische Schwerpunkte herauskristallisiert, welche die Projektarbeiten strukturierten.

- Sozio-kulturelle Milieus in Luxemburg (Kap. 3): Zur differenzierten und transversalen Betrachtung von angeeigneten Identitäten wurden zunächst soziokulturelle Milieus in der Luxemburger Gesellschaft empirisch ermittelt. Die Kategorie der Milieus beschreibt Personen mit jeweils charakteristischen Einstellungen und Lebensorientierungen und fasst diese zu sozialen Gruppen mit entsprechend ähnlichen Wertorientierungen, Lebenszielen und Lebensstilen zusammen. Die Pionierarbeit der Milieuermittlung folgt dem Ansatz der sozialen Milieus nach Pierre Bourdieu, der von Michael Vester (vgl. Vester et al. 2001) weiterentwickelt und im Rahmen des IDENT-Projekts hinsichtlich der pluralen Verhältnisse in Luxemburg angepasst wurde. Die ermittelten sozio-kulturellen Milieus - bzw. die sich hierin widerspiegelnden angeeigneten Identitäten - sind 
integrativer Bestandteil des IDENT-Projekts, wie durch die beständige Bezugnahme auf die Milieugliederung durch die Autoren/-innen der folgenden thematischen Schwerpunkte deutlich wird.

- Sprachen und Identitäten (Kap. 4): Einen weiteren Themenschwerpunkt bildet die Frage nach den sprachlichen Identitäten im multilingualen Luxemburg. Dies betrifft zunächst Fragen des Sprachkontakts sowie des Erlebens von Mehrsprachigkeit, die - aufgefächert nach sozio-kulturellen Milieus - Einblicke in sprachenbezogene Identitäten geben. Angeeigneten Identitäten wird ebenso im Zusammenhang mit der Frage nachgegangen, welche Konfliktlinien und Positionen die gesellschaftliche Debatte um Mehrsprachigkeit markieren. Schließlich werden >gelebte < bzw. >gewünschte < sprachliche Identitäten nach ihren Artikulationsformen im öffentlichen Raum befragt.

- Räume und Identitäten (Kap. 5): Dieser Themenschwerpunkt widmet sich der Frage nach räumlichen Identitäten, wobei zugeschriebene Identitäten, wie sie sich im öffentlichen touristischen Diskurs artikulieren, im Zentrum stehen. Die Betrachtungen anhand einschlägiger Kommunikationsmedien, die sich von der lokalen bis zur interregionalen Ebene erstrecken, werden den Raumperzeptionen und räumlichen Praktiken der Luxemburger Wohnbevölkerung gegenübergestellt, so dass hier auf unterschiedlichen Maßstabsebenen Diskrepanzen zwischen zugeschriebenen und angeeigneten Identitäten herausgearbeitet werden können.

- Bilder und Identitäten (Kap. 6): Dieser Schwerpunkt betrifft sprachlich und visuell verfasste Selbst- und Fremdbilder $>$ Luxemburgs $<$, wie sie in hoch- und alltagskulturellen Medien vorzufinden sind. Er geht der Frage nach, welche Schlüsse die jeweils vermittelten Aussagen sowie der Entstehungsprozess der betrachteten Bilder auf identitätsbezogene Aneignungs- und Zuschreibungsprozesse zulassen. Die hier durchgeführten Projektarbeiten stellen besonders den analytischen Charakter der konzeptionellen Kategorien heraus, die aufgrund der Rezeption der betrachteten Medien zusammenfallen (können).

- Alltagskulturen und Identitäten (Kap 7): Dieses Themenfeld fokussiert auf angeeignete und zugeschriebene Identitäten in den alltagsweltlichen - jedoch politisch und wirtschaftlich überformten - Kontexten von Gender, Konsum und Alterität. Von besonderem Interesse sind hier die betrachteten Identitäten in verschiedenen sozio-kulturellen Milieus unter dem Aspekt ihrer ambivalenten Aneignung in der alltagskulturellen Praxis, in der >geklärte Widersprüche<, aber auch Überschneidungen in Erscheinung treten. 


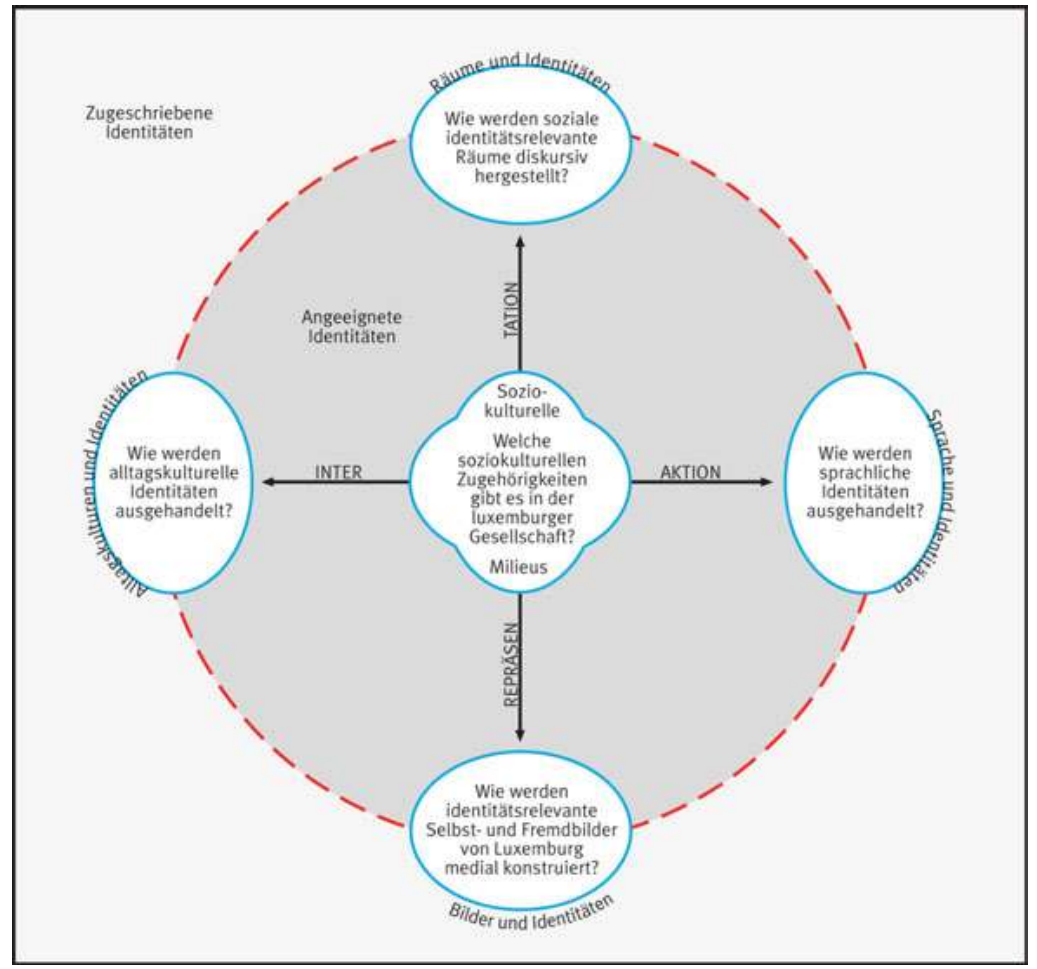

Abbildung 2: Themenschwerpunkte und konzeptioneller Rahmen

Die oben erläuterte Verortung der Projektarbeiten innerhalb der wissenschaftlichen Diskussion um Identität(en) und die Entwicklung analytischer Kategorien erwiesen sich während der dreijährigen Forschungsaktivitäten als unverzichtbar, um eine gemeinsame Grundlage für die interdisziplinäre Zusammenarbeit bereitstellen zu können. Im Folgenden sollen mit Blick auf die Untersuchungsgegenstände in den genannten thematischen Schwerpunkten die verwendeten Methoden erläutert und anschließend die Zusammenarbeit der Wissenschaftler/-innen unter forschungspraktischen Gesichtspunkten vorgestellt werden.

\subsection{MethodiK Und INTERdisziplinäre ZUSAmmenarbeit}

Aufbauend auf dem skizzierten thematisch-konzeptionellen Rahmen werden im Weiteren die verwendeten Methoden erläutert und Einblicke in den Aufbau und in die Umsetzung der gemeinschaftlich bearbeiteten Projektetappen - insbesondere der empirischen Erhebungsphasen - gegeben. Zunächst sollen die in den thematischen Schwerpunkten untersuchten Gegenstände überblicksartig dargelegt werden. 
- Sozio-kulturelle Milieus: Die Ermittlung sozio-kultureller Milieus in der Luxemburger Wohnbevölkerung zur Betrachtung angeeigneter Identitäten basierte auf der Analyse von quantitativen statistischen Daten, die im Rahmen des IDENTProjekts erhoben wurden.

- Sprachen und Identitäten: Zur Betrachtung von Identitäten im Sprachkontakt und -erleben wurde zunächst auf quantitative statistische Daten aus der vorliegenden Untersuchung zurückgegriffen; des Weiteren wurden mittels textanalytischer Verfahren Leserbriefe in einschlägigen Printmedien Luxemburgs hinsichtlich der hier vertretenen Positionen untersucht. Einer quantitativ orientierten Analyse wurde schließlich die öffentliche Beschilderung in Luxemburg unterzogen, um angeeignete und zugeschriebene sprachliche Identitäten aufzudecken.

- Bilder und Identitäten: Selbst- und Fremdbilder als Zuschreibung und/oder Aneignung von Identitäten wurden hier in alltagskulturellen und hochkulturellen Medien aufgespürt und diskutiert, wie etwa in Tageszeitungen, im Comic, in einem Ausstellungskatalog, in Printwerbung oder in Fotografien. Hier kamen weitgehend inhaltsanalytische Verfahren zur Anwendung, die durch quantitative statistische Daten aus der vorliegenden Untersuchung ergänzt wurden.

- Räume und Identitäten: Die Zuschreibung von räumlichen Identitäten wurde in diesem Themenschwerpunkt anhand von touristischen Broschüren, topografischen Karten, Reiseführern und journalistischen Publikationen diskursanalytisch dekonstruiert. Ihr gegenübergestellt wurden angeeignete räumliche Identitäten der Luxemburger Wohnbevölkerung, die auf Basis der Daten der eigenen quantitativen und qualitativen Befragung herausgearbeitet wurden.

- Alltagskulturen und Identitäten: Die hier perspektivierten Identitäten der Alltagspraxis unter dem Aspekt der Aneignung und jenem der Zuschreibung bzgl. Gender-Konstruktionen, Ansichten über sutes< Essen und Erfahrungen mit grenzüberschreitenden Arbeitnehmern/-innen basieren auf den Daten eigener quantitativer und qualitativer Befragungen und auf entsprechenden Inhaltsanalysen, in deren Rahmen offizielle Broschüren und Gesetzestexte berücksichtigt wurden.

Die Darlegung der in den einzelnen thematischen Schwerpunkten untersuchten Gegenstände zeigt ein breites Spektrum an >Analyseobjekten falt wurde mit einem ähnlich breiten methodischen Instrumentarium und einem arbeitsteiligen Vorgehen begegnet, das nur durch die Erfahrung und durch die breite Methodenkompetenz der in der Forschungseinheit IPSE vertretenen Disziplinen möglich geworden ist. Die angewandten methodischen Verfahren lassen sich unter den Projektmodulen »quantitative Befragung «, »qualitative Interviews « und »Inhaltsanalysen« zusammenfassen. 


\section{Quantitative Befragung}

Einen zentralen Bestandteil des IDENT-Projekts bildete die quantitative Repräsentativbefragung der Luxemburger Wohnbevölkerung. Mit ihr wurden zwei Zielsetzungen verfolgt: Zum einen sollten über dieses Verfahren sozio-kulturelle Milieus der Luxemburger Gesellschaft und die hier vorzufindenden Identitätsmuster ermittelt werden; zum anderen sollten spezifischen Fragestellungen der Themenschwerpunkte Sprachen, Selbst- und Fremdbilder, Räume und Alltagskulturen unter dem Aspekt der Identitätskonstruktion bearbeitet werden. Dementsprechend untergliedert sich der verwendete und gemeinsam entwickelte Fragebogen in verschiedene Themenbereiche ${ }^{8}$, die mithilfe von offenen, halboffenen und geschlossenen Fragen operationalisiert wurden. Aufgrund der Mehrsprachigkeit im Großherzogtum Luxemburg musste der Fragebogen (ebenso wie der unten vorgestellte Interviewleitfaden) in luxemburgischer, deutscher, französischer, englischer und portugiesischer Sprache angefertigt werden. Die Befragungsergebnisse konnten jedoch nur zueinander in Beziehung gesetzt werden, wenn über die Befragungsinstrumente die gleiche Bedeutung der behandelten Themen bzw. Schlüsselbegriffe sichergestellt war, was in der methodischen Diskussion unter dem Begriff der >funktionalen Äquivalenzく (vgl. Braun 2006) diskutiert wird. Angesprochen wird damit jeweils das vielschichtige und sozio-kulturell verankerte Bedeutungsspektrum von lexikalischen Elementen, das bei der Übersetzung von Untersuchungsinstrumenten die Frage aufwirft, inwiefern bzw. ob die Zielsprache eine äquivalente Formulierung bereithält, die semantische Verschiebungen ausschließt. ${ }^{9}$ Dies betrifft sowohl die Ebene der Denotation wie z.B. bei Bildungsabschlüssen als auch der Konnotation wie etwa bei sozial aufgeladenen Konzepten (vgl. Lüsebrink 2005: 47). Eine Möglichkeit der Äquivalenzprüfung besteht in der Rückübersetzung, die den Transfer eines Fragebogens bzw. Interviewleitfadens aus der Zielsprache zurück in die Ausgangssprache durch nicht vorbelastete Übersetzer/-innen bezeichnet, um sodann anhand der Ausgangsversion des jeweiligen Untersuchungsinstruments vergleichend semantische Verschiebungen ausschließen zu können. Darüber hinaus existieren diskursive Verfahren, in deren Rahmen die Übersetzungen von Untersuchungsinstrumenten mit äquivalenzsensiblen Personen bzw. Muttersprachlern/-innen diskutiert werden. Im Rahmen des IDENT-Projekts wurde aufgrund der zur Verfügung stehenden äquivalenzsensiblen Muttersprachler/-innen, bzw. Personen mit z.T. zwei oder mehr Muttersprachen, das diskursive Verfahren der Äquivalenzprüfung gewählt, das in mehreren gemeinsamen und strukturierten Sitzungen zum Einsatz kam.

8 | Vgl. ausführlich Abschnitt 3.2.

9 | Anzumerken ist, dass aufgrund subjektiver Rezeptionsmechanismen auch im einsprachigen Untersuchungskontext nur sehr eingeschränkt von der Rekonstruktion identischer Bedeutungen durch die Befragten ausgegangen werden kann. 
Die Zusammenstellung der Stichprobe wurde von dem in Luxemburg führenden Meinungsforschungsinstitut übernommen, das sich ebenso für die Durchführung der quantitativen Befragung verantwortlich zeichnet. So wurden zwischen Oktober 2008 und Februar 2009 insgesamt 1579 Personen im Alter von 16 Jahren und mehr und mit Wohnsitz im Großherzogtum Luxemburg befragt. Zwei Drittel der Befragungen wurden per Internet (>Computer Assisted Web Interview<) durchgeführt, das verbleibende Drittel wurde telefonisch (>Computer Assisted Telephone Interview <) befragt, um sowohl medienkompetente als auch Personen mit eher traditionellem Mediengebrauch erreichen zu können. Die Stichprobe wurde nach den Variablen Alter, Geschlecht, Region, Nationalität und Erwerbstätigkeit (Status) gewichtet und ist repräsentativ für die Einwohner/-innen Luxemburgs. Die Auswertung der erhobenen Daten erfolgte über Verfahren der deskriptiven und multivariaten $^{10}$ Statistik. $^{11}$

\section{Qualitative Interviews}

Aufbauend auf der erläuterten quantitativen Repräsentativbefragung wurden im Frühjahr 2009 ergänzend Interviews mit Einwohnern/-innen Luxemburgs durchgeführt, die sich hierzu im quantitativen Fragebogen freiwillig bereit erklärt hatten. Die Entscheidung für eine groß angelegte qualitative Befragung lag in dem Anliegen begründet, Aussagen über subjektive Sinnzuschreibungen und -deutungen zu treffen, für deren Erhebung ein quantitatives Verfahren nicht geeignet erschien. Vor diesem Hintergrund haben sich die Projektbeteiligten zur gemeinsamen Durchführung von fokussierten Interviews entschieden. Dabei handelt es sich um ein offenes bis teilstandardisiertes Verfahren, das durch einen Interviewleitfaden unterstützt wird und keine Repräsentativität, sondern Repräsentanz (Lamnek 2005) anstrebt.

Aufgrund des hohen Forschungsinteresses im Rahmen der Interviewbefragung wurden drei thematische Interviewleitfäden (»Alltagskulturen«, »Selbst- und Fremdbilder«, »Sprachen und Räume«) entwickelt, in denen kurze, ausformulierte Fragen und gesprächsgenerierende Impulse vermerkt wurden. Zusätzlich waren die thematischen Arbeitsgruppen, die die einzelnen Fragen entwickelt hatten, gebeten, stichpunktartig den Hintergrund bzw. die Zielsetzung der jeweiligen Frage zu notieren. Dieses Vorgehen ermöglichte es den Interviewern/-innen - welche zwar durch den gemeinsamen Arbeitsprozess mit den Fragen vertraut, jedoch nicht zwangsweise die >Stoßrichtung < aller Fragen verinnerlicht hatten - sich im Rahmen der arbeitsteiligen Interviewdurchführung individuell auf die Interviews vorzubereiten. Hierfür wurden die ausführlichen Interviewleitfäden von den Wissenschaftlern/-innen in Stichpunkte überführt, was einen flexiblen und weitge-

10 | Vgl. ausführlich Abschnitt 3.2.

11 | Der komplette Datenband inklusive Fragebogen ist in der Bibliothek der Universität Luxemburg einsehbar. 
hend offenen Interviewverlauf ermöglichte. Zwei Treffen wurden organisiert, um den Dialog zwischen den Autoren/-innen der Fragen und den Interviewern/-innen, die nicht zwangsläufig die gleichen waren, zu fördern.

Die Interviewbefragung verfolgte nicht das Ziel, bestimmte Häufigkeiten von Phänomenen zu ermitteln, sondern ein vertieftes Verständnis sozialer Phänomene zu erlangen, d.h. nach deren Repräsentanz zu fragen. Dementsprechend bestand das Interesse nicht an einer Zufallsstichprobe, um später Rückschlüsse auf eine Grundgesamtheit ziehen zu können, sondern am Studium einzelner und vielfältiger Fälle, um ein Phänomen möglichst facettenreich zu erfassen. Diese Ausrichtung erforderte bei der Stichprobenziehung »[...] eine Entscheidung gegen den $\mathrm{Zu}-$ fall und eine theoretisch-systematische Auswahl [der zu Befragenden] (Lamnek 2005: 186). Damit spricht Lamnek die bewussten Verfahren der qualitativen Stichprobenrealisierung an, zu denen u.a. die Vorab-Festlegung der Sample-Struktur zählt. (Weischer 2007: 207-209) Zu den zentralen Kriterien der bewussten Stichprobenzusammenstellung zählten das Geschlecht, das Alter und die Nationalität, welche die Pluralität der Luxemburger Wohnbevölkerung abbilden sollten. Dies ist mit der Interviewstichprobe, die 75 Personen zählt, weitgehend gelungen.

Die Durchführung der Interviews wurde unter den Wissenschaftlern/-innen nach sprachlichen Vorlieben, zeitlicher Verfügbarkeit und thematischem Interesse aufgeteilt, so dass die Projektbeteiligten jeweils ca. fünf Personen interviewten. Ein zentrales Anliegen war die interdisziplinäre Verteilung der Interviewenden, die deshalb mit Leitfäden arbeiteten, die jeweils von einer anderen Arbeitsgruppe entwickelt worden waren. Die Interviews fanden nach Terminabsprache bei den Befragten zuhause in ihrer vertrauten Umgebung und oftmals während den Tagesrandzeiten statt. Im ca. ein- bis anderthalbstündigen Gespräch wurde dem Interviewleitfaden folgend auf einen bestimmten Gegenstand mit seinen Teilaspekten fokussiert und die Befragten bekamen die Möglichkeit, sich frei, aber in einem bestimmten thematischen Rahmen zu äußern. So konnten die Interpretationen und Reaktionen der Befragten in relativ offener Form erhoben werden und die von den Forschenden nicht antizipierten Gesichtspunkte in Erscheinung treten. Zur Aufbereitung der digital aufgezeichneten Interviewdaten wurde das Transkriptionsverfahren der standardsprachlichen Umschrift (Mayring 2007: 91) gewählt, da die inhaltlich-thematische Ebene der Interviews im Vordergrund stand. Über dieses Vorgehen, welches u.a. im minimalen Beheben von Satzbaufehlern und im Glätten des Redefluss besteht, sollte die Lesbarkeit der gesprochenen Sprache verbessert werden. Die Transkriptionen der Interviews in luxemburgischer, deutscher, französischer, englischer und portugiesischer Sprache konnten mit Unterstützung studentischer Hilfskräfte realisiert werden. 


\begin{tabular}{|c|c|c|c|c|c|c|}
\hline & $\begin{array}{l}\text { Thema: } \\
\text { Alltags- } \\
\text { kulturen }\end{array}$ & $\begin{array}{l}\text { Thema: } \\
\text { Selbst u. } \\
\text { Fremdbilder }\end{array}$ & $\begin{array}{l}\text { Thema: } \\
\text { Spra- } \\
\text { chen u. } \\
\text { Räume }\end{array}$ & $\begin{array}{l}\text { Insge- } \\
\text { samt }\end{array}$ & $\begin{array}{c}\text { Luxemburger } \\
\text { Wohn- } \\
\text { bevölkerung } \\
(2007)^{\star}\end{array}$ & $\begin{array}{l}\text { Abweichung } \\
\text { zur Grund- } \\
\text { gesamtheit }\end{array}$ \\
\hline \multirow[t]{2}{*}{ Stichprobe (N) } & $n=27$ & $n=26$ & $n=22$ & $\mathrm{~N}=75$ & 476.200 & $\begin{array}{l}\text { Prozent- } \\
\text { punkte }\end{array}$ \\
\hline & (a) & (b) & (c) & (d) & (e) & (d-e) \\
\hline \multicolumn{7}{|l|}{ Geschlecht } \\
\hline Männer & 50,0 & 48,1 & 40,9 & 46,7 & 49,5 & $-2,8$ \\
\hline Frauen & 50,0 & 51,9 & 59,1 & 53,3 & 50,5 & 2,8 \\
\hline \multicolumn{7}{|l|}{ Altersgruppen } \\
\hline $15-29$ & 23,1 & 11,1 & 13,6 & 16,0 & 22,6 & $-6,6$ \\
\hline $30-44$ & 30,8 & 44,4 & 27,3 & 34,7 & 29,8 & 4,9 \\
\hline $45-59$ & 26,9 & 25,9 & 31,8 & 28,0 & 24,7 & 3,3 \\
\hline $60+$ & 19,2 & 18,5 & 27,3 & 21,3 & 22,8 & $-1,5$ \\
\hline \multicolumn{7}{|l|}{ Nationalität } \\
\hline Luxemburger & 61,5 & 55,6 & 63,6 & 60,0 & 59 & 1,0 \\
\hline $\begin{array}{l}\text { ansässige } \\
\text { Ausländer }\end{array}$ & 38,5 & 44,4 & 36,4 & 40,0 & 41 & $-1,0$ \\
\hline \multicolumn{7}{|l|}{ davon } \\
\hline Franzosen & 10,0 & 8,3 & 12,5 & 10,0 & 12,7 & $-2,7$ \\
\hline Portugiesen & 40,0 & 33,3 & 25,0 & 33,3 & 37 & $-3,7$ \\
\hline Deutsche & 10,0 & 8,3 & 0,0 & 6,7 & 5,7 & 1,0 \\
\hline Belgier & 10,0 & 8,3 & 12,5 & 10,0 & 8,3 & 1,7 \\
\hline Italiener & 10,0 & 8,3 & 12,5 & 10,0 & 9,6 & 0,4 \\
\hline $\begin{array}{r}\text { Engländer/ } \\
\text { Niederländer }\end{array}$ & 0,0 & 8,3 & 0,0 & 3,3 & 4,4 & $-1,1$ \\
\hline andere & 20,0 & 25,0 & 37,5 & 26,7 & 8,3 & 18,4 \\
\hline
\end{tabular}

Tabelle 1: Stichproben der qualitativen Befragungen nach soziodemographischen Merkmalen (Quelle: Eigene Studie; * Statec 2007).

Die schließlich vorliegenden Interviewtranskripte wurden sodann computergestützt und durch deduktiv-selektives Codieren bzw. induktiv-freies Codieren ausgewertet. In einem ersten Schritt wurden die Transkripte von den Projektbeteiligten nach einem gemeinsam verabschiedeten Codierschema mit vordefinierten Codes und Subcodes systematisiert (Grobcodierung). Dieses Schema wurde in mehreren gemeinsamen >Codier-Sitzungen < erarbeitet, in denen der direkte Austausch zwischen den Projektbeteiligten möglich und erforderlich war. Im Rahmen des darauf folgenden induktiv-freien Codierens, das von den jeweiligen thematisch spezialisierten Kollegen/-innen vorgenommen wurde, wurden unter Einbezug von Ankerzitaten und Memotechniken die bislang nicht berücksichtigten, jedoch im Interview angesprochenen Aspekte aufgegriffen; auch die von den Interviewern/-innen nach jedem Gespräch notierten situativen Beschreibungen der Inter- 
aktion wurden per Memofunktion hinzugefügt. So konnte die Codestruktur im Rahmen der sogenannten >Feincodierung < weiter differenziert werden. Die Auswertung der so aufbereiteten Texte erfolgte über die synoptische Analyse (Kelle 2007: 491f.), welche aus methodologischer Sicht zur phänomenologischen Analyse nach Mayring (Mayring 2002: 107f.) und aus technischer Sicht zu den einfachen Retrievaltechniken zählt. Ihr Ziel ist es, über die Variation bzw. über den Prozess des Vergleichens, wie ihn Glaser und Strauss (Glaser/Strauss 2005: 107ff.) bereits in den 1970er Jahren beschreiben, inhaltliche Unterschiede, Ähnlichkeiten und Beziehungen zwischen den subjektiven Bedeutungsstrukturen herauszuarbeiten, um so zum »Wesenskern der Phänomene« (Mayring 2002: 108) vorzudringen. Hierfür wurden die Textsegmente eines oder mehrerer Codes zu einer Liste zusammengestellt (Textretrieval), die dann vergleichend das interpretative Herausarbeiten von Unterschieden und Ähnlichkeiten ermöglichte. Für das beschriebene Verfahren der computergestützten Analyse qualitativer Daten wurden verschiedene Schulungen und Workshops durchgeführt, die sowohl von den Projektbeteiligten als auch von eingeladenen Experten/-innen gestaltet wurden.

Neben der groß angelegten qualitativen Befragung wurden von einigen Wissenschaftlern/-innen zusätzlich Experten/-innen aus bestimmten Gesellschaftsfeldern befragt. Im qualitativen Experten/-inneninterview war jedoch weniger der Experte/die Expertin der Gegenstand der Analyse, d.h. nicht »die Person mit ihren Orientierungen und Einstellungen im Kontext des individuellen oder kollektiven Lebenszusammenhangs « (Meuser/Nagel 2005: 72f.). Vielmehr ging es um die Experten/-innen als Informanten/-innen über einen institutionellen oder organisatorischen Zusammenhang. (Weischer 2007: 281) Demzufolge wurden im Experten/inneninterview bestimmte Wissensbestände ermittelt, die die betreffende Person in ihrem beruflichen Kontext erworben hat und die Einblicke in ein bestimmtes Untersuchungsfeld geben. Als Experte/-in galt dabei, wer für bestimmte Formen der Problemlösung in einem Untersuchungsfeld Verantwortung trägt und/oder wer über einen privilegierten Zugang zu Informationen über Akteursgruppen verfügt. Die Vorbereitung, Durchführung und Auswertung der Experten/-inneninterviews wurde von den Wissenschaftlern/-innen sichergestellt, deren Forschungsinteresse das Themenfeld berührte, das die jeweiligen Experten/-innen repräsentierte. Dazu zählten die Bereiche Verwaltung, Kultur und Wirtschaft.

\section{Inhaltsanalysen}

Neben der erläuterten quantitativen und qualitativen Befragung kamen angesichts der verschiedenen Analysegegenstände ebenso Methoden zum Einsatz, die sich unter dem Begriff der Inhaltsanalyse zusammenfassen lassen. Dabei handelt es sich auf Basis eines weit gefassten Textverständnisses um textanalytische Verfahren, die diskursanalytische Methoden und semiotische Zugänge einschließen. Die jeweils verwendeten Methoden werden in den folgenden Kapiteln an entsprechender Stelle ausführlich erläutert. 
Wie oben bereits angedeutet, wurden im Rahmen der thematischen Schwerpunkte jeweils unterschiedliche methodische Verfahren verwendet, was zu einer Fülle empirischen Datenmaterials führte. Die verfügbaren qualitativen und quantitativen Daten wurden von den Vertretern/-innen der unterschiedlichen Disziplinen vor dem Hintergrund der thematischen Orientierungen sinnvoll zusammengeführt. Die Kombination quantitativer und qualitativer Methoden bzw. entsprechender Daten sollte zum einen die Validität der Untersuchungsergebnisse sicherstellen; zum anderen ging es jedoch um die Ergänzung der Perspektiven auf einen Untersuchungsgegenstand, bei der verschiedene Aspekte eines Phänomens komplementär zu einem Ganzen zusammengeführt werden. (Kelle/Erzberger 2007: 302ff.) Dieses Vorgehen, das in der methodischen Diskussion unter dem Stichwort der >Triangulation< (vgl. Denzin/Lincoln 2005) gefasst wird, konnte im Rahmen zahlreicher Arbeitsgruppentreffen und Workshops umgesetzt werden.

\section{Interdisziplinäre Zusammenarbeit}

Die Beiträge in diesem Buch bilden keine Zusammenstellung einzelner Forschungsarbeiten, sondern sie sind das Ergebnis einer dreijährigen und intensiven Zusammenarbeit zwischen Wissenschaftlern/-innen aus unterschiedlichen Disziplinen. Im Folgenden sollen Einblicke in die interdisziplinäre Zusammenarbeit und in die Organisation des IDENT-Projekts gegeben werden. Es wurde unter Leitung von Christian Schulz zwischen 2007 und 2010 durchgeführt, dem eine Lenkungsgruppe, bestehend aus weiteren Projektbeteiligten, eng zur Seite stand. Neben dieser strategischen Ebene wurde ein IDENT-Koordinierungsbüro eingerichtet, in dem Rachel Reckinger und Christian Wille die operativen Arbeiten koordinierten und strategische Entscheidungen vorbereiteten. Die Forschungsarbeiten wurden von der IDENT-Projektgruppe durchgeführt, zu denen Vertreter/-innen der Anglistik, Gender Studies, Geografie und Raumplanung, Germanistik, Geschichte, Interkulturellen Kommunikation, Luxemburgistik, Medienwissenschaft, Romanistik, Soziologie und Visuellen Kunst zählten. Im Folgenden soll es um die Ebene der operativen Arbeiten gehen, d.h. um die Organisationsformen und Umsetzung der Forschungsarbeiten, die sich in drei zentrale Phasen untergliedern lassen: Vorbereitungsphase (2007-2008), empirische Phase (2008-2009) und Endphase (2009-2010).

Während der Projektlaufzeit wurden verschiedene kollektive Arbeitsformen der interdisziplinären Zusammenarbeit genutzt, zu denen Arbeitsgruppen, Workshops, Werkstattgespräche und internationale Konferenzen zählen.

Arbeitsgruppen: Um einem intensiven Austausch zwischen den Projektbeteiligten zu ermöglichen, wurden verschiedene Arbeitsgruppen gebildet, die sich in dauerhafte und thematisch orientierte sowie in temporäre und methodisch orientierte Arbeitsgremien untergliedern lassen. Die über den gesamten Projektverlauf bestehenden Arbeitsgruppen behandelten jeweils einen der oben erläuterten thematischen Schwerpunkte. An den Arbeitsgruppen, die je nach Projektphase in 
einem zwei- bis dreiwöchigen Rhythmus zusammenkamen, nahmen ca. fünf bis sechs Wissenschaftler/-innen mit folgender disziplinärer Besetzung teil:

\begin{tabular}{|l|l|l|l|l|}
$\begin{array}{l}\text { AG Sozio-kul- } \\
\text { turelle Milieus }\end{array}$ & AG Sprachen & $\begin{array}{l}\text { AG Selbst- und } \\
\text { Fremdbilder }\end{array}$ & \multicolumn{1}{|c|}{ AG Räume } & $\begin{array}{c}\text { AG Alltags- } \\
\text { kulturen }\end{array}$ \\
\hline $\begin{array}{l}\text { Visuelle Kunst, } \\
\text { Soziologie, } \\
\text { Germanistik }\end{array}$ & $\begin{array}{l}\text { Germanistik, } \\
\text { Luxemburgistik }\end{array}$ & $\begin{array}{l}\text { Remanistik, } \\
\text { Geschichte, } \\
\text { Medienwissen- } \\
\text { schaft }\end{array}$ & $\begin{array}{l}\text { Geschichte, } \\
\text { Geographie } \\
\text { und Raumpla- } \\
\text { nung }\end{array}$ & $\begin{array}{l}\text { Gender } \text { dies, Anglistik, } \\
\text { Soziologie, } \\
\text { Interkulturelle } \\
\text { Kommunikation }\end{array}$ \\
\hline
\end{tabular}

Tabelle 2: Thematische Arbeitsgruppen

Die Aufgaben der thematischen Arbeitsgremien bestanden je nach Projektphase in der Entwicklung eines gemeinsamen theoretisch-konzeptionellen Rahmens und Forschungsdesigns, in der Erhebung und Aufarbeitung empirischer Daten, in der gemeinsamen Interpretation der erzielten Forschungsergebnisse und dem gemeinschaftlichen Verfassen eines Kapitels in diesem Buch. Die Forschungsarbeiten innerhalb der Arbeitsgruppen strukturierten sich entlang verschiedener Fallbeispiele, die unter einer gemeinsamen Fragestellung durchgeführt und diskutiert wurden. Die Aktivitäten der Arbeitsgruppen wurden von der Projektkoordination begleitet und moderiert, wobei eine der Herausforderungen darin bestand, die fünf Arbeitsgruppen mit ihren jeweiligen Besonderheiten in konzeptioneller und methodischer Hinsicht mit Blick auf die zentralen Projektphasen zu synchronisieren.

Weitere, lediglich während bestimmter Projektphasen ins Leben gerufene Arbeitsgruppen hatten einen methodischen Auftrag. Das bedeutet, sie sollten in engem Austausch mit den inhaltlichen Arbeitsgruppen die mehrsprachigen Untersuchungsinstrumente entwickeln. Dementsprechend sammelten und integrierten die multidisziplinär besetzten Arbeitsgruppen »Fragebogen « bzw. »Interview « die in den thematischen Arbeitsgruppen erarbeiteten Fragestellungen, prüften sie auf ihre Formulierung und bereiteten sie für die quantitativen und qualitativen Untersuchungsinstrumente auf. Ebenso fielen Pretests in ihren Verantwortungsbereich, die sich im Falle der Arbeitsgruppe »Interview « u.a. in der Organisation von zwei Schulungen zur Interviewführung mit Rollenspielen sowie einer Schulung zur computergestützten Interviewaufbereitung für die Interpretationsarbeit äußerten.

Workshops: Um einer Fragmentierung der IDENT-Projektgruppe durch das arbeitsteilige Engagement der Wissenschaftler/-innen in verschiedenen Arbeitsgruppen entgegenzuwirken, wurden zusätzlich Projektworkshops durchgeführt. Hier versammelten sich alle Projektbeteiligten im Vier- bis Acht-Wochen-Rhythmus im Plenum, das je nach Projektphase unterschiedliche Diskussionsgegenstände hatte. Während in der Projekt-Vorbereitungsphase die Workshops überwiegend dazu dienten, sich über disziplinäre Besonderheiten auszutauschen und sich 
somit einem gemeinsamen und disziplinübergreifenden Verständnis bestimmter Konzepte anzunähern, waren die Workshops ebenso zur gemeinsamen Ausarbeitung der Projektarchitektur unverzichtbar. Besonders während der empirischen Phase bildeten die Plenartreffen ein wichtiges Instrument, um sich gemeinsam über organisatorische Fragen komplexer Erhebungsprozesse zu verständigen. Die von der Projektkoordination geleiteten Workshops wurden insbesondere gegen Ende des Projekts genutzt, um sich über die Arbeitsergebnisse in den thematischen Arbeitsgruppen auszutauschen und diese miteinander zu vernetzen. Aufgrund des begrenzten zeitlichen Rahmens der Workshops wurde zusätzlich ein zweitägiges Kolloquium organisiert, um dem arbeitsgruppen- und damit disziplinübergreifenden Austausch genügend Raum zur Interpretation der Forschungsergebnisse $\mathrm{zu}$ geben.

Werkstattgespräche: Neben den regelmäßig stattfindenden Workshops wurde eine Serie von Werkstattgesprächen ins Leben gerufen, die den Austausch mit externen Experten/-innen in einer lockeren Arbeitsatmosphäre zum Ziel hatten. Insbesondere während der Projekt-Vorbereitungsphase war der Austausch mit Experten, die bereits ähnliche Fragestellungen bearbeitet hatten, sehr hilfreich zur Entwicklung des gemeinsamen theoretisch-konzeptionellen Rahmens, der Projektarchitektur und der schließlich verwendeten Untersuchungsinstrumente. Wir bedanken uns für die Unterstützung bei Michael Vester und Daniel Gardemin (Universität Hannover), bei Jean-Marie Klinkenberg (Universität Lüttich) und Jürgen Pohl (Universität Bonn).

Internationale Konferenzen: Eine weitere Plattform zum Austausch mit erfahrenen Wissenschaftlern/-innen auf dem Gebiet der Identitätsforschung boten die durchgeführten internationalen Konferenzen. Die Veranstaltungen wurden strategisch in den Projektverlauf integriert, so dass unterschieden werden kann zwischen einer >Input-Konferenz< (2009) und einer >Output-Konferenz<(2010). Zu der im Jahr 2009 organisierten Konferenz mit dem Titel »IDENT - Sozio-kulturelle Identitäten: Interaktion und Repräsentation« wurden Referenten/-innen mit dem Auftrag eingeladen, neben der Vorstellung der eigenen Arbeiten die Fragestellungen und Konzeption des IDENT-Projekts kritisch zu würdigen. Für die so gewonnenen und in den weiteren Projektverlauf integrierten Anregungen bedanken wir uns herzlich bei Brigitta Busch (Universität Wien), Thomas Dörfler (Universität Bayreuth), Britta Kalscheuer (Universität Frankfurt a.M.), Claudia Lenz (Center for Studies of Holocaust and Religious Minorities, Oslo), Albert Lévy (Université Paris VIII), Lydia Martens (Keele University), Rolf Parr (Universität Bielefeld), Albert Raasch (Universität des Saarlandes), Klaus Sachs-Hombach (TU Chemnitz) und Jürgen Straub (Ruhr-Universität Bochum). Die im Jahr 2010 organisierte Konferenz mit dem Titel »Doing Identity in Luxemburg: Subjektive Aneignungen - Institutionelle Zuschreibungen - sozio-kulturelle Milieus « wurde veranstaltet, um die vorliegenden Forschungsergebnisse einem Fachpublikum und der interessierten Öffentlichkeit vorzustellen und kritisch zu diskutieren. 
Neben diesen Formen der interdisziplinären Zusammenarbeit sollen verschiedene Erfahrungen aus Sicht der Projektkoordination an dieser Stelle festgehalten werden. So sollte den Austauschprozessen und Annäherungen zwischen verschiedenen Disziplinen in anderen Vorhaben dieser Größenordnung genügend Raum gegeben werden. Das bedeutet mit Geduld auch Verwirrungen und ein gewisses >Aneinander-vorbei-Reden zuzulassen und empathisch in Kommunikationsprozesse einzugreifen und diese ergebnisorientiert $\mathrm{zu}$ moderieren oder $\mathrm{zu}>$ übersetzen $<$ Oftmals verstellen nur unterschiedliche Begrifflichkeiten den Blick auf denselben Gegenstand. Hierfür hilfreich ist ein generalistisch-analytischer Blick auf fachliche Inhalte und ein gewisses Einfühlungsvermögen hinsichtlich der beteiligten Forscher/-innenpersönlichkeiten. Insbesondere an der Universität Luxemburg mit seinen Mitarbeitern/-innen aus verschiedenen Ländern Europas und aus Übersee müssen zusätzlich verschiedene Sprachen und universitäre Sozialisationserfahrungen in den Forschungsalltag integriert werden. Dies erfordert nicht nur interkulturelle Kompetenzen, sondern auch ein entsprechendes Zeitbudget, das die gewonnene Interdisziplinaritätserfahrung jedoch entsprechend bereichert.

\subsection{Literatur}

Abels, Heinz (2006): Identität. Über die Entstehung des Gedankens, dass der Mensch ein Individuum ist, den nicht leicht zu verwirklichenden Anspruch auf Individualität und die Tatsache, dass Identität in Zeiten der Individualisierung von der Hand in den Mund lebt, Wiesbaden: VS Verlag für Sozialwissenschaften.

Ahbe, Thomas (1997): »Ressourcen - Transformationen - Identität«, in: Heiner Keupp/Renate Höfer (Hg.): Identitätsarbeit heute. Klassische und aktuelle Perspektiven der Identitätsforschung, Frankfurt a.M.: Suhrkamp, S. 207-226.

Appadurai, Arjun (1998): »Globale ethnische Räume«, in: Ulrich Beck (Hg.): Perspektiven der Weltgesellschaft, Frankfurt a.M.: Suhrkamp, S. 11-40.

Avanza, Martina/Laferté, Gilles (2005): »Dépasser la >construction des identités<? Identification, image sociale, appartenance«, Genèses. Sciences sociales et histoire, $\mathrm{N}^{\circ}$ 61, S. 134-152.

Baltes-Löhr, Christel (2006): Migration und Identität. Portugiesische Frauen in Luxemburg, Frankfurt a.M./London: IKO-Verlag für Interkulturelle Kommunikation.

Bayart, Jean-François (1996): L'illusion identitaire, Paris: Fayard.

Bhaba, Homi (1994): The Location of Culture, London: Routledge.

Bauman, Zygmut (1999): Unbehagen in der Postmoderne, Hamburg: Hamburger Edition.

Bourdieu, Pierre (1992): Die verborgenen Mechanismen der Macht, Hamburg: VSA.

Bourdieu, Pierre (1980): Le sens pratique, Paris: Editions de Minuit. 
Bourdieu, Pierre (1972): Esquisse d'une théorie de la pratique. Précédé de trois études d'ethnologie kabyle, Paris: Le Seuil.

Braun, Michael (2006): Funktionale Äquivalenz in interkulturell vergleichenden Umfragen: Mythos und Realität, Mannheim: ZUMA.

Brubaker, Rogers (2001): »Au-delà de l'>identité««, in: Actes de la recherche en sciences sociales, $\mathrm{N}^{\circ} 139$, S. 66-85.

Chilla, Tobias (2009): »Europa in Luxemburg: Die EU-Institutionen«, in: Patrick Bousch/Tobias Chilla/Philippe Gerber et al. (Hg.): Der Luxemburg Atlas du Luxembourg, Köln: Emons, S. 16-17.

Denzin, Norman K./Lincoln, Yvonna S. (32005) (Hg.): The SAGE Handbook of Qualitative Research, London: SAGE.

Eickelpasch, Rolf/Rademacher, Claudia (2004): Identität, Bielfeld: transcript.

Elias, Norbert (1986): »Figuration«, in: Bernhard Schäfer (Hg.): Grundbegriffe der Soziologie, Opladen: Leske + Budrich, S. 88-91.

Fehlen, Fernand/Pigeron-Piroth, Isabelle (2009): Mondialisation du travail et pluralité des marchés du travail: L'exemple du Luxembourg. Skript des Diskussionsbeitrags für 12è Journées de Sociologie du Travail, 25 et 26 juin 2009, GREE, Université de Nancy.

Foucault, Michel (1994): »Technologies of the Self«, in: Paul Rabinow/Nicolas Rose: The Essential Foucault. Selections from the Essential Works of Foucault 1954-1984, New York/London: The New Press, S. 145-169.

Foucault, Michel (1984): Histoire de la sexualité. Tome 3: Le souci de soi, Paris: Gallimard.

Giddens, Anthony (1997): Die Konstitution der Gesellschaft, Frankfurt a.M./New York: Campus.

Giddens, Anthony (1991): Modernity and Self-Identity, Cambridge: Polity.

Giesen, Bernhard (1999): »Identität und Versachlichung. Unterschiedliche Theorieperspektiven auf kollektive Identität«, in: Herbert Willems/Alois Hahn: Identität und Moderne, Frankfurt a.M.: Suhrkamp, S. 389-402.

Glaser, Barney G./Strauss, Anselm L. ('2005): Grounded Theory. Strategien qualitativer Forschung, Bern: Huber.

Goffman, Erving (2003): Wir alle spielen Theater, München: Piper.

Greverus, Ina-Maria (1995): Die Anderen und Ich, Darmstadt: Wissenschaftliche Buchgesellschaft.

Halpern, Catherine (2009): Identité(s). L'individu, le groupe, la société, Paris: Editions Sciences Humaines.

Hark, Sabine (1999): Deviante Subjekte. Die paradoxe Politik der Identität, Opladen: Leske + Budrich.

Kaufmann, Jean-Claude (2004): L'invention de soi. Une théorie de l'identité, Paris: Armand Colin.

Kelle, Udo ( $\left.{ }^{6} 2007\right)$ : »Computergestützte Analyse qualitativer Daten«, in: Uwe Flick / Ernst von Kardoff/Ines Steinke (Hg.): Qualitative Forschung. Ein Handbuch, Hamburg: Rowohlt, S. 486-502. 
Kelle, Udo/Erzberger, Christian (52007): »Qualitative und quantitative Methoden: kein Gegensatz«, in: Uwe Flick/Ernst von Kardoff /Ines Steinke (Hg.): Qualitative Forschung. Ein Handbuch, Hamburg: Rowohlt, S. 299-309.

Keupp, Heiner/Höfer, Renate (1997) (Hg.): Identitätsarbeit heute. Klassische und aktuelle Perspektiven der Identitätsforschung, Frankfurt a.M.: Suhrkamp.

Keupp, Heiner/Ahbe, Thomas/Gmür, Wolfgang/Höfer, Renate/Mitzscherlich Beate/Kraus, Wolfgang/Straus, Florian (32006): Identitätskonstruktionen. Das Patchwork der Identitäten in der Spätmoderne, Reinbek bei Hamburg: Rowohlt.

Kmec, Sonja (2007): »Von Identitäten und Identifikationen«, in: Forum $\mathrm{N}^{\circ}$ 271: Lëtzebuerg?, Luxembourg, S. 39-41.

Krappmann, Lothar $\left({ }^{10} 2005\right)$ : Soziologische Dimensionen der Identität. Strukturelle Bedingungen für die Teilnahme an Interaktionsprozessen, Stuttgart: KlettCotta.

Lahire, Bernhard (1998): L'homme pluriel. Les ressorts de l'action, Paris: Nathan.

Lamnek, Siegfried (42005): Qualitative Sozialforschung. Lehrbuch, Weinheim: Beltz.

Luckmann, Thomas (2007): Lebenswelt, Identität und Gesellschaft, Konstanz: UVK Verlagsgesellschaft.

Lüsebrink, Hans-Jürgen (2005): Interkulturelle Kommunikation. Interaktion, Fremdwahrnehmung, Kulturtransfer, Weimar: Metzler.

MacClancy, Jeremy (2004), »Food, Identity, Identification«, in: Helen MacBeth/ Jeremy MacClancy (Hg.): Researching Food Habits. Methods and Problems, Oxford/New York: Berghahn Books, S. 63-73.

Mayring, Philipp (52002): Einführung in die qualitative Sozialforschung, Weinheim: Beltz.

Meuser, Michael/Nagel, Ulrike (2005): »ExpertInneninterviews - vielfach erprobt, wenig bedacht. Ein Beitrag zur qualitativen Methodendiskussion«, in: Alexander Bogner/Beate Littig/Wolfgang Menz (Hg.): Das Experteninterview. Theorie, Methode, Anwendung, Wiesbaden: VS, S. 71-93.

Meyran, Régis (2008): Le mythe de l'identité nationale, Paris: Berg International. Niethammer, Lutz (2000): Kollektive Identität. Heimliche Quellen einer unheimlichen Konjunktur, Reinbek bei Hamburg: Rowohlt.

Reckwitz, Andreas (2001): »Der Identitätsdiskurs. Zum Bedeutungswandel einer sozialwissenschaftlichen Semantik«, in: Werner Rammert (Hg.): Kollektive Identitäten und kulturelle Innovationen. Ethnologische, soziologische und historische Studien, Leipzig: S 21-38.

Ricoeur, Paul (1990): Soi-même comme un autre, Paris: Le Seuil.

Rosa, Hartmut (2007): »Identität«, in: Jürgen Straub/Arne Weidemann (Hg.): Handbuch interkulturelle Kommunikation und Kompetenz. Grundbegriffe Theorien - Anwendungsfelder, Stuttgart: J. B. Metzler.

Ruano-Borbolan, Jean-Claude (1998), L'identité. L'individu, le groupe, la société, Auxerre: Sciences Humaines. 
Sennett, Richard (1996): „Etwas ist faul in der Stadt«, in: Die Zeit, N 5, 26.01.1996, S. $47-48$.

Statec (2007): Luxemburg in Zahlen, Luxemburg.

Straub, Jürgen (2004): »Identität«, in: Friedrich Jaeger/Burkhard Liebsch: Handbuch der Kulturwissenschaften. Grundlagen und Schlüsselbegriffe, Stuttgart: J. B. Metzler.

Straub, Jürgen/Renn, Joachim (2002) (Hg.): Transitorische Identität. Der Prozesscharakter des modernen Selbst, Frankfurt a.M.: Campus.

Straub, Jürgen (1998): »Personale und kollektive Identität«, in: Aleida Assmann/ Heidrun Friese (Hg.): Identitäten, Frankfurt a.M.: Suhrkamp.

Straus, Florian/Höfer, Renate (1997): »Entwicklungslinien alltäglicher Identitätsarbeit«, in: Heiner Keupp/Renate Höfer (Hg.): Identitätsarbeit heute. Klassische und aktuelle Perspektiven der Identitätsforschung, Frankfurt a.M.: Suhrkamp, S. 270-307.

Vester, Michael/Von Oertzen, Peter/Geiling, Heiko/Hermann, Thomas/Müller, Dagmar (2001): Soziale Milieus im gesellschaftlichen Strukturwandel. Zwischen Integration und Ausgrenzung, Frankfurt a.M.: Suhrkamp.

Weinreich, Peter/Saunderson, Wendy (Hg.) (2003): Analysing Identites. CrossCultural, Societal and Clinical Contexts, London: Routledge.

Weischer, Christoph (2007): Sozialforschung, Weinheim: Beltz, S. 207-209.

Willems, Helmut/Milmeister, Paul (2008): »Migration und Integration«, in: Lorig, Wolfgang H./Hirsch, Mario (Hg.): Das politische System Luxemburg. Eine Einführung, Wiesbaden: VS, S. 62-92.

Zahlen, Paul (2008): »Arbeitsmarktpolitik«, in: Wolfgang H. Lorig/Mario Hirsch (Hg.): Das politische System Luxemburg. Eine Einführung, Wiesbaden: VS, S. $253-285$. 


\section{Sozio-kulturelle Milieus in Luxemburg}

Wilhelm Amann, Fernand Fehlen, Georg Mein

\subsection{Sozialstruktur im Wandel. Der Weg Vom Vertikal orientierten SchichtModell ZUR HORIZONTAL DIVERSIFIZIERTEN MILIEULANDSCHAFT}

Die Sozialforschung hat sich zur Beschreibung der Sozialstruktur moderner Gesellschaften lange Zeit auf das in den 1930er Jahren von Theodor Geiger entwickelte Modell der sozialen Schichten gestützt. Bei diesem Schichtmodell steht das Anliegen im Vordergrund, die Gesamtbevölkerung nach bestimmten Indikatoren (ökonomischen und sozialen) so zu gliedern, dass Gruppierungen mit ähnlicher Soziallage und damit verknüpften typischen Subkulturen und Lebenschancen entstehen. (Vgl. Geißler 2002: 117) Dabei wird - im Unterschied zu dem bekannten Diktum Karl Marx' - nicht deterministisch unterstellt, dass das Sein das Bewusstsein bestimme, dass also aus den äußeren Lebensumständen konkrete Verhaltensweisen resultieren. Aber es wird empirisch überprüft, in welchen Bereichen und wie stark innere und äußere Strukturen zusammenhängen. Dabei spielen einerseits ökonomische Indikatoren wie Einkommen, Vermögen, Beruf und andererseits soziale Indikatoren wie Bildungsstatus, Sozialprestige, Gruppenzugehörigkeit eine Rolle. Diese Gruppierungen lassen sich dann zu einem vertikal geschichteten Bild der Sozialstruktur zusammenfassen.

Aufgrund ihrer primär >vertikalen< Differenzierung sind Schichtmodelle allerdings weitgehend blind für >horizontale Ungleichheiten, wie sie etwa aus geschlechtsspezifischen, generationstypischen, lokalen und regionalen Unterschieden resultieren. Vor allem das in den 1980er Jahren vom Sinus-Institut für Markt- und Wahlforschung entwickelte Modell der >Sozialen Milieus< hat sich konsequent auch auf der horizontalen Ebene der Sozialstruktur orientiert. Während Schichtanalytiker die Bevölkerung zunächst nach objektiv ähnlichen Lebensbedingungen untergliedern, um dann zu untersuchen, welche Mentalitäten, Einstellungen, Verhaltensweisen und Lebenschancen mit diesen unterschiedlichen Lebensumständen typischerweise verknüpft sind, gruppiert der Milieuansatz die 
Menschen zunächst nach Unterschieden in ihren Wertorientierungen, alltagsästhetischen Mustern, typischen Erlebnisformen und Lebensstilen, und fragt dann, aus welchen sozialen Schichten sich die Milieus rekrutieren. In einem sozialen Milieu werden somit diejenigen Menschen zusammengefasst, die sich in Lebensauffassung und Lebensweise ähneln, die also solche äußeren Lebensbedingungen und inneren Haltungen aufweisen, dass sie einen gemeinsamen Lebensstil herausbilden. Anders ausgedrückt: Milieus betonen die subjektive Seite der Gesellschaft.

Diese theoretische Neuperspektivierung der sozialen Wirklichkeit ergibt Sinn, denn mit Blick auf die radikalen Veränderungen der sozio-kulturellen Lebenswelt stimmen viele Theoretiker darin überein, dass die drastische Verbesserung des Lebensstandards in den letzten Jahrzehnten zu einem erdrutschartigen Wandel in der grundsätzlichen Werthaltung der Bevölkerung geführt hat. Durch diesen Wandel wurde seit den $1960 e r$ Jahren ein Prozess der radikalen Individualisierung und Diversifizierung von Lebenslagen und Lebensstilen in Gang gesetzt, der Helmut Schelskys These von der nivellierten Mittelstandsgesellschaft weitestgehend zu bestätigen scheint. Schelsky geht von einer grundsätzlich hochmobilen Sozialstruktur aus, in der kollektive Auf- und Abstiegsprozesse letztlich eine Einebnung der sozialen Klassen und Schichten bedingen. Dieser Tatbestand führe schließlich »zu einer sozialen Nivellierung in einer verhältnismäßig einheitlichen Gesellschaftsschicht, die ebenso wenig proletarisch wie bürgerlich ist, d.h. durch den Verlust der Klassenspannung und sozialen Hierarchie gekennzeichnet wird « (Schelsky 1979: 327).

Hatte die korporative Gesellschaft Alt-Europas sowie die kompetitive, d.h. dem Leistungs- und Wettbewerbsprinzip huldigende Gesellschaft des bürgerlichen Zeitalters noch weithin die Gemeinsamkeit, dass für die Mehrzahl ihrer Mitglieder die Existenzsicherung den Hauptlebensinhalt ausmachte, so unterscheidet sich die gegenwärtige Gesellschaft des heutigen Europas genau in diesem Punkt sehr deutlich von den früheren Stufen ihrer Entwicklung. Der in den 1970er Jahren von Ulrich Beck publizierte Aufsatz »Jenseits von Klasse und Schicht« beschreibt sehr deutlich, dass es zwar nach wie vor gewisse Abstände zwischen den sozialen Schichten gibt, dass aber die gesamte Gesellschaft auf der Wohlstandsleiter nach oben gestiegen ist. Steigender Wohlstand und Massenkonsum haben zu einer zunehmenden Vereinheitlichung der Lebensbedingungen geführt. Die Daseinsgestaltung wird jetzt nicht mehr aus Not oder Mangel angetrieben, sondern genau das Gegenteil ist der Fall. Aus Mangel an Mangel resultiert die Herausforderung, die Freizeit mit Inhalt und Sinn zu füllen.

Diese Homogenisierung der Lebensbedingungen führt einerseits zu regelrechten Sinn- und Orientierungskrisen; sie führt andererseits zu einer Auflösung der schichttypischen Subkulturen. Dieser sozioökonomische Wandel bewirkt wiederum einen enormen Individualisierungsschub, da sich durch den Wegfall traditioneller Bindungen die Handlungsspielräume radikal erweitern. Die daraus resultierende Pluralisierung der Lebensstile führt umgekehrt zu einer Entkopplung von 
den äußeren Lebensbedingungen, so dass die Vorstellung von Schicht- oder Klassenzugehörigkeit immer stärker aus dem alltäglichen Bewusstsein verschwindet. Ulrich Becks Programmschrift »Risikogesellschaft« und die darin vertretene These eines »Kapitalismus ohne Klassen« bringen diesen Tatbestand auf den Punkt. Beck schreibt:

Wir leben trotz fortbestehender und neu entstehender Ungleichheiten heute in der Bundesrepublik bereits in Verhältnissen jenseits der Klassengesellschaft, in denen das Bild der Klassengesellschaft nur noch mangels einer besseren Alternative am Leben erhalten wird. [...] In der Konsequenz werden subkulturelle Klassenidentitäten und -bindungen ausgedünnt oder aufgelöst. Gleichzeitig wird ein Prozess der Individualisierung und Diversifizierung von Lebenslagen und Lebensstilen in Gang gesetzt, der das Hierarchiemodell sozialer Klassen und Schichten unterläuft und in seinem Wirklichkeitsgehalt in Frage stellt. (Beck 1986: 121)

Ungleichheiten werden jetzt vermehrt auf der Horizontalen wahrgenommen, wo neue Zuweisungskriterien die Lebensverhältnisse diversifizieren: Geschlecht, Alter, Religion, Familienverhältnisse, Generationszugehörigkeit und vieles mehr. Die traditionellen Sozialzusammenhänge verlieren mehr und mehr an Bedeutung, kulturelle Kohäsionskräfte lösen sich auf. Die Gesellschaft scheint aufgesplittet in die unterschiedlichsten Milieus, die aufgrund ihrer konträren Lebensstile eher nebeneinander als miteinander leben.

Folgerichtig hat sich, seit Jürgen Habermas Mitte der 1980er Jahre die deutsche Sozialstruktur mit dem Etikett der >Neuen Unübersichtlichkeit< (Habermas 1985 ) versehen hatte, in der Soziologie mit der Milieu- und Lebensstilanalyse ein völlig neuer Zugang zur Ordnung und Analyse sozialstruktureller Vielfalt ausgebreitet. Im Unterschied zu den traditionellen Schicht- oder Lagenmodellen setzen diese kultursoziologisch motivierten Forschungsansätze bei einer differenzierten Beschreibung der Wertorientierungen, Einstellungen, Verhaltensweisen, Interaktionsmuster und Bildungsressourcen gesellschaftlicher Individuen an.

Die aus den kulturellen Mustern geclusterten Milieus und Lebensstile erweisen sich dabei aufgrund ihrer unterschiedlichen alltagsästhetischen Präferenzen als äußerst heterogen. Insbesondere die von Gerhard Schulze vorgelegten Milieuanalysen stellen, quasi exemplarisch für eine bestimmte Richtung der Lebensstilforschung, die sozialpsychologischen Auslöser für Gruppenbildungsprozesse und Identitätskonstruktionen in den Mittelpunkt, hinter denen dann die objektiven Soziallagen weitgehend zurücktreten. Viele Soziologen vertreten daher die Auffassung, dass man die gegenwärtige westeuropäische Gesellschaft nicht länger als >geschichtete< Gesellschaft bezeichnen kann, weil sie eine nicht mehr an klassische Vertikallagen gebundene Vergemeinschaftungsform darstelle. Das Milieu als ein Kernelement dieser neu formierten Sozialgemeinschaft übernimmt dabei - quasi als Gussform moderner Individualitätsbemühungen - zugleich auch wichtige Integrationsfunktionen. (Vgl. Meyer 2001: 258) 
Milieus bestehen aus Personengruppen, die sich durch spezifische Existenzund Weltsichten sowie durch erhöhte Binnenkommunikation von anderen Gruppen abheben. Und sie entstehen immer auch durch kollektive soziale Typisierung, nach der andere Personen sozial wahrgenommen, eingeordnet und für Interaktionen ausgewählt werden. Beziehungspartner im Alltag werden hierbei nach Ähnlichkeitsmustern ausgewählt, wobei sich Selektions- und Sozialisationseffekte verschränken. So entstandene und sich reproduzierende bzw. ausdifferenzierende Milieus geben Mentalitäten und kulturelle Orientierungsmuster vor. Jedem Milieu können also bestimmte Eigenschaftsprofile, Mentalitäten und kulturelle Orientierungen seiner Mitglieder zugeordnet werden, wobei der Begriff des Milieus all dies gleichsam heuristisch bündelt.

\section{Milieus in der 'Erlebnisgesellschaftı. Der Ansatz von Gerhard Schulze}

Obwohl sinn- und orientierungsstiftende Instanzen wie Gott, Natur, Geschichte und Vernunft in der modernen Gesellschaft deutlich an Wirkungskraft verloren haben, hält die Intuition wider besseren philosophischen Wissens unbeirrbar an der Vorstellung einer gegebenen Ordnung fest. Freilich lässt sich dieser Zustand auch als eine Form neuer Autonomie goutieren, die allerdings beständig vom Kollaps durch die Überlastung der Reflexionsansprüche bedroht ist. Denn die Abnahme der Orientierungsverbindlichkeit wird flankiert von der Einsicht in die Fragilität von Identitätsentwürfen. So zwingt eine Wirklichkeit, in der die Zeichen immer hektischer fluktuieren, das Individuum zu ständigen Orientierungsleistungen im Dienste der Selbstvergewisserung der eigenen Position. Mittlerweile jedoch ist eine historische Phase erreicht, in der das Festhalten an Sicherheitsillusionen in keinem Verhältnis mehr zu den ökonomischen und psychischen Kosten steht, die für sie aufzubringen sind. Wenn aber externe Orientierungsmechanismen obsolet werden, müssen diese intern produziert werden. Es kommt zu einer Wende des Denkens von außen nach innen, zu einer »Verschiebung des Brennpunkts der Zieldefinition« (Schulze 1994: 108). In seiner einschlägigen Studie »Die Erlebnisgesellschaft« führt Gerhard Schulze dazu aus:

Außenorientierte Lebensauffassungen zielen primär auf eine Wirklichkeit ab, die sich der Mensch außerhalb seiner selbst vorstellt, innengerichtete Lebensauffassungen verweisen auf das Subjekt. (Schulze 2000: 37)

Auf welche Weise aber verschafft das Umstellen auf eine primär innenorientierte Lebensauffassung überhaupt Orientierung und was sind die Bedingungen für diesen Wechsel? Orientierung lässt sich zunächst als eine Wiederholungsbewegung begreifen, die subjektive Prozesse mit außersubjektiven Ereignissen auf ähnliche Weise verknüpft. Situationen werden dadurch beherrschbar, dass einmal erkannte Grundmuster appliziert werden können. Orientierung erscheint somit als Form subjektiver Sinnzuschreibung in situativen Kontexten, die umso einfacher ist, je 
weniger variabel die Situationen und Kontexte sind. Schulze verdeutlicht die Umstellung auf eine primär innenorientierte Lebensauffassung anhand der Sphäre der Produkte. Produkte erscheinen in der Lebenswelt unter dem Aspekt des Konsums, in den Naturwissenschaften unter dem Aspekt der Theorieentwicklung und in den Wirtschaftsorganisationen unter dem Aspekt korporativen Überlebens. (Es liegt auf der Hand, dass hier letztlich mit materialistischen Kategorien operiert wird: Die Verdinglichung der Warenwelt wird in einer schmucken Umkehr über die Kategorie des Produkts nicht länger als Verblendungszusammenhang, sondern als Orientierungsinstanz gewertet ${ }^{1}$.) Dass Produkte als gemeinsames und übergreifendes Thema durchaus heterogener Denksysteme so attraktiv sind, liegt an ihrem Orientierungsanreiz, den sie als Gegenstandsbereich sozial organisierten Denkens deshalb in besonderem Maße bieten, weil in ihnen objektive und subjektive Elemente verschmelzen. Sie sind zum einen Ergebnis des Handelns und in diesem Sinne Ausdruck von Subjektivität, haben andererseits aber auch gegenständlichen Charakter, womit sie ohne Schwierigkeiten zum Bezugspunkt intersubjektiver Diskurse und kollektiver Orientierungsanstrengungen gemacht werden können. (Vgl. Schulze 1994: 100) Durch die Ausrichtung der Denksysteme auf die Produktsphäre greifen die Systeme selbst wie Zahnräder eines Uhrwerks ineinander und stabilisieren sich gegenseitig. Historische Ereignisfolgen können so als ein Gesamtzusammenhang von Produkt-, Konsum-, Technik- und Wissenschaftsgeschichte interpretiert werden, in dem sich zwar alles verändert, aber dennoch niemand die Orientierung verliert, da sich die Transformation in geordneten Bahnen vollzieht.

Ein wesentlicher Grund dafür, dass dieses Uhrwerksmodell nicht mehr funktioniert, liegt in der Zunahme der Produkte selbst. Denn prekärerweise muss in einer Wirklichkeit, die sich nach linearem Zeitmodell formiert hat, auch die Geschichte der Produkte teleologisch ausgerichtet sein, sie muss als Fortschrittsgeschichte begriffen werden. Fortschritt ist hier quantitativ und qualitativ denkbar, wobei mit Blick auf reale Konsumgüter das Innovationspotential in vielen Fällen schnell ausgereizt ist. Mit anderen Worten, wenn Bedürfnisse erfüllt sind, geht die Selbstverständlichkeit von Nutzendefinitionen allmählich verloren. Ein auf den ersten Blick vollkommen widersinniges Problem tritt auf den Plan, das darin besteht, Zwecke für Produkte zu >konstruieren<. Doch im fortgeschrittenen Stadium der Warenwelt existieren schlichtweg kaum noch Nischen für neue Nutzendefinitionen.

Die Produktentwickler sind aus der ursprünglichen anthropologischen Bodenhaftung entlassen und müssen ihre Konstruktionen ins Vakuum der Unbestimmtheit hineinbauen; man braucht keine Praktiker mehr, sondern Phantasten. (Schulze 1994: 104)

1 | Ein Gedanke, der schon von Hannah Arendt formuliert wurde: "Die Werktätigkeit von Homo Faber, der die Welt herstellt, vollzieht sich als Verdinglichung." (Arendt 1997: 165) 
Auf der Ebene der Konsumenten bedeutet das, dass Entscheidungen für dieses oder jenes Produkt nicht auf der Ebene des primären Nutzens gefällt werden, der als selbstverständlich vorausgesetzt wird. Traditionelle, an die Produktsphäre gekoppelte Leitkategorien wie Nutzen, Qualität und Reichtum verlieren damit ihre Orientierungsfunktion.

Genau an dieser Stelle kommt die bereits erwähnte Umstellung von einer außenorientierten Lebensauffassung zu einer innenorientierten ins Spiel. Wenn es nicht mehr um den Nutzen einer Sache geht, der über Besitz gesichert werden kann (Außenorientierung), rückt das Erlebnis in den Vordergrund, das durch diese Sache im Konsumenten ausgelöst wird (Innenorientierung). Insofern kann Schulze Innenorientierung als Erlebnisorientierung definieren und die prägnante Formel aufstellen: »Das Projekt des schönen Lebens ist das Projekt, etwas zu erleben.« (Schulze 2000: 38) Dieses Projekt bedarf angesichts eines schier unüberschaubar gewordenen Erlebnisangebots der konsequenten Steuerung. Der Mensch wird regelrecht zum Manager seiner eigenen Subjektivität, indem er Situationen zu Erlebniszwecken instrumentalisiert und mittels Erlebnisrationalität systematisiert. (Schulze 2000: 40) Damit kristallisiert sich ein neues Grundmuster der Beziehung von Subjekt und Situation heraus, da Situationen nicht mehr im orientierungsstiftenden Modus eines substanziellen Mangels (Geld, Eigentum, Bildung usw.) fokussiert werden, die der Handlungsökonomie natürliche Grenzen setzen würde. Die außenorientierten Kategorien Nutzen, Qualität und Reichtum wurden daher auf einen innenorientierten Bezugsrahmen appliziert, nämlich vom Haben zum Sein. Es geht mit anderen Worten um eine durchgehende >Ästhetisierung des Alltagslebens<.

Das Problem liegt dabei darin, dass Erlebnisse jedweder Art erst durch Reflexion goutiert werden können. »Reflexion ist der Versuch des Subjekts, seiner selbst habhaft zu werden.« (Schulze 2000: 45) Dieser Akt der gelingenden Selbstreflexion ist jedoch nur bedingt manipulierbar, womit Erlebnisfortschritt langfristig keineswegs garantiert ist.

Wir können nicht unbegrenzt gewünschte Formen des Seins anhäufen. In reicher Zahl hält die soziale Wirklichkeit Beispiele sowohl für den Versuch der Erlebnisvermehrung als auch für das Scheitern dieses Versuchs bereit: zwischen Dutzenden von Programmen springende Telekonsumenten; angestrengte Urlauber in ununterbrochener Erlebnisarbeit; Trends zur Verkürzung und zeitlichen Überlagerung von Erlebnisepisoden; Museumsbesucher, die vor lauter Bildern nichts mehr sehen; Schränke voller unbenutzter Kleider und Schuhe, Regale voller ungelesener Bücher; wechselnde Partnerschaften mit wenig Tiefe; gutes Essen in Menge mit wenig Genuß; pausenlose Musik, ohne daß man noch mehr wahrnehmen würde als ein Geräusch. (Schulze 1994: 116)

Erlebnisreichtum lässt sich gerade nicht durch Erlebnisquantität steigern. Genau das Gegenteil ist der Fall. Erlebnisse entfalten sich erst in Reflexionsprozessen. Diese aber benötigen eine äußerst knappe Ressource: Zeit! Wenn die durchgän- 
gige Ästhetisierung des Alltagslebens auf der einen Seite als ein - medial produzierter und inszenierter - Überschuss an Raum gedacht werden muss, so steht auf der anderen Seite der Mangel an Zeit. Letzterer ist darauf zurückzuführen, dass die Zeit für die Gegenwart keine Trägerin von Sinn mehr ist. Sie kann nur noch unter dem Aspekt der Begrenzung bzw. Einschränkung rezipiert werden, die einer immer gieriger voranschreitenden Akkumulation von Erlebnissen restriktiv entgegensteht. Erlebnisvermehrung führt insofern konsequenterweise dazu, dass die Einzelerlebnisse nur noch unzureichend reflektiert werden und daher ihren Reichtum und ihre Tiefe nicht mehr entfalten können. Getrieben von der Sehnsucht nach Erlebnisreichtum werden dann in hektischer Folge immer schneller die Erlebnisangebote aneinandergereiht. Seins-Steigerung soll durch Habens-Steigerung bewirkt werden. Genau diese Tendenz führt aber zur Erlebnisverarmung. Das Scheitern des Projekts, sein Leben als eine Folge glücklicher Episoden zu erleben, führt notwendigerweise zu Unsicherheit und Enttäuschung, eine Beobachtung, die von vielen aufmerksamen Beobachtern der Moderne geteilt wird. In philosophischer Hinsicht könnte man die paradoxe Einsicht formulieren, dass es uns deshalb so schlecht geht, weil es uns viel zu gut geht. Denn unter materiell harten, ggf. sogar existenzbedrohenden Bedingungen stellen sich Ziele, Perspektiven und Erfüllung von alleine ein. Menschen unter solchen Bedingungen verfügen ganz selbstverständlich über einen integrierenden und identitätsstiftenden Faktor, der aus der Spannung zwischen unerfüllten Bedürfnissen und objektiver Lebenssituation resultiert. Ein aus nackter Not geborenes Gebot der Stunde kann aber in unserer gegenwärtigen Wohlstandsgesellschaft schlechterdings nicht formuliert werden. Denn bei allem Krisenbewusstsein gilt das Leben in unserer Gesellschaft letztlich doch als garantiert, daher kommt es jetzt darauf an, »es so zu verbringen, daß man das Gefühl hat, es lohne sich « (vgl. Schulze 2000: Gof.). Erlebnisorientierung erweist sich somit nicht als die Absicht, etwas zu haben, sondern als die Absicht, jemand zu sein.

Allerdings ist der Aufbau konstanter Seins- und Selbstentwürfe durchaus problematisch, da die beständig ansteigende Flut von Gegenständen, Sinnesreizen und stimulierenden Situationen eine stabile Zuordnung von Zeichen und Bedeutung erschwert. Soziale Milieus lassen sich vor diesem Hintergrund als Stabilisierungsmechanismen begreifen, indem sie quasi standardisierte Zuordnungsmuster von Zeichen und Bedeutung bereitstellen. Solche Zuordnungsmuster haben zugleich distinktives Potential, so dass die soziale Abgrenzung nicht länger primär auf der klassisch-vertikalen Ebene der Gesellschaft ausgehandelt wird, sondern vor allem auf der Horizontalen zu den Nachbarmilieus.

\section{Pierre Bourdieus Konzept des sozialen Raumes}

Ein zentraler Vorwurf gegen die These von der Auflösung klassischer Vertikallagen besteht darin, dass der Wandel in den Strukturen sozialer Ungleichheit nicht mit deren Verschwinden verwechselt werden dürfe. Vielmehr werde durch die Plura- 
lität der Milieus lediglich verdeckt, dass diese sich nach wie vor auch von unten nach oben und damit eben schichtförmig anordnen lassen. Auch horizontale Differenzierung lasse genug Spielraum für Exklusionsmechanismen, die inzwischen bei größeren Bevölkerungsteilen in eine dauerhafte Prekarisierung von Arbeitsund Lebensverhältnissen umgeschlagen seien, bei einigen sogar zur Marginalisierung geführt haben. Ganz in diesem Sinne kritisiert Geißler die >Kulturalisierung klassischer Ungleichheitslagen durch eine primär lebensstilorientierte Sozialforschung: »Die Kritik an sozialen Ungerechtigkeiten weicht der Freude über die bunte Vielfalt.« (Geißler 1996: 323) Tatsächlich lassen sich vor allem im Bereich der empirischen Bildungsforschung - und insbesondere die PISA-Studien haben dies für viele europäische Länder eindrucksvoll nachgewiesen - trotz der konstatierten Vielfalt auch weiterhin schichtspezifisch ungleich verteilte Bildungschancen ausmachen. Die zentrale Frage lautet also: Wie lassen sich die Erklärungskraft von Lebensstil- und Milieuforschung mit klassen- bzw. schichttheoretischen Befunden und diese wiederum mit metatheoretisch geprägten Vorstellungen über die Richtung des gesellschaftlichen Wandels verbinden?

Beispielhaft für eine solche Herangehensweise ist Pierre Bourdieus Modell des sozialen Raumes, das er in seiner richtungsweisenden Studie »La distinction: Critique sociale du jugement « (Bourdieu 1979) entfaltet. ${ }^{2}$ Bourdieu hält hier auf der einen Seite am Fortbestehen von >objektiven< Klassenstrukturen fest, postuliert aber darüber hinaus einen systematischen Zusammenhang zwischen Klassenzugehörigkeit - samt der damit verbundenen Verfügung über ökonomische, kulturelle und soziale Ressourcen - und Formen der symbolischen Lebensführung (Lebensstile). Auf diese Weise verbindet sein Konzept des sozialen Raumes nicht nur soziale Positionen bzw. Lagen mit unterschiedlichen kulturellen Praktiken, sondern macht auch die diskreten Klassen und Klassenfraktionen sichtbar, die sich hinter diesen Praktiken verbergen. Auf diese Weise können kulturelle Praktiken als Elemente unterschiedlicher Lebensstile mit herkömmlichen sozialstrukturellen Analysekategorien verbunden werden: Konkrete - und wenn man so will: schichtspezifisch bestimmbare - Lebensbedingungen werden dann als Konstituenten von Habitusformen interpretiert, die ihrerseits aus offenen oder latenten Mustern des Denkens, Wahrnehmens, Bewertens und Handelns bestehen. Mit dem Begriff des >Habitus< sucht Bourdieu die Gesamtheit der psychischen Dispositionen von Menschen oder Gruppen zu erfassen, d.h. ihre Orientierungen, Einstellungen, Wahrnehmungs-, Denk- und Beurteilungsschemata. Bourdieu schreibt:

Wer den Habitus einer Person kennt, der spürt oder weiß intuitiv, welches Verhalten dieser Person verwehrt ist. Mit anderen Worten: Der Habitus ist ein System von Grenzen [...]. Deshalb sind für inn bestimmte Dinge einfach undenkbar, unmöglich; es gibt Sachen, die inn

2 | Die Studie erschien auf Deutsch unter dem Titel: Die feinen Unterschiede. Kritik der gesellschaftlichen Urteilskraft, Frankfurt a.M. 1982. 
aufbringen oder schockieren. Aber innerhalb dieser Grenzen ist er durchaus erfinderisch, sind seine Reaktionen keineswegs immer schon im Voraus bekannt. (Bourdieu 1992a: 33)

Der Habitus ermöglicht oder beschränkt also kulturelle Praktiken, die mit einem Set von Lebensstilelementen erfassbar sind: Ansichten und Vorlieben in Bezug auf Kunst, Musik, Literatur, Malerei, Sport, Essen, Wohnungseinrichtungen usw. Kulturelle Praktiken gewinnen damit, obwohl Produkte menschlichen Handelns, eine gewisse Eigenlogik und treten den Menschen als etwas Strukturierendes entgegen. Mit anderen Worten, die allgemeine Homogenisierung der Lebensbedingungen eröffnet zwar weite Handlungsspielräume, mit dem Verhältnis von Sein und Schein zu spielen und über Lebensstilinszenierungen Positionsverbesserungen im sozialen Raum anzustreben; doch macht insbesondere Bourdieus Kapitaltheorie deutlich, dass jede symbolisch inszenierte >Simulation < gesellschaftlicher Verhältnisse am Ende doch begrenzt wird durch die zur Verfügung stehenden objektiven Ressourcen.

$\mathrm{Zu}$ fragen wäre also, wie sich die Demarkationslinien sowohl in vertikaler als auch in horizontaler Ebene ausbilden und damit die Topologie des sozialen Raumes bestimmen. Als Konstruktionsprinzipien fungieren hier die verschiedenen Formen von Kapital, die innerhalb der einzelnen Felder jeweils in Kurs sind: »Kapital - in seiner objektivierten Form als materielles Eigentum wie in seiner inkorporierten Form zum Beispiel als kulturelles Kapital [...] stellt Verfügungsmacht im Rahmen eines Feldes dar.« (Bourdieu 1995: 10) Diese Verfügungsmacht kann das Kapital nur deshalb entfalten, weil es nicht nur unterschiedlich strukturiert - als ökonomisches, soziales und kulturelles Kapital -, sondern auch höchst ungleich verteilt ist. (Vgl. Bourdieu 1992b) Insgesamt ergibt sich das Bild eines symbolischen Systems, das sich gemäß der Logik von Differenzen organisiert. Diese Differenzen werden als Distinktion im Sinne von Unterscheidung wahrgenommen, d.h., die den Lebensstil einer Klasse charakterisierende Distinktion resultiert aus dessen differenzieller Beziehung zu den Lebensstilen der übrigen Klassen. Distinktion impliziert allerdings nicht notwendig das bewusste Streben nach Distinktion, vielmehr ist jeder Konsumakt oder allgemeiner: jede Praxis »sichtbar, gleichviel ob sie vollzogen wurde, um gesehen zu werden, oder nicht« (Bourdieu 1995: 21).

Wenn man die unterschiedlichen Positionen, die über den Gesamtumfang und das besondere Profil des Kapitals definiert werden, zueinander in Beziehung setzt, so fallen zahlreiche Agglomerationen und Clusterbildungen ins Auge. Obwohl diese Cluster auf verwandte Modi des Handelns, Wahrnehmens und Bewertens verweisen und hohe Übereinstimmungen des Lebensstils indizieren, können sie freilich nicht als Indiz für real existierende soziale Klassen gelten. Denn obwohl sich in den gesellschaftlichen Machtkämpfen herrschende und beherrschte Fraktionen gegenüberstehen, existieren soziale Klassen doch gleichsam nur in einem virtuellen Modus. Bourdieus Modell zeichnet den hierarchisch strukturierten, sozialen Raum als das Bild eines vibrierenden Gesellschaftskörpers, der von dem Bemühen um die Akkumulation von Kapital, dem Ringen um die Definitionshoheit und dem Streben nach Einfluss auf die Wechselkurse der unterschiedlichen Kapitalsorten 
geprägt ist - und das über keinerlei trostspendende Rahmung verfügt. Vielmehr wird ein differenziertes, an den einzelnen Kapitalsorten orientiertes Spektrum sozialer Zugehörigkeiten generiert. Anhand sozialer Positions- bzw. Raumlagen wird damit die horizontale und vertikale Pluralität unterschiedlicher sozialer Lagen erfassbar und mithilfe sozialstruktureller Daten wie Bildung, Beruf etc. rekonstruierbar. Dies ermöglicht es, im Raum der Milieus auch Praktiken und Objekte der symbolischen Lebensführung zu erfassen, die sich ihrerseits bestimmten sozialen Positionen zuordnen lassen.

\section{Der Ansatz von Michael Vester}

Das bourdieusche Modell des sozialen Raumes wurde für die Bundesrepublik Deutschland vor allem von Michael Vester adaptiert, der milieuspezifische Lebensstile und Individualisierungstendenzen nicht als völlige Neuschaffung, sondern als Rekonversion oder »als einen relativen Umbau der Mentalitäten und Milieus « interpretiert. (Vester 2001: 145) Ausgangspunkt ist auch hier die Annahme eines mehrdimensionalen sozialen Raumes, der im Kern eine Verlängerung der älteren sozialen Milieus darstellt, die im Verlaufe ihrer Entwicklung entweder auf- oder absteigen. Wichtig für Vester ist vor allem eine Analyse der >Veränderungsrichtung « der zeitgenössischen Entwicklungspfade sozialer Milieus, die gängigerweise mit den Stichworten Tertiärisierung, Wissensgesellschaft, Postmaterialismus oder Wertewandel umschrieben wird. Seine Grundthese ist dabei, dass Tendenzen der Individualisierung und Diversifizierung von Lebenslagen zwar ohne Zweifel existieren, dass diese Tendenzen allerdings nicht zu einer vollständigen Auflösung der Strukturmerkmale der >alten< Klassengesellschaft geführt haben, sondern vielmehr zu einer vertikalen und horizontalen Pluralisierung. In Anlehnung an Bourdieus Konzept des sozialen Raumes und des darin sichtbaren Habitus werden vor diesem Hintergrund von Vester zunächst Identitäten sozialer Großgruppen analysiert. Menschen bilden demzufolge ihre Gruppenidentitäten nach wie vor entlang von vier vertikalen Zugehörigkeitsprofilen heraus, denen unterschiedliche Habitusformen zugehörig sind, und die ihrerseits in unterschiedliche, in sich >geschichtete < horizontale Milieus zerfallen. Auf jeder der drei Rangebenen hat sich allerdings eine horizontale Differenzierung nach Modernisierungsgraden ausgeprägt. Hier sind spezifische Verhaltensstandards in den Milieus sozialer Großgruppen verankert, die auf bestimmte Orientierungsmuster und Mentalitäten verweisen.

\section{Fazit}

Hinsichtlich der Herausarbeitung alltagsästhetischer Zeichen bestehen insbesondere im Rahmen von Schulzes und Vesters Milieuanalysen klare Zusammenhänge. So wird von beiden konstatiert, dass die konkrete Lebensführung entlang der skizzierten Schemata erfolge, was jedoch nicht zwangsläufig aus der jeweiligen Le- 
benssituation und ihren Begrenzungen heraus resultiere, sondern immer stärker entlang von Selbstentscheidungsprozessen: Man sucht aus, mit wem man interagiert und an wem man sich orientiert. Milieus bestehen insofern aus Personengruppen, die sich durch spezifische Existenz- und Weltsichten sowie durch erhöhte Binnenkommunikation von anderen Gruppen abheben. Und sie entstehen immer auch durch kollektive soziale Typisierung, nach der andere Personen sozial wahrgenommen, eingeordnet und für Interaktionen ausgewählt werden. Beziehungspartner im Alltag werden hierbei nach Ähnlichkeitsmustern ausgewählt, wobei sich Selektions- und Sozialisationseffekte verschränken.

Während Schulzes >Erlebnisgesellschaft< den Blick eher auf psychophysisch motivierte Orientierungsmuster als kulturelle Vorlieben richtet, bietet Vesters Modell den Vorteil, auch Wanderungsbewegungen in und zwischen den unterschiedlichen Milieus sichtbar zu machen (und damit in begrenztem Umfang auch Prognosen darüber abgeben zu können, wohin Milieumitglieder streben). Zum einen nämlich erfüllt Vesters Ansatz die bis auf Geiger (1932) zurückreichende Forderung, in sozialstrukturellen Analysen die System- und Strukturebene mit der Ebene sozialer Lagen und Mentalitäten zu verknüpfen. Zum anderen ist das Modell flexibel genug, um auf mögliche Schließungen oder (Klassen-)Spaltungen reagieren zu können, so dass Anschlussmöglichkeiten an die Diskussionen um erneut wachsende Ungleichheiten und (neue) Formen sozialer Exklusion gewährleistet sind. Damit berücksichtigt Vester in seinen Studien auch und vor allem die auf Politik und Macht verweisenden Aspekte der Vergesellschaftung.

Vesters Ansatz trägt damit nicht nur der horizontalen Diversifizierung von Lebenslagen und -stilen Rechnung, sondern ist darüber hinaus auch heuristisch dazu in der Lage, zeitgenössische Ungleichheits- und Sozialstrukturen in den Blick zu nehmen. Aus diesem Grund hat sich die im Folgenden dargestellte Milieubeschreibung der Luxemburger Sozialstruktur an diesem Ansatz orientiert.

\subsection{Profile sozio-kUltureller Milieus in LuXemburg}

\section{Die IDENT-Umfrage}

Vorbild für das Luxemburger Projekt ist die unter der Leitung des Sozialwissenschaftlers Michael Vester et al. in der Bundesrepublik Deutschland durchgeführte Studie, die unter dem Titel »Soziale Milieus im gesellschaftlichen Strukturwandel. Zwischen Integration und Ausgrenzung« erstmals 1993 veröffentlicht wurde und mittlerweile zu den >Klassikern < der neueren empirischen Sozialforschung zählt. Die Arbeitsgrundlage bildete allerdings nicht diese Version der Studie, sondern eine vollständig überarbeitete, erweiterte und aktualisierte Fassung. (Vgl. Vester et al. 2001) In Vesters Studie kam - wie in Abschnitt 3.1 bereits ausführlich dargestellt wurde - ein neues empirisch-theoretisches Konzept zur Anwendung, das dem rapiden Veränderungsdruck moderner Gesellschaften Rechnung trägt. Dieses Konzept 
wurde am Beispiel der deutschen Gesellschaft erprobt, so dass die Studie selbst als >Momentaufnahme< der deutschen Verhältnisse gelesen werden kann. Prinzipiell ist das Analyseverfahren, das auf einer engen Verbindung von Struktur- und Mentalitätswandel beruht, aber auch auf andere >westliche < Gesellschaften übertragbar. Bei der Übertragung, der Neufassung und dem Abgleich von Grundlagen und Ergebnissen dieser Studie auf die Gegebenheiten in Luxemburg stand Michael Vester den Projektmitarbeitern/-innen beratend zur Seite. Daniel Gardemin, Mitglied der Arbeitsgruppe interdisziplinäre Sozialstrukturforschung (agis) der Universität Hannover, hat die statistischen Auswertungen durchgeführt.

Ausgangspunkt unserer empirischen Erhebung bildete der Originalfragebogen (Vester 2001: 222-243 und 546-557), der mit Blick auf Luxemburg gekürzt und teilweise angepasst wurde. (Vgl. Kasten 1)

\section{Habitus (Mentalitätstypen) (31 Fragen)}

Faktor 1: Rebellischer oder idealistischer Hedonismus

Faktor 2: Realistischer Hedonismus

Faktor 3: Underdog-Frustration

Faktor 4: Autoritäre Leistungsideologie

Faktor 5: Moderner Leistungsoptimismus

Faktor 6: Kulturpessimistische Fortschrittskritik

Faktor 7: Anspruchsvolle Selbstverwirklichung

Faktor 8: Pseudo-Konformismus

Faktor 9: Konsumhedonismus

Faktor 10: Bequemes Arrangement

\section{Soziale Kohäsion und Gesellungsstile (10 Fragen)}

Faktor 1: Emotionale Erlebnisorientierung

Faktor 2: Konventionelle zurückhaltende Familienzentrierung

Faktor 3: Anspruchsvolle, außenorientierte Kommunikation

\section{Freizeit Grundeinstellungen und Gesellungspraktiken (20 Fragen)}

Faktor 1: Peer Group

Faktor 2: Politische Öffentlichkeit

Faktor 3: Kulturelle Öffentlichkeit

Faktor 4: Individuelle Freizeitgestaltung

\section{Politische Partizipation und Politikstile (16 Fragen)}

Faktor 1: Insel-Syndrom

Faktor 2: Leistungsideologie

Faktor 3: Politische Enttäuschung

Faktor 4: Arbeitnehmerorientierung

Faktor 5: Politische Teilnahme 
77 Einzelstatements erlauben es, vier Ebenen der alltäglichen Lebensführung und der gesellschaftspolitischen Orientierungen abzubilden:

- Grundeinstellungen zu verschiedenen Bereichen des Alltagsleben (Habitus, Mentalitätstypen);

- Soziale Kohäsion und Gesellungsstile;

- Grundeinstellungen zur gesellschaftlichen und politischen Ordnung sowie Gesellungspraktiken;

- Politische Partizipation und Politikstile.

Der zweite Teil der Befragung beschreibt die objektiven sozialen Lagen. Dazu wurden die üblichen soziodemografischen Variablen abgefragt, wie z.B. Haushalts- und Familienform, Geschlecht, Alter, Wohnort und Religion, Quellen des Lebensunterhalts und monatliches Nettoeinkommen. Dazu kamen Fragen nach dem höchsten Schulabschluss, derzeitiger oder zuletzt ausgeübter Beruf, Tätigkeitsfeld im Beruf, Gewerkschaftszugehörigkeit und Wahlverhalten. Das soziale Umfeld und den sozialen Werdegang beschreiben Indikatoren zum Sozialstatus der Eltern und des/der Partners/-in (Beruf, Schulabschluss).

Um den besonderen multikulturellen und plurilingualen Gegebenheiten Luxemburgs gerecht zu werden, wurden zusätzlich Fragen zu Nationalität(en), Migrationsgeschichte und Sprachkompetenz zur ursprünglich Fragenbatterie hinzugefügt.

Diese Milieustudie im engeren Sinne wurde durch weitere Themenbereiche ergänzt, um spezielle Teilaspekte, die in den Kapiteln 4-7 ausführlich erörtert werden, abzudecken und statistisch zu erfassen: Genderproblematik, Konsumgewohnheiten, Perzeption von Raum und Grenzen sowie sprachpolitische Fragen (vgl. Kasten 2).

1579 Personen im Alter von mindestens 16 Jahren wurden im Zeitraum von Oktober 2008 bis Februar 2009 durch ein luxemburgisches Meinungsforschungsinstitut befragt. ${ }^{3}$ Die Stichprobe ist repräsentativ für die Wohnbevölkerung Luxemburgs und wurde nach Alter, Geschlecht, Region, Nationalität und Teilnahme am Berufsleben (als dichotome Variable) gewichtet.

3 | Zwei Drittel der Interviews wurden über Internet (Computer Assisted Web Interview), ein Drittel über Telefon (Computer Assisted Telephone Interview) durchgeführt. 


\section{Konsumgewohnheiten (31 Fragen)}

Essen und Trinken

Einkauf

Werbung

Kampagne »Gesunde Ernährung«

Genderproblematik (24 Fragen)

Geschlechterrollen

Spachgebrauch in Luxemburg (12 Fragen)

Perzeption von Regionen und Nachbarländern (12 Fragen)

Verschiedenes (9 Fragen)

Zusammenleben

Grenzgänger/-innen

Zukunftsvisionen usw.

Kasten 2: Ergänzende Themenbereiche der quantitativen Befragung

\section{Die Milieuanalyse}

Eine multivariate Diskriminanzanalyse erlaubt es, verschiedene sozio-kulturelle Milieus zu bilden. ${ }^{4}$ Die Milieukarte in Abbildung 1 zeigt die räumliche Gliederung der neun Milieus und deren Positionierung in einem zweidimensionalen Raum: Die vertikale Achse entspricht einem ansteigenden Grad sozialer Herrschaft, während die horizontale Achse eine Ausdifferenzierung der ökonomischen Hierarchisierung im Sinne einer Pluralisierung der Klassengesellschaft darstellt.

Während die Milieus in Vester et al. (2001: 48-54) als fett eingerahmte Rechtecke abgebildet sind, haben wir eine Darstellung als sich teilweise überlappende Farbwolken mit unscharfen Umrandungen vorgezogen, um darauf hinzuweisen, dass die Milieuzugehörigkeit im Einzelfall nicht zwingend bestimmbar ist.

Die Ausdehnung der Wolken entspricht nicht der Anzahl der ihr zugeordneten Bevölkerung, sondern der sozialräumlichen Streuung der Positionen der Individuen, die ihnen jeweils zugeordnet werden können. Die Prozentanteile der Milieus werden annähernd in Abbildung 2 dargestellt.

4 | "Die Diskriminanzanalyse ist ein multivariates Verfahren zur Analyse von Gruppenunterschieden." (Backhaus et al. 2008: 156) Sie gehört zu den Klassifikationsverfahren und erlaubt es, Objekte mit ähnlichen Merkmalsausprägungen einer Klasse zuzuordnen. (Vgl. Backhaus et al. 2008: 155-228) Zu Einzelheiten der Methode und auch zur Milieukartierung siehe Vester et al. (2001). 


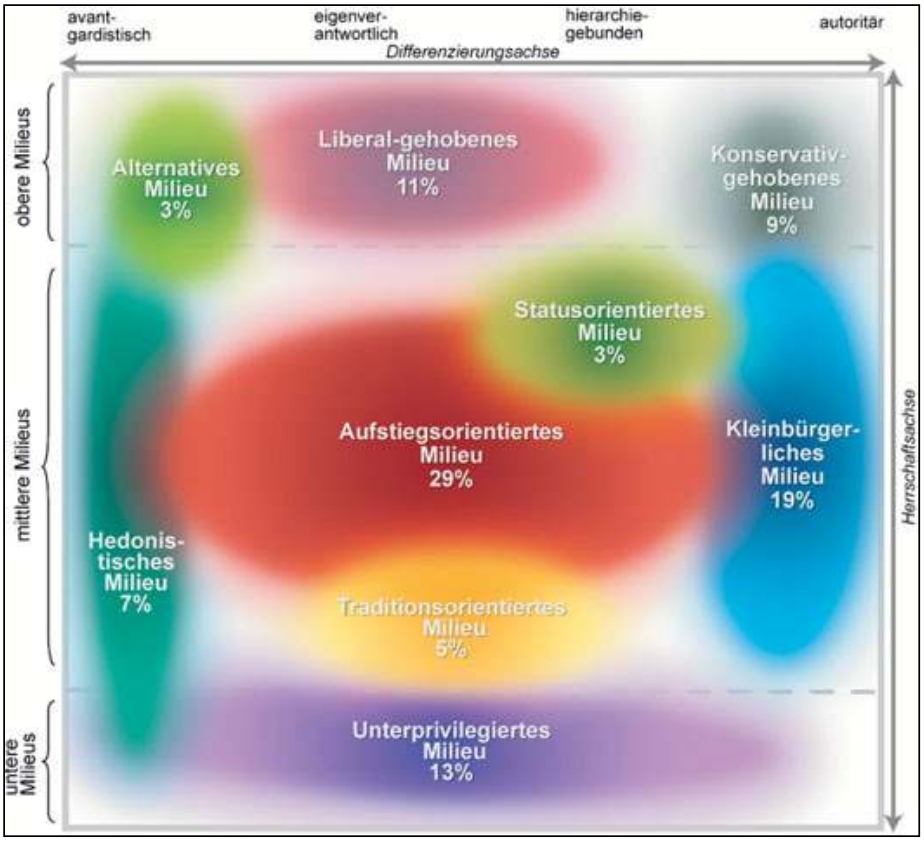

Abbildung 1: Sozio-kulturelle Milieus in Luxemburg (sozialräumliche Gliederung), Entwurf: IDENT unter Mitarbeit von Marie-Line Glesener

Die folgenden neun Milieuskizzen beschreiben in Anlehnung an Vesters Typologie (Vester 2001: 503-525) jeweils die soziale Lage, den Lebensstil auf der Grundlage von insgesamt 31 Aussagen zu verschiedenen Aspekten des Alltagslebens (siehe Kasten 1 oben) sowie den Politikstil des jeweiligen Milieus. Bei der Beschreibung werden jeweils signifikante Abweichung (z.B: >stark unterrepräsentiert<, >stark überrepräsentiert $<$ ) von den Durchschnittswerten aller Milieus besonders betont. 


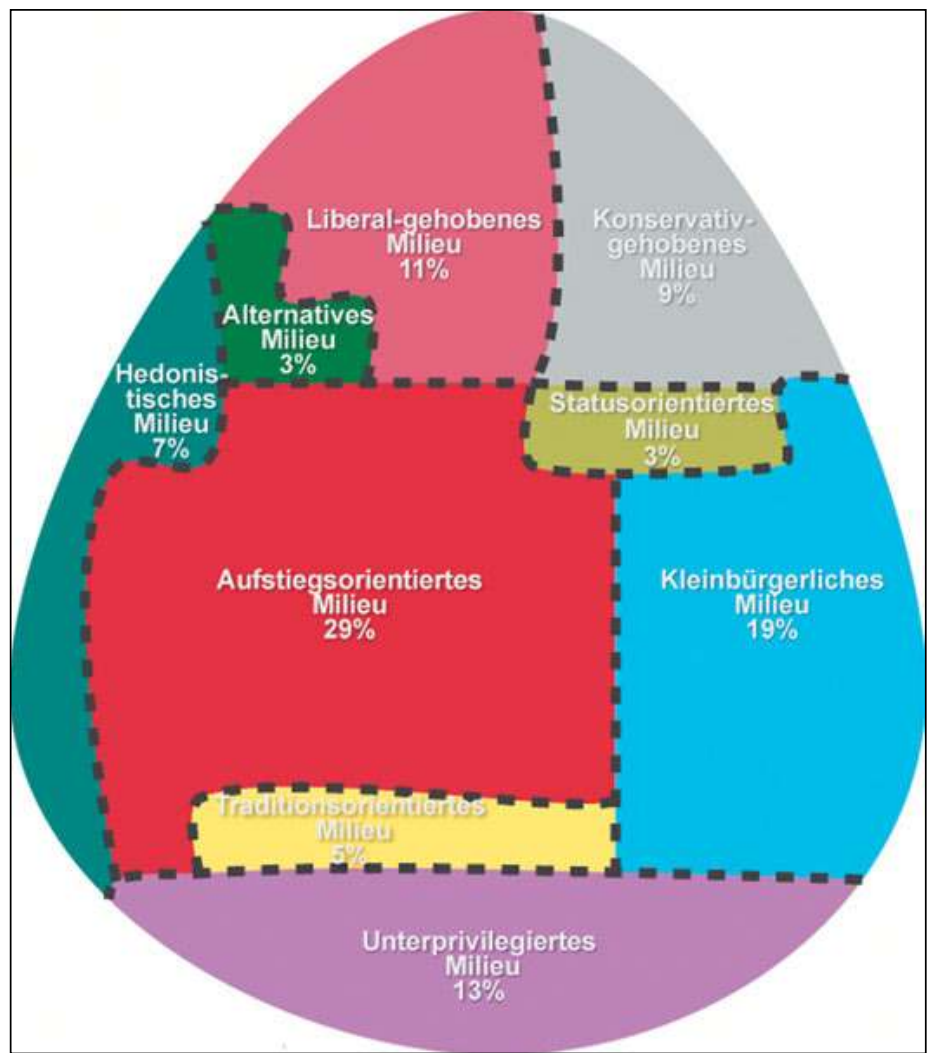

Abbildung 2: Sozio-kulturelle Milieus in Luxemburg (Anteile in \%), Entwurf: IDENT unter Mitarbeit von Marie-Line Glesener

\section{Aufstiegsorientiertes Milieu (29\%)}

Soziale Lage: Fast ein Drittel der Luxemburger Wohnbevölkerung gehört zu diesem großen, auch räumlich breit verteilten Milieu, das in einem gewissen Sinne die Mitte der Gesellschaft darstellt. Deshalb ist es nicht verwunderlich, dass sich hier eine breite Einkommensverteilung findet, wobei die unteren genau wie die oberen Einkommen etwas unterrepräsentiert sind. $40 \%$ der leitenden Angestellten gehören zu diesem Milieu. Auch sind Studierende hier besonders hoch vertreten. Im Ruhestand befinden sich $17 \%$. Der Bildungsstand entspricht praktisch dem Durchschnittsprofil, nur ist der Anteil der Personen, die das Abitur als höchsten Abschluss angeben, höher.

Lebensstil: In diesem Milieu spielt noch stärker als im statusorientierten Milieu der moderne Leistungsoptimismus eine große Rolle. Es gibt ein großes Vertrauen in die eigene Leistungsfähigkeit, die gleichen Anstrengungen werden auch von an- 
deren erwartet. Die Identifikation mit der Berufstätigkeit ist sehr hoch, Selbstverwirklichung findet vor allem hier statt, die Familie fungiert nicht als privater Rückzugsraum. Man leistet sich etwas, weil man es verdient hat, das Thema Grundbesitz steht aber nicht im Vordergrund. Mit dem Staat ist man einverstanden, gegenüber dem gesellschaftlichen Fortschritt aufgeschlossen. Ein kritisches gesellschaftliches Engagement wird nicht für nötig befunden, für kulturelles Kapital gibt es keine besondere Wertschätzung. Bürgerliche Tugenden müssen offenbar nicht betont werden, die damit verbundene Pflichtethik ist anscheinend selbstverständlich verinnerlicht worden.

Politikstil: In diesem Milieu erhalten die Grünen nur halb so viel Zuspruch wie in der Gesamtbevölkerung, während DP und LSAP besser abschneiden. ${ }^{5}$

\section{Kleinbürgerliches Milieu (19 \%)}

Soziale Lage: Zusammen mit dem konservativ-gehobenen Milieu stellt das kleinbürgerliche Milieu ein Milieu der Älteren dar. Auch wenn der Altersdurchschnitt geringer ist als im konservativ-gehobenen Milieu, unterscheiden beide sich gemeinsam von den sieben anderen, die alle im Schnitt wesentlich jünger sind. $24 \%$ der Angehörigen des kleinbürgerlichen Milieus befinden sich im Ruhestand. In keinem anderen Milieu differiert die Geschlechterzugehörigkeit so stark wie hier: Der Frauenanteil beträgt 6o \%. In diesem Milieu findet man eine breite Einkommensverteilung, die mehr oder weniger dem Durchschnitt aller Milieus entspricht. Besonders zahlreich sind allerdings jene, die ihr Einkommen nicht angeben. Alle Bildungsabschlüsse sind vertreten, jedoch sind die höheren Abschlüsse weniger und die unteren besser vertreten als für die Gesamtstichprobe. Das kleinbürgerliche Milieu ist im Süden des Landes überproportional vertreten, am schwächsten in der Hauptstadt.

Lebensstil: Im kleinbürgerlichen Milieu ist eine ausgeprägte Mentalität der Pflichterfüllung vorherrschend, die als Ausdruck der Anpassung an die Gegebenheiten verstanden werden kann. Wichtig erscheinen hier für den Zusammenhalt des Milieus nach innen die traditionelle Rollenaufteilung in der Familie sowie christliche Wertvorstellungen, beides steht mit der Ablehnung eines modernen Lebensstils in Verbindung. Die Abweisung hedonistischer Vorstellungen verweist auf einen z.B. vom unterprivilegierten Milieu sich abgrenzenden Verzicht auf Selbstverwirklichung zugunsten der Einordnung in eine vorgegebene soziale (Arbeits-) Hierarchie. In diesem Kontext erscheint der Erwerb von kulturellem Kapital wenig lohnenswert, an Kunst und Kultur ist kein Interesse vorhanden. Das starke Streben

5 | Luxemburger Parteien und ihre politische Ausrichtung: ADR: Alternativ Demokratesch Reformpartei (rechtspopulistisch); CSV: Chrëschtlech Sozial Vollekspartei (christdemokratisch); DP: Demokratesch Partei (liberal); Déi Gréng (ökologisch, grün); Déi Lénk (sozialistisch, links); KPL: Kommunistesch Partei Lëtzebuerg (kommunistisch); LSAP: Lëtzebuerger Sozialistesch Arbechterpartei (sozialdemokratisch). 
nach materieller Sicherheit zieht die Zurückstellung eigener, spontaner Bedürfnisse nach sich. Das schließt auch die Bereitschaft zum Verzicht auf Lebensgenuss zugunsten von Grundbesitz ein. Man verhält sich staatskonform. Generell wichtig ist es, seinen Platz einzunehmen, Fortkommen durch eigene Leistung spielt keine besondere Rolle. Die Abstiegsangst scheint für den Augenblick gebannt zu sein. Für ein gewisses Selbstbewusstsein hierbei spricht, dass der Arbeitsalltag ohne besondere Frustrationsneigung durchgestanden wird.

Politikstil: CSV-Wähler sind in diesem Milieu überproportional vertreten, wenn auch nicht so stark wie im konservativ-gehobenen Milieu. Auch die DP-Wähler/innen sind hier weniger zahlreich, Grüne erzielen gute Ergebnisse. Der Hauptunterschied zum konservativ-gehobenen Milieu ist jedoch die bessere Position der Sozialistischen Partei.

\section{Unterprivilegiertes Milieu (13\%)}

Soziale Lage: Mit 6o \% ist das unterprivilegierte Milieu das Milieu mit dem höchsten Ausländer/-innenanteil, von denen die Portugiesen/-innen mehr als die Hälfte stellen. Fast $40 \%$ der unqualifizierten Arbeiter/-innen und $30 \%$ der Facharbeiter/-innen finden sich in diesem Milieu. Auch die Büroangestellten, von denen sich immerhin ein Fünftel in diesem Milieu finden, sind hier überrepräsentiert. Im unterprivilegierten Milieu, das etwas jünger ist als der Durchschnitt, sind die 20- bis 45-Jährigen besonders gut vertreten. Im Ruhestand sind 13\%. Das Einkommen ist dementsprechend niedrig. Im unterprivilegierten Milieu ist das formale Bildungsniveau am geringsten von allen Milieus: Fast dreimal mehr als für die Gesamtstichprobe geben hier die Grundschule als höchsten Abschluss an. Wie für das traditionsorientierte Milieu stellt für das unterprivilegierte Milieu der Norden des Landes einen räumlichen Schwerpunkt dar.

Lebensstil: Ähnlich wie im traditionsorientierten Milieu gehört für das unterprivilegierte Milieu das Gefühl von Frustration im Arbeitsalltag zu einer Art Grundstimmung. Man fühlt sich stark in der Arbeitshierarchie eingebunden. Nach wie vor legt man Wert auf überkommene Arbeitstugenden. Insgesamt hat man sich aber von Aufstiegschancen im Arbeitsbereich verabschiedet, partizipiert aber noch an der autoritären Leistungsideologie, indem man sich nach sunten< deutlich abgrenzt. Selbstverwirklichung findet eher in der Freizeit statt, hier bietet die Familie noch einen gewissen Rückhalt, obwohl man sich offenbar nicht mehr für sie >krumm legen< will. Charakteristisch ist das offene Bekenntnis zum Hedonismus. Die im kleinbürgerlichen und im traditionsorientierten Milieu noch vorherrschende Bescheidenheitsethik ist aufgegeben. Das Sicherheitsdenken oder die Bereitschaft zum Verzicht zugunsten langfristiger Verpflichtungen, etwa für Grundbesitz, ist nicht vorhanden. Hoch ist dagegen die Bereitschaft zum spontanen Konsum, der offenbar auch die Unzufriedenheit über die eigene materielle Lage nach sich zieht. Ein gewisses ideelles Gegengewicht stellt die deutlich skeptische Einstellung zum technischen Fortschritt 
sowie die Wertschätzung des politischen und sozialen Engagements dar. Hier spielt offenkundig der für dieses Milieu festzustellende hohe gewerkschaftliche Organisationsgrad eine Rolle.

Politikstil: Berücksichtigt man nur die Luxemburger/-innen, dann ist das unterprivilegierte Milieu das Milieu der Politikverdrossenheit. Ein Drittel verweigert die Antwort auf die Frage nach der Parteiennähe.

\section{Liberal-gehobenes Milieu (11\%)}

Soziale Lage: Die Einkommensverteilung deckt zwar die ganze Bandbreite des Spektrums ab, doch sind die obere Mitte und die Spitzeneinkommen besonders gut vertreten, so dass das Durchschnittseinkommen dieses Milieus an dritter Stelle hinter den zwei reichsten Milieus, statusorientiertes und konservativ-gehobenes Milieu, steht. Lehrer/-innen sind in diesem Milieu doppelt so zahlreich wie in der Gesamtbevölkerung und auch leitende Angestellte sind überrepräsentiert. Wie im kleinbürgerlichen und im traditionsorientierten Milieu sind Frauen hier überdurchschnittlich vertreten, ihr Anteil ist mit 58,1\% am zweithöchsten. Das formale Bildungsniveau ist sehr hoch. Man findet im Vergleich zu allen anderen Milieus hier am wenigsten Grundschulabschlüsse als einzigen Schulabschluss. Im Gegensatz zu dem ebenfalls mit hohen Bildungsqualifikationen ausgestatteten konservativ-gehobenen Milieu sind hier jedoch Fachhochschulabschlüsse zahlreicher als Universitätsabschlüsse. Wie das konservativ-gehobene Milieu ist das liberal-gehobene Milieu im Süden des Landes unterdurchschnittlich vertreten.

Lebensstil: Das liberal-gehobene Milieu bezieht sein Selbstbewusstsein aus einer starken Leistungsmotivation und einem hohen Arbeitsethos. Beides geht aber nicht in einer Leistungsideologie auf, es gibt offenbar kein entsprechendes gesellschaftliches Sendungsbewusstsein. Darin, aber auch in der Ablehnung überkommener bürgerlicher Tugenden, grenzt sich das liberal-gehobene Milieu von eher konservativ ausgerichteten Milieus (konservativ-gehobenes, kleinbürgerliches und unterprivilegiertes Milieu) ab und stimmt eher mit den neuen Arbeitnehmern/-innen (statusorientiertes Milieu) überein. Die Konzentration auf die eigene Leistungsfähigkeit hat einen nüchtern-realistischen Zug, man rechnet mit Verwerfungen im Berufsleben. Dem Staat gegenüber wahrt man Distanz, die Familie bildet einen Schutzraum des Privaten, hier wird die traditionelle Rollenaufteilung zwischen Frau und Mann aber ausdrücklich als überholt angesehen. Auf der Basis einer gesicherten materiellen Ausstattung gibt man sich wenig konsumorientiert. Auffallend ist die deutliche Affinität zur Beschäftigung mit Kultur und Kunst, also der Bereitschaft zur Ausweitung des kulturellen Kapitals, wie sie sonst nur im konservativ-gehobenen und im alternativen Milieu vorhanden ist.

Politikstil: In diesem Milieu wählt man zweieinhalbmal mehr die Grünen als im Durchschnitt. Die CSV ist stark unterrepräsentiert. 


\section{Konservativ-gehobenes Milieu (9 \%)}

Soziale Lage: Die Angehörigen des konservativ-gehobenen Milieus sind durchweg älter als die Gesamtbevölkerung, der Altersdurchschnitt liegt zehn Jahre über dem der Gesamtbevölkerung. 39 \% dieses Milieus befinden sich im Ruhestand, während es für die Gesamtheit $19 \%$ sind. $42 \%$ des konservativ-gehobenen Milieus sind älter als 60 Jahre, unter den zu diesem Milieu zuzurechnenden Luxemburgern/-innen sind es sogar $57 \%$. Am Durchschnittsverdienst gemessen nimmt das konservativ-gehobene Milieu die erste Stelle unter allen Milieus ein. Besonders die Einkommensgruppen über $9000 €$ sind stark vertreten. Knapp dahinter folgen das statusorientierte Milieu sowie das liberal-gehobene Milieu, zusammen bilden sie eine weit vor allen anderen Milieus rangierende Einkommensspitze. Im konservativ-gehobenen Milieu findet man die meisten Akademiker/-innen. Es ist besonders im Zentrum des Landes, genauer in der Hauptstadt selber, vertreten, im Süden des Landes hingegen unterdurchschnittlich.

Lebensstil: Zurückhaltung und Vermeidung übertriebener Selbstdarstellung zeigt sich im konservativ-gehobenen Milieu indirekt in der durchgehenden, dezidierten Ablehnung der verschiedenen Formen des Hedonismus (des ideellen wie des realistischen). Die betonte individuelle Leistungsbereitschaft ist Ausdruck der Haltung innerer Pflichterfüllung, der berufliche Erfolg beruht auf einem ausgeprägten Arbeitsethos, aus ihm leitet sich ein quasi natürliches Selbstbewusstsein ab. Grundlage hierfür ist die Orientierung an gewachsenen Strukturen, obgleich die traditionelle Rollenverteilung in der Familie, überhaupt die Familie als geschützter Raum, nicht (mehr) gesondert betont wird (was evtl. mit dem hohen Anteil an Ruheständlern/-innen zu tun hat). Das konservativ-gehobene Milieu gibt sich aufgeschlossen gegenüber dem technischen Fortschritt und versteht sich ausdrücklich als staatstragend. Eine große Rolle spielt die gesellschaftliche Repräsentationsfähigkeit, das Prestige zählt. Selbstverwirklichung findet neben der Arbeit vor allem im gehobenen kulturellen Sektor statt, dieser bildet einen Gegenpol zu den als oberflächlich angesehenen Reizen des materiellen Konsums. Materielle Sicherheiten sind wie selbstverständlich vorhanden, müssen aber auch bewahrt und vermehrt werden, wie die große Bereitschaft zu Einschränkungen für den Grundbesitz zeigt.

Politikstil: Dieses Milieu kann man getrost als konservativ bezeichnen. Die CSV ${ }^{6}$ hat fast doppelt so viele Wähler/-innen wie in der Gesamtbevölkerung. Auch die ADR ist leicht überrepräsentiert. Es erstaunt, dass die liberalen, zumindest, wenn es um die Hauptparteienpräferenz geht, in der Minderheit sind. Noch schwerer hat es jedoch die LSAP in diesem Milieu.

6 | Luxemburger Parteien und ihre politische Ausrichtung: ADR: Alternativ Demokratesch Reformpartei (rechtspopulistisch); CSV: Chrëschtlech Sozial Vollekspartei (christdemokratisch); DP: Demokratesch Partei (liberal); Déi Gréng (ökologisch, grün); Déi Lénk (sozialistisch, links); KPL: Kommunistesch Partei Lëtzebuerg (kommunistisch); LSAP: Lëtzebuerger Sozialistesch Arbechterpartei (sozialdemokratisch). 


\section{Hedonistisches Milieu (7 \%)}

Soziale Lage: Das hedonistische Milieu ist ein junges Milieu, in dem die unter 30-Jährigen und noch stärker die unter 20-Jährigen überrepräsentiert sind, mit einem Einkommen, das nur knapp über dem Durchschnitt liegt. Dem Alter entsprechend liegt der Anteil der im Ruhestand befindlichen nur bei $14 \%$. Der öffentliche Dienst ist stark präsent, allerdings vertreten durch die Beamten der mittleren Laufbahn. Die Angehörigen des hedonistischen Milieus entsprechen weitgehend dem durchschnittlichen Bildungsprofil. Akademiker/-innen sind weniger stark, Abiturienten/innen überproportional vertreten. Von allen Milieus ist das hedonistische Milieu räumlich am geringsten im Norden des Landes vertreten. Der Anteil der Singlehaushalte, genauso wie der Paare ohne Kinder, ist in diesem Milieu am höchsten. Auch wenn die gesetzlich geregelte Lebenspartnerschaft (PACS, d.h. > pacte civil de solidarité $)$ noch ein marginales Problem darstellt (1,5\% bei allen Befragten), so sind in diesem Milieu die Lebenspartnerschaften mehr als doppelt so häufig.

Lebensstil: Die Hochschätzung des materiellen wie ideellen Hedonismus und demzufolge auch die Selbstverwirklichung in Freizeit und Konsum hat in diesem Milieu eine nicht weiter hinterfragte gesicherte ökonomischen Basis. So geht die Bereitschaft zum spontanen Konsum nicht wie in den anderen Milieus mit einer Unzufriedenheit mit den finanziellen Möglichkeiten einher, was auf ein gehobenes Herkunftsmilieu hindeutet. Arbeit ist Mittel zum Zweck, beruflicher Erfolg erscheint unwichtig, kritisch-gesellschaftliches Engagement wird abgelehnt, gehobene Kultur ist kein wesentlicher Faktor. Die deutliche Wendung gegen christliche Wertvorstellungen könnte direkt gegen diese ideellen Klammerwerte von konservativ-gehobenem und kleinbürgerlichem Milieu gerichtet sein, aus denen offenbar die Generation des hedonistischen Milieus hervorgegangen ist. Die Tendenz zur Identifizierung mit Frustrationseinstellungen ist wahrscheinlich durch die drohende Konfrontation mit möglichen Leistungsanforderungen (Konflikte mit Elterngeneration) zu erklären. Auffallendstes Merkmal: Im hedonistischen Milieu gibt man sich staatstragend. Hier werden offenkundig Grundeinstellungen des Herkunftsmilieus reproduziert, die aus materiellem Kalkül geteilt werden. Vermutlich wird hier auch die Rolle staatlicher Unterstützungsleistungen mitbedacht, die eine Aufrechterhaltung des Lebensstils so lange wie möglich sichern.

Politikstil: LSAP-Wähler/-innen sind leicht überrepräsentiert, CSV und Grüne leicht unterrepräsentiert.

\section{Traditionsorientiertes Milieu (5\%)}

Soziale Lage: In diesem Milieu mit vielen Arbeitern/-innen und kleinen Angestellten ist der Verdienst gering. Aber man scheut sich nicht, dies auch zuzugeben, vielleicht auch weil man in klaren Lohnabhängigkeitsverhältnissen lebt. So kommen Kleinsteinkommen kaum vor, da es keine Teilzeitjobs oder prekäre Arbeitsverhältnisse zu geben scheint. Dies ist das Milieu, in dem Invalidenrentner/-innen 
und Arbeitslose am stärksten überrepräsentiert sind. Im Ruhestand befinden sich $17 \%$. Das traditionsorientierte Milieu weist mit 57,9 \% einen hohen Frauenanteil auf. Genau wie im kleinbürgerlichen Milieu findet sich die ganze Bandbreite der Bildungsabschlüsse, jedoch sind die höheren Abschlüsse weniger und die unteren besser vertreten als für die Gesamtstichprobe. Räumlich ist das traditionsorientierte Milieu im Norden des Landes überrepräsentiert.

Lebensstil: Im Gegensatz zum kleinbürgerlichen Milieu durchzieht das traditionsorientierte Milieu eine deutlich pessimistischere Grundeinstellung. Materielle Sicherheit ist ein hoher Wert, man wägt den Konsum nach den vorhandenen Möglichkeiten ab und ist unzufrieden mit der eigenen Lage. Die Frustrationsneigung im Arbeitsleben ist hoch. Man fühlt sich ausgenutzt, in starre Hierarchien eingezwängt, durch eigene Leistung scheint die Sicherung der Zukunft kaum möglich. Diese Einschätzung der eigenen Lage geht mit einer Ablehnung des technischen Fortschritts einher, die Zukunftssorgen des Milieus gelten als Spiegelbild der allgemeinen gesellschaftlichen Entwicklung. Aus Enttäuschung verhält man sich distanziert gegenüber dem Staat, auf gesellschaftliches Ansehen wird kein Wert gelegt. Die Teilhabe am kulturellen Leben erscheint nutzlos. Zwar schätzt man wie im kleinbürgerlichen Milieu nach wie vor die bürgerlichen Tugenden, die traditionelle Rollenaufteilung in der Familie ist noch stark ausgeprägt. Aber anders als im kleinbürgerlichen Milieu bilden christliche Wertvorstellungen keine besonders hervorzuhebende Klammer mehr.

Politikstil: Dieses Milieu ist eine ausgesprochene ADR-Hochburg. Die Sympathien für diese Partei gehen auf Kosten der CSV, die hier fast nur die Hälfte der Wahlaussagen erhält als im Durchschnitt, während die LSAP knapp über ihrem Gesamtergebnis liegt. Allerdings liegt die Zahl der Unentschlossenen und der Auskunftsverweigerer hier besonders hoch.

\section{Statusorientiertes Milieu (3\%)}

Soziale Lage: Betrachtet man nur den Einkommensdurchschnitt, so ist er im statusorientierten Milieu, nach dem konservativ-gehobenen Milieu, am zweithöchsten von allen Milieus. In beiden geben immerhin $8 \%$ der Befragten ein Monatsgehalt von über 10.000 Euro an. Zusammen mit diesem und dem liberal-gehobenen Milieu bilden sie ein Spitzentrio, das die anderen Milieus weit hinter sich lässt. Das statusorientierte Milieu hat den geringsten Ausländer/-innenanteil (nur $17 \%$ ) und ist das jüngste Milieu, in dem die unter 20-Jährigen (14\% gegenüber $7 \%$ in der Gesamtheit), also auch die Studierenden, besonders gut vertreten sind. Im Kontrast zum kleinbürgerlichen Milieu, als dem Milieu mit dem höchsten Frauenanteil, ist im statusorientierten Milieu mit 61,5\% der Männeranteil am höchsten. Im Ruhestand befinden sich $12 \%$ (Gesamtheit $19 \%$ ). Im statusorientierten Milieu ist der öffentliche Dienst, besonders auch die Lehrer/-innen, stark überrepräsentiert. Ansonsten finden sich hier viele leitende Angestellte. Die häufigste Bildungsqualifikation ist der Fachhochschulabschluss. 
Lebensstil: Ähnlich wie im benachbarten aufstiegsorientierten Milieu steht hier das Vertrauen in die eigene Leistungsfähigkeit im Vordergrund. Im statusorientierten Milieu ist man bereit, sich fachlich fortzubilden, um verantwortungsvolle Tätigkeiten zu übernehmen; man verbindet damit jedoch keine autoritäre Leistungsideologie mehr, sondern bleibt eher individualistisch. Die Selbstverwirklichung im beruflichen Bereich wird allerdings nicht verbissen betrieben. Die Pflichtethik wird eher gelassen gehandhabt. Materiell ist man gut ausgestattet. Im Zweifelsfall steht das Privatleben im Vordergrund. Hier gelten traditionelle Familienwerte als überholt, und aus der ebenso deutlichen Ablehnung christlicher Wertvorstellungen lässt sich auf eine Aufgeschlossenheit für unkonventionelle Lebensformen schließen. Der eigene Leistungsoptimismus geht mit einem allgemeinen technischen Fortschrittsoptimismus einher, gegenüber dem Staat bleibt man aber in kritischer Distanz. Mit Kunst und Kultur kann man nichts anfangen, für konkretes gesellschaftliches Engagement lässt man sich nicht vereinnahmen. Insgesamt zeigt sich das statusorientierte Milieu selbstbewusster und unabhängiger von allgemeinen Entwicklungen, im beruflichen Sektor auch realistisch-nüchterner als das aufstiegsorientierte Milieu.

Politikstil: Im statusorientierten Milieu wählt man eher Grün und LSAP, CSV ist besonders unterrepräsentiert.

\section{Alternatives Milieu (3\%)}

Soziale Lage: Die meisten Vertreter/-innen des alternativen Milieus stehen voll im erwerbsfähigen Alter, der Anteil von Frauen und Männern ist in diesem Milieu exakt ausgeglichen. Das Einkommen liegt unter dem Durchschnitt. Das Fehlen hoher Einkommen deutet auf diesbezügliche mangelnde Auskunftsbereitschaft hin (Zwang zur antimateriellen Einstellung). Der Umstand, dass knapp $30 \%$, also fast so viel wie im kleinbürgerlichen Milieu, ihre Einkünfte nicht angeben, dürfte aber auch mit einem fehlenden kontinuierlichen Einkommen zusammenhängen. Das alternative Milieu ist ein überdurchschnittlich gebildetes Milieu, in dem Hochschul- und Fachhochschulabsolventen sehr gut vertreten sind und in dem praktisch niemand präsent ist, der nur einen Grundschulabschluss hat. Dieses Milieu ist besonders im direkten Umland der Hauptstadt vertreten.

Lebensstil: Obwohl der Leitwert in diesem Milieu eindeutig die Kritik am technologischen Fortschrittsdenken bildet, verbindet man damit nicht mehr so eindeutig die mögliche Zerstörung der Lebensgrundlagen. Dieses Indiz für ein schwindendes gesellschaftliches Sendungsbewusstsein (z.B. über die Umweltpolitik) wird durch eine Verlagerung der Selbstverwirklichung vom politisch-gesellschaftlichen Engagement in den Bereich von Kunst und Kultur bekräftigt. Auffällig ist auch hier, wie im hedonistischen Milieu die deutlich staatstragende Einstellung. Offenbar hat man sich mit den Verhältnissen arrangiert, insoweit genügend Raum für den eigenen postmateriellen Lebensstil jenseits der autoritären Leistungsideologie, traditioneller Geschlechterrollen und der Zwänge der Arbeitshierarchie vorhanden 
ist. Die starke Bereitschaft zum Ausstieg aus der Gesellschaft dürfte daher wohl eher ein Lippenbekenntnis bzw. eine Reminiszenz an die Wurzeln des Milieus in der Oppositionsbewegung darstellen.

Politikstile: Grüne- und DP-Wähler sind stark über-, CSV-Wähler stark unterrepräsentiert. Praktisch keine Stimmen erhalten ADR und LSAP.

\subsection{Schlussbemerkung}

Mit Blick auf die folgenden Kapitel der vorliegenden Publikation, die auf der Basis der Milieuanalyse die Herausarbeitung einzelner Aspekte leisten, stellt das beschriebene Set an Milieus den allgemeinen heuristischen Rahmen bereit. Die Milieukartografie der Luxemburger Sozialstruktur muss daher komplementär zu den qualitativen Ansätzen gelesen werden, die in diesem Band vorgestellt werden.

Die Verfasser möchten betonen, dass die hier vorgestellten Ergebnisse ein (!) mögliches Modell sind, die soziale Wirklichkeit in Luxemburg zu beschreiben. Insofern dürfen die präsentierten Ergebnisse nicht mit der Wirklichkeit verwechselt werden, sondern stellen mit Blick auf die Komplexität der Luxemburger Sozialstruktur einen ersten, tentativen Zugang dar, der durch weitere und vertiefende Analysen ausgebaut und fundiert werden muss. Zudem bergen auch die bereits erhobenen Daten eine Fülle weiterer Informationen, die noch ausgewertet werden müssen.

Grundsätzlich und abschließend sei darauf hingewiesen, dass die angegebenen Prozentzahlen - und dies gilt für die Ergebnisse jeder stichprobenbasierten Erhebung - immer mit Ungenauigkeit behaftet und daher relativ zu betrachten sind.

\subsection{LITERATURVERZEICHNIS}

Arendt, Hannah (1997): Vita activa oder Vom tätigen Leben, München: Piper.

Backhaus, Klaus et al. (2008): Multivariate Analysemethoden. Eine anwendungsorientierte Einführung, Berlin: Springer.

Beck, Ulrich (1986): Risikogesellschaft. Auf dem Weg in eine andere Moderne, Frankfurt a.M.: Suhrkamp.

Bourdieu, Pierre (1979): La distinction. Critique sociale du jugement, Paris: Minuit. (1982 auf Deutsch erschienen unter dem Titel: Die feinen Unterschiede. Kritik der gesellschaftlichen Urteilskraft, Frankfurt a.M.: Suhrkamp.)

Bourdieu, Pierre (1992a): »Die feinen Unterschiede«, in: Ders.: Die verborgenen Mechanismen der Macht. Schriften zur Politik \& Kultur I, hg. von Margareta Steinbrücke, Hamburg: VSA, S. 31-48.

Bourdieu, Pierre (1992b): »Ökonomisches Kapital, kulturelles Kapital, soziales Kapital«, in: Ders.: Die verborgenen Mechanismen der Macht. Schriften zur Politik \& Kultur I, hg. von Margareta Steinbrücke, Hamburg: VSA, S. 49-79. 
Bourdieu, Pierre (1995): Sozialer Raum und >Klassen<. Leçon sur la leçon. 2 Vorlesungen, Frankfurt a.M.: Suhrkamp.

Geißler, Rainer (1996): »Kein Abschied von Klasse und Schicht. Ideologische Gefahren der deutschen Sozialstrukturanalyse«, in: Kölner Zeitschrift für Soziologie und Sozialpsychologie 48, S. 319-338.

Geißler, Rainer (2002): Die Sozialstruktur Deutschlands. Die gesellschaftliche Entwicklung vor und nach der Vereinigung, Bonn: Westdeutscher Verlag.

Habermas, Jürgen (1985): Die Neue Unübersichtlichkeit, Frankfurt a.M.: Suhrkamp.

Meyer, Thomas (2001): »Das Konzept der Lebensstile in der Sozialstrukturforschung - eine kritische Bilanz«, in: Soziale Welt 52, S. 255-272.

Schelsky, Helmut (1979): Auf der Suche nach Wirklichkeit, München: Goldmann.

Schulze, Gerhard (1994): »Gehen ohne Grund. Eine Skizze zur Kulturgeschichte des Denkens«, in: Andreas Kuhlmann (Hg.): Philosophische Ansichten der Kultur der Moderne, Frankfurt a.M.: Fischer, S. 79-130.

Schulze, Gerhard (2000): Die Erlebnisgesellschaft. Kultursoziologie der Gegenwart, Frankfurt a.M.: Campus.

Vester, Michael et al. (2001): Soziale Milieus im gesellschaftlichen Strukturwandel. Zwischen Integration und Ausgrenzung, Frankfurt a.M.: Suhrkamp. 



\title{
4. Sprachen und Identitäten
}

\author{
Peter Gilles, Sebastian Sefla, Heinz Sieburg, Melanie Wagner
}

\subsection{Einleitung: Diglossie zWischen Germania UND ROMANIA}

Das Phänomen der Mehrsprachigkeit gehört in Luxemburg zu den historisch gewachsenen sozialen Realitäten. Die Lage Luxemburgs im Kontakt- und Vermittlungsraum zwischen Germania und Romania spiegelt sich seit dem Mittelalter in einer Diglossiesituation wider, die von einem Nebeneinander des Deutschen, bzw. regionaler Varietäten des Deutschen, und des Französischen geprägt ist. Über die Jahrhunderte und infolge unterschiedlicher territorialer Zugehörigkeiten und Verschiebungen haben sich die Bedingungen der Luxemburger Mehrsprachigkeit mehrfach grundlegend geändert. Die wechselnde Folge französischer, niederländischer und deutscher Vereinnahmungsversuche hatte ihren Ausdruck auch in unterschiedlichen sprachhegemonialen Bestrebungen. Dem stand der Wille der Luxemburger Bevölkerung entgegen, sich nicht durch eine Seite vereinnahmen zu lassen und somit die Grundvoraussetzung nationaler Souveränität und Identität zu wahren.

Spätestens ab dem 19. Jahrhundert tritt das Luxemburgische als eigenständige und im Bewusstsein der Bewohner Luxemburgs eigene Sprache mit ausgeprägt identitätsstiftender Funktion zunehmend stärker hervor, wobei zunächst noch die enge sprachgenealogische Verwandtschaft zum Deutschen (>lëtzebuerger Däitsch`) betont wurde. Grundlage des Luxemburgischen ist der westmoselfränkische Dialekt, der jedoch inzwischen zu einer Vollsprache ausgebaut wurde, annähernd alle Sprachdomänen abdeckt, eine normierte Schriftlichkeit besitzt und seit der Sprachgesetzgebung von 1984 auch als offizielle Nationalsprache Luxemburgs fungiert.

Flankiert wird das Luxemburgische durch die im Sprachgesetz von 1984 ebenfalls genannten offiziellen Sprachen Luxemburgs, nämlich Französisch und Deutsch, so dass von einer Triglossiesituation ausgegangen werden kann. Eine Sonderrolle kommt dem Französischen zu, das als Gesetzessprache alleinige Gültigkeit besitzt. (Zum Sprachgesetz vgl. Gilles/Moulin 2003) Ein zentrales Ziel 
dieser vornehmlich politischen Aufwertung war die Steigerung des soziolinguistischen Status des Luxemburgischen gegenüber dem Französischen und dem Deutschen. (Naglo 2007) Jedoch hatte diese Erhebung zur Nationalsprache bislang kaum praktische Konsequenzen zur Folge, und zwar weder hinsichtlich einer weiteren Standardisierung noch bzgl. eines vermehrten Gebrauchs im Schulsystem, in dem das Medium lediglich als Hilfssprache im Unterricht dient. (Gilles 1999: 9; Kraemer 1993) Vielmehr wurde die Sprache politisch und juristisch als Symbol und Ausdruck einer nationalen luxemburgischen Identität festgelegt.

Zwar sind die Vorzüge einer solchen juristisch legitimierten Mehrsprachigkeit gerade vor dem Hintergrund der Entwicklung der EU und der proklamierten Forderung nach Multilingualität unmittelbar einsichtig, andererseits konfrontiert diese Mehrsprachigkeit die Einwohner Luxemburgs aber auch mit ganz besonderen Anforderungen, so etwa im Bereich des Schulunterrichts.

Komplexer wird die Situation noch durch den Umstand, dass Luxemburg als Einwanderungsland auch große Bevölkerungsanteile mit weiteren (Mutter-)Sprachen wie Italienisch, gegenwärtig aber stärker noch Portugiesisch und im Zuge des Ausbaus des Landes als Verwaltungs- und Bankenplatz auch Englisch beherbergt. Hinzu kommen unterschiedliche Sprachen Ex-Jugoslawiens.

Sprachfragen bilden also einen wesentlichen Faktor im gesellschaftlichen Gefüge des Großherzogtums, auch, weil sie als wichtige Konstituente für die Identitäten der Einwohner Luxemburgs anzusehen sind. Damit waren in der vorliegenden Studie Fragen nach der rezenten Sprachsituation im Land nicht nur naheliegend, sondern geradezu ein zwingendes Erfordernis.

Es darf nicht übersehen werden, dass die Bedeutung von Sprachen keineswegs auf die Vermittlung von Kommunikationsinhalten eingeschränkt ist. Eine solche monofunktionale Betrachtungsweise verkennt die Relevanz von Sprache als grundlegendes Sozialsymbol, dem vielschichtige gesellschaftliche Wertzuschreibungen jenseits der kommunikativen Leistungsfähigkeit zukommen. (Vgl. Mattheier 1991) Dabei sind Fragen von Prestige und Stigma ebenso zu beachten wie die von Selbstbehauptung oder auch Fremdbestimmung. Sprachfragen sind somit immer auch politisch-soziale Fragen und insofern mitunter ebenso brisant wie kontrovers. Sie können mit unterschiedlichsten Emotionen und Wertzuweisungen verknüpft sein, wobei häufig genug die Sprache genannt wird, aber deren Sprecher gemeint sind.

Auch in Luxemburg spiegeln die wechselnden Sprachdiskurse häufig tief verankerte Gefühls- und Bewusstseinslagen wider, die sich in unterschiedlichen Abstufungen von Betroffenheit, Besorgnis oder Bedrohung ausdrücken oder, ins Offensive gewendet, in Abgrenzung, Zurückdrängung und Geltungsanspruch umschlagen können. Nahezu immer sind dabei als Bezugsgröße Aspekte von Identität, seien es zugeschriebene oder angeeignete, auszumachen.

Darauf deutet auch die im Rahmen der vorliegenden Untersuchung unternommene Fall-Studie zur Auswertung von Leserbriefen. In diesen zeigt sich exemplarisch, dass das Nebeneinander der Sprachen Luxemburgs keineswegs immer konfliktfrei verläuft. Schon weil Luxemburg ein wirtschaftlich und auch demografisch 
äußerst dynamisches Land ist, ist das Verhältnis der Landessprachen - auch abhängig von der Zahl und Nationalität der Neubürger und Grenzpendler - immer ein Stück weit von teils offenen, teils verdeckten Aushandlungsprozessen abhängig, die durchaus Momente der Sprachkonkurrenz aufweisen können.

Dabei zeigt sich, um ein zentrales Ergebnis vorwegzunehmen, als Konstante die ausgeprägte Loyalität der Luxemburger gegenüber dem Luxemburgischen, das nicht nur verordnete Nationalsprache ist, sondern sich gerade auch in den vergangenen Jahrzehnten zu einem wesentlichen Identifikations- und Integrationsfaktor entwickelt hat. Wegen der schon in der Sprecherzahl begründeten verhältnismäßig eingeschränkten kommunikativen Reichweite gilt das Luxemburgische vielen seiner Sprecher als potentiell gefährdet und daher umso mehr förderungswürdig. Nicht selten zeigen sich dabei auch Abwehrreflexe gegenüber den beiden flankierenden Landessprachen Französisch und Deutsch, wobei erstere oft als im normalen Sprachalltag zu dominant und letztere, zumindest in Teilen der älteren Generation, nicht selten als mit dem Stigma der Besatzungssprache verhaftet erscheint.

Allerdings wäre das Bild verzerrt, würde man das Konflikthafte allzu sehr in den Vordergrund rücken. Denn bei allen Schwierigkeiten, auch dies zeigen die Untersuchungsergebnisse, darf auf der anderen Seite nicht der beträchtliche Mehrwert der Mehrsprachigkeit übersehen werden. Mehrsprachigkeit ist nicht nur eine Voraussetzung für die prosperierende Wirtschaft im Land, die eben auch von den ausländischen Beschäftigten aus unterschiedlichen Herkunftsländern mitgetragen wird. Vielmehr erschließt die Mehrsprachigkeit, und hier insbesondere die Französisch- und Deutschkompetenz, den Luxemburgern einen barrierefreien Kommunikationsraum in einer Dimension, über die kein anderes europäisches Land verfügt. Auch dies, so zeigen die Analysedaten, ist den Luxemburgern durchaus bewusst. Einen anschaulichen Eindruck über das lebendige und damit auch dynamische Verhältnis der Sprachen Luxemburgs bietet auch die Fallstudie, welche die öffentliche Beschilderung zum Untersuchungsgegenstand hat (Abschnitt 4.4). Diese zeigt anschaulich, wie sich Multilingualität in Schriftform dokumentiert.

Von besonderer Relevanz, dies gilt es nochmals zu betonen, ist bei all dem die Frage nach dem Zusammenhang von Sprache und Identität. Es ist ein Gemeinplatz, dass Identitäten in Form von sprachlichen Konstruktionen aufgebaut, bestätigt und verändert werden. Die Frage, in welcher Art und Weise sich diese identitären Prozesse jedoch entwickeln, erweist sich als eine äußerst komplexe Forschungsaufgabe. Im Hinblick auf den Komplex einer >nationalen Identität< - die nicht als monolithisches Konzept, sondern als ein komplexes Bündel identitärer Aushandlungsprozesse ${ }^{1}$ zu sehen ist, die zudem in der Gesellschaft unterschiedlich gewichtet und evaluiert werden - ist das Vorhandensein und die Akzeptanz einer Nationalsprache ausschlaggebend. (Vgl. Joseph 2004) Inwieweit Luxemburgisch schon im 19. Jahrhundert als Nationalsprache auf breiter Basis etabliert war, lässt sich zurzeit noch nicht abschließend beantworten. Spätestens seit der ersten

1 | Vgl. auch Abschnitt 6.2. 
Hälfte des 20. Jahrhunderts jedoch kann davon ausgegangen werden, dass Luxemburgisch für die gesamte Bevölkerung nationalsymbolische Bedeutung bekommt. (Gilles/Moulin 2003; Moulin 2006) Kumulationspunkt der Entwicklung dürfte dann die Verabschiedung des Sprachgesetzes im Jahr 1984 gewesen sein.

Das Bündel nationaler Identitätsaspekte gründet sich jedoch nicht ausschließlich auf das Luxemburgische, vielmehr spielt - zumindest für einen Teil der Bevölkerung heute - auch die Mehrsprachigkeit als solche eine Rolle. Im Gegensatz zur territorialen Mehrsprachigkeit, wie sie in Belgien oder der Schweiz vorliegt, setzt sich in Luxemburg mehr und mehr das Selbstverständnis durch, dass Mehrsprachigkeit auf eine tiefergreifende Weise im Alltag relevant wird, als dies noch bis in die 1970er Jahre hinein der Fall war. Insbesondere bei jüngeren Altersgruppen wird damit diese soziale Mehrsprachigkeit, in der ein nicht-konkurrierendes Verhältnis der beteiligten Sprachen zueinander gefördert wird, als ein zentrales identitätsstiftendes Element prononciert.

Darüber hinaus wird natürlich bei allen sprachlichen Aspekten die Differenzierung nach >angeeigneten < und >zugeschriebenen Identitäten < relevant. So gehört eine mustergültige Kompetenz in den drei (oder vier, wenn man das Englische dazunimmt, das eine immer größer werdende Rolle spielt) Landessprachen zu den >zugeschriebenen Identitäten<, die z.B. in offiziellen Verlautbarungen zur Gesellschaftsstruktur oder zum Sprachenunterricht in den Schulen zum Ausdruck gebracht wird. Die >angeeignete Identität $<$ kann jedoch von diesem Ideal abweichen. Das so entstehende Spannungsverhältnis zwischen >angeeigneter < und >zugeschriebener Identität < wird sich im Folgenden deutlich in der Analyse der öffentlichen Beschilderung zeigen, denn die Sprachenpraxis auf offiziellen Schildern unterscheidet sich beträchtlich von derjenigen auf privaten, nicht-institutionellen Schildern.

Dieses Kapitel ist wie folgt gegliedert: In Abschnitt 4.2 werden die Kernaussagen einer repräsentativen, quantitativen Befragung vorgestellt, in der sich u.a. auch der herausgehobene Stellenwert des Luxemburgischen bestätigt. Abschnitt 4.3 widmet sich den öffentlichen Diskursen zur Sprachsituation anhand einer exemplarischen Analyse von Leserbriefen. Dabei kristallisiert sich heraus, dass der öffentliche Diskurs zwischen einer ausgewogenen Mehrsprachigkeitssituation und einer forcierten, zuweilen militanten Relevanzsetzung und Unterstützung des Luxemburgischen (auf Kosten anderer Sprachen) oszilliert. Im dritten Teil schließlich (Abschnitt 4.4) folgt eine Analyse der Mehrsprachigkeit, wie sie sich in der öffentlichen Beschilderung manifestiert (sog. >Linguistic Landscape<).

\subsection{Zentrale Befragungsergebnisse ZUR SPRACHENSITUATION}

Im Folgenden findet sich zunächst die Auswertung der repräsentativen, quantitativen Befragung, soweit diese auf Fragen zur Sprachsituation in Luxemburg bezogen ist. Ziel ist, so den Status quo bezogen auf einige zentrale Fragestellungen hin zu 
dokumentieren. ${ }^{2}$ Die präsentierten Ergebnisse können zugleich als Ausweis >angeeigneter Identitäten < verstanden werden, wo sie Sprachwerturteile implizieren. Mittelbar wird auf >zugeschriebene Identitäten < verwiesen, wo es um die Erhebung geforderter Sprachkompetenzen und staatlicher Lenkungsmaßnahmen geht.

Vorauszuschicken ist, dass alle erhobenen Sprachdaten subjektive Daten sind und somit mit allen Unwägbarkeiten behaftet, die diesem Datentyp nun einmal eigen sind.

Erhoben wurden in erster Linie Selbsteinschätzungs- sowie Einstellungsdaten, von denen im Vorfeld und auf Grundlage der Erfahrung und Forschung angenommen werden konnte, dass diese zur Beschreibung des Forschungsgegenstandes relevant sein würden. Zum einen waren dies Fragen, die eine sprachliche Rangfolge nach unterschiedlichen Kriterien (Kompetenz, Nützlichkeit, Sympathie etc.) erkennen lassen, zum anderen aber auch solche, die anhand von Zustimmungswerten für bestimmte Items (»Ich habe mehrere Muttersprachen« etc.) Rückschlüsse auf die sprachlichen Einstellungen der Luxemburger Wohnbevölkerung erlauben. Daneben wurden etwa auch Fragen nach der Orientierung auf die Nachbarländer gestellt. Auch hieraus, so kann angenommen werden, lassen sich Rückschlüsse auf die sprachlichen Präferenzen der Probanden ziehen.

Die Zahl der in Luxemburg verwendeten Sprachen geht über die der drei >offiziellen<, weil im Luxemburger Sprachgesetz von 1984 benannten Sprachen Luxemburgisch, Französisch und Deutsch weit hinaus. Die Studie trägt dem Rechnung, indem eben auch nach dem Portugiesischen, dem Englischen, dem Italienischen und als Sammelkategorie den sanderen Sprachen< gefragt wurde. Die Auswertung und Datenpräsentation folgt dem grundsätzlich, allerdings unter Beschränkung auf die zentralen Ergebnisse und der Vernachlässigung eher marginaler Größen.

\section{Mehrsprachigkeit als Mehrwert}

Die Luxemburger Bevölkerung steht der Mehrsprachigkeit insgesamt äußerst positiv gegenüber. Eine sehr deutliche Mehrheit der Befragten (95\%) sieht es als vorteilhaft an, so einen reibungslosen Kontakt mit vielen Menschen pflegen zu können. Beinahe die Gesamtheit der Befragten (95\%) sieht in der Mehrsprachigkeit zudem eine kulturelle Bereicherung. Signifikante Unterschiede hinsichtlich der nationalen, altersmäßigen oder milieubezogenen Untergruppen existieren nicht.

2 | Ergänzend sei in diesem Zusammenhang verwiesen auf die repräsentative Erhebung von Fernand Fehlen (2009): Une enquête sur un marché linguistique multilingue en profonde mutation. Luxemburgs Sprachenmarkt im Wandel, Luxemburg. 


\section{Geforderte Sprachkompetenzen}

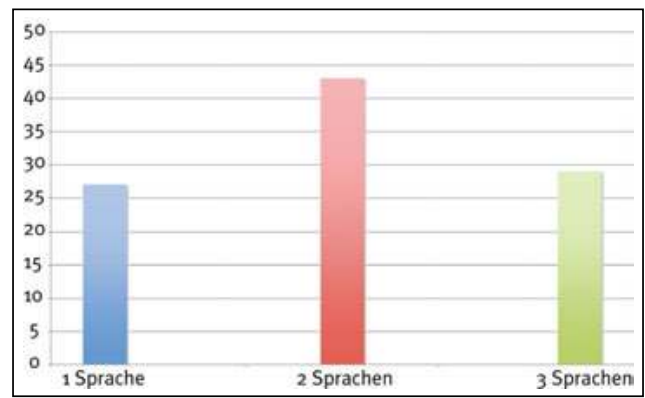

Abbildung 1: Wie viele der drei Landessprachen sollte jeder beherrschen?

Bezogen auf die drei offiziellen Sprachen Luxemburgs wurde die Zustimmung im Hinblick auf die Aussagen überprüft, ob jeder Einwohner Luxemburgs alle drei, mindestens zwei Sprachen oder mindestens eine Sprache beherrschen (und für die andere[n] über eine passive Kompetenz verfügen) soll. Eine relative Mehrheit von $43 \%$ spricht sich für ein mittleres Anforderungsniveau (zwei Sprachen) aus, und zwar zu gleichen Anteilen bei den Luxemburgern und Ausländern. Die Forderung, alle drei Sprachen zu beherrschen (29 \%) bzw. die, nur eine zu beherrschen $(27 \%)$, wird von etwa gleichen Anteilen der Befragten geäußert.

\section{Geforderte Luxemburgisch-Kompetenzen}

Die Studie spiegelt eine sehr ausgeprägte Loyalität der Befragten gegenüber dem Luxemburgischen wider. Darüber hinaus, so kann angenommen werden, wird der Nationalsprache eine entscheidende Bedeutung für den gesellschaftlichen Zusammenhalt beigemessen.

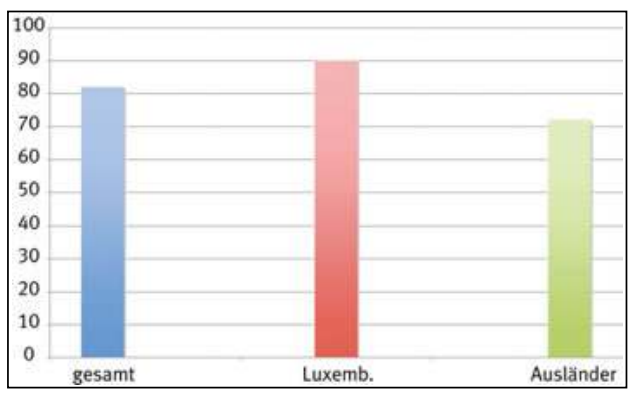

Abbildung 2: »Luxemburgisch sollte aktiv beherrscht werden.« 
Das schlägt sich in der Mehrheitsmeinung (82 \%) nieder, die die Forderung unterstützt, alle Einwohner des Großherzogtums sollten aktiv Luxemburgisch beherrschen. Differenziert nach Nationalität liegt die Zustimmung aufseiten der Luxemburger kaum überraschend am höchsten (9०\%). Aber auch die überwiegenden Mehrheiten der Ausländer (72 \%) stimmen zu, und zwar in einer Spanne von $86 \%$ (Deutsche) bis $63 \%$ (Franzosen). Differenziert nach Milieus und bezogen auf die gesamte Stichprobe zeigen sich die höchsten Zustimmungswerte beim kleinbürgerlichen Milieu (86 \%), die niedrigsten Werte dagegen beim liberal-gehobenen Milieu (71\%).

Die Forderung nach einer zumindest passiven Kompetenz des Luxemburgischen wird auch gegenüber den Grenzpendlern erhoben. Insgesamt $86 \%$ sind dieser Meinung, wobei in der Altersstaffelung die über 6o-Jährigen die höchsten Zustimmungswerte aufweisen (93\%). In der nationalen Differenzierung zeigt sich auch hier wieder der Höchstwert bei den Luxemburgern (94\%), gefolgt von den Deutschen (93\%). Am geringsten wird die Forderung an die Grenzpendler, Luxemburgisch zumindest verstehen zu können, mit jeweils 73 \% von Portugiesen und Franzosen vertreten. Hierbei ist sicherlich der unterschiedliche systemische Abstand zwischen dem Luxemburgischen und dem Deutschen einerseits sowie dem Luxemburgischen und den romanofonen Sprachen andererseits in Betracht zu ziehen.

Im Verhältnis der nationalen Zugehörigkeiten ergeben sich Übereinstimmungen insofern, als die Zustimmungsrate beim kleinbürgerlichen Milieu jeweils am höchsten ist, die des alternativen Milieus jeweils am niedrigsten. So fordert zwar immer noch eine Mehrheit der ausländischen Befragten des alternativen Milieus (57\%) passive Luxemburgisch-Kompetenzen bei den Grenzpendlern, aber immerhin $43 \%$ stimmen gegen diese Forderung.

\section{Sprachkompetenzen}

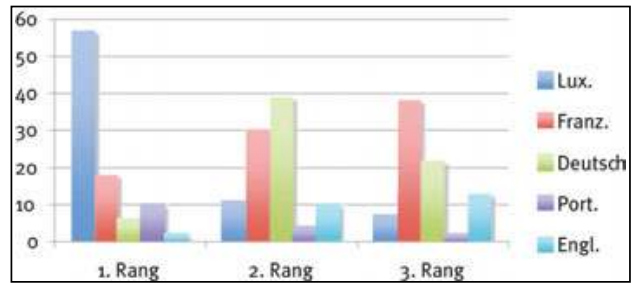

Abbildung 3: Rangfolge der am besten beherrschten Sprachen/Gesamtstichprobe

Gefragt danach, welche Sprache am besten beherrscht werde, nennt eine Mehrheit von 57 \% das Luxemburgische, mit deutlichem Abstand vor dem Französischen (18\%), dem Portugiesischen (10\%), dem Deutschen (6\%) und dem Englischen ( $2 \%)$. Auf die Frage nach der am zweitbesten beherrschten Sprache wird mit einer 
relativen Mehrheit von 39 \% das Deutsche (vor dem Französischen, 30 \%) genannt. Die Frage nach der am drittbesten beherrschten Sprache ergibt eine relative Mehrheit von 38 \% für das Französische (vor dem Deutschen 22 \%).

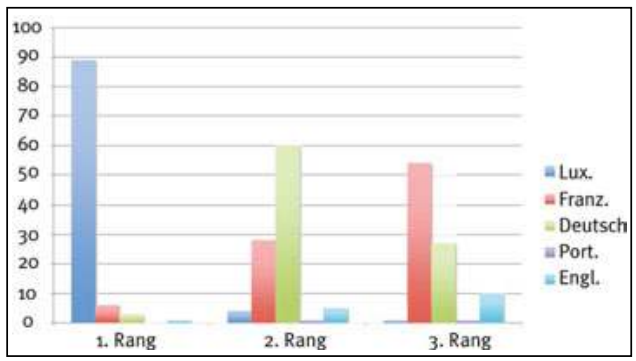

Abbildung 4: Rangfolge der am besten beherrschten Sprachen/Luxemburger

Die Unterscheidung nach nationaler Zugehörigkeit ergibt bezogen auf die Luxemburger eine deutliche Ausprofilierung des genannten Befundes. So ist für einen Anteil von 89 \% das Luxemburgische die am besten beherrschte Sprache, 60\% der Luxemburger geben zudem an, das Deutsche am zweitbesten zu beherrschen, und $54 \%$ nennen das Französische als die am drittbesten beherrschte Sprache.

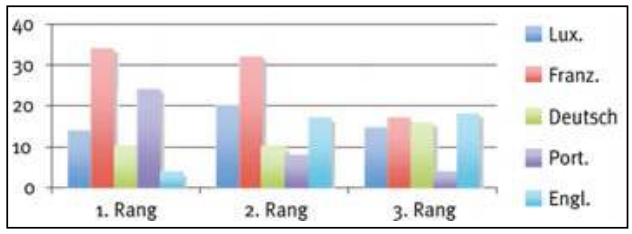

Abbildung 5: Rangfolge der am besten

beherrschten Sprachen/Ausländer

Die Sprachkompetenz der ausländischen Bevölkerung unterscheidet sich demgegenüber drastisch. Das Luxemburgische verliert hier seine dominante Rolle zugunsten des Französischen. Eine relative Mehrheit (34 \%) gibt an, das Französische am besten $\mathrm{zu}$ beherrschen, immerhin gefolgt von $24 \%$ für das Portugiesische, während das Luxemburgische hier nur einen Wert von 14 \% erreicht. Als am zweitbesten beherrschte Sprache wird ebenfalls das Französische in relativer Mehrheit (32\%) genannt, vor Luxemburgisch (20\%) und Deutsch (10\%). Als die am drittbesten beherrschte Sprache erweist sich das Englische (18\%), knapp vor dem Französischen (17\%), dem Deutschen (16\%) und Luxemburgischen (15\%).

Insgesamt ergibt sich demnach eine unterschiedlich gerichtete sprachliche Orientierung zwischen den Luxemburgern einerseits, die eine deutliche Präferenz für das Luxemburgische zeigen und für die das Deutsche mehrheitlich die am 
besten beherrschte Zweitsprache ist, und den ausländischen Einwohnern andererseits. Bei diesen zeigen sich insgesamt eine klare Präferenz für das Französische und zudem relativ hohe Werte für das Portugiesische. Ein Befund, der sich mit den demografischen Verhältnissen im Großherzogtum deckt.

\section{Muttersprache Luxemburgisch}

Der Aussage »Ich habe mehrere Muttersprachen« stimmen insgesamt $26 \% \mathrm{zu}$, aber $73 \%$ verneinen diese. Die Zustimmungsrate der Luxemburger liegt hier bei $21 \%$, die der Ausländer dagegen bei immerhin 33\%, wobei sich allerdings eine deutliche Spreizung zwischen $19 \%$ bei den Portugiesen und $44 \%$ bei den Belgiern bzw. sogar $55 \%$ bei den Italienern zeigt. Das Antwortverhalten der Luxemburger verweist auf eine deutliche (emotionale) Privilegierung des Luxemburgischen gegenüber den beiden anderen Amtssprachen Französisch und Deutsch.

\section{Sprachnützlichkeit im Alltag}

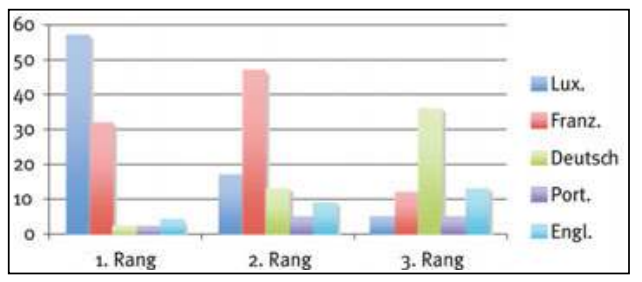

Abbildung 6: Rangfolge der nützlichsten Sprachen im Alltag

Gefragt nach der nützlichsten Sprache im Alltag, dominiert das Luxemburgische mit 57 \%. Immerhin 32 \% der Befragten geben Französisch an. Alle anderen Sprachen fallen demgegenüber weit ab. Das Französische überwiegt bei der Nennung der zweitnützlichsten Sprache (47\%), während eine relative Mehrheit von $36 \%$ das Deutsche als die drittnützlichste Sprache bezeichnet.

Nach Nationalität aufgeschlüsselt und bezogen auf die nützlichste Sprache (Rang 1) ergibt sich ein deutlicher Gegensatz. Während die Luxemburger das Luxemburgische mit $76 \%$ als die nützlichste Sprache bezeichnen, bezeichnet die (relative) Mehrheit der Ausländer (49\%) das Französische als die nützlichste Sprache. Differenziert nach Milieus ergeben sich für die Gesamtstichprobe nur beim unterprivilegierten Milieu (44\%) und dem alternativen Milieu (40 \%) Anteile von weniger als der Hälfte der Befragten, die das Luxemburgische als die nützlichste Sprache im Alltag bezeichnen. 


\section{Sympathischste Sprache}

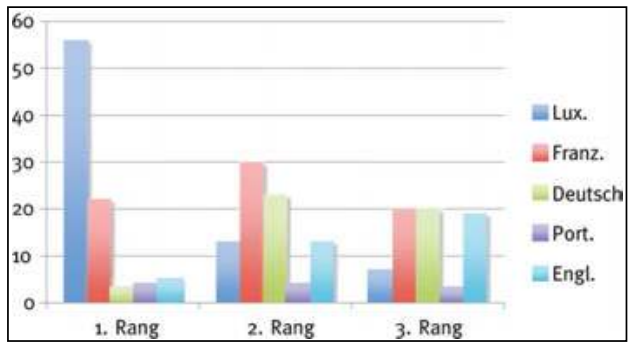

Abbildung 7: Rangfolge der sympathischsten Sprachen

Als >sympathischste Sprache « wird mit großem Abstand das Luxemburgische genannt (56\%), und zwar unabhängig vom Alter der Befragten. Deutliche Diskrepanzen in Hinblick auf die Sympathiezuweisungen ergeben sich allerdings in der Verteilung nach Nationalitäten. So bewerten die Luxemburger das Luxemburgische mit $77 \%$ als sympathischste Sprache, während die Ausländer nur zu 28 \% entsprechend votieren. Differenziert man weiter, zeigen sich allerdings auch hier wieder deutliche nationale Differenzen. Am bemerkenswertesten ist wohl, dass immerhin auch 49 \% der Deutschen das Luxemburgische als sympathischste Sprache bezeichnen. Insgesamt gibt demgegenüber allerdings eine relative Mehrheit der ausländischen Befragten (37\%) das Französische als sympathischste Sprache an.

In der Milieudifferenzierung ergeben sich bezogen auf die Gesamtstichprobe die höchsten Sympathiewerte für das Luxemburgische im statusorientierten Milieu (68\%), die niedrigsten beim unterprivilegierten Milieu (37\%).

Rang 2 der >Sympathieskala< belegt mit $30 \%$ das Französische, gefolgt von 23 \% bezogen auf das Deutsche. Bei den Luxemburgern rangiert das Französische (34 \%) mit dem Deutschen (32 \%) als zweitsympathischste Sprache annähernd gleichauf.

Bezogen auf die Grundgesamtheit und nach Milieus differenziert, rangiert das Französische mit unterschiedlichen Differenzabständen in allen Milieus vor dem Deutschen, nur beim statusorientierten Milieu ergibt sich die umgekehrte Rangfolge.

Auffällig ist hier allerdings eine deutliche Alterskorrelation. So verringern sich in der Gesamtstichprobe die Sympathiewerte des Französischen mit abnehmendem Alter drastisch. Die entsprechende Quote sinkt, angefangen von $46 \%$ bei den über 60-Jährigen über 31 \% bei den 45-59-Jährigen, 22 \% bei den 30-44-Jährigen und $23 \%$ bei den 21-29-Jährigen, bis auf nur $15 \%$ bei den 16-20-Jährigen. Demgegenüber steigen die entsprechenden Werte bezogen auf das Deutsche mit abnehmendem Alter insgesamt deutlich an, und zwar von $19 \%$ bei der ältesten auf $33 \%$ bei der jüngsten Altersgruppe. 


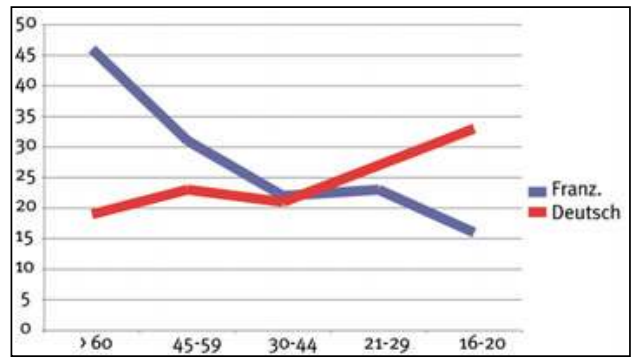

Abbildung 8: Sympathiewerte für die deutsche und französische Sprache nach Altersgruppen

Die Erklärung für dieses Phänomen ist nicht leicht. Zu fragen wäre, inwieweit es sich um ein transitorisches, also ein Durchgangs-Phänomen handelt, wonach mit zunehmendem Alter ein Rückgang der Sympathiewerte bezogen auf das Deutsche und eine entsprechende Steigerung für das Französische anzunehmen wäre. Wahrscheinlicher ist vermutlich, dass hier ein grundsätzlicher Wandel der Haltungen gegenüber den betrachteten Sprachen ablesbar wird. Anzunehmen ist auch, dass die Stigmatisierung der deutschen Sprache als Reaktion auf die Okkupation Luxemburgs durch Nazi-Deutschland im Zweiten Weltkrieg für die jüngeren Generationen immer weniger gilt.

Bezogen auf die Angaben zur drittsympathischste Sprache sind die Anteile für das Deutsche (20\%), das Französische (20\%) und das Englische (19\%) nahezu identisch.

\section{Sprachwichtigkeit in der Zukunft}

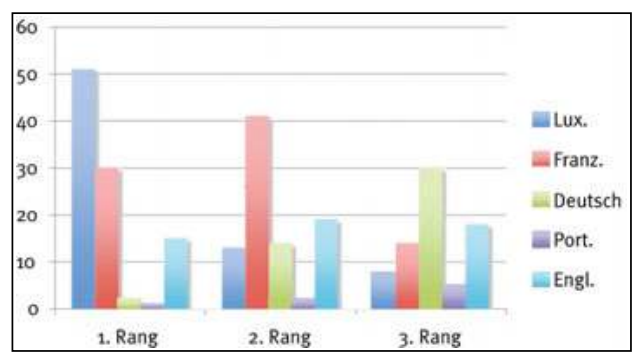

Abbildung 9: Wichtigste Sprache in der Zukunft

Als wichtigste Sprache in der Zukunft gilt einer Mehrheit von $51 \%$ das Luxemburgische. Etwa ein Drittel der Befragten (30\%) bezeichnet das Französische als zukünftig wichtigste Sprache und $15 \%$ das Englische. Höchstwerte zugunsten des Luxemburgischen finden sich im traditionsorientierten Milieu ( $\left.\sigma_{1} \%\right)$, dem alter- 
nativen Milieu und dem konservativ-gehobenen Milieu (jeweils $58 \%$ ), geringste Werte dagegen beim statusorientierten Milieu (37\%).

Zweitwichtigste Sprache in der Zukunft ist mit einer deutlichen relativen Mehrheit von 41 \% das Französische, gefolgt vom Englischen (19\%). Als drittwichtigste Sprache für die Zukunft wird mit $30 \%$ das Deutsche benannt. Auch hier ergibt sich wieder ein relativ hoher Wert für das Englische (18\%).

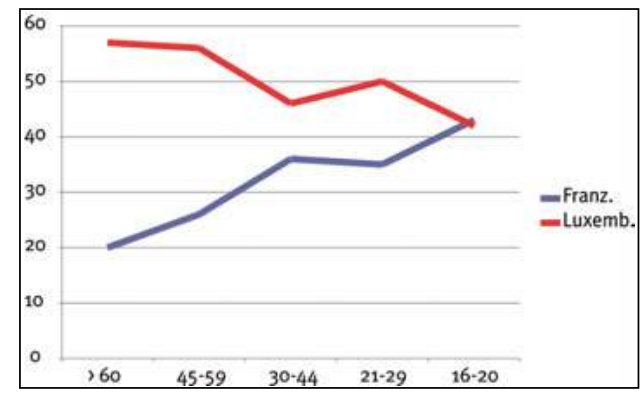

Abbildung 10: Wichtigste Sprachen in der Zukunft nach Alter

In der Altersdifferenzierung nimmt die (prognostizierte) Wichtigkeit des Luxemburgischen mit absteigendem Alter deutlich ab, die des Französischen dagegen stark zu. So sinken die entsprechenden Werte bezogen auf das Luxemburgische von $57 \%$ bei den über 6o-Jährigen auf $42 \%$ bei den 16-20-Jährigen, wohingegen die Werte bezogen auf das Französische von $20 \%$ auf $43 \%$ ansteigen.

Vor allem die Luxemburger selbst sehen das Luxemburgische mit $63 \%$ als die zukünftig wichtigste Sprache an, während die relative Mehrheit der Ausländer (42 \%) das Französische an erster Stelle sieht. Allerdings erweist sich die Teilgesamtheit der Nicht-Luxemburger auch hier wieder als sehr heterogen. Höchstwerte bezogen auf das Französische zeigen sich bei den Portugiesen (50\%) und Franzosen (49\%), während die relative Mehrheit der Deutschen (48\%) und die Gruppe der nicht weiter spezifizierten >anderen Ausländer $(46 \%)$ die zukünftige Wichtigkeit des Luxemburgischen behauptet. 


\section{Beliebteste Fernsehsprache}

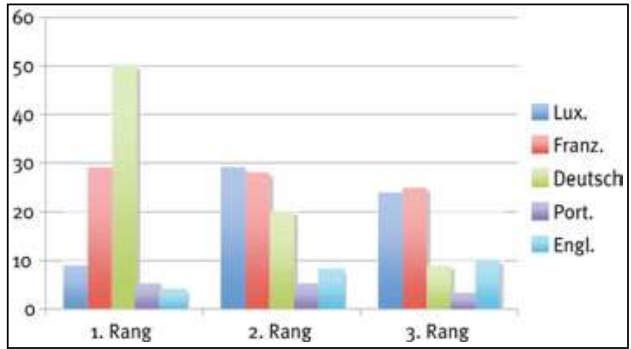

Abbildung 11: Beliebteste Fernsehsprache

Mit deutlichem Abstand fungiert das Deutsche als primäre Sprache beim Fernsehkonsum: 50 \% (deutsch) gegenüber $29 \%$ (französisch) und 9 \% (luxemburgisch). Getragen wird dieses Verhältnis vor allem durch den Fernsehkonsum der Luxemburger ( $69 \%$ deutsch, $14 \%$ luxemburgisch, $13 \%$ französisch), wohingegen die ausländischen Einwohner insgesamt eindeutig das französischsprachige Fernsehen präferieren (50\%). Eine leicht erklärliche Ausnahme sind hierbei die Deutschen, die deutschsprachiges Fernsehen zu 91 \% bevorzugen, sowie die Angehörigen der nicht weiter differenzierten >anderen Nationalitäten<, für die Deutsch (37\%) vor Französisch (25\%) und Englisch (16\%) rangiert.

Deutsch ist die erste Fernsehsprache in allen Milieus, allerdings mit unterschiedlich starker Ausprägung. Höchstwerte ergeben sich hier für das statusorientierte Milieu (66\%), gefolgt vom traditionsorientierten Milieu (55\%) und dem kleinbürgerlichen Milieu (55\%), niedrigste Werte ergeben sich für das unterprivilegierte Milieu (38\%) und das alternative Milieu (36\%).

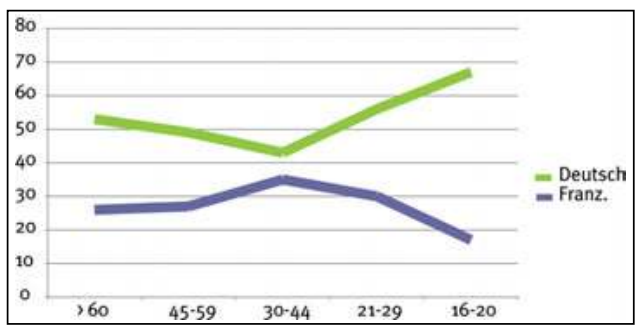

Abbildung 12: Beliebteste Fernsehsprache nach Alter

Die Grafik verdeutlicht, dass der präferierte Konsum des deutschsprachigen Fernsehens sich in den jüngeren Generationen offensichtlich verstärkt. Bei den 16-20-Jährigen finden sich die höchsten Werte bezogen auf das deutschsprachige Fernsehen $(67 \%)$ und die niedrigsten für das französischsprachige (17\%). 
In der Rangfolge schließt sich das Luxemburgische (29 \%) knapp vor dem Französischen (28\%) als zweithäufigste >Fernsehsprache< an. Die dritthäufigste Sprache beim Fernsehkonsum ist mit einem relativen Höchstwert von 25 \% das Französische, knapp vor dem Luxemburgischen (24\%).

\section{Schulsituation}

Bezogen auf die Frage, ob der Sprachunterricht in der Schule die luxemburgischen Schüler überfordere, stimmen $27 \% \mathrm{zu}$, aber eine deutliche Mehrheit von etwa zwei Dritteln der Befragten (65\%) sieht offenbar keine Überforderung - mit einem Höchstwert bei den Befragten mit Luxemburger Nationalität. Allerdings korrelieren die Aussagen mit dem erzielten Bildungsabschluss. So zeigt sich ein signifikanter Unterschied zwischen den Befragten mit Universitätsabschluss, die zu $70 \%$ keine Überforderung sehen, gegenüber nur $51 \%$ bei denjenigen mit Abschlüssen auf Grundschulniveau.

Eine in der Bildungsdiskussion häufig gestellte Frage ist die nach den Bildungschancen portugiesischer Schüler, gerade vor dem Hintergrund der komplexen und anspruchsvollen Sprachsituation an Luxemburger Schulen und der Primäralphabetisierung mittels der deutschen Sprache. Der portugiesische Bevölkerungsanteil in Luxemburg ist aufgrund der Arbeitsimmigration der letzten Jahrzehnte relativ hoch und übertrifft den der anderen ausländischen Bevölkerungsgruppen bei weitem. Bezogen auf die Frage, ob der Sprachunterricht in luxemburgischen Schulen die portugiesischen Schüler überfordere, zeigt sich ein differenziertes Bild. Eine relative Mehrheit von 49 \% verneint die Frage, allerdings stehen dem 41 \% gegenüber, die dem zustimmen. Die Binnendifferenzierung nach Altersstufen zeigt, dass vor allem die jüngere Altersgruppe (16-20 Jahre) die Schulsituation als am wenigsten überfordernd bewertet. Die Portugiesen selber sind in ihrem Antwortverhalten (ebenfalls) ambivalent: So sehen 48 \% der Befragten keine Überforderung, aber $50 \%$ sind der Ansicht, dass eine Überforderung portugiesischer Kinder bestehe.

\section{Staatliche Intervention}

Staatliche Interventionen in Bezug auf den Sprachgebrauch werden von knapp der Hälfte der Befragten (48 \%) zwar gefordert, zu etwa gleichen Prozentsätzen (46\%) aber abgelehnt - eine deutliche Spaltung in zwei >Lager . Höchste Zustimmungswerte für eine Forderung nach staatlicher Sprachreglementierung finden sich insbesondere bei den Bewohnern des Luxemburger Nordens (53\%), bei den 16-20-Jährigen (57\%) sowie bei mithelfenden Familienmitgliedern (72 \%), beim unterprivilegierten Milieu (57\%), dem kleinbürgerlichen Milieu und dem traditionsorientierten Milieu (jeweils 53 \%). In der nationalen Betrachtung wird die Forderung nach staatlicher Sprachsteuerung vergleichsweise stark von den Luxemburgern erhoben (51\%), nur noch übertroffen von den Italienern (59\%). Außerdem zeigt sich eine Korrelation mit den Bildungsabschlüssen: Je höher diese 
sind, umso geringer ist die Forderung nach staatlichen Eingriffen in den Sprachgebrauch.

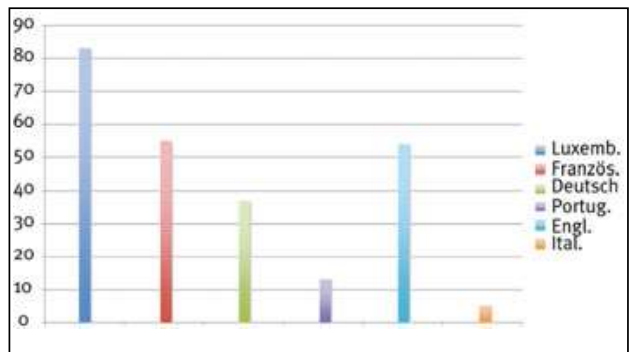

Abbildung 13: Sprachen, die vom Staat gefördert werden sollten

Bezogen auf die Frage, welche Sprachen vom Staat stärker gefördert werden sollten, erweist sich ein weiteres Mal die starke Loyalität gegenüber dem Luxemburgischen. So fordern insgesamt $83 \%$ aus der Gruppe derjenigen, die eine staatliche Förderung wünscht, diese zugunsten der Nationalsprache. Vor allem die Luxemburger selbst fordern dies zu $90 \%$, knapp gefolgt von den Deutschen (89\%), während hier die Belgier mit $59 \%$ die relativ geringste Quote aufweisen. In der Milieudifferenzierung sind es vor allem das traditionsorientierte Milieu (92\%), das aufstiegsorientierte Milieu und das liberal-gehobene Milieu (jeweils $88 \%$ ), die die Förderung des Luxemburgischen befürworten.

Etwa gleichauf in Bezug auf die Frage, welche Sprachen durch den Staat gefördert werden sollten, liegen das Französische (55 \%) und das Englische (54\%), deutlich vor dem Deutschen (37\%) und nochmals mit großem Abstand vor dem Portugiesischen (13\%) und Italienischen (5\%).

\section{Nachbarländer: Besuchshäufigkeit}

Der (regelmäßige) Besuch der Nachbarländer ist für die meisten Einwohner Luxemburgs offenbar gängige Praxis. Etwa $70 \%$ besuchen Frankreich, Belgien und Deutschland mindestens mehrmals pro Jahr. Nach Nationalität differenziert, erweist sich für die Luxemburger Deutschland als das meistbesuchte Nachbarland. Nur $17 \%$ der Luxemburger geben an, Deutschland »nie oder fast nie « zu besuchen. Dem stehen entsprechende Werte von $31 \%$ bezogen auf Frankreich und $32 \%$ bezogen auf Belgien gegenüber. Nicht nur die Zahl der luxemburgischen Besucher ist bezogen auf Deutschland am höchsten, sondern auch die Häufigkeit der Besuche. 


\section{Nachbarländer: ‘Wohlfühlcharakterı}

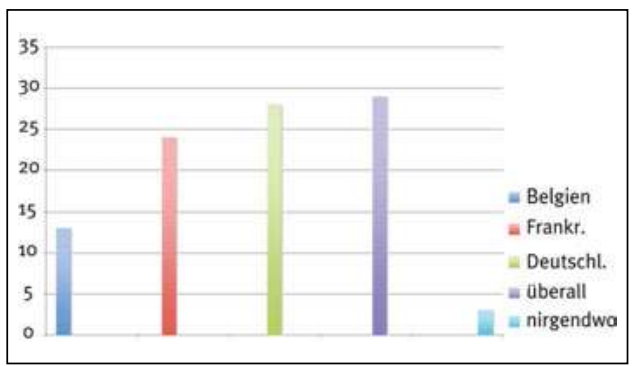

Abbildung 14: »In welchem Land fühlen Sie sich am wohlsten?«

Eine gewisse Präferenz für das Nachbarland Deutschland zeigt sich auch in Bezug auf die Frage »In welchem Nachbarland fühlen Sie sich am wohlsten?« Eine relative Mehrheit von 29 \% gibt hier zwar »überall« an, in der Länderverteilung liegt hier allerdings Deutschland (28 \%) knapp vor Frankreich (24\%) und Belgien $(13 \%)$.

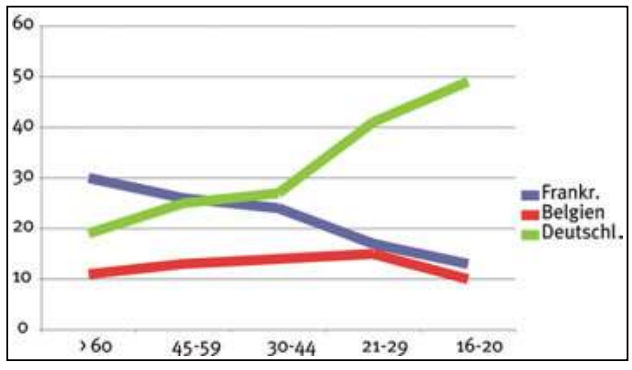

Abbildung 15: > Wohlfühlcharakter< nach Alter

Klare Veränderungen zeigen sich in der Altersverteilung. So steigen die Werte des Sich-Wohlfühlens kontinuierlich und deutlich mit dem abnehmenden Alter der Befragten. Gerade bei den jüngsten Altersgruppen (bis 29 Jahre) ist die >Favoritenrolle Deutschlands klar ausgeprägt. Bei den 16-20-Jährigen ergeben sich Werte von 49 \% für Deutschland, $13 \%$ für Frankreich und $10 \%$ für Belgien. Allerdings zeigt sich bei dieser Frage gleichzeitig eine deutliche Generationenverschiebung insofern, als bei den Älteren ( $\mathrm{ab} 45$ Jahre) Frankreich den höchsten Zuspruch erfährt.

Nach Nationalität differenziert, erweist sich für die Luxemburger Deutschland ebenfalls mit deutlichem Abstand als das Nachbarland mit dem höchsten >Wohlfühl-Wert< (37\% vs. $21 \%$ bezogen auf Frankreich und $9 \%$ bezogen auf Belgien). Portugiesische, französische und italienische Einwohner fühlen sich demgegenüber in Frankreich am wohlsten. Ein Zusammenhang zwischen Nationalität und Sprache ist im gegebenen Fragezusammenhang evident. 


\section{Resümee}

Vor dem Hintergrund der erhobenen Daten erweist sich die Luxemburger Dreisprachigkeit als insgesamt stabil und positiv bewertet. Insbesondere wird ihr ein deutlicher kommunikativer Mehrwert zugesprochen. Dabei ist die Position des Luxemburgischen deutlich exponiert. Luxemburgisch ist (für die Luxemburger) >die< Muttersprache, mit der sich ein hoher Grad von Loyalität verbindet. Man wird also kaum fehlgehen, dem Luxemburgischen eine stark identitätsstiftende Funktion beizumessen.

Der Rang des Französischen als vorherrschende Sprache des öffentlichen Raumes und in der formellen Kommunikation ist unbestritten. Zugleich erweist sich das Französische als die klar präferierte Sprache der Mehrheit der ausländischen Einwohner Luxemburgs. Die emotionale Bindung wie auch die rationale Beziehung zum Französischen rangiert bei den Luxemburgern zwar deutlich hinter dem Luxemburgischen, gleichzeitig aber vor der deutschen Sprache, - wenngleich sich die Relationen gegenwärtig (in der jüngeren Generation) zu verschieben scheinen.

Das Deutsche hat für die Luxemburger offensichtlich einen hohen Gebrauchswert in eher informellen Lebenszusammenhängen. Deutschland ist das Nachbarland, das am stärksten besucht wird, in dem man sich >am wohlsten< fühlt; das deutschsprachige Fernsehen prägt zudem den Medienkonsum. Besonders stark ausgeprägt zeigt sich dies bei den jüngeren Luxemburgern. Die Bewertung des Deutschen in der jüngeren Generation fällt zudem deutlich positiver aus als in der älteren Generation. Zugleich ist das Deutsche nach dem Luxemburgischen die Sprache, die am besten beherrscht wird. Die emotionale Bindung gegenüber dem Deutschen ist dagegen allenfalls schwach ausgebildet. Auch die rationale Bewertung fällt gegenüber dem faktischen Gebrauchswert stark ab.

\subsection{Kontroversen zUr Sprachensituation in Leserbriefen}

\section{Vorbemerkungen}

Gegenstand der vorliegenden Analyse sind Leserbriefe, die in unterschiedlichen luxemburgischen Zeitungen zu den Themenfeldern »Sprache« und »Mehrsprachigkeit« erschienen sind. Auf Grundlage dieses Diskursmaterials wurde versucht, mögliche Rückschlüsse auf angeeignete Identitäten in Bezug auf Sprache zu ziehen. Der Leitgedanke war, dass Sprachen ein enormes identitätsstiftendes Potential besitzen, gerade in einem so multikulturellen und multilingualen Raum wie Luxemburg. Selbstverständlich stand dabei das Luxemburgische im Zentrum des Interesses, da dieses als herausragendes Distinktionsmerkmal gegenüber den unmittelbaren Nachbarn Deutschland und Frankreich im Mittelpunkt steht. 


\section{Materialauswahl}

Zunächst wurden $16_{4}$ rezente Artikel und $6_{3}$ Leserbriefe, die sich mit Sprache beschäftigen, aus 19 verschiedenen luxemburgischen Zeitungen und Zeitschriften über einen Zeitraum von knapp über einem Jahr gesammelt und katalogisiert. Ziel der Studie ist es, angeeignete Identitäten der luxemburgischen Bevölkerung zu ermitteln, und daher wurde entschieden, sich auf die Leserbriefe zu konzentrieren. Durch deren Analyse erhält man ein schlaglichtartiges Bild der Positionen und Meinungen zu einzelnen Themen. Menschen, die Leserbriefe schreiben, sind keine professionellen Journalisten und daher erscheinen die Stimmen raus der Mitte der Bevölkerung besonders unmittelbar und sprechen Themen an, die einzelnen Personen am Herzen liegen.

Aus dieser Gesamtheit wurden insgesamt 54 Leserbriefe, die im Zeitraum von März 2008 bis April 2009 in den Zeitungen »Tageblatt«, »Luxemburger Wort«, »Journal«, »La Voix«, »Le jeudi«, »Lëtzebuerger Journal«, »d'Land« und »Kulturissimo « erschienen sind, ausgewertet. Dabei wurden nicht nur klassische Leserbriefe berücksichtigt, in welchen Leser eine kurze Stellungnahme zu einem zuvor erschienenen Artikel abgeben, sondern auch selbstinitiierte Artikel oder Kommentare von Leserinnen und Lesern, die den Rahmen eines gewöhnlichen Leserbriefes sprengen. In diesem Zusammenhang ist zu erläutern, dass es für die luxemburgische Medienlandschaft charakteristisch zu sein scheint, dass es möglich ist, seine Meinung oder Expertise einem größeren Lesepublikum relativ problemlos mitzuteilen. Solche Artikel wurden deshalb auch berücksichtigt, da sie ausführlichere Rückschlüsse auf die angeeigneten Identitäten zum Thema Sprache erlauben. ${ }^{3}$

Alle offiziellen Sprachen Luxemburgs - Luxemburgisch, Deutsch und Französisch - finden sich auch in den Leserbriefen wieder, wobei auffälligerweise z.T. dieselben Schreiber/-innen in verschiedenen Zeitungen oder zu verschiedenen Themen unterschiedliche Sprachen verwenden. Weiterhin bemerkenswert und eventuell eine luxemburgische Besonderheit ist, dass einige Leserbriefe in mehreren Zeitungen publiziert wurden und es daher zu Mehrfachzählungen kommt (in der unten angeführten Tabelle wird in Klammern die Anzahl der Leserbriefe angegeben, die auch in einer anderen Zeitung erschienen sind). Zu Erwähnen ist außerdem, dass nur drei Frauen, dahingegen aber 26 Männer Leserbriefe zum Thema Sprache(n) verfasst haben.

3 | Insgesamt sind im betrachteten Zeitraum mehr als die analysierten 54 Leserbriefe publiziert worden, die sich im weiteren Sinne mit den Themen Sprache und Mehrsprachigkeit beschäftigen. Aus Gründen der Objektivität wurden verschiedene Leserbriefe jedoch nicht berücksichtigt. 


\begin{tabular}{|c|c|c|c|c|}
\hline \multirow[b]{2}{*}{$\begin{array}{l}\text { Berücksichtige } \\
\text { Printmedien }\end{array}$} & \multicolumn{4}{|c|}{ Sprache des Artikels } \\
\hline & luxemburgisch & deutsch & französisch & gesamt \\
\hline Luxemburger Wort & $19(4)$ & 5 & $4(1)$ & $28(5)$ \\
\hline $\begin{array}{l}\text { Lëtzebuerger } \\
\text { Journal }\end{array}$ & $8(4)$ & 3 & 1 & $12(4)$ \\
\hline Tageblatt & $3(2)$ & $7(1)$ & 1 & $11(3)$ \\
\hline La Voix & & & 2 & 2 \\
\hline Le Jeudi & & & 1 & 1 \\
\hline d'Land & & 2 & & 2 \\
\hline Journal & $1(1)$ & $2(1)$ & $3(1)$ & $6(3)$ \\
\hline Kulturissimo & & 1 & & 1 \\
\hline Gesamt & 31 & 20 & 12 & 63 \\
\hline
\end{tabular}

In Klammern: Anzahl der Leserbriefe, die auch in anderen Zeitungen erschienen

\section{Tabelle 1: Sprache der Leserbriefe in den jeweiligen Printmedien}

Auch wenn über die Leserbriefanalyse eine Annäherung an das gesellschaftliche Sprachenbewusstsein möglich ist, muss man dennoch vorsichtig sein und sich der Tatsache bewusst bleiben, dass selbst eine umfassende Auswertung von Leserbriefen wie die vorliegende nur einen Ausschnitt der gesellschaftlichen Interessen und Meinungen wiedergibt. Gesamtgesellschaftlich gesehen schreiben nur die wenigsten Menschen Leserbriefe. Die Forschung darüber ist bislang rudimentär und hat bisher keine genauen Profile der Autoren/-innen erarbeitet. Es kann jedoch unterschieden werden zwischen der spontanen Meinungsäußerung von Laien zu bestimmten Themen, die von besonderem, oft persönlichem Interesse für einzelne Menschen sind, und der Zusendung von Leserbriefen oder Artikeln von Experten/innen auf verschiedenen Gebieten. Beispielsweise wenn sich eine luxemburgische Leserbriefschreiberin zu Wort meldet und von ihren guten Erfahrungen mit der Mehrsprachigkeit beim Einkauf in Trier berichtet ${ }^{4}$ oder etwa, wenn Wissenschaftler eine Stellungnahme zum Status des Luxemburgischen abgeben. Darüber hinaus sind es oft Leute, die regelmäßig Leserbriefe schreiben. Hier gilt es zu unterscheiden zwischen jenen, die meinen, eine Mehrheitsmeinung zu vertreten, und solchen, die ganz klar extremere Positionen einnehmen. Außerdem gelangen bei weitem nicht alle an die Redaktionen gesandten Briefe auch in die Zeitungen, und wenn doch, können sie geändert oder gekürzt erscheinen. (Vgl. Drewnowska-Vargáné 2001: 2)

4 | Vgl. Bauer, Léonie: "In welcher Sprache möchten Sie beraten werden? ", in: Tageblatt 15./16.03.2008, S. 72. 


\section{Textsorte "Leserbrief"}

Aufgrund des Umstands, dass Leserbriefe als Textsorte noch nicht umfassend erforscht sind, gibt es auch keine kanonische Definition für >Leserbriefe <. (Drewnowska-Vargáné 2001: 2) Dennoch gibt es einen gewissen Konsens darüber, was als textsortenspezifische Charakteristik angesehen werden kann. Besonders hervorzuheben ist die Intertextualität (vgl. Piirainen/Yli-Kojola 1983: 111f.), da Leserbriefe sich i.d.R. auf bereits erschienene Artikel oder aber auf andere Leserbriefe beziehen, die sie ihrerseits kommentieren, kritisieren oder ergänzen. Des Weiteren typisch ist eine spezifische >Emittent-Rezipient-Konstellation $<$. Da Leserbriefe im Vergleich zu herkömmlichen Briefen öffentlich sind, haben sie einen sehr breiten Rezipientenkreis. Dieser ist nicht mit dem Adressatenkreis identisch und dem Autor meistens nicht persönlich bekannt. (Vgl. Drewnowska-Vargáné 2001: 2)

Im hier bearbeiteten Material lassen sich feste Komponenten finden, die Sandig als konstitutiv für Leserbriefe konstatiert: thematischer Hinweis als Hauptüberschrift, Verweis auf einen vorangegangenen Zeitungsartikel als Zweitüberschrift, der Text selbst, Unterschrift: Name und Wohnort des Schreibers. (Vgl. Sandig 1986: 185) Durch diese Mittel wird der Bezug zu vorangegangenen Texten hergestellt, wodurch »indirekte Dialoge in schriftlicher Form« (vgl. Bucher 1986: 147-160) entstehen. Außerdem wird ein »Kohärenzmanagement« (Bucher 1989: 290f.) betrieben, nämlich wenn der Autor angibt:

1. Womit sein Brief zusammenhängt (durch das Zitieren der betreffenden Textstelle oder Angabe des sprachlichen Ausdrucks, auf den er Bezug nimmt); 2. Wie sein Brief gemeint ist (welches Thema behandelt wird und welche Ansichten er verfolgt); 3. "eine Handlung ausführt, die als regelhafte Anschlusshandlung auf den vorausgegangenen Beitrag gilt", z.B. wenn er den Beitrag lobt. (Bucher 1986: 149, zitiert nach: Drewnowska-Vargáné 2001: 3)

Die neuere Leserbriefforschung orientiert sich verstärkt an textlinguistisch ausgerichteten kontrastiven Untersuchungen der Leserbriefe aus unterschiedlichen Kommunikationskulturen. (Vgl. Drewnowska-Vargáné 2001: 3) In diesem Sinne könnte die vorliegende Analyse einen ersten Schritt zur Erforschung der luxemburgischen Kommunikationskultur darstellen. Aufgrund der spezifischen Gegebenheiten, etwa einer zahlenmäßig überschaubaren Leser- und Schreiberschaft, scheint es, als wenn die Leserbriefe hier im Land z.T. persönlicher als beispielsweise in Deutschland sind. So kommt es durchaus vor, dass jemand seinen Leserbrief, mit dem er auf einen anderen reagiert, mit den Worten abschließt: »Merci fir d'Dokumentatioun vun Ärem Respekt vru menger Aarbecht fir eis Sprooch; ech hoffe just, datt deen esou éierlech ass wéi meng Bewonnerung vun Ärer Leeschtung als Lëtzebuerger Historiker. « ${ }^{5}$ Auch der Wahl der Sprache kommt in Luxemburg eine

5 | Roth, Lex: "Keng Hetzcampagne, Här Prof. Dr. Michel Pauly", in: Lëtzebuerger Journal, 31.03.2009, S. 9. ("Danke für die Bekundung ihres Respekts für meine Arbeit für unsere 
besondere Bedeutung zu. Manchmal sind die Briefe auf Deutsch verfasst, vermutlich, um sicherzustellen, dass der Adressat die Argumente auch versteht, oder weil die Diskussion in derselben Sprache weitergeführt wird. Es gibt aber auch Leserbriefe, die bewusst und/oder aus grundsätzlichen Erwägungen heraus auf luxemburgisch verfasst sind. So beginnt beispielsweise ein Leserbrief mit der Passage: »Meng Identitéit als iwerzeechter Lëtzebuerger verbidd mer des puer Wierder op Däitsch ze schreiwen. Mäi Papp a meng Grousspappen genge mer et net verzeien. ${ }^{6}$ In einem Postscriptum wird dann auf deutsch eingeräumt, »es wird sich wohl jemand finden, der Herr[n] Müntefering dies zu übersetzen weiß«.

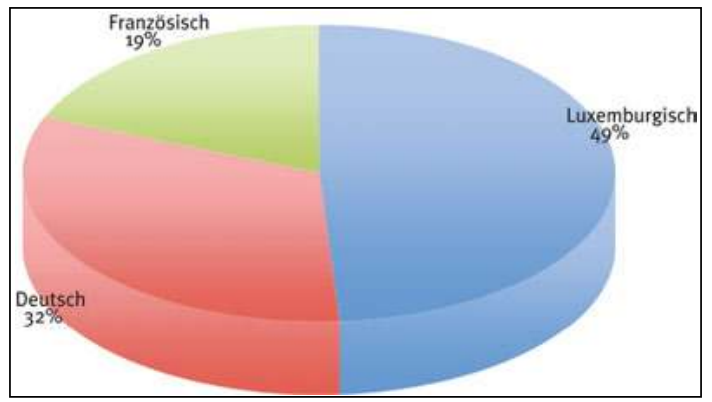

Abbildung 16: Sprachverteilung der Leserbriefe

\section{Themen}

Um auf die Zusammenhänge von Sprache und Identität schließen zu können, wurden nur solche Leserbriefe ausgewählt, die sich mit ebensolchen Themen beschäftigen. Zum einen sind dies einzelne Briefe, die sich auf einen Artikel in einer älteren Ausgabe der Zeitung beziehen, zum anderen aber auch ganze Serien von Briefen, die auf ein bestimmtes Thema abzielen. Dies kann entweder ein neues Gesetz betreffen, einen Gesetzesvorschlag, aktuell diskutierte Themen oder aber einen vorangegangenen Leserbrief, der kommentiert oder kritisiert wird. Daran schließt sich häufig eine Replik an, die wiederum oft beantwortet wird, woraus ganze Argumentationsketten oder sogar regelrechte >Fehden< entstehen können.

Die wichtigsten Themengebiete, die sich mit der Sprache beschäftigen, sind Integration, Sprache und Identität, Luxemburgisch in der Verfassung, Sprachschu-

Sprache; ich hoffe nur, dass dieser genauso ehrlich gemeint ist wie meine Bewunderung ihrer Leistung als Luxemburger Historiker.")

6 | Lenz, Guy: "Nët esou, Här Müntefering", in: Lëtzebuerger Journal, 21.03.2009, S. 7 und in: Luxemburger Wort, 21.03.2009, S. 21. ("Meine Identität als überzeugter Luxemburger verbietet es mir, diese paar Wörter auf Deutsch zu schreiben. Mein Vater und mein Großvater würden es mir nicht verzeihen.") 
len, ein folgenreicher Versprecher im Radio, sprachliches Selbstbewusstsein, Aufwertung des Luxemburgischen oder Bedrohung der Sprache.

\section{Argumentationsstränge und Konfliktlinien}

Allgemein kann man vier Argumentationslinien in den Leserbriefdebatten zum Thema Sprache in Luxemburg feststellen: Es gibt a) eine pro-luxemburgische, b) eine deutsch-kritische oder c) französisch-kritische und d) eine pro-multilinguale Position. Auf diese z.T. sehr drastisch klingenden Kategorisierungen soll im Folgenden durch genaue Beschreibung und illustrierende Beispiele näher eingegangen werden.

\section{a) pro-luxemburgisch}

Dem Luxemburgischen wird in nahezu allen Leserbriefen ein hoher Stellenwert beigemessen. Zum einen als identitätsstiftendes Merkmal, etwa wenn ganz selbstverständlich konstatiert wird, »[e]is Sprooch ass eis Identitéit«7, oder wenn gefordert wird, mehr landeskundliche Themen in luxemburgischer Sprache in Schulen zu unterrichten:

Ich hatte in letzter Zeit verschiedentlich die Gelegenheit, mich mit Primärschülern der letzten Klassen zu unterhalten. Dabei musste ich feststellen, dass kein einziger den Text geschweige denn die Melodie unserer Nationalhymne kannte. Von andern schönen luxemburgischen Liedern ganz zu schweigen. [...] Was soll in der "Chamber" das große Getue um unsere nationale Identität, wenn nicht an der Basis begonnen wird diese zu lehren? Und gibt es nicht viele luxemburgische Lieder, die einen "Ausflug" in die luxemburgische Geschichte, Geographie und Botanik ermöglichen? ${ }^{8}$

Da manche Leserbriefschreiber den Untergang ihrer Sprache befürchten, sollte Luxemburgisch natürlich auch auf dem Lehrplan stehen: »Ich bin 77 Jahre alt und verstehe immer noch nicht, warum in unseren Schulen unsere Sprache nicht gelehrt wird. Denn wenn es so weitergeht, geht unsere Sprache verloren, und das wäre sehr schade.«9 Zum anderen wird das Luxemburgische als Integrationssprache angesehen, die den vielen Ausländern im Land im Alltag nützlich wäre. So schlagen Schüler eines Luxemburgisch-Kurses vor: »Naturellement, si un étranger décide de rester au Luxembourg, la langue luxembourgeoise sera un facteur incontournable qui lui permettra d'atteindre son but: celui de son intégration socio-professionelle. ${ }^{10}$ Und die Communauté Vie Chrétienne au Luxembourg fin-

7 | Weirich, Jos: "Eis Sprooch ass eis Identitéit", in: Luxemburger Wort, 09.08.2008, S. 12. ("Unsere Sprache ist unsere Identität.")

8 | J.G.: "Luxemburgisch an der Basis lehren ", in: Lëtzebuerger Journal, 23.10.2008, S. 6.

9 | L.V.E.: "Zur luxemburgischen Sprache", in: Tageblatt, 13.02.2009, S. 65.

10 | Leserbrief der Schüler eines Luxemburgisch-Kurses: „Vivre dans une société multicul- 
det: »Nous ne nions pas le fait que le luxembourgeois reste un facteur important d'integration au Grand-Duché. ${ }^{11}$ Luxemburgisch wird also im positiven Sinne als Integrationssprache angesehen, es kann aber auch zur Bedingung gemacht werden, wenn etwa gefordert wird, »dass man, dort wo man sein Geld verdient, sich ein bisschen Mühe geben sollte, die Sprache zu erlernen $\aleph^{12}$. Besonders nachdrücklich wird diese Forderung vertreten, wenn es um den Erwerb der luxemburgischen Staatsbürgerschaft geht: »D'Sproochefuerderunge fir Lëtzebuerger ze ginn, hu fir jiddereen ze gëllen, egal wéi laang een hei geliewt huet $[. ..] . \ll^{13}$

Aufgrund der allgemein hohen Zustimmung zum Luxemburgischen ist es nicht verwunderlich, dass der Vorschlag, Luxemburgisch in der Verfassung zu verankern, ${ }^{14}$ in den meisten Leserbriefen positiv aufgenommen wird: »Elo ass jo viru kuerzem d'Proposition op den Dësch komm, eist Lëtzebuergesch als Nationalsprooch an der Verfassung ze verankeren - eng gudd Iddi, wéi mir schéngt. «5

\section{b) deutsch-kritisch}

Die Akzeptanz des Luxemburgischen ist demnach enorm hoch und es wird häufig gefordert, sich stärker für die eigene Sprache einzusetzen. Diese Stärkung des Luxemburgischen geschieht an manchen Stellen auf Kosten der anderen im Land gesprochenen Sprachen, z.B. des Deutschen. Dies spiegelt sich in sprachpflegerischen Forderungen wider, wie etwa keine Wörter unnötig aus anderen Sprachen zu übernehmen, sondern vorhandene luxemburgische Wörter zu verwenden:

Also missten déi Leit, déi am Radio poteren (inklusiv déi honorabel Politiker a Gewerkschaftler) emol hir Mammesprooch zerguttstert léieren. Innerhalb, ausserhalb, schwul, schwanger, fënnt statt ab ..., im nachhinein, nach wie vor, zumindest, zahlreich, am gesetzleche Rahmen, mindestensı sinn nëmmen e puer Beispiller. Dat ass kee Lëtzebuergescht, dat sinn op Däitsch geduechten an iwwerem braddelen op lëtzebuergesch iwwersate Sätz,

turelle au quotidien“, in: Le Jeudi, 10.07.2008, S. 44. ("Natürlich, wenn ein Ausländer beschließt, in Luxemburg zu bleiben, ist die luxemburgische Sprache ein unumgänglicher Faktor, um sein Ziel zu erreichen, nämlich die gesellschaftliche und berufliche Integration.")

11 | Communauté Vie Chrétienne au Luxembourg: "Lettre ouverte aux députés", in: La Voix, 24.052008, S. 48. ("Wir verneinen nicht, dass das Luxemburgische ein wichtiger Faktor für die Integration im Großherzogtum ist.")

12 | L.V.E. (Düdelingen): "Zur luxemburgischen Sprache", in: Tageblatt 13.02.2009, S. 65.

13 | Watgen, Fernand: "Lëtzebuerger ouni Lëtzebuergesch ...", in: Luxemburger Wort, 21.05.2008, S. 18. ("Die Sprachenanforderungen, um Luxemburger zu werden, haben für jeden zu gelten, egal, wie lange einer hier gelebt hat.")

14 | Vgl. Benoit, Jos und Jos Weirich: "Eis Sprooch ass eis Identitßeit, duerfir eis Sprooch an d'Constitution “, in: Tageblatt, 25./26.07.2008, S. 56.

15 | lucy lux : "Lëtzebuergesch, franséisch, däitsch oder wat?", in: Journal, 06.02.2009, S. 6. ("Nun ist vor kurzem der Vorschlag auf den Tisch gekommen, unser Luxemburgisch als Nationalsprache in der Verfassung zu verankern - eine gute Idee, wie mir scheint.") 
déi do erauskommen. [...] An dann och nach dat elei: Wieder, déi et vun Aalst hier am lëtzebuergesche gëtt, musse bleiwen. (Seejomes, net Ameis, Gehaansfénkelchen, net Glühwürmchen, Päiperlek, net Schmetterling). Dat ass jo fir Ekzema an d'Oueren ze kréien! ${ }^{16}$

Et wär villäicht éischter néidig, dergéint ze protestéieren, datt eis Sprooch am Alldeeglechen vun aflossräiche Leit an och vun de geschwate Medien öffentlech mat engem franséische ,style précieux، oder engem kumpelhaften Däitsch entstallt gëtt, obwohl genuch gutt Lëtzebuerger Wierder parat stinn. ${ }^{17}$

Auch wird moniert, dass durch den Konsum deutschsprachiger Fernsehprogramme von der »überwältigende[n] Mehrheit der Schulkinder« deren »>schnarrendeく deutsche Aussprache« stamme und »leider gereicht das dem Luxemburgischen nicht unbedingt zum Vorteil ${ }^{18}{ }^{18}$

Im Radio wurde das Luxemburgische von einem Moderator als »Kauderwelsch« bezeichnet, woraufhin sich viele Leser darüber beschwerten und mehr sprachliches Selbstbewusstsein verlangten. Denn bei einigen Leserbriefschreibern gehen die Ressentiments gegen das Deutsche zurück auf die deutsche Besatzung Luxemburgs während des Zweiten Weltkriegs und bei dem Wort »Kauderwelsch« werden automatisch entsprechende Assoziationen geweckt - »[v]iru 67 Joër goufe mir Lëtzebuerger schon mat dem Wuert >Kauderwelsch< vum Gauleiter Simon a senge Kollaborateuren konfrontéiert an affrontéiert« -, verbunden mit der Frage, »Gi mer se wierklech ni lass!? «19. Manche Kommentare sind daher sehr emo-

16 | Grethen, Änder: "D’Lëtzebuerger an hir Sprooch", in: Luxemburger Wort, 22.11.2008, S. 21. ("Also müssten die Leute, die im Radio reden - inklusive der ehrwürdigen Politiker und Gewerkschafter - ihre Muttersprache zuerst einmal Iernen. Innerhalb, ausserhalb, schwul, schwanger, fënnt statt ab ..., im nachhinein, nach wie vor, zumindest, zahlreich, am gesetzleche Rahmen, mindestens، sind nur ein paar Beispiele. Das ist kein Luxemburgisch, das sind auf deutsch gedachte und übers Schwätzen auf luxemburgisch übersetzte Sätze, die so herauskommen. [...] Und dann auch noch das hier: Wörter, die es seit Ewigkeiten im Luxemburgischen gibt, müssen bleiben. [...] Davon bekommt man ja Ekzeme an den Ohren! «)

17 | Thewes, Nico: "Eis Sproochen", in: Luxemburger Wort, 24.03.2009, S. 14. ("Es wäre vielleicht eher nötig, dagegen zu protestieren, dass unsere Sprache im Alltag von einflussreichen Leuten und auch von den gesprochenen Medien öffentlich mit einem französischen style précieux، oder einem kumpelhaften Deutsch entstellt wird, obwohl genug gute luxemburgische Wörter zur Verfügung stehen.")

18 | Roth, Lex: "Deutsch und wir", in: Lëtzebuerger Journal, 15.11.2008, S. 6.

19 | Weirich, Jos/Benoit, Jos: "Eis Sprooch an d'Constitution", in: Journal, 23.07.2008, S. 6. - Genauso: "Ass dat een Epigom vum Nazi-Gauleiter Gustav Simon, dee viru 60 Joer posaunt huet: 'Schluss mit dem fremden Kauderwelsch? « aus: "Fewa: Säin eegent Sproochennascht bedr...", in: Luxemburger Wort, 12.07.2008, S. 23. ("Vor 67 Jahren wurden wir Luxemburger schon einmal mit dem Wort ,Kauderwelsch، vom Gauleiter Simon und seinen Kollaborateuren konfrontiert und beleidigt. [...] Werden wir sie wirklich nie los!?») 
tional und proklamieren, »[n]ët aleng déi Persoun, och de Radio 100,7 huet sech blaméiert an disqualifizéiert ${ }^{20}$. Es scheint, als ob der Stolz und die Achtung für die eigene Sprache bei einigen Luxemburgern eng mit dem Widerstand gegen die deutschen Besatzer verknüpft ist, wenn beispielsweise gesagt wird: »Trotz Repressalien a Prisong, hu mir eis fir ons Sprooch an Onofhängegkeet agesat, déi mat Blutt bezuelt goufen. $\aleph^{21}$ Und daher ist es vielleicht nicht verwunderlich, dass entsprechende Ressentiments gegen das Deutsche in älteren Generationen immer noch vorhanden sind.

\section{c) französisch-kritisch}

Einige Leserbriefe wenden sich gegen einen immer größer werdenden Einfluss des Französischen in Luxemburg. Oftmals sei es unmöglich, ohne Französischkenntnisse einzukaufen:»Wenn ich einkaufen gehe und dann jemanden bitte, mir zu helfen, bekomme ich gleich zur Antwort: >en français, s'il vous plâit.« $\ll^{22}$ Daraus folgt, dass man sich fremd im eigenen Land fühlt. »Wéi oft hunn sech Lëtzebuerger scho schrëftlech a mündlech driwwer opgereegt, dass een iwwerall mat dem berühmte Satz >en français s.v.p. < konfronteíert gëtt? «23 Gerade ältere Menschen hätten oft Probleme, da ein Großteil des Pflegepersonals in Krankenhäusern und Seniorenheimen nur französisch spreche:

Was ich sehr schlimm finde, ist die Situation in den Spitälern, in Altenheimen und überhaupt überall dort, wo ältere Menschen in Pflege sind. Besonders hier müsste das Personal unsere Sprache beherrschen. Oder müssen ältere Leute noch andere Sprachen lernen, damit man sie versteht? ${ }^{24}$

Wie es scheint, ist der Umgang mit dem Französischen im Alltag also nicht so problemlos, wie man meinen könnte, wenn man von Luxemburg als einem dreisprachigen Land ausgeht:

Mir perséinlech fällt och op, dass an der leschter Zäit och an den Zeitungen (an zwar net nëmmen an deenen, déi extra op Franséisch verëffentlecht ginn) mee och an deenen tra-

20 | Weirich, Jos/Benoit, Jos: "Eis Sprooch an d'Constitution", in: Journal, 23.07.2008, S. 6. ("Nicht allein die Person, auch Radio 10,7 hat sich blamiert und disqualifiziert.")

21 | Weirich, Jos/Benoit, Jos: "Eis Sprooch an d'Constitution“, in: Journal, 23.07.2008, S. 6. ("Trotz Repressalien und Gefängnis haben wir uns für unsere Sprache und Unabhängigkeit eingesetzt, die mit Blut bezahlt wurden.")

22 | L.V.E (Düdelingen): "Zur luxemburgischen Sprache", in: Tageblatt, 12.02.2009, S. 65. ("auf Französisch bitte")

23 | lucy lux: "Lëtzebuergesch, franséisch, däitsch oder wat?", in: Journal, 06.02.2009, S. 6. ("Wie oft haben sich Luxemburger schon schriftlich und mündlich darüber aufgeregt, dass man überall mit dem berühmten Satz rauf Französisch bitte ‘ konfrontiert wird?")

24 | L.V.E (Düdelingen): "Zur luxemburgischen Sprache“, in: Tageblatt, 12.02.2009, S. 65. 
ditionellen Zeitungen, déi fréier praktesch nëmmen däitsch Artikelen verëffentlecht hunn, ëmmer méi franséisch Texter optauchen. An och das iergert de richtige Lëtzebuerger, deen zwar ganz gären eppes iwwert dat interessant Thema ging liesen, wat am Titel ugekënnegt gëtt, awer keng Loscht huet, sech duerch eng ganz Zeitungssäit franséisch Text zu wullen, zumol wenn dat Thema a bësse méi komplizéiert ass. ${ }^{25}$

Dazu kommt die Haltung vieler im Land arbeitender Ausländer, die anscheinend ganz selbstverständlich französisch sprechen und das auch von den Luxemburgern erwarten:

Fir ze wëssen, a watfir enger Sprooch ech mat him misst schwätzen, hunn ech héiflech gefrot "Vouz parlez français?" wourops ech ganz onfrëndlech ugebaupst si ginn "Eh bien oui, puisque c'est la langue administrative“. Das ass jo erëm eng Kéier typisch! ${ }^{26}$

Einzelne Schreiber wenden sich explizit gegen den Vorschlag, Französisch in der Verfassung weiter zu verankern, da es »politisch onvirsichteg« sei, »[e]ng friem Sprooch an d'Constitution ze setzen $\ll^{27}$.

\section{d) pro-multilingual}

Die pro-multilinguale Position richtet sich gegen sprachlich einseitige Tendenzen und weist sie zugunsten gelebter Mehrsprachigkeit zurück. Die beständige Konzentration auf das Luxemburgische mancher Leserbriefschreiber wird daher kritisch kommentiert, etwa wenn ein besonders engagierter Verfechter des Luxemburgischen als »eisen nationalen Här R[...] « ${ }^{28}$ bezeichnet wird, der »net iwwert eis däitsch Noperen an hir Sprooch schreiwen [kann], ouni e Gauleiter oder soss eppes

25 | lucy lux: "Lëtzebuergesch, franséisch, däitsch oder wat?", in: Journal, 06.02.2009, S. 6. ("Mir persönlich fällt auch auf, dass in der letzten Zeit auch in den Zeitungen (und zwar nicht nur in denen, die extra auf Französisch veröffentlicht werden) sondern auch in den traditionellen Zeitungen, die früher praktisch nur deutsche Artikel veröffentlicht haben, immer mehr französische Texte auftauchen. Und auch das ärgert die richtigen Luxemburger, die zwar gerne etwas über das interessante Thema lesen würden, welches der Titel ankündigt, aber keine Lust haben, sich durch eine ganze Zeitungsseite französischen Text zu kämpfen, zumal, wenn das Thema ein bisschen komplizierter ist.")

26 | lucy lux: "Lëtzebuergesch, franséisch, däitsch oder wat?", in: Journal, 06.02.2009, S. 6. ("Um zu wissen, in welcher Sprache ich mit inm reden sollte, habe ich höflich gefragt, 'sprechen Sie Französisch?`, woraufhin ich unfreundlich angeschnauzt wurde: Ja natürlich, das ist ja schließlich die Verwaltungssprache. Das ist ja mal wieder typisch!")

27 | Thewes, Nico: "Eis Sproochen", in: Luxemburger Wort, 24.03.2009, S. 14f. ("da es politisch unvorsichtig sei, eine fremde Sprache in der Verfassung festzusetzen ")

28 | lucy lux: "Lëtzebuergesch, franséisch, däitsch oder wat?", in: Journal, 06.02.2009, S. 6. ("unseren nationalen Herrn R[...]") 
vun den Naziën an d'Spiel ze bréngen $^{29}$ oder sich der Autor eines anderen Leserbriefs angesichts des Vorschlags, Luxemburgisch auf EU-Ebene zu stärken, fragt: »Wie weit kann man Nationalismus treiben? $\ll^{\circ}$

Ein Charakteristikum des modernen Luxemburg als internationaler Finanzund Bankenplatz und Standort wichtiger EU-Institutionen ist die Multikulturalität. Dieser Aspekt wird von manchen Leserbriefschreibern explizit herausgestellt: »La société luxembourgeoise a choisi de comprendre et d'intégrer les autres cultures. En bref, elle est une société dynamique et héterogène. « ${ }^{11}$ Neben den Positionen, die sich für eine bestimmte Sprache aussprechen, bzw. sie gegenüber anderen Sprachen aufwerten und stärken wollen, gibt es daher auch solche, die gerade die Mehrsprachigkeit Luxemburgs als großen Standortvorteil sehen. »Luxemburg hat drei offizielle Sprachen und wird für diese Sprachengewandtheit bewundert. \$ $^{32}$

Durch die Multilingualität der luxemburgischen Bevölkerung sei es erst möglich, dass einem so kleinem Land geradezu eine Schlüsselposition in der Europäischen Union und in der internationalen Finanzwelt zufiele. Außerdem sei gerade durch gute Deutsch- und Französischkenntnisse gewährleistet, dass Luxemburg sich gegenüber seinen großen Nachbarn Deutschland und Frankreich selbstbewusst behaupten kann, da es nicht ausschließlich eine Sprache spricht und somit weder der germanofonen noch der frankofonen Seite zugeschrieben werden kann. Darüber hinaus sichere die Mehrsprachigkeit das gute Verhältnis zu beiden Nachbarländern, das sich unter anderem in guten Handelsbeziehungen und einem grenzüberschreitenden Arbeitsmarkt widerspiegelt.33

\section{Resümee}

Die Tatsache, dass sich über einen überschaubaren Zeitraum immerhin ${ }_{16} 4$ Artikel und $6_{3}$ Leserbriefe im weiteren Sinne zum Thema »Sprache« finden ließen, lässt auf einen lebendigen Diskurs zu diesem Thema schließen. Auffällig ist ebenfalls, dass das Luxemburgische thematisch und als Sprache der Leserbriefe sehr präsent ist, was für eine starke Identifikation der Leserbriefschreiber mit dieser Sprache spricht. Das fällt auch den Leserbriefschreibern selbst auf: »Seele wor ons

29 | Lucy lux: "Kuerz Äntwort op den Här Roth (Journal vum 13. Februar)", in: Lëtzebuerger Journal, 25.2.2009, S. 6. ("der nicht über unsere deutschen Nachbarn und ihre Sprache schreiben kann, ohne einen Gauleiter oder sonst etwas von den Nazis ins Spiel zu bringen ") 30 | Pütz, M.: "Lissabon-Vertrag auf Luxemburgisch?", in: Tageblatt, 08.07.2008, S. 63.

31 | Leserbrief der Schüler eines Luxemburgisch-Kurses "Vivre dans une société multiculturelle au quotidien", in: Le Jeudi, 10.07.2008, S. 44. ("Die luxemburgische Gesellschaft hat sich dafür entschieden, die anderen Kulturen zu verstehen und zu integrieren. Kurz gesagt sie ist eine dynamische und heterogene Gesellschaft.")

32 | Pütz, M.: "Lissabon-Vertrag auf Luxemburgisch?«, in: Tageblatt, 08.07.2008, S. 63.

33 | Vgl. Fehlen, Fernand: "Wat schwätzt d'Majorité silencieuse?", in: Luxemburger Wort, 28.03.2009, S. 24. 
Sprooch esou an der Diskussioun wéi an de leschte Wochen a Méint. [...] Dat ass och gutt esou. «34 Man muss also davon ausgehen, dass das Luxemburgische eine entscheidende Rolle für das Selbstverständnis der Menschen im Land und damit für ihre Identität spielt.

Die Analyse der Leserbriefe zeigt, dass das Thema »Sprache« hoch emotional besetzt ist. Der Sprache wird eine bedeutende Rolle bei der nationalen Identitätsbildung zugeschrieben, Phrasen wie »Eis Sprooch ass eis Identitéit« 35 finden sich sehr häufig. Daher ist es nicht überraschend, dass sich evtl. vorhandene xenophobe Tendenzen besonders deutlich in Leserbriefen zum Thema »Sprache« finden lassen. Viele Schreiber zeigen sich wenig kompromissbereit, von ihrer Position abzurücken, aus Angst, ihre Identität zu verlieren oder ihre Vorfahren zu »verraten«, die sich für den Erhalt des Luxemburgischen eingesetzt hatten. So ist es nicht verwunderlich, dass manche Briefschreiber sehr persönlich und teilweise auch verletzend werden, gerade wenn sie sich gegen Artikel oder Leserbriefe wenden, die eine andere Position als die ihre vertreten.

Andererseits gilt es aber auch festhalten, dass sich viele Leserbriefe nüchtern, rational und argumentativ mit dem Thema »Sprache « und »Sprachen « in Luxemburg auseinandersetzen. Hier wird z.B. unterschieden zwischen »Nationalsprache« (Luxemburgisch) und »Verwaltungssprache« (Deutsch, Französisch). ${ }^{36}$

Auch gegen allzu polemische Positionen wird in manchen Leserbriefen Position bezogen, indem falsche Behauptungen korrigiert oder gemäßigte Gegenpositionen vertreten werden. ${ }^{37}$ Den Schreibern dieser Art von Leserbriefen wird jedoch des Öfteren in nachfolgenden Leserbriefen vorgeworfen, dass sie die luxemburgische Sprache »verraten« würden oder schlichtweg nicht qualifiziert seien, sich zu diesem Thema zu äußern.

Man sieht, die Konfliktlinien lassen sich sehr wohl entlang der Sprachen und Sprachenverwendung nachzeichnen. Gleichwohl darf aber nicht übersehen werden, dass es, wie in einem Leserbrief beschrieben, eine »Majorité silencieuse « ${ }^{38}$ gibt, die anscheinend die offiziell in Luxemburg gesprochenen Sprachen beherrscht und diese ganz selbstverständlich benutzt. Die in den Leserbriefen erhitzt

34 | Grethen, Änder: "D’Lëtzebuerger an hir Sprooch “, in: Luxemburger Wort, 22.11.2008, S. 21. ("Selten war unsere Sprache so in der Diskussion wie in den letzten Wochen und Monaten. [...] Das ist auch gut so.")

35 | Vgl. Weirich, Jos: "Eis Sprooch ass eis Identitéit", in: Luxemburger Wort, 09.08.2008, S. 12.

36 | Vgl. Watgen, Fernand: "Lëtzebuerger ouni Lëtzebuergesch ...? «, in: Luxemburger Wort, 21.05.2008, S. 18.

37 | Vgl. dazu "lucy luc": "Lëtzebuergesch, franséisch, däitsch oder wat?", in: Journal, 06.02.1009, S. 6 oder Pauly, Michel: "Net all Däitsch an een Dëppe geheien", in: Luxemburger Wort, 28.03.2009, S. 22.

38 | Fehlen, Fernand: "Wat schwätzt d'Majorité silencieuse?", in: Luxemburger Wort, 28.03.2009, S. 24. ("schweigende Mehrheit") 
geführten Debatten könnten also z.T. nur Scheingefechte sein, in denen einzelne, teilweise extreme Positionen vertreten werden.

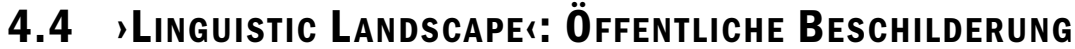 UND MEHRSPRACHIGKEIT}

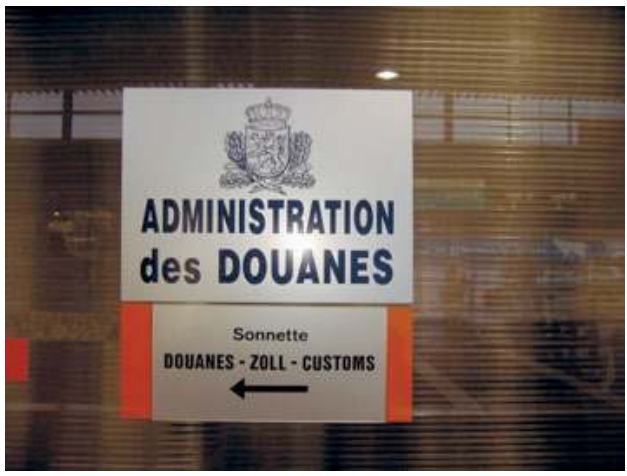

Abbildung 17: Zollbehörde am Luxemburger Flughafen

\section{Einleitung}

Gegenstand dieses Beitrags sind Beispiele visueller Mehrsprachigkeit, wie sie sich auf Schildern, Postern, Aushängen usw. manifestiert. Die Forschungsrichtung des >Linguistic Landscaping (im Folgenden: LL) - ein noch relativ neuer Ansatz innerhalb der (interdisziplinär ausgerichteten) Soziolinguistik und Teil der visuellen Ethnografie - analysiert die Struktur und Kontextualisierung sichtbarer >Zeichen< im öffentlichen Raum (Backhaus 2007: 9) und bietet neue Erkenntnisse zu Sprachpolitik, Mehrsprachigkeit, Sprachverwendung und Sprachdominanz. Geradezu emblematisch verdichten sich in der einleitenden Abbildung die - unserer Meinung nach - für Luxemburg charakteristischen Aspekte (a) der Präferenz des Französischen und (b) der Mehrsprachigkeit, in der das Englische zunehmend eine Rolle spielt.

\section{Studien in visueller Mehrsprachigkeit}

In der Soziolinguistik wurde die Struktur öffentlich sichtbarer Textzeugen lange Zeit schlichtweg ignoriert. Ausgehend von komplexen urbanen Räumen mit mehrsprachiger Bevölkerung begann erst in den 1990er Jahren die Forschung zum LL. Eine der ersten Arbeiten stammt von Landry und Bourhis (1997), die die Wahrnehmung mehrsprachiger, öffentlicher Zeichen in Kanada thematisieren. Die zentralen Forschungsfragen kreisen um den Sprachgebrauch im öffentlichen Raum einer Stadt oder einer Region, wie er sich z.B. auf Straßenschildern, Anzeigetafeln, Straßennamen, Ortsnamen, Geschäftsschildern und Schildern an öffentlichen Ge- 
bäuden usw. manifestiert. Die Analyse dieser Zeichen fokussiert dann auf Aspekte wie Adressatenorientiertheit, präsupponierte Sprachkompetenzen, zugrunde liegende Sprachenpräferenzen oder identitätsrelevante Spracheinstellungen. Quantitative Resultate erlauben es zudem, aktuelle Entwicklungstendenzen zu formulieren (z.B. Zunahme/Rückgang einer Sprache, Veränderungen in der Wertigkeit einer Sprache usw.). In Backhaus (2007) wurde eine Typologie mehrsprachiger Zeichen entwickelt und am urbanen Raum von Tokio exemplifiziert, die auch im vorliegenden Beitrag übernommen wird. Weitere Untersuchungen erweitern das Feld und analysieren Zeichenhaftigkeit in einem allgemeinen geo-semiotischen Rahmen (etwa Scollon/Scollon 2003). Mit Shohamy und Gorter (2009) liegen aktuelle Beispieluntersuchungen vor, in denen die visuelle Mehrsprachigkeit und ihre Implikationen für Sprachpolitik, Sprachidentität und Sprachbewusstsein beleuchtet werden.

\section{Methodik und Material}

Die Datenbasis dieses Fallbeispiels besteht insgesamt aus ca. 600 digitalen Fotos, die in fünf Ortschaften (Luxemburg-Stadt, Wiltz, Vianden, Esch/Alzette und Junglinster) zwischen Oktober 2007 und Juni 2009 unter studentischer Beteiligung aufgenommen wurden. Dabei wurde angestrebt, in einem umgrenzten Areal im Ortszentrum möglichst alle visuellen Zeichen mit Schriftbeteiligung zu erheben. Bei den meisten analysierten visuellen Zeichen handelt es sich um offizielle Beschilderung, Geschäftsbeschilderung, Aushänge, Poster oder private Schilder. Ausgeschlossen bleiben Adressschilder, Speisekarten, Reklamen für bestimmte Produkte oder Graffiti (sog. >transgressive Zeichen ${ }^{39}$ ). Dabei zeigte sich, dass die Abgrenzung zwischen institutionellen Hinweisen und Reklame nicht immer ohne weiteres zu leisten ist. Ist z.B. eine Hinweistafel zu bestimmten Dienstleitungen der Eisenbahngesellschaft Sociéte nationale des chemins de fer luxembourgois CFL als ein Top-down-Zeichen ${ }^{40}$ oder als Werbung zu klassifizieren? Hier müssen in Zukunft noch feinere Analyseraster entwickelt werden.

In der folgenden quantitativen Analyse liegt der Schwerpunkt auf der Sprachverteilung, sowie der Rollen der verschiedenen Sprachen. Die Ergebnisse werden daraufhin im Hinblick auf die Sprachsituation des Landes und seines linguistischen Profils interpretiert.

\section{Analyse des Korpus}

Die Zeichen werden zunächst danach untergliedert, ob es sich um ein- oder mehrsprachige Zeichen handelt. Des Weiteren wird nach dem Urheber/Verfasser des

39 | Zeichen, das ohne Berechtigung absichtlich die eigentliche Semiotik des Platzes stört, z.B. Graffiti, Tags, absichtlich weggeworfener Müll.

40 | Zentral und meist einheitlich erstelltes Zeichen: z.B. Informationsaushänge, Angebotstafeln oder Werbekampagnen (interne und externe Produzenten). 
Zeichens differenziert: Bei Top-down-Zeichen handelt es sich um Zeichen, die von staatlichen oder quasi-staatlichen Institutionen erstellt werden (z.B. Informationsaushänge der Administration, Ortseingangsschilder u.a.). Im Gegensatz dazu sind Bottom-up-Zeichen von Privatpersonen, Geschäften oder Firmen erstellt. Es wird sich zeigen, dass diese Differenzierung einen zentralen Steuerungsfaktor für die sprachliche Struktur der Zeichen darstellt.

Hinsichtlich dieser Merkmale setzt sich das Korpus wie in Tabelle 2 aufgeführt zusammen.

\begin{tabular}{|l|r|c|}
\hline & $\begin{array}{c}\text { Top-down-Zeichen } \\
\text { (institutionelle Zeichen) }\end{array}$ & \multicolumn{2}{|c|}{$\begin{array}{c}\text { Bottom-up-Zeichen } \\
\text { (kommerziell, privat) }\end{array}$} \\
\hline monolingual & 95 & 227 \\
\hline multilingual* $^{*}$ & 103 & 159 \\
\hline
\end{tabular}

Tabelle 2: Verteilung von monolingualen/multilingualen und Top-down-/

Bottom-up-Zeichen (* zwei- und dreisprachig)

Als erste Tendenz zeichnet sich ab, dass Bottom-up-Zeichen seltener multilingual sind als Top-down-Zeichen. Bei den Top-down-Zeichen ist das Verhältnis zwischen einsprachigen und mehrsprachigen Schildern hingegen annährend ausgeglichen.

\section{Analyse der monolingualen Zeichen}

Die quantitative Verteilung der monolingualen Zeichen stellt sich wie folgt dar.

\begin{tabular}{|c|c|c|c|c|c|c|c|c|}
\hline & $\begin{array}{l}\text { Luxem- } \\
\text { burgisch }\end{array}$ & Französisch & Deutsch & Englisch & $\begin{array}{l}\text { Portu- } \\
\text { giesisch }\end{array}$ & $\begin{array}{l}\text { Italie- } \\
\text { nisch }\end{array}$ & $\begin{array}{l}\text { Chine- } \\
\text { sisch }\end{array}$ & Summe \\
\hline $\begin{array}{l}\text { Top- } \\
\text { down- } \\
\text { Zeichen }\end{array}$ & 16 & 64 & 6 & 8 & 1 & 0 & 0 & 95 \\
\hline $\begin{array}{l}\text { Anteil } \\
\text { in } \%\end{array}$ & 16,84 & 67,37 & 6,32 & 8,42 & 1,05 & 0,00 & 0,00 & 100,00 \\
\hline $\begin{array}{l}\text { Bottom- } \\
\text { up- } \\
\text { Zeichen }\end{array}$ & 30 & 132 & 25 & 33 & 2 & 3 & 2 & 227 \\
\hline $\begin{array}{l}\text { Anteil } \\
\text { in } \%\end{array}$ & 13,22 & 58,15 & 11,01 & 14,54 & 0,88 & 1,32 & 0,88 & 100,00 \\
\hline
\end{tabular}

Tabelle 3: Sprachenverteilung auf monolingualen Zeichen

Wie zu erwarten kommt das Französische am häufigsten vor (67\%), während das Luxemburgische - mit großem Abstand - überwiegend an zweiter Stelle liegt. Charakteristisch ist, dass bei den Top-down-Zeichen das Französische um $10 \%$ höher vertreten ist als im Bottom-up-Bereich. Die höhere Präsenz des Deutschen und auch des Englischen im Bottom-up-Bereich erklärt sich durch den hohen Grad 
an Globalisierung. Insbesondere im Flughafen Luxemburg lassen sich viele ausschließlich englischsprachige Zeichen feststellen.

Trotz der Dominanz des Französischen manifestiert sich schon bei der Sprachenverteilung der einsprachigen Zeichen eine grundsätzliche Mehrsprachigkeit, da auch die selteneren Sprachen (Luxemburgisch, Deutsch, Englisch) mit nicht zu vernachlässigender Häufigkeit vertreten sind.

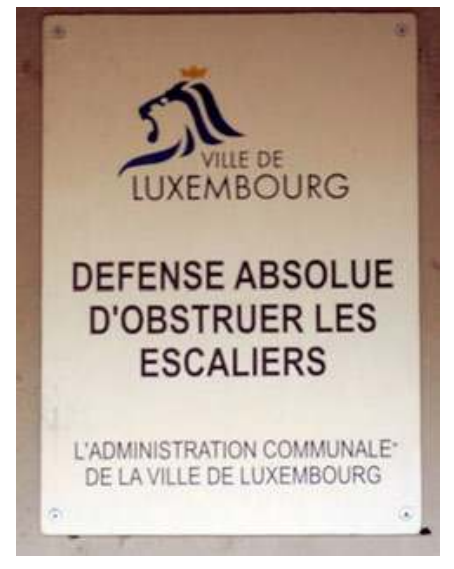

\begin{abstract}
Abbildung 18: Hinweisschild
der Stadt Luxemburg: Top-down, einsprachig, Französisch
\end{abstract}

\title{
Analyse mehrsprachiger Zeichen
}

Um eine große Anzahl an mehrsprachigen Zeichen analysieren zu können, sind terminologische Vereinfachungen für eine einheitliche und eindeutige Auswertung notwendig. Dies gilt besonders für mehrsprachige Räume oder Länder mit hohem Sprachkontakt. Im Folgenden werden die Aspekte der Sprachenpräferenz sowie des Zeichentyps behandelt.

\section{Einblick in die Sprachenpräferenz}

Hierunter wird die Reihenfolge und allgemeine Präsenz der Sprachen auf mehrsprachigen Zeichen verstanden. Häufig lässt sich beobachten, dass eine Sprache durch die Art der räumlichen Anordnung und/oder durch die grafische Gestaltung hervorgehoben wird (u.a. durch Größe, Farbe, Schrifttype), woraus sich eine gewisse Rangordnung und Wertigkeit der Sprachen rekonstruieren lässt. Diese CodePräferenz wird in der vorliegenden Untersuchung nicht systematisch beachtet und bleibt einer Folgeuntersuchung vorbehalten. Ein für Luxemburg charakteristisches Beispiel stellen die Ortsschilder dar, die zunächst in Fettdruck und Majuskeln mit dem französischen Namen beginnen, auf den dann in kursiver und kleinerer Schrift der luxemburgische Name folgt. 


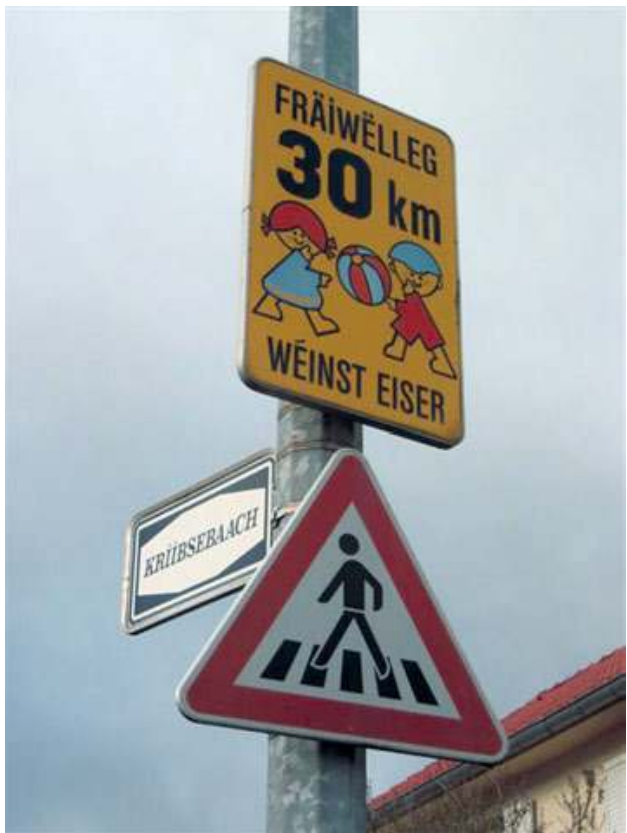

Abbildung 19: Straßenschild in der Stadt Luxemburg: Top-down, einsprachig, Luxemburgisch

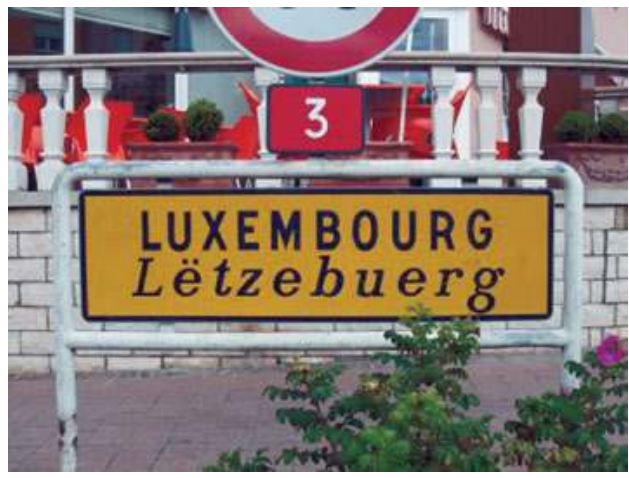

Abbildung 20: Ortseingangsschild der Stadt Luxemburg

Eingehender untersucht werden im Folgenden die Verteilungen und Reihenfolge der Sprachen. Die Tabellen zeigen die Verteilungen der Sprachenkombinationen für zwei- und dreisprachige Zeichen. Die Reihenfolge der Sprachenkürzel entspricht der Reihenfolge auf dem jeweiligen Schild. 


\begin{tabular}{|c|c|c|c|c|c|c|c|c|c|c|c|}
\hline & FR-LU & LU-FR & LU-DE & FR-DE & DE-FR & FR-EN & EN-FR & DE-EN & FR-NL & sonstige & Summe \\
\hline $\begin{array}{l}\text { Top-down- } \\
\text { Zeichen }\end{array}$ & 4 & 10 & 0 & 40 & 3 & 13 & 7 & 0 & 0 & 3 & 80 \\
\hline Anteil in\% & 5,00 & 12,5 & 0,00 & 50,00 & 3,75 & 16,3 & 8,75 & 0,00 & 0,00 & 3,75 & 100,00 \\
\hline $\begin{array}{l}\text { Bottom- } \\
\text { up- } \\
\text { Zeichen }\end{array}$ & 16 & 5 & 0 & 25 & 4 & 18 & 13 & 6 & 15 & 26 & 128 \\
\hline Anteil in\% & 12,50 & 3,91 & 0,00 & 19,53 & 3,13 & 14,06 & 10,16 & 4,69 & 11,72 & 20,31 & 100,00 \\
\hline
\end{tabular}

Tabelle 4: Sprachenverteilung zweisprachiger Schilder

\begin{tabular}{|l|r|r|r|r|r|r|}
\hline & FR-DE-EN & FR-EN-DE & EN-FR-DE & EN-DE-FR & sonstige & Summe \\
\hline $\begin{array}{l}\text { Top-down- } \\
\text { Zeichen }\end{array}$ & 12 & 3 & 2 & 2 & 4 \\
\hline Anteil in \% & $\mathbf{5 2 , 1 7}$ & $\mathbf{1 3 , 0 4}$ & $\mathbf{8 , 7 0}$ & $\mathbf{8 , 7 0}$ & $\mathbf{1 7 , 3 9}$ & $\mathbf{1 0 0 , 0 0}$ \\
\hline $\begin{array}{l}\text { Bottom-up- } \\
\text { Zeichen }\end{array}$ & 11 & 1 & 3 & 4 & 9 \\
\hline Anteil in \% & $\mathbf{3 9 , 2 9}$ & $\mathbf{3 , 5 7}$ & $\mathbf{1 0 , 7 1}$ & $\mathbf{1 4 , 2 9}$ & $\mathbf{3 2 , 1 4}$ & $\mathbf{1 0 0 , 0 0}$ \\
\hline
\end{tabular}

Tabelle 5: Sprachenverteilung dreisprachiger Schilder

Die erste Auffälligkeit betrifft die Sprachenkombinationen: Während bei den Topdown-Zeichen die Kombination FR-DE (50 \%) bzw. FR-DE-EN (52 \%) am häufigsten ist, zeichnen sich die Bottom-up-Schilder durch größere Heterogenität aus. Und in der Tat wird durch diese Zeichen der heterogen-mehrsprachige Charakter der luxemburgischen Beschilderung deutlich (vgl. auch den hohen Anteil in der Kategorie >Sonstige<, in der alle möglichen Sprachkombinationen zusammengefasst sind). Umgekehrt ist der Anteil von luxemburgisch-französischen Schildern im Top-down-Bereich höher als im Bottom-up-Bereich (12,5 \% vs. 3,9 \% bei den zweisprachigen Schildern). Auf den meisten Schildern ist das Französische die erstverwendete Sprache, wohingegen das Deutsche auf dieser Position praktisch nie auszumachen ist; tatsächlich ist das Englische häufiger auf der ersten Position $\mathrm{zu}$ finden.

In Tabelle 6 ist das Sprachenvorkommen auf zwei- und dreisprachigen Zeichen unabhängig von der Reihenfolge zusammengerechnet, um einen Eindruck von der Verwendungshäufigkeit der Sprachen zu erhalten. Die Spitzenstellung des Französischen ist klar erkennbar, das in allen Kategorien fast durchgehend vertreten ist. Bei zweisprachigen Top-down-Zeichen ist in ca. der Hälfte der Fälle das Deutsche anzutreffen, wohingegen dieser Anteil bei den Bottom-up-Zeichen zugunsten des Englischen geringer ist. 


\begin{tabular}{|c|c|c|c|c|c|}
\hline & FR & LU & DE & EN & Summe \\
\hline $\begin{array}{l}\text { zweispr. } \\
\text { Top-down-Zeichen }\end{array}$ & 79 & 14 & 44 & 20 & 80 \\
\hline Anteil in \% & 98,75 & 17,5 & 55,00 & 25,00 & \\
\hline $\begin{array}{l}\text { dreispr. } \\
\text { Top-down-Zeichen }\end{array}$ & 23 & 2 & 22 & 21 & 23 \\
\hline Anteil in \% & 100,00 & 8,70 & 95,65 & 91,30 & \\
\hline $\begin{array}{l}\text { zweispr. } \\
\text { Bottom-up-Zeichen }\end{array}$ & 105 & 21 & 40 & 40 & 119 \\
\hline Anteil in \% & 88,24 & 17,65 & 33,61 & 33,61 & \\
\hline $\begin{array}{l}\text { dreispr. } \\
\text { Bottom-up-Zeichen }\end{array}$ & 30 & 4 & 26 & 27 & 31 \\
\hline Anteil in \% & 96,77 & 12,90 & 83,87 & 87,10 & \\
\hline
\end{tabular}

Tabelle 6: Vorkommen der vier meistgenannten Sprachen auf mehrsprachigen Schildern

Somit manifestiert sich für beide Schildertypen eine Dreisprachigkeit aus Französisch, Deutsch und Englisch, wohingegen das Luxemburgische nur zu 8 und $18 \%$ verwendet wird. Die Zahlen belegen deutlich, dass das Englische in diesem Bereich bereits einen festen Platz eingenommen hat.

\section{Analyse der verschiedenen Zeichentypen}

Im Folgenden soll für die mehrsprachigen Zeichen das Verhältnis der einzelnen Sprachen zueinander analysiert werden. In Anlehnung an Backhaus (2007) werden dabei drei Zeichentypen unterschieden:

1. Homofones Zeichen: Die Information wird vollständig in mehrere Sprachen übersetzt. Die Sprachenverteilung ist auf solchen Schildern sozusagen ideal, da - außer durch die Reihenfolge - keine Sprache bevorzugt wird. Ein homofones Zeichen erfordert vom Leser keine mehrsprachige Kompetenz, da die Information in >seiner Sprache vorhanden ist.

2. Gemischtes Zeichen: Bei diesen Zeichen werden nur Teile der Information übersetzt.

3. Polyfones Zeichen: Das Zeichen enthält Informationen, die ohne Übersetzung auf mehrere Sprachen verteilt sind. Noch stärker als bei den gemischten Zeichen ist für das Verstehen polyfoner Zeichen eine mehrsprachige Kompetenz erforderlich.

Für unser luxemburgisches Sample ergeben sich interessante Unterschiede zwischen den Top-down- und den Bottom-up-Zeichen, denn bei Ersteren ist der Anteil der homofonen Zeichen deutlich höher, während in Letzteren mehr polyfone Zeichen zu finden sind (57\% und $31 \%$ ). Während auf homofonen Zeichen die Information identisch in den beteiligten Sprachen ist und somit auf demokratische Wei- 
se allen Bevölkerungsgruppen zur Verfügung steht, müssen die Leser polyfoner Zeichen die verwendeten Sprachen beherrschen, um die vollständige Information zu decodieren. Es ist offensichtlich, dass polyfone Zeichen höhere Mehrsprachigkeitskompetenzen erfordern als homofone.

\begin{tabular}{|l|r|r|}
\hline & Top-down-Zeichen & Bottom-up-Zeichen \\
\hline gemischt & 17 & 14 \\
\hline homophon & 57 & 31 \\
\hline polyfon & 26 & 55 \\
\hline
\end{tabular}

Tabelle 7: Verteilung der Zeichen nach Typen

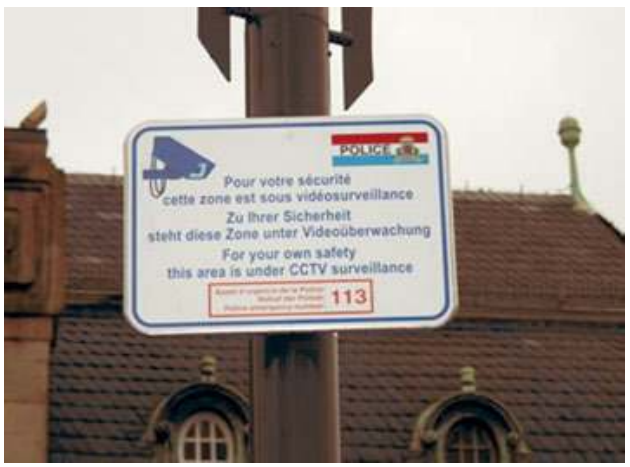

Abbildung 21: Schild der Polizei: homofon, Top-down, dreisprachig (Bahnhof Luxemburg)

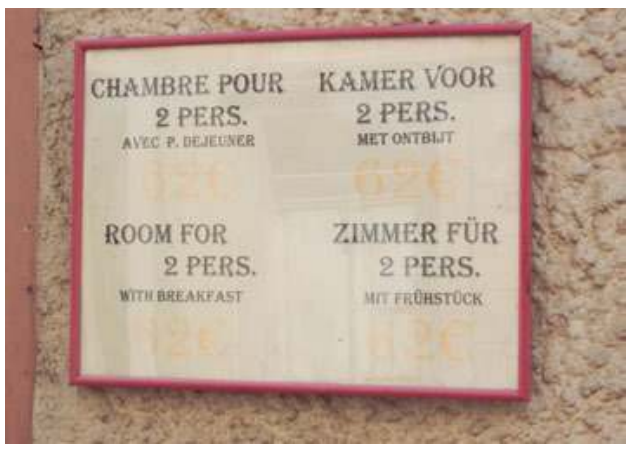

Abbildung 22: Hotel in Vianden: homofon, Bottom-up, viersprachig

Die Beispiele der Abbildungen 21 und 22 repräsentieren den klassischen Fall des homofonen Zeichens, auf dem der Inhalt vollständig in die anderen Sprachen übersetzt ist. Oft wird dies auch durch eine parallele grafische Gestaltung unterstützt. 


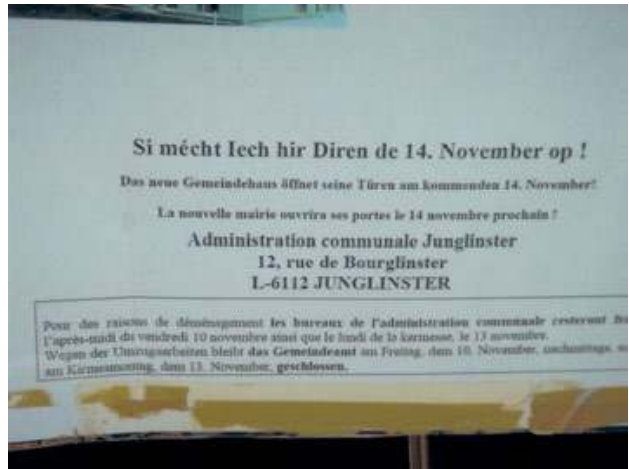

Abbildung 23: Aushang der Gemeinde Junglinster: gemischtes Zeichen, Top-down, dreisprachig (Junglinster)

Während der obere Teil des Anschlags in Abbildung 23 am Rathaus von Junglinster homofone Züge trägt, ist das >Kleingedruckte < im unteren Teil nur auf Französisch und Deutsch verfügbar.

Um bei dem Zeichen in Abbildung 24 alle Informationen aufnehmen zu können, muss der Leser Französisch (>Expo<, >Bourse <), Deutsch (>Modellbau Tauschbörse $<$ ) und Luxemburgisch (>Auer $<$ in der Nennung der Öffnungszeit) (zumindest passiv) beherrschen.

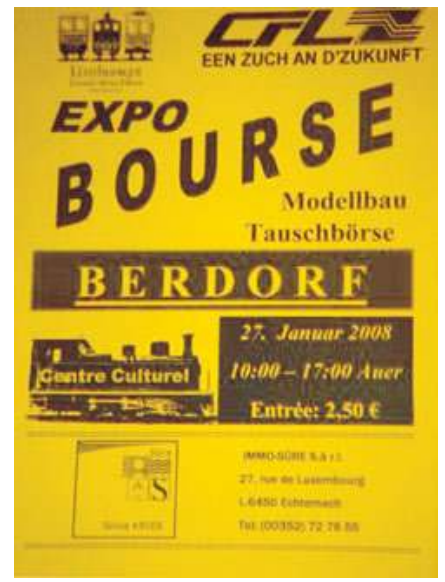

Abbildung 24: Werbeplakat in Junglinster: polyfones Zeichen, Bottom-up, dreisprachig (Junglinster) 


\section{Resümee}

Die Analyse der bislang weitgehend vernachlässigten Textsorten, wie sie sich auf öffentlichen Schildern und Aushängen manifestiert, erbrachte die folgenden Resultate:

- Quantitativ betrachtet ist ungefähr die Hälfte der öffentlichen Beschilderung multilingual gestaltet. Bei einsprachigen Zeichen dominiert das Französische, während Luxemburgisch, Englisch und Deutsch deutlich seltener als alleinige Sprachen vertreten sind.

- Auf mehrsprachigen Zeichen ist das Französische fast durchgehend vertreten. Die häufigsten Kombinationen sind >Französisch-Deutsch $<$ und >FranzösischDeutsch-Englisch<.

- Top-down-Zeichen sind häufiger mehrsprachig als Bottom-up-Zeichen. Darin lässt sich ein sprachpolitisches Element erkennen, wonach die Mehrsprachigkeit des Landes auch auf der offiziellen Beschilderung reflektiert werden soll.

- Besonders interessant ist das Aufkommen des Englischen: Wenn auf einem Schild drei Sprachen vorkommen, dann ist die dritte stets das Englische. Luxemburgisch als dritte Sprache scheint hier nicht infrage zu kommen.

- Top-down-Zeichen tendieren dazu, die Informationen identisch in den beteiligten Sprachen wiederzugeben (homofone Zeichen), wobei allerdings in der häufigen Erstsetzung des Französischen dennoch eine Präferenz ausgedrückt wird. Bottom-up-Zeichen hingegen weisen größere Heterogenität in der Sprachenwahl auf und auch im Umgang mit der Übersetzung, d.h., dass sich hier häufiger polyfone Zeichen finden, für deren Dekodierung eine höhere mehrsprachige Kompetenz erforderlich ist.

- Das Luxemburgische wird insgesamt relativ selten auf öffentlichen Schildern verwendet, wobei im Bottom-up-Bereich mehr Luxemburgisch zu finden ist als im Top-down-Bereich. Für viele Luxemburger übernimmt das Standarddeutsche nach wie vor die Funktion der Schriftsprache, so dass also eine parallele Verwendung der eng verwandten Sprache Deutsch und Luxemburgisch als redundant empfunden wird.

In Bezug auf die Identitätskonstruktionen scheint die Fallstudie des LL - oberflächlich betrachtet - den Ergebnissen der oben vorgestellten Fragebogenuntersuchung und der Leserbriefanalyse zu widersprechen, wurde doch dort der immense Stellenwert des Luxemburgischen in Identitätsbildungsprozessen herausgearbeitet. Tatsächlich ist die öffentliche Schriftlichkeit durch die Dominanz des Französischen bzw. durch die Mehrsprachigkeit ohne Luxemburgisch geprägt. Das weitgehende Fehlen der Nationalsprache Luxemburgisch hat verschiedene Gründe: Durch die noch immer stark wirkende mediale Diglossie ist das Luxemburgische weitgehend der Mündlichkeit vorbehalten und dringt nur zögerlich in die schriftlich-öffentliche Domäne vor. Dazu kommt, dass der mittlere Standardisierungs- 
grad des Luxemburgischen oftmals als Hindernisgrund gesehen wird, diese Sprache auf einem öffentlichen Schild zu verwenden. $\mathrm{Zu}$ groß ist die Unsicherheit, einen Fehler zu produzieren. Zwar ist das Deutsche auf Schildern nicht so selten vertreten, wie man meinen möchte, doch tritt es niemals als alleinige Sprache auf (monofone Schilder), es wird vielmehr vom Französischen und auch vom Englischen begleitet. Der Grund hierfür dürfte in der radikalen Ablehnung alles Deutschen unmittelbar nach dem Ende des Zweiten Weltkrieges liegen.

\subsection{Schlussfolgerung: Sprachliche Polynomie als Chance}

Die vorgestellten Ergebnisse bestätigen eindrücklich die Relevanz des Themas Mehrsprachigkeit in Luxemburg. Das Mit-, Neben- und mitunter auch Gegeneinander unterschiedlicher Sprachen gehört zu den konstitutiven Bedingungen der Luxemburger Gesellschaft. Die Komplexität der Sprachenthematik zeigt sich etwa dadurch, dass dem Luxemburgischen einerseits zwar eine herausragende Bedeutung beigemessen wird, sich diese im öffentlichen Sprachgebrauch aber nur sehr bedingt widerspiegelt. Nicht zuletzt gerade dadurch scheint dem Luxemburgischen aber seine Funktion als identitätsstiftende Sprache zuzukommen, ein Mehrwert, den weder das Französische noch das Deutsche für sich beanspruchen können. Diese werden, obwohl über Jahrhunderte im Großherzogtum verwendet und in Schule, Presse und Verwaltung maßgebend, in weiten Teilen der Bevölkerung nicht als (weitere) Muttersprachen und damit als legitimer Eigenbesitz angesehen, sondern als Sprachen der Nachbarn.

Nichtsdestotrotz wird die (spezifische) Luxemburger Mehrsprachigkeit sehr wohl als vorteilhaft für die kulturellen und wirtschaftlichen Belange des Landes erkannt. Sie stellt ein Alleinstellungsmerkmal dar, das Luxemburg zu einem europäischen, wenn nicht weltweiten Modellfall macht. Auch hierin, so zeigt die Studie, ist ein Faktor der Luxemburger Identität begründet.

Luxemburg kann als einsprachiges Land nicht funktionieren, sondern nur aufgrund eines Sprachkonsenses, der (mindestens) die drei offiziellen Sprachen des Landes einbezieht. Nur unter dieser Voraussetzung ist die kommunikative Leistungsfähigkeit des Landes und die Einbeziehung der großen ausländischen Bevölkerungsteile zu gewährleisten - bei aller Problematik im Einzelnen. Von einem Sprachkonflikt, wie er z.B. in Belgien, Südtirol oder dem Baskenland vorliegt, kann in Luxemburg jedoch nicht die Rede sein.

Gemäß den Prämissen dieser Untersuchung wird Identität immer als sich permanent aktualisierende Identitätskonstruktion(en) verstanden, die sich aus öffentlichen und privaten Diskursen speist. Ein essentialistischer Zugang zur Identität ist daher eine Aporie und verbietet sich für diese Untersuchung. Die luxemburgische Gesellschaft, und nicht nur diese, ist in ihrem Aufbau so komplex, dass einfache Gleichsetzungen zwischen Sprache und Identität nicht möglich sind: 
Eine Benennung des Luxemburgischen als Nationalsprache bedeutet daher also nicht automatisch, dass alle Einwohner diese Sprache sprechen, dass der Staat diese sprachenpolitische Setzung bildungspolitisch umsetzt oder dass alle Bevölkerungsgruppen den ersten Artikel des Sprachengesetzes von 1984 (»La langue nationale des Luxembourgeois est le luxembourgeois«) in der gleichen Art und Weise interpretieren. Gleiches gilt für das Leitbild der Mehrsprachigkeit: Zwar wird, dies belegen die quantitativen Resultate im Abschnitt 4.2, von nahezu allen Luxemburgern/-innen die Mehrsprachigkeit als zentrale kulturelle und gesellschaftliche Konstante angesehen, doch zeigen die gleichen Resultate auch, dass beträchtliche Unterschiede existieren, wie diese Mehrsprachigkeit auszugestalten ist: So ist die Mehrheit (43 \%) der Meinung, dass eine Zweisprachigkeit ausreichend ist, während sich nur $20 \%$ für die (offiziell deklarierte) Dreisprachigkeit aussprechen. Es ist nicht nur der hohe Ausländer/-innenanteil, der für diese Widersprüchlichkeit und Heterogenität verantwortlich ist, vielmehr wird offensichtlich, dass alle gesellschaftlichen und kulturellen Differenzierungsparameter (i.e. Milieuzugehörigkeit, Alter, Geschlecht usw.) zu sich ständig verändernden Identitätskonstruktionen führen können, in denen >zugeschriebene < wie auch >angeeignete Identitäten k konfligieren. Daraus resultieren zwangsläufig unterschiedliche Vorstellungen über die >gute< und >richtige< Struktur der Mehrsprachigkeit, wie die Diskussion der Leserbriefdiskurse gezeigt hat. Eine sprachliche Identität erwächst also nicht aus den beteiligten Sprachen selbst, sondern aus den je verschiedenen Vorstellungen und vermittelten und vermittelnden Diskursen über die Sprachen: »In this view, processes of identification take place through practice, which is not limited to the use of language, but includes representations of language (like discourses of endangerment).«(Jaffe 2007: 70)

Nun mag man möglicherweise bedauern, dass eine einfache Zuordnung von Sprache(n) und Identität nicht möglich ist und, dass auch für Luxemburg keine einfache Formel für eine wie auch immer geartete >nationale Identität< angegeben werden kann. Tatsächlich bedarf allerdings eine Gesellschaft der Flexibilität. Insbesondere für mehrsprachige Gemeinschaften mit kleinen Regionalsprachen zeigt sich, dass sprachliche und damit auch gesellschaftliche Konflikte vermieden werden können, wenn >Polynomie< (Marcellesi 2003) zugelassen wird, d.h. die gesellschaftliche Akzeptanz verschiedener sprachlicher Normen und mehrsprachiger Konstellationen.

\subsection{LiteratuRVERZEICHNIS}

Backhaus, Peter (2007): Linguistic Landscapes. A Comparative Study of Urban Multilingualism in Tokyo, Clevedon: Multilingual Matters.

Bucher, Hans-Jürgen (1986): Pressekommunikation. Grundstrukturen einer öffentlichen Form der Kommunikation aus linguistischer Sicht, Tübingen: Niemeyer. 
Bucher, Hans-Jürgen (1989): »Zeitungen und Leser im Dialog. Ein Beitrag zur kommunikativen Analyse von Pressetexten«, in: Edda Weigand/Franz Hundsnurscher (Hg.): Dialoganalyse II. Referate der 2. Arbeitstagung Bochum 1988. Bd. 1, S. 287-303.

Drewnowska-Vargáné, Eva (2001): »Kohärenzmanagement und Emittent-Rezipient-Konstellationen in deutsch- polnisch- und ungarischsprachigen Leserbriefen«, in: Ulla Fix/Stephan Habscheid/Josef Klein (Hg.): Zur Kulturspezifik von Textsorten, Textsorten; 3, Tübingen: Staufenburg, S. 89-108.

Gilles, Peter (1999): Dialektausgleich im Lëtzebuergeschen: zur phonetisch-phonologischen Fokussierung einer Nationalsprache, Tübingen: Niemeyer.

Gilles, Peter/Moulin, Claudine (2003): »Luxembourgish«, in: Ana Deumert/Wim Vandenbussche (Hg.): Germanic Standardizations - Past to Present. Amsterdam: de Gruyter, S. 303-329.

Jaffe, Alexandra (2007): »Discourses of Endangerment. Contexts and Consequences of Essentializing Discourses«, in: Alexandre Duchêne/Monica Heller (Hg.): Discourses of Endangerment: Ideology and Interest in the Defence of Languages, London: Continuum, S. 57-74.

Joseph, John E. (2004): Language and Identity. National, Ethnic, Religious. Houndmills: Palgrave Macmillan.

Kraemer, Jean-Pierre (1993): »Luxembourg«, in: Sociolinguistica 7 (Multilingual Concepts in the Schools of Europe), S. 162-73.

Landry, Rodrigue/Bourhis, Richard Y. (1997): Linguistic Landscape and Ethnolinguistic Vitality, in: Journal of Language and Social Psychology, Vol. 16, No. 1, S. 23-49.

Marcellesi, Jean-Baptiste/Bulot, Thierry/Blanchet, Philippe (2003): Sociolinguistique: Épistémologie, Langues Régionales, Polynomie, Paris: L'Harmattan.

Mattheier, Klaus J. (1991): »Standardsprache als Sozialsymbol. Über kommunikative Folgen gesellschaftlichen Wandels«, in: Rainer Wimmer: Das 19. Jahrhundert. Sprachgeschichtliche Wurzeln des heutigen Deutsch (Jahrbuch 1990 des Instituts für deutsche Sprache), Berlin/New York, S. 41-73.

Moulin, Claudine (2006): »Grammatisierung und Standardisierung des Luxemburgischen. Eine grammatikographisch-sprachhistorische Annäherung«, in: Damaris Nübling/Claudine Moulin (Hg.): Perspektiven einer linguistischen Luxemburgistik, Heidelberg: Winter, S. 277-311.

Naglo, Kristian (2007): Rollen von Sprache in Identitätsbildungsprozessen multilingualer Gesellschaften in Europa. Eine vergleichende Betrachtung Luxemburgs, Südtirols und des Baskenlands, Frankfurt: Peter Lang.

Piirainen, Ilpo Tapani/Yli-Kojola, Anneli (1983): Untersuchungen zur Sprache der Leserbriefe im Hamburger Abendblatt, Vaasa.

Sandig, Barbara (1986): Stilistik der deutschen Sprache, Berlin/New York: de Gruyter.

Scollon, Ron/Scollon, Suzie (2003): Discourses in place: language in the material world, London: Routledge. 
Shohamy, Elana/Gorter, Durk (Hg.) (2009): Linguistic Landscape. Expanding the Scenery, New York/London: Routledge.

Wagner, Melanie/Davies, Wini V. (2009): »The Role of World War II in the Development of Luxembourgish as a National Language«, in: Journal of Language Planning and Language Policy, S. 112-131. 


\section{Räume und Identitäten}

Marion Colas-Blaise, Sylvie Freyermuth, Sonja Kmec, Gian Maria Tore, Christian Schulz

\subsection{Einleitung: Die geografischen Diskurse LuXemburgs UND tOURISTISChe Praxis}

Während Fragen der räumlichen Dimension schon lange zentrale Bestandteile der (sozial-)geografischen Identitätsforschung sind, fanden sie erst in den letzten Jahren vermehrt Eingang in geistes- und sozialwissenschaftliche Forschungsansätze (vgl. Döring/Thielmann 2009; Lossau/Lippuner 2004). Parallel zu dieser auch als >spatial turn < bezeichneten Hinwendung zu konzeptionellen Grundlagen der Geografie vollzog Letztere ihrerseits einen >social< oder >cultural turn<, d.h. einen zunehmenden Import von konzeptionellen und methodologischen Perspektiven aus den einschlägigen Nachbardisziplinen. Dies hat insgesamt zu einer weiten Verbreitung diskursanalytischer und semiotischer Ansätze mit dezidiert räumlicher Perspektive geführt. (Vgl. Blunt et al. 2003; Gebhard et al. 2003; Glasze/Matissek 2009; Shurmer-Smith 2002)

Gemäß Weichharts stark sozialpsychologisch inspirierter Konzeption raumbezogener Identitäten (1990), kann zwischen zwei grundlegenden Begriffsverständnissen unterschieden werden: Einmal die >Identität eines Raumes<, d.h. die kognitiv-emotionale Repräsentation einzeln oder kollektiv wahrgenommener Identität eines bestimmten Raumausschnitts (Bsp.: Besonderheiten einer Grenzregion in der Wahrnehmung ihrer Bevölkerung). Zum Zweiten das Verständnis von >Raum als Bestandteil individueller und/oder kollektiver Identitäten $<$ d.h. diejenigen gedanklichen Repräsentationen und emotional-affektiven Bewertungen eines Raumausschnittes, die Teil des individuellen >Selbst- $<$ bzw. kollektiven >Wir-Konzepts werden - oder als >Sie-Konzept< eine Fremdgruppenidentität bestimmen (Bsp.: Die alltagsweltliche Relevanz der Besonderheiten einer Grenzregion bildet die Grundlage für spezifische Gruppenidentitäten, etwa: »Wir/Die Grenzgänger«).

In beiden Fällen sind die physisch-materiellen Ausprägungen des Raumes nachrangig bzw. dienen diese - in ihrer selektiven Wahrnehmung - vor allem als 
Projektionsfläche für Bedeutungszuschreibungen. Letztere konstituieren sich sowohl aus Handlungsmustern als auch aus diskursiver Praxis - beides im Sinne »alltäglicher Regionalisierungen« nach Werlen (1995/1997). Räumliche Identitätsaspekte werden so über soziale Praktiken zum Bestandteil symbolischer Sozialsysteme und damit Teil gesellschaftlicher Konstruktionen der Wirklichkeit. (Berger/Luckmann 1966) Der bewusstseinsprägende Diskurs wird dabei nicht nur durch Elemente sozialer Kommunikation über raumbezogene Phänomene (Sprache inkl. Literatur, Film etc. sowie Karten, statistische Daten u.Ä.) konstituiert. Er kann durchaus auch beeinflusst sein durch materielle Umwelten (z.B. Bausubstanz eines Stadtviertels, Ästhetik einer Landschaftsformation, Verkehrsbelastung), ohne dass diese deterministischen Charakter erlangen. Einbezogen werden auch weitere semiotische Elemente wie Beschilderungen, Flaggen/Embleme, architektonische Stilelemente u.Ä. Angesichts dieses breiten Spektrums von Untersuchungskorpora und -perspektiven betonen die vorgenannten Autoren den Mehrwert interdisziplinärer Untersuchungsdesigns, die den konzeptionellen und methodischen Anforderungen zeitgemäßer Forschungsansätze eher gerecht werden als rein disziplinäre Zugänge.

Das vorliegende Kapitel hat die Aufgabe, sich eingehend mit einer solchen >geografischen< Problemstellung zu beschäftigen, indem es beleuchtet, wie Luxemburg als Raum beschrieben, gezeichnet und verstanden wird: Es geht im vorliegenden Fall darum, genau jene Diskurse zu untersuchen, die den Raum Luxemburg sherstellen städtischer, regionaler, nationaler oder internationaler Raum begriffen? Wie sind die einzelnen Bestandteile dieses Raumes organisiert, wie fügt man diese wieder zu einem Ganzen zusammen, wie nimmt der Raum Autonomie für sich in Anspruch und wie stellt man ihn einer Exteriorität gegenüber? Wie werden seine Wege und Grenzen verstanden und wie werden seine interne Ausdehnung und seine Verbindungen mit anderen Ländern zur Geltung gebracht? Die außergewöhnliche Vielfalt der Diskurse, die diese Aufgabe erfüllen, stellt für eine erschöpfende, selbst für eine nur repräsentative Studie, von vornherein eine große Hürde dar. Es ist jedoch anzumerken, dass die vielen Diskurse sich auf eine begrenzte Anzahl von möglichen Praktiken zurückführen lassen: Der Raumausschnitt Land ist entweder Gegenstand von Sprache oder er wird erst durch Sprache konstituiert, besonders wenn man mit Praktiken aus Politik, Wirtschaft, Bildung und Tourismus zu tun hat. Man debattiert über den Raum, für den geplant wird, über den doziert wird, für den geworben wird. Die in diesem Kapitel entwickelten Analysen konzentrieren sich auf die Darstellung, mit der der Raum in den Diskursen tourismusbezogener Praktiken zur Geltung gebracht wird.

Zwei Hauptgründe haben zu dieser Entscheidung geführt. Zunächst einmal scheint es zu den Wesensmerkmalen von Tourismusdiskursen zu gehören, über den Raum als solchen zu sprechen, während beispielsweise in Politik oder Pädagogik der Raum nur eines von vielen Diskursobjekten ist. Genauer gesagt, der Tourismus setzt, deutlich offener und nachdrücklicher als es die Politik oder die 
Pädagogik tun, auf die Aufwertung, die Definition und Charakterisierung des Raumes. Insgesamt gesehen ist der Tourismus in seiner Eigenschaft als Untersuchungsgegenstand von unmittelbarer Bedeutung, wenn man sein Augenmerk auf die Identitätsfragen richtet, im vorliegenden Fall auf die Dynamik der diskursiven Konstituierung dessen, was >Luxemburg< ist. Denn das Ziel eines solchen Diskurses ist Folgendes: Er wirbt für das Land, während er es beschreiben, erklären, veranschaulichen sollte. Anders gesagt: Er soll von der Bedeutung des Landes überzeugen, während er vorgibt, einen Zustandsbericht zu liefern. Das erklärt die Bedeutung, die die Diskurse der Tourismus-Praktiken für eine Studie über Bedeutungszuschreibungen einnehmen: Sie informieren über etwas, da sie dessen Bedeutung hervorheben, dessen Einzigartigkeit oder dessen Authentizität, indem sie zugleich argumentieren; die >Objektivität< ist hier offensichtlich eine konstruierte, eine vereinbarte.

Letztendlich wurde die theoretische Bedeutung der Studie zu den TourismusDiskursen durch einen Zustandsbericht gesichert: Die Gesamtheit aller Karten, Führer, Broschüren und touristischen Bücher über Luxemburg, die untersucht worden sind, bildet ein stichhaltiges Korpus, das trotz Reichhaltigkeit und Vielfalt seine homogene und genau umrissene Eigenschaft bewahrt hat und damit Stichhaltigkeit gewährleistet. Die dargelegten Untersuchungen stützen sich auf Vorgehensweisen, deren Eklektizismus eine logische Argumentation nicht infrage stellt; sie bilden eine Einheit basierend auf Konvergenzen hin zum finalen Diskurs-Objekt.

\section{Die Ebenen des Diskurses über Luxemburg}

Der vorrangige Grund für die (relative) Heterogenität der Ansätze, denen nachgegangen wurde, um die Tourismus-Diskurse von Luxemburg zu untersuchen, liegt im eigentlichen logischen Aufbau des Korpus. Auf der Grundlage der zu untersuchenden Materialien hat sich rasch herausgestellt, dass Luxemburg auf mindestens fünf unterschiedlichen Diskursebenen >existiert<: Auf der Ebene der Stadtviertel, der Ebene der Stadt als solcher, der Ebene seiner Regionen, auf der Ebene der nationalen Einheit und auf der Ebene seiner Rolle innerhalb der Großregion. Demnach stellen sich die oben erwähnten Fragen (Grenzen, interne Zusammensetzung, erkennbare Wege, Charakterisierungen usw.) jedes Mal anders dar; überdies ändern sich die diskursiven Grundlagen und die jeweilige Textualität. Daraus ergibt sich, dass auch die Ansätze unterschieden und den jeweiligen Korpora und angesprochenen Problemstellungen angepasst werden mussten.

In allen Fällen ging es darum, die Darstellung des Raumes in den Tourismustexten zu untersuchen, d.h., inwiefern die Orts- und Gebietsangaben sinnvoll sind und Aussagekraft besitzen. In diesem Sinne wird die unterste der betrachteten geografischen Ebenen, die der Stadtviertel der Hauptstadt, in den Broschüren und Prospekten des Luxembourg City Tourist Office textualisiert, indem Teile der Stadt Luxemburg anhand von kartografierten Wegen und sprachlichen Beschreibungen gezeigt und gepriesen werden. Insofern hat man auf eine Untersuchung über den 
sich einander bedingenden Zusammenhang von verbaler und visueller Darstellung und unterschwelligen Inhalten zurückgegriffen: Man hat die Instrumente der Semio-Linguistik, aber auch die der Pragmatik und des Äußerungsaktes bemüht; man hat die Übereinstimmungen von >offiziellem « Diskurs (der das epideiktische Genre der Lobrede und das ratgebende Genre der Empfehlung heranzieht) und den >spontanen • Diskursen der im Rahmen von zwei Umfragen (quantitativ und qualitativ) befragten Personen untersucht. Die nächstfolgende geografische Ebene, die die Stadt Luxemburg betrifft, wird vor allem über die Gesamtheit der Stadtpläne erfasst. So ging es hier um eine Studie zur visuellen Semiotik, die tabellenbasierte oder bildbezogene Darstellungsmethoden der Stadt untersucht, d.h. deren Reduzierung auf eine Fläche und deren Visualisierung; dies neben einem manchmal vorhandenen >Quasi-Text<, der aus fotografierten oder gezeichneten Bildern besteht. Die Kohärenz einer solchen Gesamtheit von Visualisierungen, ihrer Beständigkeit und damit ihrer Wirksamkeit wurde mit Fragen zum kommunikativen Stellenwert in Verbindung gesetzt.

Die Ebene der Regionen des Landes wurde über Diskurse angegangen, deren Grundlagen Broschüren mit sprachlichen und bildhaften Inhalten sind und die vom Office National du Tourisme angeboten werden; ihre Bestandteilanalyse stützt sich auf die Stilistik, die Rhetorik und die Diskursanalyse. In vorliegendem Fall hat man dank dieser drei miteinander in Verbindung stehenden Felder deutlich jene Interaktion herausarbeiten können, die Schrift und visuelle Ikonizität mit dem Kern einer Beziehung verbindet, die auf Redundanz und der Generierung von Prototypen beruht. Dies geschieht besonders in einer subtilen Verwendung von Farben und dem Einsatz von hyperbolischen Sprechakten.

Die nationale Ebene wurde anhand eines Korpus untersucht, das aus einer Reihe von Führern besteht, die eine sich fortentwickelnde Darstellung des Großherzogtums Luxemburg widerspiegeln. Damit hat die Untersuchung eine diskursive historische Studie ermöglicht. Die Aufmerksamkeit richtet sich hier auf die Identitäts-Spielarten eines Landes, das von anderen Ländern gesehen wird oder mit diesen in Verbindung steht. Diese Betrachtungsweise hat vor allem eine vielschichtige und oft widersprüchliche Thematisierung von Luxemburg deutlich werden lassen. Letztendlich wurde dann die Ebene der Großregion anhand von mischförmigen Werken erfasst, die eine Art Werbe-Beschreibung unternehmen; es handelt sich dabei um Bücher, die eine >geografische Realität< vertreten, die nicht als anerkannt gilt.

Jede der Ebenen sieht unterschiedliche Themen angesprochen. Themen, deren Aufwertung über die Bereitstellung der argumentativen Strategien läuft. So hat jede Feinanalyse des Kartenmaterials, der Broschüren, der Führer und der sonstigen Bücher berücksichtigen müssen, in welcher Form die Identitäten der Orte entworfen werden, im Gegensatz zu der Form, in der die Bewohner des Landes angeben, sich diese Orte anzueignen. Oder anders gesagt, wie die Bewohner eine Vorstellung von diesen Orten entwickeln, wie sie sie charakterisieren und wie sie ihnen einen Stellenwert in ihrem Leben zuweisen. Um sich den letzten Aspekt zu vergegenwärtigen und um die Studien über die Tourismus-Textualitäten zu dialek- 
tisieren, hat jede der auf den fünf Ebenen durchgeführten Untersuchungen die Ergebnisse der qualitativen und quantitativen Erhebungen aufgegriffen, die mit den Einwohnern Luxemburgs durchgeführt wurden.

\subsection{ZWISCHEN VERFÜHRUNG UND IWIRKLICHKEIT: Die Stadt LUXembURG UND IHRE DisKURSE}

»Le mode selon lequel j'écris - ou même rêve - l'espace, rien ne«, schreibt François Wahl (1980: 46), »saurait en dire plus long sur le mode que j'ai d'habiter mon lieu «. Wie verwandeln die im Rahmen der quantitativen Erhebung befragten Personen den Raum der Stadt Luxemburg und deren Stadtviertel in einen >Ort<? Worin unterscheiden sich die >spontanen < Diskurse, die bei den qualitativen Befragungen ebenfalls erfasst werden und die einen Zugang zu angeeigneten Identitäten geben, von den Darstellungen, die vom offiziellen Tourismus-Diskurs, dem Zubringer für zugeschriebene Identitäten, vermittelt werden? Eher auf die Ausländer zielend versucht Letzterer Wissen zu vermitteln, aber auch etwas spürbar werden $\mathrm{zu}$ lassen und Handlungen $\mathrm{zu}$ initiieren, indem er $\mathrm{zu}$ einem pragmatischen Verhalten aufruft.

Die Überlegungen zu dieser Fragestellung gliedern sich in zwei Bereiche: (1) Wir werden die einzelnen Antworten auf die Frage nach den »Orten, die man ausländischen Freunden vorrangig zeigt« und die Analysevorgaben zur Stadt Luxemburg vergleichen, die in zwei Broschüren und zwei Prospekten - herausgegeben vom Luxembourg City Tourist Office ${ }^{2}$ - angeboten werden; (2) Wir werden die Bilder der Stadtviertel Bonnevoie, Grund, Limpertsberg und Kirchberg, so wie sie von den interviewten Personen beschrieben werden, mit den entsprechenden offiziellen Texten ${ }^{3}$ vergleichen.

Zu diesem Zweck bemühen wir die Bereiche der Semiotik, der Rhetorik, der Pragmatik sowie die der linguistischen Theorie des Äußerungsaktes. Um zu zeigen, wie der lobende Tourismus-Diskurs das Bild einer Stadt prägt, muss man im Hinblick auf seine verbalen und visuellen Äußerungen die Übereinstimmung zwischen Inhaltsebene und Ausdrucksebene untersuchen.

1 | Deutsch: "Die Art, in der ich den Raum erschreibe - oder sogar erträume - nichts könnte über die Art, in der ich meinen Ort bewohne aussagekräftiger sein."

2 | Wir stützen uns auf die Broschüren "Luxembourg la ville, bonjour!", "Luxemburg, Vivez la ville!" sowie auf zwei Prospekte: "Panorama City Map" und "City Promenade".

3 | Siehe die Prospekte "Le circuit des roses du Limpertsberg 'RosaLi « und "Luxembourg, une capitale européenne". Im Rahmen dieser Studie beziehen wir uns auf die jeweils französischsprachige Version. 


\section{Wenn man mir die Stadt Luxemburg zeigen würde ...}

Die im Rahmen der quantitativen Untersuchung gestellte Frage impliziert auf Anhieb eine selektive< Strategie (Fontanille 1999: 41-61): Der Blickwinkel, aus dem heraus bestimmte Aspekte eines Landes wahrgenommen werden, der die Interaktion zwischen dem Subjekt und dem infrage kommenden Objekt bestimmt, entscheidet über das >bestmögliche Beispiel<, den Beitrag, der durch seine ihm innewohnende >Strahlkraft< repräsentativ ist, den sinnbildlichen Ort, der für alle anderen steht. Es geht dabei nicht darum, sich mit einem einzelnen Detail zu begnügen, selbst wenn dieses als malerisch betrachtet wird, auch nicht darum, Überraschung zu erzeugen, vielmehr soll das Element bestimmt werden, das sich mit einem >identifizierenden < Wert verbinden lässt: So soll dieses Element, das den Glanz des Spektakulären mit der Verankerung in der Zeit vereint, dem Fremden gegenüber eine Einzigartigkeit hervorheben und einen >Wiedererkennungswert< schaffen.

Es ist nicht unwichtig, dass $71 \%$ der befragten Personen allgemein die Stadt Luxemburg dem Rest des Landes ${ }^{4}$ vorziehen oder in der Reihenfolge Altstadt (51\%), Grund/Corniche (7\%), den Großherzoglichen Palast (5\%), die Bockkasematten/Clausen (3\%), das Viadukt über die Pétrusse, die Kathedrale, die Museen/Philharmonie, den Kirchberg und das Monument der Gëlle Frau (jeweils $1 \%)^{5}$ auswählen. Ohne die angesichts der Abwesenheit von signifikanteren Unterschieden bestehende Gefahr einer Überinterpretation zu verkennen, stellt man fest, dass man mit der Hervorhebung mehreren Dimensionen begegnet und die Identitätsauswirkungen einer Ethik und einer Ästhetik unterwirft. Tatsächlich zeigen sich gewisse Tendenzen: Die Wahl fällt auf die Breite der geschichtlichen Vergangenheit (die Wiege der Stadt als UNESCO-Weltkulturerbe, aber auch, allerdings weniger ausgeprägt, die Gëlle Fra), bevor sie sich der Gegenwart zuwendet (nämlich dem Kirchberg); Tradition, Beständigkeit und Wille zur Vereinigung (etwa am Beispiel des Großherzoglichen Palastes oder der Kathedrale) scheinen allgemein über die Moderne und die Öffnung gegenüber Europa zu

4 | Vianden liegt bei $6 \%$, die Kleine Luxemburgische Schweiz/Müllerthal bei $3 \%$, Nordluxemburg (Eisleck), Remich/die Mosel, Echternach, die Obere Sauer und Südluxemburg bei jeweils $2 \%$; Diekirch und Clervaux erreichen $1 \%$.

5 | Berücksichtigt man das Alter, stellt man fest, dass diese Orte von $74 \%$ der 60 -Jährigen und älter gewählt werden; Frauen sind dabei in der Überzahl. Junge Leute (16 bis 20 Jahre) und Personen im Alter von 30 bis 44 Jahre liegen auf der zweiten Position (68 \%). Man stellt weiterhin fest, dass diese Orte von $77 \%$ der Personen genannt werden, die dem statusorientierten Milieu zuzurechnen sind, gegenüber 73 \% für das konservativ-gehobene Milieu, 72 \% für das liberal-gehobene Milieu, 71 \% für das kleinbürgerliche Milieu und 70 \% für das aufstiegsorientierte Milieu; das alternative Milieu erreicht 69 \%, während das unterprivilegierte Milieu auf 67 \% kommt; das hedonistische Milieu (62 \%) liegt vor dem traditionsorientierten Milieu (58 \%). 
siegen; die Schönheit der Landschaft (die Pétrusse und das Viadukt) und künstlerische Schwerpunkte (die Museen, die Philharmonie) werden in gleicher Weise hervorgehoben.

Der Vergleich dieser Daten mit dem Tourismus-Diskurs muss die pragmatische Zielsetzung der verschiedenen von offiziellen Einrichtungen herausgegebenen Broschüren und Prospekte berücksichtigen. Diese äußern sich, da sie ähnlich wie Marketingtexte für die Stadt werben sollen, in »deux genres rhétoriques de discours $\aleph^{6}$, wie Marc Bonhomme es nennt:

[...] deux genres rhétoriques de discours. D’une part, le discours épidictique, fondé sur le macro-acte de l'éloge et axé sur le présent de la célébration du produit proposé. D'autre part, le discours délibératif, [...], qui repose sur le macro-acte du conseil en vue d'une décision future du récepteur quant à l'achat/utilisation du produit en question. ${ }^{7}$ (Bonhomme 2003: 13)

In dieser Eigenschaft bemühen sie drei Äußerungsakte bei der grundlegenden >Schematisierung der Stadt: Neben der Auswahl und der Versinnbildlichung, die beide auch von der quantitativen Erhebung beabsichtigt sind, erhöht die $>$ Rationalisierung die Erkennbarkeit des Gebietes; sie hat als thymisches Korrelat das Gefühl, das Gelände zu beherrschen und ein Gefühl von Sicherheit. (Bonhomme 2003: 14-15)

Da die Broschüren »Luxembourg la ville, bonjour!« und »Luxembourg, Vivez la ville! « sich an potentielle >Konsumenten richten, verbinden sie elektive und einschließende Strategien: Indem sie die Angriffsflächen vielfältig gestalten, versuchen sie weniger, alle Aspekte aufzuzählen und Vollständigkeit zu erreichen, als vielmehr, zwischen dem Detail, das aufhorchen lässt, und der Einbettung in eine Gesamtheit, die die Schaffung von Kohärenz begünstigt, zu pendeln. Es geht zugleich darum, den Besucher die Stadt >mit einem Blick < erfassen zu lassen und ihn, indem man aus den Orten eine kognitive, aber auch eine pathematische und somatische Erfahrung macht, also eine affektive und eine physische, ihn einzuladen, sich die angebotene > Realität< anzueignen. So ist auch der allgemeine Aufbau der Broschüren zu verstehen, die, indem sie einen vorgezeichneten $\mathrm{Weg}^{8}$ beschrei-

6 | Deutsch:"zwei rhetorischen Diskurs-Genres".

7 | Deutsch: "[...] zwei rhetorischen Diskurs-Genres. Einerseits dem epideiktischen Diskurs, der sich auf den Makro-Akt des Lobes stützt und sich auf die in der Gegenwart angesiedelte Würdigung des angebotenen Produktes richtet. Andererseits im ratgebenden Diskurs [...], der auf dem Makro-Akt der Empfehlung im Hinblick auf eine zukünftige Entscheidung hinsichtlich des Kaufes/der Verwendung des infrage kommenden Produkts beruht."

8 | Siehe die Umschlagseite von "Luxembourg, Vivez la ville!", die mit einem Pfeil eine Erwartungshaltung in Richtung Innenseiten der Broschüre weckt. 
ben, im Einleitungstext oder auch auf den Umschlagseiten ${ }^{9}$ paradigmatisch zusammengetragene Punkte umfassend abarbeiten. Man kann so feststellen, dass im Hinblick auf die verwendeten Inhalte systematisch und immer wieder aufs Neue antithetische Begriffspaare verwendet werden: Vergangenheit und Gegenwart, Tradition - im Zusammenhang mit Volksfesten beispielsweise - sowie Modernität, Kultur und Natur, Kunst und Finanzwesen bzw. Handel, Nationales und Internationales. Dabei bemüht der Sprecher die axiologischen Sphären des Guten, des Schönen und des Wahren. Auf der Ausdrucksebene passen Systematisierung und Ästhetisierung zusammen: Eher deskriptiv oder auffordernd vervielfachen Titel, Vorspanne und Hingucker die rhetorischen Mittel (Metapher, Verallgemeinerung, Hyperbel); sie werden von der Farbgestaltung ${ }^{10}$ unterstützt, von ganzseitigen Abbildungen oder kleinen Zierbildern, die neben kleinen Textfeldern stehen, ebenfalls bemüht, der Logik des lobenden Diskurses ${ }^{11}$ zu dienen, besonders über bewertende Adjektive und Zitate bekannter Personen.

Was sodann auffällt, ist die große Übereinstimmung zwischen den von den befragten Personen genannten Präferenzen und der vom Tourismus-Diskurs vorgenommenen Auswahl. ${ }^{12}$ Letzterer erwähnt nicht nur alle mündlich ausgewählten Identifizierungs->Kandidaten<, sondern er verstärkt deren Unterscheidungswert

9 | Die vier Bildbänder auf der ersten und vierten Umschlagseite der Broschüre "Luxembourg la ville, bonjour! « zeigen das Riesenrad, das Museum für Stadtgeschichte der Stadt Luxemburg, das Staatsarchiv mit der Corniche und einen Teil von Grund und der Alzette.

10 | Die Broschüre "Luxembourg la ville, bonjour! « wählt für das jeweils besprochene Thema eine andere Farbe.

11 Siehe vor allem folgende Beispiele: "Gibraltar des Nordens", "Ein Finanzplatz internationalen Ranges" (vgl. "Luxembourg la ville, bonjour!"), "Massives Mauerwerk, Welterbe der UNESCO. Erleben Sie 1000 Jahre Festungsgeschichte!", "Internationaler Treffpunkt. Erleben Sie eine Finanzstadt, Kongresse und eine dynamische Wirtschaft!“, "Die Wiege der europäischen Einigung. Erleben Sie eine weltoffene Hauptstadt!", "Feste und Traditionen. Erleben Sie Veranstaltungen à gogo!", "Ausflüge in das 'grüne Herz.. Erleben Sie die Natur in Reinform!“ (Vgl. "Luxembourg, vivez la ville! ") Zahlreich sind übrigens die subjektiven Adjektive, die auf den (anonymen) Sprecher verweisen und die Zustimmung, die Einwilligung des Lesers oder der Leserin vorwegnehmen: "[...] Sie werden, lieber Besucher, herrliche Wanderungen unternehmen ", "Der Entwurf für einige der bemerkenswertesten Bauten der Hauptstadt stammt von anerkannten Architekten." (Vgl. "Luxembourg la ville, bonjour!")

12 Siehe Linden \& Thewes zum Thema einer "Übereinstimmung von Tourismus-Werbung und Werbung für das Land, von 'Premium`-Sehenswürdigkeiten und idealisierter Selbstwahrnehmung". Sie fügen hinzu: "Die Darstellungen beeinflussen nicht nur die Sichtweise der Touristen. Sie bestimmen letztlich auch die Wahrnehmung, die die Luxemburger von ihrem eigenen Land haben, von dessen Vergangenheit und dessen Geografie." (Linden/ Thewes 2007: 44) 
noch, indem er Abbildungen anbietet. ${ }^{13}$ Vor allem die beiden Broschüren beginnen mit großformatigen Fotos der Corniche und der illuminierten Altstadt (verteilt über mehr als eine DIN-A4-Seite) ${ }^{14}$. Wenn die Variablen, die als für den >Stil< der Stadt Luxemburg grundlegend betrachtet werden, hier und dort eingestreut werden, so sind sie bezeichnenderweise nicht nur darauf abgestimmt, gleich zu Beginn die Kontraste herauszustellen, die als bedeutsam anzusehen sind (»kleines Land, große Anziehungskraft«, vgl. »Luxembourg la ville, bonjour!«; »Vivez une ville pleine de contrastes!«; »Luxembourg, Vivez la ville!«), sondern darüber hinaus auch den Relikten der Vergangenheit einen privilegierten Platz einzuräumen, indem sie etwas Erzählerisches oder sogar Fiktionales ${ }^{15}$ anregen. Die Tatsache, dass die Wahl der einen und die Wahl der anderen quasi auf einer Wellenlänge liegen, kann den Eindruck entstehen lassen, dass gegenüber dem Fremden die Idealisierung über inszenierte Überreste einer bewegten, ja sogar spektakulären Geschichte auf breitem Konsens beruht: Die Geschichte und mit ihr eine tatsächliche Inszenierung vom Werden der Stadt, Schritt für Schritt wieder aufgebaut anhand der Umrisse und Schichtungen, scheint als ein sehr überzeugender > Zugang < betrachtet zu werden.

Als letzter Beweis für diese übereinstimmende Sichtweise kann der Prospekt mit dem Titel »City promenade« angesehen werden. Nicht nur hält sich die einleitende Aussage an die Regel, indem sie sich für den historischen Bericht entscheidet und so nebenbei altbekannte Metaphern bemüht - »die Wiege der Stadt Luxemburg«, das »europäische Machtspiel« - oder von Historikern/-innen eine Formulierung übernimmt, die ins Schwarze treffen soll (»Gibraltar des Nordens«). Vor allem der Stadtplan auf der zweiten und dritten inneren Bildtafel stellt die >Wirklichkeit< wieder her, (vgl. Marin 1994) indem er Aufwertungen zeigt: Für einige Orte und Gebäude der Innenstadt bietet er nämlich eine perspektivische Darstellung an, die mit den übrigen flächigen Zeichnungen kontrastiert. Im Wesentlichen handelt es sich dabei um Orte, die die befragten Personen spontan genannt haben: Die Bockkasematten, die Corniche, der Grund, der Großherzogliche Palast, die Kathedrale Notre Dame, das alte Jesuiten-Kolleg und das Monument der Gëlle Fra. Die hohe Dichte von Referenzen verstärkt so den empfehlenden Eindruck und ruft, indem eine (Wieder-)Erkennung ermöglicht wird - etwa die, dass man nicht allgemein eine Kathedrale vor sich hat, sondern die von Luxemburg -, eine sinnli-

13 | Jedoch nimmt die "Panorama City Map" auf der Ebene der Abbildungen weder die Place de la Constitution noch die Gëlle Fra auf.

14 | In der Broschüre "Luxembourg la ville, bonjour!" ragt das kleinere Foto der Corniche und der Altstadt über ein Band mit zwei kleinformatigen Fotos hinaus; das erste zeigt die Place de la Constitution mit der Gëlle Fra, das zweite das Rathaus.

15 | So kann man in der Broschüre "Luxembourg la ville, bonjour! “ lesen: „Früher hallten in den verwinkelten Gassen der Kanonendonner und das Klirren der Schwerter. Heute können die Besucher auf den Spuren listiger Eroberer und heldenhafter Verteidiger wandeln [...]." 
che Vorstellung hervor. Schon vor jeder Begriffsbildung kann die Aufmerksamkeit auf die plastischen Eigenschaften des >konkreten Bildes $<$ fokussiert werden.

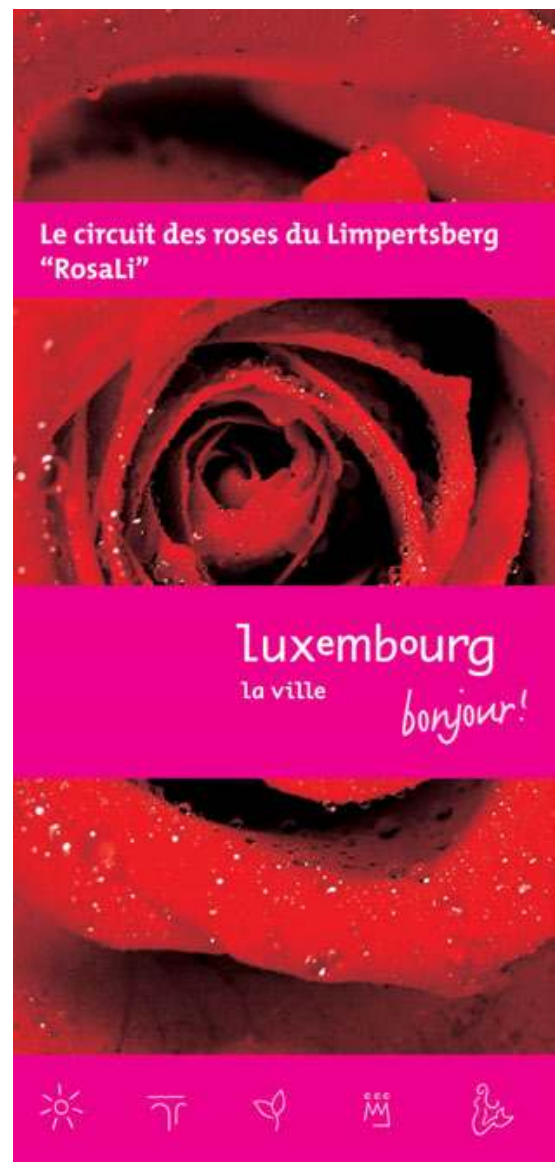

Abbildung 1: Le circuit des roses du Limpertsberg >RosaLi«« (Deckblatt), mit freundlicher Genehmigung des Luxembourg City Tourist Office

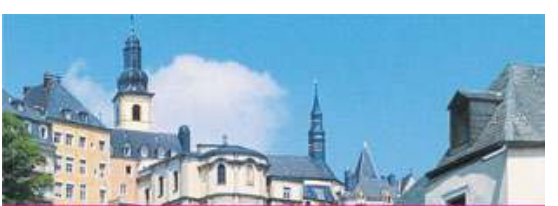

City promenade

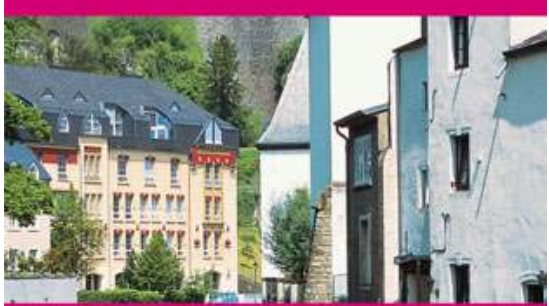

luxembourg die stadt bonjour!

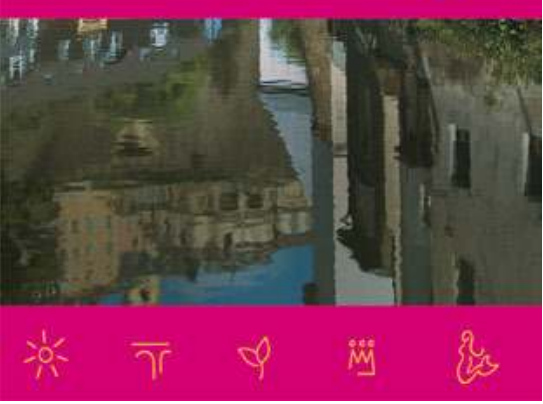

Abbildung 2: City promenade (Deckblatt), mit freundlicher Genehmigung des Luxembourg City Tourist Office

\section{Das Stadtzentrum und sein >Darüberhinausı}

Wie benennt man den Raum jenseits der Schienen, des Grüngürtels, der Pont Grande-Duchesse Charlotte oder der Oberstadt? Wir vergleichen für die Stadtviertel Bonnevoie, Limpertsberg, Kirchberg und Grund ${ }^{16}$ den Tourismus-Diskurs mit

16 | Im Gegensatz zu Limpertsberg und Kirchberg ist Bonnevoie nicht Gegenstand einer eigenen Broschüre oder eines Prospektes, herausgegeben vom Luxembourg City Tourist 
den >spontanen « mündlichen beschreibenden Diskursen. Es lassen sich bedeutsame Akzentverschiebungen erwarten: Die >spontanen< Diskurse reflektieren eher etwas >Erlebtes $<$, angeeignete Identitäten, und umgehen zumindest teilweise die Rhetorik des Lobes, die von den offiziellen Stellen gerne verwendet wird und dadurch Identitäten zuschreibt.

Man trifft zunächst auf eine doppelte Trennlinie: Die äußere erlaubt eine Unterscheidung zwischen den Wohnvierteln (Bonnevoie und Limpertsberg) und jenen Vierteln, die man eher als Durchgangsviertel oder Viertel der Begegnung bezeichnen könnte (Grund und Kirchberg); innerhalb der Wohnviertel wird auf der Grundlage einer räumlichen Differenzierung jeweils zwischen zwei Zonen unterschieden: einmal der obere Teil und der untere Teil von Bonnevoie, zweitens Limpertsberg und das Glacis; Letzteres schließt sich - wegen seines Jahrmarktsgeländes, den Bars, Cafés, Restaurants, wegen des Kinos oder des Theaters als NichtWohnviertel betrachtet - eher dem Raum der Begegnung und der Durchmischung der Bevölkerung an.

Betrachten wir zuerst das Viertel Bonnevoie, das in unserem Tourismus-Diskurs nicht erwähnt wird. Mit großer Beständigkeit geben die befragten Personen begriffliche Paare an, deren antithetische Elemente sich sozusagen automatisch ergeben: Der untere Teil von Bonnevoie ist für sie ein Viertel des einfachen Volkes, teilweise etwas heruntergekommen, ein Ort der Durchmischung, mit älteren Menschen, Ausländern (Portugiesen, Afrikanern ...), Obdachlosen; für manche ist es ein Ort der Prostitution, Drogen und Kriminalität, während für andere das Viertel ein Leben in Gemeinschaft begünstigt. Hingegen wird der obere Teil von Bonnevoie als das genaue Gegenteil beschrieben: Großbürgerlich, neu gestaltet, wird dieser Teil, so sagt man, von Personen aller Altersgruppen bewohnt, dem Bürgertum zugehörig und meist luxemburgischer Staatsangehörigkeit. Darf man vermuten, dass dieses trennscharfe Bild weniger die >Wirklichkeit< abbildet als vielmehr das Wiederholen von starren Repräsentationen? Manche der befragten Personen schließen nicht aus, dass sie so von den Medien benutzte Stereotype verwenden.

Was den Limpertsberg betrifft, so wird er in der kollektiven Vorstellung vornehmlich als Wohnviertel betrachtet, dessen Grünflächen die Lebensqualität steigern, bürgerlich, chic und teuer, nahe am Zentrum gelegen, von unerwarteten Veränderungen verschont. Am Wochenende (zu) ruhig, zeigt sich das Viertel durch zahlreiche dort ansässige Schulen an den Wochentagen lebhaft.

Im Gegensatz zu anderen Wohnvierteln ist dem Limpertsberg ein Tourismusprospekt gewidmet: »Circuit des roses du Limpertsberg >RosaLi««. Der Titel ist bezeichnend für ein Bestreben nach Ästhetisierung, das die anderen Facetten nicht

Office. Das Stadtviertel wird auch nicht in den Broschüren "Luxembourg, Vivez la ville!» und "Luxembourg la ville, bonjour! « erwähnt. (Vgl. Stadtviertel Grund) Wenn wir die verschiedenen Stadtviertel untereinander vergleichen, verwenden wir die Kriterien auf der Grundlage der vorgenommenen Auswahl. 
verhüllt, sondern sie in eine bestimmte Perspektive stellt. ${ }^{17}$ Zunächst arbeitet der einleitende Text zur Kultur der Rosen im 19. Jahrhundert mit einer objektivierenden Distanznahme (>débrayage`); dann regen der als Plan gezeichnete Rundgang, die verbale Schlussbemerkung, die die aufgeführten Punkte erläutert, aber auch die groß- und mittelflächigen Abbildungen den Besucher dazu an, sich den Raum nicht nur über eine kognitive Vorstellung, sondern auch über eine somatische, pathematische und ästhetische anzueignen. ${ }^{18}$ Die Karte und der ermunternde Diskurs verkörpern ein >Mittel im Hinblick auf die auszuführende Handlung, die die Leistung der Besucher oder Besucherinnen darstellt, wenn diese ihren Weg in Übereinstimmung mit den Vorgaben des Plans nehmen und ihn so zu einer Route machen.

Sollte man den Unterschied zwischen der so im Prospekt zugeschriebenen Identität des Viertels und der erlebten Identität kritisieren? Sie entsprechen unterschiedlichen Orts-Erfahrungen, aber in beiden Fällen knüpft das Individuum eine enge Beziehung zum Raum.

Was das Kirchberg-Plateau betrifft, so wird es, im Gegensatz zu den Wohnvierteln des >alten « Kirchberg, zu Weimerskirch oder zu Weimershof, als weit vom Zentrum entfernt betrachtet, als teuer, unpersönlich, tagsüber überfüllt, abends verlassen, kurz als wenig wohnlich betrachtet. Im Gegenzug werden die europäischen Einrichtungen hervorgehoben, die internationale Bedeutung von RTL ${ }^{19}$ ebenso wie die Banken, in gleicher Weise wie die konsumrelevanten Orte (Supermarkt Auchan, Messegelände) und die Unterhaltungsmöglichkeiten (Kino). Die Philharmonie und das MUDAM $^{20}$ verleihen dem Viertel eine nicht zu leugnende künstlerische Qualität, auch wenn etwas mehr Platz die Gebäude noch besser zur Geltung bringen würde.

So scheint das Kirchberg-Plateau seinen festen Platz im Alltag ${ }^{21} \mathrm{zu}_{\text {haben, auch }}$ wenn es einem ständigen Kommen und Gehen ausgesetzt ist und sich seine Form für ein Wohnviertel nicht eignet. Punktuell kann der Kirchberg als Vermittler auftreten, sogar als Austauschpunkt zwischen den Ausländern, die in den Büros der europäischen Institutionen arbeiten, und dem Rest der Bevölkerung.

Die europäische Dimension behandelt der Prospekt »Luxembourg, une capitale européenne ${ }^{22}$ auf Kosten der anderen: Der lobende Diskurs verbirgt den $\gg n i c h t-$

17 | So wird z.B. der Campus der Universität als "Campus des roses" erwähnt.

18 | Siehe den vereinheitlichenden farblichen Reim, den das kräftige Rosa für die Karte und die Einrahmung der Fotos bildet.

19 | Radio Télévision Luxembourg.

20 | Musée d'Art Moderne Grand-Duc Jean.

21 | Das Krankenhaus wird mehrfach erwähnt. Einige Personen heben die Grünanlagen hervor.

22 | Wir zählen zwölf Stationen: Das Rathaus, der Cercle Municipal, das Europahaus, das Robert-Schuman-Geburtshaus, der Rat der Europäischen Union, das Europäische Parlament, der Europäische Rechnungshof, die Europäische Schule, die Europäische Kommissi- 
europäischen Teil, dessen Bedeutung die befragten Personen jedoch hervorgehoben haben. Um seine Besonderheit besser zu verstehen, vergleichen wir ihn mit »Circuit des roses du Limpertsberg >RosaLi«« im Hinblick auf die Positionierung der Karte, der Abbildungen und Personen-Marker.

Während in dem Prospekt »Circuit des roses du Limpertsberg >RosaLi«« die Karte von Textpassagen umschlossen ist, befindet sie sich bei »Luxembourg, une capitale européenne « am Ende der Broschüre, grafisch deutlich durch eine kräftige Linie begrenzt; sie wird von einer Liste mit Ortsnamen begleitet. Verbindet man die Darstellungsebene mit der Inhaltsebene, kann man davon ausgehen, dass die Karte in »Circuit des roses du Limpertsberg >RosaLi«« aktiv zur Vorstellung des Raumes beim Spaziergänger oder der Spaziergängerin beiträgt, indem die Karte immer wieder neue Möglichkeiten vorschlägt und der Begleittext die sehenswerten Elemente aufzählt. Bezeichnenderweise stimmt der Endpunkt des Rundweges nicht mit dem Beginn überein. Der Weg ergibt sich aus dieser Öffnung und der daraus resultierenden Spannung.

In »Luxembourg, une capitale européenne« bietet die Karte zusammenfassend Zugang zu einer objektiven >Gegebenheit<. In einer Gesamtansicht von oben - es geht darum, einen »Überblick über die Europäische Union « zu geben - und damit auch mit der Möglichkeit, den Raum kognitiv zu beherrschen, übernimmt die Karte eine vornehmlich didaktische Rolle: Sie zielt darauf ab, das vorzustellen, was sich mit der Kraft der Offenkundigkeit aufdrängt. Man ist nicht überrascht, dass das Ende der Tour mit ihrem Startpunkt übereinstimmt; der Rundweg schließt sich und der Initiative des reisenden Lesers sind Grenzen gesetzt.

Dieser Eindruck wird durch die Abbildungen verstärkt. Die Prospekte enthalten Bildbänder, die in »Circuit des roses du Limpertsberg >RosaLi«« am unteren Seitenrand und in »Luxembourg, une capitale européenne« am oberen Rand gesetzt sind. In beiden Fällen ist die Funktion der Fotos grundverschieden. Der sechsseitige Faltprospekt »Circuit des roses du Limpertsberg >RosaLi«« ermöglicht es dem spazierenden Leser, die Fotos in ihrer Bewegung zu entdecken und seinen Blick beständig anzupassen. ${ }^{23}$ Der Leser ist unterwegs über seine Betrachtung direkt an der Erarbeitung des Weges beteiligt, der sich nicht gleichförmig gestaltet, sondern Wechsel in Tempo und Spannung enthält. (Vgl. Fontanille/Zilberberg 1998; Zilberberg 2006)

Der Prospekt »Luxembourg, une capitale européenne« hingegen präsentiert sich wie ein Büchlein, dessen Seiten in der Mitte sorgfältig geheftet sind. Die meis-

on, der Europäische Gerichtshof, die Europäische Investitionsbank, das Robert-SchumanDenkmal.

23 | Man kommt von der Großaufnahme einer Rose zu halbnahen Aufnahmen (z.B. dem oberen Teil eines Grabmals), aber auch zu halbtotalen (eine Skulptur) oder Totaleinstellungen, das Zentralgebäude der Universität als herausragendes Element, dann wieder eine Großaufnahme, diesmal unmittelbar gefolgt vom Foto eines Wasserturms. Auch im weiteren Verlauf zeigt der Prospekt diesen Wechsel von Großaufnahmen und Totaleinstellungen. 
ten Abbildungen entsprechen Aufnahmen in der Totalen oder Halbtotalen (zu sehen sind Gebäude mit oder ohne angrenzende Umgebung), die vor allem eine beschreibende Funktion ausüben und dem Blick keine Dynamik abverlangen.

Schließlich wird die Analyse noch besonders durch die Personen der Verbformen bestätigt. (Vgl. Benveniste 1966) Durch die Verwendung des > Sie < im französischen Text als Anrede eines Ansprechpartners und durch die vielfachen Empfehlungen im Imperativ bekommt der Diskurs des Prospekts »Circuit des roses du Limpertsberg >RosaLi « einen deutlich ermunternden und auffordernden Charakter: Es geht darum, den Leser mit der erforderlichen Kompetenz zu ermutigen, den Raum >neu zu erfinden $<$, und diesem durch eine andersartige Kennzeichnung eine Form zu geben. Im Gegensatz dazu bemerkt man bei dem Prospekt »Luxembourg, une capitale européenne«, dass das Pronomen >wir< vorherrscht (»Au rond-point, nous pouvons décider de parcourir le chemin d'environ $2 \mathrm{~km}$ menant, à travers le

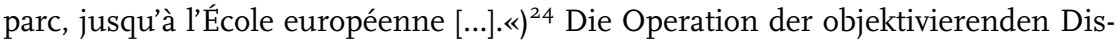
tanznahme (>débrayage <), der Lösung aus der >Ichく-Sphäre und die Distanz-Schaffung wird durch eine streng auf die erste Person bezogene Rückbindung blockiert: Nach Émile Benveniste »drückt das >wirく, das dem >ich < eine ununterscheidbare Gesamtheit anderer Personen hinzufügt [...] eine vergrößerte und ungenaue Person aus «25 (Benveniste 1966: 235). Demnach befinden sich der Verfasser der Broschüre und der Leser in einem Beziehungsnetz, das zwischen beiden den Konsens sicherstellen soll, ja sogar Einverständnis und Empathie. Insbesondere die Verwendung des Infinitivs (»Quoi de plus logique que de commencer au tout début, dans le système scolaire? $\ll^{26}$ ), der weder Zeit noch Person in sich trägt, begünstigt eine enunziative In-Differenzierung, die dem Äußerungsadressaten keine andere Wahl lässt, als sich den Standpunkt des sich Äußernden zu eigen zu machen, Zustimmung durch enunziative Kontinuität. Obschon die europäischen Institutionen eine Öffnung anstreben, versucht der Prospekt den Leser oder die Leserin in einem diffusen >wir < festzuhalten: Anstatt die Leser auf ihrem Rundgang zu begleiten, anstatt das Objekt >europäische Stadt< Schritt für Schritt aufzubauen, gibt er vor, etwas >schon Gegebenes $<\mathrm{zu}$ beschreiben, dessen unterschiedliche Facetten er aufzählt. Die Karte liefert dazu einen Gesamtüberblick, sozusagen unabhängig von der Besichtigung selbst. Nunmehr geht es darum, sich im Inneren einer durch visuelle und verbale Äußerungen eng umgrenzten Sphäre zu situieren; vielleicht kann sich das Imaginäre aus dieser Position heraus entfalten. Tatsächlich kann man angesichts des >wir<, das den mit dem Tourismus-Diskurs betrauten Sprecher und den reisenden Leser umfasst, von einem nicht-dargestellten Adressaten aus-

24 | Deutsch: „Im Kreisverkehr können wir uns entscheiden, ob wir den etwa 2 km längeren Weg durch den Park bis zur Europäischen Schule gehen [...]."

25 | Originalzitat: "[Le ’nousı] annexe au 'je ‘ une globalité indistincte d'autres personnes [...] exprime une personne amplifiée et diffuse."

26 | Deutsch: "Was lag da näher, als ganz am Anfang anzusetzen, nämlich beim Schulsystem?" 
gehen: Die Adressaten gehören Europa an und sind einer Welt zuzuordnen, der sich das > Wir < gerade eben vorstellt.

Diesen kurzen Überlegungen wird man entnehmen können, dass, auch wenn keine perfekte Übereinstimmung zwischen der Meinung der interviewten Personen und den entsprechenden Prospekten, zwischen den angeeigneten und den zugeschriebenen Identitäten besteht, der >Grundakkord<stimmt: Die Erfahrung, zu der der spazierende Leser in Limpertsberg gelangt, vermittelt etwas von der Beziehung der vertrauten Komplizenschaft, die den oder die Bewohner/-innen mit seinem/ihrem Wohnort verbindet; ähnlich strebt der Prospekt »Luxembourg, une capitale européenne«, indem er das >Sie< (>vous $<$ ) im >wir < mit einschließt und dem Empfänger die Möglichkeit von Exteriorität verwehrt, einen Konsens an, sogar eine Empathie gegenüber dem Adressaten, der nicht explizit formuliert wird (Europa und die Welt); das widerspricht sicher auch nicht dem Wesen des Kirchberg, der spontan oft als ein internationaler und zugleich unpersönlicher Ort erlebt wird.

Zum Abschluss zeigen sich beim Stadtviertel Grund deutliche Übereinstimmungen der Ansichten: Auch außerhalb der >Blues 'n' Jazz Ralley< lädt die Unterstadt mit ihren Terrassen ein zu einem »Moment der Entspannung« (vgl. »Luxemburg, Vivez la ville! «); die befragten Personen bestätigen dies auf breiter Ebene und heben weniger den Wohncharakter als vielmehr die gastliche Seite dieses sanierten Stadtteils hervor, der viele Ausländer (oft anglofone) anzieht. Teilweise betrachtet man auch das kulturelle Angebot als herausragendes Merkmal, ebenso wie die Schönheit des Viertels, die zu Spaziergängen einlädt.

Zum Abschluss dieser kurzen Betrachtungen stellt man zwei Dinge fest. Einerseits gehören die Logik des Tourismus-Diskurses und das Stattfinden dieses Diskurses, wenn jemand einem ausländischen Freund die Stadt Luxemburg zeigt, dem gleichen Makro-Akt der lobenden Positivierung, genauer noch, dem der Ästhetisierung an. Es ist deutlich geworden, dass die hier und da gewählten Präferenzen zum großen Teil übereinstimmen, und dass zumindest für einen Teil der Bevölkerung die Festung und die historische Altstadt zur >1. Wahl< gehören. Angesichts der Tatsache, dass die befragten Personen nicht in derselben Lage wie der Sprecher des Tourismus-Diskurses sind, waren im Hinblick auf die Stadtviertel andererseits gewisse Abweichungen zwischen reichhaltig ausgefülltem Alltagsleben und tourismusbezogener Schematisierung vorauszusehen. Man kann vor allem festhalten, dass die dem Limpertsberg und dem Kirchberg gewidmeten Prospekte einmal ästhetische Kriterien herausstellen, im anderen Fall die Gegensätze von >Tradition und Moderne<, >national und international<, die den offiziellen Diskurs über den Kirchberg untermauern. Das Stadtviertel Bonnevoie fällt aus dieser Charakterisierung heraus, es gehört nicht zu den >sehenswerten < Orten.

In jedem Fall besitzen die Diskurse ihre eigene Kohärenz. Ob es sich um zugeschriebene oder angeeignete Identitäten handelt, letztlich schaffen sie, jede auf ihre Weise, Artefakte, in denen sich > Wirklichkeit < und Repräsentation vermischen. 


\subsection{Die Stadt darstellen und Aufwerten: Geo-grafie Und VISUELLE IDENTITÄT DER STADT LUXEMBURG}

\section{Vorbemerkung: Die Stadt als semiotisches Konstrukt}

Eine Stadt ist nicht die Summe ihrer Straßen und Gebäude, ihrer Grünanlagen und Plätze; sie stellt vielmehr einen Sachverhalt dar, der es erlaubt, diese als zusammengehörend zu betrachten, als Teile eines Ganzen. Die Stadt ist ein semiotisches Konstrukt: Eine Betrachtungsweise, die mehreren Elementen eine Einheit verleiht, sie wie die Aspekte ein und derselben Identität (wieder) einander annähert. Man könnte irrtümlicherweise meinen, dass die Existenz einer Stadt lediglich das Ergebnis einer Gebietsaufteilung sei, die von einer anerkannten Autorität vorgenommen wird, indem ein Ort bestimmt wird: >hier beginnt oder endet die Stadt<. Aber selbst bei einem solch reduzierten Ansatz (der meint, es genüge, etwas zu umreißen, um es zu identifizieren) geht es um >semiotisches Handeln $<$ Man müsse einen Ort >bestimmen<, davon gingen wir aus, einen Raum >markieren<; dazu brauche man auch eine Autorität, von der man die Formulierung eines solchen Diskurses erwarten könne. Bereits auf dieser Ebene ist man weit von der physischen Gegenwart eines Gebäudes oder einer Kreuzung entfernt.

Auf den folgenden Seiten werden wir diese Frage vertiefen. Wir werden untersuchen, wie man eine Stadt zeigt, und zwar mithilfe dieser besonderen semiotischen Handlung, die wir >geo-grafische Darstellung< nennen. Wir werden prüfen, wie die Tourismus-Einrichtungen die Stadt Luxemburg anhand ihrer Karten darstellen. Schon jetzt ist es wichtig, den Begriff der >Darstellung < hervorzuheben, da es beim >Darstellen< darum geht, etwas >nachzubilden< (neu zu präsentieren) oder (aufs Neue) >wieder-zugeben $<$. Da eine Karte keine fertige Stadt vorfindet, muss sie diese mithilfe einer Menge visueller Kunstgriffe reproduzieren; damit stellt die Karte einen Konstruktionsraum für leseorientiertes Sehen dar.

Die Karte ist ein Text; die Karten, die wir untersuchen werden, stextualisieren< (Greimas/Courtés 1979) die Stadt Luxemburg. Das bedeutet, dass die sogenannte physische Realität der >Stadt Luxemburg < (die die Karten ebenso wie andere semiotische Vermittlungen darstellen und reproduzieren sollen) auf eine zweidimensionale Ebene gebracht wird, auf eine begrenzte Oberfläche, und dann mit folgenden semiotischen Zwängen konfrontiert wird: Sie muss eingegrenzt werden, bevor sie eine bestimmte Anzahl von Zeichenelementen der Stadt darstellen kann, die zusammenpassen müssen und gelesen werden. (Vgl. Shapiro 1969) Insgesamt durchläuft die >Stadt Luxemburg < eine Reihe von semiotischen Zwängen, die mit dem Medium der geografischen Karte und mit deren Verwendungspraxis zu tun haben (vgl. Goodwin 2003) - die im Gegenzug das >Wesen $<$ selbst der >Stadt Luxemburg < beeinflussen. Alles was im Rahmen der Karte erscheint, was in bestimmter Weise farbig ist, an bestimmten Vektoren ausgerichtet ist, was sich an bestimmten Schriftbildern orientiert, über Symbole bebildert ist, all das schafft in 
einer >performativen ${ }^{27}$ Weise (Austin 1972; Ducrot 1984) die Stadt Luxemburg. Die touristische Karte wird mit ihren eigenen Mitteln aktiv, um ein Ganzes zu schaffen, das sie >Stadt Luxemburg « nennt; sie trägt zu dem offenen Spiel der >zulässigen Identifizierungen< dessen, was die Stadt ist, bei.

In diesem doppelten Sinn werden wir uns der Art nähern, in der die Stadt Luxemburg in Form einer touristischen Karte textualisiert wird. Einerseits betrachten wir die Reihe von >Objektivierungen < der Stadt: Welche Merkmale Luxemburgs hat man berücksichtigt und benutzt, um die Karten zu erstellen, welche Aspekte und welche Perspektiven hat man im Laufe der Anpassungs- und Orientierungsschritte ausgewählt? Andererseits interessiert uns das eventuelle Erscheinen einer >subjektiven Stadt< als Ergebnis jener Merkmale, die man für die Stadtdarstellung angibt: Welches >Wesen oder zumindest welche Struktur und welches Stadtmodell werden hinter der Gesamtheit der einzelnen Teilverkörperungen sichtbar? Man könnte zusammenfassend und vereinfacht sagen, dass wir einerseits die sgezeichneter Stadt untersuchen und andererseits die >beabsichtigte< Stadt. (Vgl. Marin 1983)

Dazu untersuchen wir ein Korpus, das, wenn auch nicht erschöpfend, so doch repräsentativ ist: Einen unterschiedlichen Fundus an Karten von Luxemburg, die im Laufe der letzten zwei Jahrzehnte erschienen sind und unterschiedliche touristische Zielsetzungen haben - mal liegt das Ziel in der allgemeinen Erkundung des Landes durch einen fremden Reisenden, mal geht es um die Katalogisierung wichtiger Orte für Verbraucher, die in Luxemburg leben, mal um einen einfachen Straßenplan der Hauptstadt. ${ }^{28}$ Manchmal entspricht die Karte einem Stadtplan, manchmal einer Seite in einem Fremdenführer: Wir haben diese Unterschiede außer Acht gelassen, da, wie wir noch sehen werden, die entstandenen semiotischen Wirkungen verwandt sind. Allerdings haben wir die Bilder, ob Fotos oder Zeichnungen, berücksichtigt. Sie begleiten die Karten und tragen daher dazu bei, die Stadt mit der Karte gemeinsam zu zeigen und >wieder-zugeben<. Wenn die Karte mit all ihren Figuren, die sie enthält, einen >Text< bildet, dann stellen die Bilder und ihre vorangehenden Figuren den >Paratext $<$ dar. (Vgl. Genette 1987)

27 | 'Performativ bedeutet: etwas durch einen Sprechakt entstehen lassen. So existiert ein Versprechen, weil jemand verspricht, ein Name, weil jemand benennt; aber auch eine Liebe, weil jemand (durch unterschiedliche und multimodale Sprechakte) erklärt und verspricht.

28 | Hier die Liste der untersuchten Unterlagen (Karten oder Reiseführer mit Kartenmaterial): Baedekers Allianz Reiseführer, Luxemburg/Ostfildern-Kemnat: Mairs Geographischer Verlag, 1990; Discover Luxembourg, 1995; Marco Polo. Luxemburg: Reiseführer mit Insider-Tips, Ostfildern: Mairs Geographischer Verlag, 1995; Luxemburg. Ni vu ni connu, Luxembourg City Tourist Office, 2000; Michelin. Belgien/Luxemburg, Zellik: Le Guide Michelin, 2007; Panorama City Map. Luxemburg: La ville, Luxembourg City Tourist Office, 2008; Plan de la ville de Luxembourg, Luxembourg City Tourist Office, 2008. 


\section{Das Profil der Stadt Luxemburg im Spiegel seiner Karten: 'Der Aufstieg zur Enklaver}

Die erste Annäherung an die Wieder-gabe der Stadt befasst sich damit, wie diese >profiliert< ist. In dieser Hinsicht ist es erstaunlich festzustellen, wie häufig der Stadt systematisch nur ein einziges >Profil < gegeben wird: Es wird als >Aufstieg zu einer höher gelegenen Insel< (wobei >die Insel< das Stadtzentrum, die sogenannte $>$ Oberstadt<, meint) dargestellt.

Man setzt Konstanten und Variablen ein, die alle dazu tendieren, denselben Sinn hervorzubringen. Im Hinblick auf die konstante Taktik ist zu beobachten, dass: (i) der Paratext (d.h. die Fotos oder gezeichneten Bilder, die die Karte begleiten) immer ein Brückenbauwerk oder eine Bastion enthält; (ii) der Text (d.h. die eigentliche Karte) unverändert den Zugang zum Stadtzentrum zeigt.

Ein Paratext lenkt die Lektüre vom Text, den er begleitet, ab. Hinter den Figuren der Brücken und der Bastionen verbirgt sich hier eine klare semiotische Absicht: Der Paratext transportiert den Gedanken des >Zugangs zu einem höher gelegenen eingeschlossenen Ort<. Wir werden sehen, dass die Text-Karte genau den gleichen Inhalt wiederholt, allerdings mit anderen Mitteln. Was unverändert bleibt, ist die Darstellung des >Zugangs < zum Zentrum: Die Karte zeigt eher den Zugang als das Stadtzentrum in seiner Gesamtheit selbst. Ansonsten zeigt sie einerseits oft nur den süd-östlichen Teil des Zentrums, andererseits zeigt sie das Zentrum mit den umliegenden Stadtvierteln vom Bahnhof, Belair, Kirchberg, Grund usw. Man kann auch feststellen, dass die Brücken und Bastionen nicht die bevorzugten Zeichen im Text der Karte sind: Sie sind entweder nur angedeutet oder fehlen ganz. Man stellt auch fest, dass die Bereitstellung der Mittel für die Wieder-gabe der >Stadt< nicht selbstverständlich ist: Die Veränderlichkeit ihres Signifikanten wird erkennbar, ebenso das Zusammenspiel unterschiedlicher semiotischer Vorgehensweisen, die die Stadt bezeichnen und sie in bestimmter Weise aufwerten.

Doch kommen wir zu den variablen Vorgehensweisen, die dennoch alle auf das gleiche Ziel hinsteuern: Es geht darum, das Zentrum zu zeigen als >eine isolierte Enklave, zu der man hinstrebt<. Die Vorgehensweisen der Karte, etwas zu visualisieren, lassen sich in drei Typen unterteilen: (i) farblich, (ii) bildlich und (iii) eidetisch (d.h., dass die Vorgehensweisen etwas zeigen über (i) Farben, (ii) figürliche Darstellung, (iii) Linien und Achsen, die der Textoberfläche Form geben).

Die Verwendung von Farbe zeigt die Stadt als eine >isolierte Enklaveく. Eine mehr oder weniger schwarze, aber immer gut sichtbare Linie, die die Bahnstrecke symbolisiert, erzeugt eine umschließende Wirkung des Stadtzentrums im Osten. Ein grüner Streifen, ebenfalls mit sehr unterschiedlichen Farbwerten, verkörpert den Park und scheint das Stadtzentrum im Westen abzuschließen. Je nach Karte besitzt die für den Park stehende grüne Fläche unterschiedliche Ausdehnung von Westen nach Norden: Das bedeutet, dass es weniger darum geht, eine physische Realität abzubilden, als vielmehr darum, die Stadt in einer bestimmten Weise nachzustellen. 
Die bildhaften Vorgehensweisen haben ein zweifaches Ziel: Sie verstehen sich nicht nur als ergänzende Wirkung für die >Isolierung < der >Enklave< des Zentrums, sondern sie drücken auch das >Hinstreben< zu diesem Ort aus. Was das erste Ziel betrifft, kann man feststellen, dass die Darstellung der Mauern und Bastionen, wenn sie sich südlich vom Stadtzentrum befinden, recht ausgeprägt ist (je nach Karte unterschiedlich), während dieselben Mauern und Bastionen, wenn sie das Zentrum nicht umschließen, kaum skizziert, oft fast nicht vorhanden sind. Andererseits zeigen sich alle Monumente, wenn sie dreidimensional gezeichnet sind, immer in Süd-Nord-Richtung, von unten nach oben: Das entspricht dem Gedanken der aufsteigenden Orientierung der Stadt, eines Weges, der sichtbar nach oben führt.

Schließlich umgeben die eidetischen Vorgehensweisen selbst das Zentrum und machen es zu einem Konvergenzpunkt. Einerseits erscheint alles so, als ob die Zeichnung und die Hervorhebung bestimmter Straßen eine feste Umschließung des Zentrums bedeute. In manchen Fällen erfüllt das Quadrat mit der Ausschnittsvergrößerung für das Stadtzentrum die gleiche Funktion. Andererseits steht der Name »Luxemburg« nicht oberhalb des gesamten Stadtplans, sondern in der Nähe des Zentrums. Es erscheint fast so, als ob er als Legende allein für das Stadtzentrum aufträte, als ob er die Stadt Luxemburg per Antonomasie als Konvergenzpunkt erzeuge.

Zusammenfassend lässt sich festhalten, dass diese Vielfalt und Redundanz der semiotischen Verfahren (farblich, bildlich, eidetisch), die unterschiedlich kombiniert werden, ein und denselben Endzweck verfolgen.

\section{Das Profil der Stadt Luxemburg im Spiegel seiner Karten: Das Fehlen einer starken Identität}

Die erste Annäherung an die Wieder-gabe der Stadt hat versucht aufzuzeigen, wie diese gestaltet ist, wie sie jedes Mal als >Phänomen< angegangen wird; die zweite Annäherung wird sich nun der Hervorhebung eines Objektes >Stadt< als >Ding an sich< (>Noumenon<) zuwenden: Keine Sammlung von Eigenschaften, sondern eine Modellvorstellung; keine Absichten, sondern eine Zeichnung. Hier tritt das vielleicht erstaunlichste Ergebnis der Korpusanalyse zutage: Es scheint von Luxemburg keine gedankliche Modellvorstellung zu geben. Alles erweckt den Eindruck, als ob hinter der Vielfalt der Zeichnungen, hinter den Merkmalen der Stadt keine Absicht stünde, keine Idealvorstellung, keine starke Identifikation mit der Stadt selbst.

Um eine solche semiotische Wirkung zu verstehen, ist es wichtig, die beiden Ansätze der Wieder-gabe zu unterscheiden, obwohl beide sich ergänzen. Bisher haben wir uns bei dem Bild des >Anstiegs zu einer weiter oben gelegenen Insel< aufgehalten - ein mühsamer und steiler Anstieg: Die Stadt wird durch diesen Aufstieg erfasst, mit Sinn versehen und aufgewertet. Und - eine solche bildliche Darstellung zeigt die Stadt, bildet sie ab. Wir werden nun versuchen, ein Modell zu 
entwickeln, das die Stadt >lesbar < macht, sie symbolisiert. Die erste Annäherung hat sich auf eine >Verräumlichung < konzentriert (einen Vorstellungs->Prozess<, also auch Prozess der Wiedergabe eines bestimmten Raumes); die zweite konzentriert sich auf eine Topologie (einen >Zustand <, der von den phänomenalen Eigenschaften, den Wegen, abstrahiert und dafür ein System liefert: eine Art absolutes Objekt >Stadt<, eine Stadt >an $\operatorname{sich}<)^{29}$.

Die Modellvorstellung einer Darbietung der Stadt Luxemburg scheint nicht zu existieren. Erstens gibt es nur einen Ort, der nicht nur ein räumlicher Bezugspunkt ist, sondern die Stadt auch dauerhaft vertreten und sie daher auch symbolisieren kann: die Kathedrale. Zweitens wird die Stadt ziemlich unscharf eingegrenzt: Jede Karte zentriert sie etwas anders - wir haben gesehen, dass die einzige Konstante die Tatsache ist, dass ein oder mehrere Zugänge zum Zentrum gezeigt werden, das Zentrum selbst erfährt in den Texten der Karten keine feste Ortszuweisung. Drittens sind die Umrisse der Stadt ebenfalls ziemlich verschwommen definiert; Man muss fast sagen, dass sie so gut wie nicht definiert sind: Obwohl die Stadt Luxemburg immer als ein zum Zentrum nach oben führender Weg dargestellt wird, bleiben die Grenzen Luxemburgs, seine Ausdehnung und seine Ränder stets unbestimmt.

\section{Schlussfolgerung: Die problematische Kommunikation über 'die Stadt,}

Oft kennen wir die Form einer Stadt und wir wissen, wie sich ihr Kern um eine Gesamtheit von Bezugspunkten (Monumente, Plätze, Kreuzungen usw.) aufbaut. Eine solche Stadt lässt die Formen, die Strukturen und die Bezugspunkte wirksam sprechen; dadurch spricht sie sich selbst die Bedeutung einer Stadt zu. Sie befördert ein Modell ihres >Stadt-Wesens < - ein Modell, das jede Karte, aber auch jedes Logo, jedes Foto, jedes Bild insgesamt in eben so vielen Variationen wiederzugeben bemüht ist. Jedoch für Luxemburg scheint dieser Prozess der Bedeutungszuschreibung, der >die Stadt < mit einer > visuellen Identität < hervorbringt, zu fehlen.

>Geo-grafie bedeutet immer eine kommunikative Praxis, im weitesten Sinne des Wortes. Sie ist etwas Geschriebenes, eine Textualisierung und damit eine Vorstellung, also eine Bedeutung und eine Aufwertung. >Wieder-geben $<$, dies ist ein Sprechakt, der darin besteht zu zeigen; wir haben gesehen, dass es dabei um eine Gesamtheit aufeinander abgestimmter Vorgehensweisen geht. Daher stellen die

29 | Wir beziehen uns auf die Identitätstheorie von Ricœur (1990), die den Begriff der Identitäten problematisiert und unterscheidet zwischen einer Identität ,ipse، (Identität, die entlang einer Reihe von Veränderungen akzeptiert wird, z.B. in einem Versprechen: Selbst wenn meine Stimmung oder meine Lebensbedingungen sich ändern, werde ich immer derjenige sein, der dir das Versprochene schuldet) und einer Identität sidem، (eine strukturelle, unveränderliche und tiefgehende Identität, z.B. die eines Eigennamens, eines genetischen Codes usw.). 
Geo-grafie einer Stadt, deren Wieder-gabe auf einer Karte, eine bestimmte visuelle Identität her, die sich als Antrieb für allgemeinere >kulturelle Strategien< eignet. Im Hinblick auf die Darstellungen der Stadt Luxemburg, die wir hervorgehoben haben, kann man sich Fragen stellen hinsichtlich ihrer Zweckmäßigkeit, ihrer Effektivität und ihrer optimalen Prägung. (Fontanille 2008) Anders gesagt, ist der >Zugang zu einer höher gelegenen Insel< (also die >zu überwindende Spannung einerseits, die >Isolation < des zentralen Gegenstandes andererseits) angemessen? Unter Berufung auf welche Strategien zur Aufwertung der Stadt? Und ist das Fehlen eines Stadtmodells und einer >wesenhaften< Identität auch zweckmäßig? Wenn ja, warum und zu welchem Zweck?

\subsection{Das luXemburgische Herz ZWischen nationaler EINHEIT UND REGIONALEM INDIVIDUALISMUS}

Wenn man die benachbarten Bereiche der Rhetorik (vgl. Perelman/Olbrechts-Tyteca 2000), der Stilistik (vgl. Adam 1990; Adam 1997; Herschberg-Pierrot 2003) und der Diskursanalyse (vgl. Adam 1999; Amossy/Maingueneau 2003) zugrunde legt, zeigt sich die hier durchgeführte Untersuchung von drei luxemburgischen Tourismus-Broschüren ${ }^{30}$ in einem bewusst kreativen Rahmen, der sich darüber hinaus mit der Interaktion zwischen schriftlicher Einheit und bildlicher Bedeutung verbindet. Die beiden betrachteten Verfahren der Kodierung und Dekodierung gehen von der Absicht aus, auf andere einzuwirken, was auch eine Brücke zur Pragmatik (vgl. Bonhomme 2005; Bonhomme 2006) und zur Sprechakttheorie (vgl. Ducrot 1984; Searle 1972) schlägt. Wir interessieren uns für die von diesem TourismusDiskurs hergestellte Bedeutung und dafür, wie diese zustande kommt (siehe Bonhomme/Adam 1997), wobei wir allerdings die Klippen des radikalen Konstruktivismus meiden. Es geht also darum, die Funktionsweise eines Diskurses zu erfassen, der vom luxemburgischen Tourismusbüro, dem Office National du Tourisme, ausgeht (zugeschriebene Identitäten). Und auch die Funktionsweise all dessen, was die institutionelle Einrichtung für die Ausformung einer individuellen Vorstellung vom Land (angeeignete Identitäten) darstellt.

\section{Eine scheinbare Uniformität, eine sichere Ergänzung}

Die beiden Bände »Hôtels Restaurants et Arrangements« und »Sites et Attractions« legen bei ihren Informationen eine nur scheinbare Neutralität an den Tag.

30 | Office National du Tourisme Luxembourg (2006): Les Produits du Terroir et les Métiers d'Art. Mersch: Druckerei François Faber; Office National du Tourisme Luxembourg (2008): Hôtels Restaurants et Arrangements. (11/2007 für das Jahr 2008), Luxemburg: Druckerei Saint-Paul; Office National du Tourisme Luxembourg (2008): Sites et Attractions. (12/2007 für das Jahr 2008), Luxemburg: Druckerei Saint-Paul. 
Dadurch dass die Informationen nach Regionen aufbereitet und angeboten sind, entsteht der Eindruck einer Sortierung nach Größe, von der Region Luxemburg Stadt und dem Zentrum nach Süden, Minetteregion; der dritte Band »Les Produits du Terroir et les Métiers d'Art« hingegen zieht die thematische Klassifizierung der alphabetischen und regionalen vor. Eine Gemeinsamkeit haben jedoch alle drei Broschüren: Die gleiche Karte, die das Land zeigt und die die verschiedenen Regionen farblich unterscheidet (wir werden auf genau diesen Punkt weiter unten zu sprechen kommen). Es scheint einerseits, dass die drei angesprochenen, sich einander ergänzenden Themenbereiche alle Informationen abdecken, die den neugierigen und pragmatisch orientierten Touristen interessieren könnten, andererseits, dass die Komplementarität dieser Informationsquellen garantiert, dass die vielfachen Aktualisierungsformen einer zugeschriebenen Identität schlüssig sind. Linden und Thewes behaupten, dass bereits bei der Weltausstellung von 1937 »die Werbung für Land, Tourismus und Wirtschaft sich vermisch[t]en [...] in einer breiten Bemühung um Zusammenführung « ${ }^{31}$ (Linden/Thewes 2007: 44).

Das Feld dieser Studie ist, wie das Korpus zeigt, an der Schnittstelle von Land und Region angesiedelt und wir werden zeigen, dass die quantitative Untersuchung diese extreme Zersplitterung bestätigt, die zu einem Zeitpunkt, an dem vielerorts vom Aufbau der Großregion geredet wird, umso mehr erstaunt. Anders gesagt, man wird feststellen, wie die zugeschriebenen und die angeeigneten Identitäten miteinander in Dialog treten.

\section{Regionale oder regionalistische Annäherung?}

Die quantitative Umfrage hat ergeben, dass die befragten Personen großen Wert auf den Begriff der Nähe legen, eine seltsame Haltung in einem Land, in dem aufgrund seiner Größe eigentlich alles in der Nähe liegt. Auf die Frage »Wie oft besuchen Sie in Ihrer Freizeit folgende Orte?« - in diesem Fall ging es um das Ösling - haben $46 \%$ der Personen, die angaben, diese bewaldete Gegend mehrmals wöchentlich aufzusuchen, ihren Wohnsitz im Norden und sind im Wesentlichen den Milieus außerhalb der >Mitte der Gesellschaft< zuzurechnen. ${ }^{32}$ Die restliche Bevölkerung begibt sich niemals oder fast niemals dorthin, ${ }^{33}$ mit einer Rekordquote bei den Bewohnern im Osten (51 \%) - die vor der eigenen Tür schon schöne Landschaften haben -, gefolgt von den Bewohnern der Stadt Luxemburg und des

31 | Originalzitat: "[...] promotion nationale, touristique et économique s'entremêl[ai]ent [...] dans un vaste effort de synthèse."

32 Statusorientiertes Milieu: $12 \%$; unterprivilegiertes Milieu, aufstiegsorientiertes Milieu und alternatives Milieu: jeweils $10 \%$. Die befragten Personen, die zu diesen Kategorien gehören, verteilen sich zu fast gleichen Teilen zwischen Männern (47 \%) und Frauen (45\%).

33 | Auch hier ist die Verteilung nach Geschlecht mit 34 \% für die Männer und 37 \% für die Frauen ausgeglichen. 
Südens (jeweils $40 \%$ ) 34 . Das Verhältnis zwischen Einwohnern aus der Hauptstadt, die sich mehrmals im Jahr dorthin begeben, und denen, die nie oder fast nie dorthin fahren, ist mit $51 \%$ für die erste Kategorie und $40 \%$ für die zweite relativ ausgeglichen.

Dieser Hang, seine eigene Wohnregion zu bevorzugen, bestätigt sich für die Minetteregion, also den südlichsten Landesteil, für Luxemburg-Stadt und schließlich für die Moselregion. So leben 53 \% der befragten Personen, die mehrfach in der Woche in ihrer Freizeit die Minetteregion aufsuchen, im Süden ${ }^{35}$, gegenüber $3 \%$, die im Norden wohnen; zu $41 \%$ sind sie italienischer Herkunft, was vermutlich auf die durch den Bergbau bestimmte Vergangenheit der Region zurückzuführen ist, die durch eine starke wirtschaftlich bedingte Einwanderung gekennzeichnet ist. Aus Luxemburg-Stadt, die nicht sehr weit entfernt ist, werden nur $8 \%$ Besucher für diese Gegend verzeichnet. Von allen Befragten insgesamt besuchen $57 \%$ der Bewohner aus dem Norden und $45 \%$ aus dem Osten niemals oder fast nie die Minetteregion ${ }^{36}$. Das gleiche Szenario gilt für die Einwohner der Moselregion im Südosten, die in ihrer Freizeit ihre Region zu $49 \% 37$ besuchen, während die des Nordens dort nur sehr selten hingehen (39\%); man muss jedoch festhalten, dass über die Hälfte der Landesbevölkerung diese Weingegend mehrmals im Jahr besucht. Für Luxemburg-Stadt kehren sich die Werte hingegen um: Nur $4 \%$ aller Befragten haben nie oder fast nie die Hauptstadt zum Ziel. Unter ihnen kommen $10 \%$ aus dem Norden, 4 \% sind Männer, $9 \%$ Frauen von 60 Jahren oder älter. Was die häufigen Freizeit-Besuche Luxemburgs betrifft, stammen $66 \%$ der Befragten aus der Stadt selbst, $44 \%$ aus der übrigen Zentralregion, $31 \%$ aus dem Süden, $28 \%$ aus dem Osten und $12 \%$ aus dem Norden. Die größte Anzahl der Besucher kommt aus dem konservativ-gehobenen Milieu (51 \%), gegenüber $10 \%$ aus dem traditionsorientierten Milieu. Auch hier wiederum hebt sich Luxemburg deutlich ab, was angesichts der kulturellen und wirtschaftlichen Ressourcen einer jeden Hauptstadt nachvollziehbar erscheint.

Auf der Ebene des Tourismus-Diskurses wird die Reihenfolge, in der die Regionen in den Broschüren abgehandelt werden (im Uhrzeigersinn bei Luxemburg und Umgebung startend), durch die Antworten der Befragten bestätigt, besonders im Hinblick auf die herausragende Rolle der Hauptstadt und ihrer Umgebung. Die zugeschriebenen Identitäten, die sich aus den Informationen in den Heften ergeben, lassen eine zentralistische Metropole hervortreten, die geografisch aller-

34 | Unter innen stammen $46 \%$ der Personen aus dem traditionsorientierten Milieu und $44 \%$ aus dem unterprivilegierten Milieu.

35 | Sie verteilen sich wie folgt: 28 \% der Personen gehören dem hedonistischen Milieu an, gefolgt von $27 \%$ aus dem kleinbürgerlichen und dem traditionsorientierten Milieu.

36 | Diese finden sich mehrheitlich (36 \%) im traditionsorientierten Milieu, im unterprivilegierten Milieu und im liberal-gehobenen Milieu.

37 | Die meisten Besucher der Region stammen aus dem hedonistischen und dem alternativen Milieu, wenig repräsentiert hingegen sind die traditionellen Milieus. 
dings nach Süden verschoben ist. Diese Identitäten finden sich, verinnerlicht und angeeignet, bei den Befragten wieder, wie man an dem auf die Stadt Luxemburg gerichteten Interesse, das auf Kosten anderer Orte geht, ablesen kann. Andererseits vermittelt die Art der regionalen Sichtweise des nationalen Raumes, die Identitäten zuschreibt, den Eindruck einer weit reichenden Fragmentarisierung. Das Echo, das diese in den angeeigneten Identitäten findet, erweckt den Eindruck einer regionalistischen Haltung aufseiten der Befragten. Man konnte also feststellen, dass die bevorzugten Orte vor allem in der eigenen Wohnregion liegen und dass die Befragten sich kaum für die anderen Landesteile interessieren (außer für die Hauptstadt), die angesichts der Ausdehnung des Landes allerdings leicht zu erreichen wären. Ein solcher Rückzug auf sich selbst ähnelt einem regionalistischen Verhalten. Mit anderen Worten, dem offiziellen regionalen Tourismus-Diskurs entspricht eine individuelle regionalistische Haltung. Linden und Thewes sind im Bereich der Werbung zum gleichen Schluss gekommen:

En dépit de la superficie très limitée du Luxembourg, les campagnes publicitaires distinguent plusieurs régions spécifiques [...]. Ces représentations n'influencent pas seulement la vision des touristes. Elles finissent par déterminer la perception que les Luxembourgeois ont de leur propre pays, de son passé et de sa géographie. La propagande touristique reflète finalement les histoires que les Luxembourgeois aiment raconter sur eux-mêmes, tout autant qu'elle leur sert de moule. ${ }^{38}$ (Linden/Thewes 2007: 44)

Nun soll es um die Frage gehen, ob die Analyse der rhetorischen Verfahren und deren Interaktion mit den Bildinformationen sich in dieser ersten Schlussfolgerung wiederfinden.

\section{Passende Farben}

In allen drei untersuchten Broschüren soll die Verwendung von Farben die regional unterteilte Darstellung des Landes verstärken. Die Farben sind mit einer symbolischen Bedeutung aufgeladen, die als gedächtnisrelevantes Mittel wirkt. Dieses Verfahren trägt dazu bei, jene Redundanz zu erzielen, die den diskursiven Stil dieser Hefte in weiten Teilen ausmacht.

Jedes einzelne Heft zeigt die gleiche Karte des Großherzogtums: Der Region »Ardennen und Naturparks« ist die Farbe Grün zugewiesen, besonders für die Darstellung von Natur und Flora geeignet, die die Hauptanziehungskraft dieser

38 | Deutsch: "Trotz der sehr begrenzten Fläche Luxemburgs unterscheiden die Werbekampagnen mehrere spezielle Regionen [...]. Die Darstellungen beeinflussen nicht nur die Sichtweise der Touristen. Sie bestimmen letztlich auch die Wahrnehmung, die die Luxemburger von ihrem eigenen Land haben, von dessen Vergangenheit und dessen Geografie. Die touristische Propaganda reflektiert letztlich die Geschichten, die die Luxemburger sich gerne über sich selbst erzählen, und die innen zugleich als Schablone dient." 
Gegend charakterisieren. Die Region »Müllerthal« trägt das Braun der Felsen, während die »Stadt Luxemburg« und ihre Umgebung in Gold gehalten sind. Die »Moselregion« zeigt sich im Blau des Flusses und des Himmels bei schönem Wetter, während das »Bergbau-Land der Roten Erde« die Ziegelfarbe seiner Mineralien widerspiegelt, als ob die Bodenschätze aus der Tiefe von vornherein keine andere Farbe zuließen. Diese Assoziation stützt sich im Wesentlichen auf eine prototypische Darstellung der landschaftlichen Farbgebung und ruft beim Leser eine schematische Wahrnehmung der regionalen Unterschiede hervor, indem die stärkste repräsentative Eigenschaft einer jeden Region herausgestellt wird. Bekräftigt wird dies durch die verkleinerte Wiedergabe jeder Region im unteren Bereich der Seite, benannt in vier Sprachen (französisch, deutsch, niederländisch und englisch), das Ganze in der symbolischen Farbe der Region.

Was die beiden Titel »Hôtels Restaurants et Arrangements « sowie »Sites et Attractions « betrifft, so wird die beispielhafte Bedeutung der Farben in der Karte des Landes einerseits im Inhaltsverzeichnis für die Regionen wieder aufgenommen, andererseits in der Seitennummerierung (oben links und rechts), die in einem schiefen Viereck eingeschlossen ist, in dem das gesamte Land umrissartig abgebildet wird. Der Teil der Umrisszeichnung, der die gerade thematisierte Region abbildet, ist farblich entsprechend der in der großen Karte des Landes gewählten Farbe hervorgehoben; anzumerken ist, dass dieses Viereck einen Bezug zwischen dem Inhalt des Führers und seiner Umschlagseite herstellt: Es befindet sich auf dem Streifen der drei Titelseiten und spielt mit der inversen Darstellung von roter Schrift auf weißem Grund und weißer Schrift auf rotem Grund, wodurch die Buchstabenkombination >lu< als Bestandteil der Internetadresse hervorgehoben wird. Die zweifarbige Index-Übersicht von »Hôtels Restaurants et Arrangements« in blau und weiß entwickelt sich in »Sites et Attractions« weiter: Die Örtlichkeiten können einer Region anhand der Symbolfarben auf einen Blick zugeordnet werden. Im Gegensatz dazu benutzt der Führer »Les Produits du Terroir et les Métiers d'Art« diese feinen Unterschiede nicht in der gleichen Weise; diese signalisieren nicht mehr die Zugehörigkeit zu einer Region, sondern die Art eines Produktes (was semiotisch gesehen als Teil eines Beweggrundes gilt): Z.B. bedeutet ein klares Grün ein Weinbau-Produkt, während ein warmes Orange sich auf Schnäpse und Liköre bezieht und die Farbe Ocker (Variante von Orange) die heimischen Biere meint. Wenn man den Umschlag und den Innenteil der Broschüre miteinander vergleicht, stellt man fest, dass die verwendeten Farben genau denen des Titelfotos entsprechen, jede durch einen Ton-in-Ton-Effekt leicht variiert.

Die Farben treten fast als Überzeugungswaffe auf. Untersucht man, wie sie eingesetzt werden, so zeigt sich darin eine schematische Betrachtung des Landes, die nicht mehr aus der regionalen Perspektive (pragmatisch neutral), sondern aus der regionalistischen Perspektive (pragmatisch Stellung beziehend) geführt wird und deren Zweck darin besteht, dem Leser bei der Einschätzung des Raumes zu helfen, indem man diesen rationalisiert. Bonhomme hat dies mit einer Untersuchung eines Tourismus-Prospektes der Stadt Bern sehr gut gezeigt: »Schématiser 
la ville, c'est en retenir les aspects les plus typiques à travers trois actes énonciatifs[:] a) Sélectionner [...] b) Emblématiser [...] c) Rationaliser [...].«39 (Bonhomme 2003: 14) Die luxemburgischen Hefte stützen ihre Verwendung der Farben auf Gegebenheiten, deren prototypische Eigenschaften ${ }^{40}$ sie aufnehmen, und steuern die Entschlüsselung des Lesers mithilfe eines Redundanzverfahrens, das bis hin zu dem verweisenden Bezug von Umschlag/Innenteil des Bandes reicht. Diese Vorgehensweise passt zu einer um die Pragmatik erweiterten Rhetorik, dergestalt wie sie Perelman und Olbrechts-Tyteca (2000) konzipiert haben, nämlich als spezielle Art, ein Objekt mit der Absicht herzustellen, die Deutung durch den Leser zu steuern. So betont Georges Molinié treffend die Bedeutung der perlokutiven Funktion von Rhetorik: »[E]lle vise à faire penser ou croire aux gens ce que spontanément ils ne pensent ou ne croient pas, [...] à leur faire même désirer ce qu'ils n'imaginent même pas de désirer. $^{41}$ (Molinié 1993: 2) Es ist also einfach, diesen Typ eines epideiktischen Diskurses der Bestimmung des Werbediskurses anzunähern, der ein Objekt anpreist, um es für den Kauf zu empfehlen.

Die Untersuchung der Umschlagseiten, die wir an dieser Stelle leider nicht weiter vertiefen können, bestätigt in überzeugender Weise die Verwendung der Farbsymbolik, der >Drittel-Linien < und der Perspektiven, die bei den Fotos mit sorgfältig erarbeiteten Einstellungen regelrecht in Szene gesetzt sind. Der so aufgeteilte Raum lenkt die Blickrichtung des Lesers und suggeriert eine Ideologie, die sich bemüht, antithetische Bedeutungen in einem Ort zusammenfließen zu lassen: einerseits eine starke und konservative Bindung an die eigenen Wurzeln, andererseits die Versuchung, dem Ruf jener Räume nachzugeben, die von futuristischen Erwartungen beherrscht werden.

\section{Die Rhetorik des Tourismus}

Wenn deutlich wird, dass der Einsatz von Bildern durch einen hohen Grad an Geschicklichkeit geprägt ist, so ist dieses Merkmal jedoch nicht das einzige, das den $>$ modus operandi< dieser Tourismus-Broschüren kennzeichnet. Auch die verwendete Rhetorik spielt eine wichtige Rolle, wie die Textanalyse von »Luxembourg, la capitale et ses environs « beispielhaft für den Stil der untersuchten Veröffentlichungen zeigt. So weisen die Führer »Hôtels Restaurants et Arrangements « und »Sites

39 | Deutsch: "Eine Stadt schematisch darstellen heißt, die typischsten Merkmale über drei Äußerungsakte festhalten[:] a) Auswählen [...] b) Symbolisieren [...] c) Rationalisieren $[\ldots] . "$

40 | Z.B. die Farbe der Mineralien für die passend benannten Terres Rouges oder die Farbe der Vegetation für die Region der Naturparks.

41 | Deutsch: "Sie zielt darauf ab, Menschen etwas denken oder glauben zu lassen, was sie spontan nicht denken oder glauben, [...] sie sogar etwas wünschen zu lassen, was sie sich noch nicht einmal vorstellen können zu wünschen." 
et Attractions « ähnliche Einführungstexte auf, die deutlich von poetisch-touristischen Klischees gekennzeichnet sind.

Luxembourg, aux contrastes enchanteurs, est une ville chargée d'histoire, débordant de trésors artistiques et culturels qui ne demandent qu'à être admirés, regorgeant de sites intéressants et de coins pittoresques qui ne demandent qu'à être explorés, truffée d'anecdotes et de légendes qui ne demandent qu'à être racontées.

Et puis, quelles sont les autres capitales européennes qui peuvent se targuer d'être à quelques jets de pierre seulement de paysages verts impressionnants, où d'accueillants villages ont conservé leurs caractères?

A ce propos, le Prix Europa Nostra décerné au village restauré d'Useldange ne doit rien au hasard. Comme son nom l'indique, la Vallée des Sept châteaux invite à un périple à travers I'histoire et la nature, depuis Koerich et son église baroque jusqu'à Mersch. ${ }^{42}(11 / 2007$ : 10 und 12/2007: 6)

Die Lyrik, die sich im Laufe der wenigen Textzeilen ausdrückt, verwendet im ersten Teil den Dreierrhythmus, der sich auf die syntaktische, die morphologische und die lexikalische Ebene erstreckt. Dabei handelt es sich um eine Bewegung, die dem Leser ein beruhigendes und harmonisches Tempo vermittelt.

Die Erwähnung der Stadt zu Beginn wird über eine Personifizierung realisiert, die sich auf ihre Eigenschaften und Qualitäten stützt, die selbst wieder über ihre Verben personifiziert werden. So verfügen z.B. die Schätze, die das geschichtliche und künstlerische Erbe der Hauptstadt ausmachen, über eine wahre Seele: Sie bitten um die Aufmerksamkeit des Touristen und um Hilfe, die ihnen aus der Sprache heraus zu einer Existenz verhelfen würde. Die Verschachtelung wird durch eine dreigliedrige Aneinanderreihung erzielt. Man beginnt mit der Gesamtheit Luxemburgs, zu dessen Eigenschaften die »bezaubernden Gegensätze« (1) gehören; dann wird die reiche Geschichte angesprochen (2), die sich wiederum aus der Gesamtheit ihrer »künstlerischen und kulturellen Schätze» (3) zusammensetzt. Letztere selbst werden durch drei Besonderheiten gekennzeichnet, wodurch sich eine informationsgesättigte Wirkung ergibt, die sich als möglichst umfassend und

42 | Deutsch: "Luxemburg, voller bezaubernder Gegensätze, ist eine geschichtsträchtige Stadt, überschäumend von künstlerischen und kulturellen Schätzen, die nur darauf warten, bewundert zu werden, überfließend von interessanten Stätten und pittoresken Winkeln, die darauf warten, erkundet zu werden, umrankt von Anekdoten und Legenden, die darauf warten, erzählt zu werden. Und dann, wo gibt es eine andere europäische Hauptstadt, die sich rühmen kann, nur einen Steinwurf von beeindruckenden grünen Landschaften entfernt zu sein oder von gastfreundlichen Dörfern, die ihren Charakter bewahrt haben? In diesem Zusammenhang ist der Preis Europa Nostra, der dem restaurierten Dorf Useldange verliehen wurde, kein Zufall. Von Koerich mit seiner Barockkirche bis Mersch lädt das Tal mit dem bezeichnenden Namen Tal der sieben Schlösser zu einer Reise durch Geschichte und Natur ein." 
verlockend versteht. Bonhomme erinnert: »Loin d'obéir à des procédures logicodéductives plus ou moins complexes (du genre Démontrer $\rightarrow$ Convaincre), cette orientation persuasive est de nature empathique, faisant appel à la séduction (Grize 1981) et à l' >évocation< (Dominicy/Michaux 2001).«43 (Bonhomme 2003: 18) Es ist dennoch zweifelhaft, dass der Einführungstext das angestrebte Ziel erreicht, denn er ähnelt eher der Karikatur einer Lobrede, wie wir im Folgenden zeigen werden.

Der erste Absatz, der die Hälfte des Vorstellungstextes umfasst, besteht aus einem komplexen Satz:

[Luxemburg], [Apposition erster Ordnung: voller bezaubernder Gegensätze], [Kopula, die die Verbindung zwischen der Stadt und deren Prädikat herstellt: ist] [nominales Prädikat, das dem Subjekt zugesprochen wird und die Stadt als historisch reich bezeichnet: eine geschichtsträchtige Stadt]

Diese Aussage, die man mit einem einfachen Satz hätte ausdrücken können, dient vielmehr als Eröffnung für drei Partizipialkonstruktionen, für welche sie als Verteiler fungiert. Die Weitschweifigkeit wird durch die Wiederholung von zwei Formen des Partizip Präsens erreicht: >überschäumend/überfließend<sowie von einer Form des Partizip Perfekt >umrankt von<, wodurch ein Dreierrhythmus erreicht wird. Jedes Partizip führt eine Objektgruppe mit sich und jede Objektgruppe ist nach dem gleichen Muster aufgebaut, wodurch sich die oben erwähnte Baumstruktur ergibt. Die drei Objektgruppen werden durch die Präposition >von< eingeleitet: Im ersten Teil wird das Nomen >Schätze< durch zwei nebengeordnete Adjektive näher bestimmt; der zweite Teil besteht aus zwei nebengeordneten Nomen, jedes durch ein Adjektiv näher bestimmt; schließlich wird der dritte Teil durch zwei nebengeordnete Nomen gebildet. Insgesamt ergibt sich daraus das unten stehende Schema ${ }^{44}$ :

[von künstlerischen und kulturellen Schätzen] $=1 \mathrm{~N}+2$ beigeordnete Adj. [von interessanten Stätten und pittoresken Winkeln] = 2 NS +2 beigeordnete Adj. [von Anekdoten und Legenden] = 2 beigeordnete $\mathrm{N}$

Hier wird erkennbar, wie sich der Zweierrhythmus eines jeden Syntagmas mit dem Dreierrhythmus verbindet, der die Sequenz homogen macht. Dadurch wird die Stärke der Information vom Lesetakt unterstützt und die Aufnahme der Information erleichtert.

43 | Deutsch: "Weit davon entfernt, mehr oder weniger komplexen logisch-deduktiven Verfahren zu folgen (etwa der Art Beweisen $\rightarrow$ Überzeugen), ist diese persuasive Ausrichtung empathischer Natur und appelliert an die Verführung (Grize 1981) und an die 'Evokation. (Dominicy/Michaux 2001)."

44 | Für den syntaktischen Kommentar des Textes gelten folgende Abkürzungen: $\mathrm{N}$ = Nomen; Adj. = Adjektiv; NS = Nominalsyntagma; V = Verb. 
Auf lexikalischer Ebene hat der Sprecher einen hyperbolischen Diskurs mit einigen Ausdrücken hervorgebracht, die nicht einer gewissen Trivialität entbehren (»Luxemburg schäumt über von«, »fließt über von«, »ist umrankt von«). Überall zeigen sich Unmengen, sogar Überfluss, ein Reichtum gewissermaßen, der umgekehrt proportional zur Größe des Landes steht. Diese Generosität wird auch vom Dreierrhythmus gestützt. Die drei Objektgruppen (deren syntaktische Struktur wir oben beschrieben haben) werden jeweils durch einen gleich lautenden Relativsatz erweitert (»die darauf warten $\mathrm{zu} . . . \ll$ ), der wiederum durch einen passiven Infinitiv ergänzt wird (»bewundert zu werden«, »erkundet zu werden«, »erzählt zu werden«). Diese strukturelle Redundanz (drei Vorkommen in viereinhalb Zeilen) erscheint kontraproduktiv und schafft eher Verwirrung als die angestrebte Anziehungskraft.

In der Folge ändert der Text seinen Stil grundlegend: Von einer übertriebenen Lyrik wechselt er zu einem spontanen Diskurs, die Sätze sind kürzer, sie sprechen den Leser durch die illokutionäre Wirkung der Frage an und scheinen >vom Hölzchen zum Stöckchen zu kommen. Diese Wirkung entsteht aus den Konnektoren einer textuellen >Wegmarkierung ( vgl. Freyermuth 2003) »Und dann«, »In diesem Zusammenhang«, die auf eine deutliche Oralisierung des Diskurses schließen lassen. Im zweiten Absatz wird der Stil fordernder, denn er spricht den Leser an, der aufgefordert wird, auf die pragmatische Frage zu antworten (vgl. Searle 1982; Kerbrat-Orecchioni 1991), und von dem erwartet wird, dass er die Rechtmäßigkeit der von der Hauptstadt des Großherzogtums gebotenen Vorzüge anerkennt. Die Notwendigkeit, einen solchen Sprechakt einzusetzen, deutet auf eine negative Implikation, als ob der Sprecher eine gewisse Zurückhaltung gegenüber der anpreisenden Rede aufseiten des Lesers vorwegnehmen würde. ${ }^{45}$ So wie die Frage gestellt wird, verbirgt sie einen der Gründe für eine solche Nähe der Stadt Luxemburg gegenüber den »beeindruckenden grünen Landschaften«: Z.B. liegt das sehr idyllische und romantische Städtchen Esch-sur-Sûre (im Norden des Landes) weniger als 50 Kilometer von der Hauptstadt entfernt. Dies anders zu formulieren würde bedeuten, dass man Gefahr liefe, die Bedeutung des Landes im Hinblick auf seine Fläche in den Augen des potentiellen Touristen zu verringern. Dieser Absatz endet mit dem Hinweis auf den starken Charakter der umgebenden Dörfer. Damit ist ein perfekter Übergang zum dritten Absatz geschaffen, der den vorangehenden mit der anaphorischen Redewendung >In diesem Zusammenhang < wieder aufnimmt. In der Einbindung des Lesers, mit dem das Gespräch über dieses und jenes fortgesetzt wird, ist eine neue Stufe erreicht: Der Preis Europa Nostra wird zum zusätzlichen Beleg für den historischen Wert des luxemburgischen Architekturerbes.

Der lexikalische Aspekt des Textes verstärkt schließlich dessen Heterogenität.

45 | Diese Einstellung schließt sich dem an, was im englischsprachigen Raum als Theory of Mind، oder 'Mentalisierung، bezeichnet wird. Die Übersetzung 'Theorie der mentalen Vorstellungen، ist vorzuziehen. (Vgl. Freyermuth 2008) 
Kein Stereotyp einer pseudo-poetischen Rhetorik wird ausgelassen ${ }^{46}$, ob es sich um die >bezaubernden Gegensätze < handelt, die >pittoresken Winkel<, die >grünen Landschaften $<$ oder andere >gastfreundliche Dörfer . Diese Allgemeinplätze werden noch durch eine Anhäufung von Ungeschicklichkeiten in der Verwendung der Register verstärkt und zeugen von einer Trivialität, die dem scheinbar gewählten stilistischen Effekt der vorangehenden Zeilen schadet. Kurz gesagt, der kleine, im ersten Teil hyperbolische und überfrachtete Text wandelt sich im letzten Teil zu einem tonalen und lexikalischen Flickwerk, was sich darin auswirken könnte, dass das Ziel der Broschüren, den potentiellen Touristen zu gewinnen, in Gefahr gerät.

Insgesamt wird durch die (zu) kurze Analyse von drei Heften des Luxemburgischen Tourismusbüros ein Hang zur Weitschweifigkeit deutlich, der sich darüber hinaus auf einen Rationalisierungsprozess und die Reduktion von Informationen auf prototypische Schemata stützt, mit dem Ziel, den Zugang zu den Gegebenheiten zu erleichtern. Der gleichzeitige Gebrauch von sowohl skripturalen als auch bildlichen Hyperbeln beeinträchtigt die erhoffte Hilfsfunktion und enttäuscht, vielleicht zu nachdrücklich, den brennenden Wunsch, Werbung um jeden Preis zu machen.

\subsection{LuXemburg, ein Land der Gegensätze}

Die Darstellung des luxemburgischen Gebietes in den Tourismusführern verlangt auch eine diachronische Untersuchung. Dieser Teil befasst sich mit der Frage, wie sich diese zugeschriebene Identität im Laufe des 20. Jahrhunderts innerhalb des Tourismus-Diskurses entwickelt hat und stellt die Frage nach Kontinuitäten und Diskontinuitäten. Auf den ersten Blick sind Tourismusführer nicht bestrebt, Grenzen zu ziehen, sondern vielmehr, diese zu überwinden, indem sie den internationalen Verkehr von Personen und Kapital fördern. Damit aber diese Grenzen überwunden werden können, müssen sie zuvor diskursiv errichtet werden. Luxemburg wird demnach auf zwei Ebenen als >anders < hingestellt: Einerseits wird dem Land mit Verweis auf seine ländliche Vergangenheit und seine kleine Ausdehnung eine Aura der Authentizität verliehen; andererseits werden seine moderne Wirtschaft und seine kulturelle Öffnung hervorgehoben. Diese gegensätzlichen Züge verleihen dem Land etwas Einzigartiges, das aus Sicht der Führer die touristische Bedeutung rechtfertigt. Die Darstellung des Gegensatzes Authentizität/Modernität verändert sich im Laufe der Zeit und wird immer unverträglicher, während das Bild einer Brückenfunktion zwischen dem deutschen und dem französischen Kulturraum immer deutlicher in den Vordergrund tritt und Luxemburg als europäisches Modell bestimmt.

Die vorliegende historische Analyse stützt sich auf sechs Tourismusführer, die wegen ihres sehr unterschiedlichen Entstehungskontextes ausgewählt wurden:

46 | Siehe Abschnitt 5.5. 
Dabei geht es einmal um das goldene Zeitalter der Eisenbahn ${ }^{47}$, dann um den Vorabend des Ersten Weltkriegs ${ }^{4}$, die Zwischenkriegszeit ${ }^{49}$, die $1960 e r-J a h r e$, die durch die 1000-Jahr-Feier der Stadt Luxemburg im Jahre $1963^{50}$ geprägt waren, sowie andere Jahre mit offensiven Tourismuskampagnen: 1995 (»Luxemburg - europäische Kulturhauptstadt «) $)^{51}$ und 2007 (»Luxemburg und die Großregion - europäische Kulturhauptstadt « ${ }^{2}$ ). Ein zweites gewähltes Auswahlkriterium liegt in der Möglichkeit, anhand der Führer »Woerl« $(1914,1934)$ und »Merian« $(1964,1995$, 2007) zu verfolgen, wie die Darstellung von Luxemburg im Laufe des 20. Jahrhunderts verlaufen ist. Die Auswahl wird durch den ersten Tourismusführer von Luxemburg ergänzt, veröffentlicht von Mathias Erasmy im Jahre 1861, sowie durch ein kurzes Porträt Luxemburgs, in 14 Sprachen erschienen im »Reader's Digest « im Jahre $1967^{53}$. Abgesehen von Erasmys Werk sind alle Texte vom Standpunkt eines Außenstehenden verfasst (meist auf Deutsch), wenngleich der Merian von 1967 auch Artikel luxemburgischer Autoren beinhaltet. Da die Führer nicht vom Office national du tourisme stammen, sind sie auch nicht darauf ausgerichtet, möglichst viele Besucher nach Luxemburg zu holen, sondern möchten eher die größtmögliche Anzahl von Leserinnen und Lesern erreichen. Eine Ausnahme macht der Führer von 1892 in dem Bemühen, für die Eisenbahnlinien zu werben. Das >Produkt< ist also nicht Luxemburg, sondern das Buch oder das Heft selbst, deren Aufgabe es ist, Begeisterung und Interesse zu wecken und sich gut zu verkaufen.

Dieses Korpus wird den qualitativen Gesprächen und besonders denjenigen Antworten gegenübergestellt, die die in Luxemburg ansässigen europäischen In-

47 | Erasmy, Mathieu (1861): Le guide du voyageur dans le Grand Duché de Luxembourg, Luxemburg: V. Bück; Anonyme (1892): De Luxembourg en Écosse. Guide du Touriste à travers Le Luxembourg. - L'Ardenne Belge. - Les Flandres. La Mer du Nord et la Manche. Le Comté de Kent. - Le Pays de Galles - Les Lacs Anglais. L'Écosse. Avec 100 dessins originaux de MM. Hoeterickx, Van Gelder etc. Publié par S. de Ruette, représentant du London Chatham \& Dover Railway [à Bruxelles], avec la haute approbation des Compagnies du London \& North Western Railway, du London Chatham \& Dover Railway, des Chemins de fer Prince-Henri et des Chemins de fer du Nord de France, Luxembourg: Pierre Bruck.

48 | Renwick, George (1913): Luxembourg. The Grand-Duchy and its People, London: T. Fisher Unwin; Anonyme (1914): Woerl's Reisehandbücher. Illustrierter Führer durch das Großherzogtum Luxemburg. Mit Plan der Stadt Luxemburg, 7 Kartenbeilagen und 65 Abbildungen, 2. Auflage, Leipzig: Woerl's Reisebücher-Verlag. Kaiserl. U Königl. Hofbuchhandlung. 49 | Anonyme (1934): Woerl Reisehandbücher. Großherzogtum Luxemburg [mit Plan der Stadt Luxemburg, 7 Karten und 29 Abbildungen], 3. Auflage, Leipzig: Woerl's ReisebücherVerlag.

50 | Merian (1964): Luxemburg. Merian XVII/7.

51 | Schröder, Dirk (1995): Merian live! Luxemburg, Munich: Gräfe und Unzer.

52 | Merian (2007): Luxemburg. Merian 60/03.

53 | Schisgall, Oscar (1967): "Le Luxembourg, pays de paradoxes", in: Sélection du Reader's Digest, 01.1967, S. 62-69. 
stitutionen betreffen. So soll erforscht werden, wie das Bild Luxemburgs als ein europäisches Musterland und als ein offener und multikultureller Raum von den Einwohnern/-innen rezipiert wird. Das Gegenbild, die Vorstellung eines ländlich geprägten und in seinen Traditionen verhafteten Landes, taucht in den Gesprächen nicht auf. Aufgrund der unterschiedlichen Quellen ist es schwierig, die zugeschriebenen kollektiven räumlichen Identitäten (Darstellungen u.a. aus den Tourismusführern) mit den angeeigneten individuellen räumlichen Identitäten zu vergleichen. Wenn man sich, wie wir es tun, auf die qualitativen Gespräche beschränkt, kann man die individuellen Aussagen zur räumlichen Identität nur mit dem jüngsten Führer, dem von 2007, vergleichen. Wir können höchstens überprüfen, ob die befragten Personen diese oder andere, ältere Stereotypen aufnehmen oder ob ihre Diskurse von denen der Tourismusführer abweichen.

Die Problemstellung ist neu; allgemein widmen sich die historischen, soziologischen oder anthropologischen Studien zum Tourismus in erster Linie den wirtschaftlichen Auswirkungen und den touristischen Praktiken. Sie beziehen sich vorwiegend auf den Ferntourismus, der unter dem Gesichtspunkt der Begegnung mit dem anderen untersucht wird, (vgl. Cohen 2004: 229-316) während der Nahtourismus unter diesem Aspekt wenig erforscht ist. Die Tourismusführer über Luxemburg richten sich jedoch eindeutig an eine Öffentlichkeit aus der näheren Umgebung: Mehr als zwei Drittel der Besucher stammen aus einem angrenzenden Land, aus den Niederlanden oder aus Großbritannien; nur $10 \%$ kommen aus einem außereuropäischen Land. Diese Zahlen haben sich zwischen 1980 und 2006 kaum verändert (Statec 2007: 213, 228). Nichtsdestoweniger schüren die Tourismusführer die Suche des Reisenden nach dem > Exotischen $<$, denn eines der vorrangigen Ziele des Touristen bleibt nun mal die physische und mentale Erfahrung des Unterschiedes. (Vgl. Hennig 1997) Die Führer werden den Erwartungen der Reisenden implizit gerecht, indem sie ihnen den Anblick >außergewöhnlicher Orte bieten, die in deutlichem Kontrast zur Welt der Arbeit stehen, Orte, die sie filmisch festhalten und so gleichsam zu Landschaften umformen können. (Urry 1990: 2f.) Urry und MacCannell betrachten übrigens die touristische Erfahrung, gekennzeichnet durch die Suche nach Authentizität, als bezeichnend für die (Post-)Moderne, die der Vergangenheit zugewandt ist (sichtbar auch in der Rolle der Museen), aber auch anderen Ländern, in denen das Leben als einfacher und ursprünglicher angesehen wird. (Urry 1990: 82, 104-134; MacCannell 1976: $103 \mathrm{ff}$. 48; Cohen 2004: 159-178) Eine Funktion der Tourismusführer besteht demnach darin, den Durst nach Authentizität zu stillen. Unsere Untersuchung wird zunächst zeigen, mit welchen Begriffen dieser >genius loci< beschrieben wird, und anschließend untersuchen, ob die >Eingeborenen « sich dieser Beschreibung anschließen und sich mit ihr identifizieren (oder auch nicht). 


\section{Die Suche nach Authentizität}

Die zugeschriebene Identität Luxemburgs als letzte friedliche Insel wird im Werk von Renwick übersteigert. Das erste Kapitel mit dem Titel »A Ruritania of today« ist von der nostalgischen Sehnsucht nach einer verloren gegangenen Welt geprägt: »The world appears to be fast outgrowing its Ruritanias [...]. The Empire of Speed [...] is wrecking Arcadia.« (Renwick 1913: 13) Dem Kult von Geschwindigkeit und Modernität, den das »Manifeste du Futurisme« von Marinetti (1909) eingeleitet hat, stellt der Autor die Reise ohne Eile, ohne Karte und Wegbeschreibung und vor allem ohne touristische Führungen entgegen: »This tiny paradise, this little unspoiled corner of earth which has known Nature's most happy inspirations, is for those whose guide is Wanderlust.« (Renwick 1913: 14) Die Industrialisierung wird jedoch nicht verteufelt. Das Urteil »the canton of Esch is blackened and blurred by furnances, forges and foundries « entbehrt nicht einer gewissen Poesie (Alliteration) und führt zu der Feststellung, dass das Großherzogtum nicht weniger als ein Vierzigstel der weltweiten Stahlproduktion liefert: »einen enormen Anteil« (Renwick 1913: 19). Dieser Gegensatz wird in der Darstellung Luxemburgs Ende der 196oer Jahre im »Reader's Digest« noch vertieft: Dort wird Luxemburg als Sitz der europäischen Institutionen und als »eine moderne und blühende Nation, die auf der Rangliste der europäischen Stahlhersteller auf dem siebten Rang liegt« beschrieben, bleibt aber zugleich weiterhin ein »idyllisches Fleckchen Erde«, das geradewegs einem Märchen entsprungen ist. Man sieht den letzten Zufluchtsort der Geruhsamkeit vor sich: »Le monde d'aujourd'hui a grand besoin d'un pays comme le grand-duché, ne serait-ce que pour prouver qu'il est encore possible de vivre tranquille sur notre planète. «44 (Schisgall 1967: 64)

Diese Seelenruhe wird von Gliedner (vgl. Merian 1964) allerdings lächerlich gemacht. In seinem Essay »Ellinger Blätter« prangert er die Provinzialität und die Faulheit (»Biertischgemütlichkeit«, »Fettbäuche«, »bourgeoise eingemachte Gurkenideen«) der Luxemburger an. Der Boden bleibe nur mithilfe von Kunstdünger fruchtbar und die Landschaft sei in sich selbst dermaßen lyrisch, dass Dichter überflüssig werden. Das Essay von Gliedner findet man am Ende des »Merian« von 1964, dessen Ikonografie zu einer subtilen Unterteilung in einen ersten straditionellen 'Teil führt, illustriert mit Fotos von Schlössern und Aufnahmen der Altstadt, und zu einem zweiten >modernen< Teil, in den Abbildungen von Fabriken und Werbeseiten eingestreut sind. Der Geschichte wird der wirtschaftliche Fortschritt gegenübergestellt. In der Mitte findet sich ein Höhepunkt der erstgenannten Richtung: Ein Schwarz-Weiß-Foto einer alten Bäuerin, das den bezeichnenden Titel »Beim Kartoffelschälen - Küchenidyll in einem alten Öslinger Bauernhaus« trägt und das auf der linken Buchseite, gegenüber dem auf der rechten Seite stehenden

54 | Deutsch: "Die heutige Welt braucht mehr denn je ein Land wie das Großherzogtum, und sei es nur, um zu zeigen, dass es noch möglich ist, ungestört auf unserem Planeten zu leben." 
Essay »Mir wële bleiwe wat mer $\sin$ «5 $^{55}$ des Ethnografen Joseph Hess zu sehen ist. (Merian 1964: 58-59) Hess erklärt dort, dass die Mentalität der Luxemburger trotz des Wohlstands und der Verstädterung ungehobelt ist und aufrichtig bleibt. Im »Merian« von 1964 herrscht also das >Traditionelle< vor, wie übrigens auch an der malerisch anmutenden Karte des Landes erkennbar ist. Sie ist mit Wappen und kleinen Zeichnungen geschmückt, die Schlösser, Kirchen und Industriestätten in den gleichen Farben und im gleichen Stil darstellen, so dass man sie auf den ersten Blick nicht unterscheiden kann. Die Industriestätten werden trotz der von einigen Autoren angesprochenen Unvereinbarkeiten dem arkadischen Bild Luxemburgs einverleibt.

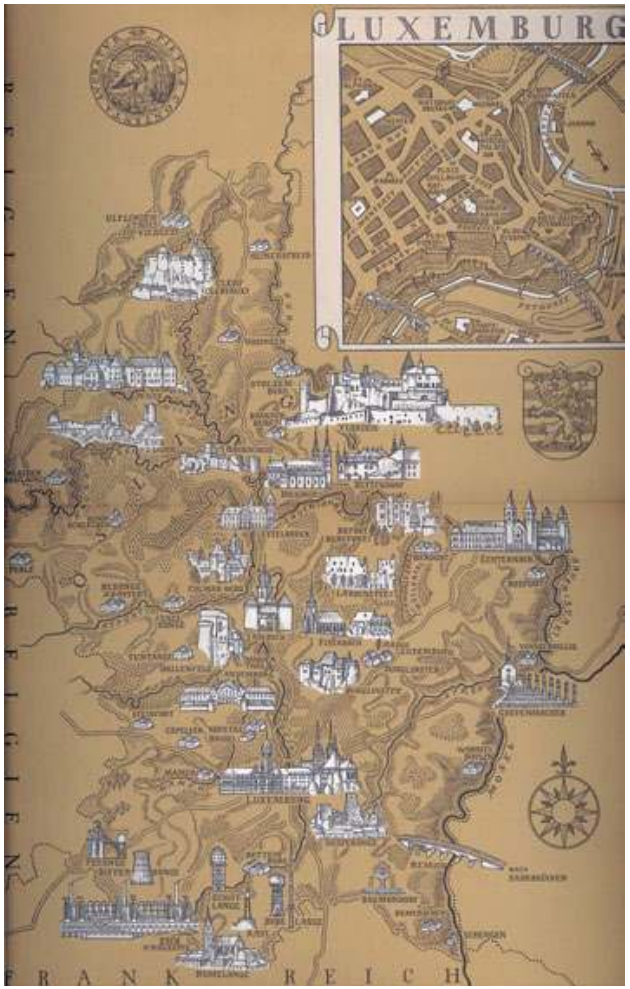

Abbildung 3: Malerische Karte des Landes (Merian 1964: 97)

Der »Merian« von 1995 greift die Werbestrategie des >Syndicat d'initiative touristique $<$ (heute >Office national du tourisme< genannt) auf, der seit den $1960 e r$ Jahren gleichzeitig auf den >sanften< Nahtourismus und den >harten< Geschäftstourismus

55 | Deutsch: "Wir wollen bleiben, was wir sind." 


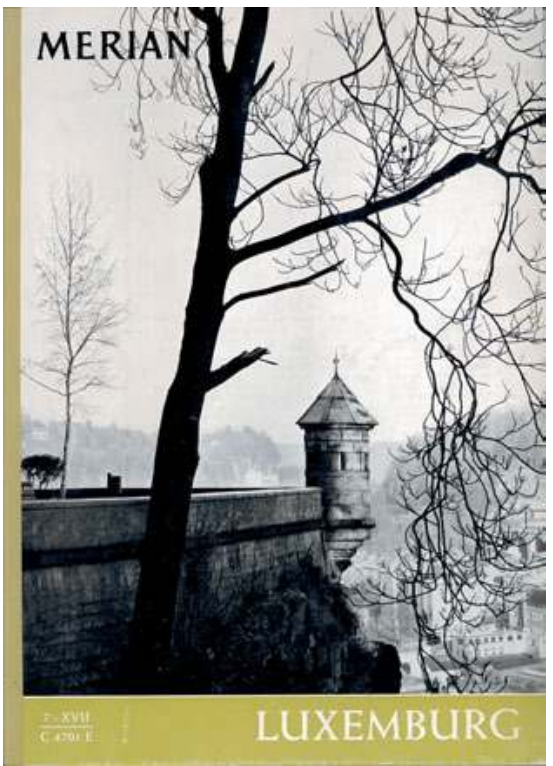

Abbildung 4: Titelseite des Merian 1964

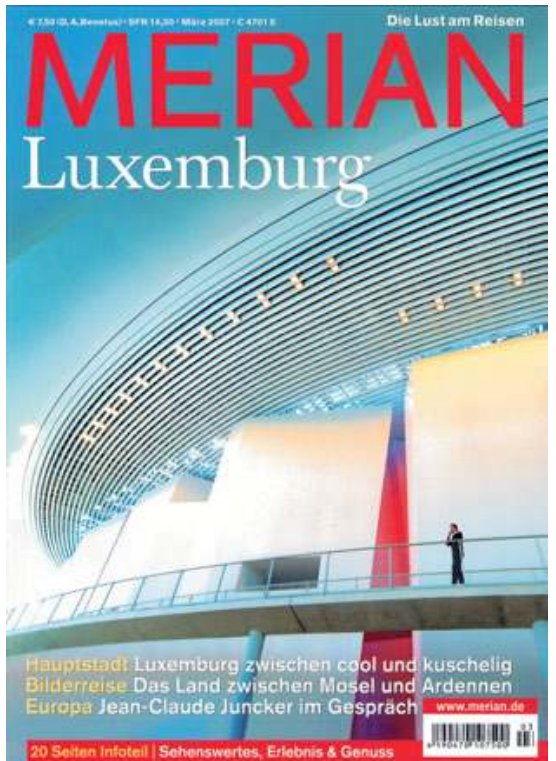

Abbildung 5: Titelseite des Merian 2007

setzt. Die Werbesprüche »Découvrez Luxembourg, le cœur vert de l'Europe« und »Luxembourg, a must for congress and incentive (Pinnel 1989: 951) spiegeln sich wider in den nebeneinandergestellten Bildern einer verglasten Bankfassade (»Am Boulevard Royal, auch >Wall Street< genannt«) und von Kühen auf einer grünen Weide (»Beschaulich: Idyll im Ösling«) (Merian 1995: 6f.). Im Jahre 2007 wird diese idyllische und dörfliche Landschaft, ein Sinnbild für Authentizität und Unschuld, nicht mehr an erster Stelle genannt. Die Hyperrealität wird über klischeehafte romantische Vorstellungen, einschließlich Schwäne und Nebelschwaden, eingebracht und erinnert an das Hameau von Marie-Antoinette oder das Schloss Neuschwanstein. (Vgl. Baudrillard 1994) Der sehr bukolische Titel, sowohl von den Illustrationen als auch vom Titel »In der Ruhe liegt die Kraft« her, hat als Untertitel »Sinnlich, still und leise zeigt Luxemburg seine Stärken: ein Land zum Auftanken« (Merian 2007: 86) und verweist damit, zumindest als Assoziation, auf den vollen Tank und damit auf das Auto und »the Empire of Speed«, das Renwick zurückweist. Mitschwingende Ironie ist aus den Untertiteln herauszuhören, etwa beim »Burgen-Land «, das eine Anspielung auf das österreichische Burgenland ist, oder aus dem Beitrag von Stölb »Das Land der Schlichter und Banker« als Anspielung auf Deutschland als »Land der Dichter und Denker « (Merian 2007: 14-15). Wie der »Merian« von 1964 stellt die Ausgabe von 2007 das alte Erbe und Innovationen nebeneinander, er vertauscht allerdings die Reihenfolge, indem er mit dem modernen und kosmopolitischen Teil beginnt (»Kulturhauptstadt«, »Banker«, »weltoffen «, »feiern«, »Nassauer«, »Kirchberg«, »Europa«, »Migranten«, »berühmt«), um sich dann dem auf die Vergangenheit gerichteten Teil zu widmen (»Familien- 
erbe«, »Sigismund «, »Ruhe«, »Sattel«, »Steinzeit«, »Vianden«, »bodenständig«) (Merian 2007: 4). Diese Schlüsselwörter, die aus den Artikelüberschriften des Inhaltsverzeichnisses stammen, zeigen Ironie: Die »Steinzeit « bezieht sich auf die Anfänge von RTL, d.h. die Vorgeschichte meint nicht die Zeit vor der Erfindung der Schrift, sondern die Zeit vor den modernen Medien.

Im Gegensatz zur Titelseite des »Merian« von 1964, der einen Teil einer Festungsanlage zeigt, bildet die Ausgabe von 2007 das Innere der Philharmonie ${ }^{5} \mathrm{ab}$ und unterstreicht den Titeltext »Luxemburg zwischen cool und kuschelig « durch die Wahl der Farben: Blau (kalte Farbe, »cool«) und rot (warme Farbe, »kuschelig«). Zusammen mit dem Weiß der Pfeiler ergibt sich die Trikolore Rot-Weiß-Blau. Die zugeschriebene Identität ist entweder die eines sich im Umbruch befindlichen Landes oder eines Landes, das sich in einem Zwiespalt zwischen Traditionen und Ambitionen befindet. Dieser Zwiespalt ist nicht nur temporärer Art (zwischen Vergangenheit und Zukunft), sondern auch räumlicher Art (zwischen einheimischer und globaler Ebene) und sogar mentaler Art (zwischen Rückzug und Öffnung). Die Öffnung ergibt sich aus der Tatsache, dass man sprachlich und kulturell »zwischen zwei Stühlen sitzt« (zwischen dem Deutschen und dem Französischen), eine Eigenheit, die den »außergewöhnlichen« Charakter Luxemburgs hervorhebt.

\section{Das Bild eines offenen und multikulturellen Landes}

Das hybride Wesen Luxemburgs ist ein Topos, der bis zum ersten Tourismusführer zurückreicht. Obschon Erasmy die Luxemburger/-innen als eine »deutsche Rasse germanischen Ursprungs« bezeichnet, so hebt er auch die Völkermischung hervor und beurteilt die einheimische Mentalität als einen Deut $\mathrm{zu}$ »nachahmend « im Hinblick auf die Nachbarn. Die Beschreibung des physischen Raumes ergibt sich ebenfalls aus den benachbarten Gebieten:

Les montagnes luxembourgeoises appartiennent au système des Ardennes et des Vosges [...]. Le noyau des Ardennes se trouve dans le Luxembourg belge [...]. Les sources des fleuves entre les bassins du Rhin et de la Meuse. La principale chaîne de montagne franchit la frontière et rattache Ardennes à Eifel. ${ }^{57}$ (Erasmy 1861: 3)

Auch Renwick unterstreicht die Verschmelzung von Völkern und Sprachen. Er meint, dass der/die Luxemburger/-in zweisprachig ist (französisch-deutsch) und darüber hinaus noch einen seltsamen Dialekt spricht:

56 | Siehe Abschnitt 5.4.

57 | Deutsch: "Die Luxemburgischen Berge gehören zu den Gebirgszügen der Ardennen und der Vogesen [...]. Der Kern der Ardennen liegt im belgischen Luxemburg [...]. Die Quellen der Flüsse zwischen den Flussgebieten von Rhein und Maas. Die Hauptbergkette reicht über die Grenze hinaus und verbindet die Ardennen mit der Eifel." 
And what a mixture that patois is! Just as the country itself carries traces of all the peoples who have swept across it and dwelt in it, so the people's language has borrowed from at least a dozen tongues [...]. Spoken, the patois sounds like curious Dutch and bad German coming from a worn gramophone. (Renwick 1913: 21)

Der »Merian« von 1964 stellt die Mehrsprachigkeit dem »Europäisch-Sein« zur Seite, beides sieht er personifiziert in dem >großen Europäer< Robert Schuman, der in Luxemburg geboren wurde. Friedländer formuliert dies in seinem Beitrag »Unser Europa begann in Luxemburg« folgendermaßen:

Diese [moselfränkische] Mundart war wohl seine eigentliche Muttersprache, denn er sprach deutsch nicht ganz wie ein Deutscher und französisch nicht ganz wie ein Franzose. In Schuman waren zwei nationale Kulturen verschmolzen. (Merian 1964: 70)

In der gleichen Ausgabe beschreibt der Schriftsteller Nic Weber, Mitbegründer der »Cahiers luxembourgeois« und der »Journées littéraires de Mondorf«, den kulturellen Spagat humoristisch als »Löwe zwischen Geflügel«, illustriert mit einer Federzeichnung, die den Löwen des luxemburgischen Wappens zeigt, wie er sich mit dem französischen Hahn und dem deutschen Adler zankt. (Merian 1964: 5) Keine Spur von Streit hingegen in dem Werk von 1995, das Luxemburg als Geburtsort von Robert Schuman, dem »Vater des europäischen Gedankens« vorstellt, »überzeugt vom Geist eines gemeinsamen Europas«, und der daraus ableitet, dass in Luxemburg »der europäische Geist tief verwurzelt ist« (Schröder 1995: 8) und dass die kulturellen Grenzen gefallen sind (Schröder 1995: 7). Das erste Kapitel nennt sich dann logischerweise auch »Eine Region stellt sich vor « (Schröder 1995: 4-9) und nicht etwa »Ein Land stellt sich vor«. Die natürlichen Grenzen sind ebenso abgeschafft: Die Mosel wird als Beispiel dafür genannt, dass eine Grenze nicht mehr teilt, sondern vereint (Schröder 1995: 6). In der chronologischen Übersicht wird das Jahr 1995, das Jahr, in dem Luxemburg europäische Kulturhauptstadt ist, folgendermaßen beschrieben: »Mit dem grenzüberschreitenden Programm spiegelt sich der Gedanke einer multikulturellen Gesellschaft in Kunst und Kultur wider« (Schröder 1995: 123).

Der »Merian« von 2007 schließt die Portugiesen/-innen in Luxemburg in dieses multikulturelle Konzept mit ein, präsentiert sie aber unter einem ethnisierenden Blickwinkel, »Saudade an der Sauer« (Merian 2007: 60-68). Im Editorial steht zu lesen, dass Luxemburg ein »Zwischenland « ist und das Cover titelt »Bilderreise: Das Land zwischen Mosel und Ardennen«. Als >typisch luxemburgisch « wird immer wieder das Bild der Brücke, der Vermittlung und der Durchmischung benutzt. (Merian 2007: 4) Die europäische Gesinnung ist eines der Schlüsselthemen des »Merian« von 2007. Die Titelseite kündigt ein Gespräch mit Jean-Claude Juncker über Europa an. Der Premierminister wird im Inhaltsverzeichnis als »Visionär« bezeichnet, der »für ein vereintes Europa kämpft«. Daneben sieht man ihn in einer Profilaufnahme, den Blick nach vorne gerichtet, flankiert von der europäischen 
Flagge. (Merian 2007: 5) Das Gespräch selbst trägt den Titel »Grenzen in Europa? Ein Horror! « und endet mit einem Abriss der Beziehungen zwischen Luxemburg und der EU unter dem Titel: »Das Zugpferdchen - Luxemburg ist Vorreiter der Europäischen Union und einer seiner stärksten Antreiber« (Merian 2007: 58). Wir haben die zugeschriebene Identität, die in den Führern des Jahres 2007 formuliert wird, in den Vordergrund gestellt, weil sie die einzige ist, die mit der angeeigneten Identität, so wie diese in den qualitativen Gesprächen artikuliert wird, verglichen werden kann.

\section{Ein zweideutiges und politisch (un-)korrektes Bild}

Die Umfragen von Eurobarometer zeigen, dass die Bindung Luxemburgs an die Europäische Union relativ stark ist. Anfang 2009 antworten $74 \%$ zustimmend auf die Frage »Generally speaking, do you think that [your country]'s membership of the European Union is ...? A good thing «, verglichen mit $52 \%$ im europäischen Mittel. (vgl. Commission européenne 2009: 85) Jedoch verbirgt diese umfassende Beurteilung Unzufriedenheiten und wesentlich ambivalentere Einschätzungen, die sich im Rahmen der qualitativen Gespräche in einem »ja, aber ...« ausgedrückt haben. Sie bestätigen zu einem großen Teil die Ergebnisse einer Studie, die 2006 über die Abstimmung für oder gegen den Vertrag für eine Verfassung für Europa durchgeführt wurde. Dem Vertrag stimmten 56,5\% im Juli $2005 \mathrm{zu}$. (Dumont et al. 2007) Die darauffolgende Untersuchung von vier Diskussionsgruppen zeigt Antwortstrategien auf, die darauf abzielen, als »politisch korrekt« (Dumont et al. 2007: 150) zu gelten. Ein ähnliches Meinungsbild hat die Analyse der qualitativen Gespräche vom Frühjahr 2009 ergeben. So wurde eine Frage unserer Studie, die als sheikel< galt - nämlich »Welche Bedeutung haben für Sie die europäischen Institutionen in Luxemburg? «zurückhaltend beantwortet. Die Antworten folgten einer Rechtfertigung oder wurden als unaussprechlich, verboten oder schrecklich betrachtet:

Mit zunehmendem Alter denke ich, hm (zögert) ist's ... normalerweise streng verboten, man ist sofort in der rechtsextremen Kiste, ausländerfeindlich und und und. Ich seh' trotzdem nicht, wo denn die Gemeinsamkeiten zwischen hier, den Luxemburgern, den Deutschen, den Franzosen hier von Nordfrankreich, den Belgiern und so weiter und den Portugiesen zum Beispiel sind. Weder in der Kultur noch in der Sprache noch in gar nix. Außer, dass alle gute Christen sein sollten. (Weiblich, 50 Jahre, Luxemburgerin, Rambrouch)

Voilà, mais avec des interférences aussi qui ne sont pas sur le plan politique non plus, mais sur le plan très concret. C'est-à-dire que euh [rire gêné], mais, ah, mon dieu, c'est horrible de dire ça comme ça, mais c'est, euh, quand on cherche un appartement à LuxembourgVille, euh, euh, dans, dans, de discu, enfin, je sais pas si c'est une légende urbaine ou si c'est authentique, que certains prix élevés de l'immobilier à la location au Luxembourg 
vient également de la présence de ces fonctionnaires européens. ${ }^{58}$ (Männlich, 30 Jahre, Franzose, Esch-sur-Alzette)

Diese Antworten zeigen zwei sich wiederholende Elemente: Die Beharrung auf kultureller Diversität, die durch die Europäisierung als bedroht betrachtet wird, und durchaus konkretere Beschwerden, die sich aus dem Auftreten der europäischen Beamten auf dem Immobilienmarkt ergeben. Von 28 zur Europäischen Union befragten Personen hat sich die Hälfte zustimmend geäußert, ein Viertel zeigt sich skeptisch und ein Viertel urteilt ambivalent. Die Stichprobe ist nicht repräsentativ, erlaubt aber gemäß der qualitativen Methode, die vorgebrachten Argumente zu vertiefen. Bei den positiven Einschätzungen der europäischen Institutionen in Luxemburg herrschen zwei Argumente vor. Das erste ist an das Bild Luxemburgs als kleines Land gebunden, das vor allem als Sitz einiger Institutionen und als Gründungsmitglied gesehen wird. Diese Überlegung bestätigt sehr deutlich den Tourismus-Diskurs. Das zweite Argument ist utilitaristischer: Die Institutionen werden als bedeutender Motor für die Schaffung von Arbeitsplätzen betrachtet und damit als > vorteilhaft< für das Land oder den Staat. Dieses Argument wird auch von jenen >unentschiedenen « Befragten geäußert, die ihre Aussagen nuancieren, indem sie z.B. auf den Nutzen hinweisen, von dem nur die »Oberschichten« profitieren (männlich, 39 Jahre, Portugiese, Strassen). Im Übrigen wird der europäische und multikulturelle Charakter Luxemburgs in Zweifel gezogen:

Die normale Bevölkerung identifiziert sich nicht damit. Das ist eine einzelne Gruppe von Europäischen Bürgern, die da arbeiten, die sich unter sich aufhalten und selten integriert sind. Oder sich integrieren wollen. Und deshalb ist auch wenig Kontakt dann da, von Menschen die da arbeiten mit der richtige Bevölkerung. Und deshalb, ja, das sind zwei Welten, die so parallel laufen. Ja, habe ich den Eindruck.

"Und meinen Sie denn, dass das für das Land selber eher vorteilig oder nachteilig ist?"

Ist schade, eigentlich - finde ich. Es ist zu verstehen, aber es fordert sicher nicht die multikulturelle Gesellschaft und Akzeptanz von Ausländern in Luxemburg. Macht das eher noch wieder ein Beispiel mehr, wo keine sich integriert und sich nicht anpasst. Ich glaube die Bevölkerung ist da eher ... Ja, es bringt ... Luxemburg ist mal wieder in den Schlagzeilen, wenn da irgendwas los ist. Aber sonst? (Weiblich, 30, Niederländerin, Lintgen)

58 | Deutsch: "So, aber mit Überschneidungen, die sich auch nicht auf politischer Ebene befinden, sondern auf einer sehr konkreten Ebene. Das heißt, dass ehm [verlegenes Lachen], aber, ach, mein Gott, es ist schrecklich das so zu sagen, aber es ist, ehm, wenn man eine Wohnung in Luxemburg-Stadt sucht, ehm, ehm, in, in Gesprächen, also, ich weiß nicht ob das eine urban legend، ist oder ob es stimmt, dass die hohen Immobilienpreise in Luxemburg auch auf die starke Präsenz der EU-Beamten zurückzuführen sind.“ 
Der Gedanke von Parallelwelten wird ebenfalls von einer Befragten aufgenommen, die sagt:

C'était, un moment donné, j'avais lu, c'était à propos des Etats-Unis où ils disaient: ,le melting-pot est terminé, on est passé à un système de mosaïque.، Et, j'avais l'impression que ça faisait un petit peu pareil sur le Luxembourg où il y avait une petite mosaïque euro, euh, dans la mosaïque, il y avait des petits bouts européens qui ne se, à côté il y avait les gens de la finance ou les, ou les Portugais, les Français, les Luxembourgeois de souche, et les Belges. Et ça vivait côte à côte de manière sympathique et sans accrocs. Mais côte à côte, pas ensemble. ${ }^{59}$ (Männlich, 30 Jahre, Franzose, Esch-sur-Alzette)

Die Mehrheit der Befragten, die die >Integration « als schwach und die Gesellschaft als gespalten betrachtet, lebt oft selbst in einer gemischten Partnerbeziehung und in einem komplexen mehrsprachigen Umfeld. Ihre Antworten, die Erlebtes, Vorurteile und Allgemeinplätze vermischen, ermöglichen es, den touristischen (und politischen) Diskurs zu differenzieren, der aus Luxemburg ein europäisches Modell macht (vgl. Majerus 2007) und es als ein interkulturelles Land betrachtet.

Schon seit den ersten touristischen Führern im 19. Jahrhundert wird Luxemburg als eine Besonderheit dargestellt. Die Autoren (alle männlich) sehen sich als Entdecker, als Ethnologen oder Soziologen, die ein Land und dessen Leute studieren, die wie auf einer Insel der Beschaulichkeit und/oder des Wohlergehens leben. Dieses Bild steht im offenkundigen Widerspruch zu dem einer Mischung der Kulturen (germanisch und romanisch), einer Welt der sprachlichen und ethnischen Verschmelzung. Dennoch werden beide Klischeevorstellungen zusammengeführt, um den außergewöhnlichen Charakter Luxemburgs zu unterstreichen. Der Widerspruch liegt eher in dem Gegensatz der Kleinheit des Landes (oft mit bukolischen Begriffen beschrieben) und dessen wirtschaftlichem und politischen Gewicht innerhalb der Europäischen Union. Der Widerspruch vertieft sich mit den Jahren, aber die diachronische Untersuchung belegt vor allem die erstaunliche Beständigkeit der zugeschriebenen Identitäten. Was die angeeigneten Identitäten betrifft, so haben wir nur die unterschiedlichen Haltungen gegenüber dem europäischen Modell untersuchen können und feststellen müssen, dass die zusammengetragenen Meinungen sehr viel unterschiedlicher und kritischer sind. Wenn manche Personen das Bild eines offenen und toleranten Luxemburg zeichnen, so sehen andere das Hervorbrechen von Parallelgesellschaften, die keinen Kontakt miteinander haben.

59 | Deutsch: "Es war, ich hatte einmal gelesen, es war über die Vereinigten Staaten, wo man sagte: 'Der Melting-Pot ist vorbei, er wurde abgelöst vom Mosaik-System. Und ich hatte den Eindruck, dass das etwas Ähnliches sei in Luxemburg, wo es ein kleines EuroMosaik gibt, ehm, in dem Mosaik gibt es kleine europäische Stückchen, die sich nicht, daneben gibt es Leute aus dem Finanzsektor oder die, oder die Portugiesen, die Franzosen, die ,Urluxemburger und die Belgier. Und die leben nebeneinander in sympathischer Weise und ohne Schwierigkeiten. Aber nebeneinander, nicht miteinander." 


\subsection{DiE iGRosSREgIONı - RAUMKONSTRUKTIONEN ZWISCHEN WUNSCH UND WIRKLICHKEIT}

Eine Untersuchung zu den touristischen Diskursen in Luxemburg muss sich zwangsläufig auch mit der Frage beschäftigen, wie der grenzüberschreitende Kooperationsraum, die sogenannte $>$ Großregion ${ }^{60}{ }^{60}$, diskursiv repräsentiert, verhandelt und angeeignet wird. Dabei steht - anders als auf der nationalen und städtischen Ebene - kein breiter Korpus etablierter Tourismusliteratur bestehend aus allgemeinen Reiseführern, touristischen Karten und Plänen sowie thematischen Spezialführern zur Verfügung. Gleichwohl scheint gerade dieser auf eine Außendarstellung (und Imagebildung) eines neuen Regionszuschnitts bedachte Korpus besonders für die vorliegende Studie geeignet, steht er doch im Verdacht, in besonders starkem Maße Identitätszuschreibungen vorzunehmen.

\section{Untersuchungskorpus und konzeptionelle Vorbemerkungen}

Trotz langjähriger Bemühungen um eine stärkere grenzüberschreitende Koordination und Konzertation des Tourismusmarketings und -managements bleiben hier verwendbare Übersichtsdarstellungen die Ausnahme. Aus diesem Grund wurden in der vorliegenden Mikroanalyse nur zwei jüngere Werke analysiert. Zum einen ein Kompaktreiseführer ${ }^{61}$, der anlässlich des Kulturhauptstadtjahres »Luxemburg und Großregion. Europäische Kulturhauptstadt 2007 « von einem luxemburgischen Verlag herausgegeben wurde. Zum anderen ein eher essayistischer Sammelband mit einem facettenreichen Portrait der Großregion ${ }^{62}$, der von einem Autorenkollektiv aus grenzüberschreitend engagierten Wissenschaftlern/-innen, Kulturschaffenden und Journalisten/-innen gestaltet wurde. Während es sich bei dem Werk von Mendgen et al. (»Im Reich der Mitte/Le berceau de la civilisation européenne. Savoir-faire/savoir-vivre«, im Weiteren IRDM) um ein reichhaltig ausgestattetes Gemeinschaftswerk handelt, wurde die erste Quelle (»Die Großregion entdecken/ Découvrir la Grande Région«, im Weiteren DGRE) federführend von einer Autorin bearbeitet. Beide Werke sind zweisprachig deutsch-französisch gestaltet, wobei in IRDM die Manuskriptsprache der Verfasser variiert, während DGRE vollständig

60 | Die ,Großregion، bezeichnet einen grenzüberschreitenden Kooperationsraum, zu dem neben dem Großherzogtum die französische Region Lothringen, die deutschen Bundesländer Rheinland-Pfalz und Saarland sowie die belgische Region Wallonien (inkl. der Deutschsprachigen und der Französischen Gemeinschaft) gehören. Mit über elf Millionen Einwohnern und einer Fläche von über $65.000 \mathrm{~km}^{2}$ handelt es sich dabei um den größten subnationalen Kooperationsraum in Europa, siehe auch: www.grossregion.net

61 | Scholz, Ingeborg (2007): Die Großregion entdecken/Découvrir la Grande Région, Luxembourg: Guy Binsfeld.

62 | Mendgen, Eva/Hildisch, Volker/Doucet, Hervé (Hg.) (2007): Im Reich der Mitte/Le berceau de la civilisation européenne. Savoir-faire/savoir-vivre, Konstanz: Hartung-Gorre. 
auf Deutsch verfasst und dann übersetzt wurde. Die weitere Analyse stützt sich auf die jeweilige Originalfassung (zur Problematik der Zweisprachigkeit s.u.).

Bei DGRE handelt es sich um einen handlichen Reiseführer, der als >offizieller Beitrag z zum Kulturhauptstadtjahr 2007 erschien. IRDM ist eher als Hintergrundbeitrag zu verstehen, dessen redaktioneller Teil im Umfang weit über DGRE hinausgeht, und der nur im weiteren Sinne dem >touristischen Diskurs < zuzuordnen ist. Gleichwohl besitzt er zumindest teilweise den Duktus eines >promotionell< ausgerichteten Werkes - nicht zuletzt bedingt durch die Emphase und die institutionelle Position einiger Autoren/-innen.

Hinsichtlich der hier betrachteten tendenziell eher angeeigneten Identitäten flossen in den folgenden Abschnitt ferner die Ergebnisse der qualitativen Interviews wie auch der standardisierten Befragung ein.

Der mikroanalytischen Betrachtung der beiden Werke aus human- bzw. kulturgeografischer Perspektive liegt ein (de-)konstruktivistisches Verständnis zugrunde, das sich eines pragmatischen Diskursbegriffs bedient. Letzterer beschränkt sich in Weiterentwicklung zu Foucaults eher holistischem Diskursverständnis auf für den Forschungsgegenstand relevante Diskurspraktiken (vgl. Hajer 1995 und 2003), wie sie sich etwa in geschriebenen Texten oder gesprochener Sprache manifestieren und zur Entwicklung spezifischer Diskursstränge (>Story Lines<, >Narratives<) führen können. Diese konstituieren sich aus punktuellen diskursiven Ereignissen (z.B. Politikerrede) oder Diskursfragmenten (z.B. Diskursmuster einer Publikation) und erlangen durch deren Repetition und/oder Verkettung einen dominanten und damit im vorliegenden Fall identitätsprägenden Charakter. (Vgl. Strüver 2005; Mattissek 2007) Auch methodologisch wird im Weiteren eine pragmatische Form der kritischen Diskursanalyse gewählt, die sich im Wesentlichen auf der semiotischen bzw. inhaltlich-assoziativen Ebene bewegt, um über wiederkehrende Begriffe, Metaphern und Diskursfragmente (siehe Schlüsselzitate) spezifische >Story Lines< oder narrative Muster zu identifizieren und einer ersten Interpretation zuzuführen. ${ }^{63}$

Der Schwerpunkt der Untersuchung lag auf einer Betrachtung der für die Fragestellung relevanten redaktionellen Teile, d.h. in IRDM vor allem die Beiträge Mendgen/Hildisch (S. 13-20) und Gengler (S. 67-73), in DGRE vor allem die einführenden Passagen (S. 8-13).

\section{Dominierende Topoi der Identitätszuschreibung}

Eine erste Sichtung der beiden Werke lässt rasch eine Reihe wiederkehrender Diskurselemente in Form interdiskursiver Metaphern und Bilder ${ }^{64}$ erkennen, die im Wesentlichen mit den Topoi >Zentralität<, >Aneignung des Raums<, >Einzigartig-

63 | Diese Vorgehensweise kann und will jedoch nicht den Ansprüchen etwa einer linguistischen Diskursanalyse im engeren Sinne gerecht werden.

64 | Siehe auch Kapitel 6. 
keit< und >Modellcharakter < überschrieben werden können. Es handelt sich dabei mit Weichhart (1990) sowohl um »Identitätsmerkmale des Raumes«, also kognitiv-emotionale Repräsentationen der Großregion in Bewusstseinsprozessen eines Individuums oder einer Gruppe von Individuen (raumbezogene Perspektive), als auch um »Raum als Bestandteil individueller und/oder kollektiver Identitätsmuster«, d.h. diejenigen gedanklichen Repräsentationen und emotional-affektiven Bewertungen der Großregion, die Teil des individuellen >Selbst- bzw. kollektiven WirKonzepts< werden (personenbezogene Perspektive).

\section{`Herzı, `Nabelı, `Rücken` - zur Anatomie Europas und der Großregion}

Beinahe inflationäre Verwendung finden in beiden Quellen Umschreibungen, die der Großregion eine besondere geografische, kulturelle oder gar politisch-wirtschaftliche Zentralität in Europa zusprechen. Dabei wird der rein geometrischen Verortung zumeist auch eine qualitative Nuance gegeben, etwa im Sinne eines wie auch immer zu bewertenden - Bedeutungsüberschusses verglichen mit anderen Regionen Europas. Bereits der in seiner deutschen Fassung unbescheidene Titel »Im Reich der Mitte« scheint mehr als eine augenzwinkernde Anspielung auf einen ungleich größeren Kulturraum zu sein. Betrachtet man die französische Variante »Le berceau de la civilisation européenne ${ }^{65}$, so schwingen hier eindeutig ambitionierte Positionierungsversuche mit. Vergleichsweise zurückhaltend - und beinahe geografisch-konzeptionell - nimmt sich dagegen der englischsprachige Titel »The Center at the Edge« aus. Was in den Titelvarianten mehr oder weniger implizit bleibt, wird im weiteren Text unmissverständlich als Portrait »des eigentlichen geopolitischen und historischen Herzens ${ }^{66}$ Europas « (IRDM: 14) dargestellt. Die Großregion ist demnach unzweifelhaft ein »Teil des dicht besiedelten >europäischen Rückens « (DGRE: 8). Letztere - nach Kenntnis des Verfassers im Deutschen nicht verfangende Lagebezeichnung - erklärt sich womöglich aus der französischen >dorsale européenne< (Brunet 1989), in Deutschland besser bekannt als >Blaue Banane<; jener vermeintlich zentrale Wirtschafts- und Siedlungsraum in Europa.

Geradezu als ob die Bedeutung der Zugehörigkeit zum >Zentrum< noch zivilisationshistorisch unterstrichen werden solle, wird - sehr exponiert - ein Zitat des rumänischen Religionswissenschaftlers und Philosophen Mircea Eliade aus dem Jahr 1957 bemüht: »Der Mensch der vormodernen Gesellschaften will so nahe wie möglich am Zentrum der Welt leben. Er weiß, dass sein Land wirklich in der Mitte der Erde liegt, dass seine Stadt den Nabel des Universums bildet«/»l'Homme de la société pré-industrielle souhaite vivre aussi près du centre du monde. Il sait que sa patrie se situe vraiment au centre de la Terre, que sa ville est le nombril de l'univers.« (IRDM: 225) Unklar bleibt hier, ob diese vormoderne Geisteshaltung auch Gegenwartsmaxime sein soll.

65 | Deutsch: "Die Wiege der europäischen Zivilisation/Kultur".

66 | Die Kollektivsymbolik der Herz-Metapher bildet auch in der Darstellung Luxemburgs in den internationalen Medien eine tragende interdiskursive Rolle. (Vgl. Parr 2009) 


\section{Aneignungsprozesse - oder: Une réalité vécue?}

Auffallend stark wird in den beiden Quellen auch eine personenbezogene Perspektive eingenommen, um großregionale Wahrnehmungs- und Identitätsmuster - teilweise mit einem fast programmatischen Imperativ versehen - herauszustreichen. Nicht weiter belegte Aussagen wie »Die Menschen nehmen diese Großregion ohne Grenzen wie selbstverständlich an« (IRDM: 16) oder »SarLorLux< est une réalité vécue « ${ }^{67}$ (IRDM: 67) münden in der fast ethnografisch anmutenden Verkündung eines neuen Artenfundes:

A l'instar du 'SarLorLux', il existe le 'SarLorLuxois' ou la 'SarLorLuxoise،. Depuis de nombreuses années, des centaines de milliers d'individus assimilent cette vaste étendue à une véritable région. Ce chiffre ne cesse pas de croître et nous voyons, nous entendons, nous rencontrons partout ces personnes. (IRDM: 67$)^{68}$

Deutlich zurückhaltender gibt sich hier DGRE:

Qu'est-ce que les habitants de la Grande Région savent les uns des autres? Existe-t-il une conscience identitaire commune? L'absence - provisoire - de cette dernière est assez souvent regrettée. Ce petit guide touristique ne doit toutefois pas servir aux lamentations. Mieux vaut se préoccuper des opportunités de forger une conscience identitaire. Une identité n'est pas une saute d'humeur, mais un sentiment qui résulte de l'expérience, du contact, de la communication et de la connaissance. (DGRE: 13) ${ }^{69}$

Ein gemeinsames grenzüberschreitendes Identitätsbewusstsein sei demnach erst noch zu schaffen bzw. zu »schmieden«, wenngleich »Erfahrung und Kontakt [...] auf breiter Ebene bereits Gegenwart der Großregion« (DGRE: 12) sind.

\section{Einzigartige Modellregion und Laboratoire de l'Europe}

Eng mit dem Zentralitätstopos verknüpft ist die nicht seltener herausgestellte Besonderheit oder gar Einzigartigkeit der Großregion. Superlative - »die erste grenzübergreifende Kulturhauptstadt« (IRDM: 13) - werden ebenso gerne bemüht wie

67 | Deutsch etwa: "Die 'SaarLorLuxı-Idee ist (vor-)gelebe Realität."

68 | "Wie es 'SaarLorLux gibt, so auch den 'SaarLorLuxer، oder die ,SaarLorLuxerin`, die großregionale Dimension ist bereits seit langen Jahren in den Köpfen hunderttausender Menschen präsent. Ihre Zahl wächst, und man hört und sieht sie, man trifft sie überall." (IRDM: 67)

69 | "Was wissen die Bewohner der Großregion voneinander? Gibt es ein gemeinsames Bewusstsein von Identität? Dass ein solches - noch - fehlt, wird immer wieder beklagt. Der knappe Platz dieses Reiseführers soll allerdings nicht zum Lamentieren verschwendet werden. Fragen wir lieber: Wo liegen die Potenziale einer Identitätsbildung? Identität kommt nicht einfach so als wohliges Sentiment angeflogen, sondern entwickelt sich durch Erfahrung, Kontakt, Kommunikation und Wissen." (DGRE: 13) 
anmaßende Charakterisierungen, wenn beispielsweise der Bevölkerung in verklärender Weise »Mehrsprachigkeit und angeborene interkulturelle Kompetenz« (IRDM: 16) bescheinigt wird. Auch hier wird die >gemeinsame pektive bemüht, um gegenwärtige Notwendigkeiten zu identifizieren:

2.500 Jahre gemeinsamer Geschichte verbinden die einzelnen Regionen miteinander. Da mutet die Bezeichnung ,Großregion، für ein Gebilde derartiger kultureller Reichtümer an wie ein blasses Provisorium - und das ist es auch. Versuche einer klangvolleren Namensgebung sind bislang gescheitert. So muss die Großregion weiterhin mit ihrem nüchternen Namen leben, kann ihre Energien aber nun darauf verwenden, sich selber und der Mitwelt zu vermitteln, wer sie eigentlich ist. (DGRE: 8)

Einzigartigkeit wird in diesem Zusammenhang mitunter durchaus wertend verstanden, im Sinne von beispielhafter Pionierregion für grenzüberschreitende Integration. Umschreibungen wie »Mini-Europa«, »laboratoire de l'Europe« u.Ä. sind sehr häufig zu finden. Wird nicht der Großregion Modellcharakter zugeschrieben, so zumindest einem Teil ihrer Bevölkerung: »Die grenzüberschreitenden Berufspendler gelten bei Experten quasi als Avantgarde der Großregion.«(DGRE: 12)

Wie oben bereits angedeutet, wirft die zweisprachige Gestaltung der beiden untersuchten Werke zusätzliche - nicht nur methodologische - Fragen auf. Die ohnehin gebotene Vorsicht bei der diskursanalytischen Annäherung an einen mehrsprachigen Korpus gilt umso mehr, wenn zwischen den beiden Sprachfassungen mitunter Sinn erweiternde bis Sinn verzerrende Abweichungen bestehen. Die nachstehende Tabelle enthält als Beispiel einige Kapitel- und Zwischenüberschriften aus IRDM; die Originalfassung ist jeweils kursiv hervorgehoben:

\begin{tabular}{|l|l|}
\hline Deutsche Fassung & Französische Fassung \\
\hline Im Reich der Mitte & $\begin{array}{l}\text { Le berceau de la civilisation } \\
\text { européenne }\end{array}$ \\
\hline Eine (Groß-)Region rauft sich zusammen & $\begin{array}{l}\text { La Grande Région - un terrain } \\
\text { d'entente? }\end{array}$ \\
\hline Renovatio Lotharingiae? & $\begin{array}{l}\text { Renovatio Lotharingiae ou } \\
\text { l'affrontement définitif }\end{array}$ \\
\hline Erste Phase der Industrialisierung & Un esprit nouveau \\
\hline Lothringen, zwischen Frankreich und & $\begin{array}{l}\text { Entre France et Allemagne, la Lorraine } \\
\text { Déchirée }\end{array}$ \\
\hline
\end{tabular}

Tabelle 1: Beispiele für Abweichungen zwischen der deutschen und französischen Fassung von IRDM.

Da hier jedoch weder den Übersetzenden noch den Herausgebern/-innen intentionales Handeln unterstellt werden kann und es sich zudem nur um einige punk- 
tuelle Abweichungen vor allem auf Ebene der (Zwischen-)Überschriften handelt, sollen diese Effekte nicht überbewertet werden. Nur eine systematisch vergleichende Feinanalyse, die im vorliegenden Rahmen weder vorgesehen noch möglich war, könnte zeigen, ob hier Abweichungen auszumachen sind, die womöglich unterschiedliche Diskursstränge in den beiden Sprachen alimentieren.

Zusammenfassend kann für beide Quellen als hervorstechendes Diskursmuster das offenkundige Bemühen um Betonung der qualitativen Besonderheiten (historische Bedeutung, sprachlich-kulturelle Komplexität, Pioniercharakter usw.) sowie der >Zentralität< (geografisch, politisch, ökonomisch, kulturell) der Großregion identifiziert werden. Referenzrahmen ist ein mehr oder weniger abstraktes Europa (Kontinent/EU/>Kulturraum<), innerhalb dessen der Großregion und ihren Einwohnerinnen und Einwohnern eine beispielhafte Sonderrolle beigemessen wird. Die im Folgenden ausgewerteten Quellen erlauben einen ersten empirischen Blick auf die Befindlichkeiten der gerne auch als >Modelleuropäer< etikettierten Bevölkerung Luxemburgs.

\section{Wahrnehmungen der Nachbarregionen}

Ausgehend von der Annahme, dass sich grenzüberschreitende Identitätsmuster bei Individuen kaum ohne persönliche Erfahrungen mit bzw. in den jeweiligen Nachbarregionen ausbilden können, beschäftigte sich ein Teil unseres Fragebogens und der qualitativen Interviews zunächst mit Handlungsroutinen, Wahrnehmungen und Einstellungen bzgl. der umliegenden Teilräume der Großregion. Die Besuchsfrequenz der standardisiert Befragten fällt dabei für alle Nachbarländer gleich hoch aus, mit einem geringfügig höheren Wert für die deutschen Anrainerländer Rheinland-Pfalz und Saarland. Die Besuchsfrequenz ist dabei unbeeinflusst von Alter und Geschlecht der Probanden. Auch die jeweiligen Milieus (s.u.) zeigen hier keine nennenswerten Unterschiede. $\mathrm{Zu}$ den persönlichen Präferenzen hinsichtlich des > Wohlfühlens < beim Besuch der Nachbarländer äußern sich die Befragten insgesamt eher ausgeglichen. Allerdings zeigt sich hier ein deutlicher Altersgradient dergestalt, dass positive Bewertungen für Frankreich eher von älteren Befragten, positive Bewertungen bzgl. Deutschlands eher von jüngeren Befragten geäußert wurden..$^{70}$ Dies mag mit altersgruppenspezifischem Konsum- und Freizeitverhalten zusammenhängen, spiegelt aber möglicherweise auch historisch bedingte Vorurteile und Ressentiments wider. Dieser Gradient findet sich jedoch nicht in der Beantwortung der Gegenfrage nach dem Nicht-Wohlfühlen, deren Ergebnis noch ausgeglichener ausfällt.

Stärker noch als der Altersgradient zeigen sich hier jedoch auch milieuspezifische Präferenzmuster, die etwa im Falle des traditionsorientierten Milieus eindeutig zugunsten Deutschlands ausfallen (42\% gegenüber $18 \%$ für Frankreich, $15 \%$ für Belgien und $18 \%$ unspezifisch). Dies gilt in ähnlicher Weise für das alternative

70 | Siehe ausführlicher Abschnitt 4.2. 
(32 \% für Deutschland, $16 \%$ für Frankreich, $8 \%$ für Belgien und $36 \%$ unspezifisch) und das statusorientierte Milieu (41\% für Deutschland, $26 \%$ für Frankreich, $7 \%$ für Belgien und $19 \%$ unspezifisch). Auch hier fällt das Antwortmuster zur Gegenfrage ausgewogener aus, wenngleich das unterprivilegierte Milieu (28\% für Deutschland, $23 \%$ für Frankreich und $17 \%$ für Belgien) und das liberal-gehobene Milieu (23 \% für Deutschland, 15 \% für Frankreich und 19 \% für Belgien) deutlich größeres Unwohlsein in Deutschland empfinden.

Die Befunde der qualitativen Interviews zeigen jedoch, dass in vielen Fällen sehr nuanciert geantwortet und kommentiert wurde, was die nachstehende Auswahl von Zitaten zu illustrieren vermag:

Nee, ich bin jetzt am Überlegen, ob's Deutschland oder Frankreich ist. Wenn ich ausländerfeindliche Sachen in Deutschland beobachte, dann sage ich mir, oh, Gott sein dank wohne ich hier nicht. Und die gibt's auch in Trier. Und in Frankreich, da laufen manchmal so Gestalten rum, wo ich sage, hm, der wird dir ja jetzt nicht was tun. Also, ich würde sagen, in beiden fühle ich mich nicht unbedingt wohl. (Weiblich, 32 Jahre, Luxemburgerin, Leudelange)

Also während der Zeit, wo ich arbeitete, hab ich mich am wohlsten gefühlt in Belgien. Und wenn ich dort arbeiten und leben müsste, noch heute, wäre ich nur aus persönlichen, mir persönlichen Gründen, hätte ich mich wahrscheinlich in Belgien etabliert. Aber durch meine Frau, die mag das nicht. Nein. Aber, und wenn ich hier wählen könnte, heute, würde ich auch nicht in Luxemburg leben. Würde ich auf der deutschen Seite leben. Und ich hab in Deutschland, bekomm ich relativ schnell Kontakt, egal, wie man das jetzt sagt. Und in Belgien auch. Fran... nach Frankreich will ich nicht gehen. Auf keinen Fall. Das ist etwas ... (Männlich, 67 Jahre, Luxemburger, Echternach)

Ça a toujours été [la France], même en tant que gosse. On est parti souvent en, en France en vacances avec mes parents. Moi, je me sens bien en France. Les gens sont beaucoup plus relax qu'en Allemagne. En Allemagne, c'est toujours ehm, il faut que ce soit comme ça euh. Je ne sais pas, ils sont ... deutsche Gründlichkeit, ne? ${ }^{71}$ (Weiblich, 54 Jahre, Luxemburgerin, Walferdange)

Während derartige Pauschalurteile bezogen auf die jeweilige Nation zumeist biografisch bzw. sozialisationsbedingt geprägt sind und von der verklärten Verallgemeinerung von positiven Jugend- und Urlaubserinnerungen bis hin zur Überbewertung punktueller Negativerfahrungen (etwa als Tourist in einem Ferienort) reichen, fallen die Urteile hinsichtlich der unmittelbaren Nachbarregionen diffe-

71 | "Das war es immer [Frankreich], selbst als kleines Kind. Wir sind mit unseren Eltern oft nach, nach Frankreich in Urlaub gefahren. Ich persönlich fühle mich wohl in Frankreich. Die Leute sind sehr viel entspannter als in Deutschland. In Deutschand ist es immer ähem, es hat so zu sein, ne. Ich weiß nicht, sie sind... deutsche Gründlichkeit, ne?" (Weiblich, 54 Jahre, Luxemburgerin, Walferdange) 
renzierter und weniger stereotyp aus. Zahlreiche Interviewte betonen die Unterschiede in ihrer Haltung zur jeweiligen Grenzregion und zum Rest bzw. Zentrum des Nachbarlands (etwa Südwallonien vs. Flandrische Nordseeküste oder Brüssel, Lothringen vs. Côte d'Azur oder Paris). Dabei sind positive wie negative Urteile etwa gleich häufig anzutreffen.

Auffällig oft werden Belgien bzw. die belgische Bevölkerung als Referenz herangezogen, um zu beschreiben, was man an den beiden >großen « Nachbarn Frankreich (häufig) und Deutschland (hier seltener) nicht mag. Sympathien für den relativ kleinen Nachbarn mögen hier ähnlich zum Tragen kommen wie historische Bezüge, familiäre Verflechtungen oder Vertrautheit durch 6o Jahre Benelux-Kooperation.

Seltener führt die regionale Differenzierung zu einem integrativen Urteil über die Gemeinsamkeiten und Bezüge der gesamten Grenzregion (ohne dabei notwendigerweise die Großregion in ihrer o.g. Abgrenzung zu meinen). Sprachliche Gemeinsamkeiten sowie sonstige kulturelle oder Mentalitätsbezüge stehen hier argumentativ im Vordergrund:

[...] d'Grenzregioun einfach. Also déi eicht $50 \mathrm{~km}$ iwwert d'Grenz ass dat nämlecht wéi fir d'Belsch. Et kann een net vergläichen Frankräich ass een ganz grousst Land, et kann een net vergläichen mat Marseille, Nice, oder [...]. ${ }^{72}$ (Männlich, 36 Jahre, Luxemburger, Redange)

Dat ass zum Beispill, hei [...] an all di Streecher do, ass d'Grondsprooch vun deenen Aalen ass nach Lëtzebuergesch, also awer wierklech dat aalt Lëtzebuergescht, also di hu nach Ausdréck dran, wou nach just den Alain Atten mol op dem Radio seet, wou dohannen bei deenen Aalen, d'Fransousen hei vir, deen aalen Loutrénger nach Gang und Gebe sinn. An esou ass et och an der Belsch. Bon, d'Belsch, 't ass e Land 't ass no bei eis. ${ }^{73}$ (Männlich, 65 Jahre, Luxemburger, Dudelange)

Mä wann een awer éischter sou an Baden Baden rageet oder alles wat bei Berlin, München an sou Saachen leit. Dass eppes ganz anescht wei hei an der Géigend, well hei alles wat Moselfränkesch ass dat ass jo am Fong, Groussregioun [sic!] nennen ech dat, fir mech ass dat een Land. Och wann een seet Lëtzebuerg, Däitschland an sou mä et gehéiert zu

72 | Deutsch: "[...] einfach die Grenzregion. Also die ersten $50 \mathrm{~km}$ hinter der Grenze, da ist das genauso wie in Belgien. Das kann man nicht vergleichen, Frankreich ist ein sehr großes Land, das kann man nicht vergleichen mit Marseille, Nizza, oder [...]."

73 | Deutsch: "Das ist zum Beispiel, hier [...] in all diese Gegenden ist die Hauptsprache der Älteren noch Luxemburgisch, aber wirklich das alte Luxemburgisch, also die benutzen noch Ausdrücke, die höchstens noch Alain Atten mal im Radio gebraucht, die aber dort drüben bei den Älteren, bei den Franzosen hier vorne, bei den alten Lothringern noch ganz gebräuchlich sind. Und so ist das auch in Belgien. Gut, Belgien ist ein Land, das uns nahe ist." 
engem Ensemble. An mä wann een awer wierklech bei hinnen eran geet, et sinn aner Leit, sinn frëndlech, sinn ganz op, si laachen gären. ${ }^{74}$ (Männlich, 21 Jahre, Luxemburger, Eschsur-Alzette)

Äußerungen wie die letzte stellen gleichwohl eine Ausnahme dar. Über Gemeinsames und Verbindendes wurde weitaus weniger gesprochen als über Trennendes und Abstoßendes oder das mitunter anziehende Andere. Zwar lässt sich vielfach ein gewisses Bewusstsein für Luxemburgs Position in einer komplexen Grenzraumsituation ausmachen, Hinweise auf ein zunehmendes Selbstverständnis etwa als >Sar-Lor-Luxois ${ }^{75}$ sind jedoch auch implizit kaum zu finden.

Auch die in den Interviews eingesetzte Stumme Karte Mitteleuropas ${ }^{76}$ liefert nur wenige Kommentare zur transnationalen Dimension von Heimat- und Zugehörigkeitsgefühl. Die geringe Zahl entsprechender Interviewpassagen erlaubt hier nur eine sehr vorsichtige Einordnung der Befunde, lässt aber erste Rückschlüsse auf angeeignete Identitäten zu.

Ausdruck einer nicht an nationale Grenzen gebundenen Identifizierung mit dem Raum ist die sowohl zeichnerische als auch verbale Einbeziehung der Nachbarregionen, ohne dabei explizit Bezug auf die institutionalisierte Großregion zu nehmen. In einem Falle wird die gewählte zeichnerische Darstellung zudem bewusst als Distanzierung von nationalistischen Deutungsmöglichkeiten eingesetzt:

Und da ich nicht so orientiert bin, dass ich nur sage: 'Luxemburg ist gut oder so', das ... das bin ich nicht. Da mach ich den Kreis ein bisschen größer. (Weiblich, 55 Jahre, Luxemburgerin, Lintgen)

74 | Deutsch: "Wenn man aber eher nach Baden-Baden kommt, oder in die Gegend von Berlin oder München oder so. Das ist etwas ganz anderes als hier in der Gegend, denn alles was moselfränkisch ist, ist im Grunde genommen, Großregion nenne ich das, für mich ist das ein Land. Auch wenn man sagt Luxemburg, Deutschland und so, gehört das doch zusammen. Und wenn man wirklich dorthin geht, sind das andere Leute, sind freundlich, sind sehr offen, sie lachen gerne."

75 | Sowohl die Sperrigkeit dieses Neologismus als auch dessen kaum mögliche Übersetzung ins Deutsche (s.o.) deuten eine sehr eingeschränkte 'Alltagstauglichkeit und empirisch nicht nachweisbare Verbreitung des Begriffs an.

76 | Bei der Wahl der 'Mental Maps` als ergänzende Methode war den Autoren/-innen dieses Kapitels deren begrenzte Reichweite und die Problematik des zugrunde liegenden hypostasierenden Raumkonzepts bewusst. Ihr Einsatz ist daher eher als Interviewstimulus denn als eigenständige und tragfähige Methode zu verstehen. Jedoch wirkten gerade die begrenzten zeichnerischen Möglichkeiten der Interviewten durchaus erzählgenerierend (s. nachfolgende Zitate). Die Ergebnisse der grafischen Darstellung sind jedoch meist punktueller Natur und auf Luxemburg oder den Wohnort beschränkt. Auf eine gesonderte Darstellung wird hier verzichtet. 
Gleichwohl muss bei den grenzüberschreitenden Zuordnungen unterschieden werden zwischen einer eher pragmatisch-funktional begründeten Vertrautheit mit den Nachbarregionen, die zu einer entsprechend großzügigen Abgrenzung führen kann, sowie einer qualitativ höherwertigen, identitätsprägenden Beziehung zu den Nachbarregionen. Der häufige Besuch einer Nachbarregion aus beruflichen oder privaten Gründen sagt nicht zwangsläufig auch etwas über die emotionale Beziehung zu dem jeweiligen Raum aus bzw. muss nuanciert betrachtet werden, wie nachstehendes Zitat verdeutlicht:

L'Allemagne. Oui, bon, on y va régulièrement. C'est pas que je me sens pas bien là-bas, ça c'est pas le cas. ${ }^{77}$ (Weiblich, 54 Jahre, Luxemburgerin, Walferdange)

Häufig werden in mehreren Interviews spontan besondere Beziehungen zu ausländischen Regionen angesprochen, die nicht unmittelbar an Luxemburg grenzen. Die hier formulierten $\mathrm{Zu}$ - und Abneigungen sind nicht immer frei von Stereotypen oder in Urlauben entwickelten Verklärungen, gehen aber häufig darüber hinaus, was zumeist biografisch begründet scheint (familiäre Beziehungen, berufliche Aufenthalte und andere persönliche Erfahrungen). Die Interviewten nutzen mitunter den weiträumigen Zuschnitt der vorgelegten Karte, um entsprechende relationale Bezüge zu weiter entfernt liegenden Regionen herzustellen.

Lëtzebuerg, an dann wou ech mer nach elo kéint virstellen dat misst dann méi sou heierouter... Am Elsass. [...] An dann eh, och ech weess net, jo vläicht och an der Schwäiz, ech weess et net. [...] Éischtens emol well ech schonn oft am Elsass war, an ech fannen déi Leit hunn och eng aner Mentalitéit wéi lo, also wat hei d'ganzt Louthrengen hei ubelaangt, also do kann ech mer virstellen datt ech mech doudsécher net géif do doheem fillen, hein. Well eh also déi Mentalitéit déi, déi leit mer guer net. An dann och vun der Landschaft hier, ech nee, do géif ech mech net doheem fillen. D'Elsass kéint ech mer eventuell nach virstellen do, do ze wunnen. An dann och, an dann och d'Schwäiz. Jo vun der Mentalitéit hier, awer lo net iergendwéi, well do lo d'Bierger sinn oder, oder. ${ }^{78}$ (Weiblich, 37 Jahre, Luxemburgerin, Bascharage)

77 | Deutsch: „Deutschland. Ja, da fahren wir schon regelmäßig hin; nicht, dass ich mich da nicht wohl fühle, das ist nicht der Fall."

78 | Deutsch: "Luxemburg, und wo ich es mir jetzt noch vorstellen könnte wäre mehr hier herunter. Im Elsass. [...] Und dann eh, ach ich weiß nicht, ja vielleicht auch in der Schweiz, ich weiß es nicht. [...] Erstens einmal weil ich schon oft im Elsass war, und ich finde, die Leute haben auch eine andere Mentalität als hier, also was hier ganz Lothringen anbelangt, also da kann ich mir vorstellen, dass ich mich dort todsicher nicht zu Hause fühlen würde, ne. Weil, eh, die Mentalität, die, die liegt mir gar nicht. Und dann auch von der Landschaft her, ich, nee, da würde ich mich nicht zu Hause fühlen. Das Elsass, da könnte ich mir aber eventuell noch vorstellen zu wohnen. Und auch da, und dann auch in der Schweiz. Ja, von der Mentalität her, aber jetzt nicht irgendwie, weil da jetzt die Berge sind oder, oder." 


\section{Divergenz oder Konvergenz der Diskursmuster?}

Trotz eines relativ kleinen Textkorpus sowie nur bedingt verallgemeinerbarer Befunde aus standardisierter Befragung und qualitativen Interviews wurde offenkundig, wie wenig Übereinstimmung zwischen der postulierten Alltagsrelevanz der Großregion bzw. den grenzüberschreitenden Verflechtungen auf der einen Seite (zugeschriebene Identitäten), und den tatsächlich als handlungs- und bewusstseinsrelevante Facetten wahrgenommenen Besonderheiten dieser Lebensumwelt auf der anderen Seite (angeeignete Identitäten) zu bestehen scheint. Gleichwohl deuten mehrere Interviewpassagen an, inwieweit die unaufgeforderte Binnendifferenzierung in der Beurteilung der Nachbarländer zwar vornehmlich Alteritätsaspekte bedient, aber im Umkehrschluss auch (grenzübergreifende) Identitätsmerkmale und eine - unterschiedlich begründete - Zusammengehörigkeit aufgreift. Eine grundsätzliche Sensibilisierung für >das Gemeinsame im anderen< jenseits der Grenze ließ sich vielfach ausmachen. Ob hier jedoch eher divergente oder eher konvergente Entwicklungstendenzen, d.h. ein Auseinanderdriften oder eine Annäherung der Diskursmuster vorliegt, lässt sich mit dem zugrunde liegenden Korpus nicht ausmachen. Nur diachrone Langzeitstudien mit einem klaren Fokus auf die grenzregionalen Aspekte der Identitätsbildung und entsprechender methodischer Ausstattung können hier weiterführende Erkenntnisse liefern. Als gesichert kann jedoch gelten, dass es den >Sar-Lor-Luxois< ebenso wenig geben wird und kann, wie die Luxemburgerin oder eine nationale Identität. Zwar mögen regionsexterne Adressaten/-innen des Tourismusmarketings empfänglicher für Stereotype und klischeehafte Portraitierungen einer ihnen weniger bekannten Bevölkerung sein, die hier teilweise sehr weit reichenden Konstruktionen postulierter Grenzraumidentitäten werden durch die empirischen Befunde aus der Region jedoch teilweise konterkariert. Ob diese Konstruktionsleistung intentional geschieht, um durch Überzeichnung besonderes Interesse für die Region zu wecken, oder ob sie eher dem persönlichen Enthusiasmus einzelner Autoren/-innen geschuldet ist, kann hier nicht abschließend geklärt werden.

\subsection{SCHLUSSFOLgeRUNG: RÄUMLICHE IDENTITÄTEN - MEHRFACHE LESARTEN?}

Die Untersuchung der geografischen Darstellungen Luxemburgs, die in den fünf beschriebenen Teilanalysen in jeweils abgewandelter Form vorgenommen wurde, ist vor allem durch die touristische Praxis selbst begründet worden, die in diesem Land auf fünf räumliche Ebenen verteilt ist: Die Stadtviertel der Stadt Luxemburg, die Stadt selbst, die Regionen Luxemburgs, das Land in seiner Gesamtheit und schließlich das Land innerhalb der Großregion. Was die Teilanalysen zutage gefördert haben, hat nichts mit einer einfachen skalaren Verteilung zu tun, wir sehen uns vielmehr fünf >Geo-grafien > gegenüber, die ebenso sehr unterschiedliche Dar- 
stellungskategorien für den >Raum Luxemburg< sind wie differenzierte Wertesysteme und semiotische Strategien.

Diese Verschiedenheit ist zweifellos einem Unterschied in der touristischen Praxis selbst zuzuschreiben: Das Modell der Großregion spricht nicht die gleichen Akteure an, nicht die gleichen Temporalitäten, nicht die gleichen Lebensarten wie jene in den Stadtvierteln; die Erkundung der Regionen im Land ist nicht unbedingt für jene bestimmt, die von weither kommend über Luxemburg reisen oder die sich nur ganz allgemein über das Land informieren möchten. Dies erklärt auch die Vielfalt der Texte, die jede Ebene der touristischen Praxis >stützen<: In der Stadt genügen Reiseführer, Prospekte oder Karten für den Reiz des Entdeckens, während man einiges mehr benötigt, wenn man die Regionen oder das Land kennen lernen oder gar erkunden möchte; was die Großregion betrifft, so ist sie weniger für Spaziergänge oder Entdeckungen geeignet, man lebt in ihr und zunehmend arbeitet man in ihr: Dessen muss man sich im Hinblick auf die Grundlagentexte bewusst sein. So wird deutlich, dass das, was jenseits der unterschiedlichen Arten praktischer und kognitiver Verinnerlichung hervortritt, die Verschiedenheit, die Vielheit und die Unbeständigkeit Luxemburgs ist.

Selbst wenn die fünf auf die verschiedenen Ebenen bezogenen Untersuchungen diese Mannigfaltigkeit und Heterogenität hervortreten lassen, kann man sich vor dem Hintergrund des in diesem Werk angesprochenen allgemeinen Forschungsthemas jedoch fragen, ob sich eine >Identität herauskristallisiert: Etwas Wiederkehrendes, etwas Beständiges, irgendeine thematische Einheit oder sogar ein Wertesystem auf der einen Seite, auf der anderen Seite eine Übereinstimmung von Verinnerlichungsarten, von Darstellungsformen, von Argumentationstaktiken. Mit anderen Worten: Kann man am Ende der Untersuchungen in diesem Kapitel behaupten, dass die fünf Räume Luxemburgs oder die Diskurse, die sie thematisieren, eine substanzielle Gemeinsamkeit haben? Auf eine solche Frage zu antworten, heißt zunächst, jene Frage zu beantworten, die jede einzelne der Teiluntersuchungen im Hinblick auf die interne Geschlossenheit ihres >geografischen< Untersuchungsgegenstandes stellt: Welches sind die >diskursiven touristischen Identitäten< der Stadtviertel von Luxemburg, der Hauptstadt selbst, der Regionen Luxemburgs, des Landes als solchem und des Landes innerhalb der Großregion? Die Antwort scheint in der Tatsache zu liegen, dass die identifizierenden Merkmale - falls es welche gibt - nicht so sehr in den Themen oder den Werten zu finden sind als vielmehr in der Art und Weise, wie das Land und seine unterschiedlichen Ebenen thematisiert und hervorgehoben werden. Insgesamt gäbe es nur eine sehr geringe (oder sogar überhaupt keine) diskursive Identität des Landes; es gäbe lediglich eine gewisse Einheitlichkeit in den DiskursivisierungsStrategien, die meist auf das Lokale, das Eindeutige, das Einzelne gerichtet sind. So wird nicht nur Luxemburg je nach den fünf untersuchten Maßstabsebenen unterschiedlich dargestellt, sondern selbst innerhalb dieser Kategorien werden Unterschiede (geografisch und historisch) deutlich, Begrenzungen und Grenzen 
werden aufgestellt, man deutet auf das Außergewöhnliche, das Unterschiedliche, das Einzigartige.

Aus dieser Perspektive führt die Untersuchung der Stadtviertel zu der Feststellung, dass die touristische Praxis dieser Ebene auf demselben Makro-Akt der Hervorhebung, und im Besonderen auf dem der Ästhetisierung, beruht. Fragt man die Einwohner nach dem Ort, den sie einem ausländischen Freund gerne zuerst zeigen würden, so bestätigen die in der Befragung eingeholten Antworten die Ergebnisse der anhand der Faltblätter und Prospekte durchgeführten Untersuchung: Sowohl was die wichtigsten Orte betrifft als auch vor dem Hintergrund einer allgemeinen ästhetischen Aufwertung werden hier die Festung und die historische Altstadt genannt. Selbst die vorhersehbaren Unterschiede zwischen den Tourismus-Texten und den Äußerungen der Personen zeigen implizit die gleiche Anforderung: Dem Stadtviertel Bonnevoie wird nicht der Status einer >Attraktion< zuerkannt, da es sich nicht wie der Limpertsberg für eine Ästhetisierung eignet und auch nicht aus den Gegensätzen >Tradition vs. Moderneく oder >national vs. international< schöpfen kann, wie es der offizielle Diskurs über den Kirchberg tut.

Die Untersuchung der Stadt Luxemburg anhand ihrer Karten und Faltblätter verdeutlicht auch, dass die Frage, die sich abzeichnet, eine ausgewählte und differenzierende Hervorhebung der Stadt allgemein betrifft. Sie wird daher in Teilen und mit einem gewissen Abstand dargestellt: Einerseits zeigt man sie ständig und schwerpunktmäßig als Hinführung zum Zentrum, andererseits wird dieses Zentrum als Insel dargestellt, zu der man aufsteigen muss. Das bedeutet, dass man weder eine Gesamtheit präsentiert (da man einen Teil ausgewählt hat, nämlich den Weg ins Zentrum) noch eine beständige und wiedererkennbare Realität, denn was zählt, ist der Zugang zum Zentrum und nicht das Zentrum als solches. Und noch weniger zählt die Stadt als solche, die vergleichsweise wenige Denkmäler zählt und wenig charakteristisches Profil aufweist.

Das Fehlen einer diskursiven Identität des Landes zugunsten singularisiernder Diskursivierungen wird bestätigt und verstärkt, wenn man den Tourismus-Diskurs über die Regionen untersucht. Die Untersuchung der drei Schriften des »Office National du Tourisme « wirft ein Licht auf die Rhetorik, die die Kombination von Bildern und Text in den Dienst von Redundanz und Übertreibung stellt. Es versteht sich, dass vor allem die von den Leser/-innen zu leistende Arbeit möglichst weitgehend rationalisiert werden soll, er soll ohne Umwege zu der gewünschten Information gelangen, aber die extreme Prototypisierung der Regionen, die über eine stark gestückelte Struktur vermittelt wird, bewirkt unmittelbar, dass für ein Land geworben wird, dessen Vielfalt in einem umgekehrt proportionalen Verhältnis zur Fläche des Landes steht. Die Ergebnisse der quantitativen Untersuchung decken sich unbestreitbar mit der Wahrnehmung einer zugleich virtuellen und differenzierten Entität an der Schnittstelle zwischen Region und Land.

Auch dem nationalen Raum, dessen touristische Darstellungen diachronisch untersucht worden sind, scheint es an Einheit zu mangeln; gleichzeitig bleibt die 
Identität der Verinnerlichungsarten und der Hervorhebungsarten bestehen. Einerseits wird Luxemburg als ein offener Raum betrachtet, einladend, Sicherheit vermittelnd, vertraut, andererseits wird es als einzigartig, anders, exotisch beschrieben. Man betont besonders die spezifische Eigenschaft, die Luxemburg aufgrund seines multikulturellen und mehrsprachigen Charakters als Ort der Besonderheit und des Unterschieds kennzeichnet; was nicht daran hindert, die Geruhsamkeit als herausragendes Merkmal des Landes zu unterstreichen. Daher sei Luxemburg zugleich modern und traditionell, innovativ und ursprünglich. Die semantische Spannung wird sogar akzeptiert und hervorgehoben: Luxemburg wird immer wieder als das >Land der Gegensätze< gezeichnet, das durch seine kleine Größe und seine großen industriellen, aber im Rahmen der Europäischen Union auch politischen Ambitionen charakterisiert ist. Diese europäische Vision, von den jüngeren Ausgaben des Merian von 1995 und 2007 in den Vordergrund gestellt, spiegelt sich in der Hälfte der durchgeführten Gespräche wider, während die andere Hälfte der befragten Personen sich kritischer zeigt und den abweichenden Aspekt ihrer Meinung betont.

Die Abweichung von zugeschriebenen und angeeigneten Identitäten wird auf der Ebene der sogenannten Großregion und deren jeweiligen touristischen Diskursen noch besser sichtbar. Der idealisierende Charakter der untersuchten Publikationen scheint deutlich von dem Diskurs abzuweichen, den man bei den Einwohner/-innen sowie deren grenzüberschreitenden Praktiken und Haltungen beobachten kann. Immerhin zeigen die Letzteren mehrheitlich Identitätsmerkmale, die über die nationalen Grenzen des Großherzogtums hinausreichen. Dieser Aspekt sollte eingehender erforscht werden, wobei der Kontext der Europäisierung und Globalisierung zu berücksichtigen wäre.

Wir sind uns hinsichtlich der Gesamtheit der untersuchten Ebenen darüber im Klaren, dass ein >offizieller Tourismus-Diskurs, so wie er von den meisten untersuchten Dokumenten wiedergegeben wird, immer auf die Hervorhebung eines Raumes abzielt. Das bedeutet, dass er starke identitätsbildende Vorstellungen hervorbringt. Aus diesem Grund haben wir uns für die umgesetzten Aufwertungsstrategien interessiert: Um die zugeschriebenen Identitäten besser zu verstehen und den Unterschied zu jenen Identitäten zu begreifen, die sich die Personen aneignen, die sich in diesen Räumen aufhalten - in der Stadt Luxemburg, im Land und in der Großregion.

\subsection{LITERATURVERZEICHNIS}

\section{Primärquellen}

Anonyme (1934): Woerl Reisehandbücher. Großherzogtum Luxemburg [mit Plan der Stadt Luxemburg, 7 Karten und 29 Abbildungen], 3. Auflage, Leipzig: Woerl's Reisebücher-Verlag. 
Anonyme (1914): Woerl's Reisehandbücher. Illustrierter Führer durch das Großherzogtum Luxemburg. Mit Plan der Stadt Luxemburg, 7 Kartenbeilagen und 65 Abbildungen, 2. Auflage, Leipzig: Woerl's Reisebücher-Verlag, Kaiserl. u. Königl. Hofbuchhandlung.

Anonyme (1892): De Luxembourg en Écosse. Guide du Touriste à travers Le Luxembourg. - L'Ardenne Belge. - Les Flandres. La Mer du Nord et la Manche. Le Comté de Kent. - Le Pays de Galles. - Les Lacs Anglais. L'Écosse. Avec 100 dessins originaux de MM. Hoeterickx, Van Gelder etc. Publié par S. de Ruette, représentant du London Chatham \& Dover Railway [à Bruxelles], avec la haute approbation des Compagnies du London \& North Western Railway, du London Chatham \& Dover Railway, des Chemins de fer Prince-Henri et des Chemins de fer du Nord de France, Luxembourg: Pierre Bruck.

Baedekers Allianz Reiseführer (1990): Luxembourg, Ostfildern-Kemnat: Mairs Geographischer Verlag.

Erasmy, Mathieu (1861): Le guide du voyageur dans le Grand-Duché de Luxembourg, Luxembourg: V. Bück.

Luxembourg City Tourist Office: »Panorama City Map«, in: Luxembourg City Tourist Office (Hg.): Luxembourg la ville, die Stadt, the city, bonjour!, Luxembourg. Luxembourg City Tourist Office (2003): »Luxembourg la ville, bonjour!«, in: Luxembourg City Tourist Office (Hg.): Luxembourg la ville, bonjour!, Luxembourg: Éditions Guy Binsfeld.

Luxembourg City Tourist Office (2006): »Le circuit des roses du Limpertsberg $>$ RosaLi««, in: Luxembourg City Tourist Office (Hg.): Luxembourg la ville, bonjour!, Luxembourg.

Luxembourg City Tourist Office (2007a): »Luxembourg, une capitale européenne«, in: Représentation de la Commission européenne à Luxembourg \& Luxembourg City Tourist Office (Hg.): Luxembourg la ville, bonjour!, Luxembourg: Binsfeld Communication.

Luxembourg City Tourist Office (2007b): »City Promenade«, in: Luxembourg City Tourist Office (Hg.): Luxembourg la ville, bonjour!, Luxembourg: Éditions Guy Binsfeld.

Luxembourg City Tourist Office (2007c): »Luxembourg, Vivez la ville!«, in: Luxembourg City Tourist Office (Hg.): Luxembourg la ville, bonjour!, Luxembourg: Éditions Guy Binsfeld.

Luxembourg City Tourist Office (2000): Ni vu ni connu, Luxembourg.

Marco Polo. Luxembourg: Reiseführer mit Insider-Tips, Ostfildern: Mairs Geographischer Verlag, 1995 .

Mendgen, Eva/Hildisch, Volker/Doucet, Hervé (Hg.) (2007): Im Reich der Mitte/ Le berceau de la civilisation européenne. Savoir-faire/savoir-vivre, Konstanz: Hartung-Gorre.

Merian (1964): Luxemburg. Merian XVII/7.

Merian (2007): Luxemburg. Merian 60/03.

Michelin. Belgique/Luxembourg, Zellik: Le Guide Michelin, 2007. 
Office national du tourisme du Grand-Duché de Luxembourg (2006): Les Produits du Terroir et les Métiers d'Art, Mersch: Imprimerie François Faber.

Office national du tourisme du Grand-Duché de Luxembourg (2008): Hôtels Restaurants et Arrangements. (11/2007 pour l'année 2008), Luxembourg: Imprimerie Saint-Paul.

Office national du tourisme du Grand-Duché de Luxembourg (2008): Sites et Attractions. (12/2007 pour l'année 2008), Luxembourg: Imprimerie Saint-Paul.

Panorama City Map. Luxembourg: La ville, Luxembourg City Tourist Office, 2008. Plan de la ville de Luxembourg, Luxembourg City Tourist Office, 2008.

Renwick, George (1913): Luxembourg. The Grand-Duchy and its People, London: T. Fisher Unwin.

Schisgall, Oscar (1967): »Le Luxembourg, pays de paradoxes«, in: Sélection du Reader's Digest 01.1967, S. 62-69.

Scholz, Ingeborg (2007): Die Großregion entdecken/Découvrir la Grande Région, Luxembourg: Guy Binsfeld.

Schröder, Dirk (1995): Merian live! Luxemburg, München: Gräfe und Unzer.

\section{Sekundärquellen}

Adam, Jean-Michel (1999): Linguistique textuelle. Des genres de discours aux textes, Paris: Nathan.

Adam, Jean-Michel (1997): Le style dans la langue. Pour une reconception de la stylistique, Lausanne/Paris: Delachaux et Niestlé.

Adam, Jean-Michel (1990): »La période. De la stylistique à la linguistique textuelle«, in: Stylistique et littérature, Versants, 18, Boudry, La Baconnière, S. 5-19.

Adam, Jean-Michel/Bonhomme, Marc (1997): L'argumentation publicitaire. Rhétorique de l'éloge et de la persuasion, Paris: Nathan-Université.

Alonso, Juan A. (2009): »Espace et métalangage. Défense du territoire«, in: Nouveaux Actes Sémiotiques. URL: http://revues.unilim./nas/document. php?id=2893 (11.05.2010)

Amossy, Ruth/Maingueneau, Dominique, (Hg.) (2003): L'analyse du discours, Toulouse: Presses Universitaires du Mirail.

Austin, John L. (1962): »Performatif-Constatif«, in: Cahiers de Royaumont, Philosophie 4: La philosophie analytique, S. 271-281.

Baudrillard, Jean (1994): Simulacrum and Simulations, Chicago: University of Michigan Press.

Benveniste, Émile (1966): Problèmes de linguistique générale, 1, Paris: Gallimard. Berger, Peter L./Luckmann, Thomas (1966): The Social Construction of Reality, New York: Doubleday.

Blunt, Alison/Gruffudd, Pyrs/May, Jon/Ogborn, Miles/Pinder, David (Hg.) (2003):

Cultural Geography in Practice, Oxford: Oxford University Press.

Bonhomme, Marc (2006): Le discours métonymique, Bern: Peter Lang.

Bonhomme, Marc (2005): Pragmatique des figures du discours, Paris: Champion. 
Bonhomme, Marc (2003): »L'éloge de la ville: rhétorique d'une plaquette touristique sur Berne«, in: Pierre Marillaud/Robert Gauthier (Hg.): Les langages de la ville, Toulouse: Presses Universitaires du Mirail, C.A.L.S./C.P.S.T., S. 13-21.

Bonhomme, Marc/Adam, Jean-Michel (1997): L'argumentation publicitaire.

Rhétorique de l'éloge et de la persuasion, Paris: Nathan-Université.

Brunet, Roger (1989): Les villes »européennes«, Paris: La Documentation Française.

Bulot, Thierry/Veschambre, Vincent (Hg.) (2006): Mots, traces et marques. Dimensions spatiale et linguistique de la mémoire urbaine, Paris: L'Harmattan.

Cohen, Erik (2004): Contemporary Tourism. Diversity and Change, Amsterdam: Elsevier.

Colas-Blaise, Marion (erscheint demnächst): »Comment fonctionne le texte de vulgarisation polymédial? Éléments pour une approche sémio-linguistique du rapport texte/image dans le dépliant touristique«, in: David Banks (Hg.): L'image dans le texte scientifique, Brest.

Commission européenne (2009): Eurobaromètre 71.1, QA6a. URL: http ://ec.europa .eu/public_opinion/archives/ebs/ebs_308_en.pdf (14.12.2009)

Dominicy, Marc/Franken, Nathalie (2001): »Epidictique et discours expressif«, in: Ders. (Hg.): La mise en scène des valeurs, Neuchâtel: Delachaux et Niestlé, S. 79-106.

Döring, Jörg/Thielmann, Tristan (Hg.) (2009): Spatial Turn. Das Raumparadigma in den Kultur- und Sozialwissenschaften, Bielefeld: transcript.

Ducrot, Oswald (1984): Le dire et le dit, Paris: Les Editions de Minuit.

Dumont, Patrick/Fehlen, Fernand/Kies, Raphaël/Poirier, Philippe (2007): Le référendum sur le Traité établissant une Constitution pour l'Europe. Rapport élaboré pour la Chambre des Députés, Luxembourg: STADE/Université du Luxembourg.

Farinelli, Franco (2009): La crisi della ragione cartografica. Introduzione alla geografia della globalità, Torino: Einaudi.

Farinelli, Franco (2009): I segno del mondo. Immagine cartografica e discorso geografico in età moderna, Firenze: Accademia Universa.

Floch, Jean-Marie (1990): Sémiotique, marketing et communication. Sous les signes, les stratégies, Paris: PUF.

Floch, Jean-Marie (1995): Identités visuelles, Paris: PUF.

Fontanille, Jacques (2008): Sémiotique des pratiques, Paris: PUF.

Fontanille, Jacques (1999): Sémiotique et littérature. Essais de méthode, Paris: PUF.

Fontanille, Jacques (1998): Sémiotique du discours, Limoges: Pulim.

Fontanille, Jacques/Zilberberg, Claude (1998): Tension et signification, Hayen: Mardaga.

Freyermuth, Sylvie (2003): »Les marques de l'ordre et de la distinction dans le discours argumentatif de Pascal-Quelques remarques pragmatiques et stylistiques sur un fragment des Pensées«, in: Catherine Schnedecker/Michel Charolles 
/George Kleiber (Hg.): Actes du Colloque Ordre et distinction dans la langue et dans le discours, (Metz), Paris: Champion, S. 203-216.

Freyermuth, Sylvie (2008): »Théorie de l'esprit et temporalité subjective chez le personnage flaubertien«, in: Pierre Marillaud/Robert Gauthier (Hg.): Langages, temps, temporalité. XXVIIIe colloque d'Albi, Toulouse: Presses Universitaires du Mirail, C.A.L.S./C.P.S.T., S. 207-214.

Gebhardt, Hans/Reuber, Paul/Wolkersdorfer, Günther (Hg.) (2003): Kulturgeographie. Aktuelle Ansätze und Entwicklungen, Heidelberg: Spektrum.

Genette, Gérard (1987): Seuils, Paris: Seuil.

Glasze, Georg/Matissek, Annika (Hg.) (2009): Handbuch Diskurs und Raum, Bielefeld: transcript.

Goodwin, Charles (2003): Il senso del vedere, Roma: Meltemi.

Greimas, Algirdas J./Courtés, Joseph (1979): Sémiotique. Dictionnaire raisonné de la théorie du langage, Paris: Hachette.

Grize, Jean-Blaise (1981): »L'argumentation - explication ou séduction«, in: L'Argumentation, Lyon: Presses Universitaires de Lyon, S. 29-40.

Hajer, Maarten A. (1985): The Politics of Environmental Discourse. Ecological Modernization and the Policy Process, Oxford: Oxford University Press.

Hajer, Maarten A. (2003): »Argumentative Diskursanalyse. Auf der Suche nach Koalitionen, Praktiken und Bedeutung«, in: Reiner Keller et al. (Hg.): Handbuch Sozialwissenschaftliche Diskursanalyse, Bd. 2, Opladen: Leske + Budrich, S. 271-297.

Hennig, Christoph (1997): Reiselust. Touristen, Tourismus und Urlaubskultur, Leipzig: Insel.

Herschberg Pierrot, Anne (2003): »La question du style«, in: Ruth Amossy/ Dominique Maingueneau (Hg.): L'analyse du discours, Toulouse: Presses Universitaires du Mirail, S. 333-339.

Kerbrat-Orecchioni, Catherine (1991): »Introduction«, in: Ders. (Hg.): La question, Lyon: Presses Universitaires de Lyon, S. 5-37.

Kerbrat-Orecchioni, Catherine (1991): »L'acte de question et l'acte d'assertion - opposition discrète ou continuum?«, in: Ders. (Hg.): La question, Lyon: Presses Universitaires de Lyon, S. 87-111.

Linden, André/Thewes, Guy (2007): »Tourisme et identité nationale«, in: Forum für Politik, Gesellschaft und Kultur in Luxemburg 271, S. 42-44.

Lossau, Julia/Lippuner, Robert (2004): »Geographie und spatial turn«, in: Erdkunde 58 (3), S. 201-211.

Louvel, Liliane (2002): Texte, image. Images à lire, textes à voir, Rennes: Presses universitaires de Rennes.

MacCannell, Dean (1976): The Tourist. New Theory of the Leisure Class, New York: Schocken Books.

Majerus, Benoît (2007): »Le petit Européen parfait. L'Europe, le Luxembourg et la construction nationale«, in: N. Beaupre/C. Moine (Hg.): L'Europe de Versailles à Maastricht, Paris: Seli Arslan, S. 225-235. 
Marcos, Isabelle (Hg.) (2007): Dynamiques de la ville. Essais de sémiotique de l'espace, Paris: l'Harmattan.

Marin, Louis (1994): De la représentation, Paris: Seuil/Gallimard.

Marin, Louis (1983): »La ville dans sa carte et son portrait«, in: Cahiers de l'école normale supérieure de Fontenay 30-31, S. 11-26.

Mattissek, Annika (2007): »Diskursanalyse in der Humangeographie - >State of the Art«, in: Geographische Zeitschrift 95 (1-2), S. 37-55.

Molinié, Georges (1993): La stylistique, Paris: PUF.

Neve, Mario (1999): Virtus loci. Lineamenti fondamentali di una teoria dell'informazione spaziale, Urbino: Quattroventi.

Parr, Rolf (2009): »Wie konzipiert die (Inter-)Diskurstheorie individuelle und kollektive Identitäten. Ein theoretischer Zugriff, erläutert am Beispiel Luxemburg«, in: Forum 289, S. 11-16.

Perelman, Chaïm/Olbrechts-Tyteca, Lucie (2000): Traité de l'argumentation, Bruxelles: Éditions de l'Université.

Pinnel, Roland (1989): »Le développement du tourisme luxembourgeois après la Deuxième Guerre mondiale«, in: Mémorial 1989: la société luxembourgeoise de 1839 à 1989. Luxembourg: Les publications mosellanes, S. 941-954.

Ricœur, Paul (1990): Soi-même comme un autre, Paris: Seuil.

Searle, John (1982): Sens et expression, Paris: Minuit.

Searle, John (1972): Les actes de langage, Paris: Hermann.

Shapiro, Meyer (1969): »On Some Problems in the Semiotics of Visual Art: Field and Vehicle in Image-Signs«, in: Semiotica 1, III, S. 223-242.

Shurmer-Smith, Pamela (Hg.) (2002): Doing Cultural Geography, London: Sage.

STATEC (2007): Annuaire statistique du Luxembourg, Luxembourg: Statec.

Strüver, Anke (2005): »Stories of the >Boring Border<. The Dutch-German borderscape in people's minds«, in: Forum Politische Geographie 2, Münster: LIT.

Urry, John (1990): The Tourist Gaze: Leisure and Travel in Contemporary Societies, London: Sage.

Wahl, François (1980): »Le désir d'espace«, in: Christian Jacob (Hg.): Cartes et figures de la terre, Paris: Centre Georges Pompidou, Centre de Création Industrielle, S. 41-46.

Weichhart, Peter (1990): Raumbezogene Identität. Bausteine zu einer Theorie räumlich-sozialer Kognition und Identifikation, Stuttgart: Franz Steiner.

Werlen, Benno (1995): Sozialgeographie alltäglicher Regionalisierungen. Bd. 1: Zur Ontologie von Gesellschaft und Raum, Stuttgart: Franz Steiner.

Werlen, Benno (1997): Sozialgeographie alltäglicher Regionalisierungen. Bd. 2: Globalisierung, Region und Regionalisierung, Stuttgart: Franz Steiner.

Zilberberg, Claude (2006): Éléments de grammaire tensive, Limoges: Pulim. 



\section{Bilder und Identitäten}

Wilhelm Amann, Viviane Bourg, Paul Dell, Fabienne Lentz, Paul Di Felice, Sebastian Reddeker

\subsection{NATIONENBILDER ALS IINTERDISKURSEי. THEORETISCHE VORÜBERLEGUNGEN ZUM VERHÄLTNIS VON \BILDERN UND IDENTITÄTEN` AM BEISPIEL LUXEMBURGS}

Die gemeinsame theoretische Rahmung zur Analyse unterschiedlicher Erscheinungsformen von >Bildern und Identitäten< im sozio-kulturellen Raum Luxemburg stellt die sogenannte Interdiskursanalyse bereit. (Gerhard/Link/Parr 2004: 293f.) Sie versteht sich als eine Weiterentwicklung und Modifikation der von Michel Foucault entwickelten Diskursanalyse und ist als eine angewandte Diskurstheorie um den Bezug zur Praxis und Empirie bemüht. Während es bei den von Foucault analysierten Diskursen weitgehend um Formationen positiven Wissens und institutionalisierte Wissenschaften (Recht, Medizin, Humanwissenschaften etc.) ging, interessiert sich die Interdiskursanalyse für Diskurskomplexe, die gerade nicht durch Spezialisierung begrenzt sind, sondern übergreifend wirken und deshalb als >interdiskursiv< bezeichnet werden können. (Parr 2009) Die Bedeutung solcher Interdiskurse ergibt sich aus dem generellen Spannungsverhältnis zwischen der zunehmenden Ausdifferenzierung des modernen Wissens und der wachsenden Orientierungslosigkeit moderner Subjekte.

In diesem Sinne kann man >Luxemburg < beschreiben als ein hochkomplexes Gebilde, das sich aus besonderen Organisationsformen zusammensetzt, so z.B. des Rechts, der Wirtschaft, der Politik oder auch des Gesundheitswesens. Jeder dieser Teilbereiche entwickelt dabei i.d.R. sehr spezialisierte, auf das Fachgebiet begrenzte Redeformen mit der Folge, dass Verständigung über Probleme und wichtige Themen zwischen diesen Teilsystemen selbst nur schwer möglich ist und vor allem die Alltagswelt und das Alltagswissen der Subjekte kaum erreicht wird.

Zur Illustration ein Beispiel: Wie in allen anderen betroffenen Ländern lassen sich auch in Luxemburg die Gefahren der Finanzkrise kaum mittels der hoch spezialisierten Fachsprache der Ökonomen vermitteln. Das Thema muss stattdes- 
sen in anschauliche Bilder übersetzt werden, die oftmals anderen Spezialdiskursen entlehnt sind. So warnte anfangs der Krise in der Luxemburger Presse ein Wirtschaftswissenschaftler vor »faulen Finanzprodukten«, die sich »wie eine Art Krebsgeschwür verbreiten« (Bildfeld: Medizin). Zusätzlich zog er den Vergleich zur »Atomenergie«, »die man erfunden hätte, ohne sich Gedanken darüber zu machen, wie die neue Technik unter Kontrolle gehalten werden soll« (Bildfeld: Technik).

Solche und ähnliche interdiskursive bildhaften Redeweisen haben einen kommunikativ-integrativen Effekt, der wie im gerade gewählten Beispiel womöglich grenzüberschreitend für weite Teile der westeuropäischen Kulturen gilt. Die Interdiskurse weisen darüber hinaus auch kulturtypische Besonderheiten auf, so dass deren Analyse auch je kulturelle Spezifika einer Gesellschaft zu verdeutlichen vermag. Man kann deshalb davon ausgehen, dass diese Interdiskurse heutzutage in einem hohen Maße zur Aufrechterhaltung einer nationalen Kultur beitragen, weil sie ein kommunikativ wirkendes Identitätspotential zu Verfügung stellen.

Fragen nach der kulturellen >Identität < sind im Rahmen des vorliegenden Kapitels also nicht auf personale Subjekte und kaum verifizierbare psychische Strukturen bezogen, sondern auf (inter-) diskursiv erzeugte > Wir<-Identitätsangebote, die in Form von Bildern innerhalb einer Kultur zirkulieren und ein Wechselverhältnis von symbolisch zugeschriebenen und angeeigneten Identitäten begründen. Diese - im umfassenden Sinne sprachlichen wie visuellen - Bilder kann man auch als Kollektivsymbole einer Kultur bezeichnen, als Gesamtheit der kollektiv verankerten, mehr oder weniger stereotypen >Bildlichkeit< einer partikularen Kultur. Indem Kollektivsymboliken selektiv und stark fragmentarisch sowohl zwischen den speziellen Wissensbereichen als auch zum Alltagswissen der Subjekte Zusammenhänge herstellen sowie von unterschiedlichen sozialen Trägern/-innen benutzt werden, erzeugen sie die Vorstellung einer gemeinsamen, verfestigten kulturellen Identität einer Gesellschaft. Diese kann dann sowohl als Selbstbild wie auch als Fremdbild hergestellt werden.

Ziel des vorliegenden Kapitels ist die Rekonstruktion zentraler Elemente des in der Gegenwart kulturell parat gehaltenen Kollektivsymbolsystems für Luxemburg. Die Untersuchungsfrage lautet: Wie werden durch sprachliche und visuelle Bilder spezifische Vorstellungen einer Luxemburger Identität erzeugt? Den Arbeitsfeldern der Beiträger/-innen entsprechend ist die Untersuchung auf unterschiedliche interdiskursive Medien verteilt: Printmedien, Werbung, Comic, Kunst. Interdiskurstheoretisch handelt es sich also um einen >Mix< aus elaborierten (Kunstfotografie, Kunstdokumentation, Comic) und elementaren Interdiskursen (Werbung, Printmedien) - also einerseits um einen eher reflektierten, intellektuellen und andererseits um einen eher alläglichen Umgang mit Kollektivsymbolen. Darüber hinaus hat sich das Autoren/-innenkollektiv auf eine Reihe Themenfelder verständigt, anhand derer exemplarisch Motive und Applikationen der besonderen Luxemburger Kollektivsymboliken in den verschiedenen medialen Formen analysiert 
werden. Stichwortartig sind dies: >Europa $<$, >Bankenplatz<, >Kulturhauptstadt $<$, $>$ Mudam $<$, $>$ Migration $<$ und $>$ Arcelor/Mittal $<$.

Die Themenfelder decken dementsprechend zentrale Bereiche von Wirtschaft, Politik und Kultur ab. Es kann davon ausgegangen werden, dass sie im Alltagswissen der Luxemburger/-innen in unterschiedlicher Gewichtung präsent sind und in ebenso unterschiedlicher Form sowohl zugeschriebene als auch angeeignete Identitäten erkennen lassen. So sind die Themenfelder >Arcelor/Mittal<, >Bankenplatz< und in gewisser Weise auch >Europa< sowie >Migration< charakteristisch für den in den letzten Jahrzehnten durchlebten Strukturwandel des Landes von einer produktions- zu einer dienstleistungsorientierten Gesellschaft. Dieser innerhalb einer Generation vollzogene Wandel hat zweifelsohne zu einem enormen Wohlstand im Land geführt und Luxemburg zu einem der reichsten Länder der Welt gemacht. Allerdings wirkt sich dieser Prozess auch auf die lange Zeit als kontinuierlich erfahrenen kollektiven Identitätsbestimmungen aus. Wie sehr das Verhältnis zwischen angeeigneten und zugeschriebenen Identitäten ins Wanken geraten ist, zeigt sich z.B. daran, dass der Verkauf der Arcelor-Stahlwerke im Jahr 2006 als eine nationale Angelegenheit betrachtet wurde. Offenbar gibt es nach wie vor eine große Bereitschaft zur Identifikation mit der industriellen Vergangenheit samt der dahinterstehenden Vorstellung paternalistisch agierender Großunternehmen. Auf der anderen Seite sind Fragen nach der pro-europäischen Haltung der Luxemburger/-innen und die damit verbundenen Vorstellungen und Probleme der Öffnung der Gesellschaft beispielhaft für die große Bedeutung von zugeschriebenen Identitäten.

Vor diesem Hintergrund ist das Ereignis >Kulturhauptstadt $2007<$ auch als ein breit angelegter Versuch zu verstehen, die auseinanderdriftenden Identitätsbestimmungen zu bündeln. Das Ereignis liefert aber auch Material genug, deren einzelne Bestandteile zu sichten und mit Blick auf ihre vergangene oder auch gegenwärtige Funktion zu hinterfragen.

Im ersten Teil dieses Kapitels (6.2) wird die Entstehung und funktionale Verwendung der für Luxemburg relevanten Nationalsymbolik am Beispiel eines publizistischen und literarischen Textkorpus erörtert. Die für Luxemburg charakteristische doppelte Identitätsbewegung von Abschottung und Anpassung kommt im zentralen Nationalsymbol der >Festung « zum Ausdruck. Im Zuge der Modernisierung der Gesellschaft gerät dieses Nationalsymbol, das sowohl als Selbstbild als auch von außen für die Fremdperspektive auf Luxemburg eine zentrale Rolle spielt, zunehmend in eine Krise, die dann in der publizistischen Berichterstattung über die >Bankenkrise< oder über das Thema >Europa < offen zutage tritt. Untersucht wird schließlich auch, mit welchen Symboliken in den Printmedien die Projekte zur Erneuerung des nationalen Image (>Mudam<, >Kulturhauptstadt $<$ ) belegt werden.

Der folgende Teil (6.3) analysiert den >Superjhemp<-Comic als eine Bildsatire über gängige Klischees von Land und Leuten. In seiner Physiognomie, den privaten Verhältnissen, in seinem Beruf und seinen Vorlieben erscheint der Held Su-

1| Musée d'Art Moderne Grand-Duc Jean, Luxemburg. 
perjhemp als eine Projektionsfigur des Luxemburger Nationalcharakters. Zudem nutzt der Comic die ihm vom Genre her zustehenden Freiräume für das humoristische wie kritische Fabulieren über Luxemburger Zustände, wobei insbesondere das Spannungsverhältnis von Provinzialität und Reichtum zu den wiederkehrenden Themen gehört. Der Erfolg der Comicserie beruht nicht zuletzt darauf, dass der Leserschaft eine Art Narrenspiegel vorgehalten wird. Das satirische Selbstbild setzt aber auch deutlich ein Insiderwissen über die Verhältnisse im Land voraus und kann von Außenstehenden nicht ohne weiteres dekodiert werden.

Der Abschnitt 6.4 widmet sich dem Werbediskurs als einem den Alltag prägenden Interdiskurs. Werbung möchte zwar in erster Linie verkaufs- und imagefördernd wirken, sie kann aber auch dazu beitragen, dass spezifische Bilder mit identitätsstiftendem Potential in Luxemburg verstärkt oder erst konstruiert werden. Wie Werbung im Großherzogtum mit Bildern und Symbolen arbeitet und dadurch Formen kollektiver Identitäten fördern kann, wird an Werbebeispielen aus der Finanz- und Telekommunikationsbranche sowie dem Kulturhauptstadtjahr Luxemburg 2007 gezeigt.

Der Abschnitt 6.5 greift einen Aspekt der in den letzten Jahren verstärkt zu beobachtenden Strategie der Kulturalisierung des Selbstbildes im Land heraus. Im Kontext einer aufstrebenden Kunstszene und intensiver Kulturförderung in Luxemburg entwickelte sich die Fotografie zu einem wichtigen Medium der Reflexion über Identität. Inwieweit dabei identitäre Repräsentationen, also zugeschriebene wie angeeignete Identitätsmuster, (de-)kontextualisiert und (de-)konstruiert werden, zeigt die Analyse einer Werkauswahl von sieben ausländischen und luxemburgischen Fotografen/-innen. Ihre Auftragsarbeiten entstanden im Kontext des Kulturhauptstadtjahres Luxemburg 2007 und greifen unterschiedliche Themenbereiche auf, so dass sie eine breite analytische Basis bieten.

Der abschließende Abschnitt 6.6 widmet sich einem im Kulturhauptstadtjahr unter dem Titel »Retour de Babel« realisierten Ausstellungsprojekt. Hier sollte das etablierte Bild über die Einwanderung in Luxemburg mit einem umfassenderen Bild konfrontiert werden. Der Beitrag fragt nach dem Verständnis und der Umsetzung der für das Konzept dieser Ausstellung zentralen Symbolik von Luxemburg als >europäisches Labor<.

\subsection{Symboliken in der medialen SelbSt- UNd FrEMDWAHRNEHMUNG LUXEMBURGS}

\section{Luxemburg im Kontext europäischer Nationensymboliken}

Der folgende Abschnitt analysiert sprachbildliche Redeformen in literarischen und - überwiegend - in publizistischen Texten der letzten Jahre, in denen ein besonderer symbolischer Diskurs über die >Luxemburger Identität< zum Ausdruck kommt. Die leitende Hypothese ist dabei, dass auch die gegenwärtige Medien- 
kultur nicht ohne »Massensymbole der Nationen« (Canetti 2000) auskommt. Diese sind zwar für die traditionsreichen, etablierten europäischen Nationen seit langem bekannt ${ }^{2}$ und kommen als elementar-literarische »Halbfabrikate « (Link 1983: 9) in den unterschiedlichsten Variationen nach wie vor in den Medien zur Anwendung. Für ein kleines Land wie Luxemburg, das selbst nach dem Zweiten Weltkrieg noch mit dem Image als »Operettenstaat « ${ }^{3}$ zu kämpfen hatte, war es allerdings allein schon aus historischen Gründen schwierig, an diesem im Ensemble der großen europäischen Nationen seit langem praktizierten Brauch symbolischer Selbst- und Fremdzuschreibungen teilzuhaben oder überhaupt anerkannt zu werden. Dieses Inferioritätsgefühl dürfte auch dazu geführt haben, dass für die Luxemburger/-innen das Symbol der >Festung «, das sich ja nicht ohne weiteres in die auf besondere heroische Leistungen, auf Auseinandersetzung mit Natur und Geschichte bezogene Symboliken anderer europäischer Nationen einfügt, zentrale Bedeutung gewonnen hat.

Von dieser Kernsymbolik ausgehend, werden im Folgenden Kontexte ihrer Verwendung und Versuche ihrer Modifikation erörtert, die im Zusammenhang mit dem rapiden gesellschaftlichen Wandel stehen, von dem auch die swehrhaften Luxemburger/-innen nicht verschont geblieben sind. Mit dem Beitrag ist keine detaillierte historische Rekonstruktion der Entstehung der besonderen Luxemburger Kollektivsymboliken intendiert, im Zentrum stehen vielmehr Aspekte ihrer funktionalen Verwendung. ${ }^{4}$

\section{Das nationale Kernsymbol der 'Festung,}

Nur wenige Länder sind in der Wahrnehmung ihrer Bewohner/-innen wie auch in der Fremdwahrnehmung so sehr mit einem Bauwerk verbunden wie Luxemburg. (Kmec 2007) Die sogenannte >Festung «, das sind die noch erhalten gebliebenen Bestandteile einer gigantischen militärischen Anlage, ausgebaut von Vauban nach der französischen Eroberung von 1684 und über fast zwei Jahrhunderte wucherar-

2 | Nach Canetti ordnen sich die Engländer dem 'Meerı, die Franzosen der 'Revolution‘, die Deutschen dem 'Waldı, die Schweizer den ıBergenı und die Holländer dem 'Deichı zu. (Canetti 2000: 197-208)

3 | Ein willkürliches, aber aussagekräftiges Beispiel, in welchem Ausmaß das nationale Selbstbild in Luxemburg lange Zeit durch eine übernommene Fremdperspektive bestimmt war, findet sich im "Escher Tageblatt" vom 12.11.1947: Dort endet ein Artikel über die Wehrpflicht im Land mit der Bemerkung, ohne Armee erscheine das Land noch "mehr als Operettenstaat als je in unserer Geschichte" (S. 6). Zum Fremdbild Luxemburgs als 'Operettenstaatı vgl. Romain Kirt: "Zu klein, um überhaupt ein richtiger Staat zu sein?", in: d'Lëtzeburger Land, 17.08.2001, S. 1.

4 | Der Beitrag beruht im Wesentlichen auf einer Auswertung von Artikeln insbesondere der Luxemburger und deutschen Tagespresse der letzten Jahre. Die Artikel selbst werden in den jeweiligen Anmerkungen nachgewiesen, erscheinen aber nicht in der Bibliografie. 
tig erweitert, bis 1867 Frankreich und Preußen sich als fadenscheinige friedensstiftende Maßnahme auf ihre Schleifung einigten. Die Eckdaten sind heute touristisches Basiswissen. Vertiefen lässt es sich durch den Verweis auf die Zäsuren, die die Festungsgeschichte für die Geschichte der Stadt sowie des Landes gesetzt hat: die Zerstörung des mittelalterlichen Stadtraums, wechselnde sogenannte >Fremdherrschaften< (Margue 2007) und schließlich das allmähliche Entstehen einer Hauptstadt auf ihren Überresten. Die noch vorhandenen Anlagen sind insofern materiale Zeugnisse eines schwierigen nationalen Selbstbehauptungsprozesses.

Gemäß der Ansicht des ehemaligen Direktors des »Service des Sites et Monuments Nationaux«, George Calteux, spiegele die Festungsgeschichte eine kollektive Befindlichkeit im Land wieder, die sich durch die Erfahrungen während der beiden Weltkriege im 20. Jahrhundert zu einer nationalen Einstellung verfestigt habe. Er beschreibt die Mentalität seiner Landsleute folgendermaßen: »Viele, lange Kriege, der Druck von Herren und Fremdherrschaft haben seit dem frühen Mittelalter in Stadt und Land aus uns zähe und (manchmal) sture Menschen gemacht, die ihre Hände zu Fäusten in der Tasche ballten.« (Calteux 2005: 144)

Die Bemerkung bringt die Selbstwahrnehmung, in der sich ein stoischer Konservatismus ausdrückt, auf den Punkt: ein von den äußeren Bedingungen aufgezwungener Rückzug ins Innere einerseits und eine nur widerwillig und nur äußerlich mit den Zeitläufen konform gehende Lebensweise andererseits. Dieses Selbstwahrnehmungsmuster findet in der Festung einen adäquaten Ausdruck: >Wir sind so wehrhaft, wie die Festungsanlagen inmitten unserer Hauptstadt.< Die Namensgleichheit von Stadt und Land erleichtert die Identifikation mit dem Bauwerk, das zu einem repräsentativen Nationalsymbol aufsteigt und damit in gewisser Weise auch den Anschluss Luxemburgs an den schon seit langem praktizierten symbolischen Nationendiskurs in Europa gewährleistet.

Als Selbstzuschreibung funktioniert das Festungssymbol natürlich auch als Angebot, alle möglichen kursierenden Fremdzuschreibungen über die >Luxemburger/-innen < in einem Merkmal zu bündeln. Dabei lassen sich am Beispiel der >Festung< aber auch die zunehmenden Schwierigkeiten der Vermittlung nationalstaatlicher Symboliken im globalen Zeitalter beobachten.

Wie sehr das durch die doppelten Momente von Abschottung und Anpassung gekennzeichnete Selbstporträt besonders für die Nachkriegsidentität zutrifft, lässt sich an der Verwendung der Festungssymbolik im Werk des für diesen Zeitraum wichtigsten Schriftstellers des Landes, Roger Manderscheid, ablesen. Dazu einige Beispiele: In dem Skizzenband »Aufstand der Luxemburger Alliteraten « beschreibt Manderscheid rückblickend die kritische Funktion der Literatur im Land als eine Sisyphos-Arbeit an einer mentalen Blockade: »in mühseliger kleinarbeit haben die luxemburger literaten über jahrzehnte hinweg probiert, die festungsmauern, die unser geistiges leben gefangen hielten und immer noch halten, stein um stein abzutragen [...].« (Manderscheid 2003: 16) In dem zur Jahrtausendwende erschienenen Gedichtband »summa summarum. gedichte aus einem vergangenen jahrhundert« findet sich eine Gruppe von Gedichten über das Land unter dem Titel 
»lyrische berichte aus dem innern der festung « zusammengefasst. (Manderscheid 2000: 17) Und in dem Band »schwarze engel« heißt es an einer Stelle: »dabei sind die luxemburger die enge gewohnt. jahrhunderte haben sie hinter festungsmauern gekauert. [...] die festungsmauern haben sie durch bankenpaläste ersetzt. ich meine die grossherzoglichen.« (Manderscheid 2001: 14f.)

Der resignative Unterton deutet darauf hin, dass das Konzept einer ästhetischen Erziehung gegen die verbreitete Festungsmentalität bislang nur wenig auszurichten vermochte. En passant gibt Manderscheid auch einen Hinweis auf die Gründe hierfür. In einer modernen, pluralen Gesellschaft wie Luxemburg funktioniert die Festungssymbolik nicht mehr ausschließlich über die direkte Anbindung an einen imaginären Nationalcharakter, sondern wird in andere dominante Bildbereiche des alltäglichen Lebens übertragen: Die »festungsmauern« sind durch »bankenpaläste« ersetzt worden, die Attribute wie »verschlossen«, »geheimnisvoll«u.ä. bestehen aber weiter und können auf die veränderten Gegebenheiten hin aktualisiert werden.

Bestätigt werden diese Zusammenhänge, wenn man den Blick auf den Umgang der Medien mit der Nationalsymbolik richtet. So bedient sich beispielsweise ein Artikel in der »Revue« über die Geschichte der Bankengründungen im Land aus dem mittlerweile entstandenen Reservoir bildhafter Argumentationsformen, wenn er mit der Überschrift »Von der Festung zum Finanzplatz « ${ }^{5}$ aufwartet. Einerseits wird damit ein Wandel suggeriert, andererseits bleibt allein schon durch die Alliteration auf subtile Weise die Kontinuität zum überkommenen Nationalsymbol gewahrt.

Es verwundet auch kaum jemanden, dass in den rezenten Bankendiskussionen die Festungssymbolik besonders häufig genutzt wird: »Risse in der Festung «tituliert der »Telecran« einen Artikel über die »Aufweichung« des Bankengeheimnisses und gibt damit dem für dieses Thema verbreiteten Bild der »Steueroase« eine spezifische nationale Wendung. ${ }^{6}$ Komplementär dazu verläuft der Blick von außen: Der schweizerische »TagesAnzeiger « vergleicht die Probleme in Luxemburg mit der »Atmosphäre einer belagerten Festung «7 und knüpft damit wie selbstverständlich an ein mutmaßliches Alltagswissen über das Land an. In den Luxem-

5 | Wolf, Claude: "Von der Festung zum Finanzplatz", in: Revue, 20.09.2006, S. 21. Ähnlich auch in der belgischen Tagespresse: "Le Luxembourg, forteresse financière", in: Le Soir, 23.01.2002, S. 10; und in der luxemburgischen: "Une forteresse financière au coeur de l'Europe", in: tageblatt, 23.01.2002, S. 1.

6 | Lanners, Maryse: "Risse in der Festung", in: Telecran, 18.03.2009, S. 22. Auf die offenbar verbreitete Praxis der Zirkulation gerade dieser und ähnlich illustrativer Titelüberschriften auf dem Luxemburger Pressemarkt sei an dieser Stelle nur mit einem weiteren Beispiel hingewiesen: "Risse in der Festung, mehr nicht! " lautete die Schlagzeile von "Point 24" zum Stand der Debatte um das Bankgeheimnis am 21.10. 2009.

7 | Israel, Stephan: „Die Atmosphäre einer belagerten Festung“, in: TagesAnzeiger, 08.05. 2009, S. 10 . 
burger Medien wird das Bild der Belagerten wiederum sofort als Bestandteil der Selbstwahrnehmung aufgegriffen:

War es ein Fehler, 1867 die Festung Luxemburg zu schleifen? Liest und hört man die einheimischen Medien, so könnte man den Eindruck gewinnen, es herrsche wieder einmal ein Belagerungszustand. Doch diesmal geht es nicht um militärische, sondern um wirtschaftliche Interessen - um das Bankgeheimnis, genau gesagt. ${ }^{8}$

Diese ohne weiteres verlängerbaren Beispiele verweisen darauf, dass kollektive Symbolsysteme in der Publizistik wie generell in den Massenmedien an Bedeutung zugenommen haben, da sie an die Stelle der Erörterungen komplexer Sachverhalte getreten sind. Im Fall der Nationensymboliken wird darüber hinaus eine Kopplung an die überlieferten nationaltypischen Deutungsmuster gewährleistet.

In der globalen Medienkultur kann das häufig polemische Spiel mit Symbolsystemen allerdings auch eine schwer kontrollierbare Eigendynamik entwickeln, so dass nationale Selbstbilder in eine Krise geraten. Genau dies scheint seit geraumer Zeit für das luxemburger Symbol der >Festung « nicht nur mit Blick auf das Thema >Banken< der Fall zu sein.

\section{Luxemburg: 'Kern` der 'Festung Europa`?}

Zum näheren Verständnis dieser Problematik ist zunächst auf einen zweiten wichtigen Bestandteil des Luxemburger Selbstbildes aufmerksam zu machen, der sich anfangs unabhängig zur Festungssymbolik entwickelt hat, aber implizit darauf hin angelegt war, deren mögliche Defizite vor allem in der Fremdbildperspektive auszugleichen. Gemeint ist das in den 1950er Jahren begonnene und seit den 1980 er Jahren zunehmend verstärkte Europa-Engagement Luxemburgs, im Stadtbild der Hauptstadt präsent durch die Ansiedlung einer Reihe wichtiger europäischer Institutionen auf dem Plateau de Kirchberg. Neben der Etablierung Luxemburgs als >Finanzplatz< spielt die, wie sich in den öffentlichen Debatten gezeigt hat, hierzu nicht ohne Friktionen stehende Vorstellung des Landes als »Kern Europas« (Stoldt 2008: 19) im politischen Diskurs des Landes eine zentrale Rolle. Die intensiven Bemühungen, Luxemburg als »Akteur der europäischen Integration und sinternationale Plattform« (Stoldt 2008: 21) auszuweisen und die nationalen Belange sukzessive den dominanten supranationalen Strukturen von Wirtschaft und Politik anzugleichen, hat zu einem offiziellen Selbstbild der Luxemburger/-innen als »Avantgarde-Europäer « ${ }^{9}$ geführt.

8 | Klein, Raymond: "Bankgeheimnis. Deine Rede sei nein, nein «, in: woxx, 23.10.2009, Nr. 1029, S. 4.

9 | Werle, Gerd: "Luxemburger weiterhin Avantgarde-Europäer", in: Luxemburger Wort, 10.03.2003, S. 3. Im Übrigen belegt die im Kontext der Vorstellung der europäischen Integration und des ,Europa der zwei Geschwindigkeiten` neu zur Geltung gekommene Metapher 
Dieses mit erheblichem Aufwand propagierte Bild des modernen, fortschrittsorientierten Luxemburgs hatte vor allem über die Rituale medial inszenierter Symbolpolitik durchaus positive Auswirkungen auf die Fremdwahrnehmung. Exemplarisch kann hier die Verleihung des renommierten Karlspreises der Stadt Aachen genannt werden: 1986 wurde der Preis an das gesamte Luxemburger Volk verliehen (»Klein, aber fein«, »Das vorgelebte Leitbild eines pluralistischen Staates $\left.\aleph^{10}\right)$, im Jahr 2006 erhielt ihn der Premierminister Jean-Claude Juncker. Nach außen signalisiert gerade die Personalisierung eine generationsübergreifende ( $P$. Werner, G. Thorn, J. Santer) Kontinuität der Europa-Orientierung. Juncker konnte dabei aber auch nach innen als Symbolfigur einer Politik der Modernisierung vermittelt werden, Zuschreibungen in den einheimischen Medien wie »Vermittler«, »Brückenbauer«, »Mediator«, »Motor Europas «"11 sind also in beiderlei Richtungen lesbar. Zumindest auf dieser politisch-medialen Ebene konnten Selbstbild und Fremdbild zur Deckung gebracht werden.

Vor diesem Hintergrund muss nun aber das überkommene Nationalsymbol der >Festung< äußerst problematisch erscheinen. Bereits für das Selbstverständnis des Landes als »Kernland Europas « ${ }^{12}$ erweist es sich im Grunde als kontraproduktiv. Die interne, auf die nationalstaatliche Entität bezogene Symbolik konkurriert zusehends mit einer die Konfrontationsmentalität einer >Festung < abschüttelnden Neupositionierung als >Herz $<$, >Kern $<$ oder >Motor $<$ Europas ${ }^{13}$. (Parr 2009: 13)

Erschwerend wirkt in diesem Zusammenhang schließlich der Umstand, dass die Festungssymbolik in Verbindung mit dem gerade für Luxemburg zentralen Thema >Europa< seit Anfang der 199oer Jahre eindeutig negativ besetzt ist. Das Bild von der »Festung Europa « ${ }^{14}$ hat sich in der Zeit des Umbruchs und der Verschie-

der 'Avantgarde` den systematischen Gebrauch von Kollektivsymbolen. 'Avantgarde und das für Luxemburg herkömmliche Symbol der ,Festung، stammen beide aus dem Bereich der militärischen Sprache. Gegenüber 'Festung، verweist ,Avantgarde` auf die Opposition von Stillstand und Bewegung. Anders als die problematische Strategie der 'Abschottung, wird mit 'Avantgarde` eine offensive ,Pionier-ı bzw. 'Vorreiterrolle` gegenüber dem unabwendbaren ,Fortschritt، betont.

10 | Titel: Deutsches Allgemeines Sonntagsblatt, 11.05.1986; Süddeutsche Zeitung, 20.05.1986.

11 | Lorent, Joseph: "Vermittler, Mediator und Brückenbauer", in: Luxemburger Wort, 12.12.2005, S. 3; Montebrusco, Lucien: "Vermittler und Brückenbauer", in: tageblatt, 24.05.2006, S. 12; ders.: "Motor für Europa", in: tageblatt, 26.05.2006, S. 1.

12 | Werle, Gerd: „50 Jahre Römische Verträge. Luxemburg das Kernland Europas", in: Luxemburger Wort, 24.03.2007, S. 3.

13 | Zur ,Herz'-Symbolik z.B.: Schlammes, Marc: "Im Herzen Europas", in: Luxemburger Wort, 02.06.2006, S. 2.

14 | Aus deutscher Sicht hat das Symbol der "Festung Europa" im Übrigen eine hoch problematische Geschichte. Es gehörte zur Propagandasprache der Nationalsozialisten am Ende des Zweiten Weltkrieges und wird von Victor Klemperer in seinem Standardwerk zur 
bung des > West/Ost<- in einen >Nord/Süd<-Konflikt als Kritik an der restriktiven Migrationspolitik in der EU durchgesetzt. (Koff 2008) Es handelt sich um eine polemische Reaktion auf die offizielle politische Symbolsprache einer »europäischen Architektur« (Schäffner 1993). Während innerhalb der EU und ihren wichtigsten Verbündeten viel vom »gemeinsamen Haus Europa« die Rede ist, also offene und kommunikative Strukturen hervorgehoben werden, verweisen Kritiker/-innen mit der »Festung Europa « auf die Kehrseite der strikten Grenzziehungen und Abschottungen gegenüber anderen, >unerwünschten< Drittländern. Seit Inkrafttreten des 1985 beschlossenen »Schengener Abkommens « in den Gründungsstaaten der EU im Jahr 1990, war es prinzipiell sogar möglich, das Nationalsymbol der Festung mit der europakritischen Symbolik gleichzusetzen: Luxemburg konnte gleichsam als >Kernfestung « einer »Festung Europa« gelten.

\section{Das Mudam und die Selbstbild-Kontroverse}

Aus diesen Gründen erschien in Luxemburg eine Modifikation der in Verruf geratenen Nationalsymbolik und die stärkere Anbindung des nationalen Images an die europäische Perspektive seit langem dringend geboten. Der zweifellos spektakulärste Versuch, diesen langwierigen Prozess der Transformation nationaltypischer Einstellungsmuster entscheidend zu forcieren, stellt der 1990 von Regierungsseite gefasste Beschluss dar, den >Stararchitekten Ieoh Ming Pei auf den Fundamenten des Fort Thüngen am Rande des Plateau de Kirchberg ein »Musée d'art moderne« bauen zu lassen. ${ }^{15}$

Die jahrelangen heftigen Debatten um diesen dann schließlich im Juli 2006 eröffneten Museums-Bau sind nur verständlich vor dem Hintergrund des skizzierten, für Luxemburg nach dem Zweiten Weltkrieg charakteristischen Spannungsgefüges von Binnen- und Außenperspektivierung des nationalen Selbstbildes. Das Bestreben, diese unter Druck geratene doppelte Identitätsbestimmung symbolpolitisch in eine Richtung zu lenken, führte in der »Schlacht um Fort Thüngen ${ }^{16}$ zeitweilig zu deutlichen Rissen im nationalen Kollektiv: auf der einen Seite eine auf das europäisch-globale Projekt setzende und institutionell verankerte politische Elite, auf der anderen Seite eine durch verschiedene Bürgerinitiativen gut vernetzte Koalition von engagierten Bürgern/-innen und kritischen Intellektuellen, die sich durch eine Unterschriftenaktion weiteren Rückhalt in der Bevölkerung zu verschaffen wusste.

Sprache des Dritten Reichs erwähnt, vgl. Victor Klemperer, LTI, Notizbuch eines Philologen, Stuttgart 2007 [zuerst: 1957], S. 73.

15 | Ich danke André Bruns für die Möglichkeit der Sichtung des von inm gesammelten Materials zum Thema.

16 | Pauly, Michel (1991): "Schlacht um Fort Thüngen“, in: forum 128-129, S. 3-7. 
Die Befürworter begrüßten das geplante Kunstmuseum als Signal »der geistigen Öffnung des bislang eher weltverschlossenen Festungsgeistes der Luxemburger « ${ }^{17}$. Der selbst als Kunstwerk konzipierte avantgardistische Bau an einem lange vernachlässigten symbolträchtigen Ort sollte nicht nur innerhalb des Stadtbildes eine »Brücke zwischen Geschichte und Modernität $\aleph^{18}$ schlagen. Im Zuge der zunehmenden europäischen Städtekonkurrenz sollte das »Musée d'Art Moderne« (Mudam) zu einem Anziehungspunkt und unverwechselbaren >Icon< werden und dem Image der >Bankenfestung< eine kulturelle Wende verschaffen. Als nach außen wirksame Selbstdarstellung solle es zur Metropolisierung der Stadt beitragen und sie »in die Nähe so genannter >Stararchitecture Cities < rücken«. (Schulz 2008: 93)

Demgegenüber befürchteten die Gegner/-innen durch den in den ersten Entwürfen noch monumentalen Bau die unbedachte und nicht wieder rückgängig zu machende Zerstörung von Teilen des Fort Thüngen und damit die Entsorgung von Zeugnissen der vergangenen nationalen Identität. Beklagt wurden Kostenrisiken, ein fehlendes Sammlungskonzept, aber vor allem die Eigenschaftslosigkeit einer globalen Architektursprache, die sich über lokale und historisch spezifische Gegebenheiten hinwegsetze.

\section{'Arcelor/Mittalı im nationalen Identitätsdiskurs}

Symbolisiert das »Mudam« den Anschluss von Stadt und Land an die Globalisierung und zugleich eine Kulturalisierung der bis dahin dominierenden Vorstellung von Globalisierung als rein ökonomischem Prozess, so erfährt diese Strategie der Modernisierung des Selbstbildes noch vor der Eröffnung des Museums einen Rückschlag. In der zu Beginn des Jahres 2006 in den Medien ausgetragenen Auseinandersetzung um den Verkauf des Stahlkonzerns Arcelor ${ }^{19}$ an den indischen Unternehmer Mittal wurden ähnlich wie bei dem Streit um Fort Thüngen Fragen nach der nationalen Identität aufgeworfen, die als solche überhaupt erst an markanten Wahrzeichen greifbar werden - in diesem Fall am Verwaltungsgebäude des Konzerns sowie an den Industriegebieten im Süden des Landes als Monumente einer >großen industriellen Vergangenheit<. »Wir sind Arcelor ${ }^{20}$ titelte das »Lu-

17 | Diese Zusammenfassung der Positionen bei Krieps, Rosch (1991): "Parallelen zum Fort Thüngen ", in: forum 130, S. 22.

18 | Helweg-Nottrot, Ina: "Eine Brücke zwischen Geschichte und Modernität, in: Luxemburger Wort", 01.07.2006, S. 8.

19 | Ein zu dieser Zeit bereits multinationales, börsennotiertes Unternehmen mit repräsentativer Konzernzentrale in Luxemburg. Ein wesentlicher Bestandteil des 2001 durch Fusion entstandenen Arcelor-Konzerns war die "Arbed" (Aciéries Réunies de BurbachEich-Dudelange; Vereinigte Stahlhütten Burbach-Eich-Düdelingen), ein 1911 gegründeter Luxemburger Stahlkonzern, der lange Zeit zu den größten der Welt gehörte und für die Industriegeschichte und die nationale Identität des Landes von erheblicher Bedeutung war.

20 | Glesener, Marc: "Wir sind Arcelor", in: Luxemburger Wort, 04.02.2006, S. 5. 
xemburger Wort « bemerkenswerterweise mit deutlichen Anlehnungen an die in Deutschland im Zuge der friedlichen Revolution von 1989 und später der Papstinthronisation 2005 erprobten Transformationen von >Wir<-Gefühlen in Formen eines um Akzeptanz bemühten nationalen Bewusstseins. Nachdem in der Mudam-Debatte höchst unterschiedliche Einstellungen zum nationalen Selbstbild offen zutage getreten waren, bot die Arcelor/Mittal-Debatte Gelegenheit, die >cohésion sociale < rhetorisch zu stärken und wiederum gemeinsam gegenüber einer $\mathrm{zu}$ weit gehenden, fehlgeleiteten Modernisierung Stellung zu beziehen. Schlagzeilen wie »Juncker: Es geht um Luxemburg « ${ }^{21}$ oder der, eine Bemerkung des Premierministers aufgreifende, Titel, die Luxemburger seien auch ohne Mittal bereits »glücklich globalisiert « $^{22}$, weisen nicht nur auf die Inanspruchnahme des >Mediators < für eine gegenläufige Entwicklung und Begrenzung der bis dahin forcierten Politik der fortschrittlichen Öffnung hin. Im »Krieg«, im »Abwehrkamp^« «3, der von außen auch als »Stahlschlacht« wahrgenommen und sogar mit einem »Duell aus der Ritterzeit « ${ }^{24}$ verglichen wird, kommt unversehens die >Festungs «-Symbolik wieder zur Geltung. Darüber hinaus führt die »Front« gegen den indischen Konzern zumindest vorübergehend $\mathrm{zu}$ einer positiven Umcodierung der »Festung Europa Der Subtext lautet hier: Im Zuge des globalen Wettbewerbs darf die »globale Normalordnung « (Link 2006: 431) nicht außer Kraft gesetzt werden. Diese Ordnung sieht vor, dass die westliche Welt, und hier vor allem der >Kern Europas<, nach wie vor die obere »Normalitätsklasse« besetzt, ökonomisch aufstrebende sogenannte >Schwellenländer , wie Indien, aber aufgrund ihrer uneinheitlichen und stark zwischen unermesslich >reich < und extrem >arm < schwankenden Lebensstandards sich mit unteren Rangplätzen begnügen müssen. Auf diese Zusammenhänge rekurrieren dann diesbezüglich subtil wirkende Titulierungen des indischen Unternehmers als »Stahl-Maharadscha «"5 (vgl. 6.3). Zum anderen wird der $>$ Komplex Arcelor/Mittal< als Kampf zwischen »zwei Kulturen « ${ }^{26}$ bezeichnet und damit eine Verbindung zu der durch Samuel Huntington in den 1990er Jahren populär gewordenen essentialistischen These vom >clash of civilizations $<$ und die dadurch geschürte Angst vor dem Verlust der kulturellen Vormachtstellung hergestellt. (Huntington 1996)

21 | Zeimet, Laurent: "Juncker: ,Es geht um Luxemburg'“, in: Luxemburger Wort, 01.02. 2006, S. 3.

22 | Langner, Arne: "Glücklich globalisiert", in: Luxemburger Wort, 16.02.2006, S. 14.

23 | G. S.: "Arcelor rechnet mit monatelangem Kampf gegen drohende Übernahme", in: Zeitung vum Lëtzebuerger Vollek, 28.01.2006, S. 3.

24 | Braunberger, Gerald: "Die Ritter der Stahlschlacht führen ein hartes Duell«, in: Frankfurter Allgemeine Sonntagszeitung, 12.02. 2006, S. 39.

25 | Chaton, Cordelia: "Wo Berater versagen, kommt der Stahl-Maharadscha zum Erfolg", in: Luxemburger Wort, 01.02.2006, S. 4.

26 | Hirsch, Mario: „Zwei Kulturen«, in: d’Lëtzebuerger Land, 03.02.2006, S. 1. 
Insgesamt liefert die Mudam- wie auch die Arcelor/Mittal-Debatte den Artikulationsrahmen für ein ansonsten diffus bleibendes nationales Identitätsgefühl, das aber - und darin liegt ein wesentlicher Unterschied zu den Identitätskonstruktionen im Zeitalter des puren Nationalismus - nicht mehr vorrangig über die direkte Abgrenzung gegenüber anderen Nationen, auch nicht gegenüber den beiden großen Nachbarn, zum Ausdruck kommt. Den Gegenpol bildet vielmehr die Vorstellung von >Europa< als übermächtig gewordene supranationale Institution, die als >Einfallstor< einer unkontrollierbaren, rücksichtslosen Globalisierung erscheint. Eine Möglichkeit der Begrenzung dieser Entwicklung wird von den Medien durch die Unterscheidung zwischen einer >guten < und einer >schlechten < Globalisierung angeboten. Diese Opposition wird personifiziert durch den >ehrlichen $<$ Vermittler Juncker auf der einen Seite und den als > verschlagen< vorgestellten globalen Akteuren, den Architekten Ieoh Ming Pei und den Unternehmer Lakshmi Mittal auf der anderen Seite. ${ }^{27}$

\section{Nationale Symboliken im Kulturhauptstadtjahr 2007}

Vor allem die Mudam-Kontroverse kann als beispielhaft für den Konflikt zwischen zugeschriebenen und angeeigneten Identitäten innerhalb eines um klarere Konturen bemühten Selbstbildes verstanden werden. Obwohl die Arcelor/Mittal-Debatte negativ auf die von politisch-institutioneller Seite (>top down<) vorangetriebene Modernisierung des nationalen Image wirkt, hat sie dennoch im Vorfeld des Kulturhauptstadtjahres 2007 einen integrativen Effekt. Vor diesem Hintergrund können dann die mit diesem Ereignis verbundenen Intentionen weiter entwickelt werden. Werbetechnisch gesprochen: Dem >Branding< der neuen >Marke< Luxemburg sollte das Kulturhauptstadtjahr 2007 neue Impulse verschaffen.

Die in den beiden skizzierten Selbstbild-Kontroversen in den Medien als problematisch dargestellte >cohésion sociale< findet dabei Berücksichtigung, insofern mit dem Schwerpunktthema >Großregion< ein alltagstaugliches Identifikationspotential bereitgestellt wird. Denn das immer noch charakteristische Differenzbewusstsein zwischen >globalem $<$ Stadtraum und >lokalem< Umland wird durch die regionale Perspektivierung aufgehoben und Luxemburg als integraler Gesamtraum in der >Mitte der Großregion < präsentiert. Korrespondierend zum kollektiv-

27 | In der Mudam- wie in der Arcelor/Mittal-Berichterstattung spielt dieser Aspekt des lesbaren physiognomischen Charakters eine nicht unwichtige Rolle. Ebenso wie der Unternehmer Mittal als "der Mann des Lächelns" porträtiert wurde (Meyer, Roman: "Der Mann des Lächelns", in: Revue, 08.02.2006, S. 30), kam kein Porträt des Architekten Pei - der auch als "Architekt der Mächtigen" gilt - ohne den Verweis auf das "für Asiaten typische breite Lächeln" aus (so z.B.: Morbach, Fern: "Der große alte Mann der Architektur", in: Telécran, Nr. 40, 30.09.1995, S. 29). 
symbolischen >Kern Europas<, wird Luxemburg für seine Einwohner/-innen zum »Reich der Mitte «, das eine neue »Auslotung der Grenze« erfordert. ${ }^{28}$ (Vgl. 4.7)

Darüber hinaus wird aber auch das im Zusammenhang mit dem Mudam eingeleitete Projekt der Neupositionierung fortgesetzt. Zwischen dem alten Selbstbild (〉Festungく) und der anvisierten neuen weltoffenen Selbstdarstellung, für die sich offenkundig noch keine prägnante Kollektivsymbolik etablieren konnten, werden u.a. architektonische Bilder des Übergangs platziert. Das bevorzugte Symbol ist hier das gegenüber der >Festung< wiederum Kontinuität suchende Bild der >Brücke $<$ : Brücken bauen $<$ zwischen den Regionen, zwischen dem >Alten $<$ und $>$ Neuen $<$ zwischen Vergangenheit und Zukunft etc. (Vgl. 6.3). In immer neuen Varianten wird Luxemburg daher offensiv als >Zwischenraum<, als >europäisches Labor< oder als >Experimentierraum präsentiert, wodurch wiederum Verbindungen zu $>$ Herz-<, >Mitte-< und >Kern<-Symboliken möglich werden.

Von außen wird der Versuch, für die nationale Symbolik sowohl Anschlüsse zu sichern als auch Neuausrichtungen zu suchen, durchaus wahrgenommen. Das Land erscheint als »Europas bunte Brücke ${ }^{29}$, und es wird wahrgenommen, dass es »nicht nur Banker und Bürokraten « $3^{\circ}$ im Land gibt, allerdings betont die Alliteration dann doch wieder den Rückbezug auf überkommene Stereotypen, eine positive Bestimmung bleibt aus. Ähnlich zwiespältig ist das Bild von der »Schatzkammer der Festung Europa « ${ }^{31}$ einzuschätzen, in dem Ambivalenzen in Hinblick auf die beabsichtigte Kulturalisierung des in der öffentlichen Wahrnehmung dominierenden ökonomischen Sektors mitschwingen. Geradezu idealtypisch rezipiert hingegen die deutsche Reisezeitschrift »Merian« diese Symboliken auf der Titelseite ihres »Luxemburg«-Heftes zum Kulturhauptstadtjahr 2007 in Form eines Emblems: Als > Pictura < fungiert die geschwungene Empore der Luxemburger Philharmonie, als >Subscriptio < wird Luxemburg gleich zweifach als »Zwischen «-Land charakterisiert, im dritten Satz wird schließlich Premierminister Juncker als die ausgleichende Mittlerfigur und Garant für diesen Übergangszustand exponiert. (Vgl. 5.5)

\section{Medien als Vermittler zugeschriebener und angeeigneter Identitäten}

Es bleibt offen, welche Nationalsymbolik sich in nächster Zukunft für Luxemburg durchsetzen lässt bzw. durchgesetzt werden kann. Diese Unterscheidung ist wichtig. Die hier skizzierten medialen Diskurse haben zwar nicht zu einem Konsens

28 | Langenbrink, Christophe: "Im Reich der Mitte", in: Luxemburger Wort, 10.05.2007, S. 13; Wahl, Susanne: "Eine Auslotung der Grenze», in: Luxemburger Wort, 31.05. 2007, S. 13.

29 | Schiemann, Hans: "Europas bunte Brücke», in: Merkur, 23.02.2007, S. 29.

30 | Meyer, Ulf: „Nicht nur Banker und Bürokraten«, in: Die Welt, 20.07.2006, S. 26.

31 | Schümer, Dirk: "Die Schatzkammer der Festung Europa", in: Frankfurter Allgemeine Zeitung, 11.06.2007, S. 42. 
über die Nationalsymbolik geführt. Sie sind aber ein Feld, auf dem zugeschriebene und angeeignete kollektive Identitäten in einem Aushandlungsprozess aufeinander bezogen werden, mit dem Effekt, dass sie sich häufig nicht mehr in dieser oder jener Form so einfach auseinanderhalten lassen. Gerade die medialen Applikationen von Nationalsymboliken präsumieren jedenfalls ein enges Wechselverhältnis und machen als Schnittstelle deutlich, wie sehr beide Identitätsmuster aufeinander angewiesen sind. Das bedeutet allerdings auch, dass kollektive Identitäten auch bzw. gerade in postnationalen Zeiten der Imagekampagnen nicht ohne weiteres neu erfunden werden können, genauso wenig, wie die überkommenen einfach fortgeschrieben werden können.

\subsection{Die Superjhemp-Saga - eine ideale Projektionsfläche}

In national gefärbten >Superheldencomics< finden sich häufig Diskurse, die Konstrukte zu den Identitäten der jeweiligen Bevölkerung sind. Folglich bietet die luxemburger Comic-Serie >Superjhemp ein besonders interessantes Fallbeispiel, um den Fragen nachzugehen, welche angeeigneten und zugeschriebenen Identitäten rekurrierend thematisiert werden und wie die satirische Darstellung sich zu den tatsächlichen Identitätskonstrukten verhält. Die identitären Merkmale des Helden spielen im Hinblick auf die vorgestellten Rezipienten/-innen eine wesentliche Rolle in deren Aneignungspraxis. So scheint Luxemburgs Megaheld Superjhemp diverse Eigenschaften von ausländischen Comicsuperhelden in sich zu vereinigen. Die Figur nimmt u.a. Anleihen bei Superman, Batman, Spiderman, Popeye, Asterix, Tintin usw. Die stärkste Inspirationsquelle der Superjhemp-Autoren - Lucien Czuga und Roger Leiner - ist aber sicherlich die französische Figur des >Superduponts $<$ aus der gleichnamigen Comicserie, die in den Intentionen, dem Witz und dem generellen Charakter sehr starke Parallelen aufweist. Obschon Superjhemp - wie die zahlreichen Anleihen belegen - das Produkt einer populärkulturellen Globalisierung ist und damit wie gewissermaßen alle Comics zu den Interdiskursen gehört, weist er dennoch zahlreiche identitäre Charakterzüge auf, die ihren Ursprung im luxemburger Lokalkolorit haben.

Die Anthologie »Comics in, aus und über Luxemburg « (Haas 2007) zeigt, dass die Comiclandschaft in dem kleinen Luxemburg mit der Bevölkerungszahl einer mittelgroßen Stadt (etwa 490.000 Einwohner) recht belebt ist. Zwar sind die Superjhemp-Geschichten der letzten 20 Jahre konkurrenzlos was Erfolg, nationale Sichtbarkeit und Popularität anbelangt, aber es gab auch schon vor dieser Zeit Versuche, Langzeit-Comicserien bei der luxemburger Leserschaft zu etablieren. Die meisten dieser Produkte erschienen aber nicht als Buch, sondern wurden als Fortsetzungsgeschichte in Wochen- oder Tageszeitungen veröffentlicht. In der bereits genannten Zusammenstellung über Comics werden die luxemburger Cartoonisten miteinbezogen. Auf der Ebene der Signifikanten benutzt das Cartoon eine Formensprache, welche Figuren und Objekte komisch verzerrt, manche Einzelheiten 
aufbläst oder andere weglässt. Es entsteht eine intendierte Rückkopplung zu dem Charakter des dargestellten Signifikats. Das gilt besonders für Superjhemp, wenn es darum geht, ikonisch visuelle Ähnlichkeiten zu bekannten luxemburger Persönlichkeiten und Objekten herzustellen. Formal gehört die Serie zu der Gattung von Comics, welche konsequent die karikierenden Mittel des Cartoons einsetzt. Durch die cartoonisierte Formensprache entsteht, genau wie bei Asterix, eine gewisse Verfremdung der Wirklichkeit, die im Gegensatz zu den rein äußerlich realen Darstellungen von Figuren und Ausstattung in führenden US-Superheldensagas steht (vgl. Superman, Spiderman, generell >graphic novels<). Es gibt, international gesehen, eine relativ große Anzahl von verschiedenen Comic-Genres. Die etwas weiter in diesem Text analysierten Samples zeigen, dass man Superjhemp nicht alleine dem >Comicsuperheldenepos < zuordnen kann, sondern sie deuten darauf hin, dass er gleich in mehrere Gattungen passt, wie die weiteren Ausführungen belegen.
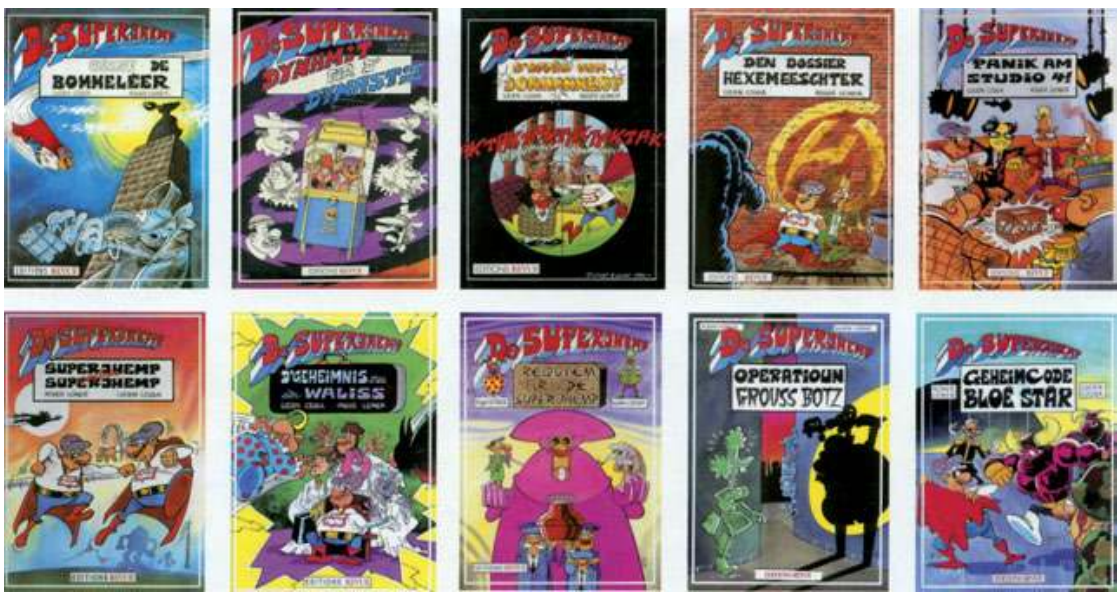

Abbildung 1: Coverbeispiele von Superjhemp-Alben (C) Editions Revue, Luxembourg)

Aufgrund der über 20-jährigen Erfolgsgeschichte drängt sich die Frage auf: womit bedienen die Autoren das luxemburger Publikum? Im Superjhemp-Comic kommt vieles vor, was das gesellschaftliche und politische Leben in Luxemburg betrifft. Die Autoren der Serie bauen im Superjhemp eine Art Parallelwelt zum Vorbild Luxemburg auf. Karikaturen von bekannten Politikern/-innen und ironische Darstellungen bringen so manche luxemburger Verhältnisse witzig auf den Punkt: Die Autoren halten den Einheimischen den Narrenspiegel vor. Und was sie darin sehen, scheint ihnen zu gefallen. Der große Verkaufserfolg der Serie - manche Bände sind vergriffen, andere wurden als Sammelausgaben neu gedruckt - zeigt, dass die satirisch verzerrte Inszenierung von luxemburger Gegebenheiten die Leserschaft begeistert. Wie der Hofnarr gegenüber seinem König formulieren Czuga und Leiner karikaturhafte Geschichten zu den Identitäten der Luxemburger/-innen. Und das sind manchmal auch unangenehme Wahrheiten; die sarkastischen 
Projektionen werden aber oft durch Sprach- und Bildwitz entschärft, so dass sie leichter zu verschmerzen sind oder sie sind so überspitzt dargestellt, dass lindernde Zweifel an der Glaubwürdigkeit entstehen.

Die Autoren thematisieren in dem fast homonymen Konstrukt »Luxusbuerg « Fremd- und Selbstbilder über Luxemburg: Identitäten, die teilweise zugeschrieben sind und solche, die Teile der Bevölkerung sich mehr oder minder angeeignet haben. Superjhemp gehört damit zum Genre des >Comicsuperheldenepos< das »als Widerspiegelung einer gesellschaftlichen Situation« (Umberto Eco zitiert nach Ditschke/Anhut 2009: 148) fungiert.

Sie [die Comicheldgeschichten] referieren zwar unterschiedlich stark auf tatsächliche soziokulturelle Ereignisse und Strukturen, trotzdem kommen die "in den Comic Books dargestellten ,Wirklichkeiten، [...] der Realität doch sehr nahe“ (Th. Sieck) - einerseits hinsichtlich zugrunde liegender gesellschaftlicher, ökonomischer und kultureller Strukturen, andrerseits hinsichtlich spezifischer Ereignisse [...] (Ditschke/Anhut 2009: 149),

die eine bedeutende soziopolitische Relevanz aufweisen. Da die Autoren von Superjhemp >dem Volk aufs Maul schauen und vieles, was sie sehen und aus der Gerüchteküche hören, karikatural transponieren, kann man annehmen, dass zahlreiche Fremd- und Selbstbilder den Luxemburgern/-innen durchaus bewusst sind und manchmal sogar - ähnlich wie in der Comic-Serie - damit kokettiert wird.

Mit seinen mehr als zwanzig Bänden, bildet die Superjhemp-Saga ein fast unerschöpfliches Repertoire von möglichen Samples, welche luxemburger Identitäten betreffen. Aus Platzgründen beschränken sich die folgenden qualitativen Analysen notwendigerweise auf einige hervorstechende identitäre Diskurse. Weil Comics eminent semiotische Geschehen sind, dient als theoretische Grundlage für die diskursanalytische Methode das triadische Modell von Peirce (vgl. Peirce 1907, deutsche Neuausgabe 2000), das durch das Konzept des kausalen Charakters von Symbolisierung und Referenzierung nach Ogden und Richards (vgl. Ogden/Richards 1923) erweitert wird. Gesellschaftlich spezifische Codes und Chiffren spielen nicht nur bei der Auswahl und Darstellung, sondern auch bei der Rezeptanz und Pragmatik der in Superjhemp eingesetzten Zeichen eine kausale Rolle. Die vernetzte Abfolge, die Jakobson in seinem Kommunikationsmodell darstellt, (vgl. Jakobson 1960) soll dazu beitragen, das Geflecht aus Comic-Produktion, gesellschaftlichen Kontexten und Diskursen (z.B. Finanzplatz, Stahlindustrie) und Superjhemp-Rezeption offenzulegen. Der Ansatz ist zwangsläufig interpretativ. Die quantitativ gewonnenen Daten werden dort wo es möglich ist mit den interpretierenden Ergebnissen in Beziehung gesetzt.

Um Näheres über die relative Wahrnehmungsintensität von Superjhemp und die soziale Struktur der Leserschaft zu erfahren, wurde bei den quantitativen Erhebungen folgende Frage gestellt: »Haben Sie schon mal Superjhemp gelesen, als Comicband oder in der Presse?« Letzteres ist relevant, weil die Vorabveröffentlichung der Bücher in einem wöchentlichen Rhythmus von jeweils zwei Seiten in 
der »Revue«, einer verhältnismäßig viel gelesenen Wochenzeitschrift, erfolgt. Die Ergebnisse deuten an, dass eine Mehrzahl (63\%) der luxemburger Wohnbevölkerung den Megahelden kennt. Der Vergleich der verschiedenen Milieus zeigt, dass der Bekanntheitsgrad sich durchwegs auf hohem Niveau bewegt und nicht stark variiert: von $69 \%$ im konservativ-gehobenen Milieu bis zu $81 \%$, sowohl im alternativen wie auch im liberal-gehobenen Milieu.

\section{Angeeignete Essenskultur}

Mit dem Herausstellen von Essensvorlieben, die zumindest von Teilen der Luxemburger/-innen als identitär angesehen werden, thematisieren die SuperjhempAutoren Aneignungen, die in einem langzeitlichen Prozess gewachsen sind. Was Spinat für Popeye und der Zaubertrank für Asterix bedeutet, ist für Superjhemp »Kachkéis « ${ }^{32}$. Von dem Stoff bekommt er die überirdischen Kräfte, die seine waghalsigen Höhenflüge erst möglich machen. »Kachkéis« wird von vielen Luxemburgern/-innen zu den nationalen Nahrungsmitteln gezählt, ist aber kein exklusiv luxemburgisches Produkt. Auch in anderen europäischen Regionen gehört er zu den traditionellen Käsesorten. Diese teilweise Aneignung entspricht einer gewissen lokalen Ernährungspraxis ${ }^{33}$ - sind doch die entsprechenden Regale im Supermarkt auch heute noch gut mit »Kachkéis « gefüllt. Bereits vor 40 Jahren wurde der Käse mit anderen Essensvorlieben im Liedrefrain des populären Kabaretttexters Pier Kremer besungen: »Kachkéis, Bouneschlupp, Quetschekraut a Mouselsbéier«34. Das Lied wurde Jahrzehnte später von der einheimischen Musikband »Cool Feet« auf Pop getrimmt und neu herausgebracht, so dass auch größere Teile der jüngeren Generationen angesprochen wurden. Während bei Superman und Asterix die Wunderkräfte einen eher kosmischen und magischen Ursprung haben, geben Czuga und Leiner ihrem Held in dieser Hinsicht bewusst einen volkstümlichen und witzigen Charakter: Als Auslöser seiner außergewöhnlichen Fähigkeiten dient der Verzehr eines banalen und massenhaft hergestellten Käses, der aber eine besondere identitäre Bedeutung im nationalen Kontext hat. In einer Episode wird »Luxusbuerg« schon im Titel in »Kachkéisien« (vgl. Czuga/Leiner 2005) umbenannt. »Kachkéis« ist dann auch der Retter in der Not, was den Erhalt der Vollbeschäftigung anbelangt: Superjhemp schlägt dem Premierminister vor, die durch den Verlust des Bankgeheimnisses drohenden Jobverluste in der Finanzbranche mit Arbeitsstellen in neu zu errichtenden »Kachkéis«-Fabriken auszugleichen. (Czuga/Leiner 2009: 6; 48) Der Vorschlag, der in dem Vorbild Luxemburg von den Entscheidungsträgern als absurd abgetan würde, wird in der Comic-Groteske zu einer plausiblen Möglichkeit, Arbeitsplätze zu schaffen, weil das identitätsstiftende Nahrungsmittel »Kachkéis « in »Luxusbuerg« zu einem wahren Wunderstoff

32 | Deutsch: "Kochkäse".

33 | Vgl. Abschnitt 7.4.

34 | Deutsch: "Kochkäse, Bohneneintopf, Zwetschgenmarmelade und Mousel-Bier". 
mutiert und - gleich einem >deus ex machina<, je nach Notlage - als multiple Verteidigungswaffe, Superklebstoff, Spiderman-Netz und Fangfolie für US-Tarnkappenbomber (Czuga/Leiner 2003: 40) eingesetzt wird.

Die Autoren zeigen auch in weiteren Geschichten, dass sie eine besondere Schwäche für die traditionelle Essenskultur der Luxemburger/-innen haben. So wird »Judd mat Gardebounen «35 gleich in mehreren Geschichten erwähnt. Diese Speise wird öfters in luxemburger Kochbüchern und Tourismusbroschüren als eine Art Nationalgericht angegeben. Die Zutat Bohnen spielt des Weiteren eine kapitale Rolle in dem Band »Superjhemp an déi grouss Gefor« (vgl. Czuga/Leiner 2007a). Der »Luxusbuerger « Held hortet sogar in seinem Keller einen beachtlichen Vorrat an »Bouneschlupp « ${ }^{36}$-Konservendosen. Und nicht zuletzt heißt er in seinem bürgerlichen Leben Charel Kuddel und ist mit Felicie Fleck verheiratet - was zusammen »Kuddelfleck« ergibt - ein wohl eher bei den älteren Generationen beliebtes Gericht auf Basis von Rinderpansen. Wenn Superjhemp flucht, sagt er »Poznennö«, die Umkehrung von »Önnenzop«, einer Zwiebelsuppe, die man wiederum nicht nur in Luxemburg findet. Der Fluch stammt nicht aus dem spontanen Wortschatz der Luxemburger, sondern ist eine Erfindung der Autoren, wohl als klangliche Anspielung auf das geläufige, jedoch vulgäre, » Nondidjö«, die luxemburgische Form von »nom de Dieu«37. »Poznennö« gehört zu den Superjhemp inhärenten speziellen Ausdrücken, die Czuga und Leiner in ihrem »Luxusbuerger Lexikon« (Czuga/Leiner 2008a) erklären. In der Parallelwelt »Luxusbuerg« werden quasi alle Namen und Bezeichnungen, die direkt auf Luxemburg verweisen, verballhornt oder leicht verdreht. Aber die bereits erwähnten Speisen haben kurioserweise ihre Originalbezeichnung behalten. In der Comicserie ist die luxemburgische Sprache die hervorstechende > unverfremdete< Identität, die den »Luxusbuergern « und Luxemburgern/-innen gemeinsam ist. Sogar das regionale Sprachkolorit - in Form von Dialekt-Varianten - findet seinen Platz.

Die von Teilen der Bevölkerung angeeigneten traditionellen und heute nur bei besonderen Gelegenheiten gekochten Nationalgerichte sind einfach und deftig sie werden mit eher billigen Zutaten hergestellt. Sie verweisen auf die Zeit im 19. Jahrhundert, als Luxemburg noch ein Bauernstaat war und die Bevölkerung so arm war, dass viele auswandern mussten. Es ist handelt sich also eher um Aneignungen, die damals aus der Not entstanden waren. Erst mit der Industrialisierung in der ersten Hälfte und später mit der Entwicklung des Bankenplatzes in der zweiten Hälfte des 20. Jahrhunderts kam der relative Reichtum. Wohlstand und Luxus sind darum auch ein weiteres zentrales Thema im Superjhemp, dessen textliche und ikonische Darstellung auf kollektivsymbolische Zusammenhänge zielt.

35 | Deutsch: "Schweinenacken mit Saubohnen".

36 | Deutsch: "Bohneneintopf“.

37 | Deutsch: "Name Gottes". 


\section{Wohlstand und Globalisierungsängste}

Das Wortspiel liegt auf der Hand; das Comic-Land heißt »Luxusbuerg « und die Einwohner sind die »Luxusbuerger«. Für die französisch sprechenden Grenzgänger/-innen, die jeden Tag nach Luxemburg zur Arbeit pendeln, ist der Ausdruck »Luxo« als Bezeichnung für »den/die Luxemburger/-in« nicht nur eine Verkürzung, sondern er ist auch konnotativ besetzt. Im Superjhemp wird dieses Kürzel von den Autoren mehrmals aufgegriffen. So führen sie im nahen französischen Grenzgebiet einen Richtungsweiser mit der Schrift »Luxoland « (Czuga/Leiner 2007a: 19) auf oder bei einer Manifestation von streikenden französischen und belgischen Arbeitern/-innen vor dem Hauptsitz der damaligen Arcelor wird ein Spruchband »A bas les Luxos « $\$^{38}$ geschwenkt. (Czuga/Leiner 2003: 3)

Das stellvertretende Symbol für Luxus und Wohlstand im Superjhemp ist »Luxonit«, ein kosmisches Element das im 19. Jahrhundert in Form eines Meteoriten in »Luxusbuerg« gelandet ist. In dem Stoff sollen alle Eigenschaften des »Luxusbürgertums « vereinigt sein. (Czuga/Leiner 1998b: 9) Das Fehlen von »Luxonit« hat Auswirkungen auf das Konsumverhalten der »Luxusbuerger«; es werden keine »Luxusbuerger Produkte« und auch sonstigen Verbrauchsartikel mehr gekauft. (Czuga/Leiner 1998b: 37) Damit wird die scheinbar wichtigste Selbstverständlichkeit in der »Luxusbuerger « Gesellschaft infrage gestellt. Mit dem »Luxonit« kreieren die Autoren ein spezifisches kollektives Symbol für »Luxusbuerg « und nehmen gleichzeitig einmal mehr eine Anleihe beim amerikanischen `Superman<. Der Wirkungsmechanismus des kosmischen Stoffes ist zwar scheinbar ähnlich, doch seine Voraussetzungen sind genau umgekehrt: während die Präsenz von grünem »Kryptonit« Superman seiner Kräfte beraubt, hindert die Absenz von »Luxonit« die »Luxusbuerger« daran, weiterhin blind zu konsumieren. Die Autoren zeigen sarkastisch mit dekonstruktiven Bild- und Textmitteln, welche >dramatischen< Konsequenzen der Verlust von Verbraucherroutinen hat, welche die oft zugeschriebene Identität sintensiver Konsument< legitimieren: Die »Luxusbuerger « werden scheinbar ziellos, leicht verwirrt und stellen so manche konsumierende Handlung infrage. (Czuga/Leiner 1998b: 37) Sie verzichten auf ihr Auto und gehen zu Fuß, tragen den Fernseher zum Recycling oder bestellen in der Kneipe Wasser anstatt Bier.

Die erst Silbe »Luxo« von Luxonit deutet auf Luxus und die bereits erwähnte französische Bezeichnung für die Luxemburger. Das bei Mineralien übliche Suffix »it« könnte auf ein imaginäres Erz verweisen und damit auf den teilweisen Ursprung des Wohlstands in Luxemburg - der Aufbau der Eisenhüttenindustrie am Anfang des 20. Jahrhunderts. Da »Luxonit« in der entsprechenden Episode wie ein brauner Stein aussieht, deutet alles auf eine imaginäre Erzsorte. Die geheimnisvolle Materie ist eine Art Katalysator und kann selber direkt keinen Reichtum produzieren. Das seltene »Luxonit« wird im Gebäude der Staatsbank - der »Luxus-

38 | Deutsch: "Nieder mit den Luxos". 
buerger « Sparkasse - gleich einem nationalen Heiligtum, dauerhaft und gut gesichert, aufbewahrt und wird somit zu einem Symbol für die in Banken gelagerten Geldreserven von Einheimischen und Ausländern/-innen. Sollte das »Luxonit« verschwinden, so fällt auch das Land in fremde Hände. (Czuga/Leiner 2008a: 52)

In dem Band »Den Dossier Hexemeeschter« zeigen Czuga und Leiner ganz konkret den Zusammenhang des relativen Reichtums der Luxemburger/-innen mit der Existenz des internationalen Finanzplatzes. Das Thema gibt den Autoren Gelegenheit sich mit mehreren zugeschriebenen und abgelehnten Identitäten zu beschäftigen. Die Banken am »King’s Boulevard « (Boulevard Royal) werden ikonisch als Waschmaschinen dargestellt, die dem Weißwaschen von ausländischem Geld dienen. (Czuga/Leiner 1991: 15) Die Assoziation Waschmaschine (Bild)/Geldwäsche (Sinn) zeigt geradezu exemplarisch den Umgang des Autorenteams mit Kollektivsymboliken.
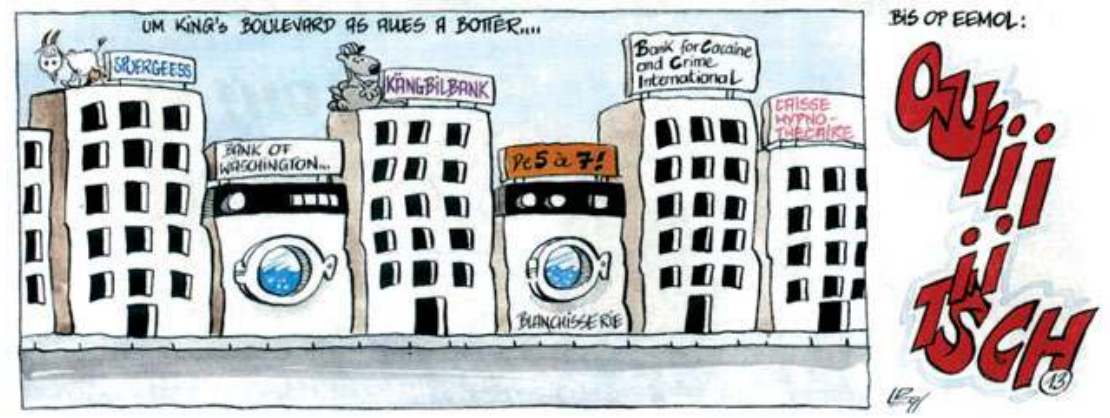

Abbildung 2: Die Banken am »King’s Boulevard«(Czuga/Leiner 1991: 15) (C) Editions Revue, Luxembourg

Aber nicht nur mit dem Bildwitz plakatieren Czuga und Leiner ganz provokativ einen stets von offizieller Seite zurückgewiesenen Charakter des Bankenplatzes, der aber andererseits in der ausländischen Presse Luxemburg immer wieder zugeschrieben wird: so heißt ein »Luxusbuerger« Finanzinstitut »Bank for Cocaine and Crime International«. Die Herkunft des Geldes wird deutlich in Bezug zum organisierten Verbrechen gesetzt. Die Abkürzung BCCI stellt die Verbindung her zu dem realen Finanzskandal der »Bank of Credit and Commerce International« (BCCI), der $1991 \mathrm{im}$ Land hohe Wellen schlug, weil auch die luxemburgische Filiale der BCCI in kriminelle Machenschaften verwickelt war. Die Silhouette von Finanzinstituten auf dem Kirchberg in dem Band »Alarm am Öro Zuch« zeigt, dass gerade die Staaten, die den Luxemburger Bankplatz als >Steuerinsel< verurteilen, mit ihren Nationalbanken präsent sind, um in der >Oase< auch Gewinne mit Steuerflüchtigen aus den eigenen Herkunftsländern zu machen: Die übliche Wortspielerei und die Verballhornung der Namen wie »Deutsche Dash Bank«, »Whyte and Wash Bank« oder »Blanchibas« lässt klar erkennen, welche Institute gemeint sind. 
Der konnotative Bezug zum Waschvorgang ist nicht zu übersehen. Ganz konkret werden auch Beispiele für die Kleinklientel, die die internationale Presse gern als stereotype Steuerhinterzieher/-innen identifiziert, gegeben: Zahnärzte/-innen aus Deutschland, belgische Notare/-innen und Viehhändler/-innen sowie französische Politiker/-innen. (Czuga/Leiner 2001: 9)

Symbolisierungen wie >Steueroase $<$ und >Geldwäsche $<$ schaden natürlich dem erwünscht seriösen Bild des Finanzplatzes. Seit dem BCCI-Skandal versucht die luxemburger Bankenaufsicht im Auftrag der jeweiligen Regierungen dubiose Finanzinstitute vom Bankplatz fernzuhalten. Die offiziellen Stellen arbeiten kontinuierlich daran, dass das Negativbild der Geldwäsche nicht mehr mit Luxemburg in Verbindung gebracht wird. Auf dem Höhepunkt der Finanzkrise im Herbst 2008 reagierte Premierminister Juncker im französischen Fernsehen sehr ungehalten auf das Bild des Weißwaschens, als man vor dem Interview mit ihm überraschend einen satirischen Film zeigte: Ein schwarzer Koffer wird in eine Waschmaschine gelegt und als weißer Koffer wieder herausgeholt. ${ }^{39}$ Zur selben Zeit wehrte sich die luxemburger Diplomatie heftig dagegen, dass das Land auf der grauen OECD-Liste der Steueroasen geführt wurde. Dementsprechend legt sich dann auch ein >Grauschleier < über »Luxusbuerg«. (Czuga/Leiner 2009: 5) Das aus der Waschmittelwerbung entlehnte Bild verweist wiederum gezielt auf Wäsche. Zwar erreichte Luxemburg die Entfernung von der grauen Liste relativ schnell, aber es bleibt die Frage, ob das Land, nachdem es als Vorleistung dem steuerlichen Informationsaustausch mit einigen Schlüsselstaaten zugestimmt hat, trotz des aufgeweichten Bankgeheimnisses, weiterhin so interessant für ausländische Kunden bleibt. Ein massiver Arbeitsabbau und Steuerausfall bei den Banken hätten eine starke reduzierende Wirkung auf den Wohlstand der luxemburger Wohnbevölkerung. Im zweiten Jahr der weltweiten Finanzkrise thematisiert der Superjhemp-Band von 2009 diese Zusammenhänge. Der ironische Titel »Cräsh am Paradäis« bezieht sich nicht nur auf das Bild des >Steuerparadieses $<$, sondern auch auf die in manchen Presseartikeln zugeschriebenen scheinbar paradiesischen Lebensumstände in Luxemburg.

Das Thema der Gefahr für den Bankenplatz taucht immer wieder in den einzelnen Geschichten auf. So wurde schon im ersten Band »De Superjhemp géint de Bommeléer« (Czuga/Leiner 1988) die damals noch rätselhafte Finalität der Serie von Bombenexplosionen in Luxemburg damit erklärt, dass der konkurrierende Finanzplatz des Fürstentums »Monastein« ein Klima der Unsicherheit in »Luxusbuerg« verbreiten wollte, damit die Banken mit ihren Kunden/-innen nach »Monastein« abwandern. Die Namensklitterung aus den beiden ersten Silben von Monaco und der letzten von Liechtenstein ist ein gezielt hintergründiges Konstrukt der Autoren. In »D’Patte wech vum Luxonit« gestehen die angeheuerten Verbrecher, dass sie im Auftrag des Fürstentums »Monastein« das »Luxonit« gestohlen haben,

39 | 21.10.2008: Le journal télévisé de 20 heures, France 2. URL: www.youtube.com/wat $c h ? v=Y w F h C d z n V w M \& f e a t u r e=$ related $(25.10 .2008)$ 
um die Identität von »Luxusbuerg« mitsamt Bankenplatz zu zerstören. (Czuga/ Leiner 1998b: 47)

Der drohende Verlust der Einnahmen des Finanzplatzes war auch 1991 ein Zentralthema im »Dossier Hexemeeschter«: Das ausländische Geld in den ansässigen Banken verschwindet plötzlich und unerklärlich. Der Sparkassendirektor aus »Luxusbuerg « bringt die Sache auf den Punkt, wenn er sagt: »Wann ons auslännesch Clienten dat gewuer gin, dann as et Pilo mat eiser Finanzplaz. «4 ${ }^{\circ}$ (Czuga Leiner 1991: 17) Die Autoren thematisieren hier die Abhängigkeit eines prekären Wohlstands von der Existenz des internationalen Bankplatzes. Die Dauerhaftigkeit der hohen Steuereinnahmen auf Benzin, Spirituosen und Tabakwaren unterliegt der gleichen Unsicherheit. Und weil das auch in Luxemburg von den Pressemedien öfters problematisiert wird, kann man annehmen, dass zahlreiche Luxemburger/-innen sich sowohl der ungewissen Zeitlichkeit dieser Einnahmen wie auch der Konsequenzen, die das Versiegen dieser Quellen für das Land hätte, bewusst sind. Mit der Aussage des Sparkassendirektors zeigen die Autoren unterschwellig eine verdrängte, aber latente Furcht vieler Luxemburger/-innen auf.

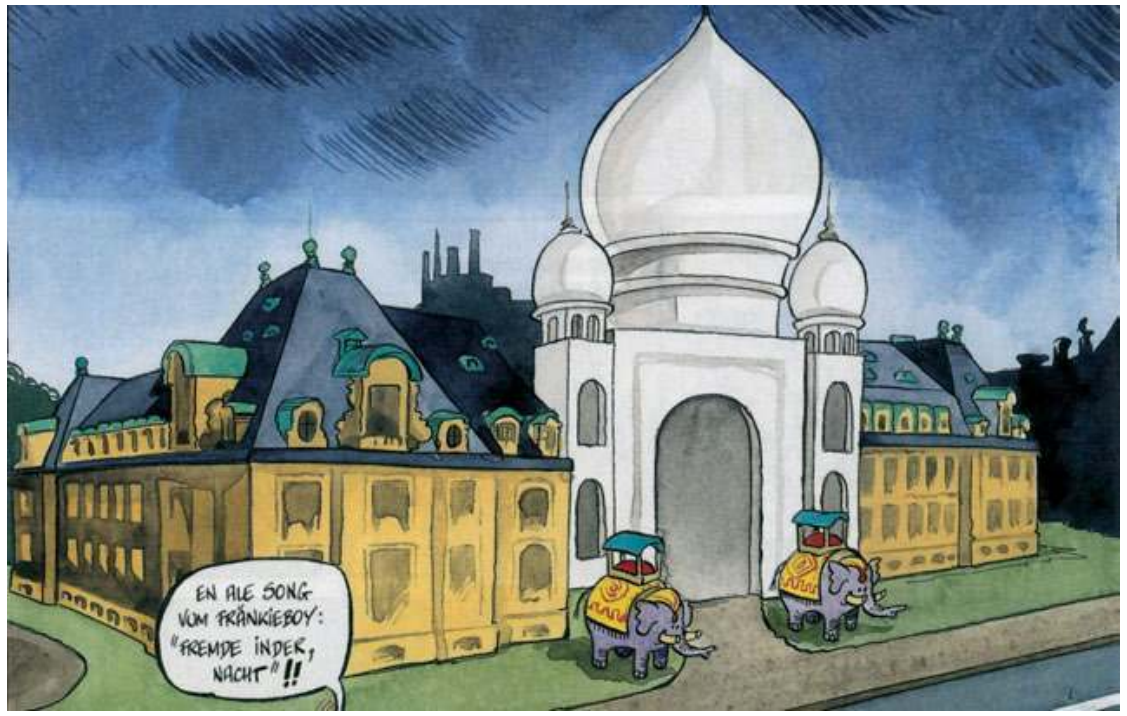

Abbildung 3: Das ehemalige Arcelor-Gebäude wird zum »Taj Mittal« (Czuga/Leiner 2006: 20). (C) Editions Revue, Luxembourg

Verlustängste, die die einheimische Stahlindustrie anbelangt, wurden auch in großen Teilen der Bevölkerung Luxemburgs ausgelöst, als 2006 die vom indischen Stahlmagnaten Lakshmi Mittal förmlich erzwungene Fusion von Arcelor mit sei-

40 | Deutsch: "Wenn unsere ausländischen Kunden das erfahren, dann ist es vorbei mit unserem Finanzplatz." 
ner eigenen Gesellschaft »Mittal Steel« über die Bühne ging. ${ }^{41}$ Mit ihrer ikonischen Darstellung zeigen Czuga und Leiner mit karikierenden Mitteln, dass im Zeitalter der Globalisierung traditionelle Identität in »Luxusbuerg « verloren geht und durch eine >fremde< ersetzt wird: So stülpt sich über die neoklassisch historisierende Architektur des ehemaligen Arcelor beziehungsweise Arbed- Sitzes ein monumentales Eingangstor im indischen Baustil, das an das Taj Mahal erinnert.

Das Gebäude, das wegen seines Verweises auf die Entstehung der luxemburger Stahlindustrie, den Charakter eines kollektiven Symbols für viele Einheimische hat, wird sarkastisch in »Taj Mittal« umbenannt. (Czuga/Leiner 2006: 20) Gleichsam als visionäre Vorwegnahme der vorgenannten Fusion, kauft in der SuperjhempEpisode von 2005 ein Diktator aus Zentralasien ganz »Luxusbuerg« auf dem Weltmarkt auf und tauft das Land - als geschützte Warenmarke - in »Kachkéisien« um. (Czuga/Leiner 2005: 23) Die Superjhemp-Saga verweist metaphorisch auf die Quellen des Wohlstands der Luxemburger/-innen und gleichzeitig auf Gefahren, die ihn kurzfristig vernichten können.

In der Auftragsarbeit, die der britische Fotograf Martin Parr für das Kulturjahr $2007^{42}$ verwirklichte, wird der bedingte Luxus der Einwohner/-innen Luxemburgs scheinbar ethnografisch dokumentiert. Genau wie die Autoren von Superjhemp hält er der luxemburger Wohnbevölkerung einen gewissen Spiegel vor. Das hohe Einkommensniveau und ostentative Konsumverhalten werden oft als >typisch luxemburgisch $<$ angesehen. Zumindest das erste Charakteristikum ist statistisch belegbar. Laut einer Studie von Eurostat von 2009 sind die Einwohner/-innen Luxemburgs die reichsten in der EU. (Weltonline 2009)

\section{Ein Land von Staatsbediensteten?}

Ein weiteres Stereotyp betrifft die Staatsbeamtenschaft: Czuga und Leiner bedienen ganz offensichtlich Vorurteile in der Bevölkerung, wenn sie den Vertretern/-innen des öffentlichen Dienstes Identitäten zuschreiben, die diese eigentlich abhalten müssten, Superjhemp zu lesen: Staatsbeamte/-innen seien faul und bestechlich - sie würden ihre private Briefmarkensammlung im Büro sortieren und ihre Arbeitszeit verschlafen. Die klischeehafte Negativdarstellung von beamteten Staatsdienern/-innen in Texten und Bildern ist eine gängige kabarettistische Praxis, die man öfters in den westlichen Demokratien findet.

Die quantitativen Erhebungen haben ergeben, dass unter allen Berufsgruppen Superjhemp bei den Beamten/-innen des öffentlichen Dienstes mit $78 \%$ die größte Leserschaft findet. Eine weitere rezente Studie (Pigeron-Piroth 2009: 3) stellt fest, dass die Zahl der Luxemburger/-innen, die im öffentlichen Dienst beschäftigt sind, mit 42,3 \% (Stand: März 2008) unverhältnismäßig groß ist, wenn man sie in Beziehung zu sämtlichen Arbeitsstellen des Landes setzt. Zieht man im Super-

41 | Vgl. Abschnitt 6.2.

42 | Vgl. Abschnitt 6.5. 
jhemp den Beruf der meisten Protagonisten in Betracht, dann geht die Gleichung fast auf: »Luxusbuerger/-in« ist gleich Staatsbedienstete/r.

Charel Kuddel arbeitet im »Ministerium für ungelöste Fälle« in der Abteilung für hoffnungslose Angelegenheiten. Er ist ein Staatsbeamter, der es sich problemlos leisten kann, oft an seiner Arbeitsstelle mit Abwesenheit zu glänzen, weil er in seiner verheimlichten Tätigkeit als Megaheld permanent Land und Leute retten muss. Superjhemp hat also eine verdeckte Persönlichkeit und unterscheidet sich in dieser Hinsicht nicht von dem generellen Identitätsmuster traditioneller amerikanischer Comicsuperhelden (vgl. Superman, Spiderman, Batman). Die zweite Silbe von Superjhemp leitet sich von dem Luxemburger Spitznamen »Jhempi « für Gendarm ab. Er ist also eine Art geheimer Superpolizist für Ausnahmefälle.

Leitende Staatsbeamte haben in »Luxusbuerg « eine Vetternwirtschaft aufgebaut: In diesem Kontext werden verschiedene Skandale, die in der luxemburger Presse angeprangert wurden, mit beißendem Humor karikiert. Es gibt allerdings einige Kriminalbeamte - Freunde Superjhemps - die in Gefahrensituationen über sich hinauswachsen und somit die Ehre ihrer Zunft retten; die Botschaft ist, dass sie trotzdem irgendwie unentbehrlich für das Land sind. Bei diesen Aktionen geht es meistens darum, bestimmte Interessen der Nation und der Dynastie zu verteidigen oder sogar »Luxusbuerg« vor dem Untergang zu bewahren. Superjhemp ist ein Kämpfer gegen so manche drohende Identitätsverluste und die damit zusammenhängenden materiellen Bedingungen.

Damit bestätigt die Serie auf inhaltlicher Ebene das Genre des >Comicsuperheldenepos <, in dem es darum geht moralische, politische, ökonomische oder gesellschaftliche Konstrukte zu erhalten und durch die Handlungen des Superhelden zu bestätigen. (Ditschke/Anhut 2009: 156) Die Autoren von Superjhemp setzen sich jedoch wesentlich von den Vorbildern aus den USA (z.B. Superman, Spiderman) ab, indem sie mit Karikatur und Parodie kritisch Stellung zu gesellschaftlichen Gegebenheiten beziehen. Der Diskurs ist in einem gewissen Sinne paradox, weil die Hauptfigur Superjhemp für dieselben Konstrukte einsteht und sie nicht infrage stellt. Er ist der treue Staatsbeamte par excellence, der dient und Autorität nicht hinterfragt. Hier wird ein Bild des Durchschnittsluxemburgers aufgestellt, der im normalen Leben ein Biedermann (Charel Kuddel) ist und nur in seinen Träumen zu visionären Höhenflügen ansetzt.

\section{Eine pointierte Darstellung der Einheimischen}

Das einzig rebellenhafte an Superjhemp ist seine lustvolle Praxis nicht durch die Tür einzufliegen, sondern durch das Fenster, welches zum größten Ärgernis seiner Vorgesetzten jedes Mal in Scherben geht. Der Superhero fällt also nicht mit der Tür, sondern plump mit dem Fenster ins Haus. Auch durch sein Äußerliches hat Superjhemp gewisse antiheldenhafte Züge. Er ist kein Athlet, sondern eher korpulent. Weil er nicht Supermans stromlinienförmige Adonis-Figur hat, wirkt die obligate Strumpfhose des Superhelden etwas lächerlich. Die lustige Knollennase 
und die drollige Figur lassen sich aus dem Kindchenschema ableiten, sie sollen Sympathien bei den Lesern/-innen wecken. Sowohl die Mütze wie auch sporadisch der Umhang haben bei Superjhemp ein Karomuster und sind in den nationalen Landesfarben (rot/weiß/blau) gehalten. Ähnliche Mützen in neutralen Farben werden oft von älteren Männern in Luxemburg getragen. Das Karo-Cape erinnert stark an ein Küchentischtuch. Charel Kuddel zieht bevorzugt - wie auch einige seiner Staatsbeamtenkollegen - eine karierte Weste an - manchmal ist es auch eine Hose mit Karomuster. Da Superhelden eine Vorbild- und Stellvertreterfunktion haben, kann man sich fragen, ob nicht über den Bildwitz von den Autoren den »Luxusbuergern « signalhaft eine gewisse Kleinkariertheit zugeschrieben wird. Dafür spricht, dass Superjhemp es auch privat eher geregelt und gemütlich mag. Abends strebt er einen ruhigen Fernsehabend mit einem kühlen Glas »Uelzechtbéier «43 an. Nachts schläft er in Bettwäsche, die dem Muster der Nationalflagge entspricht. Da Superhelden der Allgemeinheit gehören, haben sie höchstens eine unerfüllte platonische Liebe zu einer Freundin (vgl. Superman, Obelix) oder sind gänzlich asexuelle Wesen (vgl. Asterix, Tintin). Sie haben aber keinesfalls, wie Charel Kuddel, eine Frau und mehrere Kinder. »Luxusbuerger« Megahelden ticken eben anders: Sie schätzen in ihrer Freizeit den diskreten Charme eines bürgerlich beschaulichen Familiendaseins.

Die Zuschreibung der kleinbürgerlichen Idylle findet man auch in der bereits erwähnten Fotodokumentation von Martin Parr. Die Objekte, die dafür stehen, z.B. Wohnhäuser in einem nur scheinbar differenzierenden Einheitsstil, nennt er den »eitlen Stolz« (»vanities«) der Mittelklasse. Die Kleinbürgerlichkeit, die sowohl die Superjhemp-Autoren wie auch Martin Parr dem sogenannten Durchschnittsluxemburger zuschreiben, beruht natürlich auf deren subjektiver Sicht der Dinge und ist nicht frei von Klischees. Man könnte meinen, dass die Fotos von Martin Parr objektive ethnografische Beweisstücke sind. Dem ist aber nicht so, weil Parr seine Motive nach dem Kriterium der »Spießbürgerlichkeit« aussucht, teilweise inszeniert und mit den künstlerisch-formalen Möglichkeiten der Fotografie - wie Bildeinstellung, Schärfebereich und Herausstellen von Details - subjektiv beeinflusst.

In der Literatur werden Kleinbürger/-innen u.a. als Konformisten, die enggeistig, weltverschlossen und auf soziale Sicherheit bedacht sind, beschrieben. Die Thematisierung der Figur des Spießers zieht sich durch die ganze europäische Literatur und ist international ein willkommenes Sujet für Kabarettisten/-innen. In ihrer Forschung zum europäischen Kleinbürgertum stellen Haupt und Crossik die Frage, ob angesichts der empirischen Heterogenität noch von einer einheitlichen Klasse oder Sozialgruppe gesprochen werden kann. (Vgl. Haupt/Crossik 1998) Sie zeigen, dass man dem negativ konnotierten Kleinbürgertum auch positive Qualitä-

43 | Bier, das nach dem Fluss "Alzette" ("Uelzecht") benannt ist. Hier wird von den Autoren eine Analogie zu Mousel Bier konstruiert, dessen Name rein klanglich auf die luxemburgische Bezeichnung 'Musel، für den Fluss Mosel verweist. 
ten abgewinnen kann. Spießbürgerliches, so wie es als Stereotyp in der Belletristik auftritt, kann man in allen Gesellschaftsgruppen identifizieren. Im Superjhemp wird Unternehmern/-innen, Künstlern/-innen und auch Intellektuellen der Akademikerlaufbahn kleinbürgerliches Verhalten zugeschrieben. Es werden sozusagen alle sozialen Milieus auf die Schippe genommen. Mit einem sarkastischen Blick werden auch Mittel der Distinktion, die von den verschiedenen Gruppen eingesetzt werden, um sich kulturell und wirtschaftlich abzugrenzen, karikiert. Gleich einer narrativen Metalepse ziehen sich die Autoren selber mit ein, indem Sie in einigen Episoden den Fluss der Erzählung unterbrechen und sich in Wort und Bild selbstironisch inszenieren.

Aufgrund bestehender Selbst- und Fremdbilder schreiben Czuga und Leiner den Luxemburgern/-innen Identitäten zu, die z.T. ihre eigenen Projektionen sind: so wie sie die Einheimischen subjektiv wahrnehmen oder manchmal auch gerne sehen möchten, damit die Karikatur voll zum Tragen kommt. Die Persiflage lebt bevorzugt von zugeschriebenen Stereotypen - bzw. Klischees - und von als kurios empfundenen Aneignungen. Durch die gezielt hintergründige Umdeutung kommt es zu einer teilweisen Re-Kontextualisierung von Selbst- und Fremdbildern im Konstrukt »Luxusbuerg « und dementsprechend zu einer Hinterfragung von Identitäten, die Luxemburg betreffen. Die Groteske dient dabei als dekonstruktives Instrument. Schließlich erzeugt die konzentrierte Anhäufung von allen möglichen angeeigneten und zugeschriebenen Identitäten einen Diskurs, der sich gleichsam ins Unendliche reflektiert und damit auf den Spiegel im Spiegelcharakter der Superjhemp-Saga verweist. Dass die Reflexionsfläche dabei zu einem Zerrspiegel wird, der sogenannte >luxemburger Identitäten « grotesk deformiert, liegt in den dekonstruierenden Absichten der beiden Comic-Autoren.

\subsection{KolleKtivsymbole UNd (NEUE) IDENTItÄTSANGEBote IN DER LUXEMBURGISCHEN WERBUNG}

Bilder und Kollektivsymbole spielen ebenfalls eine wichtige Rolle in der Werbung, ein Interdiskurs, der aus unserem Alltag nicht mehr wegzudenken ist. Werbung wird von unterschiedlichen Akteuren/-innen eingesetzt, um verschiedene Verkaufsziele zu erreichen und Images zu erzeugen. Dazu werden bestimmte sprachliche und visuelle Bilder aufgegriffen, umgedeutet oder neu aufgeladen. Da Menschen sich der dargebotenen Informationen teils bewusst bedienen, um Wissenslücken $\mathrm{zu}$ schließen, sich $\mathrm{zu}$ orientieren und sich letztendlich in der (Konsum-)Welt zu positionieren, soll im Folgenden untersucht werden, inwieweit der Interdiskurs >Werbung< kommunikativ wirkendes Identitätspotential birgt. Von besonderem Interesse ist jene Werbung bzw. Produktkommunikation, die speziell mit Blick auf Luxemburg produziert wurde. Die darin verwendeten Bilder können die Erzeugung einer spezifischen Vorstellung einer >Luxemburger Identität< beeinflussen. Werbung, als eine Form medialer Inhalte, hält »Attribute 
für die Konstitution von Identität« (Krotz 2003: 41) bereit. Sie werden im Sinne eines Zuschreibungsprozesses reflektiert und zielgerichtet erarbeitet. Wenn die Werbung die Konsumenten/-innen erreicht, dann besteht die Möglichkeit, dass diese sie aufnehmen, verstehen und akzeptieren und sich die Diskurspositionen aneignen. In einem weiteren Schritt kann dies zu Identitätskonstruktionen beitragen.

Zunächst muss Werbung aber produziert und somit auch ein kommunikativ wirkendes Identitätspotential geschaffen werden. Für die Kreativen der Werbebranche ist die fortschreitende Entstandardisierung von Lebensläufen eine große Herausforderung. Massenmarketing erweist sich mehr und mehr als ungeeignete Basisstrategie. Zunehmend wird das Funktionieren traditioneller Werbemuster infrage gestellt. Die werbekommunikationsrelevante Problematik einer diversifizierten Gesellschaft erscheint in Luxemburg zudem im Licht einer äußerst multikulturellen und multilingualen Prägung. Ohnehin schon kleine Zielgruppen sind daher noch schwieriger zu erreichen, wenn man es mit unterschiedlichen kulturellen Codes und vielfältigen Möglichkeiten bei der Sprachwahl in Werbekommunikaten zu tun hat. Die Frage ist nun, ob und wie in der Luxemburger Werbung mit einer spezifischen luxemburgischen Bildlichkeit gearbeitet wird, die als Kollektivsymbolik zu identifizieren ist. Werbung versucht durch die diskursive Verarbeitung von Kollektivsymbolen die Grenze zwischen Produkt und Konsumenten zu überwinden. Im Idealfall vereint die Symbolik sowohl das Produkt als auch die Konsumenten/-innen im Sinne eines >assoziativen Koppelpunktes`44. In bestimmten Fällen wird dabei der nationale Raum als einheitlich imaginiert, indem beispielsweise immer wieder die gleichen bekannten Landschaften und Städteansichten oder auch Traditionen wie Volksfeste und religiöse Feste zu Werbezwecken herangezogen werden. Gerade in Luxemburg stellt sich die homogene Darstellung des nationalen Raumes als schwieriges Unterfangen dar, da die angesprochenen Konsumenten/innen sehr heterogen sind, bedingt u.a. durch das ausgeprägte Grenzgängerwesen ${ }^{45}$ und den hohen Anteil von Personen mit Migrationshintergrund ${ }^{46} .47$ Die Heterogenität zeigt sich auch im konkreten Ausleben von Konsummustern, in denen sich angeeignete Identitäten z.T. manifestieren. Anhand von Beispielen aus der Finanz- und Telekommunikationsbranche sowie dem Kulturhauptstadtjahr »Luxemburg 2007« soll im Folgenden exemplarisch verdeutlicht werden, wie luxem-

44 | Das Symbol fungiert dann als rassoziativer Koppelpunkt, wenn sowohl das beworbene Produkt an das Symbol anschlussfähig ist, als auch die anvisierten Konsumenten/innen sich mit dem Symbol assoziieren können.

45 | Vgl. Ausführungen zu Grenzgängern in Abschnitt 7.5.

46 | Vgl. Abschnitt 6.6.

47 | Entscheidend sind die zahlenmäßigen Verhältnisse: 493.500 Einwohner/-innen (01.01. 2009) bei einem Ausländeranteil von 43,7 \%, wobei die Portugiesen/-innen mit etwa 80.000 Personen den höchsten Anteil haben. Zudem gehen jeden Tag 147.599 Grenzgänger/-innen ihrer Arbeit im Großherzogtum nach. (Vgl. www.statec.lu [02.12.2009]) 
burgische Werbung als Interdiskurs mit Identitätsstiftungspotential funktionieren kann.

\section{Werbebeispiele aus der Finanz- und Telekommunikationsbranche}

Auf Unternehmensseite beziehungsweise Auftraggeberseite ist der Werbediskurs in Luxemburg geprägt von einem Mix aus staatlichen Institutionen und Ministerien, internationalen Großkonzernen, nationalen Unternehmen und lokalen Betrieben. Sie unterscheiden sich hauptsächlich im für Werbemaßnahmen veranschlagten Budget, in der Nationalitätenstruktur der Mitarbeiter/-innen, die sich in Vorlieben für gewisse Kommunikationsstile und in der Sprachwahl niederschlägt, sowie in der Konkurrenzsituation, da man sich je nach Größe des Unternehmens auf den nationalen Markt beschränkt oder auch darüber hinaus agiert. Der Luxemburger Markt als solcher ist sehr klein und dementsprechend in einigen Branchen hart umkämpft. In der qualitativen Befragung der Luxemburger Wohnbevölkerung zeigt sich, dass einige Unternehmen (mehrheitlich luxemburgische Firmen) und deren Marken Symbol für Luxemburg bzw. die Luxemburger Wirtschaft sind, und auf diesem Weg auch kollektiv verarbeitet werden. Die Unternehmen bzw. die Marken und deren Werbung werden als >typisch < für Luxemburg und für Luxemburger Werbung genannt. Diese Nennungen kann man im Sinne einer identitären Aneignung verstehen. Begründet werden sie häufig mit persönlichen Vorlieben, der landesspezifischen traditionellen Verankerung und auch der werbetechnischen Präsenz. In den Interviews wurde beispielweise die nationale Sparkasse Banque et Caisse d'Epargne de l'Etat (BCEE) genannt. Sie ist seit über 150 Jahren in Luxemburg präsent und verkörpert inzwischen die Luxemburger Bank schlechthin. Durch ihre Volksnähe konnte sie ein Image des Vertrauens aufbauen, von dem sie besonders in der Finanzkrise im Jahr 2008 profitierte. Ihr Hauptsitz in Luxemburg-Stadt wird aufgrund seines prunkvollen Äußeren und des hohen Turms von Touristen/-innen nicht selten fälschlicherweise für den großherzoglichen Palast gehalten. Durch die Integration des Bauwerks in das Unternehmenslogo unterstreicht die Bank seit Jahrzehnten ihre »Verankerung in Geschichte und Gesellschaft Luxemburgs« (Jungblut 2007: 290). Damit gehört sie zu den wenigen Finanzinstituten, die durch ihre Kommunikation den Finanzplatz Luxemburg eindeutig markieren. »Seule la BCEE perdure et laisse une trace visible, tant architecturale (par son bâtiment plateau Bourbon) qu'à travers son logo (le même bâtiment avec le pont Adolphe). $\ll^{8}$ (Auxenfants 2007: 230)

48 | Deutsch: "Nur die BCEE besteht fort und hinterlässt eine sichtbare Spur, architektonisch (durch ihr Gebäude auf dem Plateau Bourbon) wie auch durch ihr Logo (dasselbe Gebäude mit der Adolphe-Brücke)." 


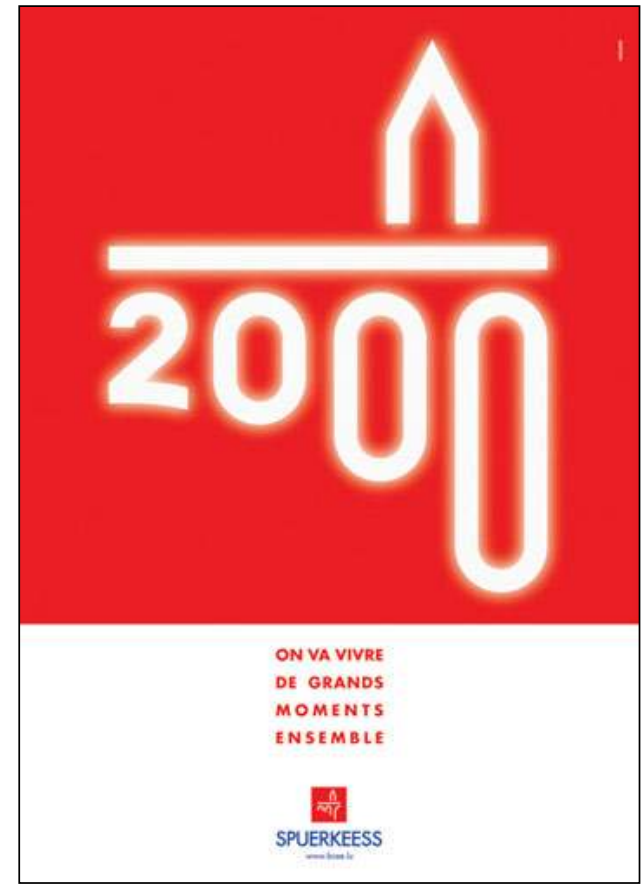

Abbildung 4: Werbung der BCEE (Mikado, Luxembourg). Abwandlung des klassischen BCEE-Logos anlässlich des Jahreswechsels $1999 / 2000$

Neben den einheimischen Sparer/-innen und Geldanleger/-innen sind aber auch die Grenzgänger/-innen wichtige Kunden/-innen für die Banken. Sie werden mit speziellen Grenzgängerangeboten umworben, die auf die persönlichen Bedürfnisse und gesetzlichen Rahmenbedingungen zugeschnitten sind. Vergleichbar mit dem Slogan »Wir machen den Weg frei« der deutschen Volks- und Raiffeisenbanken werben luxemburgische Institute damit, grenzbedingte Hindernisse aus dem Weg zu räumen und grenzüberschreitende Bankdienstleistungen anzubieten. In der Werbeanzeige der inzwischen mit der BNP Paribas fusionierten Banque Générale du Luxembourg (BGL) werden gleich mehrere Symbole integriert: die Nationalfarben, wobei Rot hier als roter Teppich fungiert, den typischen Geschäftsmann und den Torbogen, der zu beiden Seiten durchlässig ist. Auch hier wird der Weg frei gemacht, werden Grenzen aufgehoben, wobei es nicht nur um finanzielle, sondern auch um physische Grenzen geht. Die neue BGL BNP Parisbas (seit 21.09.2009) verbindet laut eigener Pressemitteilung in ihrem Namen »die tiefe nationale Verwurzelung von BGL als auch [...] die Solidität und das internationale 
Renommee von BNP Parisbas« und unterstreicht ihre zeitgemäße Aufstellung im Slogan »Die Bank für eine Welt im Wandel«49.

Auch in anderen Dienstleistungsbereichen wird mit Kollektivsymbolen gearbeitet, die luxemburgische Spezifika aufgreifen. Der Mobilfunkanbieter LuxGSM bewarb sein Roaming-Angebot mittels des Kollektivsymbols >Brückeく. Die Pont Adolphe wird als nationales Pendant zur Brücke des 25. April (Ponte 25 de Abril) in Portugal $^{50}$ dargestellt und ist gleichzeitig deren lineare Ergänzung.

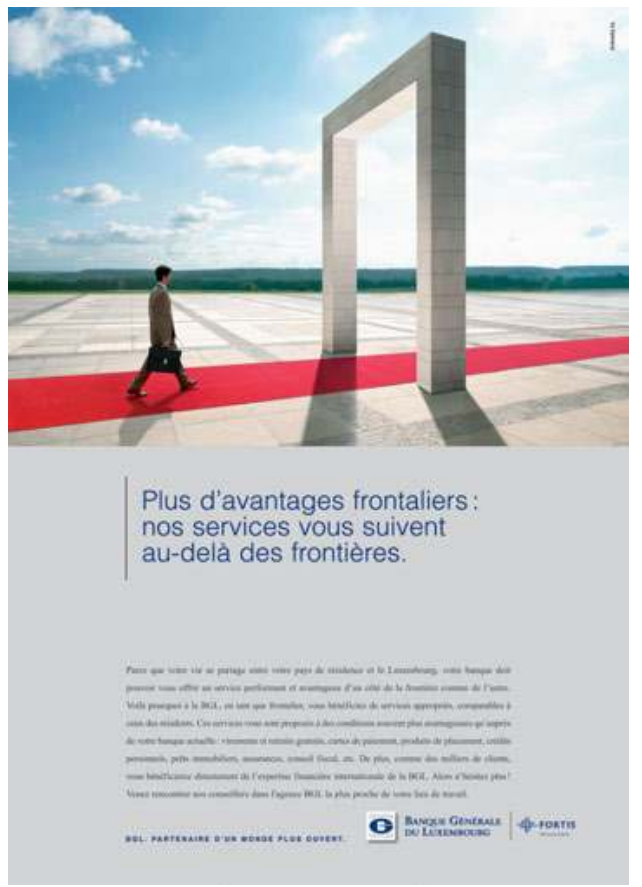

Abbildung 5: Werbung der BGL

(Mikado, Luxembourg)

49 | Pressemitteilung "Aus BGL wird 'BGL BNP Parisbas`“, Luxembourg, 21.09.2009. URL: www.bgl.lu/de/bank/corporate/die-bank/bgl-bnp-paribas/pressemitteilungen.htm (02.11.09)

50 | Die Brücke verbindet Almada mit Lissabon. Charakteristisch ist die im Hintergrund allerdings schwer - zu erkennende "Christo-Rei-Statue". Bei flüchtiger Betrachtung könnte man die Brücke auch mit der Golden Gate Bridge verwechseln. Es handelt sich daher um eine Art doppelter Bedeutung: Wird die spezifische nationale Codierung (Luxemburg/Portugal) erkannt, so entwickelt die Anzeige über die allgemein symbolische Bedeutung der Brücke hinaus noch einen potentiellen Mehrwert im national-identifikatorischen Sinne. Fungiert sie jedoch ,nur als Golden Gate Bridge oder namenlose Brücke, ist sie ein allgemeines Symbol für Verbindungen. 


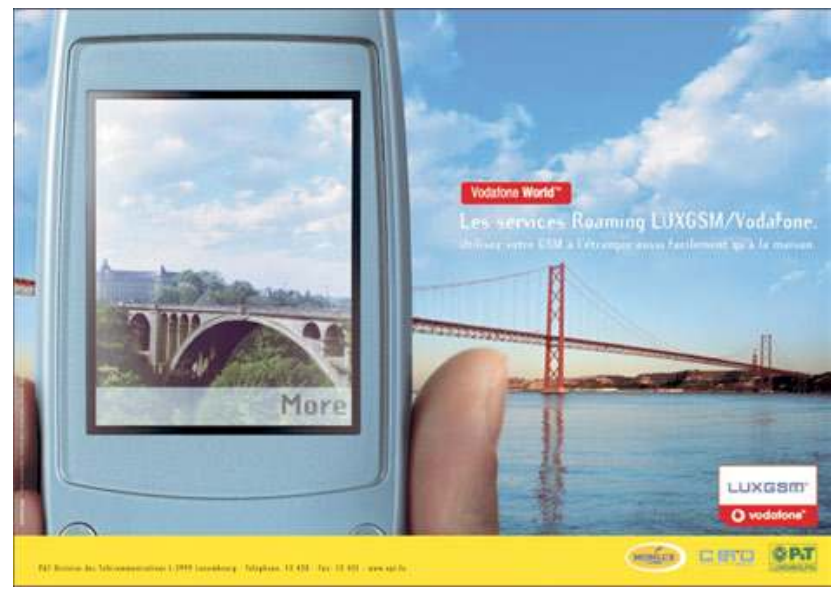

Abbildung 6: Werbung für LuxGSM/Vodafone

(Advantage, Luxembourg)

Die Brücke als Symbol für Verbindung schafft sowohl den Link zum Produkt als auch den Link zu den Konsumenten/-innen, in diesem Fall mit Blick auf die in Luxemburg lebenden Portugiesen/-innen. Die Abbildung des Bauwerks setzt auf dessen Kenntnis seitens der Konsumenten/-innen und die daraus resultierende Schaffung einer positiven Wahrnehmungsatmosphäre. Die Brücke wie auch die mobile Telekommunikation stellen eine Verbindung zwischen zwei Orten her. Auch ohne nationenspezifische Kenntnis der Brücken lässt sich die Anzeige verstehen, was die kollektivsymbolische Funktion der Brücke unterstreicht.

\section{Kommunikation und Markierung im Kulturhauptstadtjahr "Luxemburg 2007"}

Anhand der Analyse eines weiteren Beispiels soll gezeigt werden, wie Luxemburg grafisch und textlich repräsentiert wird. Das Kulturhauptstadtjahr »Luxemburg 2007《 wurde im Großherzogtum und der umgebenden Großregion veranstaltet. Aus Sicht der Marketingkommunikation und speziell der Werbung für dieses Ereignis kam es auch darauf an, Luxemburg im Kontext der Großregion im Sinne eines Zuschreibungsprozesses zu markieren. »The Greater Region, laboratory of Europe« war das verbindende Leitmotiv von »Luxemburg 2007« und gleichzeitig auch Hinweis auf alle damit verbundenen Probleme bei Konzeption und Realisation. (Vgl. Luxembourg 2007: 4-8) Das Bild des europäischen Labors lässt sich daher auch metaphorisch in vielen Bereichen des Kulturhauptstadtjahres verwenden und wurde zum verbindenden Kollektivsymbol für die Großregion. Zusätzlich zu üblichen Schwierigkeiten, die bei derartigen Großveranstaltungen auftreten können, sahen die Veranstalter/-innen das Einbinden von vier Ländern, fünf Regionen und drei Sprachen als große Herausforderung. »In these circumstances, the 
normal process of creating a coherent and accepted corporate identity became a major issue.« (Luxembourg 2007: 53) Die Konstruktion einer neuen (groß-)regionalen >Identität< und der >kulturellen Identität< der Region war ein Hauptziel der Marketing- und Kommunikationsarbeiten. Diese beruhten auf einer übergeordneten Strategie, die allerdings nicht losgelöst von den jeweiligen Inhalten, also den einzelnen Veranstaltungen und Aktionen, existierte. In den Augen der Organisatoren/-innen war »das Projekt [dennoch] nicht nur ein Kulturfestival, sondern auch eine Standortpositionierung für die Großregion im europäischen Wettbewerb« (Garcia 2004: 5). Dabei sollte die Position Luxemburgs als >Motor<von »Luxemburg 2007« herausgestellt werden. (Vgl. Luxembourg 2007: 53) Für Luxemburg speziell wollte man in der Kommunikation erreichen, die Außenwahrnehmung des Großherzogtums über die Stereotypen >Finanzzentrum<, >Banken<, >Juncker< und >Benzin< hinausgehen zu lassen. »Like the citizens of every capital, Luxembourgers sometimes tend to think they are the hub of the universe. Luxembourg may be strong on the financial and political map, but a fairly improbable cultural epicenter.« (Luxembourg 2007: 100) Von Anfang an war klar, dass eine Imageänderung ein langwieriger Prozess sei und nicht durch ein - wenn auch großes - Ereignis wie das Kulturhauptstadtjahr alleine erreicht werden könne. Auch wenn ein umfassender Imagewandel nicht realisiert wurde, so konnte zumindest die Richtung für zukünftige Entwicklungen angezeigt werden und Luxemburg konnte immerhin eine leichte Verbesserung hinsichtlich der Verbindung Kulturtourismus/Luxemburg verzeichnen. Zum Ergebnis der Pressekampagne heißt es: »For once, Luxembourg had international media coverage unrelated to banks or Mr. [sic!] Juncker.« (Luxembourg 2007: 77)

Als Logo und damit als visuelle Identität des Kulturhauptstadtjahres 2007 wählte man den >Blauen Hirsch`, der von der luxemburgischen Agentur Bizart im Rahmen des Logowettbewerbs vorgeschlagen wurde. Der Hirsch sei in der Lage, die verschiedenen Teile der Großregion zu verbinden, so die Begründung:

During the creation process, we tried to represent the impressive woods and large landscapes of the Greater Region, the nature which doesn't change from one country to the other. Of course there was this ironic touch in our graphical approach. Luxembourg is not London, Paris or New York. The blue deer represents in a very humble way the energy and the ambition of Luxembourg and the Greater Region to develop cultural life without ignoring the past of the terroir. ${ }^{51}$

Der Blaue Hirsch überrascht mit seiner außergewöhnlichen Farbe und überschreitet als Tier die Grenzen der Länder. Überraschung und Grenzüberschreitung zwei zentrale Elemente des Kulturhauptstadt-Konzeptes - wurden demnach in

51 | URL: www.bizart.lu (13.10.2009) 
einem Logo zusammengeführt. ${ }^{52}$ Die vielfältigen Diskussionen ${ }^{53}$ um die Wahl des Logos führten zu einer erhöhten Aufmerksamkeit für das Ereignis an sich, aber auch für dessen visuelles Identifikationsobjekt, den Hirsch. In Luxemburg konnte er sich dank vieler Banner, Poster und blauer Stahlskulpturen als Symbol von »Luxemburg 2007« etablieren. ${ }^{54}$

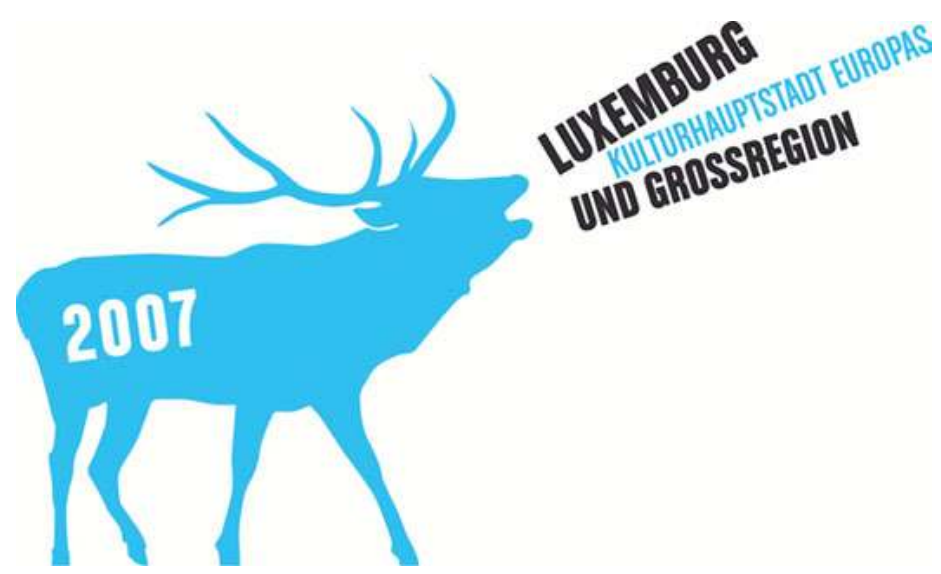

Abbildung 7: Der Blaue Hirsch, Logo des Kulturhauptstadtjahres Luxemburg 2007

Außerhalb Luxemburgs war die Wahrnehmung des Kulturhauptstadtjahres eher gering, was laut Abschlussbericht an mehreren Faktoren lag: der geografischen Ausdehnung, der hohen Anzahl an beteiligten Partnern/-innen, den drei Sprachen, die das Programm noch voller machten, als es ohnehin schon war, dem programmatischen Ansatz des Marketing, alles gleich behandeln zu wollen, 55 und der relativ späten Herausstellung von Leuchtturmprojekten (Luxembourg 2007: 65). Die Kommunikationsstrategie war vor allem mit Blick auf die Wahl der Medien spezifisch, da sie sich in erster Linie an das heimische Publikum richtete. Der Mediaplan, der ebenfalls von einer luxemburgischen Agentur entwickelt wurde,

52 | Vgl. www.granderegion.net/de/news/2005/03/20050311-2/index.html (16.12.2009) 53 | "The whole local press was scandalised and the audience just felt ridiculous to be identified by a primitive animal." URL: www.bizart.lu (13.10.2009)

$54 \mid$ "And then ... after a few month, people discovered the humour and the fun of the logo, shame turned into pride ... the blue deer which allowed 1000 and 1 interpretations became one of the strongest brands Luxembourg had ever seen. "URL: www.bizart.lu (20.02.2010) 55 | Eine derartige Gleichbehandlung wurde besonders dadurch erschwert, dass höchst unterschiedliche Veranstaltungstypen und -orte nur ansatzweise unter einem gemeinsamen konzeptionellen Dach integriert werden konnten. Abgesehen vom übergreifenden Konzept standen die Veranstaltungen in erster Linie für sich und erforderten ein je spezifisches Marketing. 
sah bei einem Budget von zwei Millionen Euro lediglich $10 \%$ für internationale Werbung vor. (Vgl. Luxembourg 2007: 54) Nicht zuletzt war dafür der Kostenfaktor >Mehrsprachigkeit< verantwortlich, da die meisten Publikationen in den drei Sprachen Deutsch, Französisch und Englisch veröffentlicht wurden. Daher flossen auch annähernd $70 \%$ der Media-Investitionen in den Printbereich. (Vgl. Luxembourg 2007: 56f.) In Luxemburg konnte man Tageszeitungen, Radio und TV am erfolgreichsten integrieren. Insgesamt wurden viele verschiedene Medien abgedeckt und viele verschiedene Kommunikationsformen genutzt.

Im PR-Bereich gab es zwei Hauptkampagnen, eine für Businesskunden/-innen und eine für das Privatpublikum. Für den Luxemburger Einzelhandel wurde die Kampagne »We support 2007 « initiiert, die insbesondere auf den Tourismussektor abzielte. Es beteiligten sich 345 Unternehmen. Zur sichtbaren Identifikation mit »Luxemburg 2007« erhielten sie einen Aufkleber mit dem Slogan der Kampagne und dem Blauer-Hirsch-Logo, sowie ein Paket mit Dekomaterial im Corporate Design des Kulturhauptstadtjahres. Der Blaue Hirsch wurde von Sponsoren/-innen der Wirtschaft häufig in Marketingaktionen genutzt, wodurch die Unternehmen ihre Identifikation mit dem Ereignis visualisierten. Einige heimische Unternehmen entwickelten spezielle Produkte oder veränderten sie mit Blick auf »Luxemburg 2007《. Der Mineralwasserproduzent Rosport markierte sein Produkt >Rosport Blue < mit dem Blauen Hirsch, die Winzergenossenschaft Domaines de Vinsmoselle entwickelte ein neues Produkt, den >Blu-Blu-Deer<-Sekt, und der Porzellanhersteller Villeroy \& Boch produzierte Tassen mit dem Hirschlogo. (Vgl. Luxembourg 2007: 75) Durch Kauf und Konsum dieser Produkte und vieler Merchandising-Artikel wurde so die Möglichkeit einer mehrdimensionalen Identifikation der Menschen mit dem Ereignis geschaffen.

Zusätzlich zur reinen Markierung des Großereignisses durch ein einheitliches Design mit dem markanten Blau und dem Hirsch sollen weitere Möglichkeiten untersucht werden, wie interdiskursive und/oder bildhafte Redeweisen im Rahmen von »Luxemburg 2007《 etabliert wurden und so Assoziationspotential zur Verfügung stellten. Die Analyse einer Auswahl des Werbe- und Informationsmaterials ${ }^{56}$ gibt einen Einblick, inwieweit Luxemburg auf eine charakteristische Weise grafisch und textlich repräsentiert wurde. Auch hier geht es in erster Linie um Zuschreibungsprozesse. Was macht laut diesem Informations- und Werbematerial die >Idenität< Luxemburgs aus? Welche Schwerpunkte werden gesetzt? Weg vom Klischeehaften, hin zu neuen Aspekten, inklusive der Kultur als translokalem Medium? In der bildlichen Symbolik bzw. bildlichen Repräsentation des Großherzogtums deuten sich zwei größere Komplexe an, die sich wiederum in

56 | Folgende Broschüren wurden berücksichtigt: "Tourisme Tourismus", "Mobil(e) 2007 ", "Panorama 2007", "Luxembourg et Grande Région, Capitale européenne de la culture", "LuxembourgCard 2007", Luxembourg and Greater Region, European Capital of Culture 2007", "Trans(ident)city", "explorator", "Avant-Programme. Nouveaux espaces - Lieux insolites", "Luxemburg und Großregion Kulturhauptstadt Europas 2007". 
kleinere, thematisch unterscheidbare Unterkomplexe gliedern lassen: >Tradition und >Moderne .

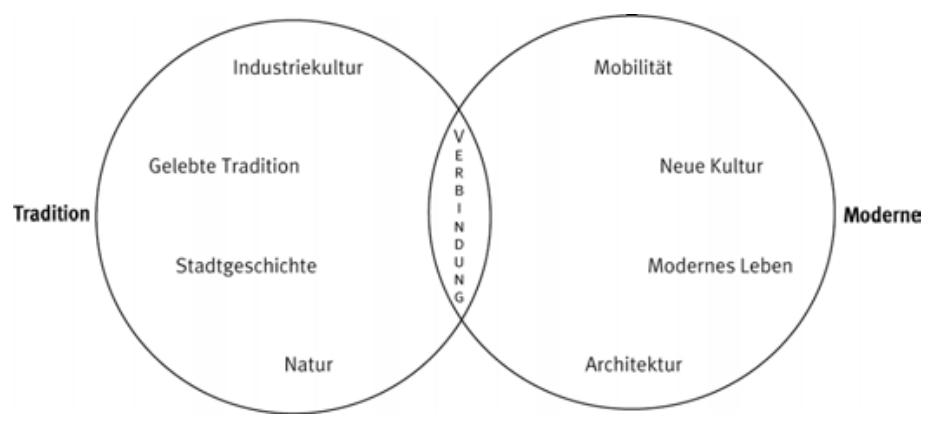

Abbildung 8: Komplexe der Luxemburg-Repräsentationen

Zum Komplex der >Tradition « kann man folgende Bereiche zählen (in Klammern jeweils Bildbeispiele, die den entsprechenden Bereichen zugeordnet werden können): Industriekultur (Industrieanlagen in Belval), Stadt-/Festungsgeschichte ${ }^{57}$ (Bockfelsen/Kasematten), Natur (Wasserlauf), gelebte Traditionen (Schobermesse, große Kirmes in Luxemburg-Stadt). Diese Bereiche manifestieren sich bildhaft in den genannten und weiteren Beispielen. Einen zweiten Komplex bildet die >Moderne <. Sie lässt sich in nachfolgende Unterbereiche gliedern: Mobilität (TGV-Anbindung an Paris), neue Kulturkonzepte (Nacht der Museen), Architektur (Rote Brücke ${ }^{5}$ ) und modernes Leben/Freizeitgestaltung (Cocque-Sport- und Veranstaltungszentrum). Einige Bilder lassen sich nicht eindeutig einer der beiden Kategorien zuordnen und können daher als eine Art Darstellung der Verbindung von Tradition und Moderne bzw. des Übergangs verstanden werden. Hierzu zählt beispielsweise ein Bild mit der Perspektive »Bockfelsen, Drei Eicheln, Mudam, Kirchberg ${ }^{59}$, welches die Chronologie der Entwicklung, die Verbindung von Altem und Neuem visualisiert.

In mehreren Broschüren ${ }^{60}$ finden sich zudem Kartendarstellungen der Großregion. Dabei gibt es zwei verschiedene Varianten. Einmal die separate Darstellung

57 | Vgl. auch Abschnitt 6.2.

58 | Wichtige Brückenverbindung zwischen Luxemburg-Stadt und dem Wirtschafts- und EU-Viertel Kirchberg.

59 | Während der Bockfelsen als erster Standort einer Befestigung seit dem Jahr 963 gilt, ist der Kirchberg mit seinen Bank- und EU-Gebäuden Repräsentant für das moderne Luxemburg. Hier steht auch das Mudam, das Musée d'Art Moderne Grand-Duc Jean. Am Übergang findet sich das wieder aufgebaute Fort 'Drei Eicheln،.

60 | "Tourisme Tourismus", "Mobil(e)2007", "Luxembourg et Grande Région, Capitale européenne de la culture", Luxembourg and Greater Region, European Capital of Culture 2007", "explorator", "Luxemburg und Großregion Kulturhauptstadt Europas 2007". 
der Großregion mit der Gliederung in die einzelnen Regionen und Länder, und außerdem die Darstellung der Großregion auf der Europakarte, bei der diese als Einheit ohne Grenzen gezeigt wird.

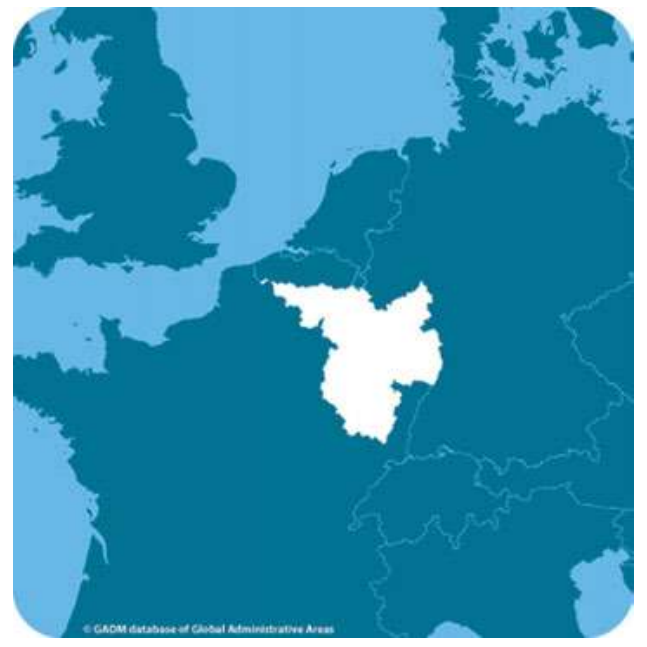

Abbildung 9: Die Großregion - Grenzenlos und im Herzen von >Kerneuropa<, wie es der kartografische Ausschnitt nahezulegen scheint.

Der Anspruch grenzüberschreitenden Arbeitens bzw. eines grenzenlosen (kulturellen) Schaffens wird auf diese Weise kartografisch symbolisch manifestiert. Den Betrachtern/-innen wird suggeriert: Die Großregion ist tatsächlich eine große Region im Zentrum Europas und hat Einheitscharakter. Die Operationalisierung des Raumes vollzieht sich mittels ikonischer bzw. symbolischer Kodierung im Sinne eines Zuschreibungsprozesses. (Vgl. Dünne 2008: 50) Es geht dabei nicht nur um die Abbildung von sozialen Verbindungen in einem Raum bzw. die Abbildung von bestehenden Territorien, sondern die Kartografie konstituiert beides. Demnach ist die Karte eine Art Medium der »Welterzeugung« (Dünne 2008: 52). Und im Globalisierungszeitalter ist sie zudem ein Hilfsmittel, den Individuen und auch Kollektiven eine Übersicht in unübersichtlichen Prozessen zu geben. Daraus abzuleiten wäre besonders der Erhalt der individuellen und kollektiven Handlungsfähigkeit im politischen Sinne. (Vgl. Dünne 2008: 53) Die Großregion als Handlungsraum ist zumindest auf sozio-ökonomischer Ebene weitgehend Realität, wenn auch teils nur kleinräumig und nicht allumfassend. Auf kultureller Ebene sollte »Luxemburg 2007« wichtige Impulse dafür geben.

Wenden wir uns der Textebene der Broschüren zu. Diese steht im Verhältnis zur Bildebene, deutet jedoch noch auf weitere Bilder und Symbole hin, die für Luxemburg (und vermutlich auch die Großregion) inzwischen als Kollektivsymbole verstanden werden können. Zwei größere diskursive Einheiten sollen erläutert 
werden: >Grenze< und >Europa<. Die Kulturhauptstadttour 2007 wird als »grenzüberschreitende >Erfahrung « im wörtlichen Sinne beschrieben. Der »Blick über den Tellerrand « impliziert diese Grenzüberschreitung, die in der Region schon eine gewisse Tradition habe, handelt es sich doch um »2000 Jahre wechselvolle, geteilte Geschichte(n)«. Somit wird die Grenzüberschreitung auch als »gemeinsame« Aktion beschrieben. Neben dem Vorgang der Überschreitung findet sich auch der Aspekt der Grenzenlosigkeit, was im Umkehrschluss eine Überschreitung unmöglich machen würde, weil keine Grenzen mehr vorhanden sind. In diesem Sinne ist auch der Anspruch zu verstehen, to »place the >unified< Greater Region on the European map« (was kartografisch bereits geschehen ist). Das grenzüberschreitende Kulturprogramm wird als vereinendes Projekt vorgestellt, bei dem »Grenzen nicht als Hindernisse, sondern (als) Beitrag zur Dynamik« zu sehen sind.

In enger Beziehung zum Grenzüberschreitungsdiskurs steht der Europadiskurs, der mit Blick auf Luxemburg und Großregion in einer spezifischen Art bedient wird. Zentral sind die sprachlich-symbolischen Repräsentationen »Europa kompakt«, »(im) Herz(en) Europas«, »mix of communities, cultural identities and emotions«, »European Laboratory«, »eine Hauptstadt Europas«. Die Bedeutung des Raumes wird mittels der Herz-Symbolik unterstrichen, die nicht nur die geografische Lage verdeutlicht, sondern auch impliziert, dass das Herz das zentrale Element eines Körpers ist. ${ }^{61}$ Die Labor-Symbolik steht im Verhältnis zum Kulturmix sowie zu »Europa kompakt«, denn es sind die beiden Attribute, die die Laborsituation in Luxemburg und Umgebung ausmachen. Nicht vermeidbar ist dabei der Hinweis auf die traditionellen Attribute >internationale Banken < und >Europaviertel<.

Ergänzend soll - analog zur Analyse der Bildsymbolik - auf textuelle Repräsentationen hingewiesen werden, die ebenfalls eine Art Übergang markieren, bzw. an Schnittstellen stehen. So wird von Altem und Neuem »in Harmonie« gesprochen oder das Kunstmuseum Mudam als Verbindung von Altem und Neuem beschrieben. In den Ausführungen zum Stahlwerk Dudelange heißt es: »Stahlschmelze als >Schmelztiegel < für Mentalitäten und Kulturen«. Industriegeschichte und Arbeitsmigration werden derart verwoben, dass sie sich an das Bild des Labors und des Mixes anschließen lassen.

Diese positiv wirkende Darstellung einer ganz und gar scheinbar vorbildlichen Situation ist das Resultat einer konsequenten Umsetzung der konzeptionellen Vorgaben von »Luxemburg 2007«. Ergebnis ist ein reduzierter Einsatz üblicher Symbole und Klischees, auf die nicht gänzlich verzichtet werden kann, da ohne sie eine eindeutige Markierung Luxemburgs außerhalb der Region vermutlich nicht funktionieren würde. Sie werden verbunden mit neuen (anderen) Identifikationsund Koppelpunkten, an deren Schlüsselstellen in der Argumentation die Kultur als Medium der Identifikation zur Geltung kommen kann.

61 | Vgl. Abschnitt 6.2 und 5.6. 


\section{Neue "Wir«-Identitätsangebote im Luxemburger Werbediskurs?}

Die Werbekommunikation - exemplarisch wurde auf den Bereich der (Finanz-) Dienstleistungswerbung und auf die Kommunikation im Rahmen von »Luxemburg 2007 « eingegangen - arbeitet in Luxemburg in bestimmten Fällen mit Konzepten, die das Großherzogtum im Sinne eines Zuschreibungsprozesses mittels einer spezifischen Bild- und Schriftsprache markieren. Dabei kommen Symboliken zum Einsatz, die sich als Kollektivsymbole identifizieren lassen, da sie auch Bestandteil anderer Interdiskurse sind, so z.B. des journalistischen Diskurses ${ }^{62}$. Durch deren Verwendung wird ein einheitlicher Raum diskursiv konstruiert. Dies erleichtert im Sinne eines Aneignungsprozesses die Assoziation von Individuen oder Kollektiven mit den Bildern und den von ihnen vermittelten Symboliken. Während die privatwirtschaftlichen Beispiele sich eher der klassischen Semantik bedienen (Tradition, Vertrauen etc.), verfolgt die Kommunikation rund um >Luxemburg 2007 < u.a. einen Imagewechsel, den sie durch Verbindung bekannter/ klassischer und neuer Bilder anregen möchte (Tradition/Moderne, Grenze/Europa). Lohnenswert für zukünftige Untersuchungen wäre die Bearbeitung der Frage, inwieweit im Luxemburger Werbediskurs neue Bilder und Symboliken - sich von traditionellen unterscheidende - verarbeitet werden. Dieser Imagewechsel könnte ein Indiz für eine Neuorientierung der Vorstellung von einer >Luxemburger Identität< sein.

\subsection{Dekontextualisierung und Dekonstruktion von IDENTITÄTSDARSTELLUNGEN AM BEISPIEL VON SIEBEN FOTOGRAFEN}

Die Entstehung einer nationalen und internationalen Kunstszene, die mit der Öffnung neuer zeitgenössischer Kunsträume zusammenfällt, und die Schaffung von europäischen künstlerischen Veranstaltungen in Luxemburg stellen ein geeignetes Studienfeld für Fragen der (De-)Konstruktion und der Dekontextualisierung von zugeschriebenen und angeeigneten Identitäten im Rahmen visueller und sprachlicher Vorstellungen dar. Einige kulturelle Ereignisse von bedeutendem Rang wie »Luxemburg und Großregion - Europäische Kulturhauptstadt 2007« ebenso wie einige mit der kulturellen Identität Luxemburgs in Verbindung stehende Aufträge wie etwa das Mudam-Projekt ${ }^{63}$ (»Portraits du Luxembourg«) oder die Initiativen einiger Städte (»Portraits d'une région, Mois de la Photo, ...«) bestimmen die Auswahl der Werke, die die Standpunkte der Künstler in diesem Zusammenhang deutlich machen.

62 | Vgl. Abschnitt 6.2.

63 | Museum für Moderne Kunst Grand-Duc Jean. 
Die aktuelle Situation, Ergebnis künstlerischer und fotografischer Bildung, die sich auf Mobilität, Interdisziplinarität und die Befreiung künstlerischer Ausdrucksformen stützt, zeigt die Bedeutung der Fotografie für die Hinterfragung der Darstellungsformen innerhalb der zeitgenössischen Kunst.

Die Werke aus dem Schaffen der sieben Fotografen aus Luxemburg und anderen Ländern, ausgewählt aufgrund ihrer fachlichen Kompetenz und ihres künstlerischen Rufes, stellen den Hintergrund verlorener künstlerischer, kultureller, politischer, sozialer und identitätsbezogener Bezugspunkte dar. Der Fall der Grenzen und die gestiegene Bedeutung der Zirkulation von Ideen, die sich für die Interkulturalität, ja sogar für die Transkulturalität als förderlich erweisen, wirken sich störend auf die Repräsentationen aus, die mit diesen Diskursen verbunden sind. Mehr noch als einen bestimmten Ort in den Fokus zu rücken, zeugen die untersuchten Bilder eher von einer kontextuellen Annäherung und zugleich von einer internationalen Öffnung, welche die Fragestellungen berücksichtigen, die mit dem Medium der Fotografie sowie dem mehrdeutigen Verhältnis von Fotografie und Realität verbunden sind.

Die heutige fotografische Praxis begnügt sich nicht damit, die Wirklichkeit aufzunehmen, und reicht damit über das Stadium der getreuen Wiedergabe hinaus. Für diesen Prozess der Transfiguration konstatieren wir mit Philippe Dubois: »La photographie est moins un contact (un indice) qu'un mouvement vers le contact (fiction). « ${ }^{64}$ (Dubois 1983, zitiert nach Labelle und Bonaccorsi 2005)

Bei den Fotografen, deren Werke wir untersuchen, liegt das Verhältnis zum Realen zwischen der Beweishaftigkeit (Indexikalität) und der Zeichenhaftigkeit (Ikonizität), der Abdruck ist nur der Ausgangspunkt, von dem aus sich der fotografische Gedanke entwickelt. Dieser Gedanke ist auch an den Kontext von Herstellung und Verbreitung gebunden; er bestimmt den Grad der Aura der Fotografie und verlangt nach einer schöpferischen Interpretation, bei der Betrachter und Betrachterin als Teilnehmer ihren ganz wesentlichen Anteil zwischen physischem Bild und mentaler Vorstellung einbringen.

Auf der Grundlage konkreter fotografischer Arbeiten, die im Rahmen institutioneller Kontexte gemacht wurden, ist diese Arbeit darauf ausgerichtet, die Besonderheiten und die Einmaligkeiten aufzudecken, die in der Beziehung der zugeschriebenen und der angeeigneten Identitäten möglich sind. Die Wahl ist auf sieben Fotografen von internationalem Renommee gefallen, deren Arbeiten in Auftrag von und deren Werke auf Ausstellungen und in jüngeren Publikationen präsentiert worden sind, speziell im Zusammenhang mit dem »Kulturjahr $2007 \ll$.

In methodologischer Hinsicht haben wir vier Untersuchungsstränge gewählt, die sich auf die Entstehung, die Beschreibung/Analyse und die Rezeption/Verbreitung des einzelnen Bildes sowie auf die Entsprechungen und Vergleiche der Bilder

64 | Deutsch: „Die Fotografie ist weniger eine Berührung (Indiz) als eine Bewegung hin zur Berührung (Fiktion)." 
untereinander beziehen. Diese Interaktion zwischen den Werken und den Autoren fördert, indem sie interdiskursive Bezüge und Anleihen verwendet, die Auseinandersetzung mit dem Betrachter in einem hermeneutischen Ansatz.

Ausgehend von dem gewählten Bildkorpus haben wir folgende Themen herausgearbeitet:

1. Vanitas ${ }^{65}$ der Mittelklasse, Konsumgesellschaft, Klischees, Stereotypen;

2. Anachronismus, Widerstand gegen den Fortschritt, Zeitlosigkeit;

3. Dekontextualisierung von Sinnbildern, Befragung von identitätsstiftenden Bezugsobjekten.

Wir haben die Themen und Autoren nach ihren Unterschieden und der Frage, inwieweit sie sich ergänzen, gruppiert, wobei wir dem Vergleich von Fotografen unterschiedlicher Nationalitäten einen besonderen Stellenwert eingeräumt haben.

\section{Inszenierung von Klischees und Stereotypen}

Unsere erste Themenkategorie zeigt, wie ein englischer Künstler und eine luxemburgische Künstlerin Klischees und Stereotypen anhand von konsumrelevanten Schauplätzen und Inszenierungen interpretieren, indem sie völlig unterschiedliche plastische Sprachen wählen.

Martin Parr war zum Ereignis »Luxemburg und Großregion, Europäische Kulturhauptstadt 2007《 eingeladen und hat die Reihe »Luxembourg 2006/Assorted Cocktail« (Auftrag der Coordination 2007 in Zusammenarbeit mit Magnum Photos) geschaffen, die Alltagsszenen darstellt. Er bleibt sich dabei selber treu, wenn er, ebenso wie er es in anderen Ländern oder anderen Städten getan hat, an >typischen< Schauplätzen Luxemburgs bedeutsame Situationen sucht: Vernissagen, Restaurants, Konditoreien, Schaufenster, Einfamilienhäuser, Gärtchen. Auf den ersten Blick erscheinen seine unterwegs gemachten Fotografien als willkürliche Momentaufnahmen, bei genauerem Hinsehen wird jedoch erkennbar, dass Martin Parr genau das beherrscht, was Cartier-Bresson beim Aufbau seiner Bilder den »entscheidenden Augenblick« nennt. Das kann man in der gesamten Reihe feststellen, die sich durch eine Mischung von Aufnahmen auszeichnet, die sich aus der Unmittelbarkeit einer Situation ergeben, und von persönlichen Vorstellungen, die die fotografische Sprache des Künstlers prägen.

65 | Wir verwenden den Begriff »Vanitas« im Sinne eines Ausdrucks von oberflächlicher Eitelkeit und Vergänglichkeit, sowie von Belanglosigkeit und Selbstgefälligkeit, insbesondere in Verbindung mit konsumorientiertem Verhalten. 


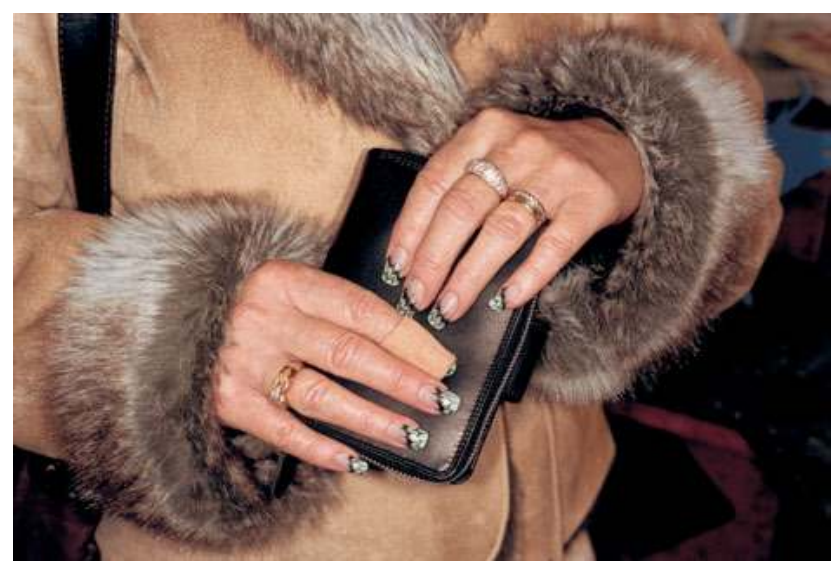

Abbildung 10: Martin Parr, »Luxembourg 2006/Assorted Cocktail« (C) Martin Parr/Magnum Photos

In der Fotografie der Hände (Abb. 10) kann man erkennen, wie Martin Parr, indem er auf einen Schnappschuss abzielt, das Bild dadurch dekonstruiert, dass er es für vielfältige plastische und ikonische Interpretationen öffnet. Im Vergleich zu den gegerbten Händen der Bäuerin in der »photographie humaniste« von Russell Lee (»Family of Man«) ${ }^{66}$, die sich eindeutig in eine monosemantische Ikonografie einbettet, verdeutlicht die Komposition von Martin Parr in diesem Bild die Dualitäten natürlich-künstlich, wahr-falsch, stark-zerbrechlich. Die Details wie der Goldschmuck oder der Ärmelrand aus Pelz stehen in einem ironischen Widerspruch zu den schwarz-weißen Zick-Zack-Motiven der Fingernägel und zu dem mit einem Pflaster versehenen Zeigefinger der rechten Hand. Die eingeschränkte Tiefenschärfe, die Nahaufnahme und die Anordnung, die sich an einer aufsteigenden Schräge orientiert, ermöglichen es dem Fotografen, die Aufmerksamkeit auf die etwas stereotype Geste der Hände zu lenken, die das Portemonnaie wie die metonymische Ersetzung der Konsumgesellschaft halten.

Wenn Martin Parr diese Fotografie dem Bild »LUX« (Abb. 11) zuordnet, auf dem man den Ausschnitt eines Erkennungszeichens sieht ${ }^{67}$, so verdeutlicht er die Trivialität des Luxus, indem er das Klischee von Luxemburg als Land der künstlichen und oberflächlichen Werte verstärkt. Der gewählte Ausschnitt aus einem Objekt, das sich auf das Wort »Luxemburg« bezieht, ist für das Vorgehen von Martin Parr

66 | Martin Parr hat für die Ausstellung "Family of Man“ Interesse gezeigt, obwohl er sich künstlerisch von ihr abhebt, indem er durch seine Einstellungen, seine Farben, das Licht die ironische und bissige Seite seines Vorgehens unterstreicht.

67 | Beide Fotografien sind gemeinsam auf einer Seite des Faltblattes erschienen, das anlässlich der Ausstellung "Luxembourg 2006/Assorted Cocktail“ im Rahmen von "Luxemburg und Großregion, europäische Kulturhauptstadt 2007“ erschienen ist. 
bezeichnend. Mit seinen Teilansichten und seiner Fokussierung auf suggestive Details beabsichtigt er, den Betrachter zum Nachdenken anzuregen.

Diese betrachtend-beobachtende Haltung wird dadurch angeregt, dass symbolische Elemente hervorgehoben werden, die ikonografische Bezüge widerspiegeln, und dadurch, dass formale und thematische Beziehungen innerhalb der Reihe »Luxembourg 2006/Assorted Cocktail« hergestellt werden.

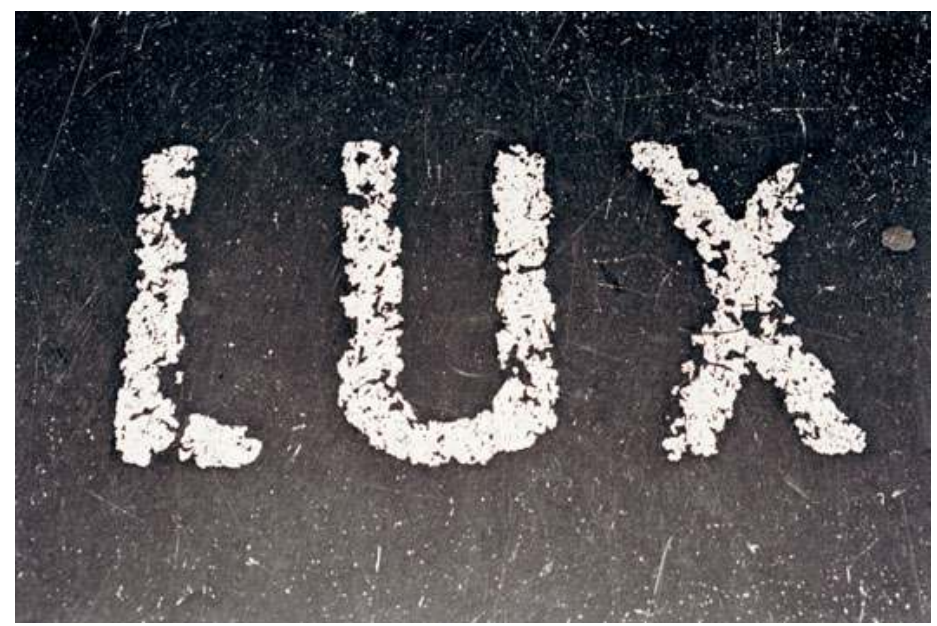

Abbildung 11: Martin Parr, »Luxembourg 2006/Assorted Cocktail« (C) Martin Parr/Magnum Photos

Die Gegenwart des Menschen beschränkt sich in dieser Reihe oft auf einzelne aktive oder inaktive Körperteile in banalen Zusammenhängen, die innerhalb des Ganzen Bedeutung erlangen. Hände haben in Parrs Fotografien verschiedene, manchmal doppelsinnige Bedeutungen. Diese Gegensätze von Formen und Inhalten sind umso wichtiger als sie ständig Assoziationen zu realen Situationen und zugeschriebenen Identitäten herstellen: In einem Foto sieht man abgearbeitete Hände, die aber durch lackierte Fingernägel übertrieben aufgewertet werden; in einem anderen abgeschnittene Hände, wobei der abwärts gerichtete Blickwinkel die Abwesenheit von Kommunikation unterstreicht. In diesem letzten Bild (Abb. 12) scheint die im Vordergrund liegende Pelzmütze die Leere zwischen der Gabel und dem Messer metaphorisch zu füllen. 


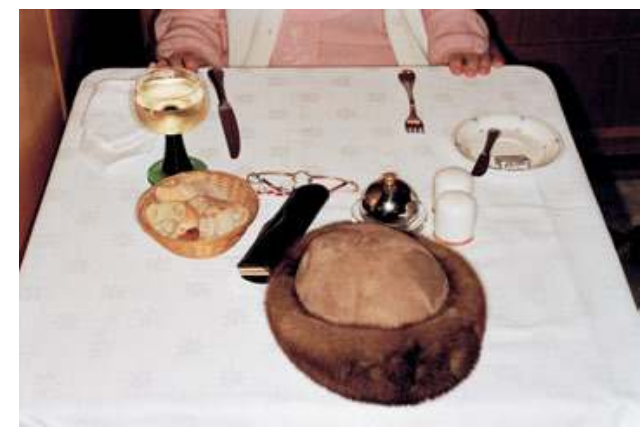

Abbildung 12: Martin Parr, »Luxembourg 2006/ Assorted Cocktail« (C) Martin Parr/Magnum Photos

Mehr noch als die Stereotypen in realen Situationen zu beobachten, schafft die luxemburgische Künstlerin Jeanine Unsen Szenen, die das Ergebnis einer langen Suche sind, bei der sie unzählige Gegenstände zusammenstellt, die zum Theater der künstlichen Situationen werden, wo Luxemburger Tourismus-Nippes und menschliche Figuren auftreten und die Bestandteile fotografischer Gemälde abgeben.

In ihrer Reihe »Odd, small and beautiful« - entstanden als Auftragsarbeit der Stadt Luxemburg im Jahre 2009 - spricht Unsen von luxemburgischen Identitäten. Sie tut dies in einer ausdrucksstarken plastischen Sprache, die aufgrund der übertriebenen Inszenierung eine gewisse Distanzierung berücksichtigt, zugleich aber auch durch die Anhäufung von Erinnerungsstücken das Gefühl der Aneignung und damit eine mögliche Personifizierung suggeriert. Die Künstlerin integriert identitätsstiftende Darstellungen in szenografischen Kontexten und Porträts, die das Dekor durch ihre stereotype Eigenschaft vervollständigen.

Angesichts von kitschigen Konsumgegenständen, die kollektive nationale Symbole darstellen (Bilder des Palastes, des großherzoglichen Paares, des Wappens mit dem roten Löwen, der Adolphe-Brücke, Teller mit Aufdruck der »Banque et Caisse d'Epargne de l'Etat«, Gläser, Tapeten, ...), entwickelt sie eine >kritische Nostalgieく, d.h. eine zugleich bissige und nostalgische Vision.

Il y a quelque chose des traditions villageoises en moi qui perdure et que je ne ridiculise aucunement, mais que je relève à travers mes mises en scène photographiques. Chaque accessoire me rappelle en quelque sorte mon enfance alors que paradoxalement ces objets sont fabriqués en tant que souvenirs pour les touristes. ${ }^{68}$ (Jeanine Unsen, zitiert in: di Felice 2009: 28)

68 | Deutsch: "Es gibt etwas aus den dörflichen Traditionen in mir, das Bestand hat und das ich in keiner Weise lächerlich mache, das ich in meinen fotografischen Inszenierungen 

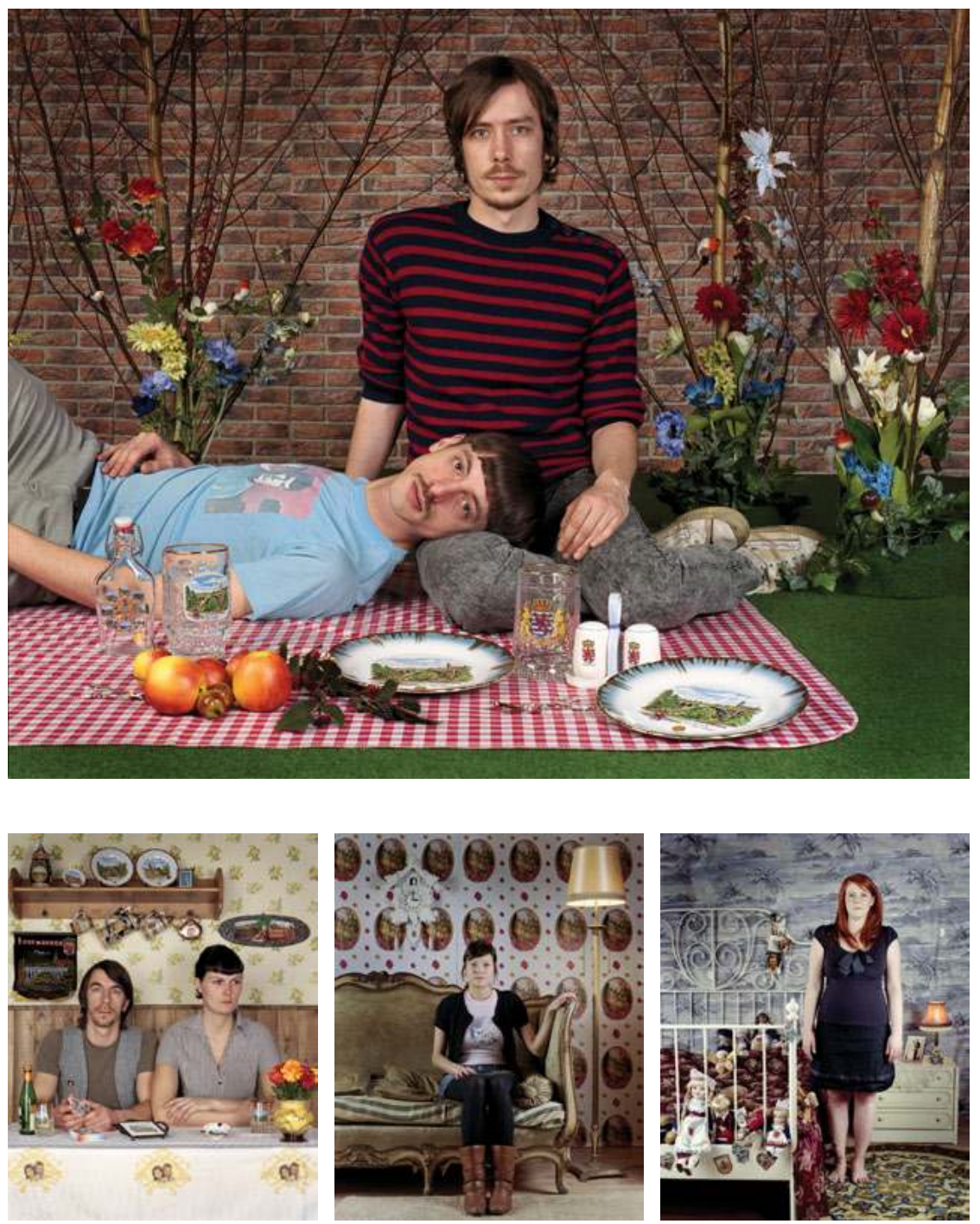

Abbildung 13-16: Jeanine Unsen, »Odd, small and beautiful «, 2009 (C) Jeanine Unsen. Mit freundlicher Genehmigung MHVL

Die in den vier Fotografien von Jeanine Unsen dargestellten Figuren (ein heterosexuelles Paar, ein homosexuelles Paar und zwei Frauenporträts; Abb. 13-16) gehören zum Dekor dieses artifiziellen Universums. Dadurch dass sie einen fiktionalen Charakter annehmen, befragen die Fotografien die Klischees. Sie tun dies über die

vielmehr erhöhe. Jedes Requisit erinnert mich in gewisser Weise an meine Kindheit, obwohl diese Gegenstände paradoxerweise als Souvenirs für die Touristen hergestellt werden." 
Erinnerungsstücke, die zum Fortreisen bestimmt sind (z.B. Postkarten und Nippes mit Bildern vom Großherzog, von der Adolphe-Brücke usw., also Andenken des Ortes), die aber in einen konstruierten Alltagskontext rück-integriert werden.

Während Martin Parr unmittelbar von der luxemburgischen Realität inspiriert wird, wenn er seine Fotografien in dem ihm eigenen Stil macht (mehr auf die Verfremdung von Verhalten und die Universalität der Kontexte ausgerichtet als auf die nationalen Identitätsunterscheidungen - Madonnenbilder in Luxemburg und in Mexiko, Bilder vom Konsum in Mexiko, Luxemburg, Irland ...), rekonstruiert Jeanine Unsen Situationen in künstlichen luxemburgischen Situationen, die sich unmittelbar auf die symbolischen Darstellungen beziehen.

Martin Parr gestattet die Interpretation von Szenen, die Besonderheiten und zugleich Allgemeinheiten im Hinblick auf den luxemburgischen Hintergrund enthalten, indem er seine Fotografien als Vanitas präsentiert, die in verschiedenen Ländern entstandene Archetypen der Mittelklasse verkörpern. Die Fotografien »Luxembourg 2006/Assorted Cocktail« zeigen die Vision eines >fremden< Künstlers über Luxemburg - wobei sich typische Merkmale, die überall der gleichen Mittelklasse zuzurechnen sind, wie ein roter Faden durch sein Werk ziehen - und ermöglichen es den Luxemburgern, in diesen stereotypen Bildern das Spiegelbild ihrer eigenen Identitätsvorstellungen zu entdecken.

\section{Aktualisierte Darstellungen einer vergangenen Epoche}

Die zweite von uns ausgewählte Themenkategorie widmet sich der Darstellung von Vergangenheit und Gegenwart im heutigen Luxemburg, betrachtet anhand der in Schwarz-Weiß gehaltenen Werke des luxemburgischen Fotografen Yvon Lambert (»photographie humaniste«) und der französischen Fotografin Valérie Belin (»photographie plasticienne«).

Mit der Reihe »Derniers feux « ${ }^{69}$, einer Auftragsarbeit der Stadt Esch-sur-Alzette, verfolgt Yvon Lambert die letzten Arbeitstage des Hochofens B in Esch-Belval und würdigt damit die Minetteregion als Ort der Erinnerung, der die Kraft der Arbeit in einer poetischen und sakralen Stimmung zeigt. (Abb. 17 und 18)

Der Hochofen erscheint nur in Teilansicht, während sich unzählige Einzelheiten (Rohre, Handschuhe, Bolzen, Gusstrichter, Gießproben, Rinnen ...) vom schwarzen Hintergrund und den unscharfen Bereichen der Schwarz-Weiß-Fotos abheben. Das Theaterlicht hebt die Sublimation der Bewegung (flou-bougé) des Arbeiters hervor (vgl. Lorgé 1998) und stellt gewissermaßen eine metaphysische Beziehung zwischen Mensch und Arbeitswelt her, die durch den Fotografen ästhetisiert wird.

Yvon Lambert wählt eine fotografische Sprache (bewusste Bildeinstellung, gesättigtes Schwarz, Lichtkontraste...), die »die greifbare Kraft einer von vornherein

69 | Diese Fotografien wurden im August 1997 aufgenommen. Der Hochofen B der "Aciéries Réunies de Burbach, Eich et Dommeldange“ (ARBED), der letzte Hochofen des Großherzogtums Luxemburg, wurde am 28. August 1997 um 19 Uhr stillgelegt. 
mehr der Produktivität als der Ästhetik verpflichteten Umgebung « ${ }^{70}$ widerspiegelt (Lambert, R. 1998: 7), einer Umgebung, mit der sich eine ganze Region identifiziert. Indem er sich für diese Art von Schwarz-Weiß-Fotografien entscheidet, versucht er, eine vergangene Zeit zeitlos werden zu lassen.

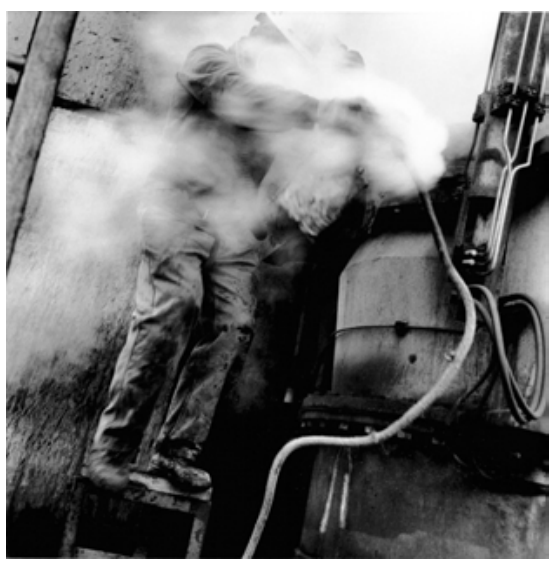

Abbildung 17: Yvon Lambert, »Coulée et granulation de laitier « ${ }^{71}, 1997$ (C) Yvon Lambert

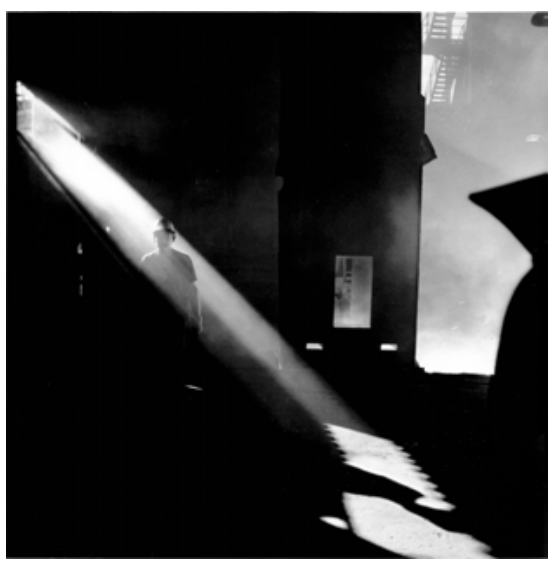

Abbildung 18: Yvon Lambert, »Nettoyage des bleeders « ${ }^{72}, 1997$ C Yvon Lambert

In der Reihe »Differdange - à la rencontre d'un lieu«, einer von der Stadt Differdange in Auftrag gegebenen und von 2003 bis 2005 durchgeführten Arbeit, begegnet uns wieder diese anachronistische Haltung und diese Art, die Zeitlichkeit durch seine Arbeitstechnik zu neutralisieren (Langsamkeit, Ortserkundung, Dokumentation ...), und sein fotografischer Stil, der alle seine Projekte prägt. (Abb. 20-22)

In einem Gespräch mit dem Journalisten Thierry Hick erzählt der Fotograf: »'ai aussi voulu montrer que cette industrie reste toujours d'actualité dans la région. Mes photos sont un trait d'union entre le passé et le présent. «73 Der Journalist fährt fort: »Dans son travail, le photographe, un Minettsdapp part à la recherche de son identité, mais aussi de ses racines. Une recherche valable tant pour l'artiste que pour le visiteur. «74 (Hick 2003: 9)

70 | Originalzitat: "la force plastique d'un environnement à priori plus soucieux de productivité que d'esthétique"

71 | Deutsch: "Anstich und Granulierung der Schlacke".

72 | Deutsch: „Reinigung der Bleeder».

73 | Deutsch: »ch wollte auch deutlich machen, dass diese Industrie für die Region immer aktuell bleiben wird. Meine Fotos stellen das Bindeglied zwischen der Vergangenheit und der Gegenwart dar."

74 | Deutsch: "Der Fotograf, ein Minettsdapp, begibt sich mit seiner Arbeit auf die Suche nach seiner Identität, aber auch nach seinen Wurzeln. Eine beachtenswerte Suche sowohl 


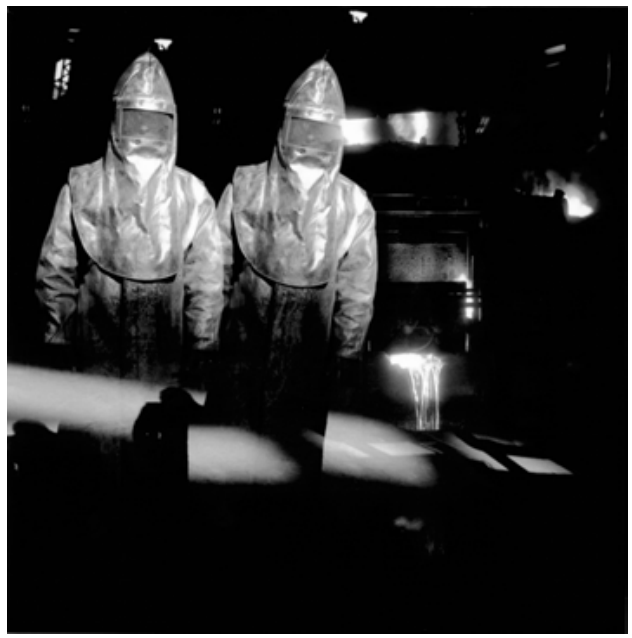

Abbildung 19: Yvon Lambert, »Differdange, Arcelor-aciérie«, 2003 (C) Yvon Lambert

Das Bild, das der Fotograf von Differdange liefert, zeigt eher die kleinen, scheinbar unbedeutenden Dinge und unspektakuläre Situationen als »ansichtskartenartige« Bilder der Stadt und zeugt von einer nostalgischen Zärtlichkeit des Künstlers gegenüber seiner Herkunft. (Abb. 20-22)

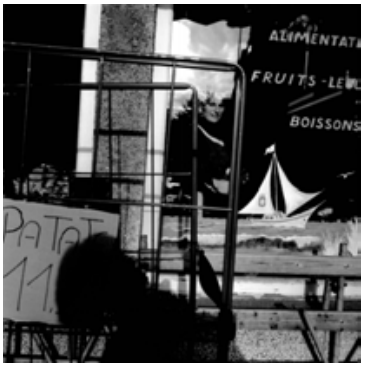

Abbildung 20: Yvon

Lambert, »Differdange,

Grand-Rue«, 2003

(C) Yvon Lambert

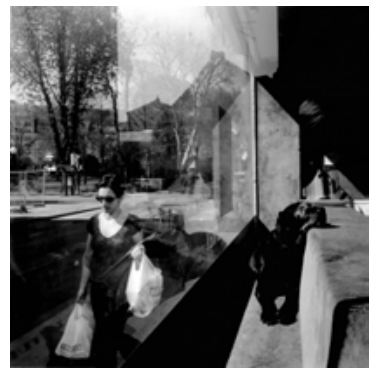

Abbilldung 21: Yvon

Lambert, »Differdange, rue

Michel Rodange«, 2004

(C) Yvon Lambert

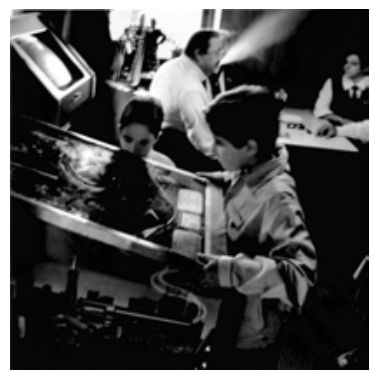

Abbildung 22: Yvon

Lambert, »Differdange,

Café Chez Quim, 2004«

(C) Yvon Lambert

Während man in den hier beschriebenen Fotografien eine gewisse Symbiose zwischen dem Fotografen und seiner Umgebung beobachten kann, zeigt im Gegensatz dazu die Reihe »Vitrines du Luxembourg« (2003) (Abb. 23-24) der französischen

für den Künstler als auch für den Betrachter." Mit dem Begriff ,Minettsdapp، bezeichnet man einen Einwohner der Minetteregion. 
Künstlerin Valérie Belin eine starke konzeptuelle Distanzierung. Diese Reihe wurde 2002 im Rahmen eines Projektes mit dem Titel »Portraits du Luxembourg « vom Mudam in Auftrag gegeben. Sie besteht aus sieben großformatigen zeitgenössischen Schwarz-Weiß-Bildern, die sieben antiquiert anmutende Schaufenster in der Stadt Luxemburg zeigen, in denen Kleidung und altmodische Gegenstände in einer an die $1960 e r$ Jahre erinnernden Dekoration ausgestellt sind.
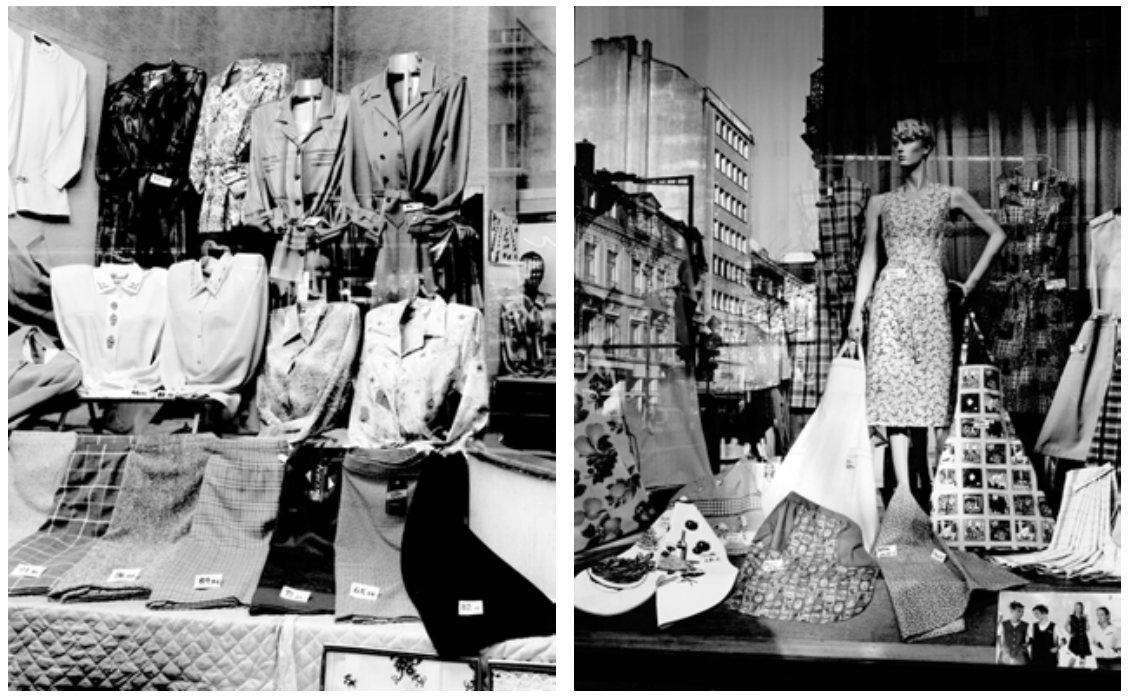

Abbildungen 23-24: Valérie Belin, »Vitrines du Luxembourg«, 2003

(C) Valérie Belin. Mit freundlicher Genehmigung von Mudam

Valérie Belin hat sich auf die Suche nach Orten gemacht, an denen das Vergangene immer noch präsent ist. Sie tut dies, indem sie die gewöhnlichen und stereotypen Aufnahmen für das Thema eines Porträts von Luxemburg meidet. Die Fotografien von Dingen, die das Luxemburg von früher verkörpern, spiegeln die Stadt von heute wider und prüfen die verborgenen Identitätsaspekte einer im Umbruch befindlichen Stadt.

Die Schaufenster von Lambert und Belin sind trotz ihrer scheinbaren Ähnlichkeit grundverschieden in dem Sinne, dass einerseits der Autor von »Differdange - à la rencontre d'un lieu « einen Vorstellungsraum schafft, in dem sich seine persönliche Biografie (>Minettsdapp $<$ ) mit den Identitätsaneignungen des Betrachters vermischt, und dass andererseits die Künstlerin von »Vitrines du Luxembourg « eine Projektionswand aufzustellen scheint, auf der sich die Dualitäten vergangengegenwärtig, alt-neu und innerlich-äußerlich zeigen.

Wenn Yvon Lambert die Bewohner der Minetteregion in den Mittelpunkt seiner Arbeit stellt, so schafft er Bilder, die die Identifizierung und die Komplizität des Betrachters mit dem Ort unterstützen. Im Gegensatz dazu ersetzt Valérie Belin 
den Menschen durch Schaufensterpuppen und Kleidungsstücke, dadurch schafft sie ein Bild der Distanzierung, das für eine identitätsstiftende Vorstellung wenig Platz lässt.

\section{Dekontextualisierungen}

Im Zusammenhang mit dem dritten Thema, der Dekontextualisierung von Emblemen, versuchen wir, die Frage nach den symbolhaften Orten zu stellen, die in den Fotografien von Andrés Lejona, Marco Godinho und Joël Tettamanti dargestellt sind.

Die Reihe des spanischen Fotografen Andrés Lejona, der seit Mitte der 1980er Jahre in Luxemburg lebt, ist anlässlich des Nationalfeiertags am 23. Juni, an dem der Geburtstag des Großherzogs gefeiert wird, unter dem Titel »F(en)êt(r)es Nationales« (Abb. 25-27) in der Zeitschrift »Rendez-Vous«75 erschienen. Die Reihe vereint zehn Farbfotografien von Schaufenstern in der Hauptstadt, in denen das großherzogliche Paar auf offiziellen Fotos oder Familienfotos gezeigt wird, z.T. innerhalb eines Dekors, das für die Nationalfeier entworfen wurde.

Andrés Lejona lässt sich - ähnlich wie Martin Parr - von der luxemburgischen Realität inspirieren, in diesem Fall im Zusammenhang mit den Nationalfeierlichkeiten. Er löst die nationalen Symbole durch eine enge Bildeinstellung aus ihrer Umgebung heraus und lässt sie in der Kombination mit anderen Details grotesk erscheinen. Das Foto des großherzoglichen Paares, mal in einem gewohnten Schaufenster-Umfeld (Kleidung, Spielzeug ...) gezeigt, mal als Teil einer ausgeklügelten Inszenierung (Luxemburger Flagge, Wappen mit rotem Löwen, Abb. 27) bildet das Leitmotiv, das den Mittelpunkt der Bilder bestimmt.
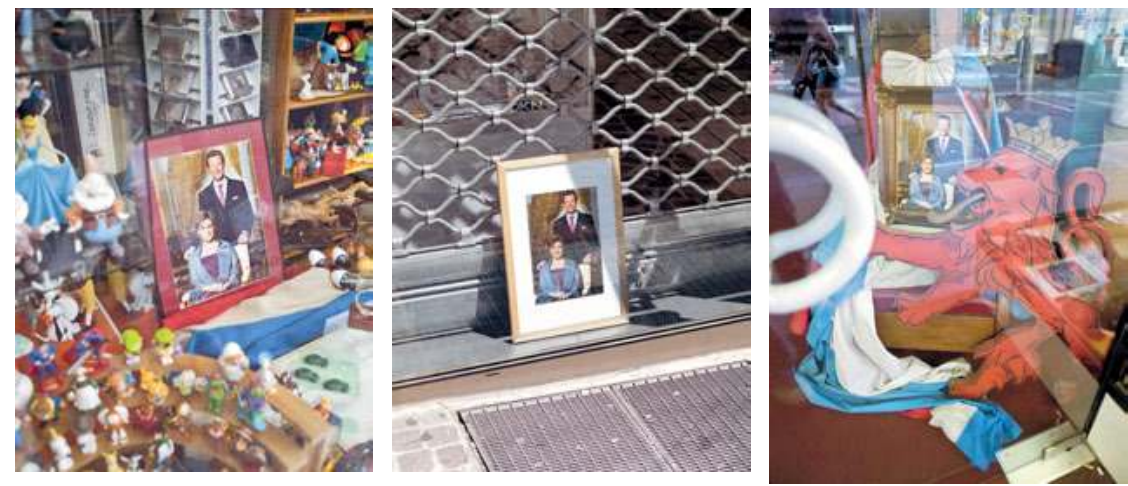

Abbildungen 25-27: Andrés Lejona, »F(en)êt(r)es Nationales«, 2009 (C) Andrés Lejona. Mit freundlicher Genehmigung von »Rendez-Vous«

75 | Lejona, Andrés (2009): "F(en)êt(r)es nationales", in: Rendez-Vous. City Magazine Luxembourg 6, S. 46-57. 
Während er sich zwischen kritischem Geist und Zuneigung bewegt, setzt der Fotograf diese Serie, die die Luxemburger würdigt und zugleich den Kult um den Großherzog hinterfragt, sehr humorvoll um. In einem der Bilder erscheint das großherzogliche Paar, als ob es Teil einer kindlichen Märchenwelt sei (Schneewittchen, Tim, Schlümpfe ...), die Inszenierung des monarchischen Kultes wird ridikülisiert (Abb. 25); in einem anderen Beispiel ist die Fotografie des Paares zwischen der Schaufensterscheibe und dem Metall-Schutzgitter eingeschlossen (Abb. 26) und erinnert an die Zweideutigkeit von Schutzmaßnahmen und Gefangensein.

Dank des Spiels mit dem Bild im Bild, der aufeinander folgenden Bildebenen und der Spiegelungen schafft Andrés Lejona eine subtile Distanzierung gegenüber den >Darstellungen der nationalen Identität<. Auch wenn die Fotografien im Rahmen der Nationalfeierlichkeiten entstanden sind, so sind sie doch bezeichnend für die Suche nach dem Außergewöhnlichen, die eine Verfremdung von realen Situationen ermöglicht und die Dekontextualisierung von Repräsentationen beabsichtigt.

Mit dem Werk »Le guide mental du Luxembourg« (2007) (Abb. 28-31), entstanden anlässlich von »Luxemburg und Großregion, europäische Kulturhauptstadt 2007《 als Auftragsarbeit des Museums für Stadtgeschichte der Stadt Luxemburg, präsentiert der luxemburgische und portugiesisch-stämmige Künstler Marco Godinho eine fotografische Installation, die aus horizontalen Fotobändern zum Thema Migration und Bevölkerungsbewegungen in Luxemburg besteht.

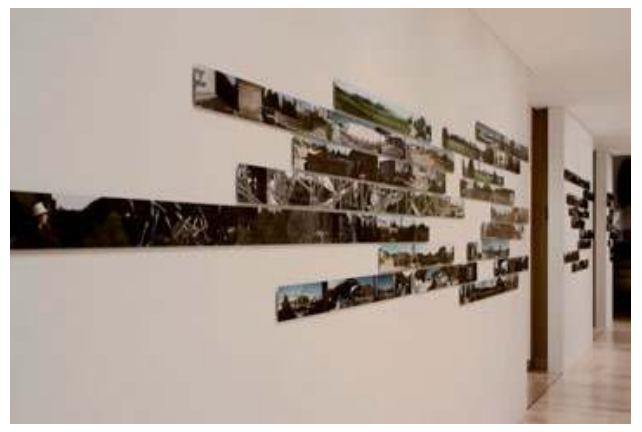

Abbildung: 28: Marco Godinho, »Le guide mental du Luxembourg«, 2007 (C) Marco Godinho, mit freundlicher Genehmigung des MHVL 

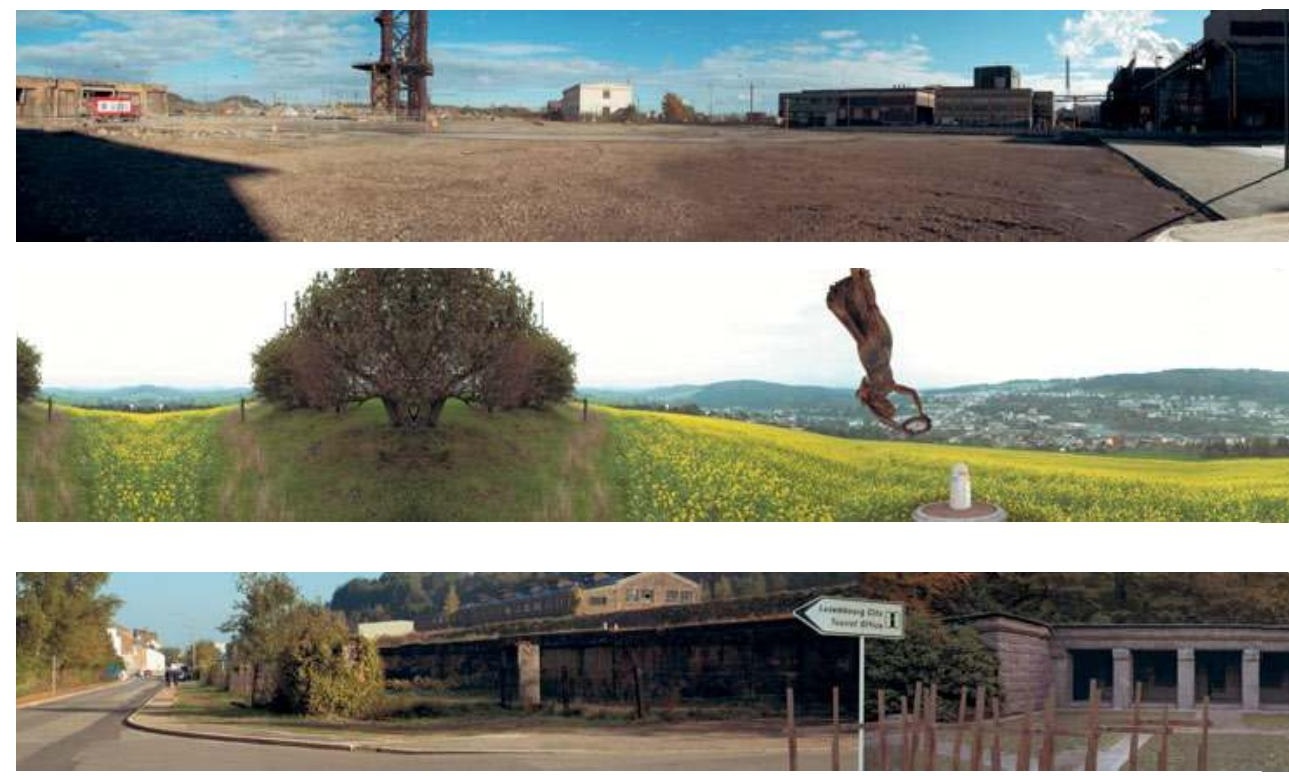

(Details, Abb. 29-31)

Mithilfe von Digitalfotos hat der Künstler Panorama-Collagen (Abb. 29-31) hergestellt, auf die er Symbole, Embleme und Signale überträgt, die er wiederholt und verschiebt, um so die Spuren der Identifizierung zu verwischen. Er erkundet die Bezugsobjekte, um sie zu dekonstruieren und sie den verschiedenen Prozessen der Transkulturalität gegenüberzustellen, indem er u.a. die Begriffe von Gebieten, Grenzen und geografischer Zugehörigkeit befragt.

L'œuvre se lit comme une carte d'un monde où les trajectoires reflètent les flux migratoires, pose la question du multiculturalisme, de l'immigration au Luxembourg et reflète l'essence même de la mobilité et des échanges interculturels, entre passé, présent et avenir. ${ }^{76}$ (Damiani o.A.)

76 | Deutsch: "Das Werk kann man wie die Karte einer Welt lesen, in der die Wege den Strömungen der Migration entsprechen; es stellt die Frage nach Multikulturalität, nach der Einwanderung nach Luxemburg und es spiegelt das Wesen der Mobilität und des interkulturellen Austauschs in Vergangenheit, Gegenwart und Zukunft." 
Die großen Fotobilder mit ihren fast abstrakt wirkenden Formen und Farben, die die Region verkörpern (rote Erde), erzählen von verfallenen Stätten und machen die Spuren einer Epoche sichtbar.

Die Fotografien zeigen wie ein Palimpsest die Spuren der Zerstörung und des Wiederaufbaus dieser Stätten, Zeugen einer industriellen Vergangenheit, welche mit der kulturellen Identität einer im Umbruch befindlichen Region verknüpft ist.

In diesem dritten Themenbereich können wir einerseits die humorvolle und ironische Verfremdung der Symbole in den Aufnahmen von Lejona und in den Collagen von Godinho festhalten, auf der anderen Seite die zeitliche und räumliche Verschiebung in den fotografischen Bildern von Tettamanti.

\section{Schlussfolgerungen}

Es lässt sich zusammenfassend festhalten, dass die zuweilen bissige Verwendung der nationalen Identitätsdarstellungen des Spaniers Lejona mit der Anhäufung von Symbolen in den lebenden Bildern der Luxemburgerin Jeanine Unsen korrespondiert, auch wenn die Werke sich in poietischer Hinsicht unterscheiden. Für eine Distanzierung sorgt der Spanier Lejona im Hinblick auf die Darstellungen des großherzoglichen Paares als nationales Symbol, aber auch anhand seiner Suche nach dem Außergewöhnlichen und der Akzentverschiebung innerhalb von realen Situationen; die Luxemburgerin Unsen hingegen distanziert sich von den darstellenden Bildern, indem sie Innenräume aufbaut, in denen die Embleme zu dekorativem Beiwerk degradiert werden.

Es gibt auch Entsprechungen zwischen den postindustriellen Landschaften von Tettamanti und den >anachronistischen $<$ Schaufenstern von Belin, die durch die Dekontextualisierung und die raum-zeitliche Verschiebung hergestellt werden, welche den Verlust von angeeigneten Identitäts-Bezugspunkten mit sich bringen. $\mathrm{Zu}$ den Palimpsesten, die die Spuren des postindustriellen Landschaftswandels verkörpern, gesellen sich die urbanen Spiegelungen, die eine immer noch spürbare Vergangenheit symbolisieren.

Während Marco Godinho, wenn er die bedeutsamen Landschaften Luxemburgs aufschlüsselt, nicht direkt Stellung bezieht, sondern vielmehr einen Raum der Hinterfragung schafft, in dem die Betrachter eine reflexive Haltung gegenüber ihren eigenen Identitäten einnehmen müssen, legt Martin Parr in seinem gewohnten fotografischen Stil den Akzent auf die Verhaltensweisen der Einwohner Luxemburgs, indem er Vulgarität, Oberflächlichkeit und Lux(us) einander gegenüberstellt. Die Reihe der Fotografien »Luxembourg 2006/Assorted Cocktail« von Parr ist näher an der >Realität< und scheint das Bild der luxemburgischen Gesellschaft widerzuspiegeln, so wie sie oft in Form kollektiver Klischees wahrgenommen und in den Abschnitten 6.2, 6.3 und 6.4 näher untersucht wird. Jedoch erlaubt die spezielle fotografische Sprache Parrs mit ihren fragmentarischen Betrachtungen und ihrem Blick auf suggestive Details, über diese stereotype Sicht 
hinauszugehen. Seine Sprache lädt zu einer gründlicheren Lektüre ein, die einen Bruch mit dem diesen Bildern innewohnenden Identifikationsprozess zur Folge hat.

Mehr noch als sich auf die Identitätsgestaltung in Luxemburg auszuwirken, verdeutlichen die gezeigten Fotografien die Bedeutung der Vorstellungen eines/r jeden Autors/Autorin für die Identitätszuschreibungen. Indem sich die Künstler von ähnlichen Kontexten und Situationen inspirieren lassen, erschaffen sie aufs Neue ein individuell gestaltetes Universum, in dem sich unterschiedliche Arten von reproduzierten, konstruierten, wieder angeeigneten oder zugeschriebenen Bildern treffen. Diese hinterfragen die Darstellungen ebenso durch die Bild-in-BildTechnik (>mise en abyme <) wie durch die Dekonstruktion der Identitätsdiskurse. Anstatt einen objektiven Blick auf die unterschiedlichen Darstellungen zu werfen, weichen die Künstler dem nationalen Kontext aus und geben ihre abweichenden Ansichten in bildlichen Konstruktionen wieder, die sie in eine persönliche formale Sprache übertragen.

Alle diese Beispiele für Fotografien, die in einem speziellen zeitgenössischen künstlerischen Zusammenhang gemacht werden, skizzieren zweifellos die Frage nach den angeeigneten und den zugeschriebenen Identitäten, aber bevor sie der Schaffung von Identitäten eine bedeutendere Rolle zuweisen, bieten sie einen Raum der Hinterfragung und der Reflexion im Hinblick auf unsere eigenen Identitäten, die sich von Bild zu Bild zunehmend dekonstruiert sehen.

\subsection{Die Darstellung italienischer Migranten/-innen Im Ausstellungskatalog "Retour de Babel"}

Wie bereits im Abschnitt 6.2. dargelegt, wurden im Zuge der Großveranstaltung »Kulturhauptstadt 2007 « verschiedene Kollektivsymbole interdiskursiv neu verhandelt. Eine zentrale Bedeutung kommt dabei der Bezeichnung dieser grenzüberschreitenden Region als >europäisches Labor $<$ zu. Sie bezieht sich u.a. auf die >multikulturelle< Zusammensetzung der Luxemburger Gesellschaft, in der der Anteil der ausländischer Bevölkerung bei über $40 \%$ liegt. Dass die Koordinationsstelle der Veranstaltung sich 2007 für das Thema »Migrationen« entschied, ist vor diesem Hintergrund nicht überraschend. Sowohl der historiografische wie auch der politische Diskurs der letzten Jahrzehnte definieren die Luxemburger Gesellschaft als >offen< und >integrationsfähig . Historische >Phasen< von Ein- und Auswanderungsbewegungen werden dabei sehr oft zitiert, um den internationalen und traditionell multikulturellen Charakter Luxemburgs zu unterstreichen. Die Ausstellung »Retour de Babel« war, gemäß der Organisatoren/-innen, das Hauptevent der »Kulturhauptstadt 2007 « zum Thema »Migration« und wurde über mehrere Monate im ehemaligen Stahlwerk der Stadt Dudelange gezeigt. Ziel war es, ein möglichst umfassendes Bild der Ein- und Auswanderung in Luxemburg zu bieten. Damit sollte nicht nur den Migranten/-innen ein Denkmal gesetzt werden, 
sondern auch die Zuschauer/-innen für die vielfältigen Formen der Migrationen sensibilisiert werden.

Es stellt sich die Frage, wie - und ob - im Katalog »Retour de Babel« den italienischen Einwanderer eine kollektive Identität zugeschrieben wird.

\section{Migrationen in Luxemburg}

\section{Historischer Überblick}

Das noch immer in der Bevölkerung stark verbreitete Bild Luxemburgs als armes Auswanderungsland bis zur Zeit der Industrialisierung und seitdem reiches Einwanderungsland wird schon seit geraumer Zeit durch die Resultate historischer Studien, aber auch in verschiedenen Artikeln des Katalogs »Retour de Babel« selbst weitgehend infrage gestellt. (Vgl. Pauly 1986; Reuter/Ruiz 2008) Dem vorherrschenden gesellschaftlichen Diskurs folgend77 beginnt die Geschichte Luxemburgs als Einwanderungsland erst in den 18 goer Jahren im Zusammenhang mit der (sogenannten) >ersten Einwanderungswelle< italienischer Arbeitssuchenden während der Industrialisierung. Historiker/-innen haben hingegen nachgewiesen, dass die ersten italienischen Industriearbeiter bereits ab den $1870 e r$ Jahren über Lothringen nach Luxemburg kamen, um vorwiegend in der Stahlindustrie zu arbeiten. Desweiteren wurde aufgezeigt, dass es bereits im Mittelalter Arbeitsmigration in Luxemburg gab. (Reuter 1995) Sesshaftigkeit wird damit zum Mythos und Migration zum Normalfall erklärt. (Hahn 2008: 16) Erst die Grenzziehungen der Nationalstaaten haben Menschen zu >In- und Ausländern< gemacht. Bilaterale zwischenstaatliche Abkommen zwischen Portugal und Jugoslawien (1972) sowie familiäre und lokale Netzwerke erklären die ausgeprägte Präsenz italienischer und portugiesischer Migranten in Luxemburg.

Die italienische Einwanderung dauerte bis in die frühen $1960 e r$ Jahre an, ab dieser Zeit stieg die portugiesische Einwanderung an. Unter den männlichen Einwanderern waren vorwiegend Stahl- und Bauarbeiter, aber auch Freiberufliche, vor allem Handwerker und Lebensmittelhändler. (Gallo 1992) Der größte Teil der Einwanderinnen waren deren Ehegattinnen, die ihren Männer nach einigen Jahren folgten; viele von ihnen waren als Verkäuferinnen, Putzfrauen und z.T. auch in der Industrie als Arbeiterinnen beschäftigt. Heute ist die italienische Einwanderung nur noch gering, und es handelt sich vor allem um hoch qualifizierte Arbeitnehmer/-innen, die in den Banken und EU-Institutionen arbeiten. (Langers 1999)

77 | In "Histoire du Luxembourg. Le destin européen d'un ıpetit paysı ist z.B. zu lesen: "En s'industrialisant, le Luxembourg, de pays rural pauvre et donc d'émigration, se transforme en terre d'immigration." ("Durch die Industrialisierung wird Luxemburg von einem armen Agrarstaat und folglich Auswanderungsland zu einem Einwanderungsland.") (Trausch 2003: 268) 


\section{Politische Diskurse zur Einwanderung in Luxemburg}

In Luxemburg hat man im Vergleich zu den beiden großen Nachbarländern Frankreich und Deutschland, zumindest auf politischer Ebene, bedeutend früher erkannt, dass das Land ein >Einwanderungsland < sei, dass es sich bei den Einwanderern/-innen also nicht um Gastarbeiter handele, sondern um Menschen, die in Luxemburg bleiben und zur Gesellschaft gehören. Diese Wahrnehmung kann man verschiedenen politischen Diskursen entnehmen, vor allem anlässlich internationaler Staatsbesuche oder generell zum Thema »Europa«, welche die Multikulturalität und traditionelle Offenheit der Luxemburger/-innen immer wieder unterstreichen. Diese Darstellung hat jedoch nur wenig Auswirkungen auf 1.) konkrete Maßnahmen für ein besseres Zusammenleben oder für eine bewusste Integrationspolitik und 2.) auf die breitere gesellschaftliche Anerkennung Luxemburgs als > Einwanderungsland .

Doch spätestens seit Ende der 1970er Jahre beschäftigen sich Teile der Zivilgesellschaft mit der Frage der Einwanderung in Luxemburg. So wurde im Jahre 1978 die »Association de soutien aux travailleurs immigrés « (ASTI) gegründet, gefolgt von dem »Comité de liaison des associations étrangères « (CLAE) (1985) und »Centre de documentation sur les migrations humaines« (CDMH) (1996), um nur die größten Organisationen zu nennen. Wenn sich diese auch durch verschiedene Zielsetzungen und politische Motivationen charakterisieren, so bleibt ihnen gemeinsam, dass sie sich für die Anerkennung der Migranten/-innen in Luxemburg einsetzen.

Es waren auch die beiden letztgenannten Organisationen, die zusammen mit der Gemeinde Dudelange die Ausstellung »Retour de Babel«, in der die verschiedenen Phasen und Formen der Migration in Luxemburg dargestellt werden sollten, vorbereiteten.

Wir wollen uns hier vorwiegend mit der Darstellung italienischer Einwanderer/-innen im Ausstellungskatalog »Retour de Babel« befassen, da es über die italienische Einwanderung ein auf Geschichte und Erinnerung bezogenes $>$ Master Narrative ${ }^{78}$ gibt, mit dem man diese Darstellungen vergleichen kann. Das im >Master Narrative< skizzierte Bild italienischer Einwanderer/-innen schreibt ihnen eine kollektive Identität zu, beruhend auf dem Bild des Stahlarbeiters und der Vorstellung der gelungenen Integration.

\section{Diskurse über italienische Einwanderung in Luxemburg}

Zur Erfassung von Diskursen über italienische Einwanderung wurden politische Reden, Publikationen sowie die Aktivitäten und Inhalte der Internetseiten ${ }^{79}$ von

78 | Auch 'Metar- oder 'Grand Narrativer genannt. Man kann das 'Master Narrativer als Hauptbild, das von einer historischen Erfahrung in der Öffentlichkeit vermittelt wird, definieren. (Vgl. Lyotard 1979)

79 | URL: www.italiani.lu; www.passaparola.info 
verschiedenen Organisationen untersucht. Der Katalog »Retour de Babel« ist ebenfalls als ein Produzent dieser Diskurse zu verstehen.

Konsensdiskurs oder >Master Narrative <: Die italienischen Einwanderer/-innen werden vor allem als Stahlarbeiter wahrgenommen, ${ }^{8 \circ}$ deren Arbeitskraft zur Industrialisierung und damit zum Reichtum Luxemburgs beigetragen hat. Dieses Bild taucht im Einleitungstext des Kataloges auf, wird aber auch auf politischer Ebene vermittelt. Bis vor kurzer Zeit wurde darauf in Reden des Staatschefs, des Regierungschefs wie auch des italienischen Staatspräsidenten während seines offiziellen Staatsbesuchs in Luxemburg Bezug genommen. Die Publikation »Il Centenario « (Gallo 1992), welche die hundertjährige italienische Einwanderung in Luxemburg feiert, ist ein weiterer wichtiger Produzent und Träger des Konsensdiskurses. Das >Master Narrative< ist konsensfähig, weil eine Mehrheit der Bevölkerung sich in ihrer Selbstwahrnehmung mit dem Bild des durch die Industrialisierung - und damit auch durch die Arbeitskraft der eingewanderten Italiener/-innen - entstandenen Reichtums identifizieren kann. Neben diesem >Master Narrative< haben wir in der Literatur zur italienischen Einwanderung in Luxemburg und den Internetseiten zwei weitere Diskurse erfassen können.

Der marginale Linksdiskurs oder antifaschistische Diskurs: er beruht auf z.T. wenig wissenschaftlich aufgearbeiteten Episoden der Zwischenkriegszeit und des Zweiten Weltkriegs. Der Katalog »Retour de Babel« macht ihn nicht nur durch die Darstellung der porträtierten Italiener/-innen, sondern auch durch seine wissenschaftlichen Artikel, die Ereignisse der antifaschistischen Bewegungen oder der Resistenz beleuchten, sichtbar. Dieser Diskurs wird von einigen Historikern/-innen und politisch links engagierten Persönlichkeiten getragen: Denis Scuto, Henry Wehenkel, Marcel Lorenzini u.a. Da das Bild des engagierten (italienischen) Antifaschisten für die Mehrheitsgesellschaft nicht identitätsstiftend ist - aus politischen und ideologischen Gründen durch die Bindung des italienischen Antifaschisten an die kommunistische Partei -, bleibt dieser Diskurs jedoch marginal.

Der kulturelle Diskurs: Er distanziert sich vom Arbeiter/-innenbild und stellt die italienische Kultur (Römisches Reich, Republiken, Künstler/-innen, Komponisten/-innen etc.) in den Mittelpunkt. Das Aufkommen dieses Diskures fällt in die $1970 e r$ Jahre, also in eine Zeit, als die Einwanderung italienischer Arbeiter/-innen durch die verstärkte Einwanderung hochqualifizierter italienischer Arbeitnehmer/-innen abgelöst wird. Generell zählen wir zu den Trägern dieses Diskurses die Organisationen »Amitiés italo-luxembourgeoises « und »italiani.lu«, die jedes Jahr einen »Miss-Italia-Luxemburg-Wettbewerb« organisieren, und das »Istituto italiano di cultura«, das der italienischen Botschaft nahesteht. Als Produzent des kulturellen Diskurses zählt auch die Organisation »Convivium«, die eine luxem-

80 | Allocution de S.A.R. le grand-duc lors du dîner offert à Son Excellence le président de la République italienne et à Madame Giorgio Napolitano (2 février 2009). Cour grandducale de Luxembourg, 28 décembre 2009. URL: www.monarchie.lu/fr/Presse/Discours/ 2009/02/VisiteEtat_Italie/index.html 
burgisch-italienische Kultur in den Vordergrund stellt. Auch wenn die Diskurse der verschiedenen Organisationen inhaltlich voneinander abweichen, so steht bei allen die Kultur im Mittelpunkt und weniger die historischen Ereignisse, wie es bei den oben genannten Diskursen der Fall ist.

\section{Die Ausstellung "Retour de Babel»}

Die Ausstellung »Retour de Babel« bot im Rahmen der »Kulturhauptstadt 2007《 einen historischen Überblick der Aus- und Einwanderungen Luxemburgs. Ziel war es, die Vielfältigkeit der Migrationen zu schildern, die gesellschaftlich nur wenig wahrgenommen wird.

\section{Der Ausstellungskatalog}

Die thematische Unterteilung des Katalogs nimmt jene der Ausstellung wieder auf: »Partir ${ }^{8_{1}}$ (Band 1), »Arriver ${ }^{82}$ (Band 2), »Rester, être ${ }^{83}$ (Band 3). Diese Verben verweisen auf Migration schlechthin: die Gründe, aus denen man ein Land verlässt, wie man sich zurechtfindet in einer neuen Welt und schließlich die Entscheidung trifft zu bleiben. Nur das letzte Verb >tanzt aus der Reihe<, da »être« (sein), einen Zustand beschreibt und nicht unbedingt zu einer prozesshaften Darstellung der Migration passt.

Neben Portraits von Einwanderern/-innen aus verschiedenen Nationen, die auch in der Ausstellung zu sehen waren, findet man im Katalog eine große Anzahl von wissenschaftlichen Artikeln, die verschiedene Themen der Migration behandeln. Des Weiteren zeigt der Katalog Portraits von verstorbenen historischen Persönlichkeiten wie Victor Hugo, der in Luxemburg politisches Asyl gefunden hatte, aber auch weniger bekannte Personen, die aus politischen oder ökonomischen Gründen nach Luxemburg einwanderten.

\section{Die Portraits}

Unter den insgesamt 57 Portraits findet man acht Italiener/-innen, alle Einwanderer/-innen oder Angehörige der sogenannten zweiten Generation. ${ }^{84}$ Diese acht

81 | Deutsch: "Weggehen “.

82 | Deutsch: "Ankommen".

83 | Deutsch: "Bleiben, sein “.

84 | Miserini, Renato/Scherre, Claudiner (2007): "Un choix de vie“, in: Reuter, Antoinette/ Ruiz, Jean-Philippe (2007): Retour de Babel. Itinéraires, Mémoires et Citoyenneté Gasperich: Retour de Babel a.s.b.I., Band 1, S. 108-116; Malvetti, Mario/Scherrer, Claudine (2007): "Plus on grandissait, plus on s'intégrait“, in: Reuter, Antoinette/Ruiz, Jean-Philippe: Retour de Babel. Itinéraires, Mémoires et Citoyenneté Gasperich: Retour de Babel a.s.b.l., Band 2, S. 58-63; Plebani, Emidio/Pairoux, Kristel (2007): "Pour qu'un pont résiste, il faut deux piliers", in: Reuter, Antoinette/Ruiz, Jean-Philippe: Retour de Babel. Itinéraires, Mémoires et Citoyenneté Gasperich: Retour de Babel a.s.b.I., Band 2, S. 154-161; Boggiani, 
Personen wurden auf italienisch oder französisch interviewt. Nach der Transkription wurden die Interviews z.T. übersetzt, zusammengefasst und schließlich für den Katalog überarbeitet. Dieser nicht unübliche Vorgang bringt jedoch mit sich, dass mehrere Stufen der Interpretation eingefügt wurden, die bestimmte Bilder der jeweiligen Portraitierten ergeben. Anders gesagt: Wenn schon keine Typen erstellt wurden, so wurden zumindest starke Akzente gesetzt. Auf diese Weise fand eine Verschiebung von der durch die Herausgeber/-innen des Katalogs beabsichtigten Darstellung angeeigneter Identitäten hin zu einer Darstellung zugeschriebener Identitäten statt, wobei diese jedoch identisch sein können. Die Zuschreibung geschieht durch die ausgewählten Titel der Interviews und die Untertitel, die die jeweiligen Texte strukturieren, die schon alleine grafisch den Blick des Lesers lenken. Das so entstehende identitätsstiftende Bild kann aber jenem, das der/die Interviewte von sich selbst hat, entsprechen.

Neben dem Text besteht jedes Portrait aus einer Fotografie, die speziell für die Ausstellung angefertigt wurde, sowie mehreren privaten Fotos und einem persönlichen Objekt, das für die Lebensgeschichte der Portraitierten wichtig ist. Schließlich sind die Migrationswege mit ihren jeweiligen Etappen grafisch dargestellt.

\section{Die inszenierten Fotos}

Auf jenen Fotos, die Andrès Lejona ${ }^{85}$ für die Ausstellung aufgenommen hat, stehen die Portraitierten jeweils in einer Ecke ihres Wohnzimmers, welche nach der Himmelsrichtung ihres Heimatlandes ausgerichtet ist. Der Fotograf versteht diese Ecke als >Pfeil<, der in Richtung der Herkunft der Portraitierten zeigt (»la direction de leur pays, de leurs origines«).

So stellt diese Ecke einen Bezug dar, der jedoch für eine/n Betrachter/-in nicht klar erkennbar ist. Zwar liegt sie räumlich hinter der eingewanderten Person, wodurch man die Symbolik so verstehen könnte, dass die Einwanderung abgeschlos-

Rosina/Scherrer, Claudine (2007): "Je ne me suis jamais vraiment sentie étrangère", in: Reuter, Antoinette/Ruiz, Jean-Philippe: Retour de Babel. Itinéraires, Mémoires et Citoyenneté Gasperich: Retour de Babel a.s.b.I., Band 2, S. 190-196; Peruzzi, Raymond/Caldognetto, Maria Louisa (2007): "A la maison des Romagnoli ...", in: Reuter, Antoinette/Ruiz, Jean-Philippe: Retour de Babel. Itinéraires, Mémoires et Citoyenneté Gasperich: Retour de Babel a.s.b.I., Band 2, S. 209-215; Piticco, Flora/Sander-Emram, Dominique (2007): "J'ai la double âme", in: Reuter, Antoinette/Ruiz, Jean-Philippe: Retour de Babel. Itinéraires, Mémoires et Citoyenneté Gasperich: Retour de Babel a.s.b.I., Band 3, S. 67-73; Rech, Louis/Scherrer, Claudine (2007); "Celui qui nie ses origines et son passé ne mérite pas l'avenir", in: Reuter, Antoinette/Ruiz, Jean-Philippe: Retour de Babel. Itinéraires, Mémoires et Citoyenneté Gasperich: Retour de Babel a.s.b.I., Band 3, S. 82-89; Rinaldis, Marinelle/ Pairoux, Kristel (2007): "Quand on regarde les gens dans leur individualité, on ne regarde pas leur nationalité“, in: Reuter, Antoinette/Ruiz, Jean-Philippe: Retour de Babel. Itinéraires, Mémoires et Citoyenneté Gasperich: Retour de Babel a.s.b.I., Band 3, S. 334-343.

85 | Vgl. auch Abschnitt 6.5. 
sen und die Bindung an Heimat und/oder Herkunft überwunden ist; doch die Erklärung des Fotografen, der zufolge damit »eine Richtung angeben« gemeint sei, stellt diese Interpretation infrage und legt vielmehr die Deutung als Nostalgie, Gebundenheit an das Ursprungsland, Unabgeschlossenheit mit der Vergangenheit, Spaltung (der Zugehörigkeit), Multikulturalität, Sich-Umschauen, ZurückGehen nahe.

\section{Italienische Einwanderung in Luxemburg: neue Perspektiven}

Das im Ausstellungskatalog »Retour de Babel« aufgefächterte Bild italienischer Einwanderung reproduziert eine ganze Reihe Klischees. Die meistgenannten sind die Großfamilie, die italienische Küche, Fußball, italienische regionale Verbände, die Mehrsprachigkeit, die katholische Religion und Luxemburg als »American Dream« im Sinne der Möglichkeiten, die Luxemburg den Einwanderern bietet. Wir werden hier nur auf einige dieser Elemente eingehen. Neben diesen üblichen Topoi sticht ein Element heraus, nämlich das gesellschaftliche und politisch links anzusiedelnde Engagement einiger der Portraitierten und/oder deren Verwandten.

Die Aspekte der »Großfamilie« und im weitesten Sinn der »Italiener als Gemeinschaft« (»Ils étaient nombreux les Italiens!«) verbunden mit der Idee der Solidarität werden u.a. von der Portraitierten Rosina Boggiani in ihrem Interview betont, anhand des Beispiels des Hausbaus als kollektivem Unternehmen. Die »Casa Grande« oder »Casa dei Romagnoli«, ein Haus im Arbeiterviertel Hiehl der Stadt Esch/Alzette, in dem über Jahrzehnte mehrere italienische Familien lebten, steht als Symbol der Großfamilie und der italienischen Gemeinschaft schlechthin. Raymond Peruzzi, dessen Interview mit »A la casa dei Romagnoli ...« betitelt ist, wuchs dort auf und bedauert, dass der Umzug in ein kleines Dorf eine regelrechte Isolierung von dieser Gemeinschaft zur Folge hatte. Ein ähnliches Beispiel findet man bei Mario Malvetti. Weitere Beispiele der Solidarität, gebunden an Wohnorte, sind bei Flora Piticco zu finden, die von der Solidarität in der Brillstraße redet und bei Louis Rech, der im italienischen Viertel in Dudelange aufgewachsen ist und dieses als eine »italienische Insel« bezeichnet. Durch diese Beschreibungen entsteht das Bild einer Einwanderungsgemeinschaft, die zusammenhält und unter sich bleibt. Zusätzlich zu den Erinnerungen, in denen mit Nostalgie an Italien gedacht wird, entsteht eine Nostalgie einer heute nicht mehr existierenden Gemeinschaft und der Solidarität, die mit ihr verschwunden ist.

Verbunden mit der Vorstellung des Zusammenlebens der italienischen Gemeinschaft ist das Bild der Mehrsprachigkeit, dargestellt als positive Dynamik oder als Schwierigkeit. Bei den in Luxemburg Aufgewachsenen wird, trotz eventueller Schwierigkeiten in der Schule, die Mehrsprachigkeit positiv und als Integrationsfaktor dargestellt. Eine Ausnahme bildet Marinella Rinaldis, die sich u.a. über das Schulsystem beschwert, in dem die Alphabetisierung auf Deutsch erfolgt.

Die italienische Küche wird oft als einer der kulturellen Beiträge der Italiener/innen in Luxemburg genannt. Für den früheren Bürgermeister Louis Rech ist die 
italienische Küche die letzte »Tradition«, die übrig geblieben ist, d.h. der letzte Bezug zur italienischen Herkunft. Für den Portraitierten Malvetti ist die italienische Küche eine zentrale Verbindung nicht nur zur Herkunft, sondern auch zu Italien als solches und vor allem zum Großvater, der den Stab, mit dem der Teig für die Pasta gemacht wird, 1924 aus Italien mitbrachte.

Was wir hier als >American Dream < bezeichnen, ist die Darstellung Luxemburgs als offenes Land, das den Eingewanderten unvergleichbare Möglichkeiten und Lebensqualität bot. Rosina Boggiani unterstreicht z.B., dass diejenigen, die nach Italien zurückgegangen sind, Luxemburg nachtrauern. Renato Miserini erzählt, dass er sich entschieden hat, nach Luxemburg auszuwandern, als er Zurückkehrende sah, die sich nach einigen Jahren in Luxemburg ein Haus in Italien bauen konnten. Trotz ihrer schlechten Erfahrungen ist auch Marinella Rinaldis der Meinung, dass sie durch ihr Aufwachsen in Luxemburg ein »anderes Schicksal vermieden ha[be]« (Rinaldis/Pairoux 2007: 341).

Im Kapitel »Rester, c'est s'engager « findet man die Portraits von Louis Rech und Flora Piticco, seines aufgrund des gewerkschaftlichen und politischen Engagements, ihres wegen der Mitarbeit in verschiedenen sich mit Migrationsfragen befassenden Verbänden. Im Übrigen sind insgesamt sechs von acht Portraitierten entweder selbst politisch engagiert, gemeinnützig tätig oder mit solchen Personen verwandt. Bei einem derart hohen Prozentsatz stellt sich die Frage, wie die Auswahl der interviewten italienischen Einwanderer/-innen im Ausstellungskatalog getroffen wurde. Denn durch diese besondere Zusammenstellung an Portraitierten bekommt der marginale Linksdiskurs einen höheren Stellenwert als üblich. So war auch Renato Miserini politisch und gewerkschaftlich aktiv und hat die Sektion der italienischen kommunistischen Partei in Luxemburg-Stadt mit aufgebaut. Raymond Peruzzi, Sohn des Widerstandskämpfers Luigi Peruzzi, betont zwar, dass er seinem Vater nicht in allem gefolgt ist, aber auch in diesem Portrait ist der Antifaschismus ein zentrales Element. Pater Emidio Plebani tritt seinerseits auf der Ebene der katholischen Kirche stark für Interkulturalität und somit für das Zusammenführen aller Gemeinschaften ein.

Allgemein vermitteln die acht Portraits den Eindruck einer progressiven, erfolgreichen >Integration<. Am häufigsten wird sie in den Kontext von Vereinen und Organisationen, beispielsweise eines Fußball- oder Gesangsvereins, gestellt. Parallel zu dieser Darstellung wird aber in den meisten Portraits die Frage der >Identität< aufgeworfen. Die Zusammenhänge zwischen >Integration< und >Identität $<$ haben für die jeweiligen Portraitierten ihre eigene Bedeutung. So meint Flora Piticco, dass die Italiener zwar »integriert« seien, aber nicht »ganz« - sie hätten sich die Luxemburger Mentalität angeeignet, aber ihre Wurzeln seien in Italien. Sie benennt diesen Zustand für sich selbst mit dem Begriff »double âme«. Unter dem Untertitel »Nous étions des étrangers« beschreibt sie weiter, dass nach dem Krieg ausländerfeindliche Bemerkungen den Italienern/-innen gegenüber abnahmen. Positive Urlaubserlebnisse der Luxemburger/-innen in Italien, aber auch der Zuzug von italienischen Mitarbeitern/-innen der Europäischen Institutionen hät- 
ten dazu beigetragen, das Italiener/-innenbild der Luxemburger/-innen positiv zu verändern. Der Titel des Interviews von Rosina Boggiani, »Je ne me suis jamais vraiment sentie étrangère « ist dabei leicht irreführend. Er suggeriert >Integration<, während ihre Erklärung, sich nicht fremd zu fühlen, sich nicht auf die luxemburgische Gesellschaft bezieht, sondern auf ihr unmittelbares Umfeld, das fast ausschließlich italienisch war. Dies wirft die Frage auf, was eigentlich gemeint ist, wenn öffentlich von der gelungenen oder >guten « Integration der Italiener gesprochen wird.

Abschließend wollen wir uns den drei weiblichen Portraits zuwenden. Bei der reinen Betrachtung der Fotos und der Portraitüberschriften entsteht der Eindruck einer gewissen Typenbildung. So stellt Rosina Boggiani das Frauenbild der >Mutter d dar, das aber vor allem durch die Fotos, bei denen es sich ausschließlich um Familienfotos handelt, entsteht. Neben diesem eher traditionellen Bild der familienorientierten >Mamma < gibt es die emanzipierte, engagierte Frau, Flora Piticco, Sprecherin der »Organisation Unione Donne Italiane«. Auf den Fotos sieht man sie auf großen Zusammenkünften mit einem Mikrofon in der Hand. Komplementär zu diesem Bild geht der Text auf das einerseits harte Arbeiter/-innenleben und andererseits auf Fremdheitserfahrungen ein. Im Kapitel »être« wird schließlich Marinella Rinaldis vorgestellt. Sie schreibt sich in keine große Rahmengeschichte ein, im Gegensatz zu den anderen Portraitierten, die oft historische Referenzen angeben. Außer einem kurzen Hinweis auf ihre Großeltern kommt kein Element der italienischen Einwanderungsgeschichte zur Sprache. Dadurch, dass sie sich konkret in der Gegenwart verortet, nehmen Bemerkungen über erfahrene Diskriminierung einen besonderen Stellenwert ein. Das Bild dieser jungen Frau entfernt sich von der öffentlichen Repräsentation der >guten Integration<, und somit wird mit ihrem Portrait zum Thema »être«, das schematisiert als Ziel und Ergebnis des erfolgreichen Migrationsprozesses »partir, arriver, rester, être« dargestellt wird, das positive Modell der italienischen Migration und Integration in Luxemburg infrage gestellt.

\section{Fazit}

Im Ausstellungskatalog »Retour de Babel« wird neben den üblichen Bildern über italienische Einwanderung in Luxemburg ein besonderer Akzent auf politisches, soziales und gewerkschaftliches Engagement gesetzt. Dementsprechend erhält der Linksdiskurs einen wichtigeren Stellenwert als üblich, was weniger mit der Art und Weise der Darstellung zu tun hat als mit der Auswahl der interviewten italienischen Einwanderer/-innen.

Die vom Konsensdiskurs zugeschriebene kollektive Identität der Italiener/-innen als Arbeiter/-innen, die sich gut integriert haben, wird einerseits durch Details bestätigt, andererseits aber auch durch die wichtige Rolle, die den antifaschistischen Organisationen und dem sozialen und politischen Engagement zugesprochen wird, angezweifelt. 
Auch die >gute Integration < der Italiener/-innen als Teil ihrer zugeschriebenen kollektiven Identität wird dadurch infrage gestellt, dass die Portraitierten Aussagen über ihre eigene Integration differenzieren und sie nicht direkt mit der Frage der eigenen Identität verbinden. Zwar spielen sowohl Herkunft als auch soziales Umfeld eine wichtige Rolle in der Selbstbeschreibung der Migranten/-innen und werden z.B. mit dem Begriff der doppelten Identität, oder »double âme«, bezeichnet.

Man muss aber bedenken, dass es ein Anliegen der Ausstellungsorganisatoren/-innen war, ein breiteres - und z.T. abweichendes - als das übliche Bild der Einwanderung in Luxemburg zu geben. So findet man bei den übrigen Portraits Nationalitäten, die noch keine Erwähnung im öffentlichen Diskurs finden. Das dargestellte Gesamtbild der Einwanderung in Luxemburg überwindet so das etablierte Bild, das in Luxemburg über Einwanderer/-innen vorherrscht: gut integrierte Italiener/-innen, eine vergleichsweise schwierigere portugiesische Einwanderung und Einwanderer aus Ex-Jugoslawien oder allgemein aus nicht EU-Ländern, die immer häufiger, parallel zu einem europäischen Illegalisierungsdiskurs, als kriminell dargestellt werden. Diesem diskriminierenden Diskurs gegenüber stehen die Portraits im Ausstellungskatalog, darunter die Geschichte einer Frau, die aus Niš (Serbien) über Frankreich nach Luxemburg einwanderte, lange bevor der Krieg dort ausbrach; oder eines Tänzers aus Brasilien; einer Mutter mit ihrem Kind, die beide ihren Kopf in einer Kiste verstecken, um nicht erkannt zu werden, weil sie ohne Papiere und daher illegal in Luxemburg leben. Außerdem bricht der Ausstellungskatalog mit seiner generell großen Zahl weiblicher Portraits mit der traditionellen Darstellung der Migration als männlich.

Die im Katalog dargestellte Vielfalt spiegelt des Weiteren das Kollektivsymbol »europäisches Labor« wieder, auch wenn die Bezeichnung nicht auftritt. Durch die Aufmachung selbst wird die Vorstellung eines offen Landes, das (fast) ohne Unbehagen mit mehr als $40 \%$ ausländischer Bevölkerung zurechtkommt.

\subsection{Schlussfolgerung: Nationale Identität IM POSTNATIONALEN ZEITALTER?}

Nationen sind »vorgestellte Gemeinschaften«, »imagined communities« (Anderson 1991). Um ein verbindliches Gefühl von Zugehörigkeit zu gewährleisten, sind sie auf die Bilderwelten der alten und neuen Medien angewiesen. Aus Sprachbildern und visuellen Bildern ist über einen langen Zeitraum hinweg ein großer Fundus an Kollektivsymbolen entstanden, aus dem sich die Nationen aus Gründen der Anerkennung, der Selbstdarstellung, aus Gründen der Abgrenzung oder der Polemik, aber auch zur Selbstverständigung und nicht zuletzt auch selbstkritisch bedienen.

Im postnationalen, globalen Zeitalter spielen die großen symbolischen Auseinandersetzungen vom Typ >tiefsinnige Deutsche< vs. >frivole Franzosen< (vgl. Florack 2001), die nicht selten Auftakt oder Begleiterscheinung realer Machtkämpfe 
bildeten, glücklicherweise keine Rolle mehr. Dennoch ist das politisch-kulturelle Handeln, das sich nach wie vor in nationalen oder regionalen Größeneinheiten bewegt, weiterhin auf symbolische Repräsentationen von Identitäten angewiesen. Die zunehmende Ausdifferenzierung der Gesellschaft, ihre Vernetzung und Spezialisierung, drängt diese Identitätsdiskurse zwar aus den zentralen Funktionsbereichen, die Frage nach dem kulturellen Zusammenhalt einer in einem überschaubaren Raum organisierten Gesellschaft wie Luxemburg stellt sich dabei aber umso drängender.

Die Beiträge zum Thema »Bilder und Identitäten« in Luxemburg haben am Beispiel unterschiedlicher Kommunikationsbereiche von Printmedien, Werbung, Comic, Kunst und Kunstdokumentation und ihren jeweils unterschiedlichen Adressaten/-innenkreisen gezeigt, dass die Suche nach und die Erörterung von Formen anschaulicher und prägnanter kollektiver Identitätsmuster zu den Alltagsdiskursen in Luxemburg gehört. Das bedeutet aber nicht, dass diese kollektiven Identitätsbilder alltäglich sind. Sie können durchaus größere Aufmerksamkeit auf sich ziehen, sind aber nicht beständig präsent. Um überhaupt generiert und wahrgenommen zu werden, bedürfen sie äußerer medialer Anlässe (das Kulturhauptstadtjahr, die Arcelor/Mittal-Debatte, die Bankendiskussion, Werbekampagnen etc.), die manchmal kaum vorhersehbar sind. Das macht positive Identitätszuschreibung schwierig und die häufig nur in negativer Form präsenten Identitätsaneignungen unübersichtlich. Man kann dahinter auch ein grundsätzliches Unbehagen im Umgang mit den besonders auf Bilder und Symbole angewiesenen Schematisierungen und Verrechnungen von Identitäten auf ein nationales Ganzes vermuten. Im 20. Jahrhundert sind die Vorstellungen kollektiver Identitäten gründlich diskreditiert worden.

Heutzutage lässt mit dem Verschwinden eines Ereignisses im Zuge der beschleunigten medialen Aufmerksamkeitskette scheinbar auch das Interesse an den daran gekoppelten Identitätsbildern nach. Der Mechanismus medialer Applikation nationaler Identitätssymboliken bestimmt in Luxemburg das Spannungsverhältnis zwischen zugeschriebenen und angeeigneten Identitäten. Auch in postnationalen Zeiten der Imagekampagnen können kollektive Identitätssymbole nicht ohne weiteres neu erfunden werden, genauso wenig aber lassen sich die überkommenen einfach fortschreiben.

\subsection{Literatur}

\section{Primärliteratur}

Czuga, Lucien/Leiner, Roger (1988): De Superjhemp géint de Bommeléer, Luxembourg: Ed. Revue (Neudruck 2001 im Sammelband 1).

Czuga, Lucien/Leiner, Roger (1989): Dynamit fir d'Dynastie, Luxembourg: Ed. Revue. 
Czuga, Lucien/Leiner, Roger (1990): D’Affair vum Jorhonnert, Luxembourg: Ed. Revue.

Czuga, Lucien/Leiner, Roger (1991): Den Dossier Hexemeeschter, Luxembourg: Ed. Revue (Neudruck 2006 im Sammelband 2).

Czuga, Lucien/Leiner, Roger (1992): Panik am Studio 2, Luxembourg: Ed. Revue.

Czuga, Lucien/Leiner, Roger (1993): Superjhemp contra Superjhemp, Luxembourg: Ed. Revue.

Czuga, Lucien/Leiner, Roger (1994): D’Geheimnis vun der Waliss, Luxembourg: Ed. Revue.

Czuga, Lucien/Leiner, Roger (1995): Requiem fir de Superjhemp, Luxembourg: Ed. Revue.

Czuga, Lucien/Leiner, Roger (1996): Operatioun grouss Botz, Luxembourg: Ed. Revue.

Czuga, Lucien/Leiner, Roger (1997): Geheimcode bloe Stär, Luxembourg: Ed. Revue.

Czuga, Lucien/Leiner, Roger (1998a): Aktioun Réiserbunny, Luxembourg: Ed. Revue.

Czuga, Lucien/Leiner, Roger (1998b): D’Patte wech vun Luxonit, Luxembourg: Ed. Revue.

Czuga, Lucien/Leiner, Roger (1999): Terror em den Troun, Luxembourg: Ed. Revue.

Czuga, Lucien/Leiner, Roger (2000): Lescht Chance fir Luxusbuerg, Luxembourg: Ed. Revue.

Czuga, Lucien/Leiner, Roger (2001): Alarm am Öro Zuch, Luxembourg: Ed. Revue.

Czuga, Lucien/Leiner, Roger (2002): S.O.S. Cosa Mia, Luxembourg: Ed. Revue.

Czuga, Lucien/Leiner, Roger (2003): D’Aaxt vum Béisen hat, Luxembourg: Ed. Revue.

Czuga, Lucien/Leiner, Roger (2004): De Fluch vun der 23, Luxembourg: Ed. Revue.

Czuga, Lucien/Leiner, Roger (2005): Countdown fir Kachkéisien, Luxembourg: Ed. Revue.

Czuga, Lucien/Leiner, Roger (2006): Déck Mënz fir de Prënz, Luxembourg: Ed. Revue.

Czuga, Lucien/Leiner, Roger (2007a): Superjhemp an déi grouss Gefor, Luxembourg: Ed. Revue.

Czuga, Lucien/Leiner, Roger (2007b): Kids, Kachkéis a Kuddelmuddel, Luxembourg: Ed. Revue.

Czuga, Lucien/Leiner, Roger (2008a): Luxusbuerger Lexikon, Luxembourg: Ed. Revue.

Czuga, Lucien/Leiner, Roger (2008b): De Kinnek vun Öropa, Luxembourg: Ed. Revue.

Czuga, Lucien/Leiner, Roger (2009): Cräsh am Paradäis, Luxembourg: Ed. Revue. 


\section{Sekundärliteratur}

Allocution de S.A.R. le grand-duc lors du dîner offert à Son Excellence le président de la République italienne et à Madame Giorgio Napolitano (2 février 2009). Cour grand-ducale de Luxembourg, 28.12.2009. URL: www.monarchie.lu/fr/ Presse/Discours/2009/02/VisiteEtat_Italie/index.html (20.11. 2009)

Anderson, Benedict 1991: Imagined Communities: Reflections on the Origin and Spread of Nationalism, London/New York: Verso.

Auxenfants, Marc (2007): »D'Finanzplaz. La place financière«, in: Sonja Kmec et al. (Hg.): Lieux de mémoire au Luxembourg. Erinnerungsorte in Luxemburg, Luxembourg: Saint Paul, S. 285-290.

Bernas, Steven (2009): La photographie et le sensible, Paris: L'Harmattan.

Calteux, Georges (2005): »Mir wëlle bleiwe wat mir sinn, mä wat sin mir?«, in: Projet Moien! (Hg.): Actes du cycle de conférences Lëtzebuergesch: Quo Vadis? Sproochenhaus Wëlwerwolz, Mamer: Wilwerwiltz, S. 143-154.

Canetti, Elias (2000[1960]): Masse und Macht, Frankfurt a.M.: Fischer.

Centre de documentation sur les migrations humaines (Hg.) (1999): LuxembourgItalie: Hommage au père Benito Gallo, Dudelange: Centre de documentation sur les migrations humaines.

Cicotti, Claudio (2007): »D'Italiener«, in: Sonja Kmec/Benoît Majerus/Michel Margue/Pit Peporte (Hg.): Lieux de mémoire au Luxembourg, Luxembourg: Imprimerie St. Paul, S. 109-114.

Damiani, Didier (o.A.): »Marco Godinho, Passeur de temps«. URL: www.galeriesdudelange.lu/artistes.php?artiste $=00121(08.10 .2009)$

di Felice, Paul (2009): »Jeanine Unsen, Odd, small and beautiful«, in: Café Crème, Mois Européen de la Photo 2009 (Hg): Luxembourg: Café Crème Edition, S. 28.

di Felice, Paul (2006): »Regards distanciés sur le Luxembourg. Les expériences photographiques de Valérie Belin, de Charles Fréger et de Joël Tettamanti«, in: Mudam Luxembourg, Eldorado, Luxembourg: Musée d'Art Moderne GrandDuc Jean, S. 6o-67.

Ditschke,Stephan/Anhut, Anjin (2009): »Menschliches, Übermenschliches. Zur narrativen Struktur von Superheldencomics«, in: Ditschke, Stephan/Kroucheva, Katerina/Stein, Daniel (Hg): Comics. Zur Geschichte und Theorie eines populärkulturellen Mediums, Bielefeld: transcript, S. 148, 149, 156.

Dubois, Philippe (1983): L'acte photographique, Paris/Bruxelles: Nathan/Labor.

Dünne, Jörg (2008): »Die Karte als Operations- und Imaginationsmix. Zur Geschichte eines Raummediums«, in: Jörg Döring/Tristan Thielmann (Hg.): Spatial Turn. Das Raumparadigma in den Kultur- und Sozialwissenschaften, Bielefeld: transcript, S. 49-70.

Florack, Ruth (2001): Tiefsinnige Deutsche, frivole Franzosen. Nationale Stereotype in deutscher und französischer Literatur, Stuttgart: Metzler. 
Gallo, Benito (1992): Centenaire. Les Italiens au Luxembourg. 1892-1992, Luxembourg: Imprimerie St. Paul.

Garcia, Robert/Stoldt, Jürgen (2004): »Kulturhauptstadt: die Zweite«, in: forum 241, S. 4-7.

Gerhard, Ute/Link, Jürgen/Parr, Rolf (2004): »Interdiskurs«, in: Metzler Lexikon Literatur- und Kulturtheorie, hg. v. Ansgar Nünning. Dritte, aktualisierte u. erweiterte Auflage, Stuttgart/Weimar: Metzler, S. 293f.

Haas, Luke (2007): Comics in aus und über Luxemburg, Luxembourg: Ed. Schortgen.

Hahn, Sylvia (2008): Migration - Arbeit - Geschlecht. Arbeitsmigration in Mitteleuropa vom 17. bis zum Beginn des 20. Jahrhunderts, Göttingen: V\&R.

Haupt, Heinz-Gerhard/Crossick, Geoffrey (1998): Das Kleinbürgertum. Eine europäische Sozialgeschichte des 19. Jhd, München: C.H. Beck.

Hick, Thierry (2003): »A la découverte d'un lieu. Exposition de photographies d'Yvon Lambert à Lasauvage. Une ville passée au crible«, in: La Voix du Luxembourg, 06.09.2003.

Huntington, Samuel (1996): The Clash of Civilizations and the Remaking of World Order, New York: Simon \& Schuster.

Jakobson, Osipovich Roman (1960): Linguistics and Poetics, Cambridge: Ed. Thomas Sebeok/MA: MIT Press.

Jungblut, Marie-Paule (2007): »D’Spuerkess«, in: Sonja Kmec et al. (Hg.): Lieux de mémoire au Luxembourg. Erinnerungsorte in Luxemburg, Luxembourg: Saint Paul.

Klemperer, Victor (2007[1957]): LTI. Notizbuch eines Philologen, Stuttgart: Reclam.

Kmec, Sonja (2007): »Gibraltar des Nordens«, in: Sonja Kmec/Benoît Majerus/ Michel Margue/Pit Péporté (Hg.), Lieux de mémoire au Luxemburg. Usages du passé et construction nationale, Luxembourg: Saint-Paul, S. 267-272.

Koff, Harlan (2008): Fortress Europe or a Europe of Fortress? The Integration of Migrants in Western Europe, Bruxelles u.a.: Peter Lang.

Krieps, Rosch (1991): »Parallelen zum Fort Thüngen«, in: forum 130, S. 22.

Krotz, Friedrich (2003): »Medien als Ressource der Konstitution von Identität. Eine konzeptionelle Klärung auf der Basis des Symbolischen Interaktionismus«, in: Carsten Winter/Tanja Thomas/Andreas Hepp (Hg.): Medienidentitäten. Identität im Kontext von Globalisierung und Medienkultur, Köln: Halem, S. 27-48.

Labelle, Sarah/Bonaccorsi, Julia: »La contribution de la photographie d'écrivain à la médiation littéraire sur le web. Le cas de Raymond Queneau«. URL: www. archimuse.com/publishing/ichimo5/labelleSELECTo5.pdf (01.10.2009)

Lambert,Yvon (1998): Derniers feux. Photographies, Esch-sur Alzette: Ville d'Eschsur-Alzette.

Lambert,Yvon/Helminger, Nico (2005): Brennweiten der Begegnung, Differdange: Ville de Differdange. 
Langers, Jean (1999): L'hétérogénéité de la main-d'œuvre étrangère au Luxembourg, Differdange: STATEC, CEPS/Instead, IGSS, Population et emploi 3, série bleue, S. 1-4.

Lejona, Andrés (2009): »F(en)ê(r)es nationales«, in: Rendez-Vous. City Magazine Luxembourg 6, S. 46-57.

Link, Jürgen (1983): Elementare Literatur und generative Diskursanalyse, München: Fink.

Link, Jürgen (2006): Versuch über den Normalismus. Wie Normalität reproduziert wird, 3. ergänzte, überarbeitete und neu gestaltete Ausgabe, Göttingen: Vandenhoek \& Ruprecht.

Lorgé, Marie-Anne (1998): »La Habana«, in: Luxemburger Wort, 05.05.1998, S. 5.

Luxembourg and Greater Region, European capital of culture 2007. Final Report, Luxembourg (2007).

Manderscheid, Roger (2000): Summa Summarum. Gedichte aus einem vergangenen Jahrhundert. Mit Zeichnungen vom Autor, Echternach: Phi-Verlag.

Manderscheid, Roger (2001): Schwarze Engel. Geschichten mit 23 Zeichnungen des Autors, Nospelt: Ed. ultimomondo.

Manderscheid, Roger (2003): Der Aufstand der Luxemburger Alliteraten. Notizen zur Entwicklung der luxemburger Literatur in der zweiten Jahrhunderthälfte, Esch: Phi-Verlag.

Margue, Michel (2007): »Les dominations étrangères«, in: Sonja Kmec/Benoît Majerus/Michel Margue/Pit Péporté (Hg.): Lieux de mémoire au Luxemburg. Usages du passé et construction nationale, Luxembourg: Saint-Paul, S. 29-34.

Ndiaye, Pap (2008): La condition noire. Essai sur une minorité française, Paris: Calmann-Lévy.

Merian Magazin: Luxemburg, Jg. 60, Nr. 03, 2007.

Noiriel, Gérard (1992): Le Creuset français. Histoire de l'immigration (XIXe-XXe siècle), Collection Points Histoire, Paris: Seuil.

Ogden, Charles Kay/Richards, Ivor Amstrong (1923): The Meaning of Meaning. 8. Auflage, New York: Harcourt, Brace \& World, Inc.

Parr, Rolf (2009): »Wie konzipiert die (Inter-)Diskurstheorie individuelle und kollektive Identitäten? Ein theoretischer Zugriff, erläutert am Beispiel Luxemburgs«, in: forum 289, S. 11-16.

Parr, Martin (2007): Luxembourg 2006/Assorted Cocktail, Luxembourg: Luxembourg et Grande Région, Capitale européenne de la Culture 2007.

Parr, Rolf (2009): »Diskursanalyse«, in: Methodengeschichte der Germanistik, hg. v. Jost Schneider, Berlin: de Gruyter, S. 89-107.

Parr, Martin (2006): Mexico, London: Chris Boot Ltd.

Pauly, Michel (1991): »Die Schlacht um Fort Thüngen«, in: forum 128-129, S. 3-7.

Pauly, Michel (2000): »Eglise et immigration. Un entretien avec le père Benito Gallo«, in: forum 197, S. 59-62. 
Pauly, Michel (1984): »L'immigration dans la longue durée«, in: Lëtzebuerg de Lëtzebuerger? Le Luxembourg face à l'immigration, Luxembourg: Guy Binsfeld, S. 7-21.

Peirce, Sanders Charles (2000): Semiotische Schriften. 3 Bände, Frankfurt a.M.: Suhrkamp.

Pigeron-Piroth, Isabelle (2009): »Le secteur public«, in: Economie et statististiques, Working Papers du Statec 34, S. 3.

Poivert, Michel (2002): La photographie contemporaine, Paris: Flammarion.

Reuter, Antoinette (1995): »Cinq siècles de présence italienne au Luxembourg XIIIe-XVIIIe siècles«, in: Antoinette Reuter/Denis Scuto (Hg.): Itinéraires croisés. Luxembourgeois à l'étranger, étrangers au Luxembourg/Luxemburger im Ausland, Fremde in Luxemburg. Menschen in Bewegung, Esch/Alzette: Ed. Le Phare, S. 46-57.

Reuter, Antoinette/Ruiz, Jean-Philippe (2007): Retour de Babel. Itinéraires, Mémoires et Citoyenneté, 3 Bände, Gasperich: Retour de Babel a.s.b.l.

Sayad, Abdelmalek (1999): La double absence. Des illusions de l'émigré aux souffrances de l'immigré, Paris: Seuil.

Schaeffer, Jean-Marie (1987): L'image précaire. Du dispositif photographique, Paris: Seuil.

Schäffner, Christina (1993): »Die europäische Architektur - Metaphern der Einigung Europas in der deutschen, britischen und amerikanischen Presse , in: Adi Grewenig (Hg.): Inszenierte Informationen. Politik und strategische Information in den Medien, Wiesbaden: Verlag für Sozialwissenschaften, S. 13-30.

Schulz, Christian (2008): »Die >Metropolisierung< Luxemburgs«, in: Wilhelm Amann/Georg Mein/Rolf Parr (Hg.): Periphere Zentren oder zentrale Peripherien? Kulturen und Regionen Europas zwischen Globalisierung und Regionalität, Heidelberg: Synchron, S. 89-98.

Scuto, Denis (2008): »Historiographie de l'immigration au Luxembourg«, in: Hémecht 60, S. 391-413.

Stoldt, Jürgen (2008): »Luxemburg - Kern Europas«, in: Aus Politik und Zeitgeschichte 8, S. 19-25.

Weltonline - Wirtschaft (25.06.2009): dpa-Meldung. URL: www.welt.de/die-welt/ article4001464/Luxemburger-sind-die-reichsten-Buerger-in-der-EU.html

Wey, Claude (2005): »Discours et politiques d'immigration et d'intégration au Luxembourg«, in: Vincent Ferry/Piero-D. Galloro/Gérard Noiriel (Hg.): 20 ans de discours sur l'intégration, Paris: L'Harmattan, S. 151-162.

Williams, Val (2004): Martin Parr, London: Phaidon Press Ltd. 


\section{Alltagskulturen und Identitäten}

Christel Baltes-Löhr, Agnes Prüm, Rachel Reckinger, Christian Wille

\subsection{Einleitung: Zur wechselseitigen Relevanz von AlLtagsKuLtUREN UND IDENTITÄTSKONSTRUKTIONEN}

Der Schwerpunkt dieses Beitrags liegt auf der empirischen Analyse von Interaktionen im sozialen Alltag. Unsere gemeinsamen Forschungsfragen lauten dabei: Wie werden alltagskulturelle Identitäten im Spannungsfeld zwischen zugeschriebenen und angeeigneten Identitäten ausgehandelt? Wie und warum artikuliert sich diese identitäre Wechselbeziehung in der Alltagspraxis? Welche Erklärungen, Legitimierungen und Interpretationen für Handeln und Einstellungen werden gegeben? Diese Fragestellungen werden von Forschern/-innen aus unterschiedlichen disziplinären Blickwinkeln anhand der Bereiche Geschlechterwahrnehmungen, >banale< Konsumformen und soziale Ordnungen beleuchtet.

Die im Folgenden diskurspraktisch (s.u.) erschlossene Alltagspraxis konstituiert sich aus kollektiv geteilten Werten, Einstellungen und Normen (>Conceptas $<$ ), sowie aus Formen des Wahrnehmens, Glaubens, Bewertens und Handelns, wie sie sich in der sozialen Interaktion manifestieren (>Perceptas<). (Bolten 2007) Dabei schwingt in den unten vorgestellten Ergebnissen stets das Kollektive im Individuellen und das Individuelle im Kollektiven mit, was nicht zuletzt eine Veränderbarkeit und Vielschichtigkeit der untersuchten Identitäten einräumt. Diese Grundlegung des Identitätsbegriffs verweist zunächst auf seine Pluridimensionalität, welche Individuen nicht auf feste Kategorien (z.B. Frau/Mann) reduziert, sondern ebenso kontextuelle und intersektionale Zugehörigkeiten (z.B. sozio-kulturelle Milieus, Altersgruppen, Affinitätsgruppen usw.) berücksichtigt.

\section{Alltagskulturen in praxeologischer Perspektive}

Die folgenden Untersuchungen fokussieren somit nicht auf vorgefertigte kognitive Bedeutungs- und Sinnstrukturen von >alltäglicher Kultur<, sondern auf ihren prak- 
tischen Einsatz - wobei es nicht darum geht, was Menschen jeden Tag tun, sondern welche Selbstverständlichkeiten und Irritationen in den Einstellungen und im Handeln von den Subjekten geteilt werden. Denn es wird davon ausgegangen, dass Kultur ihren Ausdruck in der konkreten Auseinandersetzung mit der dinglichen und sozialen Umwelt findet: »Kultur lässt sich [...] erst im Umgang mit Dingen und Körpern wirklich >dingfest<, d.h. sichtbar, aufzeigbar, nachweisbar, nachvollziehbar machen.« (Hörning/Reuter 2004: 12) Angesprochen werden damit Praxiszusammenhänge, in denen sich das Kulturelle artikuliert. Hier sind besonders Formen des Zwischenkategorialen wie etwa »Third Spaces« (Bhabha 2000) bedeutsam, die unter dem Stichwort der Ambivalenz erläutert werden. Das skizzierte Kulturverständnis wird im Folgenden unter dem Begriff >Alltagskulturen < gefasst, wobei der hier wiederum gewählte Plural die Aufmerksamkeit auf parallel verlaufende und unterschiedliche Prozesse sozialer Interaktion und deren Effekte lenken soll.

Der erste Aspekt dieses Doppelbegriffs unterstreicht die Lebensnähe unseres Ansatzes, der sich auf unhinterfragte, »vertraute Selbstverständlichkeiten, eingelebtes Alltagswissen und routinisiertes Alltagshandeln« (Hillmann 2007: 19) konzentriert. Routinen, Rituale und Ausgestaltungen sind hierbei nicht als mechanische, starre Wiederholungen von alltäglichen Einstellungen und Handlungsabläufen, sondern als permanente, geringfügige Verschiebungen im Sinne eines Wandels aufzufassen (Butler 1993; Deleuze 1968), die Derrida mit dem Konzept der >différance < beschreibt (Derrida 1968). Die hier angesprochenen Verschiebungen lassen einen individuellen Handlungsspielraum zu, stellen die Intersubjektivität in Handlungssituationen aber nicht infrage. Kollektive Wissensbestände können unsystematisch, wertend und teilweise widersprüchlich sein (Schütz 1972), d.h. eine pragmatische Vereinfachung von alltäglichen Handlungen darstellen - womit sie eine gewisse interpretative Offenheit mit einer normativen Regelhaftigkeit verbinden. Trotz der intersubjektiven Konsensfähigkeit von Alltagswissen »machen sich infolge sozialstruktureller, gruppenspezifischer und biographischer Einflüsse individuelle Differenzierungen [...] bemerkbar« (Hillmann 2007: 20), welche wiederum auf das Alltagswissen zurückwirken.

Der zweite Aspekt des Begriffs >Alltagskulturen< schließt an die kulturtheoretische Debatte an. Wie oben angedeutet, geht es hier nicht um ein normatives oder holistisches Begriffsverständnis von Kultur, vielmehr steht das wissens- und bedeutungsorientierte Kulturverständnis im Vordergrund (Reckwitz 2001; Reckwitz 2004). Dieses geht davon aus, dass vor dem Hintergrund der symbolischen Ordnungen der sozialen Welt unterschiedliche Verhaltenskomplexe entstehen, reproduziert und verändert werden und die Ebene des Kulturellen bilden. Dieser Kulturbegriff wird in den Sozial- und Kulturwissenschaften fortwährend angepasst und weiterentwickelt, wobei sich die Frage stellt, ob Kultur als eine Konfiguration von übersubjektiven symbolischen Strukturen oder als Ergebnis subjektiv-interpretativer Leistungen aufzufassen ist. Dieser Fragestellung kann mit einer praxeologischen Synthese beider Perspektiven begegnet werden. Während die strukturalistische Sicht hilft, Kultur als übersubjektive Zuschreibung aufzudecken, verengt 
diese Perspektive den Blick auf Phänomene des kulturellen Wandels oder der subjektiven Aneignung. Hier greift die Konzeptualisierung von Kultur als Ergebnis subjektiver Interpretationsleistung, etwa wenn die alltagskulturellen und innovativen Aneignungen der Subjekte untersucht werden. Diese erfolgen jedoch nicht losgelöst von sozio-kulturellen Bedingungen und Ressourcen, die den handelnden Subjekten - durch ihre verschiedenen, jedoch eingeschränkten Positionen im sozialen Raum - zur Verfügung stehen, wie es die Praxeologie impliziert. (Bourdieu 2000 [1972]; Bourdieu 1980)

Dieser Beitrag folgt dem skizzierten >praxeologischen Synthesemodell < und betrachtet Prozesse der identitären Zuschreibung im dargelegten strukturalistischen Sinn und die Aneignungsprozesse bzw. -praktiken als interpretative Leistungen der Subjekte. Über die Zusammenführung beider Perspektiven kann der Dualismus von Kultur als Struktur einerseits und Kultur als subjektive Leistung andererseits überwunden werden, indem phänomenologisch und strukturalistisch orientierte Ansätze theoretisch und empirisch miteinander verknüpft werden. Das bedeutet, hier werden die sozialstrukturellen Einbettungen im sozialen Raum gleichzeitig mit den subjektiven Einstellungen und Handlungen der Akteure gedacht. In diesem Sinne repräsentiert eine Praktik - sei es als Zuschreibung bzw. Aneignung von Genderkonzepten, Konsumformen oder gesellschaftlicher Ordnungskategorien einen Komplex von diskurspraktisch vollzogenen (s.u.), sinnhaften und wandelbaren Verhaltensroutinen, die durch eine implizite >Logik des Alltags < zusammengehalten werden. Die Beiträge in diesem Kapitel versuchen solche alltagskulturellen Logiken aufzudecken und knüpfen hierfür an die Praktiken der sozio-kulturellen Milieus der Luxemburger Wohnbevölkerung an.

\section{Konstruktionen von Identitäten als soziale Praxis}

Die alltagskulturelle Identitätsarbeit in den untersuchten Bereichen charakterisiert sich durch eine gewisse >Unsichtbarkeit<, da sie in der Alltagspraxis i.d.R. nicht hinterfragt wird und der Pragmatik des konkreten Handelns unterliegt. Erst die Befragungssituation führt zu einer reflektierten Verbalisierung bzgl. der betreffenden Thematik. ${ }^{1}$ Sie ermöglicht Einblicke in subjektive und in den Interviewinteraktionen artikulierte Sinnwelten. Sowohl dieser diskursive Akt als auch seine Bedeutungsinhalte werden mit Foucault als >Diskurspraktiken< aufgefasst:

Es handelt sich darum, die diskursiven Praktiken in ihrer Komplexität und in ihrer Dichte erscheinen zu lassen, zu zeigen, dass Sprechen etwas tun heißt - etwas anderes, als das auszudrücken, was man denkt, das zu übersetzen, was man weiß, etwas anderes auch, als die Strukturen einer Sprache spielen zu lassen; zu zeigen, dass eine Aussage einer bereits vorher existierenden Folge von Aussagen hinzuzufügen heißt, eine komplizierte und

1 | Dies bedeutet nicht, dass im Alltag nicht reflektiert wird, sondern vielmehr dass alltagskulturelle Entscheidungen während ihrer Umsetzung kaum hinterfragt werden. 
kostspielige Geste zu tun, die Bedingungen impliziert (und nicht nur eine Situation, einen Kontext, Motive) und die Regeln umfasst (die von den logischen und sprachlichen Konstruktionsregeln verschieden sind); zu zeigen, dass eine Veränderung in der Ordnung des Diskurses nicht 'neue Ideen', ein wenig Erfindungskraft und Kreativität, eine andere Mentalität, sondern Transformationen in eine Praxis [...] voraussetzt. (Foucault 1969: 297-298; )

Dies bedeutet einerseits, dass unterschiedliche Diskurse sich wechselseitig beeinflussen und andererseits, dass ihre Interaktionen performativ sind, so dass die Personen - in unserem Fall die Befragten - »etwas mit den Worten tun« (Austin 1970). Jedoch >tun< ebenso die Worte etwas mit ihnen. Dieses Ineinandergreifen bringt >Diskurspraktik< auf den Begriff und verdeutlicht, dass Diskurse die Praktiken strukturieren (Diskursivität der Praktiken) und gleichzeitig Praktiken die Diskurse strukturieren (Performativität der Diskurse). (Reckinger 2008) Diese Sichtweise hilft, Diskurse und Praktiken nicht als autonome, sozio-kulturell freischwebende Gebilde zu betrachten, sondern den Blick auf ihre konstruierte Relation zu lenken.

In diesem Sinn ist aus methodischer Sicht zu hinterfragen, inwiefern soziale Praktiken mit empirischen Erhebungstechniken erfasst werden können. Grundsätzlich ist dabei zwischen Verfahren zu unterscheiden, mit denen soziale Praktiken mittelbar bzw. unmittelbar zugänglich werden. Zu Letzteren zählen z.B. teilnehmende Beobachtungen oder experimentelle Verfahren, welche kontrollierte Rahmenbedingungen und damit eine Alltagsferne für die Befragten implizieren. Hingegen wird mit Befragungen, Interviews oder Inhaltsanalysen ein mittelbarer Zugriff möglich, der den verbalen Zugang privilegiert und daher für unsere Zwecke geeigneter ist. So war es z.B. in qualitativen Interviews und quantitativen Befragungen möglich, mit den handelnden oder an sozialen Praktiken beteiligten Subjekten in Kontakt zu treten und über ihre Bekundungen bestimmte Informationen über soziale Praktiken, ihre Regelmäßigkeit und Häufigkeit sowie über ihre subjektiven Bedeutungen zu erhalten. Ferner bietet die diskurspraktische Inhaltsanalyse die Möglichkeit, verschiedene Handlungsaspekte zu rekonstruieren, wobei sie nicht auf die Handlungen selbst fokussiert, sondern auf ihre Performativitäten. Dabei geht es hier um die Analyse von Gesetzestexten, Informationsmaterialien und statistischen Daten mit dem Ziel, Spuren sozialer Praktiken aufzudecken und Rückschlüsse auf >Logiken des Alltags < zu ziehen.

Neben dem erläuterten methodischen Aspekt gilt es, weiterführende Überlegungen hinsichtlich der betrachteten Identitäten bzw. Differenzsetzungen durch die Subjekte vorzunehmen. (Reuter/Wiesner 2008) Die Beschäftigung mit diesen Kategorien ist im Zuge von Globalisierung und Liberalisierung vielschichtiger geworden. Formen der Hybridisierung oder Bindestrichkonstruktionen stellen klassische Theorieangebote auf den Prüfstand und unterlaufen binäre Differenzvorstellungen und Ordnungskategorien. So werden die theoretischen Figuren des Zwischenkategorialen (Reuter/Wiesner 2008) im Rahmen poststrukturalistischer und postmoderner Ansätze zunehmend als ambivalente Gegenwartsphänomene (wieder-)entdeckt. Ihnen gemeinsam ist das Aufbrechen der binären Logiken von 
weiblich/männlich, gut/schlecht, fremd/vertraut usw., die im Allgemeinen als Erklärungskategorien herangezogen werden. Konzepte des Zwischenkategorialen (Bhabha 2000; Welsch 2005; Breinig/Lösch 2006) versuchen die Durchdringung von vorgängig gesetzten Differenzen zu erfassen und lenken das Augenmerk auf »prozesshafte, dynamische, flüssige Vermischungen, die sich nicht ohne weiteres feststellen lassen « (Reuter/Wiesner 2008: 132). Der so umschriebene ambivalente Zustand des Dazwischen, welcher sich gegen klare Zuordnungen sperrt, erfindet sich permanent neu.

Das Forschungsinteresse in diesem Kapitel richtet sich u.a. auf solche flüchtigen Momente der Ambivalenz auf der Ebene des Alltagskulturellen. Sie geht davon aus, dass die Binarität von Differenzen im Rahmen sozialer Praktiken aufgebrochen werden kann und einer >Logik des Alltags < folgend sich innovativ rekonfiguriert. Somit geht es nicht um die Auflösung von Differenzen, sondern um die alltagskulturelle Überschreitung von binären Kategorien. Der Blick verschiebt sich somit von der binär codierten Logik des »Entweder-oder « zur ambivalenten Logik des »Sowohl-als-auch« (vgl. Baltes-Löhr 2006: 70) als ein weitgehend vernachlässigter Aspekt alltagskultureller Praktiken. Diese Verschiebung soll nicht als Ersetzung, sondern als Erweiterung gedacht werden, da auf phänomenologischer Ebene sowohl die Rekonstruktion von Binaritäten als auch ihre Überschreitung und Neukonfiguration auszumachen ist.

Vor diesem Hintergrund werden im Folgenden in den alltagskulturellen Bereichen der Geschlechterwahrnehmung, Konsumformen und Aneignung von sozialen Ordnungen beispielhaft verschiedene Momente der Ambivalenz und die damit verknüpften Dynamiken von Identitätskonstruktionen betrachtet. Zunächst werden Wirkmächtigkeiten von Geschlechterbildern eruiert und nach der Virulenz von Geschlecht bei in Luxemburg lebenden Frauen und Männern gefragt. Hegemonial wirksame Zuschreibungsprozesse im Kontext der Konstruktionen von Geschlechteridentitäten werden ebenso betrachtet wie individuelle Aneignungsprozesse in ihren unterschiedlichen Äußerungsformen. Hierzu werden homogenisierende bzw. differenzierende Vorstellungen von Geschlechteridentitäten vor einer intersektionalen Folie beleuchtet und der Relevanz der Dichotomie »konstruktivistische vs. essentialistische Geschlechterbilder « nachgegangen. Neben der Frage nach der Bedeutung der Geschlechterzugehörigkeit im Umgang mit anderen Menschen werden Favorisierungen unterschiedlicher Modelle zur Vereinbarkeit von Beruf und Privatleben in Bezug zueinander und den jeweiligen Milieus vorgestellt. Quer hierzu wird die kategoriale Trennschärfe von Geschlecht als Analysekategorie hinsichtlich alltagskultureller Genderthematiken in ihrer Verschränkung zwischen Zuschreibungs- und Aneignungsprozessen überprüft.

Des Weiteren wird die Norm und das Verständnis von >gutem< Essen zwischen politischem Leitbild und individueller Alltagspraxis thematisiert. Die Analyse konzentriert sich in einem ersten Schritt auf ein zuschreibendes politisches Handlungskonzept, das Gesundheitsorientierung - kreativ, aber objektbezogen - in seinen Empfehlungen zu vermitteln sucht. Anschließend wird der Schwerpunkt 
auf alltägliche Ernährungspraxen gelegt, deren Aneignungsformen um Eigenverantwortung, Handlungspotential und hedonistischen Pragmatismus - in eher personenbezogener Perspektive - kreisen. Die dynamischen Wechselwirkungen zwischen diesen beiden Interpretationstypen von >gutem < Essen eröffnen kontextgebundene Spielräume für Identitätskonstruktionen.

Schließlich sollen am Beispiel des Grenzgängerwesens in Luxemburg Aussagen über Prozesse der Identitätskonstruktion getroffen werden. Dabei steht die Wahrnehmung der grenzüberschreitenden Pendler/-innen durch die Luxemburger Wohnbevölkerung im Vordergrund, die hier verschiedene Formen der Konstruktion dieses Phänomens aufzeigt. In diesem Sinne sollen Identitätsaneignungen bzw. Positionierungen der Luxemburger Wohnbevölkerung gegenüber dem Grenzgängerwesen nachgespürt werden. Hierfür werden alltagskulturelle Einund Ausschlussstrategien beleuchtet und darauf aufbauend der Status des Grenzgängers im Großherzogtum bestimmt. In einem zweiten Schritt wird der Frage nachgegangen, welche Rückschlüsse die aufgedeckten und teilweise ambivalenten Aneignungen auf Prozesse der Identitätsbildung zulassen.

\subsection{Permanente Performanzen: gender in motion}

In diesem Teil der Untersuchung werden Wirkmächtigkeiten von Geschlechterbildern eruiert. Welche Repräsentationen von Geschlecht sind bei in Luxemburg lebenden Frauen und Männern virulent? Ausgehend von Zuschreibungsprozessen von Geschlechteridentitäten via hegemoniale Geschlechterdiskurse richtet sich der Blick der Untersuchung vor allem auf Prozesse der Aneignungen von Geschlechteridentitäten und Geschlechterbildern im intersektionalen Kontext. Hiermit verbunden sind zwei forschungsleitende Aspekte: einmal die Frage, inwieweit dualistisch konnotierte Unterscheidungen zwischen essentialistischen und konstruktivistischen Geschlechterbildern im empirischen Material abgebildet sind, und zum anderen die Frage nach der Trennschärfe von Geschlecht als Analysekategorie. Inwieweit kommt also der Kategorie >Geschlecht< hinsichtlich des Antwortverhaltens der Befragten eine signifikante Bedeutung zu?

Im Rahmen einer repräsentativen Studie und 27 leitfadengestützten Interviews wurden die Vorstellungen zu Weiblichkeiten, Männlichkeiten sowie zu Homo- und Transsexualität und die Bedeutung der Kategorie >Geschlecht< für den Umgang mit anderen ebenso ermittelt wie Einschätzungen hinsichtlich der in Luxemburg existierenden Gleichstellung bzw. Diskriminierungen der Geschlechter. Daneben ging es um die von den Befragten favorisierten Gendermodelle mit Blick auf eine verbesserte Vereinbarkeit von Beruf und Privatleben und nicht zuletzt um Kenntnisse zu nationalen Gesetzen und Kampagnen rund um die Thematik >Gender ${ }^{2}$.

2 | Hierzu wurden begleitend zu diesem Forschungsprojekt die seit Ende der 1960er Jahre in Luxemburg zum Thema ,Gleichberechtigung/Gleichstellung der Geschlechter 
Die folgende Darstellung bezieht sich somit auf die Auswertung von vier statistischen Itembatterien mit insgesamt 22 Items sowie 290 Interviewsequenzen aus 27 Interviews mit Frauen und Männern.

\section{Theoretische Rahmung}

Identitäten und damit auch Geschlechtsidentitäten werden als pluridimensionale, intersektionale Repräsentationskonstruktionen betrachtet. (Vgl. McCall, 2005; Hirschauer, 2001; Klinger/Knapp 2005; Baltes-Löhr 2006: 66) Neben der Geschlechterkategorie, die lange Zeit als die gesellschaftliche Strukturkategorie betrachtet wurde, und dem körperbezogenen biologischen Geschlecht >sex < treten auch ethnische und sozio-ökonomische Aspekte ebenso wie Alter in den Vordergrund, wenn es um Konstruktionen von Identitäten geht. ${ }^{3}$ Unabhängig von der Anzahl der >Sektionen < wird die Bedeutung der Kategorie >Geschlecht < durch ihre intersektionale Kontextualisierung entdramatisiert.

Neben ihrer intersektionalen Verfasstheit sind Identitäten als Momentaufnahmen von ständig sich in Bewegung befindlichen und sich verändernden performativen Konstruktionsprozessen zu betrachten. Trotz aller Fluidität und Veränderbarkeit scheinen bestimmte identitäre Aspekte sich eher zu verflüchtigen, während andere sich sedimentieren, sei es im hegemonialen Diskurs und/oder der alltagsweltlichen individuellen Praxis. Performative Konstruktionsprozesse, performative Handlungen (Butler 1993: 123) rufen durch Benennungen und Inszenierungen sowie durch wiederholte Darbietungen in Zeit und Raum ihren Gegenstand, also auch Identitäten, hervor. In solchen sich wiederholenden performativen Handlungen liegen Möglichkeiten für Verfestigungen des Wiederholten sowie zum diskursiven Durchbrechen vorherrschender Bilder und damit zur Vervielfältigung binärer Muster, in dem z.B. andere Facetten von Geschlechtsidentitäten hervorgebracht werden.

Bergen Wiederholungen Möglichkeiten, vielfältige Aspekte eines Gegenstandes präsent werden zu lassen, so dass sich dualisierende Hierarchien verändern, verschieben und auflösen können, dann ist die Wirksamkeit von Wiederholung als enthomogenisierend und heterogenisierend $\mathrm{zu}$ beschreiben. Differenzen wird Raum gegeben. Gleichzeitig können Ähnlichkeiten erkennbar und vertieft

verabschiedeten Gesetzestexte gesichtet und analysiert. Die Ergebnisse dieser Dokumentenanalyse sind in einer eigenen Veröffentlichung zusammengefasst und werden hier nur kursorisch behandelt. Baltes-Löhr, Christel/Stricker, Susanne: Eine Untersuchung der luxemburgischen Gesetzgebung in Bezug zur Konvention CEDAW und dem ihr implizierten Gleichstellungsprinzip, Universität Luxemburg 2009.

3 | Es gibt unterschiedliche Vorstellungen darüber, welche randeren Kategorien hinsichtlich der Identitätskonstruktionen bedeutsam sind. Knapp (2005) fügt race` und 'classı hinzu. Lutz/Wenning (2001) finden 13 Differenzkategorien, betonen jedoch die Unvollständigkeit der Liste. McCall (2005) spricht von 'genderı, race`, 'class` und fügt die Kategorie region ‘ hinzu; Baltes-Löhr (2009) die Kategorie ‘körperliche Verfasstheit‘. 
werden. Neues entsteht somit immer in Bezug auf etwas, meist das Alte, das Tradierte, das Bekannte. Das Neue entsteht in langsam sich verschiebenden Veränderungen.

Ebenso wie kultureller Pluralismus und kulturelle Differenzen nicht nur zwischen den Kulturen, sondern auch innerhalb der Kulturen zu verorten sind, (Kumoll 2006: 86) kann dies auch für Identitätskonstruktionen vermutet werden. Es gilt, das Minoritär-werden (Deleuze nach Rölli 2006: 39) als differentielle Regel zu begreifen, d.h., dass faktisch vorliegende Merkmale, anhand derer beispielsweise kulturelle Zugehörigkeiten identifiziert werden, als Effekte einer ihnen vorausgehenden Sinnzuschreibung betrachtet werden. Solche Vorstellungen von Minoritär-werden grenzen sich ab gegen Annahmen von essentialistisch konnotiertem Anders-Sein bzw. Nicht-identisch-sein. Hegemoniale Identitätszuschreibungen können somit demontiert bzw. dekonstruiert werden, wenn die Prozesse des Minoritär-werdens hervorgehoben werden, so dass etablierte, selbstverständlich oder gar natürlich anmutende Strukturen der Mehrheit, und damit - im foucaultschen Sinne - oftmals der Macht, als bedingt und damit veränderbar erscheinen können. (Rölli 2006: 40)

Ähnlich wie bei differenten kulturellen Repräsentationen sind besonders die Zwischenräume oder >Third Spaces< (Rutherford 1990: 207-221; Chambers 1996: 78; Gregory 1997: 228) für Artikulationen identitärer Minoritäten oder Minderheitenpositionen bedeutsam. Sind mit Bhabha »Kulturen zwar durch das Begehren nach Stabilität und Determination, beispielsweise im Sinne einer Nation, gekennzeichnet [...], müssen aber gerade in der Instabilität, der Gleichzeitigkeit von inkommensurablen Geschichten (Narrativen und Historizitäten) und Orten gedacht werden« (Bhabha nach Bonz/Struve 2006: 141), dann lässt sich dies ebenfalls auf die Konstruktionsprozesse von Identitäten übertragen. Identitätskonstruktionen werden durch ihre Differenziertheit charakterisiert. Identitäten werden zu einem Ort der Aushandlung und der Äußerung. Identitäten und identitäre Subjekte fallen jedoch nicht ineinander, sondern können different sein. Scheinbar homogenen Identitäten wohnen somit identitäre Differenzen inne. So wie Bhabha gegen eine multikulturell orientierte kulturelle Vielfalt und für eine kulturelle Differenz plädiert, die den kulturellen Antagonismen, Widersprüchlichkeiten und Inkommensuralibilitäten gerecht wird (Bonz/Struve 2006: 149), kann die Annahme identitärer Differenzen (konstruktivistischer Ansatz) den tatsächlich gelebten und wahrgenommenen Widersprüchlichkeiten, Gegensätzen eher gerecht werden, als das Suchen und Streben nach homogenen, identitären Identitäten (essentialistischer Ansatz).

Im Folgenden wird also zu zeigen sein,

- welche homogenisierenden bzw. differenzierenden Vorstellungen von Geschlechteridentitäten zusammen mit anderen intersektionalen Komponenten erkennbar sind;

- welche Relevanz der Dichotomie >konstruktivistisch vs. essentialistisch< zukommt; 
- welche Bedeutung dem Geschlecht im Umgang mit anderen Menschen zugewiesen wird und wie mit Anderssein und anderen umgegangen wird;

- welche Modelle zur Vereinbarkeit von Beruf und Privatleben favorisiert werden;

- welche konkreten Kenntnisse in Bezug auf Maßnahmen und Gesetze zur Gleichstellung von Frauen und Männern bekannt sind.

\section{Resistente Reste. Wie stark wirken essentialistische Geschlechterbilder heute noch?}

Aus der repräsentativen Studie geht hervor, dass $90 \%$ der Befragten der Annahme einer natürlich gegebenen Unterschiedlichkeit zwischen Frauen und Männern zustimmen. Gleichzeitig gehen $77 \%$ davon aus, dass weibliches bzw. männliches Verhalten im Laufe des Lebens erlernt wird. $96 \%$ aller Befragten betonen, dass Frauen ebenso wie Männer geeignet sind, gesellschaftliche Leitungsfunktionen zu übernehmen. Hier deutet sich an, dass die oftmals aufgerufene und vertiefte Divergenz zwischen Essentialismus und Konstruktivismus nicht in der zu vermutenden dichotomen Binarität besteht, sondern beide Haltungen miteinander einhergehen können.

Die deutlich hohe Zustimmung zu der Annahme geschlechtsunabhängiger Befähigungen hinsichtlich der Übernahme von Führungspositionen geht einher mit dem Ergebnis, dass lediglich $56 \%$ äußern, dass die vermeintlich gleichen Möglichkeiten im beruflichen Feld von Frauen und Männern in Luxemburg auch tatsächlich gleichermaßen genutzt werden. Dies deutet darauf hin, dass eine faktische Geschlechterdisparität auf dem luxemburgischen Arbeitsmarkt immer noch als ein gewichtiger Faktor der Diskriminierung zwischen Frauen und Männern zu gelten scheint. Dies wird auch durch die Aussagen aus den Interviews bestätigt: dort wird Geschlechterdiskriminierung vor allem in der Unterrepräsentanz von Frauen in Leitungspositionen und der ungleichen Bezahlung gesehen.

Interessante Ergebnisse zeigen sich hinsichtlich des Antwortverhaltens von Frauen und Männern. Die natürliche Unterschiedlichkeit zwischen den Geschlechtern wird signifikant häufiger von Männern angenommen, was auf eine eher essentialistische Haltung hindeutet. Dieser Zusammenhang zeigte sich für Luxemburg erstmals in einer Untersuchung zur »Bedeutung des Geschlechteraspektes für die berufliche und persönliche Entwicklung/Biographie von zukünftigen Erziehenden und Lehrenden « ${ }^{4}$ (Baltes-Löhr et al. 2005: 87).

4 | Im März-April 2003 durchgeführte Vollerhebung bei Studierenden bzw. Schülerinnen und Schülern des damaligen ISERP (Institut Supérieur d'Etudes et de Recherches Pédagogiques), der Referendariatsausbildung für das Lehramt in der Sekundarstufe am CUNLUX (Centre Universitaire de Luxembourg) und dem damaligen IEES (Institut d'Etudes Educatives et Sociales) mit der Ausbildung und den Studien zur Erzieherin/zum Erzieher und zur Sozialpädagogin/zum Sozialpädagogen (Educatrice graduée/Educateur gradué). 
In der vorliegenden Untersuchung stimmen signifikant mehr Frauen als Männer der Annahme zu, dass beide Geschlechter gleichermaßen geeignet seien, leitende Funktionen in der Gesellschaft einzunehmen. Demgegenüber sind signifikant weniger Frauen der Ansicht, dass beide Geschlechter berufliche Aufstiegschancen in gleichem Maße nutzen. Dem entspricht, dass deutlich mehr Frauen sich bereit erklären, beruflich kürzer zu treten, um Beruf und Familie besser vereinbaren zu können. Auch hier lohnt sich wiederum ein Blick auf die Ergebnisse der o.g. Studie, dass nämlich »bei tatsächlich stattgefundenen Einstellungsveränderungen scheinbare Resistenzen bei Frauen und Männern >übrig bleiben«« (Baltes-Löhr et al. 2005: 85).

Solche >resistenten Reste< zeigen sich in der aktuellen Untersuchung darin, dass Männer in einem signifikant geringeren Ausmaß von der gleichen Eignung von Frauen für Führungspositionen ausgehen, d.h., dass aus einer männlichen Perspektive betrachtet Männer immer noch eher für die Berufswelt und dort vor allem für die Entscheidungspositionen geeignet sind. Dieses Ergebnis strahlt sozusagen sowohl auf die Vorstellungen der Männer bzgl. existierender Diskriminierungen in der Berufswelt aus als auch auf männliche Haltungen in Bezug auf das eigene Privatleben. Männer meinen im Gegensatz zu Frauen eher, dass weibliche Kollegen heutzutage im beruflichen Feld nicht diskriminiert sind, und erklären sich selbst andererseits aber signifikant seltener dazu bereit, zur verbesserten Vereinbarkeit von Beruf und Familie die eigenen beruflichen Aktivitäten zu reduzieren. Dass diese Haltungen und Einstellungen immer noch gelebten Realitäten entsprechen, zeigt ein Blick auf die Zahlen, die angeben, wie hoch der Prozentsatz derjenigen Väter ist, die den Elternurlaub in Luxemburg in Anspruch nehmen. Lag der Anteil der Männer, die Elternurlaub nehmen, 2002 bei 13\%, stieg er bis zum Jahr 2006 auf $22 \%$ an. Ebenso wie auch schon in der Untersuchung »Die Bedeutung des Geschlechteraspektes für die berufliche und persönliche Entwicklung/Biographie von zukünftigen Erziehenden und Lehrenden« resümiert wurde, »dass Frauen kritischere Haltungen gegenüber Traditionalismen einnehmen« (Baltes-Löhr et al. 2005: 86), scheinen es auch im vorliegenden Fall die Frauen zu sein, die sich von traditionellen Vorstellungen hinsichtlich der Geschlechterrollen eher verabschiedet haben als die Männer. >Resistente Reste< aus sogenannten traditionellen Vorstellungen zeigen sich bei den Frauen vor allem hinsichtlich der Zuständigkeit für die Vereinbarkeit von Beruf und Familie und bei Männern in eher naturalistischen Vorstellungen von der essentiellen Unterschiedlichkeit der Geschlechter und der vorrangigen Eignung der Männer für die Berufswelt.

In den Interviewaussagen zeigt sich zu den Fragen der gesellschaftlich wahrgenommenen Geschlechterverhältnisse die gesamte Meinungspalette von: »Es ist gut so, wie es ist und es soll sich nicht ändern« über »Wir sind im Wandel und auf einem guten Weg« bis hin zu »Es geschieht zwar etwas, aber Gleichberechtigung oder Gleichstellung der Geschlechter bleiben unerreichbare Utopien«. Sowohl von Frauen als auch von Männern unterschiedlichen Alters wird betont, dass es Veränderungen auf dem Arbeitsmarkt gibt, dass immer mehr Frauen gute Bildungs- 
abschlüsse haben, berufstätig sind und immer mehr Männer Erziehungsurlaub nehmen.

\section{Kompetenz-, Leistungs- und Entlohnungsdiskurs}

Deutlich erkennbar ist ein >Kompetenzdiskurs $<$. Immer wieder wurde in den Interviews betont, dass es nicht so sehr auf das Geschlecht ankommt, wenn es um die Frage der Eignung für eine berufliche Tätigkeit geht, sondern um Kompetenzen und Qualität der erbrachten Leistungen. Kompetenz zählt, unabhängig von Geschlecht, Hautfarbe, Staatsangehörigkeit und Alter. Leistung zählt. In den Interviews wird Folgendes betont: Genau wie Männer sich aufgrund ihres Engagements und ihrer Leistung Respekt verschaffen, sollten Frauen das ebenso tun. Diesem Diskurs entspricht zweierlei: einmal die Vorstellung von der »defizitären Frau« (vgl. Baltes-Löhr 2006; 2009), die sich den männlichen Normen anpassen soll, wenn sie ebenso wie der Mann Anerkennung erlangen will, und zum Zweiten die Annahme von der Unattraktivität der Hausarbeit. Zu sagen, dass man Hausmann ist und zuhause staubsaugt, fänden nach Meinung zahlreicher Interviewpartnerinnen und Interviewpartner viele Männer uncool und dies sei für viele Männer der Grund, warum sie tatsächlich keine Hausarbeit machen. Als eindeutige Diskriminierung wird von den Interviewten immer wieder auf die Lohnungleichheit hingewiesen. Werden Kompetenzen, Qualität der Arbeit und Leistung eher als für beide Geschlechter geltende Grundlagen für gesellschaftliche Anerkennung und Positionierungen betrachtet, dann bleibt ungleiche Bezahlung ein Indiz für noch bestehende Diskriminierungen von Frauen. Häufig wird die ökonomische Unabhängigkeit von Frauen von den Interviews als Indiz für Emanzipation gedeutet.

Essentialistische Vorstellungen von Weiblichkeit und Männlichkeit finden sich in Aussagen zu den augenscheinlich wesenhaft anderen Arbeitsformen von Frauen und Männern. Ein drastisches Beispiel: Frauen könnten bestimmte Arbeiten körperlicher Anstrengung nicht so erledigen wie Männer, deshalb sei ungleiche Bezahlung gerechtfertigt. Wenn Frauen »so stark sind wie Männer«, dann würden sie dennoch weniger effektiv bleiben als ihre männlichen Kollegen. Diese essentialistisch anmutende Genderdifferenz wird mit den Hierarchisierungen der Arbeitswelt in Verbindung gebracht: Helfer, Maurer und Vorarbeiter auf der Baustelle würden ja auch unterschiedlich entlohnt.

\section{Andersheiten und resistente Reste}

Weitere >Andersheiten $<$ werden in den Interviewaussagen deutlich: Sind bei Männern Selbstdarstellung, Betonung ihrer eigenen Wichtigkeit und Bedeutung dem tatsächlichen Tätigsein vorrangig, werden Frauen als themenzentrierter und gegenstandsbezogener erlebt und dargestellt. Frauen in Führungspositionen werden oft als spezifisch weiblich, also sanft, einfühlsam etc. und damit als >anders als männliche Chefs wahrgenommen oder es wird angenommen, sie müssten sich dem männlichen Stil anpassen. Letzteres verweist auf die Vorstellung, dass 
Geschlechterverhalten von Situationen abhängen kann, erlernbar und somit auch veränderbar ist. Die Annahme von der >spezifisch weiblichen Chefin< lässt jedoch auf eine tief verwurzelte essentialistische Vorstellung von Geschlecht schließen: Frauen bleiben auch in sogenannten männlichen Domänen stereotyp weiblich konnotiert. Stereotyp wesenhafte Weiblichkeit besteht in einem solchen Vorstellungsmuster auch hinsichtlich der Wahrnehmung in Bezug auf berufstätige Frauen ungebrochen weiter. Auch hier deuten sich wiederum >resistente Reste< an, wenn essentialistische Vorstellungen von Geschlecht nicht durch emanzipatorische Veränderungen wie die zunehmende Berufstätigkeit von Frauen gebrochen werden. Essentialistische Diskurse scheinen somit wirkmächtiger zu sein als faktische Veränderungen in Arbeitswelt und Politik. Um zu ergründen, wie genau diese Wirkmächtigkeiten konstruiert werden, wie also durch Wiederholungen Veränderungen, Verschiebungen sowie Konsistenzen und Ähnlichkeiten performativ hergestellt werden, bedarf es weiterer Forschungen.

Deutlich wird anhand der vorliegenden Daten, dass Frauen teilweise ebenfalls in ihren Residuen verharren. So betonen einige Befragte, dass Frauen ein gleichberechtigtes Miteinander der Geschlechter dadurch verhinderten, dass sie Männern beispielsweise nicht zutrauten, »Haushalt zu können«. Berufstätigkeit gilt zwar häufig, aber nicht in jedem Fall als Zeichen oder gar Symbol für Emanzipation. Frauen, die in traditionellen Rollen verbleiben wollen, möchten, so einige Interviewte, dennoch respektiert werden. Allerdings lässt sich festhalten, dass die Mehrzahl der Befragten davon ausgeht, dass mehr Frauen berufstätig seien als umgekehrt Männer in Haushalt, Erziehung und Pflege. So ist zu vermuten, dass die Durchlässigkeit zwischen den traditionellen >Geschlechterdomänen <, die Porosität der Geschlechtergrenzen ${ }^{5}$, von der sogenannten weiblichen in die sogenannte männliche Domäne ausgeprägter zu sein scheint als Grenzüberschreitungen von Männern in sogenannte weibliche Domänen wie Haushalt, Erziehung und Pflege.

\section{Umgang mit Anderssein und anderen}

\section{Die Bedeutung des Geschlechts im Umgang mit anderen Menschen}

Ausgehend von intersektional und pluridimensional verfassten Identitäten, die sich in ständigen Konstruktionsprozessen in Raum und Zeit verändern und als Ergebnisse permanenter Performanzen beschrieben werden können, wurde in der vorliegenden Untersuchung nach der Bedeutung der kulturellen Herkunft, der beruflichen Position, des Alters, des Geschlechts, der Religion sowie der sexuellen Orientierung für den Umgang mit anderen gefragt.

5 | Lässt das Bild der Porosität auf den ersten Blick eher eine gleichmäßige Fluidität und Durchlässigkeit von A nach B vermuten, dann wird dieses Bild hier insofern verschoben, als die jeweiligen Öffnungen der durchlässigen Poren als unterschiedlich groß bzw. ausgeprägt betrachtet werden. 
Es hat sich gezeigt, dass das Geschlecht an vorletzter Stelle rangiert, nur noch gefolgt von der Religion, die für den Umgang mit anderen noch unbedeutender zu sein scheint. Am wichtigsten sind das Alter und die kulturelle Herkunft, dann die berufliche Position und die sexuelle Orientierung. Das Geschlecht spielt vor allem für Männer eine Rolle, wenn es um den Umgang mit anderen geht. Signifikant wichtig ist das Geschlecht im Umgang mit anderen auch für in Luxemburg lebende Italiener sowie für diejenigen, die einen Primärschulabschluss haben. Die sexuelle Orientierung ist vor allem bedeutsam für Männer, die Befragten portugiesischer Herkunft, für diejenigen, die einen Primärschulabschluss haben, für Arbeitslose sowie ebenfalls für Rentner. In Bezug auf das Alter, die Berufstätigkeit, den Familien- und Zivilstand zeigen sich keine signifikanten Werte im Antwortverhalten der Befragten. Die Bedeutsamkeit von Geschlecht (27\% Zustimmung) und sexueller Orientierung (34\% Zustimmung) spielt im unterprivilegierten Milieu die größte Rolle. Im alternativen Milieu wird dem Geschlecht die geringste Bedeutung beigemessen (9\%). Im statusorientierten Milieu ist die sexuelle Orientierung lediglich für $5 \%$ der Befragten für den Umgang mit anderen Menschen von Bedeutung.

\section{Homosexualität}

Eine Gleichwertigkeit von Homosexualität und Heterosexualität konstatieren $65 \%$ aller Befragten. Die signifikant höchsten Zustimmungen finden sich bei den Frauen (74 \%) im Gegensatz zu den Männern (57\%), den 21-29-Jährigen, Befragten mit deutscher Nationalität, Studierenden und $>$ Gepacsten ${ }^{6}$. Im statusorientierten Milieu findet die Annahme der Gleichwertigkeit von Homo- und Heterosexualität die höchste Zustimmung (78 \%) und die geringste im konservativ-gehobenen Milieu (54 \%).

In den Interviews geben einige der Befragten an, noch nie über diese Thematik nachgedacht zu haben. Ein Interviewpartner möchte sich nicht äußern, da er von sich selbst sagt, hierzu eine »rassistische« Einstellung zu haben, die er dann doch erläutert. Er meint, dass homosexuelle Menschen nicht normal wären und Homosexualität den Fortbestand der Menschheit bedrohe: »Wenn alle so wären, dann würde es keine Kinder mehr geben.« Hier zeigt sich, wie durch Homogenisierung: »Wenn alle so wären«, in Verbindung mit Generativität sozusagen der Minderheitenstatus von Homosexuellen konstruiert wird. Hierzu gehören auch Aussagen wie: Der homosexuelle Mensch spüre immer, dass er dem natürlich vorgegebenen Muster, dass Frauen und Männer füreinander gemacht seien, nicht entspricht. In der Mehrzahl der Interviews wird jedoch eher konstatiert, dass sich in Bezug auf den Umgang mit Homosexualität und mit Homosexuellen in Luxemburg etwas ver-

6 | Mit dem Gesetz zu eingetragenen Partnerschaften wurden auch eheähnliche Gemeinschaften zwischen Gleichgeschlechtlichen ermöglicht. Gesetz vom 09.07.2004. Diejenigen, die eine solche Partnerschaft (Pacte civil de solidarité, abgekürzt PACS) haben eintragen lassen, werden im Folgenden mit dem Begriff , Gepacste، bezeichnet. 
ändert habe und dass den >anderen « mehr Toleranz entgegengebracht würde. Die zunehmende offizielle Akzeptanz entspräche jedoch nicht immer den inoffiziellen Meinungen und Haltungen. Daran habe auch die Ermöglichung einer eheähnlichen Gemeinschaft für gleichgeschlechtliche Paare (PACS) nichts grundlegend verändert: »Die Bevölkerung schaut aber immer noch schief.« Homosexuelle würden immer noch als andere, als anders und damit als komisch betrachtet. Der Begriff >schwul< tauge immer noch als Schimpfwort. Hierin spiegelt sich ein hegemonialer, heteronormativer Normalitätsdiskurs, wenn das Markierte als das >Nicht-Normale gilt. (Knapp 2010: unveröffentlichter Redebeitrag anlässlich der Gründungstagung der Fachgesellschaft Geschlechterstudien in Berlin, 29./30.01.2010; Jakobson 1971) Häufig wird betont, dass das Kennen von homosexuellen Menschen hilft, bestehende Vorurteile abzubauen. Homosexualität wird von einigen Interviewten als Diskriminierung >von außen< verstanden. Es gehöre ein starkes Selbstbewusstsein dazu, sich zu outen: »Wenn der Homosexuelle die Homosexualität als Normalität betrachtet, dann kann er sich outen, wenn er sich nach der Familie oder den Freunden richtet, dann nicht.« Das Verständnis für die Angst vor einem Outing wird aber auch quantitativ begründet: »weil das [gemeint ist die Homosexualität] eine Ausnahme ist«. Hier wird die Angst vor dem Outing mit einem vermeintlichen Minderheitenstatus konnotiert, aber auch betont, dass Homosexuelle sich selbst nicht unbedingt als Minderheit betrachten. Quantitativität als Selbstverständlichkeit mehrheitlich orientiert verfasster Demokratien scheint oftmals mit einem eher schwach ausgeprägten Verständnis oder schwach ausgeprägter Toleranz bzgl. Minderheiten (Minderheitenschutz) einherzugehen: Die Mehrheit zählt und hat damit auch Recht. Die anderen bleiben letztendlich zur Anpassung aufgefordert oder marginal. Differenzkompetente Toleranz und Akzeptanz sowie die Sensibilität dafür, dass das Eigene sowie das kollektive Subjekt in der Mehrheitsposition für die marginalisierten anderen das Nicht-Eigene darstellen, fehlen häufig. Schwule Männer werden als einfühlsamere Menschen beschrieben, nicht nur im Vergleich zu anderen Männern, sondern auch - und das ist bemerkenswert - zu Frauen. Hier wird die traditionelle, essentialistische Geschlechterdualität überschritten, indem Einfühlsamkeit nicht sozusagen automatisch, als wesenhafte Verhaltensdisposition den Frauen per se zugeschrieben wird. Obwohl niemand der Interviewten sich selbst als schwul oder lesbisch bezeichnet, wird von einigen der Befragten Geschlechternormalität an sich explizit infrage gestellt; es werden Verunsicherungen zugegeben und es wird eingeräumt: »Wer weiß schon, was normal ist.«

Wie sehr liberale Einstellungen mit ethnisch konnotierten Diskriminierungen einhergehen können, zeigt die Aussage eines Interviewten, der meint, dass die Gesellschaft heute sehr viel liberaler mit dem Thema Homosexualität und mit Homosexuellen umgeht: »Heute ist alles liberaler, außer vielleicht bei den Portugiesen, die noch Angst haben, mit ihren Eltern darüber zu sprechen. «Auch hier zeigt sich, wie leicht oftmals selbst demokratisch motivierte Mehrheitsdiskurse Minderheiten konstruieren und diesen einen marginalisierten Status zuweisen, und wie nahe Toleranz und diskriminatorische Haltungen beieinander liegen können. 


\section{Transsexualität}

$75 \%$ aller Befragten können verstehen, dass es Menschen gibt, die sich in ihrer Geschlechterrolle nicht wohl fühlen und $52 \%$ haben Verständnis für Menschen, die ihr Geschlecht ändern wollen. Beim statusorientierten Milieu ist sowohl das Verständnis für Menschen, die sich in ihrer Geschlechterrolle nicht wohl fühlen, mit $80 \%$ sehr ausgeprägt als auch dasjenige für Transsexuelle mit $73 \%$. Am niedrigsten ist dieser Wert beim kleinbürgerlichen Milieu. Dort zeigen aber immer noch 70 \% Verständnis für das Unwohlsein mit der Geschlechterrolle. Deutlich geringer ist das Verständnis für Transsexuelle, das bei 43 \% rangiert.

Folgende Tabelle zeigt die signifikant höchsten bzw. niedrigsten Werte:

\begin{tabular}{|c|c|c|c|}
\hline Kategorien & $\begin{array}{c}\text { Höchste } \\
\text { Zustimmung }\end{array}$ & Item & $\begin{array}{c}\text { Niedrigste } \\
\text { Zustimmung }\end{array}$ \\
\hline $\begin{array}{l}\text { Geschlecht } \\
\text { Nationalität } \\
\text { Alter } \\
\text { Ausbildung } \\
\text { Berufstätigkeit } \\
\text { Arbeitsverhältnis } \\
\text { Familiensituation } \\
\text { Zivilstand }\end{array}$ & $\begin{array}{l}\text { Frauen } \\
\text { n.s. } \\
45-59 \\
\text { n.s. } \\
\text { n.s. } \\
\text { Hausfrau/-mann } \\
\text { n.s. } \\
\text { Gepacste }\end{array}$ & $\begin{array}{l}\text { Verständnis für } \\
\text { Unwohlsein mit } \\
\text { Geschlechterrolle }\end{array}$ & $\begin{array}{l}\text { Männer } \\
\text { n.s. } \\
60 \text { plus } \\
\text { n.s. } \\
\text { n.s. } \\
\text { Andere Beschäftigung } \\
\text { n.s. } \\
\text { Verwitwete }\end{array}$ \\
\hline $\begin{array}{l}\text { Geschlecht } \\
\text { Nationalität } \\
\text { Alter } \\
\text { Ausbildung } \\
\text { Berufstätigkeit } \\
\text { Arbeitsverhältnis } \\
\text { Familiensituation } \\
\text { Zivilstand }\end{array}$ & $\begin{array}{l}\text { Frauen } \\
\text { Deutsch } \\
30-59 \\
\text { Universität } \\
\text { Hausfrau/-mann } \\
\text { Hausfrau/-mann } \\
\text { n.s. } \\
\text { Gepacste }\end{array}$ & $\begin{array}{l}\text { Verständnis für } \\
\text { Transsexuelle }\end{array}$ & $\begin{array}{l}\text { Männer } \\
\text { Portugiesisch } \\
60 \text { plus } \\
\text { Primär } \\
\text { Rentnerinnen/Rentner } \\
\text { Arbeitssuchende } \\
\text { n.s. } \\
\text { Verwitwete }\end{array}$ \\
\hline
\end{tabular}

Tabelle 1: Bewertungen von/Einstellungen zu Transsexualität (n.s.: nicht signifikant)

Deutlich wird zweierlei: Die Antworten zu dem Verständnis für das Unwohlsein mit der Geschlechterrolle zeigt in Bezug auf Nationalität, Ausbildung, Berufstätigkeit sowie Familiensituation keine signifikanten Werte, was darauf hindeutet, dass hier die Antworten über diese Kategorien hinweg gleich verteilt sind. Es sind die Frauen, die 45-49-Jährigen, im Haushalt Tätige und Gepacste, die hier mit deutlicher Signifikanz zustimmen.

Die Frage nach dem Verständnis für Transsexuelle polarisiert das Antwortverhalten stärker. Lediglich hinsichtlich der Familiensituation gibt es keine signifikanten Antworten. Auch hier sind es wieder die Frauen, die Altersgruppe der 30-59-Jährigen, Angehörige deutscher Nationalität, Akademikerinnen und Akademiker, zuhause Arbeitende sowie Gepacste, die in starkem Maße zustimmen. 
Geringste Zustimmung zu diesen Fragen, vor allem hinsichtlich der Einstellung zu Transsexuellen, gibt es bei Männern, der Altersgruppe der über 6o-Jährigen, Primarschulabsolventen, Verwitweten, bei Rentnerinnen und Rentnern sowie Arbeitssuchenden.

In den Interviews hat sich niemand als transsexuell bezeichnet. Lediglich drei der Interviewten geben an, Transsexuelle zu kennen. Einige erzählen, dass Bekannte Transsexuelle kennen, andere haben vage davon gehört und einige geben an, im Fernsehen Berichterstattungen darüber gesehen zu haben. Eine Befragte erinnert sich an eine Mitschülerin, die ausgesehen habe wie ein Junge, und meint, das könnte jemand sein, die später zum Jungen geworden wäre. Sie erzählt von der großen Verunsicherung, die damals vor ca. 15 Jahren im Gymnasium herrschte, wie mit dem Mädchen umzugehen sei. Generell wird Transsexualität in den Interviews in die Nähe der Homosexualität gerückt und/oder mit z.B. Brustvergrößerungen in Verbindung gebracht. Es wird oft erzählt, dass aus lauter Verunsicherung hinsichtlich des Umgangs mit transsexuellen Menschen diese häufig der Lächerlichkeit preisgegeben werden. Immer wieder wird die Wichtigkeit betont, bei ersten Anzeichen von transsexuellem Verhalten sofort Psychologen/-innen oder Ärzte/-innen hinzuzuziehen. Neben dieser Tendenz zur Pathologisierung wird auch Toleranz eingefordert und gleichzeitig unterstrichen, das sei in der Theorie einfacher als in der Praxis.

In den Interviews ist auch gefragt worden, wie die Interviewten mit einem Kind umgehen würden, das sich in seinem Geschlechtskörper nicht wohlfühlt. Die Antworten zeigen Unwissenheit, Hilflosigkeit sowie Unsicherheit im Umgang mit einem solchen Kind. Einige der Interviewten sagten, dass dies vielleicht/hoffentlich nur eine Phase sei: »Wenn es sich wieder einrenkt, ist es eben gut.« In Bezug auf Erwachsene hingegen sind andere Befragte der Meinung, es sei für diejenigen, die ihr Geschlecht verändern möchten, unbedingt notwendig, dies auch zu tun, da ein Verbleiben in einer solchen Unzufriedenheit auch krank machen könne.

Das Thema Transsexualität wird ebenso wie das der Homosexualität immer noch mit Angst in Verbindung gebracht und als Schicksalsschlag betrachtet. Je weiter das Thema in das eigene Privatleben hineinreicht, desto bedrohlicher scheint es zu werden. Es wird eine Demarkationslinie zwischen den Geschlechtern aufgebaut, wenn in den Interviews immer wieder betont wird, Männer hätten mit dieser Thematik mehr Probleme als Frauen. Aber es wird auch bemerkt, dass es möglicherweise für Mütter eine Herausforderung darstelle, wenn der Junge lieber ein Mädchen wäre oder umgekehrt. Letzteres wäre von der Mutter schwerer zu verstehen: schließlich sei sie selbst ja zufrieden damit, weiblich zu sein. Hier zeigt sich die Relevanz der Identifikation mit dem eigenen Geschlecht. Wird diese infrage gestellt, kommen Irritationen auf. So betont eine Interviewte, dass ein Vater mit dem Outing eines homosexuellen Sohnes größere Schwierigkeiten habe als eine Mutter, weil er ein Mann sei. 


\section{Gendermodelle}

\section{Favorisierte Gendermodelle: Egalitäre Rollenverteilung, solange kein Kind da ist}

Die Einstellungen zu den unterschiedlichen Gendermodellen wurden anhand folgender Fragen eruiert:

Welches Modell zur Arbeitsteilung in der Lebensgemeinschaft halten Sie für das beste?

A. Der Mann arbeitet Vollzeit, die Frau ist nicht erwerbstätig und sie kümmert sich um Haushalt und Familie

B. Der Mann arbeitet Vollzeit, die Frau in Teilzeit und kümmert sich um Haushalt und Familie

C. Beide Partner teilen sich gleichberechtigt Erwerbsarbeit, Haushalt und Familie

D. Die Frau arbeitet Vollzeit, der Mann in Teilzeit und kümmert sich um Haushalt und Familie

E. Die Frau arbeitet Vollzeit, der Mann ist nicht erwerbstätig und kümmert sich um Haushalt und Familie

Die einzelnen Modelle erhielten folgende Zustimmungen:

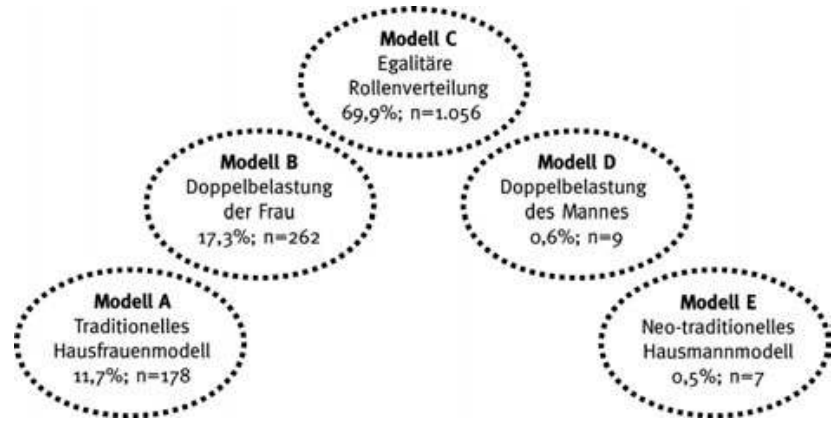

Abbildung 1: Zustimmung zu den Modellen A-E in \% und absoluten Zahlen der Zustimmenden

Da die Berechnung der Signifikanzen für die Modelle D und E aufgrund der geringen Antworten nicht möglich ist $(\mathrm{n}<30)$, soll im Folgenden kurz beschrieben werden, welche Personengruppen hier am häufigsten geantwortet haben: Modell D wird vor allem befürwortet von jungen, alleinstehenden oder in Partnerschaften mit oder ohne Kinder lebenden Studierenden, die in 3-5-Personenhaushalten, wohlmöglich in stadtnahen WGs oder in der elterlichen Wohnung, leben und französischer Herkunft sind. Diejenigen, die diesem Modell zustimmen, kommen aus allen Einkommensklassen außer derjenigen zwischen 5000 und $7000 €$ und sind im alternativen Milieu am stärksten vertreten. Das Modell E findet erstaunlicher- 
weise vor allem Zustimmung bei den über 45-Jährigen, bei Menschen, die ohne Kinder leben, Menschen, die verwitwet sind, in 1- oder 2-Personenhaushalten in Mietwohnungen oder als Eigentümer wohnen. Menschen mit einem Haushaltseinkommen von 4000-5000 € stimmen am ehesten diesem Modell zu, ebenso wie Zugehörige zu dem hedonistischen Milieu.

Überaus erstaunlich ist die Häufigkeit der Nennung von Modell C, wird doch hier die egalitäre Aufgabenverteilung zwischen den Geschlechtern eindeutig favorisiert. In Bezug auf die Modelle A, B und C unterscheidet sich das Antwortverhalten der Frauen und Männer nicht signifikant. Unterschiedliche Geschlechtermodelle repräsentieren sich also mit deutlicher Trennschärfe, ohne dass das Geschlecht des Befragten hier als diskriminierende Kategorie nachzuweisen ist. Dies deutet darauf hin, dass die Vorstellungen zu unterschiedlichen Geschlechtermodellen nicht über die Geschlechterzugehörigkeit selbst (re-)produziert werden, sondern dass möglicherweise andere Kategorisierungen hinsichtlich der Geschlechtermodelle wirkmächtiger sind. Das heißt u.a., dass Ansätze zu intersektionalen Betrachtungen angemessen zu sein scheinen, wenn es um die Erklärung bestehender Repräsentationen zu Geschlechterfragen geht.

Kinderlosigkeit scheint die Zustimmung zu dem egalitären Modell zu erhöhen, sowohl bei denjenigen, die in einer Partnerschaft als auch alleinstehend leben. Anders ausgedrückt: Sobald Kinder in Beziehungen eintreten, nimmt die Zustimmung zu egalitären Partnerschafts- und Berufsmodellen ab. Gleichberechtigung lässt sich dann am ehesten befürworten, wenn sie nicht mit der lebensweltlichen Realität der Kindererziehung konfrontiert wird. Elternschaft/Kinderlosigkeit spielen somit im Kontext der Zuschreibungs- und Aneignungsprozesse von Identitäten eine nicht zu vernachlässigende Rolle und sollten im intersektionalen Kanon Berücksichtigung finden.

Zeigen sich bei den Modellen A und C deutliche Unterschiede im Antwortverhalten, dann ist bei Modell B die prozentuale Spannbreite im Antwortverhalten gering. Hieraus ließe sich schließen, dass dieses Modell der Doppelbelastung der Frau über alle Kategorien wie Alter, Schulbildung etc. hinweg am ehesten von einer breiten Bevölkerungsgruppe akzeptiert wird.

Findet das Modell C zwar mit der egalitären Aufgabenverteilung die höchste Zustimmung, so geben allerdings lediglich $51 \%$ aller Befragten an, dass sie selbst bereit wären, beruflich kürzer zu treten, um z.B. Familie und Beruf besser miteinander vereinbaren zu können. Auch wenn die Zustimmung zu Modell C keine Geschlechtersignifikanz im Antwortverhalten erkennen lässt, dann sind jedoch immer noch signifikant mehr Frauen bereit, zur Verbesserung der Vereinbarkeit von Beruf und Familie ihre eigenen beruflichen Ambitionen zurückzustecken.

Folgende Tabelle gibt Aufschluss über höchste und geringste Zustimmungen zu den Modellen A, B und C: 


\begin{tabular}{|c|c|c|}
\hline Höchste Zustimmung & Modelle & Geringste Zustimmung \\
\hline $\begin{array}{l}\text { Über 6o-Jährige } \\
\text { Luxemburgische Nationalität } \\
\text { Partnerschaft mit Kindern } \\
\text { Verwitwete } \\
\text { mithelfende Familien- } \\
\text { angehörige } \\
\text { Primarschulabschluss }\end{array}$ & $\begin{array}{c}\text { Modell A: } \\
\text { Traditionelles } \\
\text { Hausfrauenmodell }\end{array}$ & $\begin{array}{l}\text { 20-29-Jährige } \\
\text { Französische Nationalität } \\
\text { Alleinstehend ohne Kinder } \\
\text { Getrennt, ledig, gepacst } \\
\text { Studierende, Selbständige, } \\
\text { Beamtinnen und Beamte } \\
\text { Etudes Supérieures }\end{array}$ \\
\hline $\begin{array}{l}\text { Alle Altersgruppen außer } \\
\text { 20-29-Jährige } \\
\text { Italienische Nationalität } \\
\text { Partnerschaft mit Kindern } \\
\text { Gepacste } \\
\text { Angestellte } \\
\text { Andere Bildungsabschlüsse }\end{array}$ & $\begin{array}{c}\text { Modell B : } \\
\text { Doppelbelastung der Frau }\end{array}$ & $\begin{array}{l}\text { 20-29-Jährige } \\
\text { Britische Nationalität } \\
\text { Alleinstehend ohne Kinder } \\
\text { Ledige } \\
\text { Studierende } \\
\text { Universität }\end{array}$ \\
\hline $\begin{array}{l}\text { 20-29-Jährige } \\
\text { Britische Nationalität } \\
\text { Alleinstehende ohne Kinder } \\
\text { Ledige } \\
\text { Studierende } \\
\text { Universität }\end{array}$ & $\begin{array}{l}\text { Modell C: } \\
\text { Egalitäre Rollenverteilung }\end{array}$ & $\begin{array}{l}\text { Über 6o-Jährige } \\
\text { Italienische Nationalität } \\
\text { Partnerschaft mit Kindern } \\
\text { Verwitwete } \\
\text { Mithelfende } \\
\text { Familienangehörige } \\
\text { Primarschulabschluss }\end{array}$ \\
\hline
\end{tabular}

\section{Tabelle 2: Präferenzen der Gendermodelle}

Das Modell A, traditionelles Hausfrauenmodell, wird am ehesten im kleinbürgerlichen Milieus favorisiert; Modell B, Doppelbelastung der Frau, wird mit jeweils $20 \%$ gleich von Zugehörigen mehrerer Milieus präferiert: denjenigen des kleinbürgerlichen, des unterprivilegierten, des statusorientierten und des aufstiegsorientierten Milieus. Modell C, egalitäre Rollenverteilung, wird vor allem im liberal-gehobenen Milieu favorisiert. Modell D, Doppelbelastung des Mannes, findet, wie schon weiter oben erwähnt, höchste Zustimmung im alternativen Milieu und Modell E, das neo-traditionelle Hausmannmodell, im hedonistischen Milieu.

\section{Gelebte Gendermodelle}

Aus den Interviews geht deutlich hervor, dass alle Gendermodelle vorkommen. Immer wieder wird betont, dass Frauen und Männer die Möglichkeit zur freien Entscheidung haben sollten, wie sie sich in ihrem Privatleben arrangieren. Allerdings wird auch erzählt, dass fehlende Kinderbetreuungseinrichtungen, mangelnde Qualität dieser Einrichtungen und finanzielle Zwänge die Entscheidungen, wer zuhause bleibt, beeinflussen. Viele weibliche Befragte berichten, dass sie gerne als Mutter zuhause bleiben; vereinzelt wird aber auch genau nachgezeichnet, wie sehr dann doch der Außenkontakt und eine intellektuelle Betätigung vermisst wer- 
den. Thematisiert wird auch die >Mütterkonkurrenz $<$ z.B. das Organisieren von Kindergeburtstagsfeiern als Messlatte für die >Qualität< der Mütter. Es wird immer wieder betont, dass Kindererziehung >vollen Einsatzく erfordere, dass Männer sich oft nicht dazu in der Lage sähen, den erzieherischen oder haushälterischen Anforderungen gerecht zu werden. Sie würden sich erzieherische Tätigkeiten vor allem bei Babys oder Kleinkindern - selbst wenn es ihre eigenen Kindern sind nicht zutrauen. Auch wird deutlich, dass gemeinschaftlich organisierte Haushalte sich verändern, wenn ein Kind geboren wird: Die Frauen bleiben i.d.R. zuhause. Kindererziehung ist für Männer immer noch keine Selbstverständlichkeit. Dies bestätigt die Ergebnisse, die weiter oben in dem Abschnitt »Favorisierte Gendermodelle« vorgestellt wurden, dass die partnerschaftliche Rollenverteilung eng mit Kinderlosigkeit bzw. Elternschaft verknüpft ist. Wenn Männer jedoch in Haushalt oder Kindererziehung der etwas älteren Kinder (meistens ab vier Jahren) aktiv werden, dann erzählen sowohl sie selbst als auch ihre Partnerinnen, dass dies oft mit Spaß und Freude verbunden sei.

Ein anderer Faktor, der zu einer nicht-traditionellen Verteilung von Hausarbeit und Kinderbetreuung beitragen, ist berufliche Selbstständigkeit, die eine Vereinbarkeit von Beruf und Familie erleichtert, weil hier flexible Arbeitszeiten auch Vätern ermöglichen, sich mehr um die Kinder kümmern zu können. Auch wird der Aspekt der Effektivität genannt: Wer kann was am besten und am schnellsten? Daneben rangieren Körpergröße, berufliche Belastung und der Zeitfaktor. Beim Kochen hängt auch viel davon ab, was gekocht werden soll und für wen das Essen ist, also der Geselligkeitsgrad. In einem Fall kocht die Frau bei englischsprachigem Besuch, damit sie sich nicht auf Englisch unterhalten muss, das macht dann der Mann. Hier begegnen sich Geschlecht, Kultur, Herkunft und Sprachkompetenz und Kochen: ein deutliches Beispiel dafür, wie Intersektionalität sich in der Alltagspraxis abbildet. Aber auch die goffmansche Vorder- und Hinterbühne (Goffman 1969) tauchen in den Interviews auf, wenn erzählt wird, dass in einem als partnerschaftlich deklarierten Verhältnis die Frau für die Wascharbeit im Keller zuständig ist und er für das, was sich >oben< abspielt: Sie wäscht und hängt auf; er bügelt und faltet. Dieses Bild wiederholt sich interessanterweise bei der räumlichen Aufteilung des Putzens: »Sie putzt den hinteren Teil der Wohnung und ich den vorderen.«

In Bezug auf die Frage, woher die Entscheidung für ein verändertes Gendermodell kommt, zitieren einige Interviewte ihre Eltern als Vorbild. Andere betonen, dass sie bewusst im krassen Gegensatz zu dem, was sie im Elternhaus erlebt haben, ihr eigenes Gendermodell entworfen und realisiert haben. Um diese Wege der Veränderungen jedoch genauer zu ergründen, besteht erheblicher Forschungsbedarf.

Traditionelle Gendermodelle werden oftmals als vollkommen normal und selbstverständlich dargestellt. Das Traditionelle wird nicht hinterfragt. Auch wird oft betont, dass man darüber noch nicht einmal zu reden brauche. Deutlich werden hier die Unausgesprochenheit von Normalitätsdiskursen und die damit verbundene >diskussionsfreie< Übernahme von Rollenzuschreibungen. Dies könnte 
als Beleg für die Annahme gelten, dass im hegemonialen Normalitätsdiskurs nur das Nicht-Normale markiert wird. So zeigt sich in den Interviews, dass das >noch Normale oftmals von denjenigen, die sich davon gelöst haben, belächelt zu werden scheint. Was wiederum bei den >Traditionalisten< zum Ärger über die sogenannte Arroganz der >anderen < führen kann.

\section{Kenntnisse in Bezug auf Maßnahmen und Gesetze zur Gleichstellung von Frauen und Männern}

Vor allem die über 6o-Jährigen kennen Gesetze oder luxemburgische Regelungen zur Gleichstellung von Frauen und Männern. Der »Girls' und Boys' Day« (GBD), den es für Mädchen seit 2002 und auch für Jungen seit 2005 in Luxemburg gibt, und mit dem Mädchen und Jungen für sogenannte atypische Berufe sensibilisiert werden sollen, ist hingegen eindeutig eher bei den 16-20-Jährigen bekannt. Ist der Girl's Day bei Frauen und Männern gleichermaßen bekannt, dann zeigt sich eindeutig, dass Männer eher die Gesetzesregelungen kennen und Frauen eher auch vom Boy's Day gehört haben. Die Gesetze und Regelungen sind am ehesten den in Luxemburg lebenden Deutschen bekannt; der GBD ist eindeutig der luxemburgischen Bevölkerung am ehesten bekannt. Insgesamt nimmt das Kennen von Gesetzen, Regelungen und dem GBD relativ zur Höhe des Bildungsabschlusses zu.

Aus den Interviews geht eine bemerkenswerte Unkenntnis hinsichtlich bestehender Gesetze und Regelungen hervor. Mehr oder weniger bekannt sind: Schwangerschaftsschutzregelungen, Wegweisungsgesetz, Gesetze zur gleichen Bezahlung, Personenstandsgesetze, Scheidungsgesetzgebung, eingetragene Partnerschaften. Bei aller Unwissenheit wird dennoch mehrfach mit Nachdruck behauptet, dass Gesetze die Tendenz hätten, Frauen zu favorisieren. Proportional zur Unkenntnis wird die Bedeutung der Gesetze und Regelungen für den Alltag negiert. Die Tatsache, in einer Vertragsgesellschaft zu leben, scheint den Befragten kaum bewusst zu sein. Gesetze und Regelungen scheinen nur für besondere Fälle notwendig zu sein, wie z.B. bei Gewalt in der Ehe. Auch wird betont, dass man selbst für seine Rechte kämpfen müsse, auch wenn es um Gleichberechtigung geht. Die Quotendebatte wird häufig als Nonsens abgetan. Hier zeigt sich eine interessante Querverbindung zum Kompetenzdiskurs, wenn gesagt wird, es komme auf die Kompetenz an und nicht auf das Geschlecht. Geschlechterdisparitäten seien nicht durch Regelungen abzubauen. Dies sei eine Frage der Bildung, der Erziehung und der Änderung der inneren Haltung, und das brauche Zeit.

\section{Geschlechterkategorie im polyfonen Chor der Intersektionalität}

Wurde Gender bis in die späten 199oer Jahre im wissenschaftlichen feministischen Diskurs als die Strukturkategorie betrachtet, dann zeigen die vorliegenden Ergebnisse, dass Geschlecht als Analysekategorie an Trennschärfe verloren hat. Gender wird zu einem Aspekt im polyfonen Chor der Intersektionalität und somit ent- 
dramatisiert. Je nach Situation scheinen einzelne Sektionen im Identitätsgeflecht mehr oder weniger stark auf. Mal ist das Alter, mal die berufliche Positionierung, mal die sexuelle Orientierung bedeutsamer und wird zum das Subjekt kenntlich machenden Aspekt. So nimmt Geschlecht im Umgang mit anderen Menschen nach den vorliegenden Ergebnissen eine eher nachgeordnete Rolle ein. Geht es um Annahmen zu Weiblichkeiten und Männlichkeiten, dann zeigen sich essentialistische und konstruktivistische Vorstellungen zu Identitäten und Geschlechtern als diskursiv eng verwoben. Hier deutet sich möglicherweise eine erstaunliche Nähe und Überschneidung dieser paradigmatisch oftmals dichotom verorteten Diskursfiguren an. Ähnliches gilt für Toleranz und diskriminatorische Haltungen. Hegemoniale Normalitätsdiskurse scheinen in Bezug auf die Präferenzen von Geschlechtermodellen ebenso brüchig geworden zu sein wie tradierte Vorstellungen über Homo- und Transsexualitäten, obwohl sie ihre Wirksamkeit als Instrument des Ein- und Ausschlusses noch nicht eingebüßt haben und immer noch zu Marginalisierungen von Minoritäten beitragen können. Dennoch hat sich - so die Ergebnisse - in der luxemburgischen Gesellschaft viel getan hinsichtlich einer zunehmenden Geschlechtergerechtigkeit, die Geschlechtergrenzen sind poröser geworden, wobei die Durchlässigkeit noch nicht ausbalanciert zu sein scheint: Es ist wohl einfacher, von der sogenannten weiblichen Seite auf die sogenannte männliche zu gelangen, als umgekehrt. Dennoch zeigt sich deutlich, dass traditionelle Geschlechterbilder ihre Wirkmächtigkeit keineswegs vollständig verloren haben, vor allem wenn es um Elternschaft geht. Elternschaft scheint immer noch die Umsetzung egalitärer Partnerschafts- und Berufspraxen zu erschweren. Frauen sind immer noch eher als Männer zu einer Priorisierung der Familie zuungunsten ihrer eigenen beruflichen Tätigkeit bereit. Faktische Strukturveränderungen wie zunehmende Erwerbstätigkeit von Frauen, zunehmend bessere Bildungsabschlüsse sowie zunehmende Präsenz von Frauen in Politik und Öffentlichkeit (Allegrezza et al. 2007; Ministère de l'Egalité des Chances 2006; Ministère de l'Education nationale et de la Formation professionnelle 2007) haben nachgewiesenermaßen keinen unbedingten Einfluss auf die Einstellungen der Menschen hinsichtlich der Geschlechterthematik. Es kann somit von >resistenten Resten< gesprochen werden, die sich bei Frauen eher im Bereich der Mütterlichkeit und der Zuständigkeit für die Vereinbarkeit von Beruf und Familie zeigen und bei Männern in naturalistisch begründeten Annahmen essentiell unterschiedlicher Geschlechter sowie der vorrangigen Eignung der Männer für die Berufswelt.

Solche geschlechterpolarisierende Zuschreibungsprozesse können jedoch durchaus geschlechterübergreifend von allen Geschlechtern gemacht werden. Trotz dieser >resistenten Reste< scheinen (Geschlechts-)Identitäten immer mehr als permanente Performanzen im intersektionalen Kanon auf. Sie konstruieren einen Raum, in dem sich Subjekte interaktiv verorten, in dem auch immer wieder die Geschlechtszugehörigkeiten der Agierenden eine Rolle spielen - können. Die Wirkmächtigkeit der Kategorien ist in Bewegung. Die Kategorie Geschlecht ist in Bewegung geraten: gender in motion. 


\section{3 iGutesi Essen. OSZiLlation ZWISCHEN POLITISCHEM LEITBILd UND INDIVIDUELLER ALLTAGSPRAXIS}

\section{Identitätsrelevante Essensnormen und -praxen erforschen}

Im folgenden Fallbeispiel für Identitätskonstruktionen geht es um normative Vorstellungen darüber, was unter >gutem< Essen zu verstehen sei bzw. wie dieses sozial praktiziert wird. Der sowohl qualitativ wie quantitativ ausgerichtete Analysekorpus erfasst zugleich die institutionelle und die individuelle Dynamik: Er basiert einerseits auf einem Gruppeninterview mit Experten/-innen sowie auf politischen Veröffentlichungen zum Thema und andererseits auf einer Repräsentativumfrage sowie qualitativen Interviews in der Luxemburger Wohngesellschaft.

Diese Forschungsthematik bietet die Möglichkeit, durch das banale und wiederkehrende Ernährungsbedürfnis der Subjekte das Ineinandergreifen von normativen Einflüssen mit weitgehend routinisierten und unhinterfragten Diskurspraktiken zu untersuchen. Diese werfen aufgrund des menschlichen Omnivorismus ${ }^{7}$ (Fischler 1990) Fragen über die ressourcenabhängige Nahrungsauswahl (was essen?), über deren Zubereitung und Zusammenstellung (wie essen?) sowie über den gemeinsamen Verzehr mit ausgewählten Mitmenschen (mit wem essen?) auf. Die allägliche, individuelle Bewältigung dieser Fragen (>Perceptas $<$ ) ermöglicht einen analytischen Blick auf die ihr zugrunde liegenden und kollektiv verfügbaren Werte, Einstellungen und Normen ( $>$ Conceptas $<)^{8}-$ was wiederum einen Rückschluss auf sinnstiftende und über Alltagshandeln artikulierte Prozesse der Identitätsarbeit ermöglicht. (Vgl. Keupp et al. 2006) Dabei wird der Begriff der Identitätsarbeit hier sowohl als Form der individuellen Selbstkonstruktion als auch der kollektiven Mitgestaltung auf gemeinschaftlicher bzw. gesellschaftlicher Ebene aufgefasst. Denn gerade durch körpergebundene Handlungen wie beim Essen konstruiert sich das Individuum bewusst als >Ich< mit idiosynkratischen Präferenzen und Gewohnheiten. Gleichzeitig wird es von den inkorporierten Speisen physiologisch wie auch symbolisch konstituiert. Es gestaltet kollektive Normen von >gutem< Essen, indem es sie zwar individuell interpretiert, jedoch ohne den tradierenden Rahmen zu sprengen. Gleichzeitig wird das individuelle Handlungsrepertoire - zu dem Rezepte, Menüzusammenstellungen, Geschmacksvorlieben, Tischmanieren, Mahlzeitengestaltung, Werte und Einstellungen in Bezug auf Essen oder Essensgespräche etc. zählen - von kursierenden, kollektiven (kulturellen, sozioökonomischen und milieuspezifischen) Einstellungen, Denk- und Wissensbeständen gerahmt. (Bourdieu 2000; 1994; 1980) Allägliches Essverhalten und individuelle bzw. kollektive Identitäten sind demnach eng miteinander verwoben; dennoch ist ihre Beziehung weder eindeutig noch gleichbleibend. (Scholliers et al. 2001)

7 | Die physiologische Tatsache, dass die Menschen nahezu alles essen können - jedoch in einem Rahmen des kulturell Akzeptablen.

8 | Vgl. Abschnitt 7.1. 
Besonders den selbstverständlich erscheinenden Aspekten identitätsrelevanter Diskurspraktiken haftet eine gewisse Unsichtbarkeit, Unsagbarkeit und Intimität an. Dies zieht forschungspraktische Konsequenzen nach sich, da Interviewte in einem qualitativen semidirektiven Gespräch - aufgrund des für viele ungewöhnlichen Themenfelds und der oft wenig ausgeprägten Selbstreflexivität in Bezug auf die eigene Essenspraxis - nicht ohne weiteres die uns interessierenden Sinnzusammenhänge und Wertvorstellungen verbalisieren. Eine Konsequenz für die Interviewführung bestand darin, das Gespräch auf das Thema der kollektiven Identifikationen zu lenken. So haben die Interviewten die Themenfelder Essen und Identität über ethnische, nationale oder familiäre Zugehörigkeit durch ostentatives oder festliches, oftmals stereotypisiertes Verzehren von bestimmten Gerichten oder Zutaten zu bestimmten Gelegenheiten artikuliert.9 So hieß es z.B.: »Wir Portugiesen essen viel Fisch « oder: »Quand on va boire un café dans un bistrot en Italie, dans n'importe quelle ville, on papote avec tout le monde sans problème «. ${ }^{10}$ Solche Verbalisierungen auf kollektiver Ebene erscheinen unproblematisch, geht es dabei doch lediglich um das Anknüpfen an bestehende kollektive Zuschreibungen, deren Konstruktionen bereits abgeschlossen sind und als kulturelle Identitätsangebote zur Verfügung stehen. Hingegen scheint es für die Interviewten ungleich komplexer, Essen und Identität auf individueller Ebene zu verbalisieren. Dies ist der Bereich der als problematisch empfundenen Selbstkonstruktion, d.h. der impliziten, nie abgeschlossenen Subjektivierung. Diese ist von Wahlmöglichkeiten zwischen unterschiedlichen normativen Angeboten geprägt (Corbeau/Poulain 2002) $)^{11}$ und erzeugt dementsprechend Unsicherheit aufgrund unzureichender Eindeutigkeit. Letztgenannte kann als Infragestellung des Selbst und damit als ein allgemeines Gefühl der Angreifbarkeit im Zuge der eigenverantwortlichen Modellierung von >Identity in Progress< empfunden werden.

Um die beschriebenen Problembereiche aufzufangen, wurde das Forschungsinteresse auf ein externes und kontrastives Moment gerichtet. Dieses bestand in der von den Befragten vollzogenen Positionierung der eigenen ernährungsbezogenen Diskurspraktiken hinsichtlich eines spezifischen Konzepts von >gutem< Essen.

9 | Es war nicht Ziel dieser Untersuchung, die Rolle von ,Produits du Terroir und regionaler Küche in luxemburgischen Identitätskonstruktionen zu analysieren; dies wird an anderer Stelle erfolgen.

10 | Deutsch: "Wenn man in Italien in einer Kneipe einen Kaffee trinkt, egal in welcher Stadt, plaudert man problemlos mit Jedem."

11 | Die Vielfalt der möglichen Speiseplangestaltungen soll, den Nahrungsmittelherstellern und -vertreibern nach, die privaten Entscheidungen vereinfachen, indem sie repetitive Langeweile vermeidet; tatsächlich stiften die unterschiedlichen normativen Orientierungen hinter den vielfältigen Produkten jedoch Verwirrung. So koexistieren etwa industrielle, aus Massenzüchtung gewonnene Fertiggerichte mit biologischen Konserven ohne Genmanipulation. Beide vereinfachen die Essenszubereitung, entsprechen aber diametral entgegengesetzten Produktionsketten und Konsumpositionierungen. 
Dabei handelte es sich um den von vier luxemburgischen Ministerien koordinierten $^{12}$ und im Juli 2007 verabschiedeten nationalen Aktionsplan mit dem programmatischen Titel »Gesond iessen, méi bewegen «. ${ }^{13}$ Dieser nationale Aktionsplan (im Folgenden GIMB) wird hier als die institutionelle Zuschreibung eines Identifikationsangebotes aufgefasst, das als politisch und gesellschaftlich wünschenswert dargestellt wird. Dieser Eindruck wird umso deutlicher, wenn die Grundlagen des Aktionsplans - internationale Vergleiche wissenschaftlicher Befunde sowie moralisches Regierungsbestreben im Bereich der >Public Health< (s.u.) - berücksichtigt werden. Die konvergierenden ebenso wie die divergierenden Wechselwirkungen zwischen diesen moralisch gelagerten, kollektiven Zuschreibungen einerseits und der ethisch ausgeprägten, individuellen Aneignungen andererseits ${ }^{14}$ geben Aufschluss über die dynamische Identitätsarbeit der Subjekte im luxemburgischen Kontext.

\section{Gesundheitsorientierung auf politischer Ebene}

\section{Politisches Handlungskonzept}

Zur Annäherung an die Frage, welche performative Zuschreibung im Sinne eines moralischen Identifikationsideals über GIMB vermittelt wird, gilt es zunächst das Selbstverständnis der politisch Verantwortlichen zu erläutern. Die Ausgangsannahmen für die politische (prophylaktische und ggf. korrektive) Intervention hinsichtlich >Public Health in den Bereichen der Ernährung und Bewegung basieren auf vergleichenden wissenschaftlichen Studien der Weltgesundheitsorganisation (u.a. WHO 1999; WHO 2008) und der Europäischen Kommission (u.a. CE, 2007; CE 2005). Insbesondere auf der Definition von Fettleibigkeit (Adipositas) als Epidemie, welche mit steigender Kaufkraft einhergehe

12 | Im Einzelnen handelt es sich um die zuständigen Ministerien in den Bereichen Gesundheit, Erziehung, Familie und Sport.

13 | Deutsch: "Gesund essen, sich mehr bewegen."

14 | Wir folgen Foucault in seiner Unterscheidung zwischen Moral und Ethik. Die Moral steht für "ein Ensemble von Werten und Handlungsregeln, die den Individuen und Gruppen mittels diverser Vorschreibapparate - Familie, Erziehungsinstitutionen, Kirchen usw. - vorgesetzt werden. Es kommt vor, daß diese Regeln und Werte sehr ausdrücklich in einer zusammenhängenden Lehre und in einem ausführlichen Unterricht formuliert werden. Es kann aber auch sein, daß sie in diffuser Weise übermittelt werden und daß sie kein systematisches Ganzes, sondern ein komplexes Spiel von Elementen bilden, die sich kompensieren, korrigieren, stellenweise aufheben und dergestalt Kompromisse oder Ausflüchte gestatten." (Foucault 1984a: 36) Dieser "moralische Code" geht Hand in Hand mit der "Art und Weise, wie man sich führen und halten - wie man sich selber konstituieren soll als Moralsubjekt, das in Bezug auf die den Code konstituierenden Vorschriften handelt" (Ders.: 37). 
und eine Serie von Sekundarerkrankungen ${ }^{15}$ mit sich ziehe, wird rekurrierend Bezug genommen. Auf nationaler Ebene zeigte eine vom luxemburgischen Gesundheits- und Bildungsministerium durchgeführte Studie ${ }^{16}$ ähnliche Ergebnisse wie in den Nachbarländern, was ähnliche Handlungsstrategien der politischen Entscheidungsträger im Rahmen von GIMB legitimierte - von der Einbindung in europäische und internationale Netzwerke bis hin zum Austausch über die jeweiligen Strategien.

Vor diesem Hintergrund sind etwaige Besonderheiten des Großherzogtums nicht in der Ausgangsproblematik angesiedelt, sondern in der Umsetzung der politischen Intervention ${ }^{17}$. Das institutionelle Vorgehen auf nationaler Ebene basiert auf einem Experten/-innen-Ausschuss ${ }^{18}$, einem interministeriellen Ausschuss $^{19}$ und auf öffentlichen Texten ${ }^{20}$. Konzeptionell folgt das Vorgehen den Prinzipien von $>$ Health in All Policies $<^{21}$, der Politik der >Santé $21^{22}$ und der $>$ European Charter on Counteracting Obesity ${ }^{23}$. Ausgehend von einem $»$ multifaktoriellen $\ll^{24}$

15 | Herz-Kreislauf-Erkrankungen, Krebs, Typ-2-Diabetes, Osteoporose etc.

16 | Ministère de l'Education Nationale et de la Formation Professionnelle/Ministère de la Santé/Universität Karlsruhe 2006.

17 | Zunächst bedeutet die gängige zwei- bis viersprachige Kommunikation (Deutsch und Französisch, ggf. erweitert auf Portugiesisch und Englisch) einen erheblichen Mehraufwand, aber gleichzeitig durch die Berücksichtigung unterschiedlicher kultureller Muster und Referenzen einen Mehrwert.

18 | Hier diskutierten vor allem Mediziner/-innen unterschiedlicher Fachbereiche über einen wissenschaftlich-technischen Konsens über national definierte Ernährungsrichtlinien. Insbesondere ging es um eine nachhaltige und aktive Implizierung in die Gestaltung einer ,Public-Healthı-Politik, d.h. darum, "Partner zu vereinen und sie zu begeistern".

19 | Dieser hat eine Koordinierungs- und Evaluierungsrolle der von Partnerinstitutionen eingereichten Projekte, welche unter dem Label und dem Logo GIMB laufen sollen.

20 | Insbesondere die am 05.07.2006 von vier Ministerien (Gesundheit, Erziehung, Sport, Familie) unterzeichnete "Politique commune favorisant l'alimentation saine et l'activité physique", in Form des "Programme national pour la promotion de l'alimentation saine et de l'activité physique 'Gesond iessen méi bewegen`", aber auch die Kriterien, die eingereichte Projekte aufweisen müssen, um das Benutzungsrecht des Labels und des Logos GIMB zu erhalten.

21 Leitinitiative des finnischen Ministeriums für Soziales und Gesundheit (in internationaler politischer Kooperation) während der finnischen EU-Präsidentschaft im Jahr 2006, zur Förderung von "gesunden Entscheidungen" und "Gesundheitsbildung" (Ståhl et al. 2006: 15).

22 | Die Rahmenpolitik der Weltgesundheitsorganisation bzgl. Gesundheit in der europäischen Zone (51 Staaten).

23 | Europäische ministerielle Konferenz, Istanbul, 15.-17.11.2006.

24 | Die Interviewzitate aus dem Absatz "Gesundheitsorientierung auf politischer Ebene" sind dem Experten/-innengespräch vom 16.12.2008 mit dem zuständigen Team im 
Verständnis von Gesundheit, ziehen diese Ansätze einen interdisziplinären und intersektionalen politischen Zugang nach sich. Konkret bündelt das Gesundheitsministerium thematische und zeitlich begrenzte »Aktionen« oder »Projekte«, die von Partnerinstitutionen wie Schulen, Maisons Relais pour Enfants ${ }^{25}$, Gemeinden, aber auch von Vereinen u.Ä. lanciert und unter dem Motto GIMB durchgeführt werden. Darüber hinaus bietet das Ministerium eine Plattform für den Erfahrungsaustausch und die Vernetzung dieser »Akteure im Feld«, jedoch ohne ein systematisiertes Feedback der Ergebnisse von deren Initiativen. Umso weniger besteht ein formaler Austausch zwischen dem Ministerium und der Gesamtbevölkerung, außer über den Weg der eingeschulten Kinder. Diese sollen mit staatlich gefördertem und flächendeckend an Schulen verteiltem pädagogischen Material $^{26}$ in Verbindung kommen und schließlich ihre Eltern sensibilisieren. Dieser Prozess ist - sofern er stattfindet - langfristig angelegt und auf einen gesellschaftlichen bzw. kulturellen Wandel ausgerichtet. Genau um diesen »tiefschürfenden Gewohnheitswandel« sind die Verantwortlichen des GIMB bemüht, d.h. um »Bewusstseinsmachung, die Mobilisierung, Interesse und Engagement schafft«. In den GIMB-Projekten geht es daher nicht um eine »eins-zu-eins Anwendung der ministeriellen Richtlinien, sondern um eine kreative Interpretation dessen, was in der Praxis machbar ist .

\section{Informationskampagne für die Wohnbevölkerung}

Die Verantwortlichen des GIMB sehen sich somit in erster Linie als Impulsgeber/innen für einen langfristigen und nachhaltigen Prozess, welchen sie stop-down über verschiedene Institutionen fördern und schließlich gesellschaftlich verankert sehen möchten. Als Pendant zu der >core activity< der ministeriellen Vergabe des Logos und des Labels GIMB für spezifische Aktionen wird die Wohnbevölkerung flächendeckend durch die Zusendung einer zweisprachigen Informationsbroschüre mit Richtlinien über gesundheitsförderndes Bewegen und Essen im Alltag informiert.

Die in unseren Interviews festgestellte Auseinandersetzung der Wohnbevölkerung mit GIMB zeigt, dass ausschließlich die o.g. Öffentlichkeitsarbeit wahrge-

Gesundheitsministerium entnommen (welches, wie in Luxemburg nicht unüblich, in verschiedenen Sprachen durchgeführt worden ist, jedoch der Lesbarkeit halber auf Deutsch wiedergegeben wird), während die Zitate aus dem Absatz "Eigenverantwortung in alltäglichen Ernährungspraxen “ aus den Interviews mit Personen aus der luxemburgischen Wohnbevölkerung stammen.

25 | Eine aufgrund des großherzoglichen Reglements vom 20.07.2005 gegründete Form von Kindertagesstätten.

26 | Zusammen mit dem Bonner Verlag aid wurden unterschiedliche thematische Mappen ausgearbeitet, die sich als ansprechend, praxisnah und spielerisch verstehen. Die Umsetzung in den schulischen Alltag ist dem Lehrpersonal freigestellt. 
nommen wird. Die »grüne Broschüre $\ll^{27}$ - wie die Broschüre »Freude am gesunden Essen Spaß an der Bewegung!/Le plaisir de bien manger et d'être actif!« (Ausgabe 2007) oftmals von den Interviewten bezeichnet wurde - gliedert sich in eine zweiseitige Einleitung, in Ernährungsempfehlungen, in eine Empfehlung hinsichtlich körperlicher Bewegung (anderthalb Seiten) sowie in eine Adressenübersicht zentraler Institutionen (eine halbe Seite). Der Schwerpunkt liegt mit insgesamt sieben Seiten auf der Ernährung, was vermutlich auf die zahlreichen Auswahlmöglichkeiten in diesem Bereich im Vergleich zur griffigen Maxime »Bewegen Sie sich jeden Tag wenigstens 30 Minuten « (S. 9) zurückzuführen ist.

Trotz der ernsten Hintergrundfolie der Broschüre - die falsche Ernährung und unzureichende Bewegung in statistische Korrelation mit Zivilisationskrankheiten, Krankenkassenkosten und Todesraten setzt, so dass »dringendes Handeln notwendig«(S. 1) sei - hat sich das Verfasser/-innenkollektiv um einen spielerisch-positiven und ermutigenden Tenor bemüht. Dieser wird zusätzlich von sommerlich-frischen Farben der Broschüre und lustigen Illustrationen unterstrichen. Die Empfehlungen werden weitgehend positiv formuliert, so z.B. »nehmen Sie jeden Tag Milch und Milchprodukte zu sich« (S. 5) etc. Können negative Aspekte einer Botschaft nicht umschrieben werden, so wird eine Alternative angeboten, z.B. »beschränken Sie den Konsum von fettreichen Nahrungsmitteln. Ziehen Sie pflanzliche Fette den tierischen Fetten vor « (S. 4). So existieren keine > verbotenen< Nahrungsmittel, vielmehr wird Wert auf Vielfalt und auf Nahrungsmittelzufuhr laut Ernährungspyramide gelegt, d.h. letztendlich auf die Ausrichtungen der Diätetik. ${ }^{28}$

27 | Die gewählte hellgrüne Farbe als Hintergrund für die gesamte Broschüre ist aussagekräftig, da sie einerseits Gemüse konnotiert (als Inbegriff vom Aspekt "gesond iessen", siehe auch unsere Interviewauswertung) und andererseits Natur (als Kulisse für OutdoorFreizeitaktivitäten als junge Familie bezgl. dem Aspekt "méi bewegen").

28 | Bezeichnenderweise ist die Association Nationale des Diététicien(ne)s du Luxembourg die einzige nicht-staatliche Partnerorganisation auf nationalem Niveau, die in der Broschüre aufgeführt ist. 


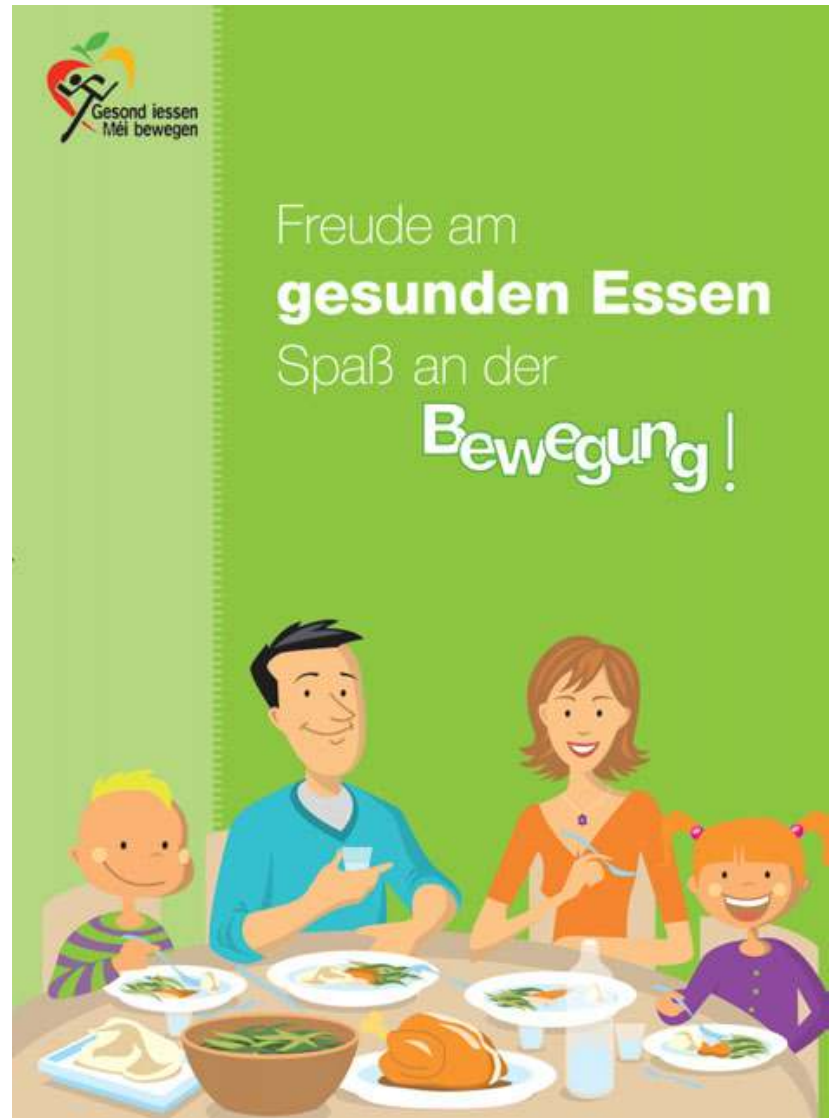

Abbildung 2: Deckblatt der Broschüre »Freude am gesunden Essen. Spaß an der Bewegung!/Le plaisir de bien manger et d'être actif!« (Ausgabe 2007)

In der Einleitung der Broschüre durch den Gesundheitsminister wird die Legitimierung der euphemistisch dargelegten Anstrengung, die die angestrebte Ernährungsumstellung mit sich bringt (»Ich wünsche Ihnen Ausdauer«), durch ein erhöhtes Selbstwertgefühl zementiert, das u.a. durch »Freude« und »Sich wohl fühlen in Ihrer Haut « herbeigeführt werden soll. So geht »Ich wünsche Ihnen Ausdauer « Hand in Hand mit dem »Spaß, neue Ess- und Bewegungsgewohnheiten zu entdecken und in Ihren Alltag aufzunehmen« (S. 1). Mit dieser Distanz schaffenden grammatikalischen Form schwingt eine Wertung mit: Ein bestimmtes >Ich zeigt sich in einer Überlegenheitsposition, die wohlwollende Ratschläge an die Leserschaft gibt. Eine einschließende Formulierung hätte eher Solidarität und Anteilnahme artikulieren können, wie sie etwa das Verfasser/-innenkollektiv der kooperierenden Institutionen formuliert: »Lassen Sie uns gemeinsam die Wohltaten und den Spaß [...] entdecken; sie sind ein wichtiger Bestandteil unseres Wohlbefindens.« 
(S. 2) Aber auch in dieser Textpassage werden die >schlechten < Gewohnheiten - die einen staatlichen Handlungsbedarf erforderlich machen - der als Alterität konstruierten Leserschaft zugeschrieben: »Für die gute Gesundheit brauchen Sie keineswegs ganz auf Ihre Lieblingsspeisen zu verzichten.« Hier erweckt eine Schein-Komplizität über das affektiv besetzte Erleben von Essen den Eindruck, die Relation zwischen Ungesundem und Bevorzugtem sei naturgemäß gegeben. Das Selbstverständnis der Broschüre lässt sich schließlich in einem Ergebnis auf den Punkt bringen, zu dem »verschiedene Studien ${ }^{29}$ kommen: »Jeder vierte Jugendliche und jeder zweite Erwachsene leidet an Übergewicht.«(S. 2) Einerseits wird Übergewicht in dieser Aussage als ein >Leiden< dargestellt, ${ }^{30}$ dessen Existenz oder Wirksamkeit von den betroffenen Personen nicht oder wenig berücksichtigt wird. ${ }^{31}$ So haben z.B. die von uns Befragten nicht ihr Körpergewicht primär als Motivation für gesundes Essensverhalten angeführt. Sie haben vielmehr das ethische Bemühen um verantwortungsvollen Umgang mit dem eigenen Gesundheitskapital im Allgemeinen thematisiert - jedoch nicht im Sinne der Prävention von spezifischen Pathologien. Formal betrachtet stellt die radikale Zuschreibung von >Leiden< einen Bruch zum reformistisch-konstruktiven Gewohnheitswandel dar, den die Broschüre durch ein respektvolles und umsichtiges Vokabular zu vermitteln sucht. Andererseits fokussiert die o.g. Aussage auf Individuen und kommt in dieser Hinsicht dem Alltagserleben von Essen nahe. Unsere Interviews bestätigen das Empfinden der Befragten, dass die Nahrungsauswahl und -zubereitung eine zumeist individuelle und komplexe Entscheidung ist, die die Einzelperson oder auch ihr unmittelbares Umfeld betrifft (z.B. wenn sie für Familie, Mitbewohner, Gäste etc. kocht). Der Anspruch auf ausgewogenes Essen wird laut den Befragungsergebnissen tendenziell durch die Präsenz von Kindern am Familientisch erhöht. Die Broschüre trägt diesem Umstand insofern Rechnung, als die Illustrationen ausschließlich junge Familien darstellen, die implizit die wichtigste Zielgruppe bilden. Inhaltlich setzt der Fokus auf einzelne Ernährungskomponenten ${ }^{32}$ ein analytisches Wissen über Kochzutaten, Speisen- und Mahlzeitenzusammenstellung voraus - obwohl sich die ministeriellen Empfehlungen im Allgemeinen eher pragmatisch orientiert sehen und konkrete Anhaltspunkte ${ }^{33}$ liefern. So können die Empfehlungen auch andere Bevölkerungsgruppen ansprechen, wie im Folgenden zu sehen sein wird.

29 | Bemerkenswerterweise werden in der französischen Version diese wissenschaftlichen Aussagen als "faits" (S. 2) übersetzt.

30 | Eine wertungsfreiere Beschreibung hätte "[...] hat Übergewicht" sein können (obwohl auch hier ein Bezug zu einem normativen Idealwert mitschwingt).

31 | Nur für den Fall der Jugendlichen liegen die international und national durchgeführten HBSC-Studien vor, die auf diese Thematik eingehen.

32 | Obst und Gemüse, Fette, Flüssigkeit, Mehlspeisen, Milchprodukte, Salz und Zucker.

33 | Etwa dass man den ungefähren Fettgehalt einer Backware an "Fettflecken auf der Verpackung“ (S. 4) einordnen kann. 


\section{Eigenverantwortung in alltäglichen Ernährungspraxen}

\section{Selektive Verinnerlichung allgemeiner Ernährungsempfehlungen}

Die Verfasser/-innen des institutionellen Identifikationsangebots GIMB begreifen >gutes< Essen als >gesundes< Essen, das vielfältig und ausgewogen ist und dessen Verzehr Freude machen soll. Das Verständnis der Interviewten weicht von diesem gesundheitsorientierten Konzept z.T. ab, bedingt durch das selektive Aufgreifen verschiedener Aspekte dieses Ansatzes unter Vernachlässigung anderer, sowie durch Erweiterungen der Kategorie des >guten< Essens um anders gelagerte, individuelle oder intersubjektive Kriterien.

Diese relativen Abweichungen lassen sich z.T. auf die Bekanntheit des GIMB zurückführen: $70 \%$ der Befragten geben an, von GIMB gehört zu haben. Von ihnen hat sich jedoch weniger als die Hälfte (43\%) aktiv damit auseinandergesetzt (vor allem aus dem liberal-gehobenen und dem alternativen Milieu, d.h. vorrangig Personen mit Hochschulabschlüssen und hohem Einkommen [5000-7000 $€]$, meistens mit Kindern). Außerdem ist die Aufmerksamkeit für die landesweite ministerielle Informationskampagne gering: »Ich habe nicht sehr darauf geachtet«, »Also schon eng Kéier duerchgekuckt, jo mä net méi« ${ }^{34}$, »Do war mol sou e Flyer «35 oder »Ech kennen nëmmen d'Iwwerschrëft « ${ }^{36}$.

Bemerkenswerterweise verweisen die Angaben der Befragten zum täglichen und wöchentlichen Speiseplan jedoch auf eine - zumindest auf diskursiver Ebene - selektive Verinnerlichung von allgemeinen Ernährungsempfehlungen, auch wenn diese nicht spezifisch mit GIMB in Verbindung gebracht werden. Dieses Ergebnis ist nur bedingt an die betrachteten sozio-kulturellen Milieus gekoppelt; insbesondere für die Kategorien Süßigkeiten und Gebäck, Obst und Gemüse, sowie Getreideprodukte herrscht Konsens in allen Milieus (keine Abweichung vom Durchschnitt von mehr oder weniger als $5 \%$ ), und für alkoholische Getränke und Fleisch in nahezu allen Milieus (eine Abweichung von mehr oder weniger als $5 \%$ ). Die am wenigsten von soziodemografischen Variablen ${ }^{37}$ beeinflussten Nahrungskategorien - deren Konsum also gesamtgesellschaftlich am beständigsten ist - sind Milch und Milchprodukte, Getreideprodukte sowie Obst und Gemüse. ${ }^{8}$ Dementsprechend gaben $81 \%$ der Befragten als am weitesten verbreitetes Ernährungsideal an: »Leichte und kurz gegarte Gerichte machen ein gutes Essen aus«.

34 | Deutsch: "Also schon mal so durchgekuckt, ja, aber nicht mehr."

35 | Deutsch: "Da war mal so ein Flyer."

36 | Deutsch: "Ich kenne nur die Überschrift."

37 | Alter, Geschlecht, Bildung, Beruf, familiäre Situation und (Nicht-)Aktivität der Befragten.

38 | Eine detaillierte Darstellung der Ergebnisse ist an dieser Stelle nicht möglich. 


\begin{tabular}{|c|c|c|c|}
\hline Nahrungskategorie & $\begin{array}{l}\text { Gesamtanteil } \\
\qquad(\mathbf{a}+\mathbf{b})\end{array}$ & Häufigste Antwort (a) & Zweithäufigste Antwort (b) \\
\hline \multicolumn{4}{|c|}{ Angaben zur täglichen Verzehrhäufigkeit } \\
\hline $\begin{array}{l}\text { Milch u. } \\
\text { Milchprodukte }\end{array}$ & $85 \%$ & zwei bis drei Mal pro Tag (43\%) & ein Mal pro Tag ( $42 \%)$ \\
\hline Obst u. Gemüse & $82 \%$ & zwei bis drei Mal pro Tag (44\%) & ein Mal pro Tag (38\%) \\
\hline Alkoholische Getränke & $78 \%$ & nie $(43 \%)$ & ein Mal pro Tag (35\%) \\
\hline Wurstaufschnitt & $77 \%$ & ein Mal pro Tag (48\%) & nie $(29 \%)$ \\
\hline Salzige Snacks & $77 \%$ & nie (52\%) & ein Mal pro Tag (25\%) \\
\hline $\begin{array}{l}\text { Süßigkeiten u. } \\
\text { Gebäck }\end{array}$ & $71 \%$ & ein Mal pro Tag (56\%) & weniger als ein Mal pro Tag (15\%) \\
\hline Soft Drinks & $65 \%$ & nie $(39 \%)$ & ein Mal pro Tag (26\%) \\
\hline \multicolumn{4}{|c|}{ Angaben zur wöchentlichen Verzehrhäufigkeit } \\
\hline Getreideprodukte & $95 \%$ & $\begin{array}{l}\text { mehr als drei Mal pro Woche } \\
(78 \%)\end{array}$ & zwei bis drei Mal pro Woche (17\%) \\
\hline Fleisch & $90 \%$ & $\begin{array}{l}\text { mehr als drei Mal pro Woche } \\
(50 \%)\end{array}$ & zwei bis drei Mal pro Woche (40\%) \\
\hline Fisch & $87 \%$ & ein Mal pro Woche (57\%) & zwei bis drei Mal pro Woche (30\%) \\
\hline Kartoffeln & $80 \%$ & zwei bis drei Mal pro Woche (44\%) & mehr als drei Mal pro Woche (36\%) \\
\hline
\end{tabular}

Tabelle 3: Tägliche und wöchentliche Verzehrhäufigkeiten der Luxemburger Wohnbevölkerung

Auf die im Interview gestellte Frage, was in Bezug auf Ernährung wichtig sei, wird die »Ausgewogenheit« als ein zentrales Anliegen genannt, ebenso der gemäßigte Konsum von sämtlichen Nahrungsmitteln. Dies ist sicherlich der innovativste Aspekt von zeitgenössischen internationalen Ernährungskampagnen (Coveney 2006), da er sich der Essenspraxis der Interviewten annähert. Sehr oft wird das Desiderat nach »mehr Obst und Gemüse« angeführt, nur gelegentlich jedoch wird dies mit dem Schlagwort »fünf am Tag« genannt. Schließlich soll die Ernährungspraxis den Befragten zufolge »nicht zu fettig« sein, wobei sich hier das Gesundheitsbestreben mit dem machtvollen Ideal der Schlankheit überschneidet. Keine oder wenig spontane Erwähnung finden demgegenüber die spezifischen Empfehlungen bzgl. Wasser und Alkohol, Mehlspeisen, Milchprodukten, Salz und Zucker. Dieses Ausklammern geht z.T. auf den hochgradigen Alltagswert von Essen zurück, der eine gewisse Resistenz gegenüber Selbstreflexion mit sich bringt.

\section{'Doing Identity، zwischen Eigenverantwortung, Handlungspotential und Pragmatismus}

Dieses Alltagserleben hinsichtlich >gutem < Essen beinhaltet identitätsrelevante Dimensionen, die über den Rahmen der technischen Essenszubereitung (so wie er im GIMB und anderen ernährungswissenschaftlichen Ansätzen vermittelt wird) hinausgehen. Diese lassen sich in drei diskurspraktische Stränge des >Doing Identity< bündeln: 
1) Am häufigsten tritt der Diskurs der Eigenverantwortung in Erscheinung, der sich in drei Varianten untergliedert (positiv, flexibel, negativ) und unterschiedliche Ansprüche erfüllt (Subjektivation, Verantwortung für Kinder, an ethischen Vorstellungen orientierter Konsum).

In der positiven Variante stellt das alltägliche Anliegen von >gutem « Essen einen herausfordernden Handlungsspielraum dar, in dem selbstbestimmte Subjektivationen ausgetestet werden (über das eigene Kochen) und sich ausprägen können (über resultierende Präferenzen). Hier geht es darum, vieles »einfach ze probéieren «99 und ohne Schuldgefühle zu genießen (»ët soll schmaachen«, »Schëllegkeet erof $\left.«^{40}\right)$. »Aimer ce qu'on mange $\ll^{41}$ wird im doppelten Sinn von Wohlgeschmack und positiver Grundeinstellung zum Essen begriffen, und die marktgängigen Wahlmöglichkeiten werden als Chance anstatt einer Selbstverständlichkeit angesehen (»mir kënnen eis kafen wat mir wëllen « ${ }^{42}$ ).

Ich bin grundsätzlich mit meiner Ernährungsweise im Alltag zufrieden. Ja. Die hängt ja von mir ab. Wenn ich was Falsches koche, dann kann ich nicht sagen, ich bin beim Kochen nicht zufrieden." (Männlich, 50 Jahre, Luxemburger, Junglinster)

Ech passen zimlech op datt et ë bëssen gesond ass, jo vill Uebst. Ech denke mer, et kann een jo awer vill maachen fir seng Gesondheet, dat kann een nët alles den Genen iwwerloossen, ech mengen et kann een och sälwer maachen." ${ }^{43}$ (Weiblich, 74 Jahre, Luxemburgerin, Dudelange)

Ech sin zefridden mat menger Ernährungsweis am Alldag, wëll ech éischtens mol doheem sälwer kachen, dat heescht ech sichen sälwer eraus wat ech iesse wëll. [...] Firdrun hun ech alles giess wat mir an d'Aën geflunn ass, ewourobber ech Loscht hat, an elo kucken ech vill méi drop wat ech genau iessen, zum Beispill méi Friichten, manner Schokela, nët zevill naschelen [...]. Am wichtegsten ass esou équilibréiert wéi méiglech, elo nët all Dag Nuddelen, mee esou vill wéi méiglech an verschidden ewéi méiglech an och ... jo nët ze vill Naschereien dertëschent, dat heescht datt ech wirklech ee fixe Plang hunn. ${ }^{44}$ (Weiblich, 18 Jahre, Portugiesin, Stadtbredimus)

39 | Deutsch: "einfach zu probieren".

40 | Deutsch: "es soll schmecken «; "ohne Schuldgefühle".

41 | Deutsch: "das was man isst lieben". Die oben kommentierte Doppelbedeutung von diesem französischen Satz findet sich im Deutschen nicht wieder, wo eher "sein Essen mögen" gesagt wird.

42 | Deutsch: "wir können uns kaufen was wir wollen".

43 | Deutsch: "Ich passe ziemlich auf, dass es ein bisschen gesund ist, ja, viel Obst. Ich denke mir, man kann doch viel für seine Gesundheit tun, das kann man nicht alles den Genen überlassen, ich meine, man kann auch selbst etwas tun."

44 | Deutsch: "Ich bin zufrieden mit meiner Ernährungsweise im Alltag, weil ich erstens zu Hause mal selbst koche, das heißt, ich wähle selbst was ich essen will. [...] Vorher aß ich 
Verbreiteter jedoch als solche dezidiert voluntaristischen Positionen sind flexible Arrangements, bei denen einzelne Abweichungen toleriert werden, wenn die Gesamtbilanz subjektiv angemessen erscheint:

Ech denke mëttlerweil datt ech also net sou schlecht ..., awer bon, 't sinn mol Saachen derbäi, déi net onbedéngt misste sinn. Mee bon, se sinn derbäi. “ ${ }^{45}$ (Weiblich, 27 Jahre, Luxemburgerin, Vianden)

Demgegenüber wird die negative Variante des Diskurses der Eigenverantwortung weitgehend mit dem Argument des »Zeitmangels«, aber auch des geschmacklichen Konservatismus hervorgebracht. Diese Legitimierungen erscheinen jedoch vielmehr als eine passive Vermeidung ${ }^{6}{ }^{6}$ der Komplexität von Selbsttechniken im kulinarischen Bereich, die Planung sowie >Ad-hoc <-Entscheidungen, Positionierung in einem Feld von nahezu unbegrenzten Wahlmöglichkeiten, Ideenreichtum, technischem Können und Zeiteinteilung bedeutet.

J'aimerais manger plus équilibré, plus ... disons que je n'ai pas trop le temps donc euh ... Et puis quand on mange au travail, on est toujours obligé de manger des choses qui vont vite euh ... - Et vous pensez que vous pouvez un jour changer ça? - Non je pense pas, mais pourquoi pas? Enfin, il faut se prendre peut-être le soir pour cuisiner quelque chose en avance et ramener au travail, euh je ne sais pas, j'ai pas encore trouvé la solution mais euh ... - Qu'est-ce qui l'empêche en fait? - Ben le temps, le temps. C'est que quand on rentre du travail le soir, on est fatigué ... Si, je pourrais, je pourrais ... mais il y a des jours où ça va, mais c'est pas régulier, ça peut être un jour où je vais manger très bien, vraiment, salade, légumes, tout ça, poisson, et l'autre jour ça va être catastrophe.." ${ }^{47}$ (Weiblich, 28 Jahre, Französin, Luxemburg-Stadt)

alles was mir in den Blick kam, worauf ich Lust hatte, und jetzt achte ich viel mehr darauf was ich genau esse. Zum Beispiel mehr Obst, weniger Schokolade, nicht zu viel naschen. [...] Das wichtigste ist, so ausgewogen wie möglich, also nicht jeden Tag Pasta, aber so viel wie möglich und so verschieden wie möglich und auch ... ja, nicht zu viele Naschereien dazwischen, das heißt, dass ich wirklich einen festen Plan habe."

45 | Deutsch: "Ich denke mittlerweile, dass ich mal gar nicht so schlecht ... aber gut, es sind mal Sachen dabei, die nicht unbedingt sein müssten. Aber gut, sie sind dabei."

46 | Es bliebe zu klären, inwiefern es sich hier um eine Selbsteinschätzung (mangelndes Selbstvertrauen) oder eine Überforderung (mangelnde Kompetenz) handelt.

47 | Deutsch: „Ich würde gerne ausgewogener essen, mehr ... Sagen wir, dass ich nicht viel Zeit habe, also eh ... Und wenn man auf der Arbeit isst, ist man gezwungen Zeug zu essen, das schnell geht eh ... - Und meinen Sie, dass Sie das eines Tages ändern können? - Nein, ich glaube nicht, aber warum nicht? Na ja, vielleicht muss man sich abends Zeit nehmen um etwas im Voraus zu kochen und mit zur Arbeit zu nehmen, eh ... Ich weiß nicht, ich habe die Lösung noch nicht gefunden aber eh... - Was hindert Sie daran? - Ach, die Zeit, die Zeit. Wenn man abends von der Arbeit zurückkommt, ist man müde ... Doch, ich könnte, ich 
Ausgewogen na ja ... aber wir essen jeden Tag etwas anderes, unterschiedliche Speisen, aber ... Ich könnte vielleicht schon noch einiges ändern, aber es müsste alles so in der gleichen Geschmacksrichtung liegen, die wir gewohnt sind. (Männlich, 38 Jahre, Portugiese, Consdorf)

Eine Person macht sich jedoch bewusst, dass der besagte Zeitmangel auf eine subjektive Prioritätensetzung in anderen Bereichen (insbesondere dem beruflichen) zurückzuführen ist:

Ech sinn eigentlech net zefridden mat menger Ernährungsart a -weis, wëll ech u sech wéisst, wéi ee sech gesond ze ernähren hätt a probéiere menge Kanner dat ze vermëttelen, mä mech selwer awer meeschtens sou ënner Drock setzen, datt ech mech net drun halen. ${ }^{48}$ (Weiblich, 30 Jahre, Luxemburgerin, Dippach)

Das Ideal der verantwortungsvollen Selbstregierung - ob sie nun mit einer gewissen Offenheit oder mit Zweifeln und Missmut praktiziert wird - gewinnt an Bedeutung, wenn es zusätzlich zum >Ich < an die eigenen Kinder herangetragen wird. Diese Ernährerrolle (im wörtlichen Sinn) scheint so weit verinnerlicht zu werden, dass sie auch noch im Zusammenhang mit erwachsenen Kindern als sinngebend genannt wird. Der Diskurs der notwendigen Vorbildrolle der Eltern für ihre Kinder beinhaltet insbesondere eine normative Aufwertung der Kategorie >Obst und Gemüse<.

Ech sinn zefridden mat der Art a Weis méi ech mech ernähren wëll dat bestëmmen ech jo selwer. An ech kucken wëll ech zwee Kanner hei am Stot hunn, bon deen een ass 18, dat anert ass 24 ... kucken ech awer datt bëssen Uebst a Geméis... a variabel, datt d'lessen net langweileg ass an datt se gär iessen, jo. ${ }^{49}$ (Weiblich, 53 Jahre, andere Nationalität, Lenningen)

Ich finde Suppe sehr wichtig für die Ernährung. [...] Und es ist eine gute Art, die Kinder dazu zu bringen, Gemüse zu essen. (Weiblich, 47 Jahre, Portugiesin, Putscheid)

könnte ... aber es gibt auch Tage wo es geht, aber es ist nicht regelmäßig, das kann ein Tag sein wo ich wirklich sehr gut esse, Salat, Gemüse, all' so was, Fisch, und ein anderer Tag wo es katastrophal ist."

48 | Deutsch: "Ich bin eigentlich nicht zufrieden mit meiner Ernährungsart und -weise, weil ich an sich wüsste, wie ich mich zu ernähren hätte und probiere, das meinen Kindern zu vermitteln, aber mich selbst meistens so unter Druck setze, dass ich mich nicht daran halte." 49 | Deutsch: "Ich bin zufrieden mit der Art und Weise wie ich mich ernähre, weil das bestimme ich ja selbst. Und ich schaue, weil ich zwei Kinder hier im Haushalt habe, gut, der eine ist 18, die andere ist 24 ... ich schaue aber, dass ein bisschen Obst und Gemüse ... und abwechselnd, dass das Essen nicht langweilig ist und dass sie gerne essen, ja." 
Mit der Sorge um die eigenen Kinder ist teilweise das Bestreben nach einer ethischen Konsumform verknüpft. Statistisch gesehen handelt es sich bei dieser Einstellung um ein Segment der Bevölkerung, das tendenziell den älteren Jahrgängen angehört, einen leicht erhöhten Anteil an Frauen zählt, eher zu den Nicht-Erwerbstätigen (Hausfrau/-mann, pensioniert) oder zu den Selbstständigen zählt, Kinder hat und Hochschulabschlüsse aufzeigen kann. Dieses Profil entspricht im Wesentlichen dem der Personen, die GIMB kennen und sich damit auseinandergesetzt haben - was bedeutet, dass GIMB vor allem von denjenigen rezipiert wird, deren Ernährungspraxis sich ohnehin schon an der >gewünschten< Richtung orientiert. Hier ist das als explizit verantwortungsvoll dargestellte Handeln entweder auf die Nahrungsmittel selbst gerichtet (etwa Rückverfolgbarkeit oder Qualität des Produkts) oder auf die gesamte Herstellungskette (etwa Saisonalität, Regionalität, biologische Landwirtschaft, fairer Handel oder artgerechte Tierhaltung). Da Bemühungen um Nachhaltigkeit durch einen wahrgenommenen Mehrwert für die eigene Gesundheit oder auch durch die wahrgenommene Affektivität bei der Herstellung motiviert sind - also durch identitätsrelevante Kriterien -, ist diese Haltung als ambivalent zu betrachten (Lamine 2008):

Ech ginn haaptsächlech an Bio Rayonen akafen. [...] Haaptsächlech Geméis muss wierklech ... do muss nach eppes dra sinn. [...] Ech sinn iwwerzeegt, datt déi industriell Nahrungsmëttel, datt do, alles wat sou ouni Léift gemaach ass, datt do wierklech just nach minimal eppes Guddes fir eis dran ass. ${ }^{50}$ (Weiblich, 41 Jahre, Luxemburgerin, Bous)

2) Ein anderer diskurspraktischer Strang ist der des hypothetischen Handlungspotentials; insbesondere im Fall unerwarteter Gesundheitsprobleme oder akuter Gewichtszunahme »wéisst een, wat een ze maachen hätt «51. Solange jedoch keine größeren Veränderungen feststellbar sind, herrscht Nachsichtigkeit bzgl. gelegentlichen >Ausrutschern < vor - wie auch immer diese in der Alltagspraxis definiert werden.

Fir déi Leit déi sech selwer iergendwéi iwwergewiichteg fannen oder déi selwer fannen datt se net gesond iessen ass dat vläicht ee flotten ... een Input, sou ze soen an si gesinn, okay wann jiddereen dat mécht, dann kann ech och dozou bäidroen, mee ech perséinlech, also nee. ${ }^{52}$ (Männlich, 18 Jahre, Italiener, Strassen)

50 | Deutsch: "Ich kaufe vor allem in Bioregalen ein. [...] Hauptsächlich Gemüse muss wirklich ... da muss noch was drin sein. [...] Ich bin überzeugt, dass diese industriellen Nahrungsmittel, dass da, alles was so ohne Liebe gemacht ist, dass da wirklich nur noch minimal etwas Gutes für uns drin ist."

$\mathbf{5 1}$ | Deutsch: "wüsste man, was man zu tun hätte“.

52 | Deutsch: "Für Leute, die sich selbst irgendwie übergewichtig finden oder die selbst finden, dass sie nicht gesund essen, ist das vielleicht ein netter ... Input, sozusagen und 
Diese Position kann bis hin zu einem bewusst beanspruchten Gegenentwurf reichen:

Da kuckt een dat [0.g. Broschüre], et stellt een awer keng Gedanken drop, da leet een et rëm op d'Säit. Wëll mir beweegen eis net sou gär! ${ }^{53}$ (Weiblich, 66 Jahre, Luxemburgerin, Ettelbruck)

Oftmals ist eine Dialektik zwischen einer allgemeinen und einer persönlichen Diskursebene feststellbar. Initiativen wie das GIMB (aber auch Informationen über >gutes< Essen in den Medien und in der Fachliteratur) werden als allgemein erstrebenswert angesehen, die Person selbst fühlt sich jedoch nicht davon angesprochen, so z.B.: »Ech fannen et gutt datt de Ministère eppes mécht. Ob et eppes déngt, dat ass eng aner Fro. ${ }^{54}$ Dies ist ein typisches alltagskulturelles Paradox, das nicht selten mit Humor umschifft wird. Die Ambivalenz der >Lösung < wird dadurch zwar alltagstauglich entschärft, aber nicht aufgehoben:

- Wie sind Sie auf das Programm "Gesond iessen méi bewegen" aufmerksam geworden? - Durch die Medien. - Und was halten Sie davon? - In unserer Gemeinde gibt's auch so ein Slogan ein Mal pro Jahr. Da werden die Straßen hier für den Verkehr abgesperrt und ich glaube das geht in dieselbe Richtung ${ }^{55}$. - Haben denn diese ...? - [unterbricht] Die bewegen aber leider bei mir nicht sehr viel (lacht)! (Männlich, 37 Jahre, Luxemburger, Bertrange)

3) Schließlich lässt sich vor dem Hintergrund der vorangegangenen diskurspraktischen Stränge der Pragmatismus angesichts widersprüchlicher kursierender Normen ausmachen. Dieser Pragmatismus stellt gewissermaßen das >banalste $<$ und weit verbreitetste Handlungsmuster in Bezug auf die alltägliche Ernährung dar - eben aufgrund der inhärenten Ambivalenz, die Widersprüche zwar anerkennt, sich jedoch mit ihnen arrangieren kann. Demgegenüber fungieren die >Eigenverantwortung < und das >hypothetische Handeln< eher als bewusste Positionierungen. Die Widersprüchlichkeit der Essensnormen wird ungleich intensiv wahrgenommen (von verwirrend über gleichgültig bis auffordernd). Sie kann außerdem zeitlich strukturiert sein - etwa wenn die Wertvorstellungen der (vergangenen) Essenssozialisation nicht mit denen der (aktuellen) Praktiken übereinstimmen. Die herausgearbeitete Ambivalenz kann des Weiteren ethisch orientiert sein, insbesondere

sie sehen, okay, wenn jeder das macht, dann kann ich auch dazu beitragen, aber ich persönlich, also nein."

53 | Deutsch: "Dann schaut man sich das an [0.g. Broschüre], man verschwendet aber keinen Gedanken daran, dann legt man es wieder zur Seite. Weil wir bewegen uns nicht so gerne!"

54 | Deutsch: "Ich finde es gut, dass das Ministerium etwas tut. Ob es was nützt, das ist eine andere Frage."

55 | Im Sinne eines thematischen Aktionstags, z.B. ein autofreier Sonntag. 
auf der Ebene der strukturellen Entwicklungen des Ernährungsangebots - etwa wenn die Industrialisierung der Nahrungsmittelherstellung Zweifel an deren Qualität aufkommen lässt. Sie kommt ebenso situationsbedingt zum Vorschein, vorzugsweise auf der Ebene der individuellen Konfrontation mit spezifischen Einschränkungen - etwa wenn Ernährungsempfehlungen und -angebot auseinanderklaffen. Folgendes Zitat bringt diese Dimensionen auf den Punkt:

On n'arrive jamais à vraiment ... Parce qu'en plus, la télé, les médecins, ils vous disent: "il faut manger comme çau, en plus maintenant la nourriture n'est plus aussi saine qu'avant alors ... [...] On vous dit tout le temps, on vous dit plus quoi manger que dans le temps. Je veux dire, nos parents, on leur disait toujours: "mange, finis ton assiette, mange bien", et nous on nous dit: "attention, ne mange pas trop"! [...] II faut faire plus attention. [...] Y a beaucoup plus de choix et puis d'un autre côté, on vous dit: "attention, n'en fais pas trop", alors on est un peu frustré. ${ }^{56}$ (Weiblich, 28 Jahre, Französin, Luxemburg-Stadt)

Doch unabhängig davon, wie hoch der kognitive Aufwand der Ernährungszusammenstellung ist, wird er im Alltagserleben durch eine pragmatische Fokussierung auf den kommunikativen Wert und die Vergemeinschaftung durch Essen kompensiert. So liegt das gemeinsame Essen »am Familljekrees« oder »all zesummen« vielen Interviewten am Herzen; »An Arbeitstagen ist Essen für mich eine gute Gelegenheit, mit anderen zusammen zu sein«, wurde dementsprechend von $74 \%$ der Befragten 57 bejaht:

't ass einfach fir zesummen ze sinn an bëssen, jo, ze schwätzen. [...] Et schafft een, also déi eng ginn schaffen, déi aner ginn an d'Schoul, d.h. et ass ee bal ni zesummen a wéinstens ass een dann, wann een ësst, dann ass een zesummen. Et soll een och e bësse Kommunikatioun hunn. ${ }^{58}$ (Männlich, 18 Jahre, Italiener, Strassen)

56 | Deutsch: "Man bringt es nie fertig, wirklich ... Weil zusätzlich sagen dir das Fernsehen und die Ärzte: ,Man muss so und so essen. Außerdem ist das Essen heute nicht mehr so gesund wie früher, daher ... [...] Man sagt Dir dauernd, man sagt dir mehr was du essen sollst als früher. Ich will damit sagen, dass man unseren Eltern immer sagte: Iß iß deinen Teller leer, iß gut, und uns sagt man: 'Achtung, iß nicht zu vielı! [...] Man muss mehr aufpassen. [...] Man hat viel mehr Auswahl und andererseits sagt man dir: "Achtung, übertreibe nicht", dann wird man etwas frustriert."

57 | Bei dieser Einstellung sind vor allem zwei Variablen statistisch tragend: das Alter und die Erwerbstätigkeit. Insbesondere die noch nicht oder nicht mehr im Erwerbsleben Stehenden sowie die nicht Erwerbstätigen legen Wert auf diese Art der alltäglichen Geselligkeit - was wiederum bestätigt, dass die emotionale Verfügbarkeit zum Essen entscheidend ist.

58 | Deutsch: "Es ist einfach um zusammen zu sein und ein bisschen, ja, zu reden. [...] Man arbeitet, also die einen gehen zur Arbeit, die anderen gehen zur Schule, d.h. man ist fast nie 


\section{Identitäre Oszillationen}

Das angeführte Fallbeispiel hat Konvergenzen und Diskrepanzen zwischen moralischer Zuschreibung und ethischer Aneignung im Bereich des sguten< Essens - als beispielhafte Kristallisierung von identitären Konzeptionen auf politisch-institutioneller und alltagskulturell-individueller Ebene - verdeutlicht. Die prozesshafte und kreativ ausgerichtete Gesundheitsorientierung auf politischer Ebene ist praxisnah gestaltet. Indem sie ausschließlich auf das Essen selbst - seine ausgewogene Zusammenstellung und das daraus resultierende sensorische Wohlbefinden - fokussiert, behandelt sie jedoch nur den analytisch-kognitiven Teil in Bezug auf >gutes< Essen der Wohnbevölkerung. Somit spricht sie insbesondere diejenigen Personen an, die sich der Herausforderung der Eigenverantwortung sowie der Subjektivierung bei der Ernährung positiv oder flexibel stellen und über die hierfür notwendigen Kapitalien verfügen. In diesen Fällen wird, im Bereich des Essens, die Identitätsarbeit im o.g. Sinn teilweise zur Selbstverwirklichung - etwa wenn eine bewusst gewählte gesundheitsorientierte Ernährungsideologie (z.B. Vollwert-Nahrungsmittel, biologische oder biologisch-dynamische Herstellung, regionale Produkte, Vegetarismus, o. ̈.) als spezifisches, distinktives Identitätsmerkmal fungiert. Demgegenüber hat die Mehrzahl der Befragten einen pragmatischen, weniger puristischen und weniger gesundheitsorientierten Zugang zur Ernährung, die weniger objektbezogen und vielmehr personenbezogen gelebt wird. Hier stehen sowohl das subjektive - eher spontane, wenig reflektierte und gesundheitstechnisch eventuell kurzsichtige - Erleben im Vordergrund, als auch intersubjektive und kulturelle Dimensionen der Vergemeinschaftung. (Reckinger 2008) Letztere zeigt sich insbesondere als Sorge um die Kinder und als kommunikativer Zusammenhalt von >meaningful others<, d.h. als affektive und soziale Identitätsarbeit in einem umfassenderen Sinn als nur Gesundheitsorientierung. Gerade diese pragmatisch-hedonistische und personenbezogene Ausrichtung auf individueller und kollektiver Ebene wird bei dem betrachteten politischen Identifikationsangebot ausgeblendet. ${ }^{59}$ Dementsprechend ist in der Interaktion zwischen den betrachteten Zuschreibungen und Aneignungen eine permanente Oszillation auszumachen, da sich das institutionelle Regierungsbestreben im foucaultschen Sinne (hier: GIMB) und die alltagskulturellen Selbstregierungen (hier: die befragte Wohnbevölkerung) teilweise überlagern und eine permanente und grundsätzlich ambivalente Variation zulassen. Die ethische Aneignung des Ideals des >guten< Essens kann sowohl mit den moralischen, intentionalen und korrigierenden Logiken der politischen Zuschreibung in Einklang stehen (>identitäre Anpassung ২),

zusammen und wenigstens ist man dann, wenn man isst, dann ist man zusammen. Man soll auch ein bisschen Kommunikation haben."

59 | Da das GIMB jedoch als langfristiger und gesellschaftlicher "Prozess" und nicht als "Projekt" mit sofortigen konkreten Auswirkungen angelegt ist (Wagener 2008: 25), wären Synergien in diese Richtung zukünftig nicht unmöglich. 
von ihnen abweichen (>identitäre Opposition<) oder ganz anderen Mustern folgen (>identitäre Eigenständigkeit<). Eben in dieser Dynamik liegt die Bedeutung des gewählten Fallbeispiels für die Gouvernementalitätsforschung ${ }^{60}$ bzw. für die Beleuchtung von identitätsbezogenen Konstruktionsprozessen.

\subsection{Die Grenzgänger als vertraute Fremde}

Die Hinwendung zum Grenzgängerwesen als ein weiteres illustratives Beispiel für Prozesse der Identitätsbildung liegt in seiner Entwicklung und Rolle für die luxemburgische Gesellschaft begründet. Mit insgesamt 147.400 Männern und Frauen (2009), die täglich aus dem Saarland und aus Rheinland-Pfalz (DE), aus Lothringen (FR) oder Wallonien (BE) nach Luxemburg an ihren Arbeitsplatz pendeln, zählt Luxemburg die meisten Grenzgänger in der EU-27 (European Commission 2009: 18-20). ${ }^{61}$ Darunter befinden sich zur Hälfte Franzosen und jeweils ein Viertel Belgier bzw. Deutsche. Ihre Gesamtzahl hat sich seit Ende der 1980er Jahre versechsfacht, wobei 1995 erstmals mehr Grenzgänger/-innen als ansässige Ausländer/-innen im Großherzogtum arbeiteten und 2001 die Zahl der Grenzgänger/-innen die der Arbeitnehmer/-innen mit luxemburgischer Staatsbürgerschaft überstieg. Heute (2009) beläuft sich der Anteil der Luxemburger/-innen am Arbeitsmarkt auf $29 \%$, der ansässigen Ausländer/-innen auf $27 \%$ und der Grenzgänger/-innen auf 44 \%. Die starke Anziehungskraft des Großherzogtums ist neben weichen Faktoren (Jobadäquation, Karrierewege usw.) vor allem auf den attraktiven Nettoverdienst und das Arbeitsplatzangebot zurückzuführen. Denn im Gegensatz zu den Nachbarregionen werden in Luxemburg trotz Konjunkturtiefs mehr Jobs geschaffen als - wie zu erläutern sein wird - mit ansässigen Arbeitskräften besetzt werden können.

\section{Theoretische Annäherung an den Status der Grenzgänger}

Angesichts des seit Jahrzehnten außergewöhnlich hohen quantitativen Gewichts und der damit verbundenen Abhängigkeit von Arbeitskräften aus den Nachbarregionen stellt sich die Frage, ob den Grenzgängern in Luxemburg der Status des Fremden oder des Vertrauten zukommt. Damit stehen hier die Wahrnehmungen

60 | Nach Foucault (1993; 1984b; 1983; 1982a; 1982b; 1978; vgl. auch Bröckling/Krasmann/Lemke [2000]) setzt sich dieser Neologismus aus 'Gouverner (Regieren) und 'Mentalité، (Denkweise) zusammen und ermöglicht eine simultane und relationale Lesart auf kollektive und individuelle Formen der identitären Regierung - in Form von moralischen und politischen Rationalitäten ebenso wie von ethischen und individuellen Selbsttechniken. So konvergiert staatliche Kontrolle mit Selbstkontrolle - diese Konvergenz ist jedoch fundamental dynamisch und veränderbar, wie vorliegendes Fallbeispiel aufzeigt.

61 Lediglich in der Schweiz arbeiten mehr Grenzgänger aus den benachbarten Ländern. 
des Grenzgängerwesens durch die Luxemburger Wohnbevölkerung im Vordergrund, welche verschiedene Formen der Aneignung bzw. der Konstruktion des Grenzgängerwesens repräsentieren. Die Beschäftigung mit dem Fremden führt auf theoretischer Ebene zunächst zu jener soziologischen Richtung, die Stichweh als »klassische Soziologie des Fremden« bezeichnet (Stichweh 2005). Dabei handelt es sich um den »Exkurs über den Fremden« von Georg Simmel, der den Fremden als Wanderer mit einer sozialen Gemeinschaft in Beziehung setzt. Er unterscheidet zwischen den Konsequenzen für die aufnehmende Gemeinschaft und der Beobachtung derselben aus der Perspektive des Fremden. (Simmel 1908) Diese Positionierungen werden von Robert Park im Konzept des >marginal man $<$ erweitert, welcher sich auf der Grenze zwischen zwei Kulturen befindet und Ressourcen entwickeln muss, um einen Kulturkonflikt zu lösen. (Park 1974) Schließlich fragt Alfred Schütz in handlungstheoretischer Perspektive nach den psychischen Prozessen, die der Fremde zu bewältigen hat, wenn er sich in ein Feld unvertrauter Zivilisationsmuster begibt. (Schütz 1971) Ebenso wie Park misst Schütz den Status des Fremden daran, ob ihm die Übernahme der in der aufnehmenden Gemeinschaft geltenden Regeln gelingt oder ob er als Fremder weder zu seiner alten noch zu seiner neuen Umgebung gehört. Die Gemeinsamkeit dieser Ansätze besteht darin, dass sie im Fremden jeweils einen >Eindringling hen, die als normativ integrierter Verband beschrieben wird. Diese Vorstellung von homogenen Ingroups, die für Outsider nur schwer zugänglich sind, geht vermutlich auf den Erfahrungshorizont unidirektionaler und dauerhafter Migration im 19. und 20. Jahrhundert zurück und lässt sich mit der dichotomen Denkfigur >Vertrauter/Fremder < auf den Begriff bringen. Mit Blick auf das Grenzgängerwesen als eine zirkuläre Mobilitätsform würde dies bedeuten, dass die Frage nach seinem Status über die normbezogene Zugehörigkeit gelöst werden könnte. Somit wären Grenzgänger als >vertraute Insider<, welche das normative Regelwerk der luxemburger Gesellschaft beherrschen, oder als >fremde Outsider < bestimmbar.

Im Zuge der Ausdehnung transnationaler Lebenswirklichkeiten (Pries 2008; Kreutzer/Roth 2006), zu deren Akteuren auch die Grenzgänger/-innen zählen, greift die auf normbezogene Zugehörigkeit beruhende Denkfigur jedoch zu kurz. Vielmehr ist zu hinterfragen, »um welche Modalitäten es sich eigentlich handelt, in denen jemand als Fremder erfahren werden kann« (vgl. Stichweh 2005: 141). Die Frage nach dem Fremden kann sich in transnationaler Perspektive also nicht mehr an nationale Superkollektive und an die der Normenübernahme unterworfenen >Eindringlinge< richten, sondern sie muss auf die von den Ansässigen vollzogenen Konstruktionen des Fremden bzw. Vertrauten fokussieren. Denn soll die Perspektive der normativ integrierten Gesellschaften aufgebrochen und das Fremde sich auch im spätmodernen Alltag als theoretische Kategorie behaupten, so ist nach den Prozessen zu fragen, die soziale Phänomene als fremd bzw. als vertraut konstruieren. Hinsichtlich der Grenzgänger/-innen muss es also darum gehen, ihren Status anhand der Aneignungen bzw. Wahrnehmungen der Luxemburger Wohnbevölkerung zu bestimmen. Konzeptionelle Anhaltspunkte hierfür liefert der An- 
satz Armin Nassehis, der die Dichotomie der positiven und negativen Aneignung (+/-) von sozialen Phänomenen einführt. (Nassehi 1995) Damit kann das Vertraute - als Kehrseite des Fremdem - zweifach besetzt werden: positiv und negativ. Dieser theoretische Zugang, der mit dem Denkmodell > Vertrauter (+/-)/Fremder< wiedergegeben werden kann, macht Formen der gesellschaftlichen Binnendifferenzierung greifbar. Andererseits behält das Denkmodell die Kategorie des Fremden bei, welche soziale Phänomene auffängt, die sich der positiven oder negativen Aneignung durch die Subjekte verweigern und damit außerhalb des Vertrauten anzusiedeln sind. Mit Blick auf den Status des Grenzgängerwesens bedeutet dies, dass der Grenzgänger als Vertrauter bestimmt werden kann, wenn die durch die Wohnbevölkerung vollzogenen Aneignungen positiv oder negativ ausfallen. Als Fremder wäre er zu bestimmen, wenn die entsprechenden Aneignungen ambivalent anzunehmen sind, d.h., wenn sich die Wohnbevölkerung positiv und negativ gegenüber dem Grenzgängerwesen positioniert. Solche Aneignungsprozesse des Fremden oder Vertrauten werden in allen Gesellschaften praktiziert, sind sie doch auf identitätskonstitutive Differenzsetzungen angewiesen, die im Alltag durch Inklusion (positive Aneignung) und Exklusion (negative Aneignung) realisiert werden. Angesprochen sind damit ein- und ausschließende Praxisstrategien, die als Formen der alltagsdiskursiven Aneignung über spezifische Semantiken kollektive Identitäten konstruieren. Vor diesem Hintergrund werden die Aneignungsprozesse der Wohnbevölkerung im Hinblick auf das Grenzgängerwesen näher betrachtet.

\section{Alltagsdiskursive Aneignungen zwischen 'Unverzichtbarkeit، und 'Bedrohung,}

Durch die erläuterte Entwicklung des Luxemburger Arbeitsmarkts rücken die Grenzgänger/-innen zunehmend in den Wahrnehmungsfokus der Wohnbevölkerung. Die Befragten sind der Überzeugung, dass das Grenzgängerwesen im Vergleich zu den 1980er Jahren heute stärker in Alltagsdiskursen thematisiert wird. Dabei geht es um die größere Präsenz der Pendler/-innen und hiermit verbunden um die Verdichtung von Themen wie etwa Arbeitsplatzkonkurrenz oder Sprachkontakt. Ferner wird angemerkt, dass Grenzgänger/-innen zunehmend als Projektionsfläche für soziale Unzufriedenheit dienen, oder wie ein Befragter es formuliert: »Et gëtt ee gesicht, dee schold ass. « ${ }^{62}$ Die folgenden Einblicke in Aneignungsstrategien hinsichtlich des Grenzgängerwesens berühren Aspekte der Wirtschaft und des Arbeitsmarkts sowie der Sprache und Kultur in Luxemburg. In den Befragungen wurden jeweils positive und negative Implikationen der Grenzgängerbeschäftigung angesprochen, um Anschlüsse an das erläuterte Denkmodell herzustellen.

Zunächst soll untersucht werden, welche Aneignungen des Grenzgängerwesens in sozio-ökonomischer Hinsicht auszumachen sind. Hierfür wurde gefragt,

62 | Deutsch: "Es wird jemand gesucht, der Schuld ist." 
ob Grenzgänger/-innen notwendig seien für die Wirtschaft Luxemburgs, und mit $87 \%$ Zustimmung ist zunächst eine positiv-einschließende Haltung gegenüber den Pendlern/-innen festzustellen. Diese basiert auf zwei Einschlussstrategien: Zum einen handelt es sich um die nützliche Arbeitskraft der Grenzgänger/-innen, die unter dem Gesichtspunkt der unzureichenden ansässigen Arbeitskräfte und der nachgefragten Qualifikationen, über die Luxemburger/-innen nur teilweise verfügen, thematisiert wird. Hintergrund dafür ist das Wirtschaftswachstum Luxemburgs, das ohne die Grenzgänger/-innen nicht möglich (gewesen) wäre. So werden seit einigen Jahren rund zwei Drittel der jährlich neu geschaffenen Arbeitsplätze mit Grenzgängern besetzt, womit nicht nur Arbeitskräfte, sondern ebenso die nachgefragten Qualifikationen ins Land geholt werden.

Dat fannen ech ganz richteg, well mir hu jo eendeiteg net genuch Leit, déi schaffe ginn; an menger Usiicht no, wa mir keng Grenzgänger hätten, hätte mir vill méi Problemer hei zu Lëtzebuerg. Da giff eis Economie och guer net fonctionnéieren; an vu que datt mir awer déi Grenzgänger hunn, hu mer eng Chance fir ze fonctionnéieren, respektiv, wat elo mat der Finanzkrise kënnt, weess ee jo awer net; also, mä et sinn och vill Lëtzebuerger, déi einfach ... bon, et wäert sécher alt, gesot: ze liddereg si fir schaffen ze goen; respektiv, si hunn einfach näischt geléiert, dat heescht si hunn op der 9ième opgehal, an ... 'Oh mir kréie jo eng Plaz‘. Mee haut kriss Du keng Plaz méi ouni, a mëttlerweil hunn d'Grenzgänger zimlech vill Chancen, well si awer vill méi Ausbildung hunn, wéi esou munnechen Lëtzebuerger. ${ }^{63}$ (Weiblich, 18 Jahre, Luxemburgerin, Heinerscheid)

Die zweite Einschlussstrategie hebt ebenso auf die Unverzichtbarkeit der Grenzgänger/-innen ab, jedoch erfahren sie hier keine direkte Aufwertung. Ihre Unverzichtbarkeit leitet sich den Befragten zufolge hier aus Tätigkeiten ab, die Luxemburger nicht übernehmen möchten. Sie bringen es auf den Begriff mit »Luxemburger seien sich zu fein«, »wollen sich nicht die Hände schmutzig machen«, weshalb die Grenzgänger/-innen für »Drecksarbeiten« gebraucht werden. Ebenfalls wird angesprochen, dass Grenzgänger/-innen insbesondere für niedrig bezahlte Tätigkeiten unverzichtbar seien, die von Luxemburger/-innen abgelehnt werden.

63 | Deutsch: "Das finde ich ganz richtig, weil wir hier ja eindeutig nicht genug Leute haben, die arbeiten gehen; meiner Ansicht nach, wenn wir keine Grenzgänger hätten, hätten wir hier in Luxemburg viel mehr Probleme. Dann würde die Wirtschaft auch gar nicht funktionieren; aber da wir die Grenzgänger haben, kann das funktionieren; beziehungsweise was jetzt passiert nach der Finanzkrise, weiß man noch nicht ...; also, es gibt auch viele Luxemburger, die ... ja, man kann schon sagen, die einfach zu faul sind arbeiten zu gehen; ... beziehungsweise die haben einfach nichts gelernt, die haben nach der 9 . Klasse die Schule beendet und... Ja, wir kriegen schon einen Job. Aber heute kriegst du keinen Job mehr ohne, und mittlerweile haben die Grenzgänger ziemlich viele Chancen, weil sie eine bessere Ausbildung haben als so mancher Luxemburger." 
An et sinn der och vill, wou verschidde Lëtzebuerger sech ze gutt sinn, fir déi ze maachen. ... Also, di Drecksaarbechten. Wann dat net bei der Gemeng ass oder esou, da si vill Lëtzebuerger, déi soen: "Oh nee dofir ginn ech awer net schaffen". Also do kennen ech der awer och, déi dat gesot hunn. Oder: "Fir déi Paie ginn ech net."64 (Weiblich, 31 Jahre, Luxemburgerin, Rambrouch)

So wie die Qualifikationen der Grenzgänger/-innen und ihre Arbeitskraft für niedrig vergütete Jobs betont werden, sehen die Befragten darin ebenso einen Wettbewerbsvorteil gegenüber Luxemburger/-innen. Das bedeutet, im Alltagsdiskurs werden ebenso ausschließende Strategien praktiziert, die sich unter dem Stichwort der >Arbeitsplatzkonkurrenz< fassen lassen. So sind ein Drittel (34 \%) der Wohnbevölkerung der Meinung, Grenzgänger/-innen nähmen den Luxemburger/-innen die Arbeitsplätze weg und aktivieren in diesem Zusammenhang verschiedene Ausschlussstrategien. Argumentiert wird mit der wachsenden Zahl der Arbeitslosen, die anstelle der Grenzgänger/-innen beschäftigt werden sollten, und mit dem niedrigeren Lohnniveau der Grenzgänger/-innen, das die Luxemburger/innen mit ihren Gehaltsvorstellungen vom Arbeitsmarkt verdränge. Ferner wird auf das Bild der Grenzgänger als »motivierte Arbeitnehmer« verwiesen, das für viele Arbeitgeber/-innen bestimmend und zum Nachteil der Luxemburger sei. Des Weiteren wird mehrfach von der »Grenzgängerisierung « von Unternehmen berichtet, in deren Zusammenhang die Einführung von »Luxemburger-Quoten« vorgeschlagen wird. Besonders ansässige Ausländer/-innen unterstreichen das Konkurrenzverhältnis mit Grenzgänger/-innen und beklagen, dass diese ebenso wenig Luxemburgisch sprächen, aber dennoch einen weitaus besseren Zugang zum Arbeitsmarkt bekommen. Hintergrund hierfür sind strukturell bedingte Selektionsmechanismen des luxemburgischen Bildungssystems und die seit 2001 steigende Arbeitslosigkeit, von der besonders Ausländer, Jugendliche und Frauen betroffen sind. (Statec 2009a: 108)

Sie könnten ja auch die Zahl der Grenzgänger irgendwie begrenzen, statt 130.000 hereinzulassen ..., wenn das so weitergeht und immer mehr Leute hereingelassen werden, dann sieht es in Luxemburg bald nicht mehr so gut aus, so ist die Lage. ... Ein Portugiese kann praktisch nicht mehr hierher kommen, wenn er kein Luxemburgisch kann, und die anderen können doch erst recht kein Luxemburgisch; warum sollen die also herkommen dürfen und wir nicht? Die Deutschen können auch kein Luxemburgisch und kommen hierher und arbeiten für die Kommunen. In diesen Fällen finde ich das nicht in Ordnung. Warum sollen die denn herkommen dürfen ...?" (Männlich, 38 Jahre, Portugiese, Consdorf)

64 | Deutsch: "Es gibt auch viele Luxemburger, die sich zu fein sind für bestimmte Arbeiten. ... Also für die Drecksarbeiten. Wenn die nicht bei der Kommune arbeiten können ... da sind viele Luxemburger, die sagen [dann zu einem]: , Oh nein, für das Gehalt gehe ich aber nicht arbeiten. A Also, da kenne ich auch welche. Oder: 'Für die Kohle gehe ich nicht los. « 
Bereits seit den 1990er Jahren ist auch auf handlungspraktischer Ebene eine Ausschlussstrategie erkennbar, welche eine Segmentierung des Arbeitsmarkts zur Folge hat. Dabei handelt es sich um die Tendenz, dass Arbeitnehmer/-innen mit Luxemburger Staatsbürgerschaft sich zunehmend aus dem privatwirtschaftlichen Sektor auf Arbeitsplätze im öffentlichen und halböffentlichen Sektor zurückziehen. (Vgl. Statec 2009b) Diese sind nicht nur arbeits- und sozialrechtlich attraktiv, ebenso bieten sie >Schutz< vor der Konkurrenz ausländischer Arbeitskräfte. Diese von Fehlen und Pigeron-Piroth (2009) als »Rückzugsstrategie« bezeichnete Entwicklung wird durch ein »nationales Verankerungskapital« möglich, zu dem neben der luxemburger Staatsbürgerschaft entsprechende Sprachkenntnisse, sozio-kulturelles Wissen und soziale Netzwerke im Land zählen, über die Grenzgänger/-innen i.d.R. nur eingeschränkt verfügen.

[Le secteur public] constitue une sorte de refuge, dans lequel les salariés luxembourgeois peuvent faire valoir leurs compétences particulières (notamment linguistiques) qui sont raréfiées sur le marché. Il se trouve ainsi à l'abri de la concurrence des travailleurs étrangers, de plus en plus nombreux et qualifiés. (Fehlen/Pigeron-Piroth 2009: 11)

Weiterführend sollen auf sozio-kulturellem Gebiet die Aneignungen mit Blick auf das Grenzgängerwesen hinsichtlich der luxemburgischen Kultur und Sprache betrachtet werden. Hierfür wurde der Wohnbevölkerung die Frage gestellt, ob Grenzgänger/-innen eine Bereicherung für die luxemburgische Kultur seien. Über die Hälfte der Befragten (55\%) ist dieser Ansicht, wobei hier noch von Effekten sozialer Erwünschtheit auszugehen ist. Denn der damit artikulierte Einschluss von Grenzgängern/-innen in die Sphäre des Vertrauten entspricht zunächst einem öffentlichen Diskurs, ${ }^{65}$ der besonders im Umfeld des Grenzgängerfestes im Jahr 2008 wirksam wurde. So heißt es in einer Pressemitteilung des Ministeriums für Kultur, Hochschulbildung und Forschung:

Unter dem Motto ZZusammen arbeiten, zusammen feiern, zusammen leben , hat das Fest zum Ziel, über die Arbeitsbeziehungen hinaus und außerhalb der Bürozeiten, einen echten interkulturellen Dialog und einen gemeinschaftlichen Geist zwischen Grenzgängern und Anwohnern, sowie unter den Grenzbewohnern selbst zu fördern. Das Fest der Grenzgänger hofft so ebenfalls zur Entwicklung einer gemeinsamen regionalen Identität beizutragen. [...] Für Luxemburg als ,Land der 100 Nationalitäten، ist die Vielfalt kein leeres Wort, und das Fest der Grenzgänger ist dazu berufen keine einmalige Initiative zu bleiben. (Ministerium für Kultur, Hochschulbildung und Forschung 2008: 1)

Im Gegensatz zu dieser einschließenden Strategie der Identitätszuschreibung werden im Rahmen der Interviews negative Aneignungen des Grenzgängerwesens deutlich. Hier wird Vertrautes durch identitätskonstitutive Differenzsetzungen

65 | Vgl. auch Abschnitt 5.5. 
konstruiert, wenn gefordert wird, dass die Grenzgänger/-innen sich an die luxemburgische Kultur anpassen bzw. den Luxemburger/-innen mehr Interesse und Respekt entgegenbringen sollten.

Et ass och fir mech een wichtegen Aspekt datt Frontalieren, wann se an Lëtzebuerg kommen, datt se net nëmmen heihinner kommen fir ze schaffen, mä datt se sech wéinstens e bëssen fir eis Kultur souzesoen interesséieren an och vläicht iergendwéi een Austausch oder kommunizéieren mat den Lëtzebuerger. Et sinn wierklech vill Frontalieren, déi gesinn Lëtzebuerg nëmmen als Staat, wou een Suen verdéngt; d.h. si kommen heihinner, si schaffen dann ginn si nees zeréck an si interesséieren sech guer net. Dat fannen ech ëmmer e bëssen blöd. Leit, déi awer dann heihinner kommen an vläicht dann eben sech integréieren an eis Gesellschaft dat fannen ech dann besser an wann si dann och nach versichen e bëssen Lëtzebuergesch ze schwätzen an dann fannen ech dat och gutt. ${ }^{66}$ (Männlich, 18 Jahre, Italiener, Strassen)

Wie im Zitat angedeutet wird auch mit Blick auf die luxemburgische Sprache eine ausschließende Aneignungspraxis vollzogen. Diese artikuliert sich in der Meinung, Grenzgänger/-innen seien eine Bedrohung für die luxemburgische Sprache (57\%) und setzt sich fort in der Aussage, Grenzgänger/-innen sollten Luxemburgisch zumindest verstehen können (86 \%). ${ }^{67}$ Die Befragten berichten, dass sie im öffentlichen Raum - insbesondere im Einzelhandel, Gaststättengewerbe und Gesundheitswesen - nicht in Luxemburgisch kommunizieren können ${ }^{68}$ und führen an, dass es selbstverständlich sei, im Ausland auch die landesübliche Sprache womit hier ausschließlich das Luxemburgische angesprochen wird - zu beherrschen. Dabei wird kein hohes Sprachniveau von Grenzgängern erwartet, jedoch sollte eine elementare Sprachkompetenz vorhanden sein bzw. reiche es aus, einen »guten Willen zu zeigen«.

Ech fannen et ganz schlëmm, datt een am 'Cactus، [luxemburgische Supermarktkette], op lëtzebuergesch keng Wirschtecher méi bestelle kann, well si een net verstinn. Also, ech

66 | Deutsch: "Das ist auch für mich ein wichtiger Aspekt, dass die Grenzgänger, wenn sie nach Luxemburg kommen, nicht nur hierher kommen zum Arbeiten, sondern dass sie sich wenigstens ein bisschen für unsere Kultur interessieren, oder dass sie sich vielleicht irgendwie mit Luxemburgern austauschen oder kommunizieren. Es gibt wirklich viele Grenzgänger, die sehen Luxemburg als Staat, wo sie Geld verdienen können; d.h. sie kommen hierher, sie arbeiten und dann fahren sie wieder nach Hause und haben überhaupt gar kein Interesse [am Land]. Das finde ich immer ein bisschen blöd. Leute, die aber hierher kommen und sich dann vielleicht in unsere Gesellschaft integrieren, das finde ich dann besser. Wenn die dann noch versuchen ein bisschen Luxemburgisch zu sprechen, das finde ich dann auch gut."

67 | Vgl. auch Abschnitt 4.2.

68 | Vgl. auch Abschnitt 4.3. 
fannen ee Minimum vu Sprooch missten si awer kënnen, well wa mir an d'Ausland ginn, do kënne mer och net soen 'Hei, mir si Lëtzebuerger, mir kommen, hei schwätzt emol lëtzebuergesch mat eis. ${ }^{69}$ (Weiblich, 31 Jahre, Luxemburgerin, Rambrouch)

Die oben vorgenommene Differenzsetzung zwischen Personen, die Luxemburgisch beherrschen und jenen, die über keine Luxemburgischkenntnisse verfügen, sowie die gleichzeitig eingeräumte Relativierung der Sprachkompetenzen lassen erkennen, dass das Luxemburgische im Kontext des Grenzgängerwesens primär als Identitätsmarkierung (Lüdi 2008: 187, 190), denn als effektives Kommunikationsmittel fungiert. Dies spiegelt sich z.T. ebenso in den Sprachverwendungsstrategien der Befragten wider, die sich hinsichtlich des Sprachkontakts mit Grenzgänger/-innen in vier Typen untergliedern lassen:

- Die Konfrontativen versuchen strategisch ausschließend Luxemburgisch mit Grenzgänger/-innen zu sprechen und verlassen beispielsweise ein Geschäft, wenn ihnen mit »En français, s'il vous plaît« oder »Comment? « erwidert wird.

- Die Konstruktiven hingegen räumen ein, dass Grenzgänger/-innen kein Luxemburgisch lernen können, wenn die Wohnbevölkerung die Sprachen der Grenzgänger/-innen aktiv spricht. Somit handeln sie einschließend und sprechen auch in schwierigen Gesprächssituationen luxemburgisch, wenn sie bemerken, dass sich Verkäufer/-innen oder Kellner/-innen »Mühe geben«.

- Die Pragmatiker bleiben ausschließend und sprechen apriori Französisch, da sie in dieser Verkehrssprache ihr kommunikatives Ziel erfahrungsgemäß erreichen.

- Schließlich bleiben die Vermittelnden, die sprachliche Informationen im Rahmen der Begrüßung oder im Gespräch mit einem anderen Kunden/Gast/Patienten aufnehmen und sich dann strategisch einschließend dem Verkäufer/ Kellner/Pfleger sprachlich anpassen.

Die Gesamtschau der Ergebnisse zeigt, dass die Luxemburger Wohnbevölkerung sowohl positiv-einschließende als auch negativ-ausschließende Strategien hinsichtlich des Grenzgängerwesens praktiziert. Auf Basis der quantitativen Daten kann weiterführend gefragt werden, welche Strategien in welchem gesellschaftlichen Feld aktiviert werden. Somit geht es um die übergreifende Betrachtung von sozio-kulturellen und sozio-ökonomischen Aspekten, wofür die jeweils positiven bzw. negativen Aussagen über das Grenzgängerwesen zusammengeführt werden (Tab. 4).

69 | Deutsch: "Ich finde es ganz schlimm, dass man im 'Cactus` [luxemburgischer Supermarkt] auf Luxemburgisch keine Würstchen mehr bestellen kann, weil sie einen dort nicht verstehen. Also, ich finde, die Grundlagen der Sprache sollten die aber kennen; weil wenn wir ins Ausland fahren, können wir auch nicht sagen ,Wir sind Luxemburger, wir kommen jetzt, sprecht mal Luxemburgisch mit uns.'" 


\begin{tabular}{|c|c|c|c|}
\hline \multicolumn{2}{|c|}{ Positive Aneignung (+) } & \multicolumn{2}{|c|}{ Negative Aneignung (-) } \\
\hline $\begin{array}{l}\text { sozio-kulturelles } \\
\text { Feld }\end{array}$ & $\begin{array}{c}\text { sozio-ökonomisches } \\
\text { Feld }\end{array}$ & $\begin{array}{l}\text { sozio-kulturelles } \\
\text { Feld }\end{array}$ & $\begin{array}{c}\text { sozio-ökonomisches } \\
\text { Feld }\end{array}$ \\
\hline $\begin{array}{l}\text { Grenzgänger sind } \\
\text { eine Bereicherung für } \\
\text { die luxemburgische } \\
\text { Kultur. }\end{array}$ & $\begin{array}{l}\text { Grenzgänger werden } \\
\text { in der luxemburgi- } \\
\text { schen Wirtschaft } \\
\text { gebraucht. }\end{array}$ & $\begin{array}{l}\text { Grenzgänger sind } \\
\text { eine Bedrohung für } \\
\text { die luxemburgische } \\
\text { Sprache. }\end{array}$ & $\begin{array}{l}\text { Grenzgänger } \\
\text { nehmen den Luxem- } \\
\text { burgern die Arbeits- } \\
\text { plätze weg. }\end{array}$ \\
\hline $55 \%$ (Zustimmung) & $87 \%$ (Zustimmung) & $57 \%$ (Zustimmung) & 34 \% (Zustimmung) \\
\hline
\end{tabular}

Tabelle 4: Positive und negative Aneignungsstrategien der Luxemburger Wohnbevölkerung

Hinsichtlich der positiv-einschließenden Aneignungsstrategie ist festzuhalten, dass diese von der Luxemburger Wohnbevölkerung vor allem im sozio-ökonomischen Feld praktiziert wird, etwa wenn es um die Unverzichtbarkeit der Grenzgänger/-innen für das nationale Wirtschaftswachstum $(87 \%)$ im Vergleich zur kulturellen Bereicherung Luxemburgs (55\%) geht. Negativ-ausschließende Aneignungsstrategien hingegen werden besonders im sozio-kulturellen Feld wirksam, etwa wenn die Frage nach der Bedrohung der eigenen Sprache durch Grenzgänger/-innen (57 \%) im Vergleich zur Arbeitsplatzkonkurrenz (34\%) gestellt wird. Diese Betrachtung gibt jedoch nur erste Hinweise auf die praktizierten Aneignungen nach gesellschaftlichen Feldern und erlaubt keine Aussagen über mögliche ambivalente Aneignungsstrategien oder über den Status des Grenzgängerwesens in sozio-kulturellen Milieus.

\section{Zum Status der Grenzgänger in sozio-kulturellen Milieus}

In Anknüpfung an die erläuterte Denkfigur >Vertrauter (+/-)/Fremder< wird im Folgenden der Status der Grenzgänger/-innen anhand der Aneignungsstrategien der Luxemburger Wohnbevölkerung bzw. in sozio-kulturellen Milieus systematisiert. Die Betrachtungen stützen sich auf quantitative Befragungsergebnisse und verweisen auf drei zentrale Status der Grenzgänger/-innen in Luxemburg. 


\begin{tabular}{|c|c|c|c|c|c|}
\hline & \multicolumn{2}{|c|}{ Sozio-kulturelles Feld } & \multicolumn{2}{|c|}{ Sozio-ökonomisches Feld } & \\
\hline & $\begin{array}{l}\text { Grenzgänger sind } \\
\text { eine Bedrohung } \\
\text { für die luxembur- } \\
\text { gische Sprache. }\end{array}$ & $\begin{array}{l}\text { Grenzgänger sind } \\
\text { eine Bereiche- } \\
\text { rung für die } \\
\text { luxemburgische } \\
\text { Kultur. }\end{array}$ & $\begin{array}{l}\text { Grenzgänger } \\
\text { nehmen den } \\
\text { Luxemburgern } \\
\text { die Arbeitsplätze } \\
\text { weg. }\end{array}$ & $\begin{array}{l}\text { Grenzgänger } \\
\text { werden in der } \\
\text { luxemburgi- } \\
\text { schen Wirtschaft } \\
\text { gebraucht. }\end{array}$ & \\
\hline Zustimmung in \% & $\begin{array}{c}\text { Negative } \\
\text { Aneignung (-) }\end{array}$ & $\begin{array}{c}\text { Positive } \\
\text { Aneignung (+) }\end{array}$ & $\begin{array}{c}\text { Negative } \\
\text { Aneignung (-) }\end{array}$ & $\begin{array}{c}\text { Positive } \\
\text { Aneignung (+) }\end{array}$ & $\begin{array}{l}\text { Aneignung } \\
\text { insgesamt }\end{array}$ \\
\hline $\begin{array}{l}\text { Luxemburger } \\
\text { Wohnbevölkerung }\end{array}$ & 57 & 55 & 34 & 87 & I \\
\hline $\begin{array}{l}\text { Status der } \\
\text { Grenzgänger/-innen }\end{array}$ & \multicolumn{2}{|c|}{ Fremde } & \multicolumn{2}{|c|}{ Vertraute+ } & Vertraute Fremde \\
\hline $\begin{array}{l}\text { Konservativ- } \\
\text { gehobenes Milieu }\end{array}$ & 37 & 62 & 15 & 93 & 1 \\
\hline $\begin{array}{l}\text { Status der } \\
\text { Grenzgänger/-innen }\end{array}$ & \multicolumn{2}{|c|}{ Vertraute+ } & \multicolumn{2}{|c|}{ Vertraute+ } & Vertraute+ \\
\hline $\begin{array}{l}\text { Kleinbügerliches } \\
\text { Milieu }\end{array}$ & 64 & 48 & 38 & 87 & 1 \\
\hline $\begin{array}{l}\text { Status der } \\
\text { Grenzgänger/-innen }\end{array}$ & \multicolumn{2}{|c|}{ Vertraute- } & \multicolumn{2}{|c|}{ Vertraute+ } & Fremde \\
\hline $\begin{array}{l}\text { Traditions- } \\
\text { orientiertes Milieu }\end{array}$ & 74 & 45 & 54 & 86 & l \\
\hline $\begin{array}{l}\text { Status der } \\
\text { Grenzgänger/-innen }\end{array}$ & \multicolumn{2}{|c|}{ Vertraute- } & \multicolumn{2}{|c|}{ Fremde } & Vertraute Fremde \\
\hline $\begin{array}{l}\text { Unterprivilegiertes } \\
\text { Milieu }\end{array}$ & 64 & 53 & 56 & 75 & l \\
\hline $\begin{array}{l}\text { Status der } \\
\text { Grenzgänger/-innen }\end{array}$ & \multicolumn{2}{|c|}{ Vertraute- } & \multicolumn{2}{|c|}{ Vertraute- } & Vertraute- \\
\hline $\begin{array}{l}\text { Aufstiegsorientiertes } \\
\text { Milieu }\end{array}$ & 59 & 58 & 30 & 91 & 1 \\
\hline $\begin{array}{l}\text { Status der } \\
\text { Grenzgänger/-innen }\end{array}$ & \multicolumn{2}{|c|}{ Fremde } & \multicolumn{2}{|c|}{ Vertraute+ } & Vertraute Fremde \\
\hline $\begin{array}{l}\text { Liberal-gehobenes } \\
\text { Milieu }\end{array}$ & 46 & 62 & 16 & 89 & 1 \\
\hline $\begin{array}{l}\text { Status der } \\
\text { Grenzgänger/-innen }\end{array}$ & \multicolumn{2}{|c|}{ Vertraute+ } & \multicolumn{2}{|c|}{ Vertraute+ } & Vertraute+ \\
\hline $\begin{array}{l}\text { Hedonistisches } \\
\text { Milieu }\end{array}$ & 59 & 50 & 49 & 82 & I \\
\hline $\begin{array}{l}\text { Status der } \\
\text { Grenzgänger/-innen }\end{array}$ & \multicolumn{2}{|c|}{ Fremde } & \multicolumn{2}{|c|}{ Vertraute- } & $\begin{array}{l}\text { Vertraute } \\
\text { Fremde }\end{array}$ \\
\hline Alternatives Milieu & 45 & 68 & 28 & 88 & 1 \\
\hline $\begin{array}{l}\text { Status der } \\
\text { Grenzgänger/-innen }\end{array}$ & \multicolumn{2}{|c|}{ Vertraute+ } & \multicolumn{2}{|c|}{ Vertraute+ } & Vertraute+ \\
\hline $\begin{array}{l}\text { Statusorientiertes } \\
\text { Milieu }\end{array}$ & 53 & 48 & 26 & 93 & I \\
\hline $\begin{array}{l}\text { Status der } \\
\text { Grenzgänger/-innen }\end{array}$ & \multicolumn{2}{|c|}{ Vertraute- } & \multicolumn{2}{|c|}{ Vertraute+ } & Fremde \\
\hline
\end{tabular}

\section{Tabelle 5: Status der Grenzgänger in sozio-kulturellen Milieus}


Grenzgänger als Vertraute: Die Aneignung der Grenzgänger/-innen als Vertraute basiert auf einer eindeutigen positiven oder negativen Konstruktion des Phänomens. Werden die Grenzgänger/-innen als >negative Vertraute` angeeignet - wie im unterprivilegierten Milieu -, so sind im sozio-kulturellen und sozio-ökonomischen Feld weitgehend negativ-ausschließende Aneignungsstrategien vorzufinden. Diese artikulieren sich im letztgenannten Feld über die Betonung der Arbeitsmarktkonkurrenz und über die Relativierung der Notwendigkeit von Grenzgänger/-innen. Hinsichtlich der Aneignung der Grenzgänger/-innen als >positive Vertraute< - die im konservativ-gehobenen, liberal-gehobenen und alternativen Milieu vorherrscht - überwiegen in den betrachteten Feldern jeweils positiv-einschließende Strategien, die über die Betonung der positiven und über die Relativierung der negativen Implikationen der Grenzgängerbeschäftigung deutlich werden.

Grenzgänger als Fremde: Die Aneignung der Grenzgänger/-innen als Fremde basiert auf einer ambivalenten Konstruktion des Phänomens. Das bedeutet, dass die Befragten sowohl positive als auch negative Aneignungsstrategien im Hinblick auf das Grenzgängerwesen praktizieren. Diese Form der Aneignung trifft auf das kleinbürgerliche und statusorientierte Milieu zu, die sich in sozio-kultureller Hinsicht eher ausschließend und in sozio-ökonomischer Hinsicht eher einschließend gegenüber dem Grenzgängerwesen positionieren. So wird hier das kulturell bereichernde Moment relativiert und von einer Sprachbedrohung durch Grenzgänger ausgegangen, was sich besonders im kleinbürgerlichen Milieu akzentuiert. Die Notwendigkeit der Grenzgänger/-innen wird jedoch bestätigt, ebenso wie - insbesondere im statusorientierten Milieu - die vermeintliche Arbeitsmarktkonkurrenz vergleichsweise niedrig bewertet wird.

Grenzgänger als vertraute Fremde: Die Aneignung der Grenzgänger/-innen als vertraute Fremde gibt den von Nassehi nicht vorgesehenen Umstand wieder, dass der Grenzgänger nach den betrachteten gesellschaftlichen Feldern sowohl ambivalent als auch eindeutig konstruiert wird. Dieser Status lässt sich an den Aneignungen der Luxemburger Wohnbevölkerung insgesamt und der Angehörigen des hedonistischen Milieus ablesen, welche das Grenzgängerwesen in sozio-kultureller Hinsicht positiv und negativ konstruieren. So gehen sie hier von einer kulturellen Bereicherung und von einer sprachlichen Bedrohung durch Grenzgänger/innen aus. Jedoch unterscheiden sich die genannten Gruppen hinsichtlich ihrer Konstruktionen auf sozio-ökonomischem Gebiet: Während die Luxemburger Wohnbevölkerung insgesamt die Unverzichtbarkeit der Grenzgänger/-innen für die Wirtschaft in einschließender Manier betont, unterstreichen hier die Hedonisten die Arbeitsplatzkonkurrenz, wodurch sie sich eher ausschließend positionieren. Der Status der Grenzgänger/-innen als vertraute Fremde lässt sich ebenso anhand der Aneignungsstrategien des traditionsorientierten Milieus bestimmen. Jedoch ist hier durch die Betonung der Arbeitsmarktkonkurrenz bei gleichzeitiger Notwendigkeit von Grenzgänger/-innen die Ambivalenz der Konstruktion auf sozio-ökonomischem Gebiet und die eher negativ-ausschließende Strategie auf sozio-kulturellem Gebiet auszumachen. 


\section{Zur Figur des (vertrauten) Fremden}

Die vorgenommene Analyse zeigt, dass die praktizierten Ein- und Ausschlussstrategien der Luxemburger Wohnbevölkerung verschiedene Aneignungsformen hinsichtlich des Grenzgängerwesens repräsentieren. Dabei überwiegt auf sozioökonomischem Gebiet tendenziell der Einschluss von Grenzgänger/-innen durch die Betonung ihrer wirtschaftlichen Unverzichtbarkeit. Hingegen werden in sozio-kultureller Hinsicht Ausschlussstrategien praktiziert, die vor allem auf das Bedrohungsmoment für die Luxemburger Sprache abheben. Diese Aneignungsprozesse, welche nach sozio-kulturellen Milieus variieren, wurden weiterführend auf Kohärenz geprüft, wodurch teilweise widersprüchliche Konstruktionen herausgearbeitet werden konnten. Diese unterscheiden sich je nach betrachtetem soziokulturellen Milieu und verweisen auf einen weitgehend ambivalenten Status der Grenzgänger/-innen in Luxemburg, der mit den Figuren des >Fremden< bzw. des >vertrauten Fremden< wiedergegeben wurde.

Dieser Befund erscheint angesichts der einleitenden Ausführungen zunächst als identitäres Dilemma, jedoch kann bei seiner Interpretation eine mögliche Logik des strategischen Zusammenspiels von alltagskulturellen Ein- und Ausschlüssen herausgearbeitet werden. Denn während im 20. Jahrhunderts die durch die Nachfrage der Unternehmen bedingte Präsenz von Immigranten/-innen und Grenzgängern/-innen »in den Köpfen zu einer Selbstverständlichkeit wurde« (vgl. Fehlen 2008: 82) und sich somit ausländerfeindliche Diskurse in Luxemburg kaum ausformten, etablierten sich besonders seit den Konjunkturumschwüngen nach der Jahrtausendwende protektionistische Strategien gegenüber der Konkurrenz ausländischer Arbeitskräfte. Diese kommen den Forschungsergebnissen folgend weniger in einer offenen und konsequenten Ablehnung von Grenzgänger/-innen im Sinne des >negativen Vertrauten < zum Ausdruck, sondern ihnen wird aus den genannten Gründen durchaus ihre wirtschaftliche Unverzichtbarkeit zugebilligt. Jedoch endet diese aus Sicht vieler Luxemburger an der Schwelle zum staatlichen und halbstaatlichen Sektor, was über die erläuterte Verankerungskompetenz reguliert wird. Vor diesem Hintergrund erschließt sich die Logik des strategischen Zusammenspiels von sozio-ökonomisch motivierten Einschlussstrategien einerseits und von sozio-kulturell motivierten Ausschlussstrategien andererseits, die auf die Sicherung von Wachstum und Wohlstand im eigenen Land und auf den >Schutz< vor Arbeitsplatzkonkurrenz abstellt. Denn laut der Ergebnisse sind Grenzgänger/innen in den Augen der Wohnbevölkerung zwar wichtig für die Wirtschaft, ihr Zugang zur luxemburgischen Sprache könnte ihnen jedoch den Eintritt in die vor der Konkurrenz ausländischer Arbeitskräfte >geschützten< Sektoren weitgehend ermöglichen. So wird das sozio-kulturelle Argument der Sprachbedrohung - insbesondere im traditionsorientierten, unterprivilegierten und kleinbürgerlichen Milieu - in ausschließender Manier praktiziert, um den Wettbewerbsvorteil gegenüber Grenzgänger/-innen auf sozio-ökonomischem Gebiet zu sichern. 
Zunächst widersprüchlich erscheinende Aneignungsprozesse im Hinblick auf das Grenzgängerwesen können demnach durchaus >Logiken des Alltags< folgen, die dem Verlangen nach Sicherheit nachgeben und hierfür Gemeinschaft durch Abgrenzung imaginieren. Daher gilt es, sich den alltagskulturellen Aneignungen des Grenzgängerwesens, welches sich in Luxemburg sowohl der Kategorie des Vertrauten als auch der des Fremden entzieht, in ihrer Qualität des Zwischenkategorialen weiterführend zuzuwenden. Das bedeutet, die Aneignungen der Wohnbevölkerung explizit mit ihren Widersprüchen in den Blick zu nehmen und so den Grenzgänger als eine ambivalente aber dennoch eigenständige Kategorie zu rekonstruieren. Auf konzeptioneller Ebene impliziert dies eine Erweiterung des Ansatzes Nassehis durch die Denkfigur >vertrauter Fremder [Vertrauter (+/-)/ Fremder]; auf empirischer Ebene gilt es, den Verortungen der Subjekte zwischen ein- und ausschließenden Aneignungsprozessen weiter nachzuspüren und somit der ambivalenten Logik des Alltagskulturellen auf die Spur zu kommen.

\subsection{Schlussfolgerung: Identitäten Und Ambivalenzen des Alltagskulturellen}

Im vorliegenden Kapitel wurden verschiedene Bereiche des Alltagskulturellen exemplarisch beleuchtet und jeweils kursierende (politische) Zuschreibungen und/oder (individuelle sowie milieu-spezifische) Aneignungen in Bezug auf identitätskonstitutives Handeln aufgezeigt. Dabei ging es um Genderbilder und -erfahrungen, um Einstellungen zur Ernährung und um Wahrnehmungen der Grenzgänger/-innen in Luxemburg. Neben ihrer Alltagsrelevanz und ihrem identitätskonstitutiven Potential konnte eine weitere Gemeinsamkeit dieser Themen herausgearbeitet werden: eine ausgeprägte Ambivalenz. So werden in der betrachteten sozialen Praxis die Binaritäten männlich/weiblich, gut/schlecht und vertraut/fremd produktiv und z.T. arbiträr aufgebrochen, wodurch unterschiedliche $>$ Logiken des Alltags < in Erscheinung treten.

Bei der Genderthematik bemerkenswert ist der Befund, dass die Befragten tendenziell nach dem Ideal der Gleichberechtigung handeln, gleichzeitig jedoch in ihren mentalen Haltungen (noch) traditionellen Mustern verhaftet bleiben - und umgekehrt Gleichberechtigung befürworten und z.B. im Falle der Elternschaft entsprechend traditioneller Muster agieren. Diese Widersprüchlichkeit findet sich wieder in den Einstellungen zu Ernährungsfragen: >Gutes< Essen wird laut der Ergebnisse auf Zuschreibungsseite eher objektbezogen behandelt (etwa in Form von Ernährungsempfehlungen), während es auf Aneignungsseite eher personenbezogen gelebt wird (etwa in Form von Subjektivierung und Vergemeinschaftung). Dabei zeigen sich Praktiken, die zugeschriebene Identifikationsmerkmale gleichzeitig selektiv, kontextgebunden und permanent variierend integrieren (etwa in Form von Adaptation, Opposition oder Autonomie gegenüber Ernährungsempfehlungen). Grenzgänger/-innen schließlich werden den Befragten zufolge als wich- 
tig für die wirtschaftliche Situation Luxemburgs angesehen, hingegen wird ihre in Alltagssituationen als unzureichend erlebte Luxemburgisch-Kompetenz kritisiert. Gleichzeitig steht dabei eher eine sprachlich zu realisierende Anerkennung des >Eigenen < im Vordergrund als die Sprachkompetenz als solche, insbesondere wenn es um die weitgehend von Luxemburgern dominierten und >geschützten< Bereiche des Arbeitsmarkts geht, über deren Zugang oftmals das Beherrschen des Luxemburgischen entscheidet.

In den hier untersuchten Fallbeispielen des Alltagskulturellen sind also Diskurspraktiken feststellbar, die eine binäre >Entweder-oder-Logik< pragmatisch überschreiten und sich in eine flexible >Sowohl-als-auch-Logik < einschreiben - so etwa, wenn im Geschlechter-Erleben sowohl Essentialismen als auch Konstruktivismen parallel praktiziert werden, wenn widersprüchliche Normen und Praxen sowohl von gesundem als auch von nachsichtigem Essen sich wechselseitig durchdringen oder wenn Grenzgänger/-innen sowohl positiv als auch negativ - und somit als >vertraute Fremde< - wahrgenommen werden. Die latenten >Logiken des Alltags<, an denen sich dynamische Identitätskonstruktionen ablesen lassen, erscheinen somit im Luxemburger Untersuchungskontext weitgehend pragmatisch und selbstbezogen: ein Selbstverständnis der Subjekte, das Gender als nur einen Aspekt der Alltagspraxis unter vielen anderen erlebt und je nach Kontext naturalistisch oder kulturell argumentiert, das eine pragmatisch-hedonistische Ernährungspraxis im Alltag privilegiert oder ein Selbstverständnis, das dem Verlangen nach dem >Eigenen < durch ambivalente Konstruktionen des >Fremden < nachgibt.

Vor diesem Hintergrund ist festzuhalten, dass dieses Kapitel sich insbesondere mit angeeigneten Identitäten beschäftigt und zusätzlich untersucht hat, inwiefern zugeschriebene Identitäten (nicht) übernommen werden, wobei Strategien von Adaptation, Opposition und Autonomie hinsichtlich Identifikationsidealen wirken. Demnach sind Identitäten weder vorausbestimmt noch festgeschrieben, sondern sie können nur als Momentaufnahme und in der konkreten Alltagspraxis nachgezeichnet werden.

\subsection{LiterATURVERZEICHNIS}

\section{Primärquellen}

Ministère de la Santé/Ministère de l'Education Nationale et de la Formation Professionnelle/Ministère de la Famille et de l'Intégration/Département Ministériel des Sports (2007): Freude am gesunden Essen. Spaß an der Bewegung!/Le plaisir de bien manger et d'être actif!, Luxembourg. 


\section{Sekundärquellen}

Allegrezza, Serge/Frising, Armande/Haag, Antoine/Langers, Jean/Reichmann, Liliane/ Schockmel, Marco (2007): »Egalité Hommes-Femmes. Mythe ou Réalité?«, in: Cahier Economique. No 105 du Statec, Luxembourg.

Antoni-Komar, Irene/Pfriem, Reinhard/Raabe, Thorsten/Spiller Achim (Hg.) (2008): Ernährung, Kultur, Lebensqualität. Wege regionaler Nachhaltigkeit, Marburg: Metropolis.

Austin John (1962): How To Do Things with Words, Oxford: University Press.

Baltes-Löhr, Christel (2006): Migration und Identität. Portugiesische Frauen in Luxemburg. Frankfurt a.M./London: IKO-Verlag für Interkulturelle Kommunikation.

Baltes-Löhr, Christel (2009): »Umgang mit Differenzen«, in: Manuel Achten; Christel Baltes-Löhr et al. (Hg.): Maison Relais pour Enfants. Le Manuel - Das Handbuch, Luxembourg: Edition le Phare 2009, S. 88-117.

Barlösius, Eva (2008): »Weibliches und männliches rund ums Essen«, in: Alois Wierlacher/Regina Bendix (Hg.): Kulinaristik. Forschung, Lehre, Praxis, Berlin: Lit, S. 35-44.

Bhabha, Homi, K. (2000): Die Verortung der Kultur, Tübingen: Stauffenburg.

Bolten, Jürgen (2007): Einführung in die Interkulturelle Wirtschaftskommunikation. Stuttgart: UTB.

Bonz, Jochen/Struve, Karen: »Homi K. Bhabha: Auf der Innenseite kultureller Differenz: >in the middle of differnces «, in: Stephan Moebius, Dirk Quadflieg (Hg.): Kultur. Theorien der Gegenwart, Wiesbaden: VS, S. 140-153.

Bourdieu, Pierre (2000 [1972]): Esquisse d'une théorie de la pratique. Précédé de trois études d'ethnologie kabyle, Paris: Le Seuil.

Bourdieu, Pierre (1994): Raisons pratiques. Sur la théorie de l'action, Paris: Le Seuil.

Bourdieu, Pierre (1980): Le sens pratique, Paris: Editions de Minuit.

Bourdieu, Pierre (2000 [1972]): Esquisse d'une théorie de la pratique. Précédé de trois études d'ethnologie kabyle, Paris: Le Seuil.

Breinig, Helmbrecht/Lösch, Klaus (2006): »Transdifference«, in: Journal for the Study of British Culture 13/2, S. 105-122.

Bröckling, Ulrich/Krasmann, Susanne/Lemke, Thomas (Hg.) (2000): Gouvernementalität der Gegenwart. Studien zur Ökonomisierung des Sozialen, Frankfurt a.M.: Suhrkamp.

Brunner, Karl Michael/Geyer, Sonja/Jelenko, Marie/Weiss, Walpurga/Astleithner, Florentina (2007): Ernährungsalltag im Wandel. Chancen für Nachhaltigkeit, Wien/New York: Springer.

Brunnett, Regina (2009): Die Hegemonie symbolischer Gesundheit. Eine Studie zum Mehrwert von Gesundheit im Postfordismus, Bielefeld: transcript.

Butler, Judith (1993): Für ein sorgfältiges Lesen, in: Benhabib, Seyla et al. (Hg.): Der Streit um Differenz, Frankfurt a.M.: Fischer, S. 123-132. 
Chambers, Iain (1996): Migration, Kultur, Identität, Tübingen: Stauffenburg.

Commission des Communautés Européennes (CE) (30.05.2007): Livre blanc. Une stratégie européenne pour les problèmes de santé liés à la nutrition, la surcharge pondérale et l'obésité, Bruxelles, COM (2007) 279 final, non publié au Journal Officiel. URL: http://europa.eu/legislation_summaries/public_health/ health_determinants_lifestyle/c11542c_fr.htm

Commission des Communautés Européennes (CE) (08.12.2005): Livre vert. Promouvoir une alimentation saine et l'activité physique. Une dimension européenne pour la prévention des surcharges pondérales, de l'obésité et des maladies chroniques, Bruxelles, COM (2005) 637 final, non publié au Journal Officiel. URL: http://europa.eu/legislation_summaries/public_health/health_ determinants_lifestyle/c11542b_fr.htm (15.01. 2010)

Corbeau, Jean-Pierre/Poulain, Jean-Pierre (2002): Penser l'alimentation. Entre imaginaire et rationalité, Paris: Privat.

Coveney, John (2006 [2000]): Food, Morals and Meaning. The Pleasure and Anxiety of Eating, London: Routledge.

Csergo, Julia (2009): Trop gros? L'obésité et ses représentations, Paris: Autrement.

Deleuze, Gilles (1968): Différence et Répétition, Paris: PUF.

Derrida, Jacques (1968): »La différance«, in: Bulletin de la Société Française de Philosophie 62.3., Paris, S. 73-120.

European Commission (2009): Scientific Report of the Mobility of Cross-Border Workers within the EU-27/EEA/EFTA Countries (Directorate General for Employment, Social Affaires and Equal Opportunities), Luxembourg.

Fehlen, Fernand (2008): »Streit um den roten Löwen. Diskurse über das nationale Selbstbild Luxemburgs im Spannungsfeld von Modernisierung und Rückwärtsgewandtheit«, in: Wilhelm Amann/Georg Mein et al. (Hg.): Periphere Zentren oder zentrale Peripherien? Kulturen und Regionen Europas zwischen Globalisierung und Regionalität, Heidelberg: Synchron Verlag, S. 61-87.

Fehlen, Fernand/Pigeron-Piroth, Isabelle (2009): Mondialisation du travail et pluralité des marchés du travail: L'exemple du Luxembourg, Diskussionsbeitrag der 12e Journées de Sociologie du Travail, 25. und 26. Juni 2009, GREE, Université de Nancy. URL: http://gree.univ-nancy2.fr/digitalAssets/53345_JIST_ Fehlen_Pigeron.pdf

Fischler, Claude (1990): L'homnivore. Le goût, la cuisine et le corps, Paris: Poches Odile Jacob.

Foucault, Michel (1993): »About the Beginnings of the Hermeneutics of the Self. Two Lectures at Dartmouth «, in: Political Theory, $N^{\circ}$ 21/2, S. 198-227.

Foucault, Michel (1984a): Histoire de la sexualité, Tome 2: L'usage des plaisirs, Paris: Gallimard.

Foucault, Michel (1984b): »L'éthique du souci de soi comme pratique de la liberté«, in: Michel Foucault (1994): Dits et écrits. Tome 4: 1980-1988, Paris: Gallimard, S. 708-729. 
Foucault, Michel (1983): »On the Genealogy of Ethics. An Overview of Work in Progress«, in: Paul Rabinow/Nicolas Rose (2003 [1994]), The Essential Foucault. Selections from the Essential Works of Foucault 1954-1984, New York/ London: The New Press, S. 102-125.

Foucault, Michel (1982a): »The Subject and Power«, in: Paul Rabinow/Nicolas Rose (2003 [1994]): The Essential Foucault. Selections from the Essential Works of Foucault 1954-1984, New York/London: The New Press, S. 126-144.

Foucault, Michel (1982b): »Technologies of the Self«, in: Paul Rabinow/Nicolas Rose (2003 [1994]): The Essential Foucault. Selections from the Essential Works of Foucault 1954-1984, New York/London: The New Press, S. 145-169.

Foucault, Michel (1978): »La gouvernementalité. Séminaire au Collège de France en 1977 et 1978: Sécurité, territoire et population«, in: Aut-Aut, $\mathrm{N}^{\circ}{ }_{167-168, \mathrm{~Pa}-}$ ris: Gallimard, S. 12-29.

Foucault Michel (1969): L'archéologie du savoir, Paris: Gallimard.

Goffman, Erving (1969): Wir alle spielen Theater, München: Piper.

Gregory, Derek (1997): »Lacan and Geography. The Production of Space Revisted «, in: Georges Benko und Ulf Strohmayer (Hg.): Space and Social Theory. Interpreting Modernity and Postmodernity, Oxford: Blackwell Publishers, S. 203234.

Hillmann, Karl-Heinz (2007): Wörterbuch der Soziologie, Stuttgart: Alfred Kröner.

Hirschauer, Stefan (2001): »Das Vergessen des Geschlechts. Zur Praxeologie einer Kategorie sozialer Ordnung«, in: Bettina Heinz (Hg.): Geschlechtersoziologie. Sonderheft der Kölner Zeitschrift für Soziologie und Sozialpsychologie, Opladen: Westdeutscher Verlag, S. 208-235.

Hörning, Karl H./Reuter, Julia (2004): »Doing Culture. Kultur als Praxis«, in: Dies. (Hg.): Doing Culture. Neue Positionen zum Verhältnis von Kultur und sozialer Praxis, Bielefeld: transcript, S. 9-15.

Jakobson, Roman (1971): »Signe zéro«, in: Ders.: Selected Writtings, II: Word and Language, Den Haag: Parin. S. 211-222.

Keupp, Heiner/Ahde, Thomas/Gmür, Wolfgang/Höfer, Renate/Mitzscherlich, Beate/Kraus, Wolfgang/Straus, Florian (2006): Identitätskonstruktionen. Das Patchwork der Identitäten in der Spätmoderne, Reinbek bei Hamburg: Rowohlt.

Klinger, Cornelia/Knapp, Gudrun-Axeli (2005): »Achsen der Ungleichheit - Achsen der Differenz - Verhältnisbestimmung von Klasse, Geschlecht, >Rasse</ Ethnizität«, in: Transit 5/2005.

Kreutzer, Florian/Roth, Silke (2006): Transnationale Karrieren. Biographien, Lebensführung und Mobilität, Wiesbaden: VS.

Kumoll, Karsten (2006): »Clifford Geertz: Die Ambivalenz kultureller Formen«, in: Stephan Moebius, Dirk Quadflieg (Hg.): Kultur. Theorien der Gegenwart. Wiesbaden: VS, S. 81-90. 
Lamine, Claire (2008): Les intermittents du bio. Pour une sociologie pragmatique des choix alimentaires émergents, Paris: Editions de la Maison des Sciences de l'Homme \& Editions Quae.

Lüdi, Georges (2008): »Der Schweizer Sprachencocktail neu gemixt. Sprache als Brücke und Barriere«, in: Daniel Jentsch-Müller (Hg.): Die neue Zuwanderung. Die Schweiz zwischen Brain-Gain und Überfremdungsangst, Zürich: Avenir Suisse, S. 185-203.

Luxemburgisches Ministerium für Kultur, Hochschulbildung und Forschung (26.09.2008): 1. Fest der Grenzgänger - Grenzenlos feiern im Namen des interkulturellen Dialogs, Pressemeldung.

McCall, Leslie (2005): »Managing the Complexity of Intersectionality«, in: Signs (2005), 30(3), S. 1771-1800.

Ministère de l'Education Nationale et de la Formation Professionnelle/Ministère de la Santé/Universität Karlsruhe (2006): Gesundheit, motorische Leistungsfähigkeit und körperlich-sportliche Aktivität von Kindern und Jugendlichen in Luxemburg. Untersuchung für die Altersgruppen 9, 14 und 18 Jahre, Abschlussbericht zum Forschungsprojekt, Luxemburg.

Ministère de l'Education nationale et de la Formation professionnelle (2007): Les chiffres clés de l'éducation nationale 2005/2006. Statistiques et indicateurs, année scolaire 2005/2006, Luxembourg.

Ministère de l'Egalité des Chances (2006): Gleiche Rechte für Mädchen und Jungen, Frauen und Männer, Luxembourg.

Ministère de la Santé/Ministère de l'Education Nationale et de la Formation Professionnelle (2005a): Das Wohlbefinden der Jugendlichen in Luxemburg im internationalen Vergleich, Luxemburg.

Ministère de la Santé/Ministère de l'Education Nationale et de la Formation Professionnelle (2005b): Das Wohlbefinden der Jugendlichen in Luxemburg, 5. und 6. Klasse, Grundschule, Luxemburg.

Ministère de la Santé/Ministère de l'Education Nationale et de la Formation Professionnelle (2002): Das Wohlbefinden der Jugendlichen in Luxemburg, Luxemburg.

Nassehi, Armin (1995): »Der Fremde als Vertrauter. Soziologische Beobachtungen zur Konstruktion von Identitäten und Differenzen«, in: Kölner Zeitschrift für Soziologie und Sozialpsychologie 47, S. 443-463.

Park, Robert (1974): »Human Migration and the Marginal Man«, in: Collected Papers of Robert Ezra Park, Band 1, New York: Arno Press, S. 345-356.

Pries, Ludger (2008): Die Transnationalisierung der sozialen Welt, Frankfurt a.M.: Suhrkamp.

Poulain, Jean-Pierre (2002): Manger aujourd'hui. Attitudes, normes et pratiques, Paris: Privat.

Reckinger, Rachel (2008): Les pratiques discursives oenophiles entre normativité et appropriation. Contribution à une sociologie des cultures alimentaires, 2 to- 
mes, thèse de doctorat en sociologie, Marseille: Ecole des Hautes Etudes en Sciences Sociales.

Reckwitz, Andreas (2004): »Die Kontingenzperspektive von Kultur. Kulturbegriffe, Kulturtheorien und das kulturwissenschaftliche Forschungsprogramm«, in: Friedrich Jaeger/Jörn Rüsen (Hg.): Handbuch der Kulturwissenschaften. Themen und Tendenzen, Stuttgart: Metzler, S. 1-20.

Reckwitz, Andreas (2001): »Multikulturalismustheorien und der Kulturbegriff: Vom Homogenitätsmodell zum Modell kultureller Interferenzen«, in: Berliner Journal für Soziologie, Heft 2, Jg. 11, S. 179-200.

Reuter, Julia/Wiesner, Matthias (2008): »Soziologie im Zwischenraum: Grenzen einer transdifferenten Perspektive«, in: Lars Allolio-Näcke/Britta Kalscheuer (Hg.): Kulturelle Differenzen begreifen. Das Konzept der Transdifferenz aus interdisziplinärer Sicht, Frankfurt a.M.: Campus, S. 129-143.

Reuter, Julia (2002): Ordnungen des Anderen. Zum Problem des Eigenen in der Soziologie des Fremden, Bielefeld: transcript.

Rölli, Marc (2006): »Gilles Deleuze: Kultur und Gegenkultur«, in: Stephan Moebius, Dirk Quadflieg (Hg.): Kultur. Theorien der Gegenwart, Wiesbaden: VS, S. 30-41.

Rose, Nicolas (1999 [1989]): Governing the Soul. The Shaping of the Private Self, London: Free Association Books.

Rutherford, Jonathan (1990): »The Third Space. Interview with Homi Bhabha«, in: Ders. (Hg.): Identity: Community, Culture, Difference, London: Lawrence and Wishart: 207-221.

Scholliers, Peter (Hg.) (2001): Food, Drink and Identity. Cooking, Eating and Drinking in Europe since the Middle Ages, Oxford/New York: Berg.

Schütz, Alfred (1972): Gesammelte Aufsätze. Band 2: Studien zur soziologischen Theorie, hg. von Arvid Brodersen, Den Haag: Nijhoff.

Schütz, Alfred (1971): »Der Fremde. Ein sozialpsychologischer Versuch«, in: Ders.: Gesammelte Aufsätze, Band 2, Den Haag: Martinus Nijhof, S. 53-69.

Simmel, Georg (1908): Soziologie. Untersuchungen über die Formen der Vergesellschaftung, Duncker \& Humblot: Berlin.

Ståhl, Timo/Wismar, Matthias/Ollila, Eeva/Lahtinen, Eero/Leppo, Kimmo (Hg.) (2006): Health in All Policies. Prospects and Potentials, Ministry of Social Affairs and Health, Finland \& European Observatory on Health Systems and Policies. URL: www.euro.who.int/document/E89260.pdf (15.01. 2010)

STATEC (2009a): L'économie luxembourgeoise. Un kaléidoscope 2008, Luxembourg.

STATEC (2009b): Le secteur public. Economie et Statistiques, Working papers du STATEC 34.

Stichweh, Rudolf (2005): Inklusion und Exklusion. Studien zur Gesellschaftstheorie, Bielefeld: transcript. 
Wagener, Yolande (2008): »Promotion de la santé au Luxembourg«, in: Promotion \& Education, Hors-Série Promotion de la santé: Besoin de recherche francophone et perspectives, $\mathrm{N}^{\circ}$, S. 22-25.

Weischer, Christoph (2007): Sozialforschung, Weinheim: Beltz.

Welsch, Wolfgang (2005): »Auf dem Weg zu transkulturellen Gesellschaften«, in: Lars Allolio-Näcke/Britta Kalscheuer/Arne Manzeschke (Hg.): Differenzen anders denken. Bausteine zu einer Kulturtheorie der Transdifferenz, Frankfurt a.M.: Campus, S. 314-341.

World Health Organisation Europe (WHO) (1999): Santé 21. La politique-cadre de la Santé pour tous dans la Région européenne de l'OMS. Série européenne de la Santé pour tous, $\mathrm{N}^{\circ} 6$.

World Health Organisation Europe (WHO) (2008): Inequalities in Young People's Health. Health Behaviour in School-Aged Children (HBSC). International Report from the 2005/2006 Survey. Health policy for children and adolescents, $\mathrm{N}^{\circ} 5$. 



\title{
8. Identitätskonstruktionen in Luxemburg
}

\author{
Rachel Reckinger, Christian Schulz, Christian Wille
}

Das Augenmerk dieses Buchs lag auf Konstruktionen von Identitäten in Luxemburg. Diese bewusst >breite< Formulierung deutet an, dass nicht primär nationale Identität(en) oder kulturelle Identität(en) im Zentrum standen, sondern Prozesse der subjekt- und akteursbezogenen alltagsweltlichen »Identitätsarbeit« (Keupp et al. $\left.{ }^{32006}\right)^{1}-$ was wir mit >Doing Identity< begrifflich fassen. Vor diesem Hintergrund wurden vier besonders relevante Themenbereiche näher untersucht: die eher auf Aushandlungsebene anzusiedelnden sprachlichen und alltagskulturellen Praktiken einerseits sowie die eher auf Repräsentationsebene vorzufindenden Raumkonstruktionen und Konfrontationen zwischen Selbst- und Fremdwahrnehmungen andererseits. Diese vier Themenfelder, die den Fokus jeweils auf Sprachen, Räume, Bilder und Alltagskulturen legen, wurden systematisch mit dem Querschnittsthema der Sozialstrukturanalyse nach Milieus (Vester et al. 2001) in Verbindung gebracht. Ein besonderes Anliegen bestand darin, den dynamischen und dialogischen Charakter von Identitätsangeboten und -konstitutionen zu erfassen. Daher wurden in den jeweiligen Kapiteln - mit unterschiedlicher Gewichtung - politisch-medial erwünschte bzw. projizierte Zuschreibungen von Identitäten (Identifikationen) und gelebte Aneignungen von Identitäten (Identifizierungen) berücksichtigt.

Diese konzeptionelle Annäherung in der Perspektive des >Doing Identity< verweist stets auf die Dimension der Gewordenheit und Wandelbarkeit von Identitäten. So wird mit Blick auf sprachliche Identitäten unweigerlich deutlich, dass eine Gleichsetzung zwischen Sprachgebrauch und Identitäten zu einer verkürzten Sichtweise führt. Vielmehr erlaubt die vorgenommene Betrachtung des praktizierten Sprachkonsenses bzw. der öffentlich verhandelten Positionen über Sprache einen Einblick in Identitätskonstruktionen, die nicht auf eine einfache Formel gebracht werden können. Sie reflektieren z.T. divergente Verhältnisse zwischen zugeschriebenen und angeeigneten Identitäten, verschiedene Praktiken der Mehrspra-

1 | Vgl. Abschnitt 2.1. 
chigkeit und ein breites Spektrum an Vorstellungen über >gute< bzw. >schlechte< Mehrsprachigkeit. Die Autoren/-innen kommen schließlich zu dem Schluss, dass eine erhöhte Kenntnisnahme und gesellschaftliche Akzeptanz der verschiedenen herausgearbeiteten sprachlichen Normen und mehrsprachigen Konstellationen einen wesentlichen Beitrag zur sozialen Kohäsion in Luxemburg leisten können. Des Weiteren wurde anhand von sprachlichen und visuellen Bildern den symbolischen Repräsentationen von Identitäten nachgegangen. Die hierfür untersuchten Kommunikationsbereiche im Kontext ausgewählter medialer Ereignisse fungierten als Vektoren für simagined communities < bzw. für verschiedene Konstruktionen Luxemburgs, die auf einem breiten Fundus an Kollektivsymbolen basieren. Bedingt durch gesellschaftlichen Wandel und Pluralität unterliegen ihre Konstitutionsmechanismen und Funktionsweisen zugleich Kontinuitäten und Diskontinuitäten, die sich medial artikulieren. Das als vielfältig zu charakterisierende Verhältnis zwischen zugeschriebenen und angeeigneten Identitäten wird in Luxemburg über diese mediale Applikation bestimmt. Ferner arbeiteten die Autoren/-innen unter dem Stichwort >Geo-grafien < unterschiedliche raumbezogene Konstruktionen Luxemburgs heraus und hinterfragten auf verschiedenen Maßstabsebenen die hier in Erscheinung tretenden Identitäten. Als Kristallisationspunkte von räumlichen Identitäten wurden zunächst die im touristischen Diskurs artikulierten Repräsentationen und semiotischen Strategien herangezogen. Dabei wurde deutlich, dass die Identitätskonstruktionen nach den betrachteten räumlichen Ebenen z.T. sehr heterogen sind, sie jedoch stets - und ebenso in diachroner Perspektive - auf eine gewisse Einzigartigkeit, Außergewöhnlichkeit und insbesondere Vielfältigkeit verweisen, die sich mit der Vorstellung einer > Einheit Luxemburg < kontrastierend und ambivalent verstrickt. Das beleuchtete Verhältnis zwischen angeeigneten und zugeschriebenen Identitäten ist angesichts des idealisierenden Charakters des öffentlichen touristischen Diskurses einerseits und der Repräsentationen bzw. sozialen Praktiken der Luxemburger Wohnbevölkerung andererseits als divergent zu charakterisieren. Solche Divergenzen sowie Ambivalenzen von Identitätskonstruktionen wurden schließlich auch in verschiedenen Bereichen des Alltagskulturellen vorgefunden. Hinsichtlich des Verhältnisses zwischen angeeigneten und zugeschriebenen Identitäten im Kontext unterschiedlicher Vorstellungen von >gutem< Essen konnte eine idealisierende Typologie (Adaptation, Opposition, Autonomie) herausgearbeitet werden. Diese reflektiert widersprüchliche >Logiken des Alltags ebenso wie das (Er-)Leben von Geschlechterrollen oder die Wahrnehmung von Grenzgängern/-innen, die jeweils binäre >Entweder-oder-Logiken< pragmatisch aufbrechen und nur aus einer Perspektive des >Sowohl-als-auch< zugänglich werden.

Die hier schlaglichtartig resümierten Forschungsergebnisse verweisen deutlich auf den Charakter von Identitäten: Die in Luxemburg empirisch erforschten Identifizierungen, Identifikationen und Identitäten sind so vielfältig wie komplex. Demnach können griffige und somit homogenisierende, statische und verkürzte Gleichungen - insbesondere hinsichtlich kollektiver Identität(en) - immer nur 
normativ und ideologisch ausfallen. Daher verzichten wir auf pauschalisierende Zusammenfassungen und verweisen auf die ermittelten konstitutiven Ambivalenzen von Identitätskonstruktionen. Zugeschriebene und angeeignete Identitätsmuster weisen generell mehr oder weniger ausgeprägte Diskrepanzen auf, die sich aus der Interaktion bzw. aus dem kommunikativen Verhältnis dieser Kategorien ableiten. Ferner sind die jeweiligen Identifikationsangebote bzw. Identifizierungspraxen als solche wandelbar, plural und polysemisch (und somit potentiell widersprüchlich). Insofern bergen Identitäten - als permanente, aber stets vorläufige Resultanten der genannten kommunikativen Prozesse - immer Infragestellungen und Veränderungspotential. Aufgrund der sozio-kulturellen Milieuzugehörigkeiten und der jeweiligen Ressourcen der Subjekte kann es sich dabei immer snur< um »Veränderung in Grenzen « (Straub 2004: 284) handeln.

Diese Dynamiken können wohlwollend (z.B. bei Tourismusdiskursen oder Werbung), kritisch (z.B. bei Genderkonstruktionen, Ernährungsnormen und -praxen, künstlerischen oder publizistischen Hinterfragungen) oder konfliktgeladen (z.B. beim Grenzgängerwesen oder bei Mehrsprachigkeit) sein. Das größte Konfliktpotential haben solche Aspekte gesellschaftlichen Zusammenlebens, die metonymisch abstrahiert werden und als konsensfähige, weil polysemische, d.h. unterschiedliche Perspektiven vereinende, Kollektivsymbole für politisch-soziale Zwecke genutzt werden können. Daher erlangt das Thema Sprache hier die aktuell höchste Brisanz. Es scheint den gesellschaftlichen Wandel Luxemburgs und die daran gekoppelten Ängste zu kondensieren, gleichwohl das Luxemburgische noch nie von so vielen Menschen wie derzeit gesprochen worden ist. Insbesondere für konfliktgeladene Bereiche gilt demnach: »Identität wird nur in ihrer Krise zum Problem« (Eickelpasch/Rademacher 2004: 5), da es hier stets um subjektive Wahrnehmungen, Zugehörigkeitsgefühle, Selbsteinschätzungen geht und weniger um objektive $>$ Fakten $<$. Dennoch ist das > Erarbeiten < von Identitäten seitens der Subjekte als eine Kontinuitätsleistung und keinesfalls als etwas substantiell Anhaftendes aufzufassen. Dieses Selbstverhältnis wird sowohl in synchronen als auch in diachronen Gesellschafts- und Welterfahrungen deutlich und narrativ verarbeitet, kommuniziert und tradiert. Dieser Prozess des >Doing Identity < zielt auf eine »flexible Kontinuität und Sinnhaftigkeit des Selbst« ab und dient weniger der Abbildung oder Beschreibung von objektiven >Fakten<. (Straub 2004: 284-286)

Die in diesem Buch gebündelten Ergebnisse der dreijährigen interdisziplinären Forschungsarbeit ${ }^{2}$ spiegeln das breite Spektrum an Identitätsangeboten in Lu-

2 | Das Projekt profitierte in hohem Maße von dem intensiven Austausch unter Forschenden unterschiedlicher Disziplinen sowie von der interaktiv gestalteten Wissenschaftskultur innerhalb der Forschungseinheit IPSE (Identités, Politiques, Sociétés, Espaces) an der Fakultät für Sprachwissenschaften und Literatur, Geisteswissenschaften, Kunst und Erziehungswissenschaften (FLSHASE) der Universität Luxemburg. In diesen Kontext wird auch ein in Vorbereitung befindliches Folgeprojekt eingebettet sein, das sich mit der grenzüberschreitenden Dimension von Identitätskonstruktionen beschäftigen wird. 
xemburg ebenso wider wie die Pluralität und Wandelbarkeit von Identifizierungspraxen. Hierbei verlieren die scheinbar traditionellen und Orientierung gebenden Ordnungskategorien ihre Bedeutung. Fragen des sozialen Zusammenhalts können sich daher - in Luxemburg und darüber hinaus - nicht länger an einer Vorstellung von Gesellschaften als normativ integrierte und homogene Kollektive ausrichten, vielmehr gilt es, sich gesellschaftlicher Pluralität und Binnennuancierungen zu öffnen und diese anzuerkennen.

Aus der Perspektive einer kritischen Begleitforschung von aktuellen gesellschaftlichen Entwicklungen müssen Fragen des sozialen Zusammenhalts somit auf die Beschaffenheit und Dynamik von gesellschaftlichen Wechselwirkungen und auf das Ineinandergreifen unterschiedlicher Identitätsentwürfe in der kulturellen Praxis abstellen. Mit den hier dargestellten Ergebnissen des Forschungsprojekts »IDENT - Sozio-kulturelle Identitäten und Identitätspolitiken in Luxemburg« hoffen die Beteiligten nicht zuletzt dazu beizutragen, die gegenwärtig intensive politische, gesellschaftliche und mediale Debatte über Identitätsfragen in Luxemburg und andernorts für differenzierendere Betrachtungen und weniger reduktionistische Argumentationsmuster zu sensibilisieren, in denen gesellschaftliche Vielfalt und multiple Identitätsmuster eher als $>$ Normalfall $<$ denn als $>$ Problem $<$ interpretiert werden. 


\section{Autoren/-innen}

Wilhelm Amann (Dr.) ist Germanist an der Universität Luxemburg. Seine Arbeitsschwerpunkte sind kulturelle Globalisierung/Regionalisierung, Diskursanalyse. Mit Georg Mein u. Rolf Parr ist er Mitherausgeber von »Gegenwartsliteratur und Globalisierung. Konstellationen - Konzepte - Perspektiven« (Synchron Verlag 2010).

Christel Baltes-Löhr (Prof. Dr.) ist universitäre Genderbeauftragte sowie Leiterin der Abteilung Gender-Studies. Sie koordiniert den »European Migration Network - National Contact Point - Luxembourg« der EU-Kommission und vertritt Luxemburg als Gender-Expertin in der EU-Helsinki Group »Women and Science«.

Viviane Bourg ist Hauptdozentin für Kunst und ihre Didaktik im Bachelorstudiengang Erziehungswissenschaften an der Universität Luxemburg. Ihre Arbeitsschwerpunkte sind Kunsterziehung in der Schule und ästhetisch-biografische Forschung in der Lehrer/-innenausbildung.

Marion Colas-Blaise (Prof. Dr.) unterrichtet französische Linguistik und Semiotik an der Universität Luxemburg. Sie hat zahlreiche Veröffentlichungen über literarische und visuelle Semiotik, Stilistik und Diskursanalyse publiziert. Sie hat kürzlich als Koautorin das Werk « Le sens de la métamorphose » (2009) veröffentlicht.

Paul Dell ist Dozent und Forscher für Visuelle Künste an der Universität Luxemburg. Bei seiner Forschungstätigkeit innerhalb des »Laboratoire d'Arts Visuels« bzw. IPSE liegt der Fokus auf zeitgenössischer Kunst. Des Weiteren beschäftigt er sich mit Medien und Mediationstechniken im Bereich Kunst.

Fernand Fehlen ist Dozent an der Universität Luxemburg mit dem Schwerpunkt empirische Sozialforschung. Soziolinguistik, Wahlforschung und Sozialstrukturanalyse bilden die drei Schwerpunkte seiner Arbeit. Zuletzt hat er die soziolinguistische Studie »Luxemburgs Sprachenmarkt im Wandel« (Sesopi 2009) veröffentlicht. 
Paul Di Felice ist Hauptdozent der Kunstgeschichte und Kunstdidaktik an der Universität Luxemburg wo er auch für das «Laboratoire d'arts visuels« verantwortlich ist. Als Kunstkritiker (Mitglied der AICA) publiziert er vor allem zur Entwicklung der zeitgenössischen Fotografie auf «lacritique.org«.

Sylvie Freyermuth (Prof. Dr.) lehrt französische Sprache und Literatur an der Universität Luxemburg. Ihre Forschungsarbeiten und Veröffentlichungen befinden sich an der Schnittstelle zwischen Literatur und Linguistik. Sie ist Spezialistin für das Werk des französischen Romanautors Jean Rouaud.

Peter Gilles (Prof. Dr.) ist Professur für Linguistik an der Universität Luxemburg. Seine Hauptarbeitsgebiete sind Phonetik/Phonologie, Variationslinguistik und Soziolinguistik des Luxemburgischen.

Sonja Kmec (Prof. Dr.) lehrt Geschichte und Kulturwissenschaften an der Universität Luxemburg. Im Bereich der Erinnerungsforschung hat sie zusammen mit Michel Margue, Pit Péporté und Benoît Majerus »Lieux de mémoire au Luxembourg « (2007) herausgegeben und »Inventing Luxembourg« (2010) verfasst.

Fabienne Lentz promoviert an der Universität Luxemburg zum Thema »Italienische Einwanderung in Luxemburg: zwischen privater Erinnerung und öffentlichen Repräsentationen«. Sie beschäftigt sich desweiteren mit der Geschichte der europäischen Integration gebunden an die Frage der nationalen Identitäten.

Georg Mein (Prof. Dr.) ist Professor für Neuere deutsche Literaturwissenschaft an der Universität Luxemburg. Seine Arbeitsschwerpunkte sind Literatur vom 18. Jahrhundert bis in die Gegenwart, Medien und Kulturtheorien, Literatursoziologie, Literalitätsforschung.

Agnès Prüm ist Dozentin für Englische Literatur an der Universität Luxemburg. Arbeitsgebiete: Interaktion zwischen Erzählung und Identitätkonstruktionen, Gender, Utopien, Dystopien, spekulative Fiktion. Publikationen im Bereich Popular Culture über CSI und Frankenstein als Motive westlicher Kulturen.

Rachel Reckinger (Dr.) ist Soziologin und arbeitet als wiss. Projektkoordinatorin an der Universität Luxemburg. Sie forscht und veröffentlicht in den Bereichen Identitätskonstruktionen, Ernährung, Nahrungsherstellung, Konsum, Expertise und Politik, Ethik und Moral, Nachhaltigkeit sowie Gouvernementalität.

Sebastian Reddeker (M.A.) ist Doktorand an der Universität Luxemburg. Er forscht schwerpunktmäßig in den Bereichen Luxemburger Werbediskurs, Identität und Werbung, sowie Normalismus- und Interdiskurstheorie. 
Christian Schulz (Prof. Dr.) ist Leiter der Forschungseinheit IPSE an der Universität Luxemburg. Seine Arbeitsschwerpunkte als Geograph liegen im Bereich der grenzüberschreitenden Regionalentwicklung und umweltorientierten Wirtschaftsgeographie. Er ist Mitherausgeber des »Atlas du Luxembourg« (2009).

Sebastian Seela (M.A.) ist Assistent am Institut für deutsche Sprache, Literatur und Interkulturalität an der Universität Luxemburg. Er unterrichtet Mittelhochdeutsch und Linguistik und promoviert zu den Tagebüchern Victor Klemperers an der Schnittstelle von Linguistik und Sozialwissenschaften.

Heinz Sieburg (Prof. Dr.) ist Professor im Bereich der germanistischen Linguistik und Mediävistik. Er forscht und publiziert u.a. zur historischen Wortbildung im mittelfränkisch-luxemburgischen Raum. Jüngst erschienen ist sein Buch »Literatur des Mittelalters« (Berlin 2010).

Gian Maria Tore (Dr.) forscht über Medien-, Film- und Kunstsemiotik an der Universität Luxemburg. Er lehrt an der Universität Metz und arbeitet regelmäßig mit der Cinémathèque de Luxembourg zusammen. Er ist Mitherausgeber von »L'expérience. En sciences de l'homme et de la société«(Limoges, Pulim 2006).

Christian Wille (M.A.) ist wiss. Projektkoordinator an der Universität Luxemburg. Er arbeitet über Raum- und Identitätskonstruktionen in europäischen Grenzräumen und publiziert zur Interkulturellen Kommunikation, grenzüberschreitenden Zusammenarbeit und Arbeitnehmermobilität in der Großregion SaarLorLux.

Melanie Wagner (Dr.) ist Postdoktorandin im »Laboratoire de linguistique et de littératures luxembourgeoises « an der Universität Luxemburg. Sie forscht hauptsächlich im Bereich Soziolinguistik und veröffentlicht zu soziolinguistischen Themen wie Sprachvariation, Normbewusstein, Spracheinstellungen und -ideologien. 


\section{Kultur und soziale Praxis}

Sylko tartana.

Alivar ianat tho.]

Das Vertraute

und das Fremids

aifterenuertahruas und

frontvarstahan is

Interkulturalltatsdinkers

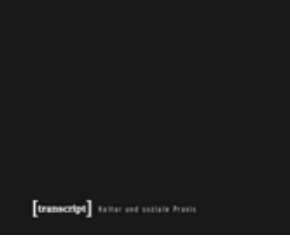

Gatrialo tapual

Bungo stinats.

daruan strant (In).

Interpretative

Sozialforsehung

und Kulturanalyse

Itrmentutik un alo

Innaration Analyas

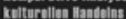
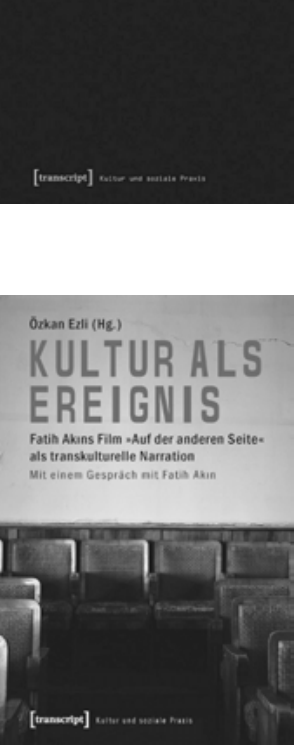

ÖZKan Ezli (Hg.)

Kultur als Ereignis

Fatih Akins Film »Auf der anderen Seite« als transkulturelle Narration

August 2010, ca. I50 Seiten, kart., ca. 22,80 €, ISBN 978-3-8376-1386-5 


\section{Kultur und soziale Praxis}

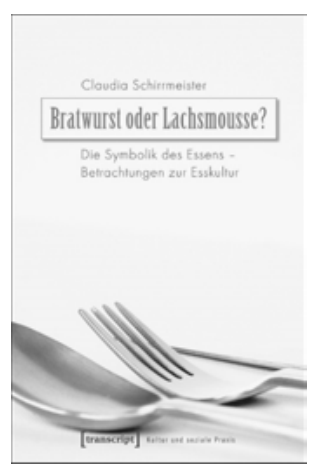

Claudia Schirrmeister

Bratwurst oder Lachsmousse?

Die Symbolik des Essens -

Betrachtungen zur Esskultur

November 20IO, ca. 204 Seiten, kart., ca. 2I,80€, ISBN 978-3-8376-1563-0

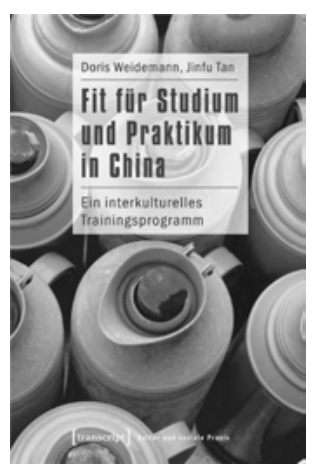

Doris Weidemann, Jinfu Tan

Fit für Studium und Praktikum in China

Ein interkulturelles Trainingsprogramm

August 20I0, ca. 200 Seiten, kart., ca. $16,80 €$, ISBN 978-3-8376-I465-7

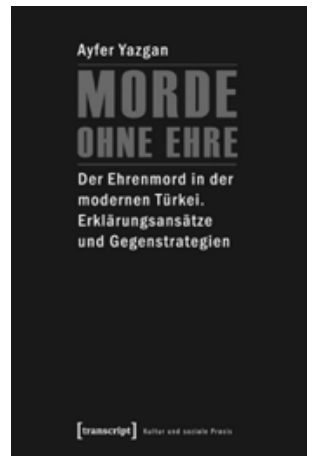

Ayfer Yazgan

Morde ohne Ehre

Der Ehrenmord in der modernen Türkei. Erklärungsansätze und Gegenstrategien

September 20Io, ca. 366 Seiten, kart., zahlr. Abb., ca. $29,80 €$,

ISBN 978-3-8376-1562-3 


\section{Kultur und soziale Praxis}

Aida Bosch

\author{
Konsum und Exklusion \\ Eine Kultursoziologie der Dinge \\ Januar 20I0, 504 Seiten, kart., \\ zahlr. farb. Abb., 33,80€, \\ ISBN 978-3-8376-I326-I
}

Anne Broden, Paul Mecheril (Hg.)

Rassismus bildet

Bildungswissenschaftliche Beiträge $\mathrm{zu}$

Normalisierung und Subjektivierung

in der Migrationsgesellschaft

Mai 20Io, 294 Seiten, kart., 28,80€,

ISBN 978-3-8376-I456-5

\section{Nesrin Z. Calagan}

Türkische Presse in Deutschland

Der deutsch-türkische Medienmarkt und seine Produzenten

August 20Io, ca. 246 Seiten,

kart., ca. $29,80 €$,

ISBN 978-3-8376-I328-5

LUCYNA DAROWSKa,

THOMAS LÜTTENBERG,

Claudia Machold (Hg.)

Hochschule als transkultureller

Raum?

Kultur, Bildung und Differenz

in der Universität

August 20I0, ca. I46 Seiten, kart., ca. I9,80€, ISBN 978-3-8376-I375-9

\section{KATHRIN DÜSENER}

Integration durch Engagement?

Migrantinnen und Migranten

auf der Suche nach Inklusion

Januar 20Io, 290 Seiten,

kart., zahlr. Abb., 29,80€,

ISBN 978-3-8376-II88-5

JÖRG GERTEL

Globalisierte Nahrungskrisen

Bruchzone Kairo

Juli 2oro, 458 Seiten,

kart., zahlr. Abb., 35,80€,

ISBN 978-3-8376-III4-4
Jörg Gertel, Ingo Breuer (Hg.)

Alltags-Mobilitäten

Aufbruch marokkanischer

Lebenswelten

Dezember 20Io, ca. 350 Seiten,

kart., zahlr. Abb., ca. 29,80 €,

ISBN 978-3-89942-928-2

Martina Grimmig

Goldene Tropen

Zur Koproduktion natürlicher

Ressourcen und kultureller

Differenz in Guayana

September 2010, ca. 320 Seiten, kart., ca. $34,80 €$,

ISBN 978-3-89942-75I-6

Anne-Christin Schondelmayer Interkulturelle Handlungskompetenz

Entwicklungshelfer und

Auslandskorrespondenten in Afrika.

Eine narrative Studie

August 2010, ca. 402 Seiten,

kart., ca. $34,80 €$,

ISBN 978-3-8376-II87-8

\section{Tina Spies}

Migration und Männlichkeit

Biographien junger Straffälliger

im Diskurs

Oktober 20Io, ca. 430 Seiten,

kart., ca. $34,80 €$,

ISBN 978-3-8376-I5I9-7

Asta Vonderau

Leben im »neuen Europa«

Konsum, Lebensstile und

Körpertechniken im Postsozialismus

Juni 20Io, 238 Seiten, kart., 26,80 €,

ISBN 978-3-8376-II89-2 